\title{
ECOLOGICAL EVALUATION OF PROPOSED DISCHARGE OF DREDGED MATERIAL FROM OAKLAND HARBOR INTO OCEAN WATERS (PHASE III B OF -42-FOOT PROJECT)
}

Volume 2 - Appendixes
N. P Kohn
J. A. Ward
H. L. Mayhew
J. Q. Word
E. S. Barrows
S. M. Goodwin
L. F. Lefkovitz

Battelle/Marine Sciences Laboratory

Sequim, Washington

June 1992

Prepared for the U.S. Army Corps of Engineers under a Related Services Agreement with the U.S. Department of Energy

Contract DE-AC06-76RLO 1830

Pacific Northwest Laboratory

Richland, Washington 99352

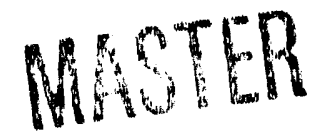




\section{PREEACE}

This is Volume 2 of a two-volume report that presents information gathered to determine the suitability' of ocean disposal of sediments dredged from Oakland Hartor. Volume 1 contains project background, materials and methods, results, discussion, and conclusions. This volume contains the Appendixes ( $A$ through $N$ ), which provide details of the data analyses and full presentation of the data and results. 


\section{SUMMARY}

The Water Resources Development Act of 1986 (Public Law 99-662) authorized the U.S. Army Corps of Engineers (USACE) San Francisco District, to deepen and widen the navigational channels of the Oakland Inner and Outer Harbors to accomodate deeper-draft vessels. The USACE is considering several disposal options for the dredged material removed during these channel improvements including open-water disposal. Dredged material proposed for open-water disposal must be evaluated to determine the porential impacis of the disposal activity on the water column and disposal site environments. The USACE requested that Battelle/Marine Sciences Laboratory (MSL) conduct studies to evaluate open-water disposal options for Oakland Harbor sediments. This request developed into the Oakland Harbor Phase III Program.

Because of the number of sites and associated evaluations, Phase III was divided into three projects: Oakland Harbor Phase II A (Inner Harbor deepening from - $38 \mathrm{ft}$ to $-42 \mathrm{ft}$ MLLW), Phase III B (Outer Harbor deepening to $-42 \mathrm{ft}$ MLLW), and Phase III $38 \mathrm{ft}$ (Inner Harbor deepening from $-35 \mathrm{ft}$ to $-38 \mathrm{ft}$ MLLW). This report summarizes the collection, chemical analysis, toxicity testing, and bioaccumulation analysis of sediments conducted during the Oakland Harbor Phase III B Project, initiated in November 1990.

During Phase III B, sediments representing dredged material from 18 Oaklarld Harbor stations were tested with and compared to sediments from six reference areas representing potential disposal sites (in the absence of a single designated disposal site). Core samples of sediment targeted for potential dredgirg operations were collected from the Oakland Harbor stations, while surface sediments were collected from reference and control sites. Test treatments (potential dredged material), reference treatments, and control treatments were tested for physical and chemical parameters, water column effects, dredged sediment toxicity, and bioaccurnulation potential.

Physical and chemical analyses of sediment consist $\mathcal{A}$ of conventional parameters [grain size, total volatile solids (TVS), total organic carbon (TOC), oil and grease, and total petroleum hydrocarbons (TPH)], metals, polynuclear aromatic hydrocarbons (PAH), chlorinated pesticides and polychlorinated biphenyls (PCB), and buryltin compounds. The results of the physical analyses for sediment conventionals showed that the highest concentrations of these parameters were present in test treatments $\mathrm{O}-\mathrm{C} 7, \mathrm{O}-\mathrm{C} 6, \mathrm{O}-\mathrm{C} 8$, reference treatment $\mathrm{R}-\mathrm{BF}$, and $\mathrm{O}-\mathrm{Cl} 3$ (in descending order). Generally, the finer-grained sediments were associated with higher TOC, TVS, oil and grease, and TPH levels. Results of chemical analyses showed some interesting and 
similar trends for some parameters. Test treatments $\mathrm{O}-\mathrm{C} 8, \mathrm{O}-\mathrm{C} 7, \mathrm{R}-\mathrm{BF}$, and $\mathrm{O}-\mathrm{Cl} 3$ hat the highest total metal concentrations of all samples. Levels of organic compounds, determined by the sum of butyltin compounds, Aroclor 1254, and PAH concentrations were found to be the highest in sediment treatments $\mathrm{O}-\mathrm{C} 13, \mathrm{O}-\mathrm{C} 6, \mathrm{O}-\mathrm{C} 8$, and $\mathrm{O}-\mathrm{C7}$. Chlorinated pesticides and PCBs other than Aroclor 1254 were not present in significant amounts in any of the sediments tested. In contrast to the consistently high contaminant concentrations in sediment treatments $\mathrm{O}-\mathrm{C} 6, \mathrm{O}-\mathrm{C} 7$, $\mathrm{O}-\mathrm{C} 8$, and $\mathrm{O}-\mathrm{C13}$, four treatments consistently showed low concentrations of the parameters measured: O-C5, O-C9, O-C12, and I-C3.

To evaluate water column effects, suspended-particulate-phase (SPP) tests were conducted using the mysid shrimp Holmesimysis sciupta, the speckled sanddab Citharichthys stigmaeus, and larvae of the mussel Mytilus edulis. SPP tests of four sediment composites representing the bulk of Outer Harbor sediments showed that none were acutely toxic to the species tested. To evaluate dredged sediment toxicity, solid-phase toxicity tests were conducted using the bent-nose clam Macoma nasuta, the polychaete worm Nephtys caecoides, the speckled sanddab C. stigmaeus, and the amphipod Rhepoxynius abronius. Acute toxicity was noted in two of the solid-phase tests: the 10-day $R$. abronius test and the 10-day $N$. caecoides test. Significant decreases in $R$. abronius and $N$. caecoides survival were observed in certain test treauments, depending on the choice of reference treatment used for comparison. Treatment $\mathrm{O}-\mathrm{Cl}$ showed significant $R$. abronius mortality relative to all six reference treatments; $\mathrm{O}-\mathrm{Cl}$ was also the only treatment that was acutely toxic to both $R$. abronius and $N$. caecoides relative to a refer, nce treatment (R-AM).

Bioaccumulation was evaluated by exposing $M$. nasuta and $N$. caecoides to solid-phase treatments for 28 days and then measuring the contaminants of concern present in their tissues. The concentrations of contaminants in the tissues were compared to existing Food and Drug Administration (FDA) limits, and were also compared using Dunn's Test to determine whether statistically significant $(\alpha=0.10)$ levels of contaminants were present in organisms exposed to iest sediments relative to those exposed to reference sediments. Bioaccumulation results showed that FDA limits, where available for compounds of interest, were not exceeded, but that statistically significant levels of contarninants were present in the tissues of $M$. masitua and $N$. caecoides exposed to test treatments relative to the six references. Three PAHs (raphthalene, fluorantherie, and pyrene) accumulated in $M$. nasufa and $N$. caecoides regardless , f which reference the test treatments were compared to. Metals aiso bioaccumulated in both $M$. nasura and $N$. caecoides from treatments relative to references, but the significant treatments were different for $M$. nasuta than for $N$. caecoides. The number and significance of the comparisons depends on the reference to which the test treatments are compared. 


\section{ACKNOWLEDGMENTS}

The authors thank Mike Barrows, Meg Pinza, Rhonda Karls, and Liam Antrim for the success of the laboratory testing. Also of valuable assistance in the laboratory were Larri Ann Smith, Terry Siebens, Vanessa Gauger, and Julianne Schindler. For many hours of careful data entry, proofreading, and preparation of data appendices we thank Mike Hamilton, Ginger Hawkins, Larri Ann Smith, Elisa Sanderson, Lisa Karle, Jenny Bridge, and Shawn Sanderson. We are especially indebted to Ginger Hawkins for her attention to detail in preparing appendix tables for final publication. Many thanks to Ron Thom, Dick Ecker, Rob Cuello, and Wayne Gorst for their careful reviews. Publication of this report would not have been possible without Michele Finney, who put it all together. 


\section{CONTENTS}

APPENDIX A MATERIALS AND METHODS USED FOR THE GEOLOGIC DESCRIPTIONS OF SEDIMENT CORES . . . . . . . . A.1

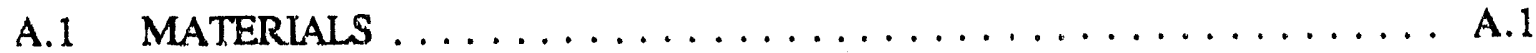

A.2 METHODS $\ldots \ldots \ldots \ldots \ldots \ldots \ldots \ldots \ldots \ldots \ldots \ldots \ldots \ldots \ldots \ldots \ldots \ldots$

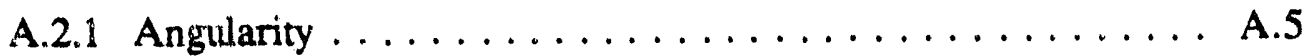

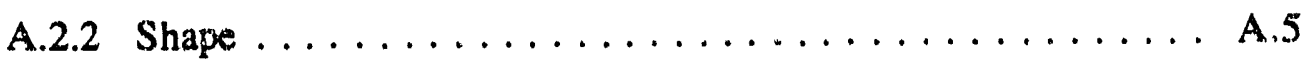

A. 2.3 Color $\ldots \ldots \ldots \ldots \ldots \ldots \ldots \ldots \ldots \ldots \ldots \ldots \ldots \ldots$

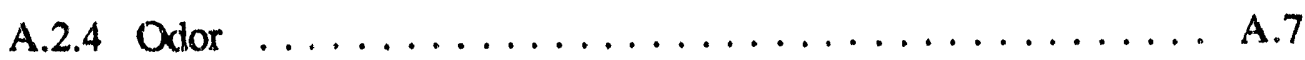

A.2.5 Moisture Condition $\ldots \ldots \ldots \ldots \ldots \ldots \ldots \ldots \ldots$ A.9

A.2.6 $\mathrm{HCl}$ Reaction $\ldots \ldots \ldots \ldots \ldots \ldots \ldots \ldots \ldots \ldots \ldots$

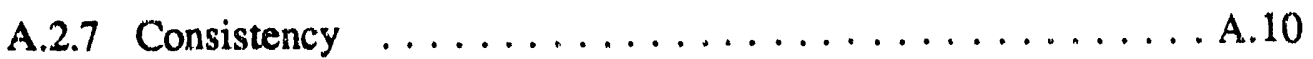

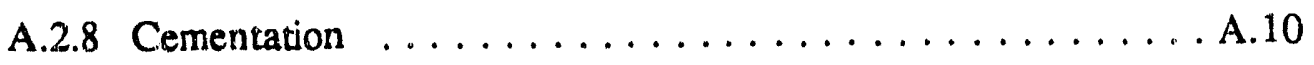

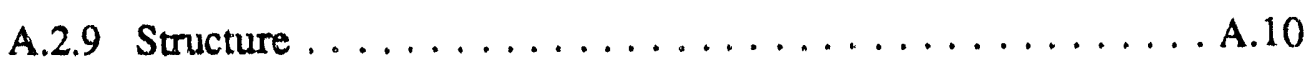

A.2.10 Sediment Classification Type . . . . . . . . . . . A.11

A.2.11 Range of Particle Size .................. A.18

A.2.12 Maxımum Particle Size . . . . . . . . . . . . . A.18

A.2.13 Dry Strength $\ldots \ldots \ldots \ldots \ldots \ldots \ldots \ldots \ldots \ldots \ldots \ldots \ldots$

A.2.14 Dilatancy ......................... A.19

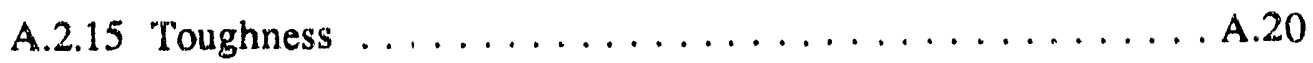

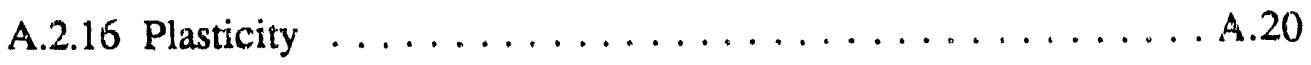

A. 3 REFERENCES $\ldots \ldots \ldots \ldots \ldots \ldots \ldots \ldots \ldots \ldots \ldots \ldots$ A. 22

APPENDIX B GEOLOGIC CORE DATA LOGS ............. B. 1

APPENDIX C SEDIMENT CHEMISTRY AND QUALITY CONTROL DATA C.1

APPENDIX D 10-DAY FLOW-THROUGH SOLID-PHASE TEST WTTH

N. CAECOIDES $\ldots \ldots \ldots \ldots \ldots \ldots \ldots \ldots \ldots$ D. 1 
APPENDIX E 28-DAY FLOW-THROUGH SOLID-PHASE TEST WITH

APPENDIX F 10-DAY STATIC SOLID-PHASE TEST WITH $R$. ABRONIUS . F.1

APPENDIX G 10-DAY FLOW-THROUGH SOLID-PHASE TEST

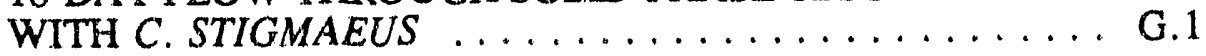

APPENDIX H 96-HOUR STATIC SUSPENDED-PARTICULATE-PHASE TEST WITH C. STIGMAEUS . . . . . . . . . . . . . H. 1

APPENDIX I 96-HOUR STATIC SUSPENDED-PARTICULATE-PHASE TEST WITH H. SCULPTA . . . . . . . . . . . . . . 1.1

APPENDIX J 48-HOUR STATIC SUSPENDED-PARTICULATE-PHASE TEST WITH LARVAL $M$. EDULIS $\ldots \ldots \ldots \ldots \ldots \ldots$ J. 1

APPENDIX $\mathrm{K} \quad$ N. CAECOIDES TISSUE CHEMISTRY AND QUALITY CONTROL DATA $\ldots \ldots \ldots \ldots \ldots \ldots \ldots \ldots \ldots \ldots \ldots \ldots$

APPENDIX L M. NASUTA TISSUE CHEMISTRY AND QUALITY CONTROL DATA $\ldots \ldots \ldots \ldots \ldots \ldots \ldots \ldots \ldots \ldots \ldots \ldots \ldots$

APPENDIX M STATISTICAL ANALYSIS OF M.NASUTA BIOACCUMULATION $\ldots \ldots \ldots \ldots \ldots \ldots \ldots \ldots . .1$

APPENDIX N STATISTICAL ANALYSIS OF N. CAECOIDES BIOACCUMULATION $\ldots \ldots \ldots \ldots \ldots \ldots \ldots \ldots$ N.I 


\section{EIGURES}

A.1 $\log$ Form Used to Record Sediment Descriptions $\ldots \ldots \ldots \ldots \ldots \ldots$. . . . .

A.2 Comparison Chart Used to Estimate the Percentages of Constituents . . . . . A.4

A.3 Charts Used to Visually Estimate (A) Roundness/Sphericity and

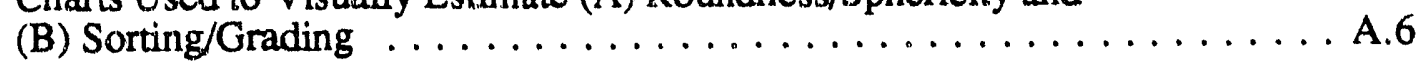

A.4 Criteria Used to Describe Particle Shape of Pebbles and Large Gravels . . . . A.7

A.5 Example of a Page from the Munsell Soil Color for Hue 7.5YR . . . . . . A.8

A.6 Abbreviated Form of the Unified Soil Classification System . . . . . . . . . A.12

A.7 Detailed Flow Chart For the Classification of Coarse- and Fine-Grained

Soils Using the Unified Soil Classification System . . . . . . . . . . . . . A.13

A.8 Grain-Size Scale Used to Determine Sedimentary Particle Size . . . . . . . . A.15

A.9 Comparison Chart Used to Distinguish Among Sand to Pebble-Size Particles A.16

A.10 Identification of Inorganic Fine-Grained Soils from Manual Tests . . . . . A. A.16

A.11 Lithologic Symbols Used on Core Log Forms . . . . . . . . . . . . . A.17

\section{TABLES}

A.1 Charts and Other Reference Materials Used to Provide Standardized

Descriptions of Sediment Characteristics . . . . . . . . . . . . A.3

A.2 Sediment Characteristics Identified in ASTM Procedure D2488-84 . . . . . A.3

C.1 Summary of Sediment Grain Size Including Quality Control Data . . . . . . . C. 1

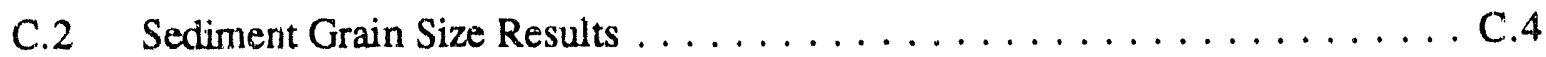

C.3 Quality Control Data for Sediment Grain Size Analysis . . . . . . . . . C.6

C.4 Sediment Total Organic Carbon (TOC) and Total Volatile Solids

(TVS) Results . . . . . . . . . . . . . . . . . . . . . . . .7

C.5 Quality Control Data for Sediment Total Organic Carbon (TOC) and

Total Volatile Solids (TVS) Analyses . . . . . . . . . . . . . . . . C.9

C.6 Sediment Oil and Grease and Total Petroleum Hydrocarbons (TPH) Results . C. 10 
C.7 Quality Control Data for Sediment Oil and Grease Total Petroleum

Hydrocarbons (TPH) Analysis . . . . . . . . . . . . . . . . 11

C.8 Total Polynuclear Aromatic Hydrocarbon (PAH) Found in Sediment

Treatments

C.9 Sediment Low Molecular Weight Polynuclear Aromatic Hydrocarbon

(LPAH) Results . . . . . . . . . . . . . . . . . . . . . . . . C 1 . 15

C.10 Sediment High Molecular Weight Polynuclear Aromatic Hydrocarbon

(HPAH) Results . . . . . . . . . . . . . . . . . . . . . . . . C.16

C.11 Quality Control Data for Sediment Low Molecular Weight Polynuclear

Aromatic Hydrocarbon (LPAH) Analysis . . . . . . . . . . . . C.18

C.12 Quality Control Dara for Sediment High Molecular Weight Polynuclear

Aromatic Hydrocarbon (HPAH) Results . . . . . . . . . . . . . . . . . C.20

C.13 Surrogate Recoveries for Sediment Polynuclear Aromatic Hydrocarbons

(PAH) Results . . . . . . . . . . . . . . . . . . . . . C.22

C. 14 Sediment Chlorinated Pesticide Results (alphabetical, Aldrin - 4,4'-DDT) . . C.24

C.15 Sediment Chlorinated Pesticides Results (alphabetical, Dieldrin -

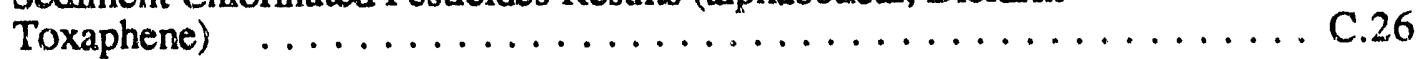

C.16 Quality Control Data for Chlorinated Pesticide Results (alphabetical,

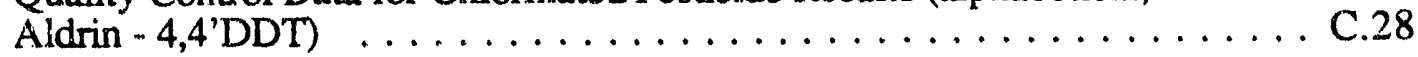

C.17 Quality Control Data for Chlorinated Pesticides (alphabetical, Dieldrin -

Toxaphene) $\ldots \ldots \ldots \ldots \ldots \ldots \ldots \ldots \ldots \ldots$. . . . . . . . . . . . .

C.18 Sediment Polychlorinated Biphenyl (PCB) Results . . . . . . . . . C.32

C.19 Quality Control Data for Polychlorinated Biphenyl (PCB) Analysis . . . . . C. C.33

C.20 Surrogate Percent Recoveries for Sediment Polychlorinated Biphenyls

(PCBs) and Chlorinated Pesticide Results . . . . . . . . . . . . . . . C.34

C.21 Sediment Metais Results $\ldots \ldots \ldots \ldots \ldots \ldots \ldots \ldots \ldots \ldots$. . . . . . . . .

C.22 Quality Control Data for Sediment Metals Analyses . . . . . . . . . C.38

C.23 Sediment Butyltin Results $\ldots \ldots \ldots \ldots \ldots \ldots \ldots \ldots \ldots \ldots$. . . . . . . . . .

C.24 Quality Control Data for Sediment Butyltin Analysis . . . . . . . . . . . . C.42

D.1 Test Results for All Replicates in 10-Day N. caecoides Solid-Phase Test . . . D.1

D.2 Rank Order Based on Mean Proportion Surviving 10-Day N. caecoides

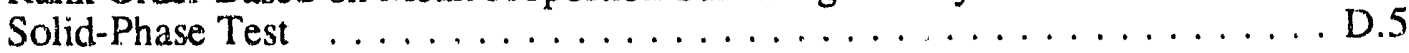

D.3 Water Quality Summary for 10-Day N. caecoides Solid-Phase Test . . . . . . D.6

PHASEIIIB $\quad$ xii 
D.4 Daily Observations of $N$. caecoides on Sediment Surface During 10-Day Solid-Phase Test . . . . . . . . . . . . . . . . . D.7

D.5 Daily Observations of $N$. caecoides Dead and Removed During 10-Day

Solid-Phase Test $\ldots \ldots \ldots \ldots \ldots \ldots \ldots \ldots \ldots \ldots \ldots$

E.1 Test Results for 28-Day $M$, nasuta Solid-Phase Test . . . . . . . . . . E.1

E.2 Rank Order Based on Mean Proportion Surviving 28-Day M. nasuta

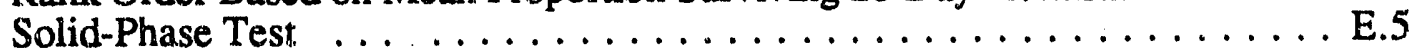

E.3 Test Results for 28 -Day $N$. caecoides Solid-Phase Test . . . . . . . . . E.6

E.4 Rank Order Based on Mean Proportion Surviving 28-Day N. caecoides

Solid-Phase Test ......................... E.10

E.5 Water Quality Summary for 28-Day M. nasuta/N. caecoides

Solid-Phase Test . . . . . . . . . . . . . . . . . . . . . E.11

E.6 Daily Observations of $M$. nasuta on Sediment Surface During 28-Day

Solid-Phase Test ........................ E.12

E.7 Daily Observations of $M$. nasuta Siphons Exposed During 28-Day

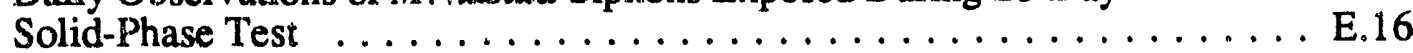

E.8 Daily Observations of $M$. nasuta Dead and Removed During 28-Day

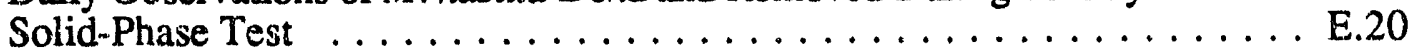

E.9 Daily Observations of $N$. caecoides on Sediment Surface During

28-Day Solid Phase-Test . . . . . . . . . . . . . . . . . . E.24

E.10 Daily Observations of $N$. caecoides Dead and Removed During 28-Day

Solid-Phase Test . . . . . . . . . . . . . . . . . . . . . . . . E.28

F.1 Test Results for All Replicates in 10-Day R. abronius Solid-Phase

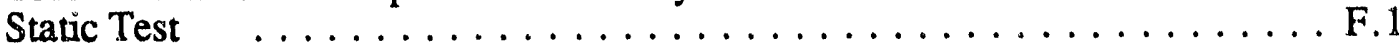

F.2 Rank Order Based on Mean Proportion Surviving for 10-Day R. abronius

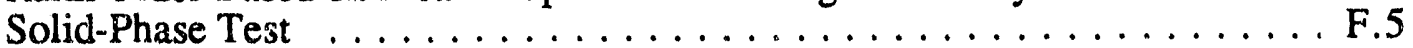

F.3 Water Quality Summary for 10-Day R. abronius Solid-Phase Test . . . . . F.6

F.4 Daily Observations of $R$. abronius on Sediment Surface During 10-Day Solid Phase Test . . . . . . . . . . . . . . . . F.7

F.5 Daily Observations of $R$. abronius Floating on Water Surface During

10-Day Solid-Phase Test . . . . . . . . . . . . . . . F.11

F.6 Survival Data for All Replicates in 4-Day R. abronius Reference

Toxicant Test .........................F.15

F.7 Water Quality Summary for 4-Day R. abronius Reference Toxicant Test . . F.16

PHASEIII B

xiii 
F.8 Daily Observations of $R$. abronius Floating on Water Surface

During 4-Day Reference Toxicant Test ................... F.17

G.1 Test Results for All Replicates in 10-Day C. stigmaeus Solid-Phase Test . . . G.1

G.2 Water Quality Summary for 10-Day C. stigmaeus Solid-Phase Test . . . . . . G.5

H.1 Survival Data for all Replicates in 4-Day C. stigmaeus Suspended-

Particulate-Phase Test $\ldots \ldots \ldots \ldots \ldots \ldots \ldots \ldots . \ldots \ldots$. . . . . . . . . . . .

H.2 Rank Order Based on Proportion Survival for 4-Day C. stigmaeus

Suspended-Particulate-Phase Test . . . . . . . . . . . . . . H.4

H.3 Water Quality Summary for 4-Day C. stigmaeus Suspended-

Particulate-Phase Test . . . . . . . . . . . . . . . . . . . H.5

H.4 Daily Observations of C. stigmaeus During 4-Day Suspended-

Particulate-Phase Test . . . . . . . . . . . . . . . . H.6

I.1 Survival Data for All Replicates in 4-Day H. sculpta Suspended-

Particulate-Phase Test . . . . . . . . . . . . . . . . . . . . I.1

I.2 Rank Order Based on Mean Proportion Surviving 4-Day H. sculpta

Suspended-Particulate-Phase Test ................... 1.4

I.3 Water Quality Summary for 4-Day $H$. scultpta Suspended-Particulate-

Phase Test $\ldots \ldots \ldots \ldots \ldots \ldots \ldots \ldots \ldots \ldots \ldots \ldots$. . . . . . . . . . . . .

I.4 Daily Observations of 4-Day $H$. scultpta Suspended-Particulate-Phase Test . I.6

I.5 Survival Data for All Replicates in 4-Day H. sculpta Reference Toxicant Test . I.9

I.6 Water Quality Summary for 4-Day H. sculpta Reference Toxicant Test . . . I.10

I.7 Daily Observations of 4-Day $H$. sculpto Reference Toxicant Test $\ldots \ldots$. . I.10

J. 1 Test Results for All Replicates in 48-Hour Larval M. edulis Suspended-

Particulate-Phase Test . . . . . . . . . . . . . . . . . . . . J.1

J.2 Survival Data for All Replicates in 48-Hour Larval M. edulis

Reference Toxicant Test . . . . . . . . . . . . . . . . . . . J.4

J.3 Water Quality Summary for 48-Hour Larval $M$. edulis Suspended-

Particulate-Phase Test . . . . . . . . . . . . . . . . . . J.5

J.4 Water Quality Summary for 48-Hour Larval $M$. edulis Reference

Toxicant Test ........................... J.6́

K.1 Total Detected Polynuclear Aromatic Hydrocarbons (PAHs), Dry Weight,

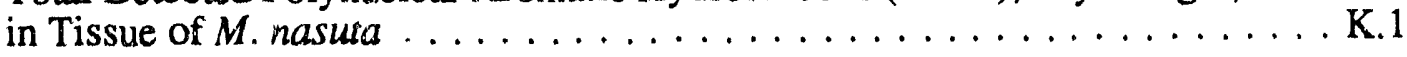

K.2 Low Molecular Weight Polyruclear Aromatic Hydrocarbons (LPAHs),

Dry Weight, in Tissue of $M$. nasuta . . . . . . . . . . . . K.4

PHASEIIIB 
K.3 High Molecular Weight Polynuclear Aromatic Hydrocarbons (HPAHs),

Dry Weight, in Tissue of $M$. nasuta $\ldots \ldots \ldots \ldots \ldots \ldots \ldots \ldots$

K.4 Total Detected Polynuclear Aromatic Hydrocarbons (PAHs), Wet Weight, in Tissue of $M$. nasuta . . . . . . . . . . . . . . . . . K.13

K.5 Low Molecular Weight Polynuclear Aromatic Hydrocarbons (LPAHs),

Wet Weight, in Tissues of $M$. nasuta . . . . . . . . . . . . K.17

K.6 High Molecular Weight Polynuclear Aromatic Hydrocarbons (HPAHs),

Wet Weight, in Tissue of $M$. nasuta . . . . . . . . . . . . . K.21

K.7 Quality Control Data for Low Molecular Weight Polynuclear Aromatic Hydrocarbons (LPAHs), Wet Weight, in Tissue of $M$. nasuta . . . . . K.26

K.8 Quality Control Data for High Molecular Weight Polynuclear Aromatic Hydrocarbons (HPAHs), Wet Weight, in Tissue of M. nasuta . . . . . . . K.29

K.9 Surrogate Recoveries for Polynuclear Aromatic Hydrocarbons (PAHs)

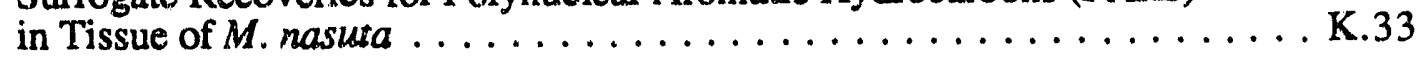

K.10 Chlorinated Pesticides (alphabetical, Aldrin - 4,4'-DDT), Dry Weight,

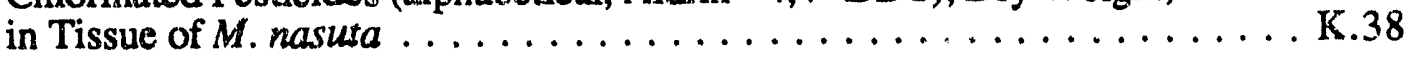

K.11 Chlorinated Pesticides (alphabetical, Dieldrin - Toxaphene), Dry Weight, in Tissue of $M$. nasuta . . . . . . . . . . . . . . . . . K.43

K.12 Chlorinated Pesticides (alphabetical, Aldrin - 4,4'-DDT), Wet Weight, in Tissue of $M$. nasuta

K.13 Chlorinated Pesticides (alphabetical, Dieldrin - Toxaphene), Wet Weight, in Tissue of $M$. nasuta . . . . . . . . . . . . . . . . . K.52

K.14 Quality Control Data for Chlorinated Pesticides (alphabetical, Aldrin - Dieldrin), Wet Weight, in Tissue of $M$. nasuta

K.15 Quality Control Data for Chlorinated Pesticides (alphabetical, Endosulfan I Toxaphene), Wet Weight, in Tissue of $M$. nasuta . . . . . . . . K.61

K.16 Polychlorianted Biphenyls (PCBs), Dry Weight, in Tissue of $M$. nasuta. Oakland Harbor Phase III B . . . . . . . . . . . . . . . . K.62

K.17 Polychlorinated Biphenyls (PCBs), Wet Weight, in Tissue of $M$. nasuta . . K.67

K.18 Quality Control Data for Polychlorinated Biphenyls (PCBs), Wet Weight, in Tissue of $M$. nasuta . . . . . . . . . . . . . . . . . K.72

K.19 Surrogate Percent Recoveries for Chlorinated Pesticides and Polychlorinated Biphenyls (PCBs) in Tissue of $M$. nasuta

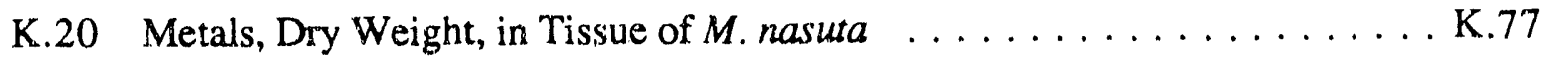

K.21 Metals, Wet Weight, in Tissue of $M$. nasuta $\ldots \ldots \ldots \ldots \ldots \ldots$ K. $\ldots 2$ 
K.22 Quality Control Data for Metals in Tissue of $M$. nasuta . . . . . . . . K.87

K.23 Butyltin Results, Dry and Wet Weight, in Tissue of $M$. nasuta . . . . . . K.92

K.24 Quality Control Data for Butyltins Analyses in Tissues of M. sasuru . . . . K.97

L.1 Total Detected Polynuclear Aromatic Hydrocarbons (PAHs), Dry

Weight, in Tissue of $N$, caecoldes . . . . . . . . . . . . . . L.1

L.2 Low Molecular Weight Polynuclear Aromatic Hydrocarbons (LPAHs),

Dry Weight, in Tissue of $N$. caecoides . . . . . . . . . . . . . L.A

L.3 High Molecular Weight Polynuclear Aromatic Hydrocarbons (HPAHs),

Dny Weight, in Tissue of $N$. caecoides . . . . . . . . . . . . . . L.7

L.4 Total Detected Polynuclear Aromatic Hydrocarbons (PAHs), Wet

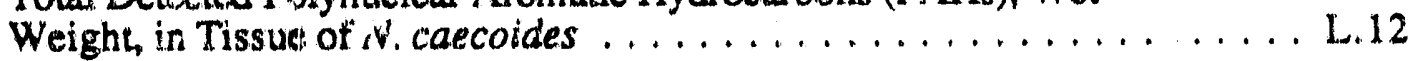

L.5 Low Molecular Weight Polynuclear Aromatic Hydrocarbons (LPAHs),

Wet Weight, in Tissue of $N$. caecoides . . . . . . . . . . . . . 15

L.6 High Molecular Weight Polynuclear Aromatic Hydrocarbons (HIPAHis),

Wet Weight, in Tissue of $N$. caecoides . . . . . . . . . . . . . . L.13

L.7 Quality Control Data for Low Molecular Weight Polynuclear Aromatic

Hydrocarbons (LPAHs), Wet Weight, in Tissue of N. caecoides . . . . . . L..23

L.8 Quality Control Data for High Molecular Weight Polynuclear Aromatic Hydrocarbons (HPAHs), Wet Weight, in Tissue of $N$, caecoides . . . . . . L.27

L.9 Surrogate Percent Recoveries for Polynuclear Aromatic Hydrocarbons

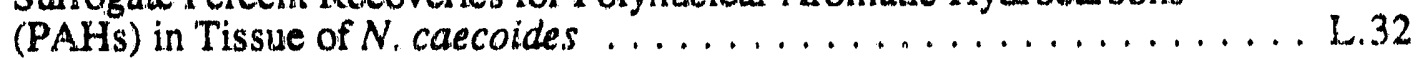

I.10 Chlorinated Pesticides (alphabetical, Aldrin - Dieldrin), Dry Weight,

in Tissue of $N$. caecoides . . . . . . . . . . . . . . . . . . L.36

L.11 Chlorinated Pesticides (alphabetical, Endosulfan I - Toxaphene),

Dry Weight, in Tissue of $N$. caecoides .

L.12 Chlorinated Pesticides (alphabetical, Aldrin - Dieldrin), Wet Weight, in Tissue of $N$ caecoides $\ldots \ldots \ldots \ldots \ldots \ldots$. . . . . . . . . . . . . . .

L.13 Chlorinated Pesticides (alphabetical, Endosulfan I - Toxaphene), Wet

Weight, in Tissues of $N$. caecoides . . . . . . . . . . . . . L.51

L. 14 Quality Control Data for Chlorinated Pesticides (alphabetical, Aldrin -

Dieldrin), Wet Weight, in Tissue of $N$. caecoides . . . . . . . . . . L.56

L.15 Quality Control Data for Chlorinated Pesticides (alphabetical, Endosulfan I -

Toxaphene), Wet Weight, in Tissue of $N$. caecoides . . . . . . . . . . L.61

L. 16 Polychlorinated Biphenyls (PCBs), Dry Weight, in Tissue of $N$ caecoides . L.63 
L.17 Polychlorinated Biphenyls (PCBs), Wet Weight, in Tissue of N. caecoides . L.68

L.18 Quality Control Data for Polychlorinated Biphenyls (PCBs), Wet Weight, in Tissue of $N$. caecoides

L.19 Surrogate Percent Recoveries for Polychlorinated Biphenyls (PCBs) and Chlorinated Pesticides in Tissue of N. caecoides . . . . . . . . . . L.78

L.20 Metals, Dry Weight, in Tissue of $N$. caecoides . . . . . . . . . . L.82

L.21 Metals, Wet Weight, in Tissue of $N$. caecoides $\ldots \ldots \ldots \ldots \ldots \ldots$. . . . . .

L.22 Quality Control Data for Metals, Dry Weight, in Tissue of N. caecoides . . . L.88

L.23 Butyltins, Wet and Dry We. ght, in Tissue of $N$. caecoides . . . . . . . L. L.94

L.24 Quality Control Data for Butyltins, Wet Weight, in Tissue of N. caecoides . . L.99

M.1 Mean Concentration and Statistical Grouping for Naphthalene in $M$. nasuta Tissue $\ldots \ldots \ldots \ldots \ldots \ldots \ldots \ldots \ldots \ldots \ldots \ldots . \ldots \ldots$

M.2 ANOVA Results of Naphthalene in M. nasuta Tissue $\ldots \ldots \ldots \ldots$ M.1

M.3 Mean Concentration and Statistical Grouping for Phenanthrene

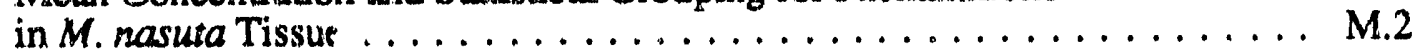

M.4 ANOVA Results for Pł.enanthrene in $M$. nasuta Tissue $\ldots \ldots \ldots \ldots$ M.2

M.5 Mean Concentration and Statistical Grcuping for Fluoranthene

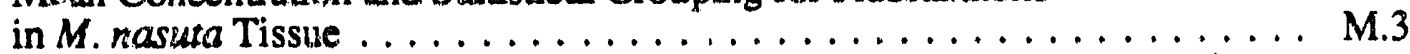

M.6 ANOVA Results for Fluoranthene in $M$. nasuta Tissue $\ldots \ldots \ldots \ldots$. . . . .

M.7 Mean Concentration and Statistical Grouping for Pyrene

M.8 ANOVA Results for Pyrene in $M$. nasuta Tissue $\ldots \ldots \ldots \ldots \ldots$ M.4

M.9 Mean Concentration and Statistical Grouping for Benzo(a)anthracene in $M$. nasuta Tissue $\ldots \ldots \ldots \ldots \ldots \ldots \ldots \ldots \ldots, \ldots \ldots . \ldots \ldots$

M.10 ANOVA Results for Benzo(a)anthracene in $M$. nasuta Tissue . . . . . . . M.5

M.11 Mean Concentration and Statistical Grouping for Chrysene in $M$. rasuta Tissue $\ldots \ldots \ldots \ldots \ldots \ldots \ldots \ldots \ldots \ldots$. 6

M.12 ANOVA Results for Chrysene in M. nasuta Tissue . . . . . . . . . M.6

M.13 Mean Concentration and Statistical Grouping for Benzo(b)fluoranthene in $M$. nasuta Tissue . . . . . . . . . . . . . . . . . . . . . . . . M.7

M.14 ANOVA Results for Benzo(b)fluoranthene in M. nasuta Tissue $\ldots \ldots$. . M.7

PHASE WLB $\quad$ xvii 
M.15 Mean Concentration and Statistical Grouping for Benzo(a)pyrene

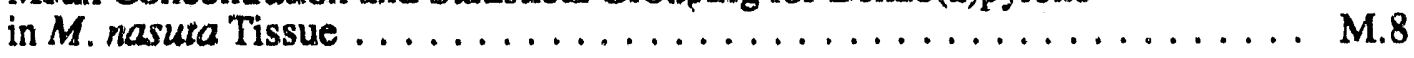

M.16 ANOVA Results for Benzo(a)pyrene in $M$. nasuta Tissue $\ldots \ldots \ldots \ldots$ M.8

M.17 Mean Concentration and Statistical Grouping for Silver

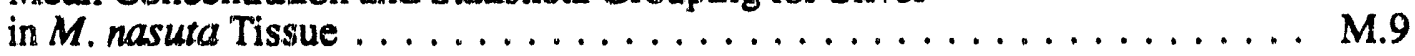

M.18 ANOVA Results for Silver in M. nasuta Tissue $\ldots \ldots \ldots \ldots \ldots \ldots$ M.9

M.19 Mean Concentration and Statistical Grouping for Arsenic in $M$. nasuta Tissue . . . . . . . . . . . . . . . . . . M.10

M.20 ANOVA Results for Arsenic in $M$. nasuta Tissue . . . . . . . . . . M.10

M.21 Mean Concentration and Statistical Grouping for Cadmium in $M$, nasuta Tissue . . . . . . . . . . . . . . . . . . . . M.11

M.22 ANOVA Results for Cadmium in M. nasuta Tissue $\ldots \ldots \ldots \ldots \ldots . . \ldots .11$

M.23 Mean Concentration and Statistical Grouping for Chromiurn

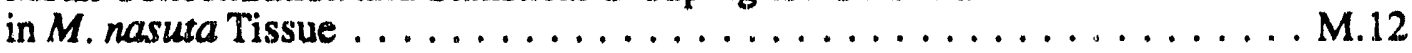

M.24 ANOVA Results for Chromium in M. nasuta Tissue ............. M.12

M.25 Mean Concentration and Statistical Groupings for Copper in

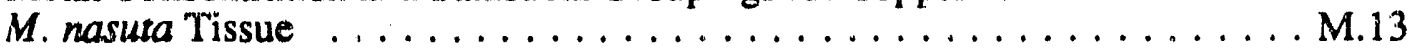

M.26 ANOVA Results for Copper in $M$. nasuta Tissue $\ldots \ldots \ldots \ldots \ldots \ldots$. . . 13

M.27 Mean Concentration and Statistical Grouping for Mercury In $M$. nasuta Tissue

M.28 ANOVA Results for Mercury in $M$. nasuta Tissue $\ldots \ldots \ldots \ldots \ldots$. 14

M.29 Mean Concentration and Statistical Grouping for Nickel in $M$. nasuta Tissue .

M.30 ANOVA Results for Nickel in M. nasuta Tissue . . . . . . . . . . . M.15

M.31 Mean Concentration and Statistical Grouping for Lead in $M$. nasuta Tissue

M.32 ANOVA Results for Lead in $M$. nasuta Tissue $\ldots \ldots \ldots \ldots \ldots \ldots \ldots$. . . . . . . . . .

M.33 Mean Concentration and Statistical Grouping for Selenium In $M$. nasuta Tissue

M.34 ANOVA Results for Selenium in $M$. nasuta Tissue . . . . . . . . . . M.17

M.35 Mean Concentration and Statistical Grouping for Zinc in $M$. nasuta Tissue 
M.36 ANOVA Results for Zinc in $M$. nasuta Tissue $\ldots \ldots \ldots \ldots \ldots \ldots . \ldots \ldots$

M.37 Mean Concentration and Statistical Grouping for Tributylin in M. nasuta Tissue . . .............................. 19

M.38 ANOVA Results for Tributyltin in $M$. nasuta Tissue . . . . . . . . . . . M.19

M.39 Mean Concentration and Statistical Grouping for Dibutyltin in

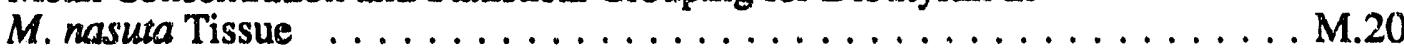

M.40 ANOVA Results for Dibutyltin in $M$. nasuta Tissue $\ldots \ldots \ldots \ldots \ldots$. . . . . .

M.41 Mean Concentration and Statistical Grouping for Monobutyltin in

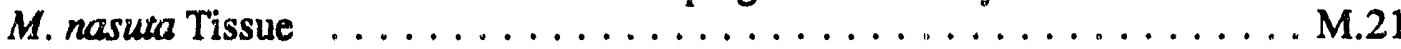

M.42 ANOVA Results for Monobutyltin in M. nasuta Tissue . . . . . . . . M.21

N.1 Mean Concentration and Statistical Grouping for Naphthalene in $N$. caecoides Tissue . . . . . . . . . . . . . . . . . . . . .

N.2 ANOVA Results of Naphthalene in $N$. caecoides Tissue $\ldots \ldots \ldots \ldots \ldots$ N.1

N.3 Mean Concentration and Statistical Grouping for Acenaphthene in $N$. caecoides Tissue . . . . . . . . . . . . . . . . . . N.2

N.4 ANOVA Results for Acenaphthene in $N$. caecoides Tissue $\ldots \ldots \ldots \ldots$ N.2

N.5 Mean Concentration and Statistical Grouping for Fluorene in $N$. caecoides Tissue . . . . . . . . . . . . . . . . . . . . N.3

N.6 ANOVA Results for Fluorene in $N$. caecoides Tissue $\ldots \ldots \ldots \ldots \ldots$ N.3

N.7 Mean Concentration and Statistical Grouping for Phenanthrene in $N$. caecoides Tissue . . . . . . . . . . . . . . . . . . . N.4

N.8 ANOVA Results for Phenanthrene in $N$. caecoides Tissue $\ldots \ldots \ldots \ldots \ldots$ N.4

N.9 Mean Concentration and Statistical Grouping for Anthracene in $N$. caecoides Tissue . . . . . . . . . . . . . . . . . . . . N.5

N.10 ANOVA Results for Anthracene in $N$. caecoides Tissue $\ldots \ldots \ldots \ldots \ldots$ N.5

N.11 Mean Concentration and Statistical Grouping for Fluoranthene

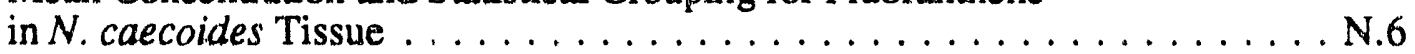

N.12 ANOVA Results for Fluoranthene in $N$. caecoides Tissue $\ldots \ldots \ldots \ldots$ N.6

N.13 Mean Concentration and Statistical Grouping for Pyrene in $N$. caecoides Tissue . . . . . . . . . . . . . . . . . . . . N.7

N.14 ANOVA Results for Pyrene in $N$. caecoides Tissue $\ldots \ldots \ldots \ldots \ldots \ldots$. . . . . .

PHASE IIUB $\quad$ xix 
N.15 Mean Concentration and Statistical Grouping for Benzo(a)anthracene

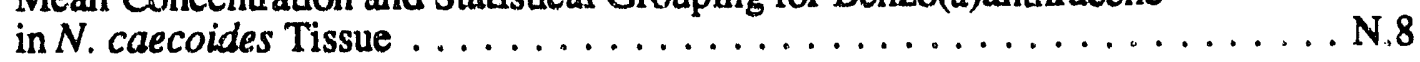

N.16 ANOVA Results for Benzo(a)anthracene in $N$. caecoides Tissue . . . . . . . N.8

N.17 Mean Concentration and Statistical Grouping for Chrysene in $N$. caecoides Tissue . . . . . . . . . . . . . . . . . N.9

N.18 ANOVA Results for Chrysene in $N$. caecoides Tissue . . . . . . . . . N.9

N.19 Mean Concentration and Statistical Grouping for Benzo(b)fluoranthene in $N$. caecoides Tissue . . . . . . . . . . . . . . . . N.10

N.20 ANOVA Results for Benzo(b)fluoranthene in N. caecoides Tissue . . . . . N.10

N.21 Mean Concentration and Statistical Grouping for Benzo(k)fluoranthene in $N$. caecoides Tissue . . . . . . . . . . . . . . . . . N.11

N.22 ANOVA Results for Benzo(k)fluoranthene in $N$. caecoides Tissue $\ldots \ldots \ldots$ N.11

N.23 Mean Concentration and Statistical Grouping for Benzo(a)pyrene in $N$. caecoides Tissue . . . . . . . . . . . . . . . . . N.12

N.24 ANOVA Results for Benzo(a)pyrene in $N$. caecoides Tissue . . . . . . . N.12

N.25 Mean Concentration and Statistical Grouping for Silver in

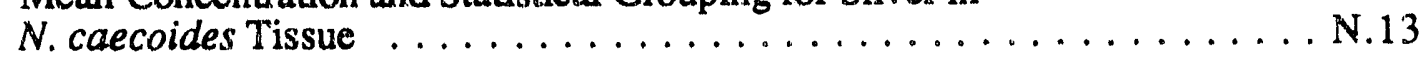

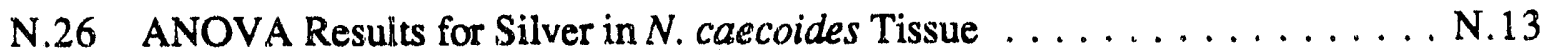

N.27 Mean Concentration and Statistical Grouping for Arsenic

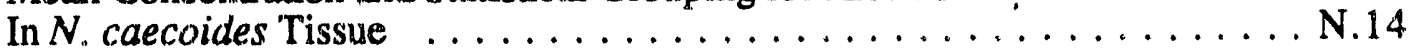

N.28 ANOVA Results for Arsenic in N. caecoides Tissue . . . . . . . . . N.14

N.29 Mean Concentration and Statistical Grouping for Cadmium in $N$. caecoides Tissue . . . . . . . . . . . . . . . . N.15

N.30 ANOVA Results for Cadmium in $N$. caecoides Tissue $\ldots \ldots \ldots \ldots \ldots$ N.15

N.31 Mean Concentration and Statistical Grouping for Chromium in

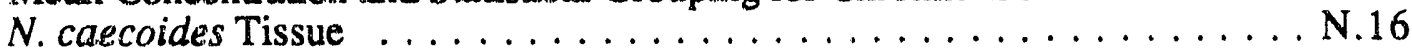

N.32 ANOVA Results for Chromiurn in $N$. caecoides Tissue . . . . . . . . N.16

N.33 Mean Concentration and Statistical Grouping for Copper

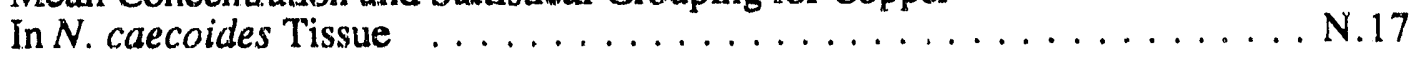

N.34 ANOVA Results for Copper in N. caecoides Tissue . . . . . . . . . . N.17

N.35 Mean Concentration and Statistical Grouping for Mercury in

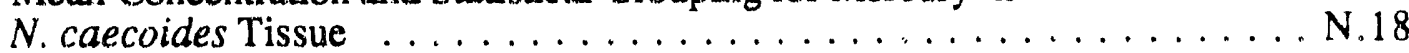


N.36 ANOVA Results for Mercury in $N$. caecoides Tissue $\ldots \ldots \ldots \ldots \ldots$ N.18

N.37 Mean Concentration and Statistical Grouping for Nickel in N. caecoides Tissue . . . . . . . . . . . . . . . . . . . . . N.19

N.38 ANOVA Results for Nickel in $N$. caecoides Tissue . . . . . . . . . . . . N.19

N.39 Mean Concentration and Statistical Grouping for Lead in

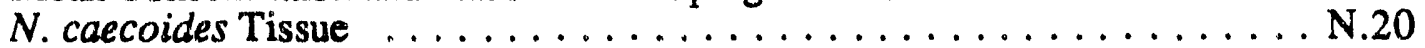

N.40 ANOVA Results for Lead in $N$. caecoides Tissue . . . . . . . . . . . . . N.20

N.41 Mean Concentration and Statistical Grouping for Selenium in

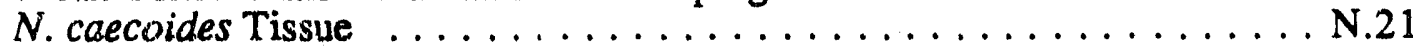

N.42 ANOVA Results for Selenium in $N$. caecoides Tissue $\ldots \ldots \ldots \ldots \ldots \ldots$ N.21

N.43 Mean Concentration and Statistical Grouping for Zinc in N. caecoides

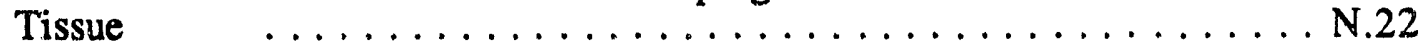

N.44 ANOVA Results for Zinc in $N$. caecoides Tissue $\ldots \ldots \ldots \ldots \ldots \ldots \ldots$. .22

N.45 Mean Concentration and Statistical Grouping for Tributyltin in

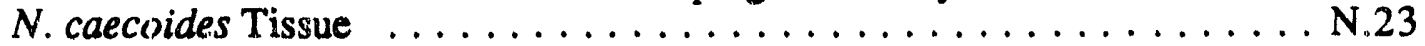

N.46 ANOVA Results for Tributyltin in $N$. caecoides Tissue $\ldots \ldots \ldots \ldots \ldots$ N.23

N.47 Mean Concentration and Statistical Grouping for Dibutyltin in N. caecoides Tissue ......................... N.24

N.48 ANOV A Results for Dibutyltin in $N$. caecoides Tissue $\ldots \ldots \ldots \ldots \ldots$ N.24

N.49 Mean C.oncentration and Statistical Grouping for Monobutyltin in

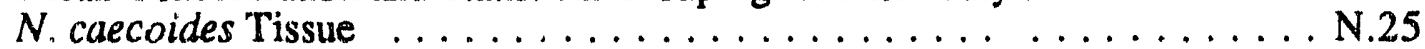

N.50 ANOVA Results for Monobutyltin in N. caecoides Tissue $\ldots \ldots \ldots \ldots \ldots \mathbb{N} .25$ 


\section{APPENDDX A}

MATERIALS AND METHODS USED FOR THE GEOLOGIC DESCRIPTIONS OE SEDIMENT CORES 


\section{APPENDIX A}

MATERIALS AND METHODS USED FOR

THE GEOLOGIC DESCRIPTIONS OF SEDIMENT CORES

\section{A.1 MATERIALS}

The following is a checklist of items and materials useful for the examination and description of sediment cores.

- ASTM Procedure D 2488-84

- Stainless-steel knife

- Hand lens (10X magnification)

- $\quad 10 \mathrm{~N}$ Hydrochloric acid (HC1)

- Ruler (scaled in 0.1-foot increments)

- Blank log forms (see Figure A.l)

- Clipboard

- AGI Data Sheets

- Munsell Color Charts

In addition, the charts and/or reference materials listed in Table A.1, and included in this appendix, are useful in the description of specific sediment characteristics.

\section{A.2 METHODS}

Descriptions of the physical, chemical, and biological features preserved in sediments aid in the interpretation of the types of geologic processes active both during and after the sediment was deposited. A total of 17 sediment characteristics, outlined in ASTM (1984), are commonily used to describe inorganic soils. These are listed in Table A.2.

Moisture condition was not routinely logged because of the saturated nature of the sediments. Furthermore, since particles were rarely larger than coarse sand, neither were angularity, particle shape, range in particle size, and hardness logged. For this reason, these sediment characteristics were not included in the $\log$ form used for the description of Oakland Phase III $B$ sediments (Figure A.1). However, in the few instances where these characteristics did apply, they were described under the "COMMENTS" column. 
Cort Data Log

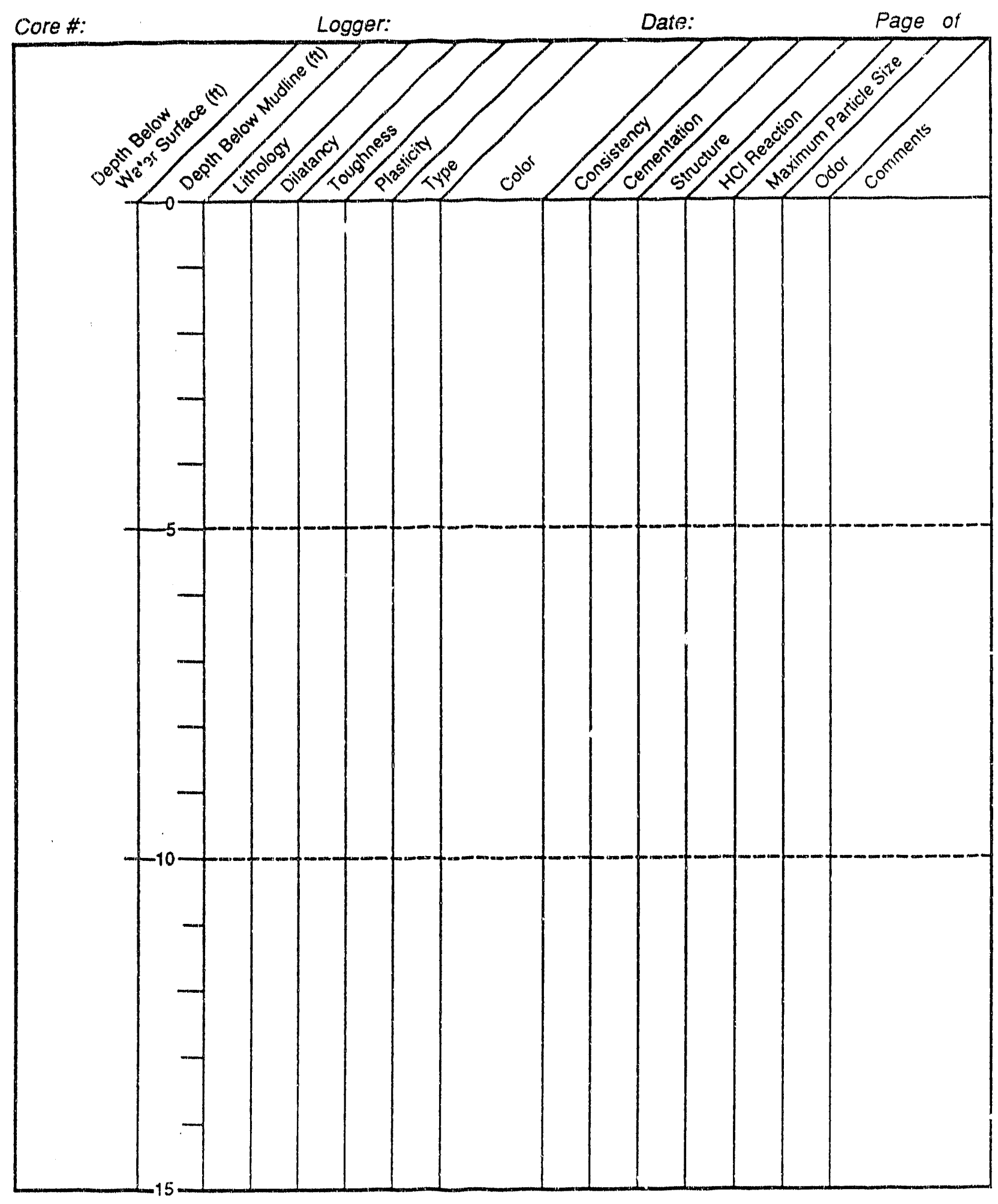

FIGURE A.1. Log Form Used to Record Sediment Descriptions

A. 2 
TABLE A.1. Charts and Other Reference Materials Used to Provide Standardized Descriptions of Sediment Characteristics

CHART/REFERENCE

- Percentagi

Estimate Chait

- Roundness Scale

- Sorting Chart

- Particle Shape

- Munsell Soil Color Charts

- Unified Soil

Classification

System

- Grain-size Scales

- Lithologic Symbols
PURPOSE

FIGURE \#

Estimate percentage of individual particles or constituents

Roundness of sand and coarser particles

Estimate of grading

Reference to describe particle shape

Soll color

Method for designating sediment type

Range of particle sizes; maximum particle size

Graphic patterns for Iithologic log
A. 3B

A. 4

A. 5

A. $6, A .7$

A. 2

A. 3 A

A. 8, A. 9

A. 10, A.11

TABLE A.2. Sediment Characteristics Identified in ASTM Procedure D2488-84

1) angularity *

2.) particle shape *

3) color

4) odor

5) moisture condition

6) $\mathrm{HCl}$ reaction

7) consistency (i.e., firmness)

8) cementation *

9) structure
10) sediment classification type

(i.e., lithology)

11) range of particle sizes *

12) maximum particle size

13) hardness *

14) dry strength **

15) dilatancy **

16) toughness **

17) plasticity **

* Applies to coarse-grained sediment (sand and larger particles)

** Applies to fine-grained sediment of mostly silt and/or clay

Features not generally logged for this study are underlined 
The definition of "soil" from the engineers standpoint (ASTM, 1984), includes any unconsolidated scdiment. The geologic definition of soil is slightly different and restricts soils to those sedimentary deposits that have undergone alteration near the land's surface by either physical, chemical, and/or biological processes; therefore, in a strict sense, not all sediments are soils. For the purposes of this discussion, however, "soils" and "sediments" will be used synonymously.

It is sometimes helpful to provide an estimate of the relative proportions of different constituents in sediments (e.g. light-versus dark-colored minerals). This is made easier and more accurate by using a percentage estimate chart, which provides a graphic reference with varying concentrations of a particular constituent (Figure A.2).

The criteria used to describe each of the 17 sediment characteristics identified in ASTM (1984) are discussed below.
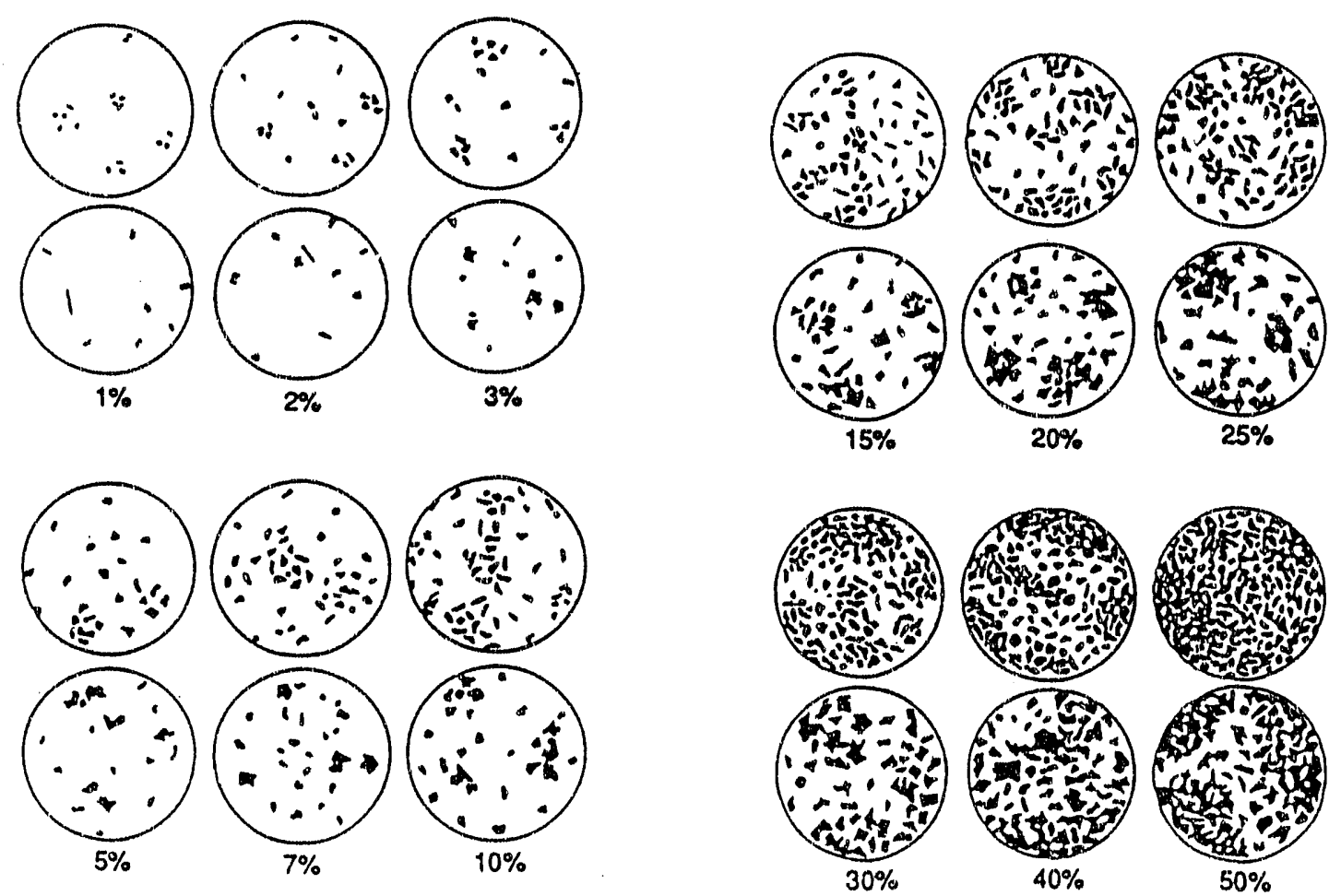

FIGURE A.2. Comparison Chart Used to Estimate the Percentages of Constituents (from AGI 1982) 


\section{A.2.1 Angularity}

The angularity of sedimentary particles is a reflection of the sedimentary environment and the amount of time that has elapsed before deposition and burial. A chart showing how to classify the angularity of sedimentary particles is presented in Figire A.3A. A range of angularity may be stated, such as: subrounded to rounded.

\section{A.2.2 Shape}

Shapes of sedimentary particles often reflect the internal characteristics (e.g., preferential parting) of the material or sometimes the type of sedimentary environment. For example gravel clasts deposited in high-energy environments, such as beaches and river bottoms, are often worn flat.

According to Figure A.4, gravel-sized clasts may be described in one of four ways. First, if the ratio of the clast's width to thickness is $>j$, it is classified as flat. Second, if the ratio of the clast's length to width is $\therefore 2$, the clast is elongata. Third, if bo:h criteria apply the clast is both flat and elungate. And last, if none of the criteria apply, then shape is not mentioned. Inalcate the fraction of the clasts that have the shape, such as: one-third of gravel clast.s are flat. Particle shape did not apply to most of the sediments logged during this project and the few pebbles that were observed were neither flat nor elongate.

\section{A.2.3 Color}

Color may be useful in identifying materials of similar geologic origin. For example, color was often a useful criteria for differentiating Younger Bay Mud from 01der Bay Mud. Sediment color was determined by comparing the wet sediment with standard sediment colors given in Munsell (1975). The advantage to using the Munsell soil color system is that it provides a consistent, standardized method for describing color and subjectivity is minimized.

The Munsell color notation consists of three simple variables that combine to describe all colors known in the Munsell soil color system. The three variables are: hue, value, and chroma (Figure A.5). The hue notation indicates the relation of the sediment color with respect to red, yellow,

\section{PHASE III B}


A

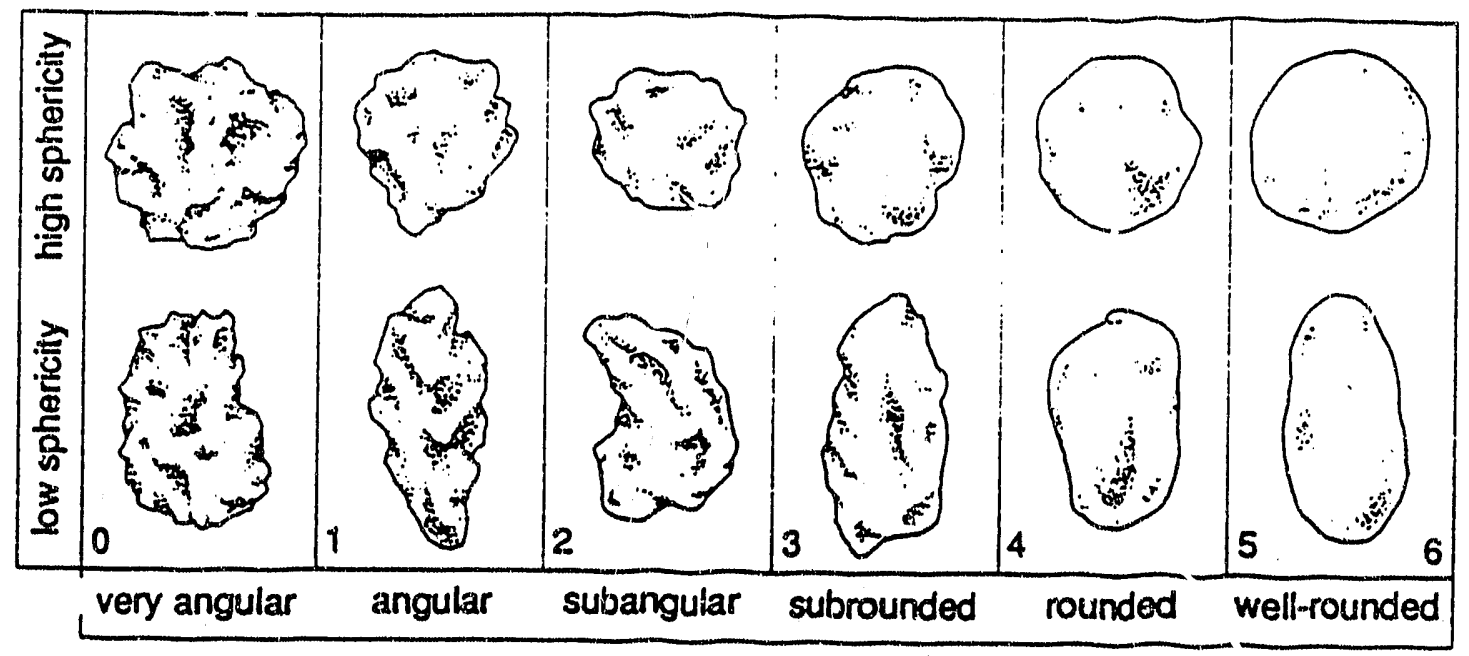

B

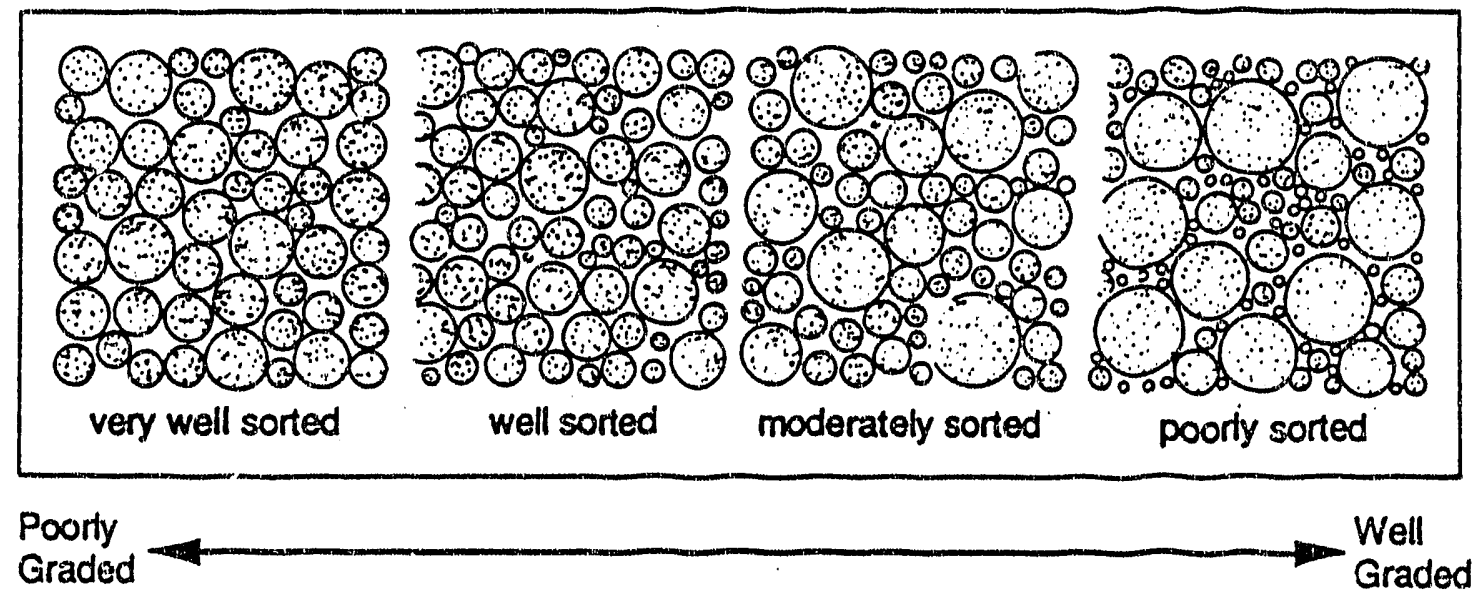

FIGURE A.3. Charts Used to Visually Estimate (A) Roundness/Sphericity and (B) Sorting/Grading 
$W=$ Width

$T=$ Thickness

$L=$ Length

Flat: $W / T>3$

Elongated: $L W>3$

Flat and Elongated: meets both criteria

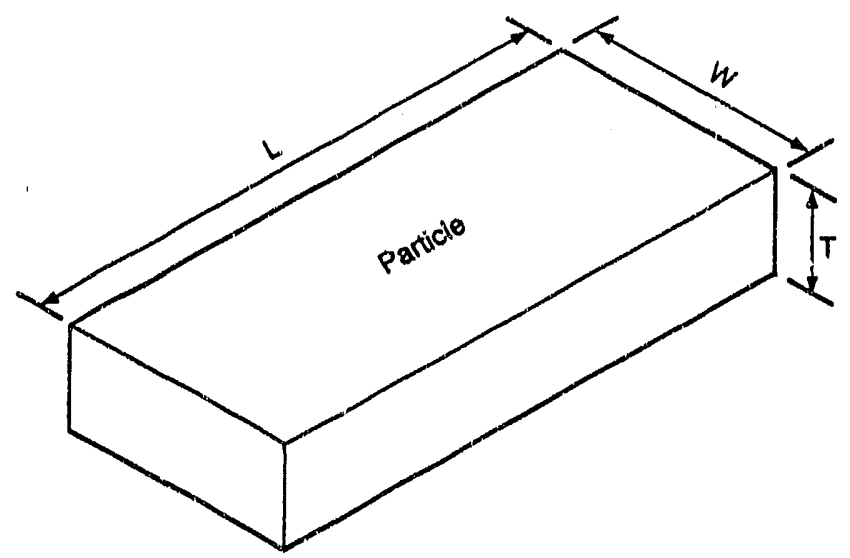

FIGURE A.4. Criteria Used to Describe Particle Shape of Pebbles and Large Gravels (From ASTM 1984)

green, blue, and purple; the value notation indicates its lightness, and the chroma notation indicates its strength (i.e., intensity).

Color can be described either by the Munsell notation (e.g. 5YR 5/3; hue $=5 Y R$, value $=5$, chroma $=3$ ) or by its equivalent color name (e.g. reddish brown). Both the color name and Munsell notation were recorded on core logs (see Appendix B). Only rarely was there not a reasonable match between the true color of the core sediment and one of the colors on a Munsell color chart.

\section{A.2.4 Odor}

Odors may indicate the presence of contaminants or be the result of the geochemical environment. Odors most frequently noted were that of petroleum hydrocarbons and the smell of rotten eggs (an indication of the presence of hydrogen sulfide). Both of these odors were restricted to the Younger Bay Mud unit. Petroleum odors may be the result of contamination of the sediments by shipping spills or industrial waste, or perhaps is derived from the abundant decaying organic matter present in these sediments. Hydrogen sulfide is a cominon natural by-product in chemically reducing environments such as the Richmond Harbor estuary. 


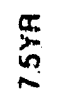

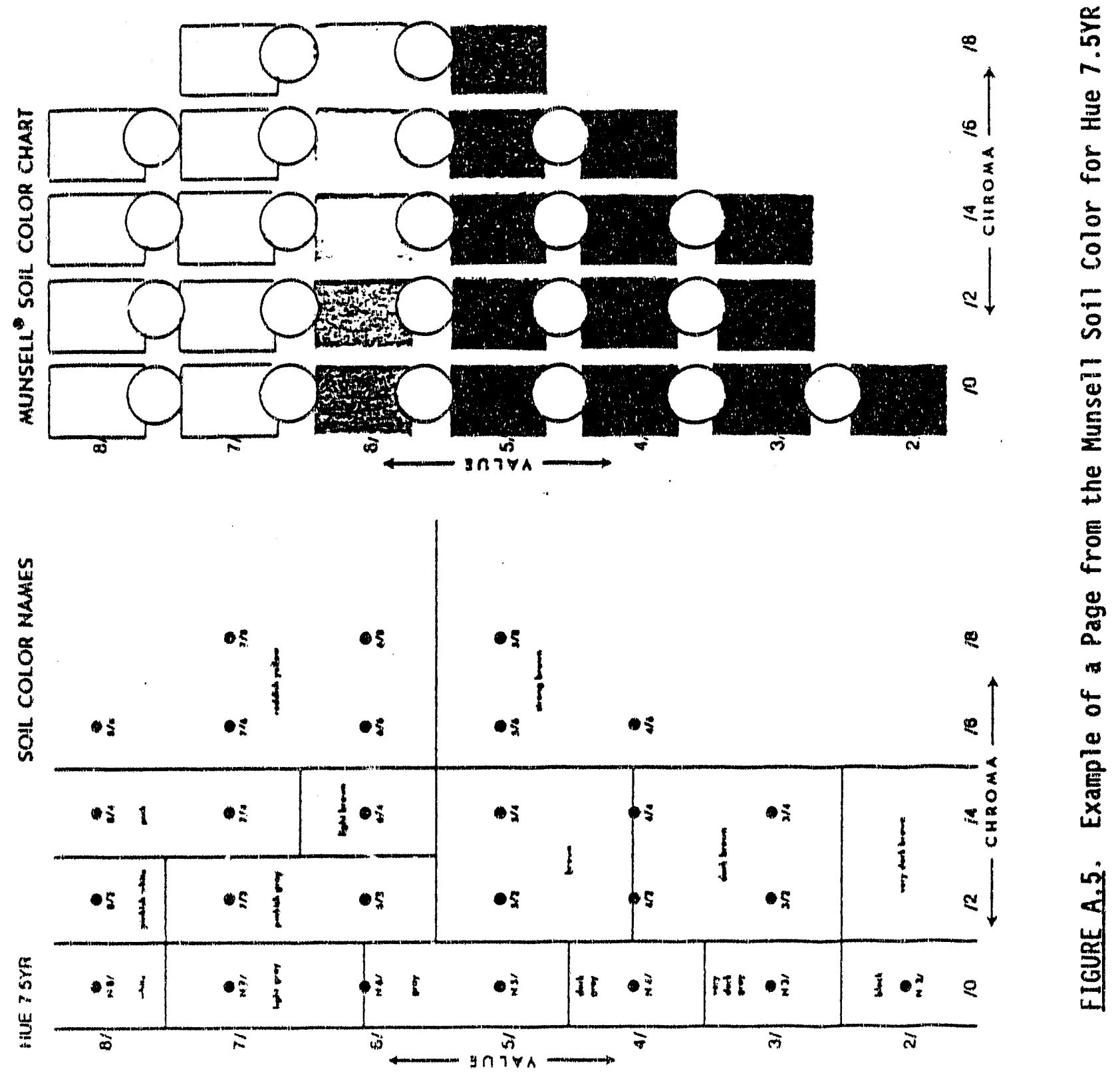

PHASE III 8

A. 8 


\section{A.2.5 Moisture Condition}

Moisture condition is described as either dry, moist, or wet according to the following criteria:

DRY Absence of moisture, dry to the touch

MOIST Damp but no visible water

WET Visible free water, usually soil is below water table (i.e., saturated)

A11 the sediments logged for this project were taken from below sea level and did not lose any significant moisture between the time they were drilled and logged. Therefore, they are all classified as wet.

\section{A.2.6 $\mathrm{HCl}$ Reaction}

The reaction (i.e., effervescence) of sedimentary material, as a result of adding dilute hydrochloric acid, is an indication of the presence of calcium carbonate. Calcium carbonate in sediments may be derived from a variety of sources including: 1) physical disintegration of preexisting carbonate rocks (e.g., limestone, marble), 2) biogenic precipitation (e.g., shell, bone), and 3) soil development. In the last example, calcium carbonate concentrations, often referred to as caliche or calcrete, may accumulate over time near the land's surface in arid climates. Where calcium carbonate concentrations occur in combination with other evidence for soil development, such as root traces and oxidation, then a pedogenic (soil forming) origin is favored. Criteria for describing the reaction with $10 \mathrm{~N} \mathrm{HCl}$ are as follows:

NONE No visible reaction

WEAK Some reaction, with bubbles forming slowly

STRONG Violent reaction, with bubbles forming immediately

A solution of $10 \mathrm{~N} \mathrm{HCl}$ is obtained by slowly adding one part of concentrated hydrochloric acid to three parts of distilled water. (To avoid a violent exothermic reaction never add water to acid).

PHASE III B 


\section{A.2.7 Consistency}

Consistency is a measure of the firmness or consolidation of sedimentary material. In general, there is a direct relationship between consistency and age of the deposit (i.e., older deposits are usually more firm because of compaction and/or cementation). Consistency is most applicable to fine-grained sediments and least applicable on sediments that contain significant amounts of gravel. The criteria used to determine consistency are as follows:

VERY SOFT Penetrometer penetrates soil greater than $4 \mathrm{~cm}$

SOFT Penetrometer penetrates soil 2.0 to $4.0 \mathrm{~cm}$

FIRM Penetrometer penetrates soit 0.25 to $2.0 \mathrm{~cm}$

HARD Penetrometer penetrates soil less than $0.25 \mathrm{~cm}$

The penetrometer used for sediment core descriptions consists of a 6 -in. nail spike attached to a clay brick for a total mass of $2.0 \mathrm{~kg}$; the nail spike is marked in centimeter increments to quantify the amount of soil penetration.

\section{A.2.8 Cementation}

Often sedimentary particles are held together with a binding cement. Three common natural cements are calcium carbonate (lime), silica, and iron-oxide compounds. Particles cemented with calcium carbonate effervesce in the presence of hydrochloric acid (see Section A.2.6 above). Sediments cemented with iron oxide are usually some shade of red, yellow, or brown. Usually there is a relationship between consistency (Section A.2.7) and cementation, in that strongly cemented deposits are also hard to very hard. criteria used to describe the degree of cemeritation are:

WEAK Crumbles or breaks with handling or light finger pressure MODERATE Crumbles or breaks with considerable finger pressure STRONG Will not crumble or break with finger pressure

\section{A.2.9 Structure}

Structures are features that originate within the layers of sediment or at the sediment/water interface in response to various physical, biologic and/or chemical processes. Structures may be classified into two categories: PHASE III B A. 10 
primary and secondary. Primary structures form as the sediment is being deposited (e.g., lamination, stratification). Secondary structures form after deposition, often as a result of compaction or other stresses (e.g., fissured, slickensided), biologic activity (e.g., root traces, mottling), and soil develapment (e.g., homogeneous, blocky, mottled). The following are some common structures observed in sedimentary deposits.

\title{
PRIMARY STRUCTURES
}

STRATIFIED Alternating layers of varying material or color with layers at least $6 \mathrm{~mm}$ thick

LAMINATED Aiternating layers of varying material or color with the layers less than $6 \mathrm{~mm}$ thick

LENSED Inclusion of small pockets of different sediment type, such as small lenses of sand scattered through a mass of clay. (This type of structure may also be secondary).

\section{SECONDARY STRUCTURES}

FISSURED Breaks along definite planes of fracture with little resistance to fracturing

SLICKENSIDED Fracture planes appear polished or glossy, sometimes striated

BLOCKY Cohesive soil that can be broken down into small angular lumps which resist further breakdown

MOTTLED Variation in color of sediments as represented by localized spots or blotches of color or shades of color

HOMOGENEOUS Same color and appearance throughout

\section{A.2.10 Sediment Classification Type}

The classification method used in this study is the Unified Soil Classification System, which consists of a two-letter designation for most soils (i.e., unconsolidated sediments). A simplified version of the Unified Soil Classification Systern is presented in Figure A.6, while a more-detailed breakdown is presented in Figure A.7.

\author{
PHASE III 8
}




\begin{tabular}{|c|c|c|c|c|}
\hline \multicolumn{3}{|c|}{ Major Divisions } & \multirow{2}{*}{\begin{tabular}{|c} 
Group \\
Symbols
\end{tabular}} & \multirow{2}{*}{$\begin{array}{l}\text { Description } \\
\begin{array}{l}\text { Well-graded (i.e., prorly sorted) gravels, } \\
\text { gravet-sand mixtures, little or no fines }\end{array}\end{array}$} \\
\hline \multirow{8}{*}{ 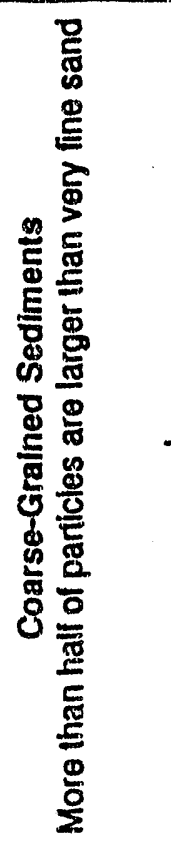 } & \multirow{4}{*}{$250 \%$ Gravel } & \multirow{2}{*}{$\begin{array}{l}\text { Clean } \\
\text { Gravels }\end{array}$} & & \\
\hline & & & GP & $\begin{array}{l}\text { Poorly graded (i.e., well sorted) gravels, } \\
\text { gravet-sand mixtures, little or no fines }\end{array}$ \\
\hline & & \multirow{2}{*}{$\begin{array}{l}\text { Gravels } \\
\text { with } \\
\text { Fines }\end{array}$} & GM & Silty gravels, gravel-sand-silt mixtures \\
\hline & & & GC & Clayey gravels, gravel-sand-clay mixtures \\
\hline & \multirow{4}{*}{$250 \%$ Gravel } & \multirow{2}{*}{$\begin{array}{l}\text { Clean } \\
\text { Sands }\end{array}$} & SW & Well-graded sands, gravelly sands, little or no fines \\
\hline & & & SP & Poorly graded sands, gravelly sands, little or no fines \\
\hline & & Sands & $S M$ & Silty sands, sand-sith mixtures \\
\hline & & Fines & SC & Clayey sands, sand-clay mixtures \\
\hline$\stackrel{\stackrel{0}{E}}{\stackrel{\Phi}{\Phi}}$ & & Low & ML & $\begin{array}{l}\text { Silts and very fine sands, sitty or clayey fine sands, } \\
\text { or clayey silts, with slight plasticity }\end{array}$ \\
\hline 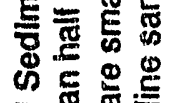 & Silts and & Limit & $\mathrm{CL}$ & $\begin{array}{l}\text { Clays of low to medium plasticity, gravelly clays, } \\
\text { sandy clays, sithy clays, lean clays }\end{array}$ \\
\hline 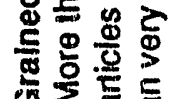 & Clays & High & MH & Silts or fine sandy silts with moderate plasticity \\
\hline$\stackrel{2}{\Phi}=$ & & Limit & $\mathrm{CH}$ & Clays of high plasticity, fat clays \\
\hline
\end{tabular}

FIGURE A.6. Abbreviated Form of the Unified Soil Classification System (From AGI 1982) 

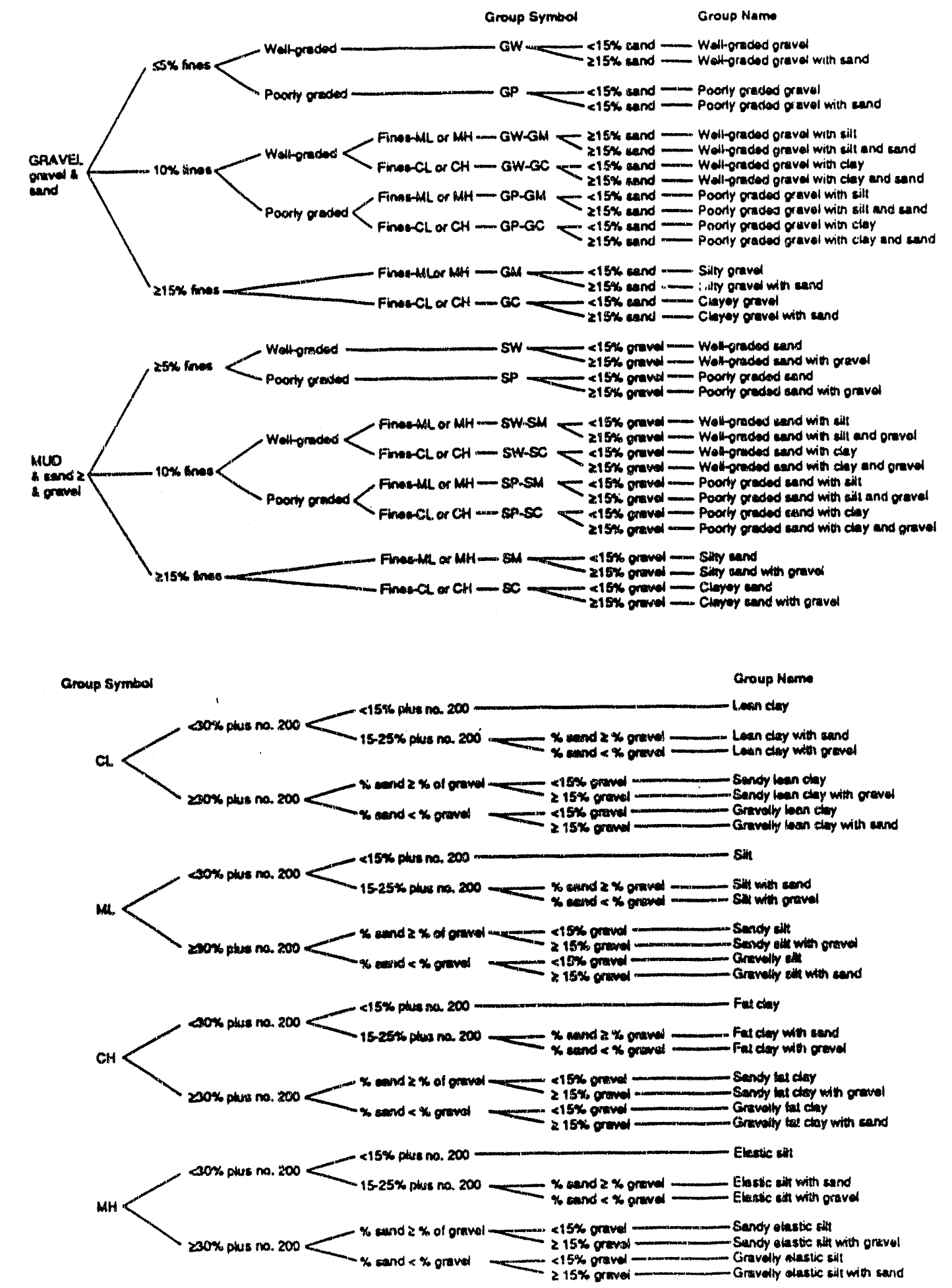

FIGURE A.7. Detailed Flow Chart For the Classification of Coarse- and FineGrained Soils Using the Unified Soil Classification System
PHASE III $\mathrm{B}$
A. 13 
According to the Unified Soil Classification System, coarse-grained sediments are classified based on grain-size distribution and grading (i.e., sorting), while fine-grained sediments are classified on the basis of grain size and liquid limit vs. plasticity.

Particle-size distribution may be determined with precision using laboratory methods (e.g., sieving of sand and coarser particles; pipette or hydrometer analysis of silt and clay). Because these methods are expensive and time-consuming, it is more desirable to estimate grain size using rapid visual-manual techniques described below. For example, sand and coarser particles are most easily identified via comparison with standard charts of grain size (Figures A.8 and A.9). Fine-grained soils, consisting of mostly silt and/or clay, $\mathrm{cn}$ the other hand, are identified based on manual tests of their dry strength, dilatancy, toughness, and plasticity (Figure A.10).

In the Unified Soil Classification System, the first letter of the sediment-type symbol represents the predominant grain-size interval, be it gravel $(G)$, sand $(S)$, silt $(M)$, or clay $(C)$. For coarse-grained sediments, the first letter (i.e., $G$ or $S$ ) may be followed by a descriptor of grading, either $W$ (well graded) or $P$ (poorly graded), or a secondary grain-size descriptor ( $M$ or $C$ ). The definition of grading is opposite that of sorting, a common geologic term. For example, a clean, well-sorted sand, consisting of particles over a narrow range in grain size, is referred to as poorly graded in the Unified Soil Classification System and would receive the designation "SP". The relationship between grading and sorting is shown graphically in Figure A.3B. The second letter in the fine-grained soil designation consists of either L (low liquid limit) or $H$ (high liquid limit).

The lithology column on the geologic log (Figure A.1) essentially represents a graphic display of sediment type, which can be utilized for quick easy reference and comparison between different cores and thus make interpretations easier. Examples of litholagic symbols in common use are presented in Figure A.11. Additional symbols may be used as long as they are graphically representative of the feature and are specifically defined and identified in a key that accompanies lithologic logs.

$$
\text { PHASE III B } \quad \text { A.14 }
$$




\begin{tabular}{|c|c|c|c|c|c|c|c|}
\hline \multicolumn{3}{|c|}{ Grade Limits } & \multirow[b]{2}{*}{ inches } & \multirow{2}{*}{$\begin{array}{c}\text { U.S. } \\
\text { Standard } \\
\text { Sieve Series }\end{array}$} & \multirow{2}{*}{\multicolumn{3}{|c|}{ Grade Name }} \\
\hline phi & $\mathrm{mm}$ & $\mathrm{mm}$ & & & & & \\
\hline-12 & $4096-$ & $\begin{array}{lll}- & - & -\end{array}$ & 161.3 & $-\quad-\quad-$ & $--\overline{-}$ & & \multirow{11}{*}{ GRAVEL } \\
\hline-11 & $2048-$ & $-\quad-\quad-$ & 80.6 & $-\quad-\quad-$ & - - & & \\
\hline-10 & $1024-$ & $-\quad-$ & 40.3 & $-\quad-$ & ${ }_{\text {medium }}^{-}-$ & Boulders & \\
\hline-9 & $512-$ & $-\quad-$ & 20.2 & $-\quad-$ & $-\overline{\text { small }}-\overline{-}$ & & \\
\hline-8 & $256-$ & $-\quad-$ & 10.1 & $-\quad--$ & large & & \\
\hline-7 & $128-$ & $-\quad-$ & 5.0 & $-\quad-\quad-$ & $-\overline{\text { small }}-$ & Cobbles & \\
\hline-6 & $64-$ & $-\quad-\quad-$ & 2.52 & $63 \mathrm{~mm}-$ & very coarse & \multirow{5}{*}{ Pebbles } & \\
\hline-5 & $32-$ & & 1.26 & $31.5 \mathrm{~mm}$ & coarse & & \\
\hline-4 & $16-$ & $-\quad-$ & 0.63 & $16 \mathrm{~mm}-$ & $\overline{\text { medium }}^{-}$ & & \\
\hline-3 & $8-$ & $-\quad-$ & 0.32 & $8 \mathrm{~mm}-$ & $-\overline{f i n e}^{-}-$ & & \\
\hline .2 & $4-$ & $-\quad-\quad-$ & 0.16 & No. 5 & $\overline{\text { very }}$ line & & \\
\hline \multicolumn{3}{|l|}{$--1=$} & \multirow{2}{*}{$\begin{array}{l}0.08 \\
0.04\end{array}$} & \multirow{3}{*}{$\begin{array}{l}\text { No. } 10- \\
\text { No. } 18 \text { - } \\
\text { No. } 35 \text { - }\end{array}$} & very coarse & \multirow{5}{*}{ Sand } & \multirow{5}{*}{ SAND } \\
\hline 0 & $1-$ & $-\quad-$ & & & $-\overline{c o a r s e}-$ & & \\
\hline+1 & $1 / 2-$ & $0.500-$ & - & & ${ }_{\text {medium }}^{-}-$ & & \\
\hline+2 & $1 / 4=$ & $0.250-$ & - & No. 60 - & fine - & & \\
\hline+3 & $1 / 3$ & $0.125-$ & $-\quad-$ & No. 120 & - very fine & & \\
\hline$\$-$ & $-1 / 16-$ & 0.062 & & - No. $230-$ & $\operatorname{coars} \theta$ & \multirow{4}{*}{ Sitt } & \multirow{8}{*}{ MUD } \\
\hline+5 & $1 / 32-$ & $0.031-$ & $-\quad-$ & $-\quad-$ & - & & \\
\hline+6 & $1 / 64-$ & $0.016-$ & $-\quad-$ & $-\quad-$ & $\overline{f i n e}^{-}-$ & & \\
\hline+7 & $1 / 128-$ & $0.008-$ & - & $-\quad-$ & very fine & & \\
\hline+8 & $1 / 256-$ & $0.004-$ & $=-$ & $-\quad-\quad-$ & $\operatorname{coars} \theta$ & \multirow{4}{*}{ Clay Sizo } & \\
\hline+9 & $1 / 512-$ & $0.002-$ & - & $-\quad-$ & - $\overline{\text { medium }}-$ & & \\
\hline+10 & $1 / 1024$ & $0.001-$ & - & $-\quad-$ & $\overline{f i n e}^{-}-$ & & \\
\hline+11 & $1 / 2048$ & $0.0005-$ & $-\quad-$ & $-\quad-$ & - & & \\
\hline-+12 & $-1 / 4096-$ & -0.00025 & & & & & \\
\hline
\end{tabular}

FIGURE A.8. Grain-size Scale Used to Determine Sedimentary Particle Size 


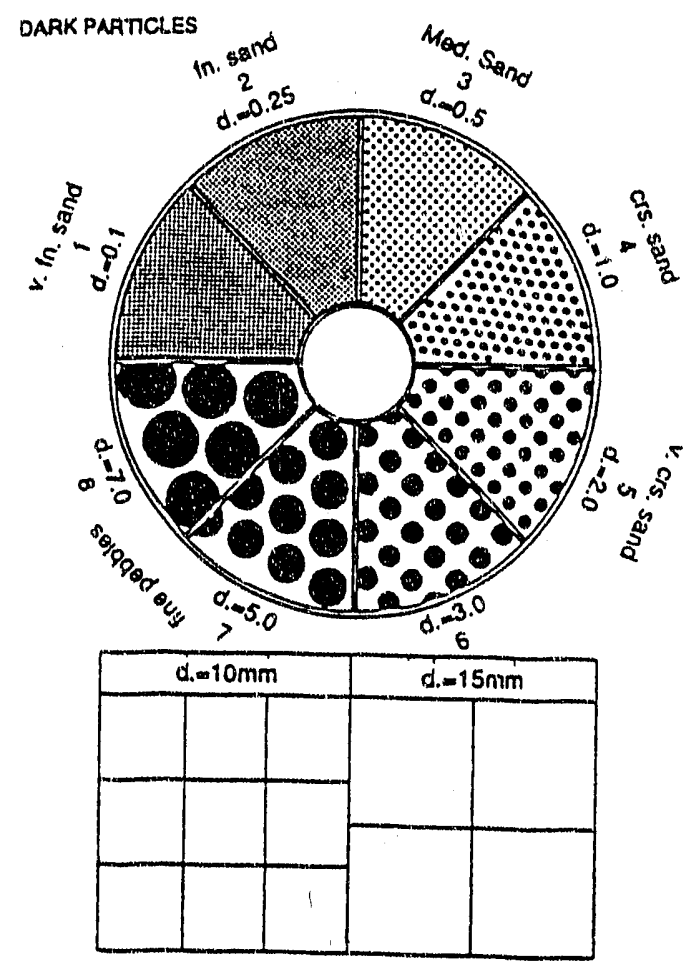

FIGURE A.9. Comparison Chart Used to Distinguish Among Sand to Pebble-size Particles (From AGI 1982). For larger particles, refer to

Figure A.8; for smaller particles, refer to Sections A.2.13 and A.2.16 in this Appendix

\begin{tabular}{|c|c|c|c|c|c|c|c|c|}
\hline \multirow{2}{*}{$\begin{array}{l}\text { Sediment } \\
\text { Type }\end{array}$} & Dry Strength & \multicolumn{3}{|c|}{ Dilatancy } & \multicolumn{3}{|c|}{ Toughness } & Plasticity \\
\hline & 量高兽 & 愛 & के & $\begin{array}{l}\frac{7}{8} \\
\text { o } \\
\text { ㄸ }\end{array}$ & $\underline{\xi}$ & E⿱士⿻尸丨 & 点 & 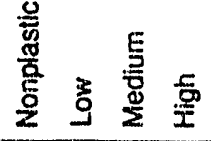 \\
\hline $\begin{array}{l}\text { ML } \\
\text { (Silit) }\end{array}$ & & & & & & & & \\
\hline $\begin{array}{c}\text { MH } \\
\text { (Elastic Silt) }\end{array}$ & & & & & & & & \\
\hline $\begin{array}{c}\mathrm{CL} \\
\text { (Lean Clay) }\end{array}$ & & & & & & & & \\
\hline $\begin{array}{c}\mathrm{CH} \\
\text { (Fat Clay) }\end{array}$ & & & & & & & & \\
\hline
\end{tabular}

FIGURE A.10. Identification of Inorganic Fine-grained Soils From Manual Tests PHASE III B

A. 16 


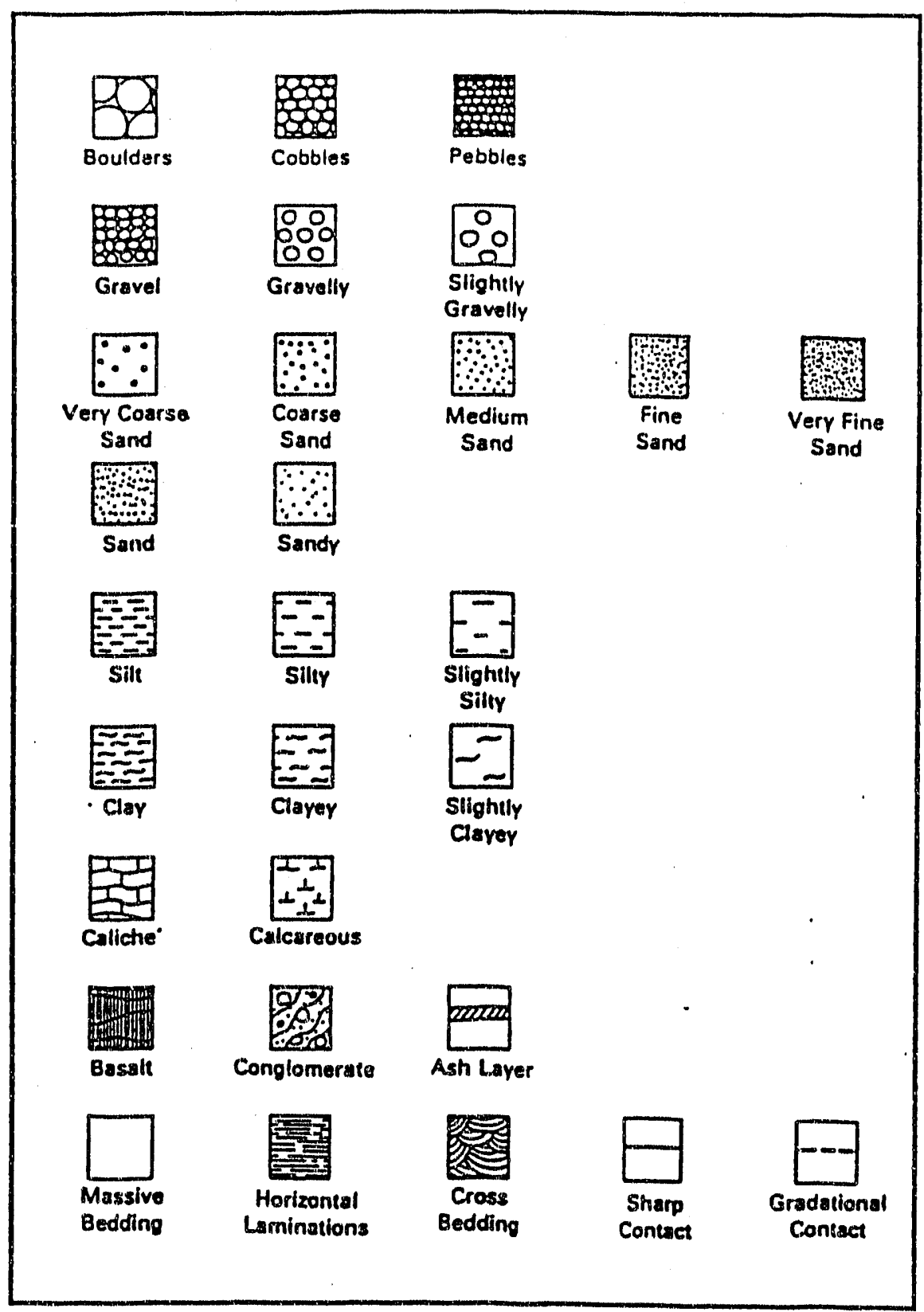

FIGURE A.11. Lithologic Symbols Used on Core Log Forms 


\section{A.2.11 Range of Particle Size}

For gravel- and sand-sized particles, the range of particle sizes within each component is defined. For example, $20 \%$ fine to coarse grave1, $40 \%$ fine to coarse sand. The sizes of particles corresponding to the different size components are presented in Figures A.8 and A.9.

\section{A.2.12 Maximum Particle Size}

Maximum particle size is significant because it gives a general indication of the amount of turbulence or energy associated with deposition. If the maximum particle size is sand, it should be described as either fine, medium, or coarse sand. If the maximum particle size is in the gravel range, the largest particle is measured and its width recorded along the narrowest axis. The sizes of particles cormespording to the different size components are presented in Figures $A .8$ and $A .9$.

The maximum grain size observed for the Younger Bay Muds ranged from silt to medium sand, while the 01der Bay Mud usually ranged from fine sand to coarse sand. The largest particles observed anywhere were fine pebbles in the Older Bay Mud unit.

\section{A.2.13 Dry Strength}

Dry strength, along with dilatancy, toughness, and plasticity are physical characteristics used to distinguish fine-grained inorganic soils, consisting of mostly silt and/or clay. Basically, the more clay present in a soil the greater its dry strength (Figure A.10). To perform a manual test of dry strength enough material must be selected in order to mold into a ball about $1 \mathrm{in}$. in diameter. Mold the material until it has the consistency of putty, adding water if necessary. From the molded material, make at least three test specimens each about $1 / 2$ in. in diameter. Allow the test specimens to dry in air, sun or by artificial means, as long as the temperature does not exceed $60^{\circ} \mathrm{C}$ (ASTM, 1984). The criteria for determining dry strength are as follows:

NONE The dry specimen crumbles into powder with mere pressure of handling

LOW The dry specimen crumbles into powder with light finger pressure

$$
\text { PHASE III B } \quad \text { A. } 18
$$


MEDIUM The dry specimen breaks into pieces or crumbles with considerable finger pressure

HIGH The dry specimen cannot be broken with finger pressure. Specimen will break into pieces between thumb and a hard surface

VERY HIGH The dry specimen cannot be broken between the thumb and a hard surface

Dry strength was determined for 0akland Phase III B cores by sampling selected intervals and allowing the samples to air dry overnight. Dry strength was determined the next day and noted in the comments column of the geologic log.

\section{A.2.14 Dilatancy}

Dilatancy is a measure of how easily a soil gives up water when shaken. For example, some clays have the ability to absorb and retain large amounts of water into their crystal lattice. "Fat" clays tend to retain their water even under stress whereas "lean" clays and silt tend to release water when shaken.

To test for dilatancy select enough material to mold into a ball about $1 / 2$ in. in diameter. Mold the material, adding water if necessary, until it has a soft, but not sticky consistency. Smooth the soil ball in the palm of the hand with a blade of a knife or small spatula. Shake horizontally, striking the side of the hand vigorously against the other several times. Note the reaction of water appearing on the surface of the soil. Squeeze the sample by closing the hand or pinching the soil between the fingers, and note the reaction. Specimens with high dilatancy will quickly yield water when shaken and absorb water when squeezed. The criteria for describing dilatancy are:

NONE No visible change in the specimen

SLOW Water appears slowly on the surface of the specimen during shaking and does not disappear or disappears slowly upon squeezing

RAPID Water appears quick'y on the surface of the specimen during shaking and disappears quickly upon squeezing 
The range of dilatancy for the different fine-grained sediment types is shown in Figure A.10. From this figure it is apparent that dilatancy decreases with decreasing grain size.

\title{
A.2.15 Toughness
}

After completion of the dilatancy test, shape the same specimen into an elongated pat and roll by hand on a smooth surface or between the palms into a thread about $1 / 8 \mathrm{in.}(3 \mathrm{~mm}$ ) in diameter. (If the sample is too wet to roll easily, it should be spread into a thin layer and allowed to lose some water by evaporation.) Fold the sample threads and reroll repeatedly until the thread crumbles at a diameter of about $1 / 8 \mathrm{in}$. The thread will crumble at a diameter of $1 / 8 \mathrm{in}$. When the soil is near the plastic limit. Note the pressure required to roll the thread near the plastic limit. Also, note the strength of the thread. After the thread crumbles, the pieces should be lumped together and kneaded until the lump crumbles. Note the toughness of the material during kneading and classify into one of the following categories.

LOW Only slight pressure is required to roll the thread near the plastic limit. The thread and lump are weak and soft

MEDIUM Medium pressure is required to roll the thread to near the plastic limit. The thread and iump have medium stiffness.

HIGH Considerable pressure is required to roll the thread to near the plastic limit. The thread and the lump have very high stiffness.

The range of toughness for the different fine-grained sediment types is shown in Figure A.10. From this figure it is apparent that toughness increases with a decrease in particle size.

\section{A.2.16 Plasticity}

On the basis of observations made during the toughness test, describe the plasticity of the material according to the following criteria:

NONPLASTIC A $1 / 8$ in. thread cannot be rolled at any water content

LOW The thread can barely be rolled and the lump cannot be formed when drier than the plastic limit.

\author{
$\begin{array}{ll}\text { PHASE III B } & \text { A.20 }\end{array}$
}


MEDIUM The thread is easy to roll and not much time is required to reach the plastic limit. The thread cannot be rerolled after reaching the plastic limit. The lump crumbles when drier than the plastic limit.

HIGH It takes considerable time rolling and kneading to reach the plastic limit. The thread can be rerolled several times after reaching the plastic limit. The lump can be formed without crumbling when drier than the plastic limit.

The range of plasticity for the different fine-grained sediment types is shown in Figure A.10. From this figure it is apparent that an increase in plasticity accompanies a decrease in grain size. 


\section{A.3 REFERFNCES}

AGI. 1982. AGI Data Sheets for Geology in the Field, Laboratory, and Office. American Geological Institute, Falls Church, Virginia, 22041

ASTM. 1984. Standard Practice for Description and Identification of Soils (Visual-Manual Procedure): Procedure D 2488-84. American Society for Testing and Materials, Philadelphia, Pennsylvania, pp. 293-302.

Compton, R. R. 1962. Manual of Field Geology. John Wiley and Sons, New York, $378 \mathrm{p}$.

Last, G. V., and Liikala, T. L. 1987. A Fjeld Guide for Well-Site

Geologists: Cable-Tool Drilling. PNL-6392. Pacific Northwest Laboratory, Richland, Washington.

Munse 11. 1975. Munsell Soil Color Charts. Macbeth, a Division of Kollmorgen Co., Baltimore, Maryland, 7 charts.

Tucker, M. E. 1982. The Field Description of Sedimentary Rocks. Johri Wiley and Sons, New York, p. 112. 
APF'NDIX B

GEOLOGIC CORE DATAILOGS 


\section{Oakland Harbor-Phase IIIB}

Key to Core Data Logs (After ASTM Procedule D2488-84)

\section{Lithology:}

\begin{tabular}{|c|c|}
\hline $\bar{E}$ & Clay \\
\hline $\bar{E}$ & Sitty clay to clayey silt \\
\hline$= \pm$ & Sitt \\
\hline$=5$ & Sandy sitt to silty sand \\
\hline & Sand \\
\hline & Pebbles \\
\hline & Mollusk shells \\
\hline & Concentrated organic matter \\
\hline & Iron-oxide concentrations \\
\hline$\lambda \lambda$ & Root traces \\
\hline
\end{tabular}

Silt/Clay Characteristics

Dilatancy:

$$
\begin{aligned}
& N=\text { none } \\
& S=\text { slow } \\
& R=\text { rapid }
\end{aligned}
$$

\section{Toughness:}

$$
\begin{aligned}
& L=\text { low } \\
& M=\text { medium } \\
& H=\text { high }
\end{aligned}
$$

$$
\begin{gathered}
\text { Plasticity: } \\
\begin{aligned}
N=\text { none } \\
L=\text { low } \\
M=\text { medium } \\
H=\text { High }
\end{aligned}
\end{gathered}
$$

Type:

See soil group classificatior in ASTM D2488-84

Color (wet):

Selected from Munsell Soil Color Chart

\section{Consistency:}

$H=$ hard $=<0.25 \mathrm{~cm}$

$F=$ firm $=0.25-2.0 \mathrm{~cm}$

$S=$ soft $=2.0-4.0 \mathrm{~cm}$

VS $=$ very soft $=>4.0 \mathrm{~cm}$

\section{Cementation:}

$\mathrm{N}=$ not cemented

$W=$ weakly cernented

$M=$ moderately cemented

$S=$ strongly cemented

\section{Structure:}

$S=$ stratified

$L$ = laminated

$F$ - fissured

$S L$ = slickensided

$\operatorname{Ln}=$ lensed

$\mathrm{BI}=$ blocky

$M=$ mottled

$H=$ homogeneous

\section{HCI Reaction:}

$$
\begin{aligned}
& N=\text { none } \\
& W=\text { weak } \\
& S=\text { strong }
\end{aligned}
$$

\section{Maximum Particle Size:}

$C P=$ coarse pebble

$\mathrm{CS}$ = coarse sand

MS medium sand

FS = fine sand

VFS = very fine sand

Odor:

$N=$ none

$S=$ sulfide

$\mathrm{P}=$ petroleum

$O=$ other

$w / H C l=$ odor apparent after $\mathrm{HCl}$ application

Comments:

$Y B M=$ Younger Bay Mud unit $\mathrm{OBM}=$ Older Bay Muó unit 
Core Data Log

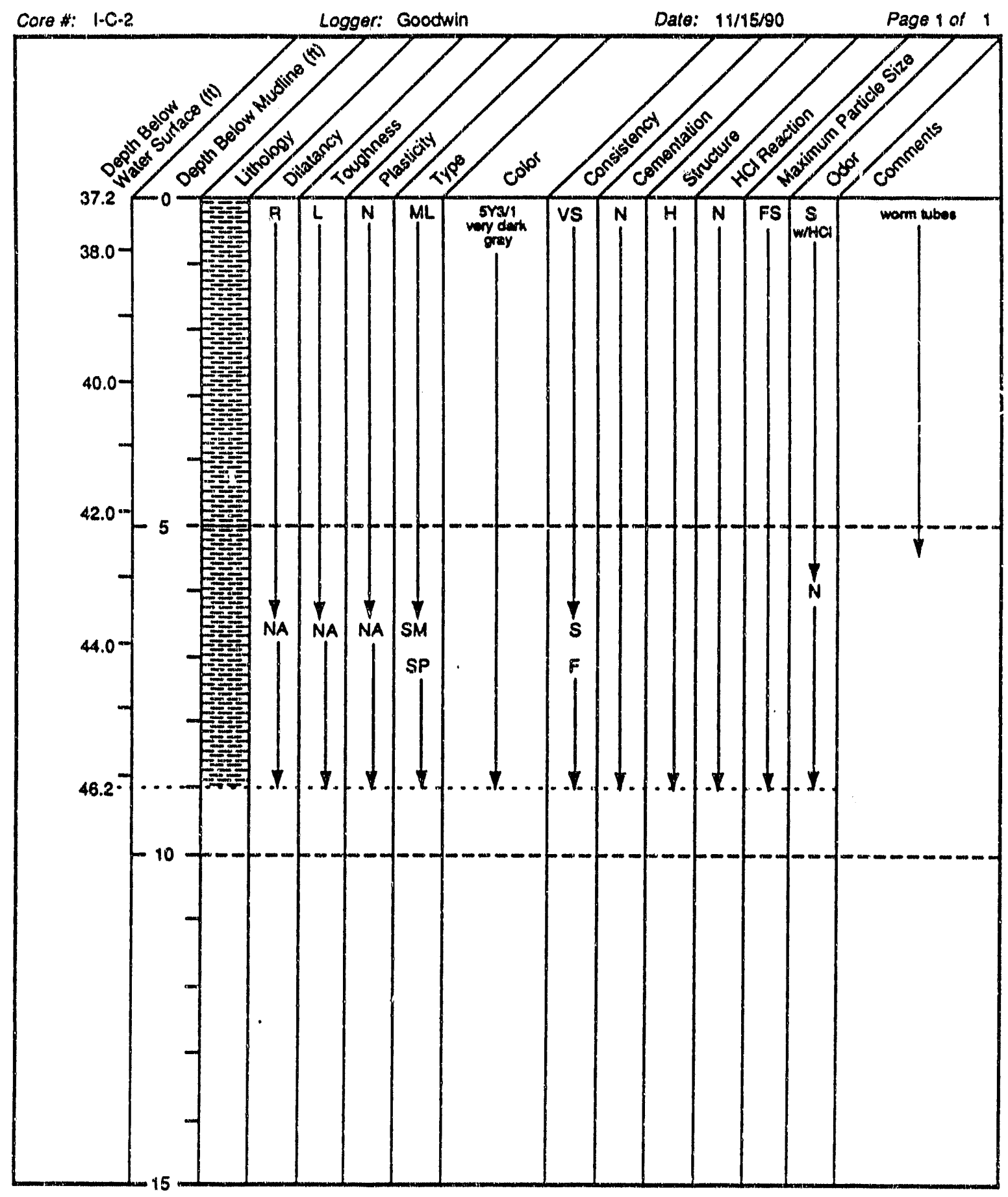




\section{Core Data Log}

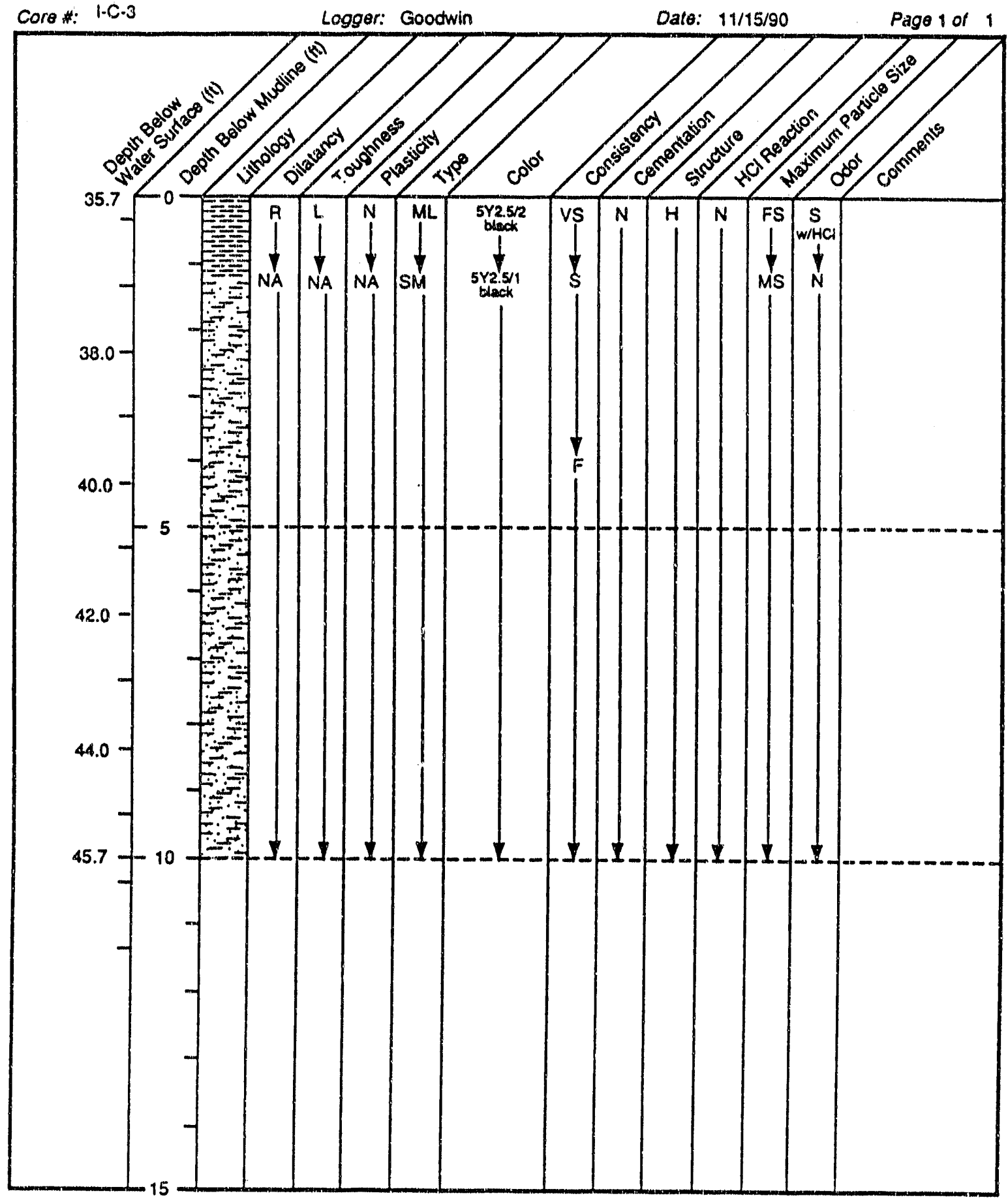


Core Data Log

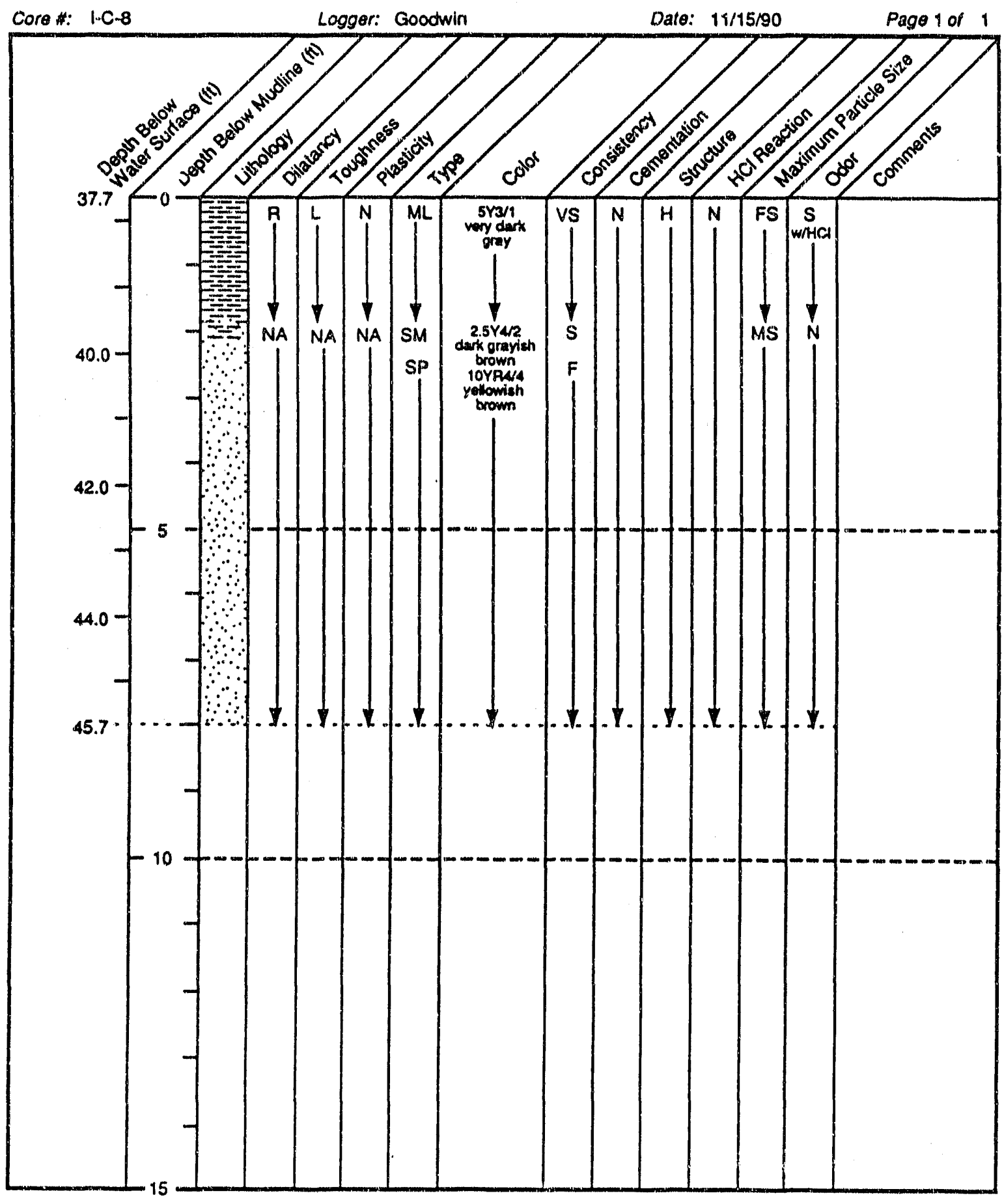

PHASE III B

B. 4 


\section{Core Data Log}

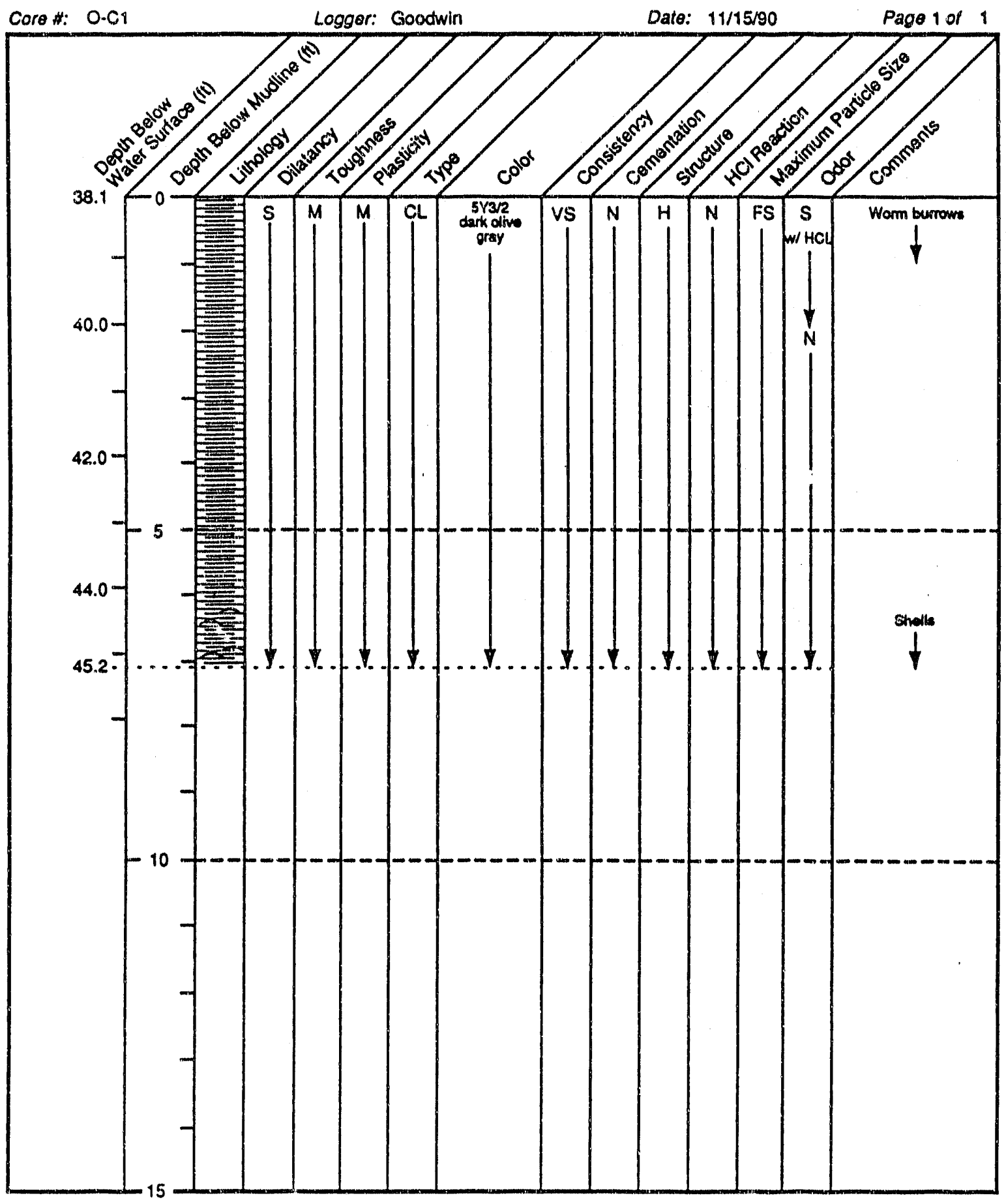


Core Data Log

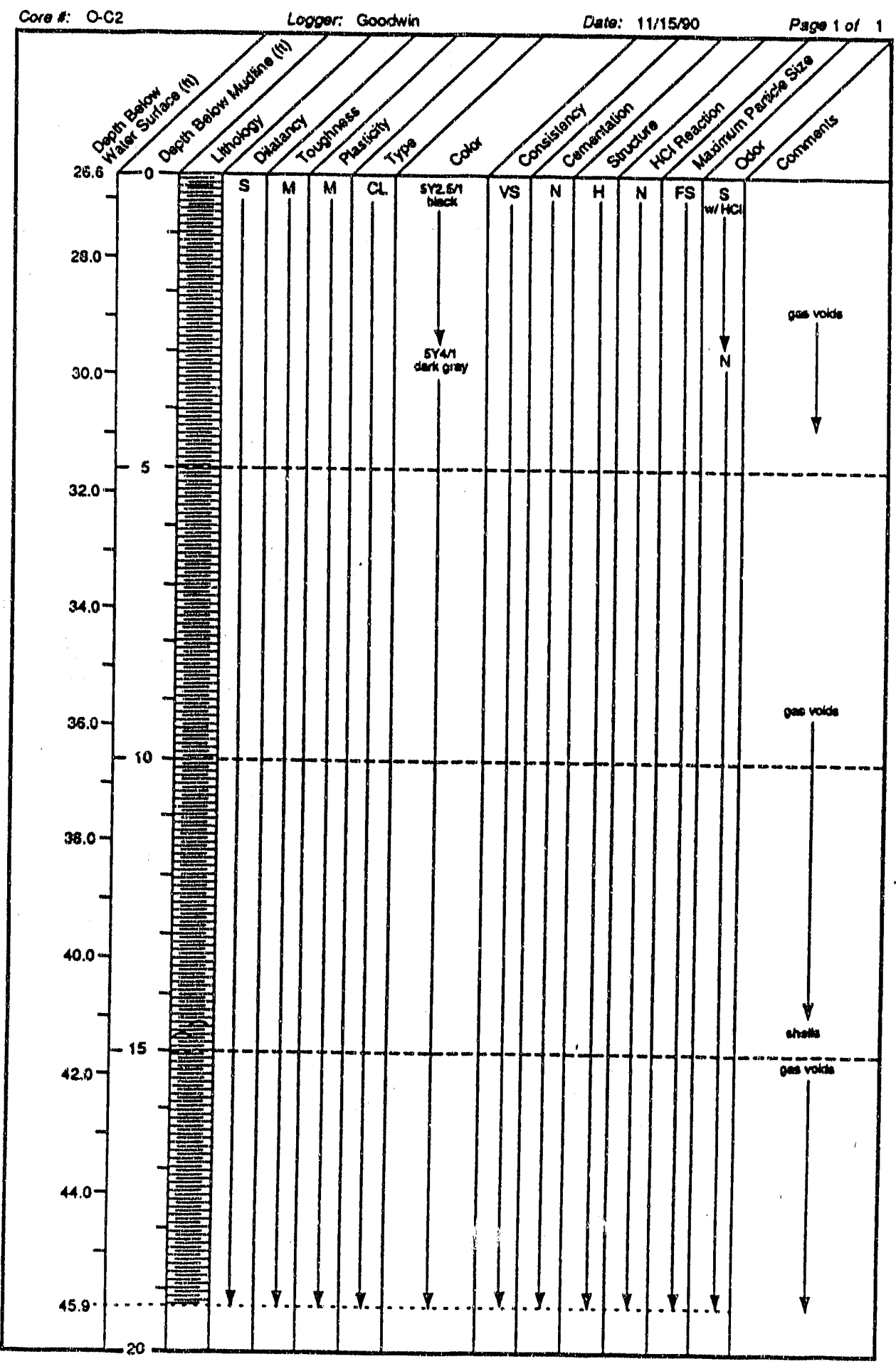


Core Data Log

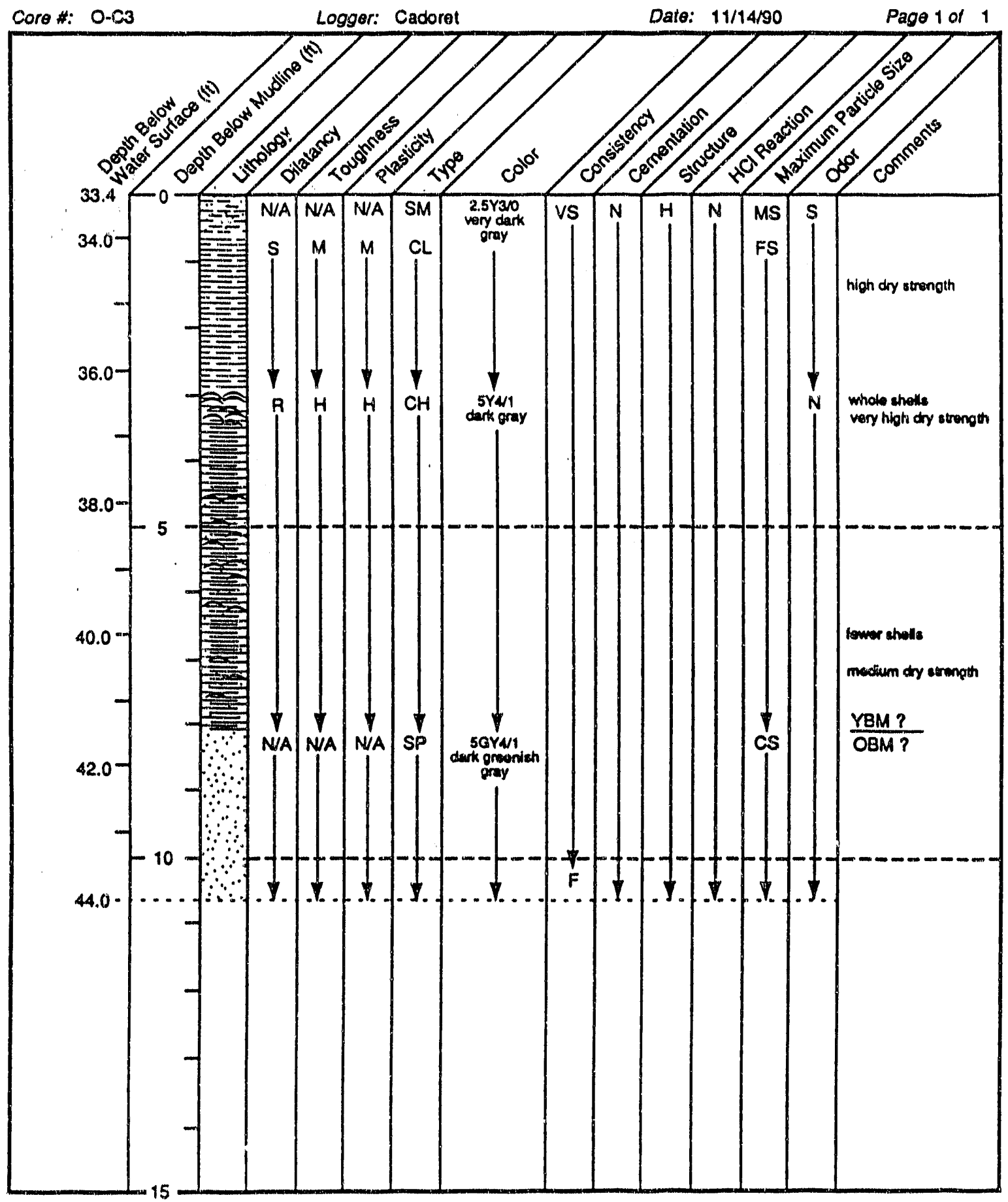

PHASE III B

B.7 
Core Data Log

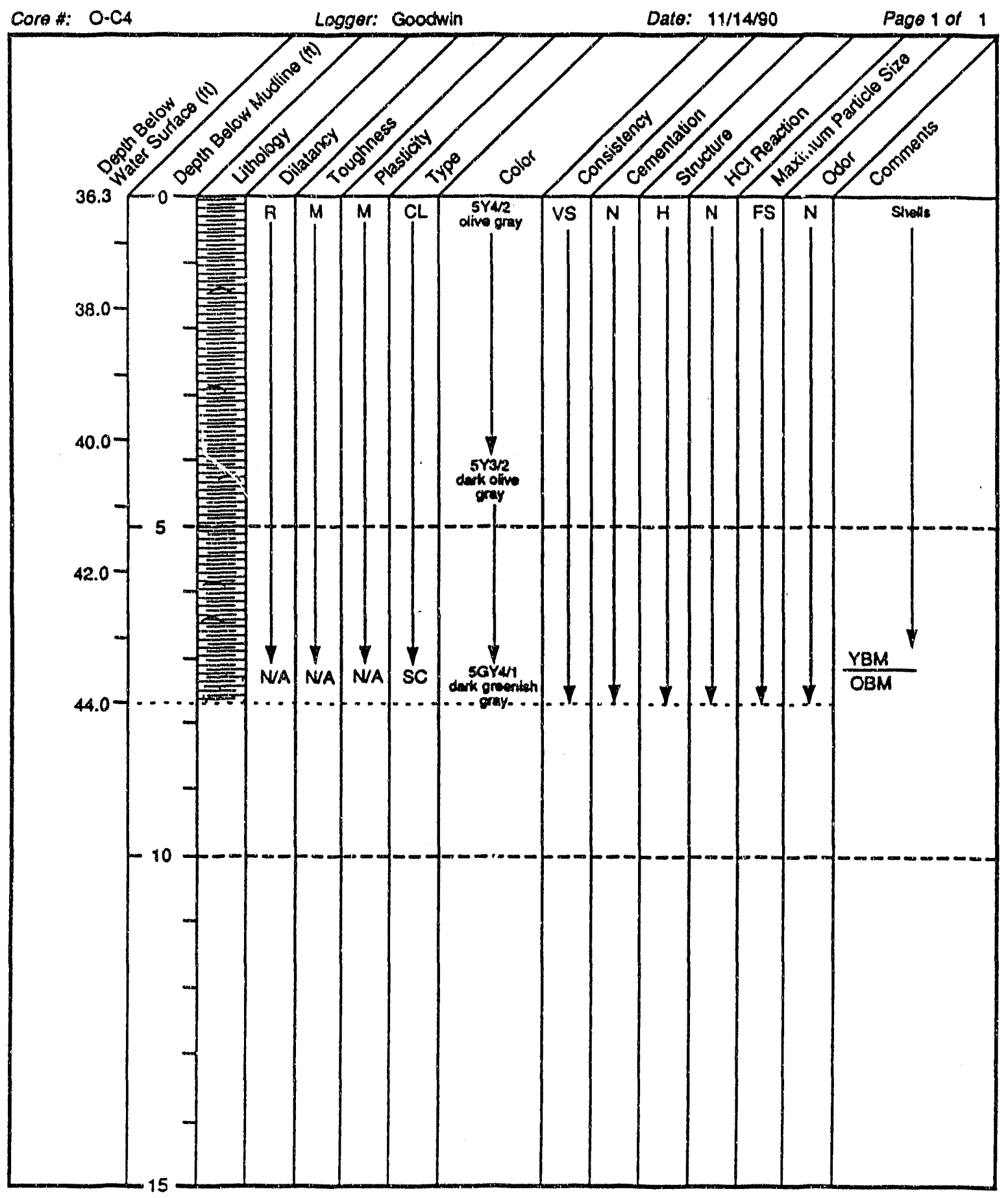


Core Data Log

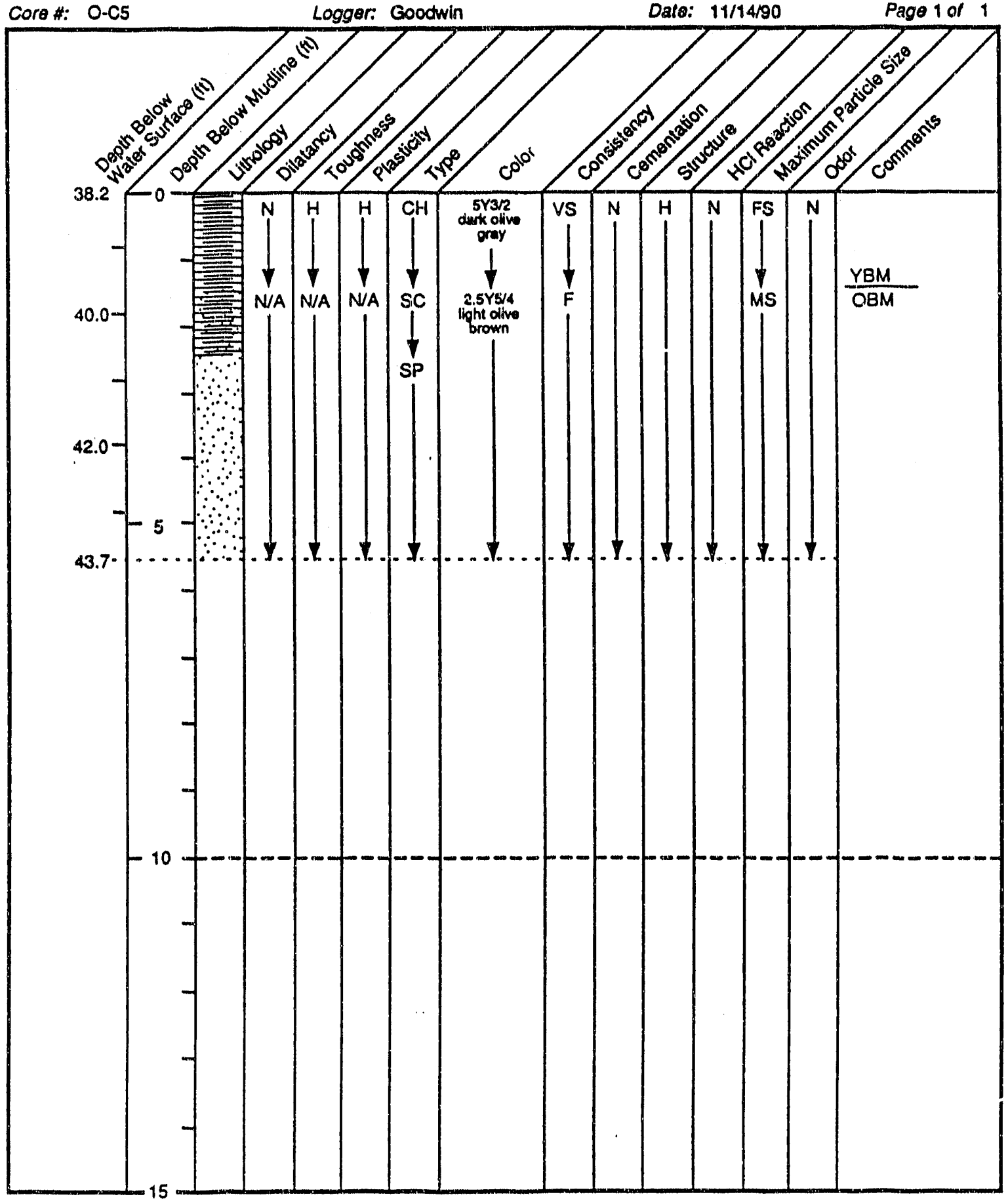




\section{Core Data Log}

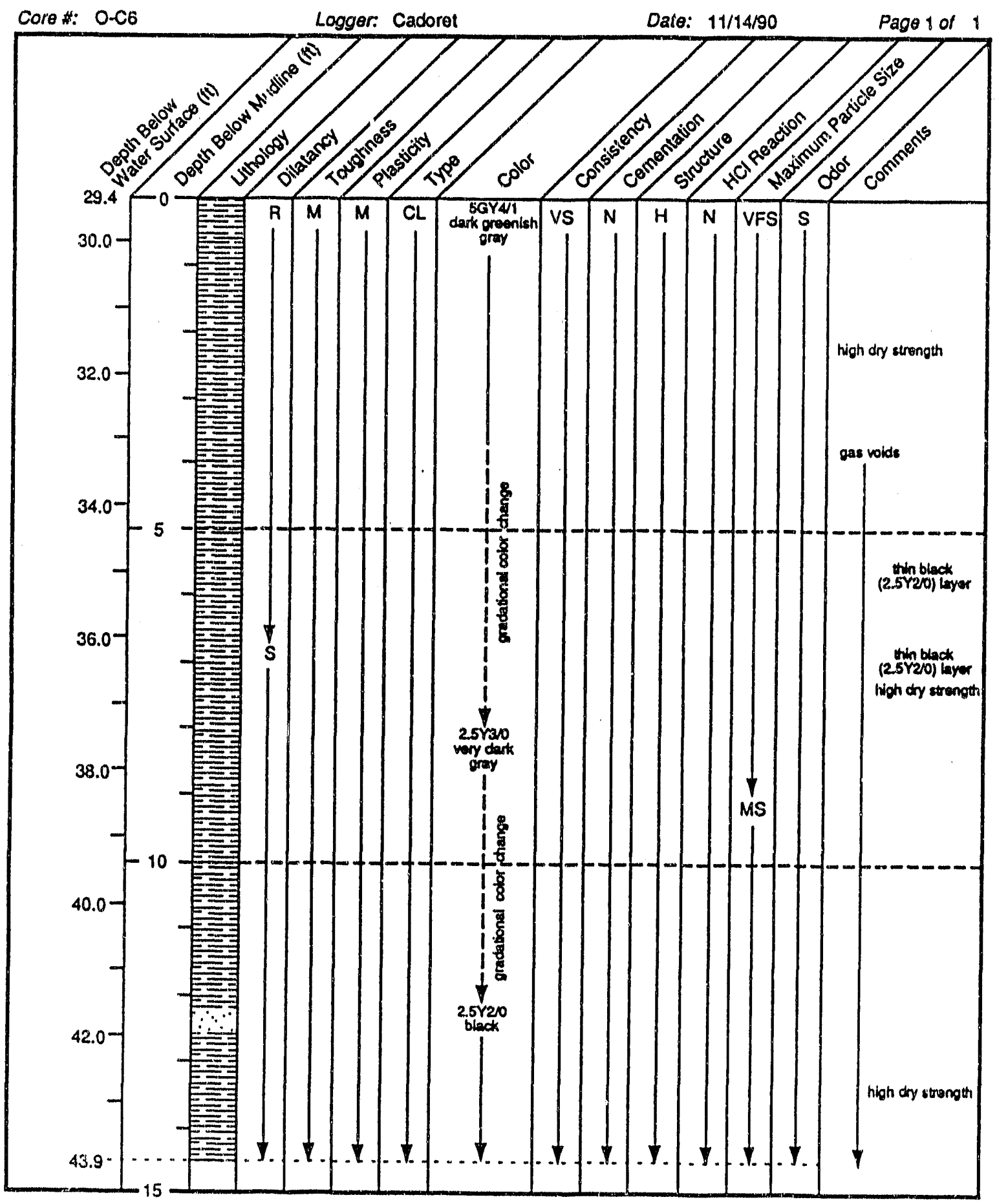


Core Data Log

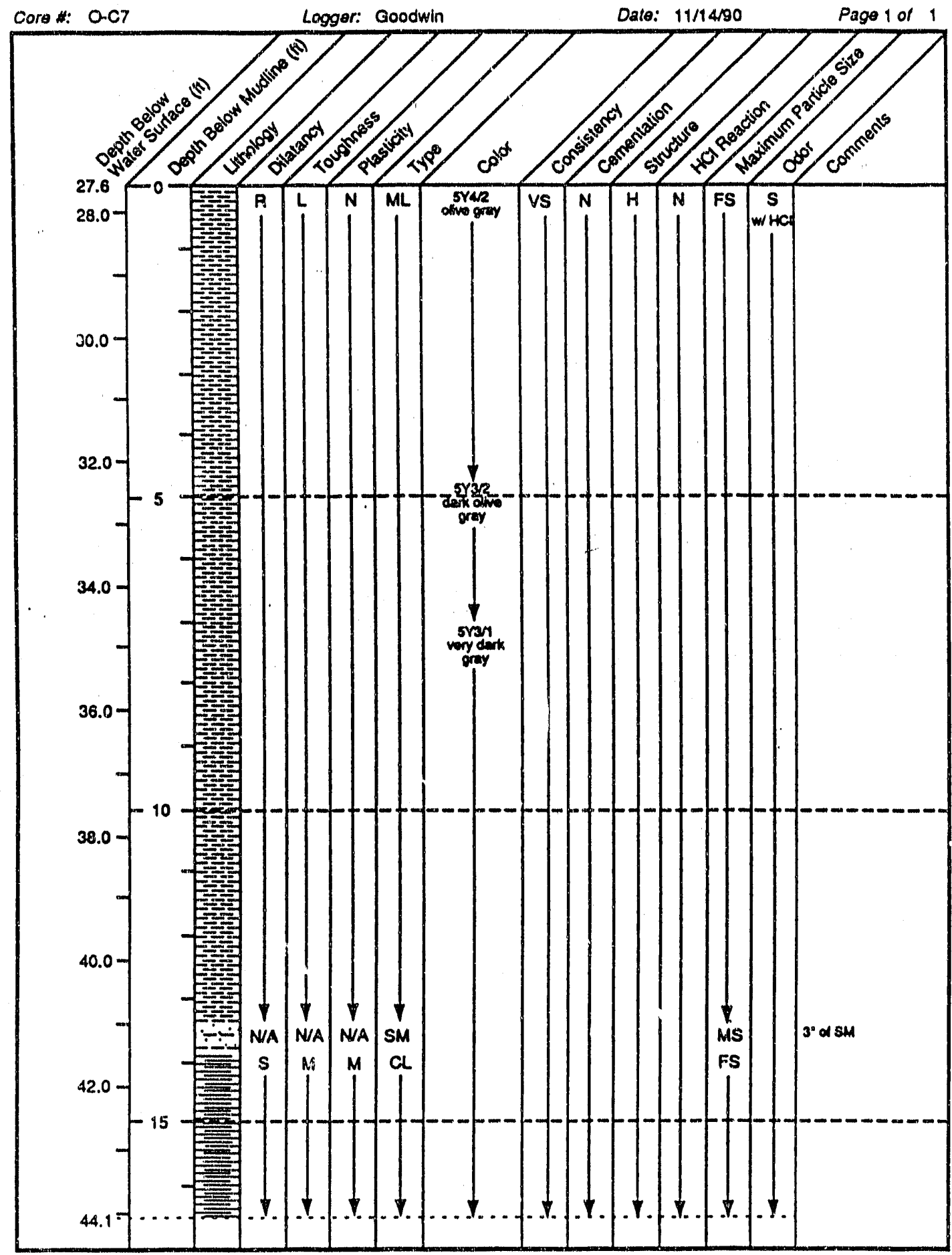




\section{Core Data Log}

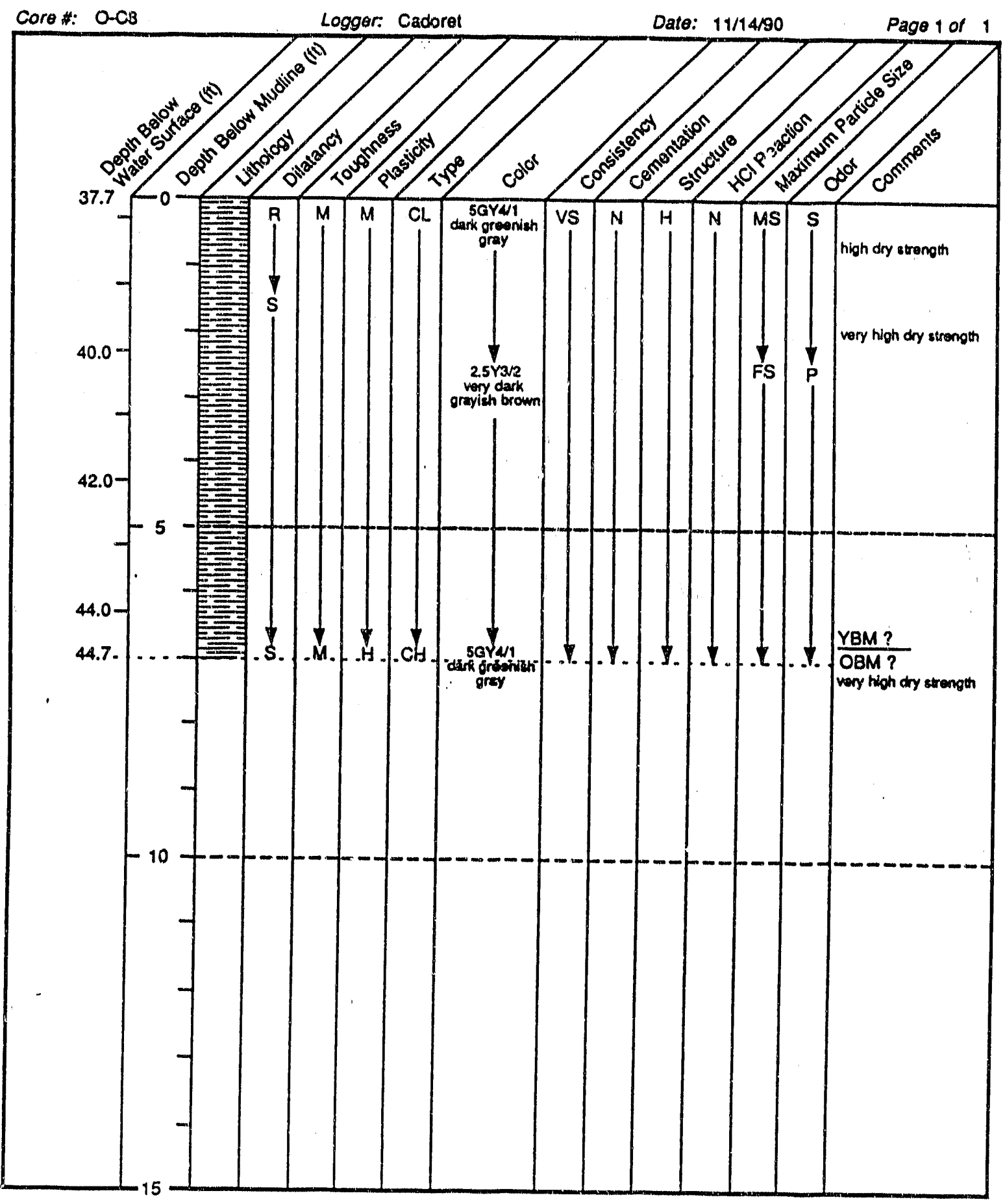


Core Data Log

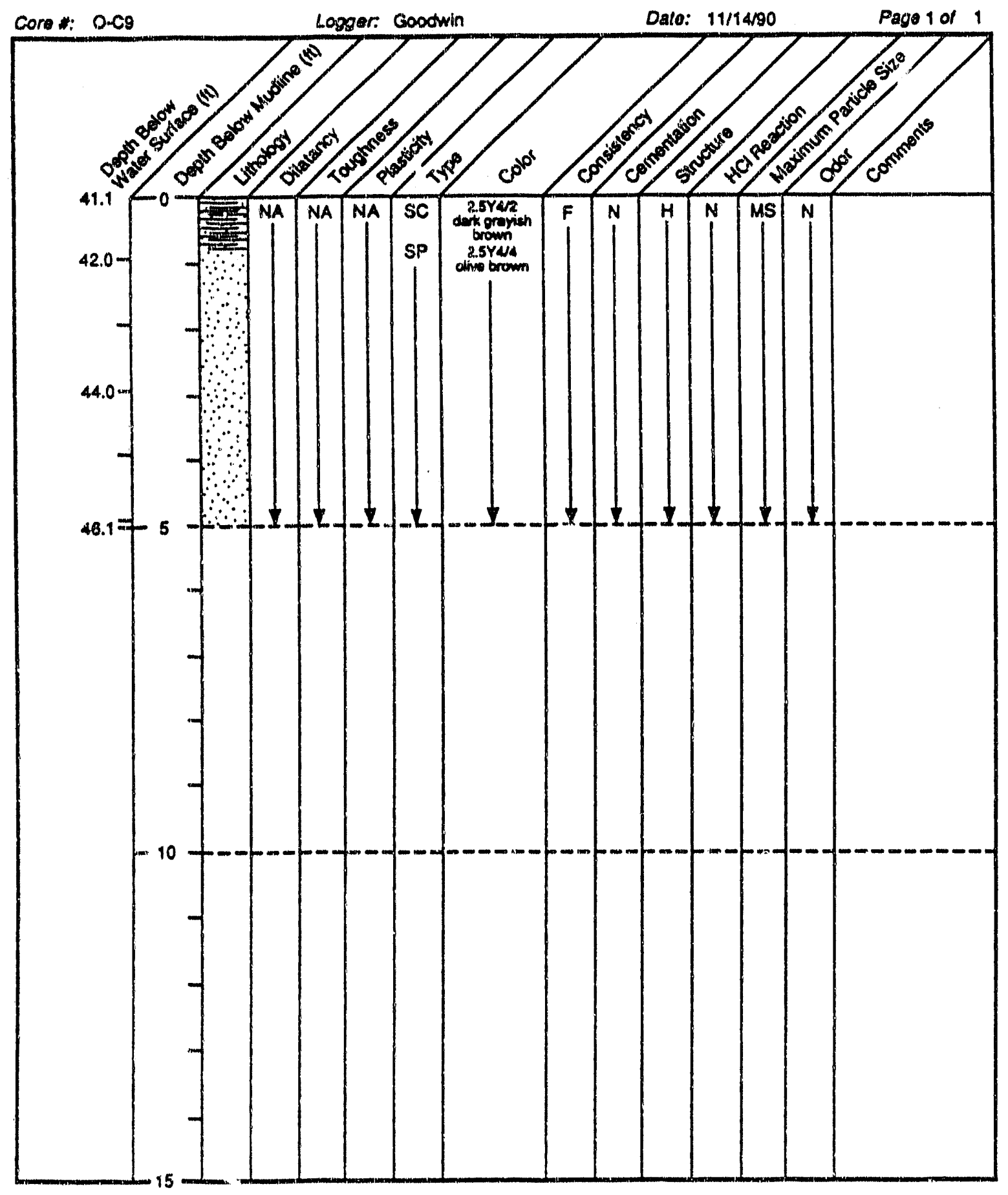

PHASE III $B$

B. 13 
Core Data Log

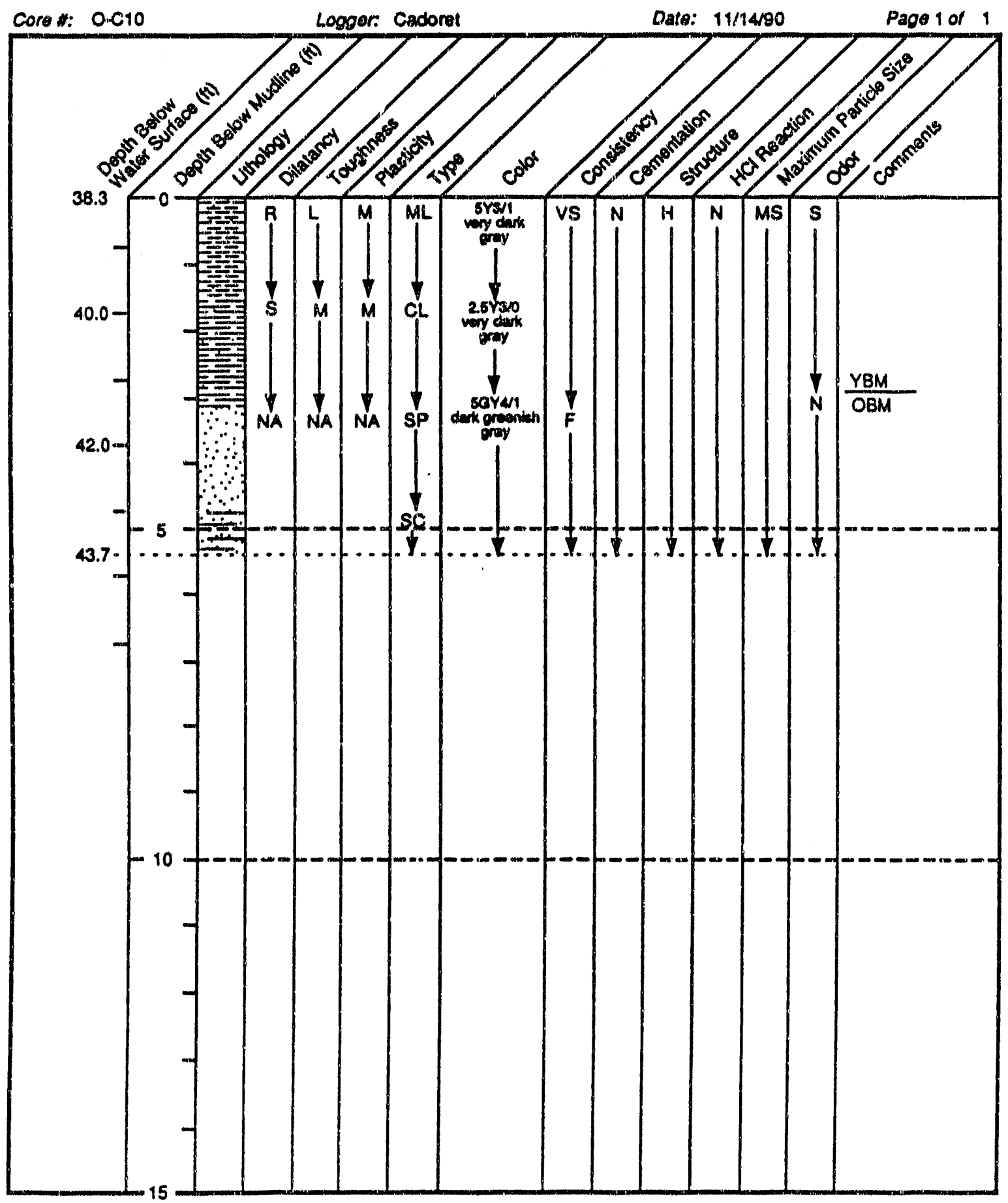




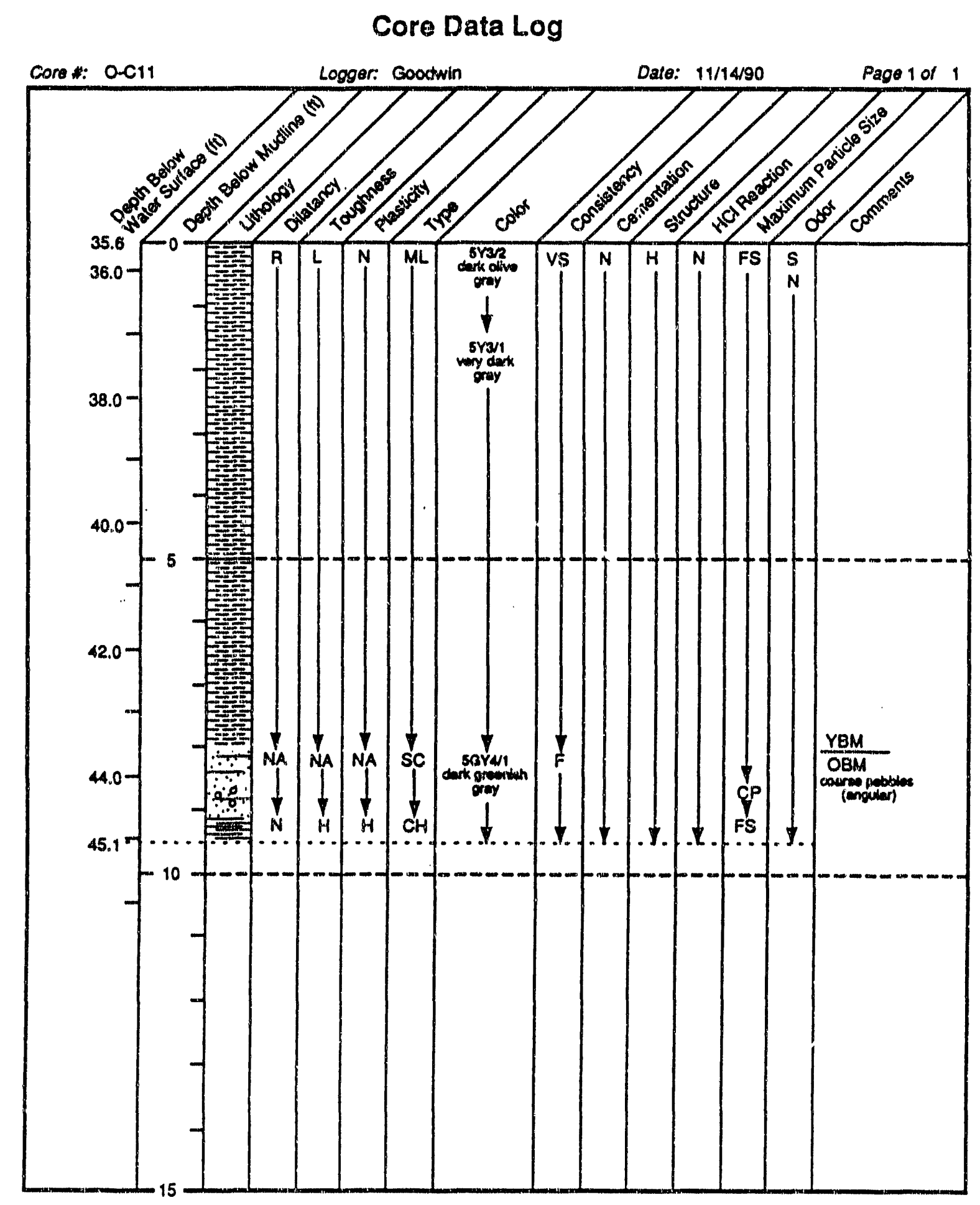


Core Data Log

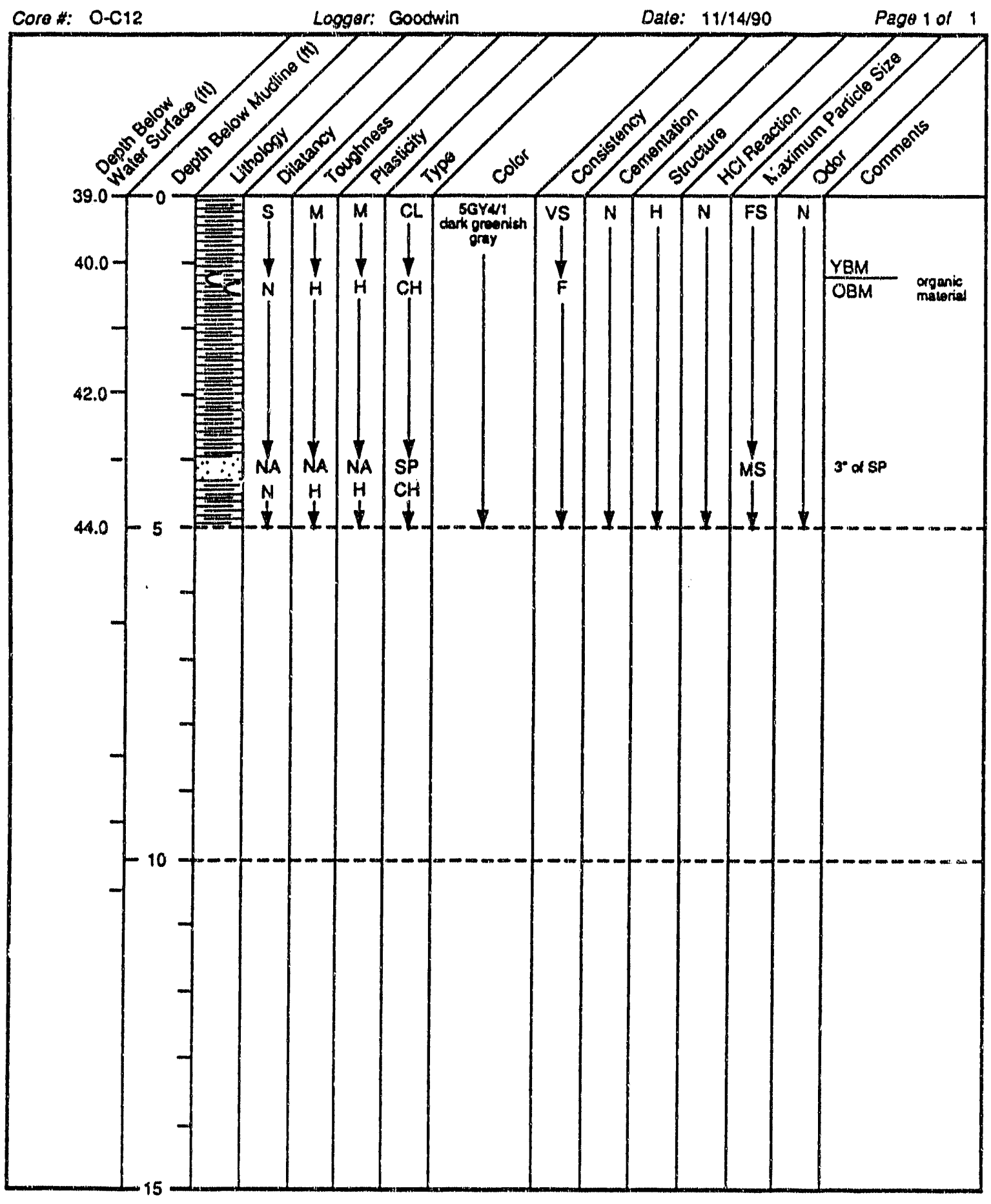


Core Data Log

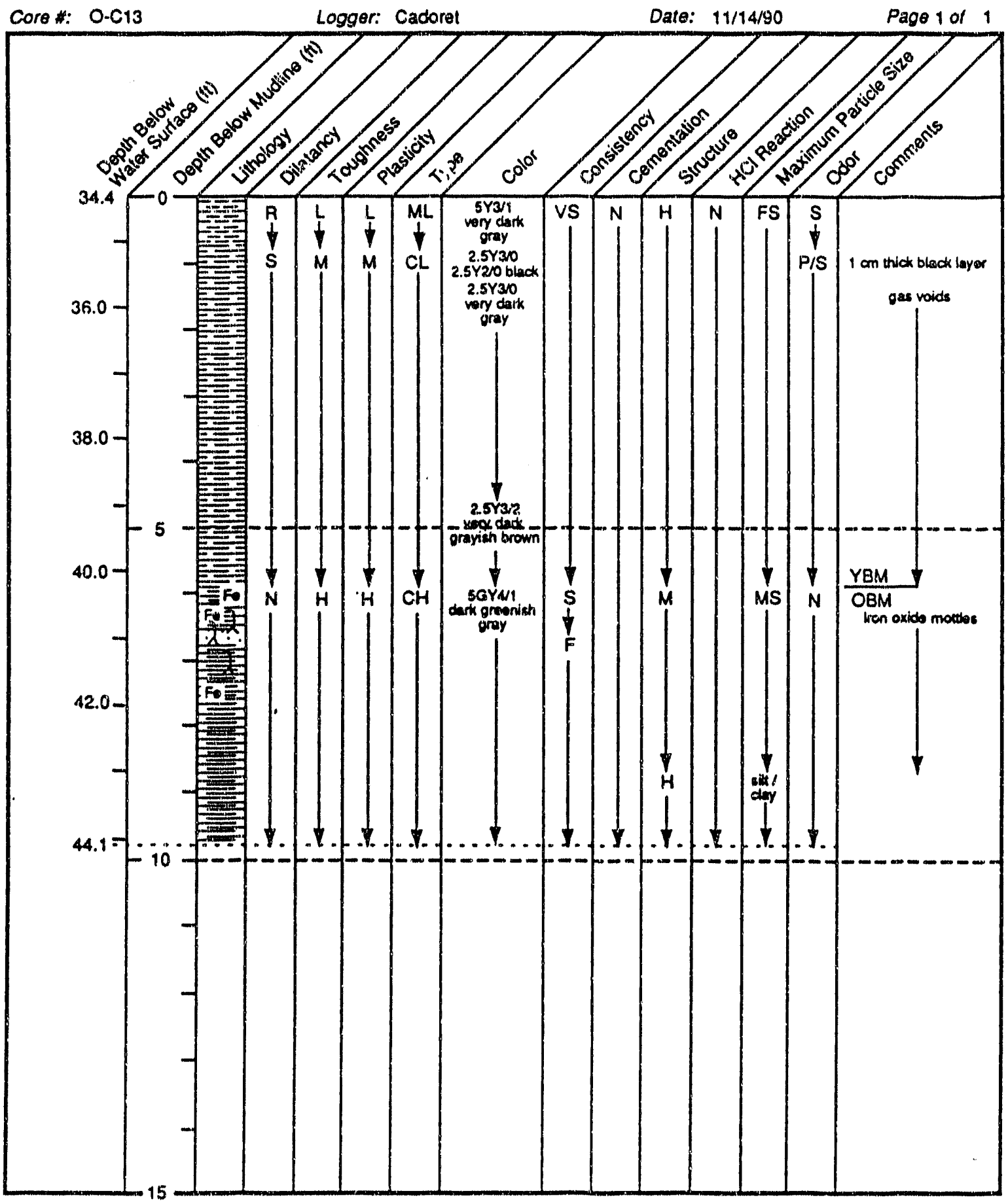


APPENDIX C

SEDIMENT CHEMISTRY AND QUALITY CONTROL DATA 


\section{QA/QC SUMMARY}

PROGRAM:

PARAMETER:

LABORATORY:

MATRIX:

SAMPLES NUMBER:

HOLDING TIMES

DETECTION LIMITS

METHOD BLANKS

SURROGATES

MATRDX SPIKES

\section{REPLICATES}

SRMs
Oakland Harbor Phase III B

Butyltins

Battelle/Marine Science Laboratory

Sediment

36

Holding times for butyltin compounds in sediment are not established. Samples were kept frozen until extraction. Analyses were performed within 2 months of sample receipt at the laboratory as recommended for other organic analyses.

Target detection limits were met.

Four method blanks were analyzed. Low levels of tri-, di- and monobutyltin were detected in these blanks. Levels detected in the method blanks were less than $1 \mu \mathrm{g} / \mathrm{kg}$ in all cases except for one di-butyltin blank which contained $1.3 \mu \mathrm{g} / \mathrm{kg}$.

One compound, tripentyltin, was added prior to extraction as a surrogate to assess the efficiency of the analysis. Surrogate recoveries ranged from $45 \%$ to $110 \%$ within the QC guidelines of $40 \%$. Note, one sample was reported with a surrogate recovery of $198 \%$; possibly due to inadvertantly spiking the sample twice. The results are not affected and were reported with no qualifications.

Three matrix spikes were analyzed. Three butyltin compounds, mono-, di-, and tributyltin were spiked to assess the accuracy of the analysis. Matrix Spike Recoveries (MSR) ranged from $25 \%$ to $71 \%$. The lowest recoveries were found for monobutyltin. This is due to the relatively high volatility of monobutyltin compared to the other butyltin compounds. All recoveries for tributyltin were within the $10 \%$ to $120 \%$ guidelines.

Analytical duplicate anlayses were performed on five samples. Precision was measured by Relative Percent Difference (RPD) between the two replicates. RPDs ranged from $2 \%$ to $62 \%$. Values reported over $5 \mu \mathrm{g} / \mathrm{kg}$, generally had much better agreement with RPDs less than $15 \%$.

Two Standard Reference Material (SRM) samples, PACS-1, obtained from the National Research Council of Canada, were analysed to assess the accuracy of butyltin analyses. (Note: values reported were corrected for surrogate recoveries for those samples. This lab participated in the certification of this SRM and values reported were required to be corrected for tripropyltin recoveries.) Recoveries for tri 
and dibutyltin were below the certified range of values. Past experience in this lab has resulted in recoveries similar to those reported here. and therefore no corrective action was taken.

\section{COMPOSITING} EFFICIENCY

Two blind compositing duplicates were analyzed. Precision was measured by RPD between replicates. RPD values for these compositing duplicates ranged from $18 \%$ to $129 \%$.

Higher RPD values were found for sample O-C10 (56\% to $129 \%)$. This may indicate lower compositing efficiency for butyltins due to nonhomogenous distribution of butyltin containing material in the sample. 
PROGRAM:

PARAMETER:

LABORATORY:

MATRIX:

SAMPLE NUMBER:

HOLDING TIMES

DETECTION LIMITS

METHOD BLANKS

SURROGATES

MATRIX SPIKES

REPLICATES

SRMs

COMPOSITING EFFICIENCY
Oakland Harbor Phase III B

Total Organic Carbon (TOC)

Global Geochemistry Corp.

Sediment

36

Holding times for TOC analyses of sediments are not established. All samples were freeze dried upon receipt at the lab. Samples were analyzed within 2 months of sample receipt.

Target detection limits were met $(<0.1 \%)$

Two method blanks were analyzed. TOC was measured at less than $0.01 \%$ in the blanks. These values do not affect the sample results.

NOT APPLICABLE

NOT APPLICABLE

Two samples were analyzed in triplicate. Precision was measured by Relative Standard Deviation (RSD)between replicates. The RSDs for the replicates ranged from $1.6 \%$ to $1.84 \%$, indicating acceptable precision for the analysis.

NOT APPLICABLE

Two blind compositing duplicates were analyzed. Precision was measured by Relative Percent Difference (RPD) between replicates. RIP values for these compositing duplicates ranged from $0 \%$ to $3 \%$, indicating acceptable analytical precision on blind samples and efficient sample compositing. 
PROGRAM:

PARAMETER:

LABORATORY:

MATRIX:

SAMPLES NUMBER:

HOLDING TIMES

DETECTION LIMITS

METHOD BLANKS

SURROGATES

MATRIX SPIKES

REPLICATES

SRMs

COMPOSITING EFFICIENCY
Oakland Harbor Phase III B

Total Volatile Solids

Battelle/Marine Sciences Laboratory

Sediment

36

Holding times for TVS analyses of sediments are not established. Samples were held frozen or freeze dried until analysis. All analyses were completed within 2 months of sample receipt at the lab.

Target detection limits were met $(<0.1 \%)$

One method blank was analyzed with these samples. Volatile solids were undetected in the blank.

NOT APPLICABLE

\section{NOT APPLICABLE}

Two samples were analyzed in triplicate. Precision was measured by Relative Standard Deviation (RSD) between replicates. The RSDs for the two sets of triplicates were less than $2 \%$, indicating acceptable precision of the analysis.

\section{NOT APPLICABLE}

Two blind compositing duplicates were analyzed. Precision was measured by Relative Percent Difference (RPD) between replicates. RPD values for thee compositing duplicates ranged from $6 \%$ to $7 \%$, indicating acceptable analytical precision on blind samples and efficient sample compositing. 


\section{QA/QC SUMMARY}

PROGRAM:

PARAMETER:

LABORATORY:

MATRIX:

SAMPLE NUMBER:

HOLDING TIMES

DETECTION LIMITS

METHOD BLANKS

SURROGATES

MATRIX SPIKES

REPLICATES

SRMs
Oakland Harbor Phase III B

Polynuclear Aromatic Hydrocarbons (PAHs)

Alder Analytical, Inc.

Sediment

36

PAH analyses were completed within our recommended holding time limits of 2 months. Samples were kept frozen until extraction and all extracts were analyzed within the 40 day EPA (1986) recommended holding time for extracts.

Target detection limits for each PAH compound were met $(<20 \mu \mathrm{g} / \mathrm{kg})$.

Two method blanks were analyzed. PAH compounds were undetected in these blanks. Surrogate recoveries of four radiolabeled cornpounds added to blanks ranged from $76 \%$ to $120 \%$ which are within acceptable ranges of $50 \%$ to $150 \%$.

Four radiolabeled compounds were added to samples prior to extraction to assess the efficiency of the analysis. Recoveries for all 35 samples were within the $50 \%$ to $150 \%$ QC range with the exception of one sample, O-C4. This sample had recoveries of $45 \%$ and $42 \%$ for two of the four surrogates. The other two surrogates were over $50 \%$ and because no recoveries were below $20 \%$, reanalysis was not required.

Analytical accuracy was partially assessed through use of PAH Matrix Spikes. Two samples, C-WB and $\mathrm{O}-\mathrm{C} 12$ were spiked in duplicate with the PAH compounds evaluated. Matrix spike recoveries ranged from $83 \%$ to $161 \%$. These recoveries, except for one, were within the QC recovery range of $50 \%$ to $150 \%$; therefore, further action was not taken.

Two sets of triplicate analyses were performed. Precision was measured by determining the Relative Standard Deviation (RSD). Replicate analyses showed good agreement, with RSDs ranging from $6 \%$ to $58 \%$. All compounds were measured within the QC limit of $20 \%$, except for one replicate of the first set of triplicates which consistently had higher values (1.58-fold) of each of the PAH compounds. Because all other measurements were within the QC limits, no further action was taken.

One SRM compound, HS-4 (NRCC) was analyzed twice for all PAH compounds. Seven of the 17 compounds exceeded the range of certified values for these compounds. The concentrations of the compounds that exceeded the certified ranges were generally present at levels 5 to 10 times those measured in the samples. Dilution of the SRM was required and therefore provided additional levels of error to the measurements. These exceedences, however, were generally within $30 \%$ of the certiried mean value and no further action was taken. 
COMPOSITING EFFICIENCY
Two blind compositing duplicates were analyzed. Precision was measured by Relative l'ercent Differences (RPD) between replicates. RPD values for these compositing duplicates ranged from $0 \%$ to $30 \%$ for the PAH compounds, indicating acceptable analytical precision on blind samples and efficient sample compositing. 


\section{QA/QC SUMMARY}

PROGRAM:

PARAMETER:

LABORATORY:

MATRIX:

SAMPLE NUMBER:

DETECTION LIMTTS

METHOD BLANKS

SURROGATES

MATRIX SPIKES

REPLICATES

SRMs

COMPOSITING EFFICIENCY
Oakland Harbor Phase III B

Grain Size

Soil Technologies, Inc.

Sediment

35

NOT APPLICABLE

NOT APPLICABLE

NOT APPLICABLE

NOT APPLICABLE

Five samples were analyzed in duplicate. Precision was measured by Relative Percent Difference (RIPD) between replicates. Comparison of gravel, sand, silt and clay summations resulted in low RPD values ranging from $0 \%$ to $10 \%$ for those fractions representing greater than $10 \%$ of the sediment dry weight. RPD values between duplicate values for fractions representing less than $10 \%$ of the sediments dry weight were higher $(<29 \%)$ due to the small amounts in these fractions. When individual fractions contained less than $10 \%$, RPDs tend to become exaggerated.

NOT APPLICABLE

Two blind compositing duplicates were analyzed. Precision was measured by RPDs between replicates. RPD values for these compositing duplicates ranged for $0 \%$ to $14 \%$, indicating acceptable analytical precision on blind samples and efficient sample compositing. 
PROGRAM:

PARAMETER:

LABORATORY:

MATRIX:

SAMPLES NUMBER:

HOLDING TIMES

DETECTION LIMITS

METHOD BLANKS

SURROGATES

MATRIX SPIKES

REPLICATES
Oakland Harbor Phase III B

Metals (Ag, As, Cd, Cr, Cu, Hg, Ni, Pb, Se Zn)

Battelle/Marine Sciences Laboratory

Sediment

35

Metals were analyzed within the allowed holding times. Samples were freeze dried immediately upon receipt and digested and analyzed within the 6 month recommended holding time for metals in sediment (EPA/USACE, 1991).

Target detection limit goals were met for all metals except selenium. Selenium DL's were slightly higher $(<10 \%)$ than the achieved target of $0.1 \mathrm{mg} / \mathrm{kg}$.

Low levels of silver and mercury were detected in method blanks. All values reported were corrected for blank concentrations. (Note: method blanks do not apply to $\mathrm{As}, \mathrm{Cr}, \mathrm{Cu}, \mathrm{Ni}, \mathrm{Pb}$ and $\mathrm{Zn}$ due to the method of analysis $X R F$, which precludes use of method blanks.)

\section{NOT APPLICABLE}

Matrix Spikes apply to non-XRF metals only, $\mathrm{Ag}, \mathrm{Cd}, \mathrm{Hg}$ and Se. Two samples were spiked with these compounds. Recoveries for these metals ranged from $81 \%$ to $105 \%$ which is within the quality control limits of $75 \%$ to $125 \%$

Triplicate analyses were performed on two samples to access precision. Precision was measured by Relative Standard Deviation (RSD) between the values. RSDs ranged from $1.26 \%$. These results indicated acceptable precision of both $\mathrm{AA}$ and $\mathrm{XRF}$ metal analyses.

Four different SRMs were analyzed (a minimum of 1 SRM per 10 samples) to assess accuracy of metals analyzed. The different SRM samples were analyzed to include certified values for all metals and to cover the range of concentrations of metals expected in the project samples. From the eight SRM analyses, $\mathrm{Cd}, \mathrm{Cr}, \mathrm{Cu}$, and $\mathrm{Hg}$ were out of certified range for one or more of the SRMs certified for that metal. In general, the outlier values were within $15 \%$ of the certified values and at least three SRMs for each metal were within the certified range; therefore, no further action was taken. 
Two blind compositing duplicates were analyzed. Precision was measured by Relative Percent Differences (RPD) between replicates. RPD values for these compositing duplicates ranged from $2 \%$ to $64 \%$. $\mathrm{Hg}, \mathrm{Pb}$, and As had the largest differences (20\% to 64\%). All other metals had RPDs less than $13 \%$, indicated acceptable analytical precision on blind samples and efficient sample compositing. 


\section{QAVC SUMMARY}

PROGRAM:

PARAMETER:

LABORATORY:

MATRDX:

SAMPLES NUMBER:

HOLDING TIMES

DETECTION LIMITS

METHOD BLANKS

SURROGATES

MATRIX SPIKES

REPLICATES

SRMs

COMPOSITING EFFICIENCX
Oakland Harbor Phase III B

Oil and Grease/Total Petroleum Hydrocarbons

Twin City Testing

Sediment

36

No holding times for oil and grease analyses of sediments have been established. All samples were held frozen $\left(<4^{\circ} \mathrm{C}\right)$ and analyzed within 2 months of receipt at Battelle MSL.

Target detection limits were met $(<20 \mathrm{mg} / \mathrm{kg}$ )

Seven method blanks were analyzed with the samples. Oil and grease levels in the method blanks ranged from 2 to $15 \mathrm{mg} / \mathrm{kg}$ and from $<0.5$ to $4 \mathrm{mg} / \mathrm{kg}$ for TPH.

\section{NOT APPLICABLE}

Two sets of matrix spike/matrix spike duplicate samples were analyzed. Matrix spike for oil and grease ranged from $109 \%$ to $156 \%$ and from $72 \%$ to $153 \%$ for petroleum hydrocarbons. Relative Percent Differences (RPD) between the recoveries were good, ranging from $3 \%$ to $4 \%$ for oil and grease and $6 \%$ to $8 \%$ for petroleum hydrocarbons. No QC goals are available for recoveries of oil and grease and TPH spikes although the recoveries are within the range set for other organic analyses (50\% to $150 \%)$

Two samples were analyzed in triplicate. Relative Standard Deviation (RSD) between replicates ranged from $25 \%$ to $100 \%$. These values exceed our goal of $10 \%$ for precision, however, the RSDs reflect the differences between low values that are less than the target detection limits ( $<20 \mathrm{mg} / \mathrm{kg}$ ).

NOT APPLICABLE

Two blind compositing duplicates were analyzed. Precision was measured by RPD between replicates. RPD values for these compositing duplicates ranged from $2 \%$ to $3 \%$ for oil and grease and from $6 \%$ to $17 \%$ for petroleum hydrocarbons, indicating acceptable analytical precision on blind samples and efficient sample compositing. 
PROGRAM:

PARAMETER:

LABORATORY:

MATRIX:

SAMPLE NUMBER:

HOLDING TIMES

DETECIION LIMTS

METHOD BLANKS

SURROGATES

MATRD SPIKES

REPLICATES

SRMs
Oakland Harbor Phase III B

Polychlorinated Biphenyls (PCBs) and Chlorinated Pesticides

Alden Analytical Laboratories, Inc.

Sediment

36

PCB and pesticide analyses were completed within holding time limits of 2 months. Samples were kept frozen until extraction and all extracts were analyzed within the 40 day EPA (1986) recommended holding time.

Target detection limits for PCBs of $20 \mu \mathrm{g} / \mathrm{kg}$ were exceeded. Achieved DL's were $50 \mu \mathrm{g} / \mathrm{kg}$ dry weight. Detection limits for toxaphene were higher due to the coelution of this multi-component pesticide with other pesticide and PCB peaks on the chromatogram. This detection limit was set to assure accurate quantitation of toxaphene and to avoid false positive detections.

Two method blanks were analyzed. No pesticides or PCBs were detected.

One surrogate compound, dibuty' hlorendate (DBC) was added to all samples prior to analyses for $\mathrm{PCBs}$ and pesticides. All recoveries were greater than $70 \%$ with the exception of two samples with DBC recoveries of $32 \%$ and $34 \%$. No PCBs or pesticides were detected in these samples and because the recovery was greater than $20 \%$, reanalysis was not required.

Two samples were spiked in duplicate with 2 pesticides, Aldrin and Dieldrin. One Matrix Spike Duplicate (MSD) was lost during extraction. Recoveries for the single comparison ranged from $95 \%$ to $155 \%$. The recovery for Dieldrin exceeded the QC limits of $50 \%$ to $150 \%$. This slight exceedance did not require qualification of the data.

Two samples were analyzed in triplicate to assess precision. Precision was measured by Relative Standard Deviation (RSD). No PCBs or pesticides were detected in any of the samples, therefore, calculations were not performed.

NOT APPLICABLE 


\section{COMPOSITING}

EFFICIENCY
Two blind compositing duplicates were analyzed. Precision was to be measured by RPD between replicates. No pesticides or PCB's were detected in either replicate of the two composites. 


\section{REFERENCES}

U.S. Environmental Protection Agency (EPA). 1986. Test Methods for Evaluating Solid Waste: Physical/Chemical Methods. EPA-955-001-00000. U.S. Environmental Protection Agency, Government Printing Office, Washington, D.C. 
IABLE C.1. Summary of Sediment Grain Size Including Quality Control Data, Oakl and Harbor Phase III B Project

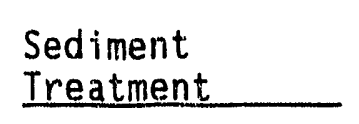

$\mathrm{BC}-7$

$0-C 13$

$0-\mathrm{Cl} 2$

$0-C 11$

$0-C 10$

$0-$ C10 Cdup $^{(a)}$

$B C-5$

$0-C 9$ Rep 1

$0-C 9$ Rep 2

$0-C 8$ Rep 1

$0-C 8$ Rep 2

$0-\mathrm{C} 7$

$0-\mathrm{C} 6$

$B C-8$

$B C-8$ Cdup

$0-C 5$

$0-C 4$ Rep 1

$0-C 4$ Rep 2

$B C-6$

$0-\mathrm{C} 3$

$0-\mathrm{Cl}$

$\mathrm{I}-\mathrm{C} 2$

$0-\mathrm{C2}$

I - $\mathrm{C} .3$

$\mathrm{I}-\mathrm{C} 8$

I-C8 top 6 in. Rep 1

I-C8 top 6 in. Rep 2

I $-\mathrm{C} 4$

I $-\mathrm{TS}$

$R-A C$

$R-A M$

$R-B F$

$R-O S$

$R-P C$

$R-P F$ Rep 1

R-PF Rep 2

\begin{tabular}{|c|c|c|c|}
\hline $\begin{array}{c}\text { Gravel } \\
\geq 2000 \mathrm{\mu m} \\
\end{array}$ & $\begin{array}{l}\text { Sand } \\
62.5- \\
2000 \mathrm{\mu m} \\
\end{array}$ & $\begin{array}{r}\text { Silt } \\
3.9- \\
62.5 \mu \mathrm{m}\end{array}$ & $\begin{array}{r}\text { Clay } \\
\leq 3.9 \mu \mathrm{m} \\
\end{array}$ \\
\hline $\begin{array}{l}0 \\
0 \\
0 \\
5 \\
0 \\
0\end{array}$ & $\begin{array}{l}40 \\
12 \\
64 \\
25 \\
52 \\
52\end{array}$ & $\begin{array}{l}23 \\
33 \\
14 \\
27 \\
18 \\
21\end{array}$ & $\begin{array}{l}37 \\
55 \\
22 \\
43 \\
30 \\
27\end{array}$ \\
\hline $\begin{array}{l}0 \\
0 \\
0 \\
0 \\
1 \\
0 \\
0\end{array}$ & $\begin{array}{r}40 \\
87 \\
87 \\
11 \\
11 \\
6 \\
5\end{array}$ & $\begin{array}{r}24 \\
6 \\
8 \\
31 \\
30 \\
35 \\
38\end{array}$ & $\begin{array}{r}36 \\
7 \\
6 \\
58 \\
58 \\
59 \\
57\end{array}$ \\
\hline
\end{tabular}

62.

64

87

19

19

18

16
7

38

39

$23 \quad 39$

$28 \quad 32$

$9 \quad 49$

$27 \quad 35$

7

92

86

52

52

66

85

45

5

5

19

18

13

98

91

2

60

97

62

63
21

20

6
43

42

27

36

6

58
58

59

7

38

40

42

40

48

5

30

30

21

$1]$.

2
6
63
13
3
11
10


TABLE C.1. (contd)

Total Percent

Sediment

Treatment

C-SB

C-NE

$C-W B$

C-SD
Sand

62.5 -

$2000 \mu \mathrm{m}$

11

97

98

99

Silt

3.9-

$62.5 \mu \mathrm{m}$

53

1

0

0
Clay

$<3.9 \mu \mathrm{m}$

36

2

2

Sample

Analytical Duplicates

0-C9 Rep 1
0-C9 Rep 2
RPD

I-STAT

0-C8 Rep 1

0-C8 Rep?

RPD

I-STAT

I-C8 Rep 1

I-C8 Rep2

RPD

I-STAT

0-C4 Rep 1

0-C4 Rep 2

RPD

I-STAT

R-PF Rep 1

$R$-PF Rep 2

.. RPD

I - STAT
0
0
$0 \%$
0.00

0
1
$200 \%$

1.00

0

$0 \%$

0.00

0

$0 \%$

0.00

0
0
$0 \%$
0.00

Compositing Duplicates
$0-\mathrm{C} 10$

$0-$ C10 Cdup

RPD

I - STAT
0
0
$0 \%$
0.00
87

87

0.00

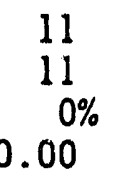

52

52

$0 \%$

0.00

19

19

$0 \%$

0.00

62
63
$2 \%$
0.01

62

$2 \%$

0.01
6
8
$29 \%$
0.14

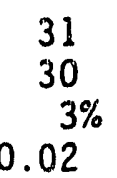

0.02

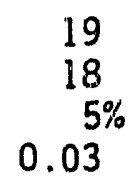

\section{8}

39

$3 \%$

0.01

27
27
$0 \%$
0.00

27

$0 \%$

0.00
$15 \%$

0.08

58
58
$0 \%$
0.00

\section{0}

30

0.00

$0 \%$

43

42

0.01

11

10

$10 \%$
0.05 $\%$ 
IABLE C.1. (contd)

Sediment

Treatiment

$B C-8$

BC-8 Cdup

RPD

I-STAT

\begin{tabular}{cccc}
\multicolumn{4}{c}{ Total Percent } \\
\hline Grave1 & Sand & Silt & Clay \\
$\geq 2000 \mu \mathrm{m}$ & $62.5-$ & $3.9-$ & $\leq 3.9 \mu \mathrm{m}$ \\
\hline 0 & $2000 \mu \mathrm{m}$ & $\underline{62.5 \mu \mathrm{m}}$ & \multicolumn{3}{c}{} \\
0 & 62 & 18 & 21 \\
$0 \%$ & 64 & 16 & 20 \\
0.00 & $3 \%$ & $12 \%$ & $5 \%$ \\
\cline { 2 - 4 } & 0.02 & 0.06 & 0.02
\end{tabular}

(a) Compositing duplicate. 


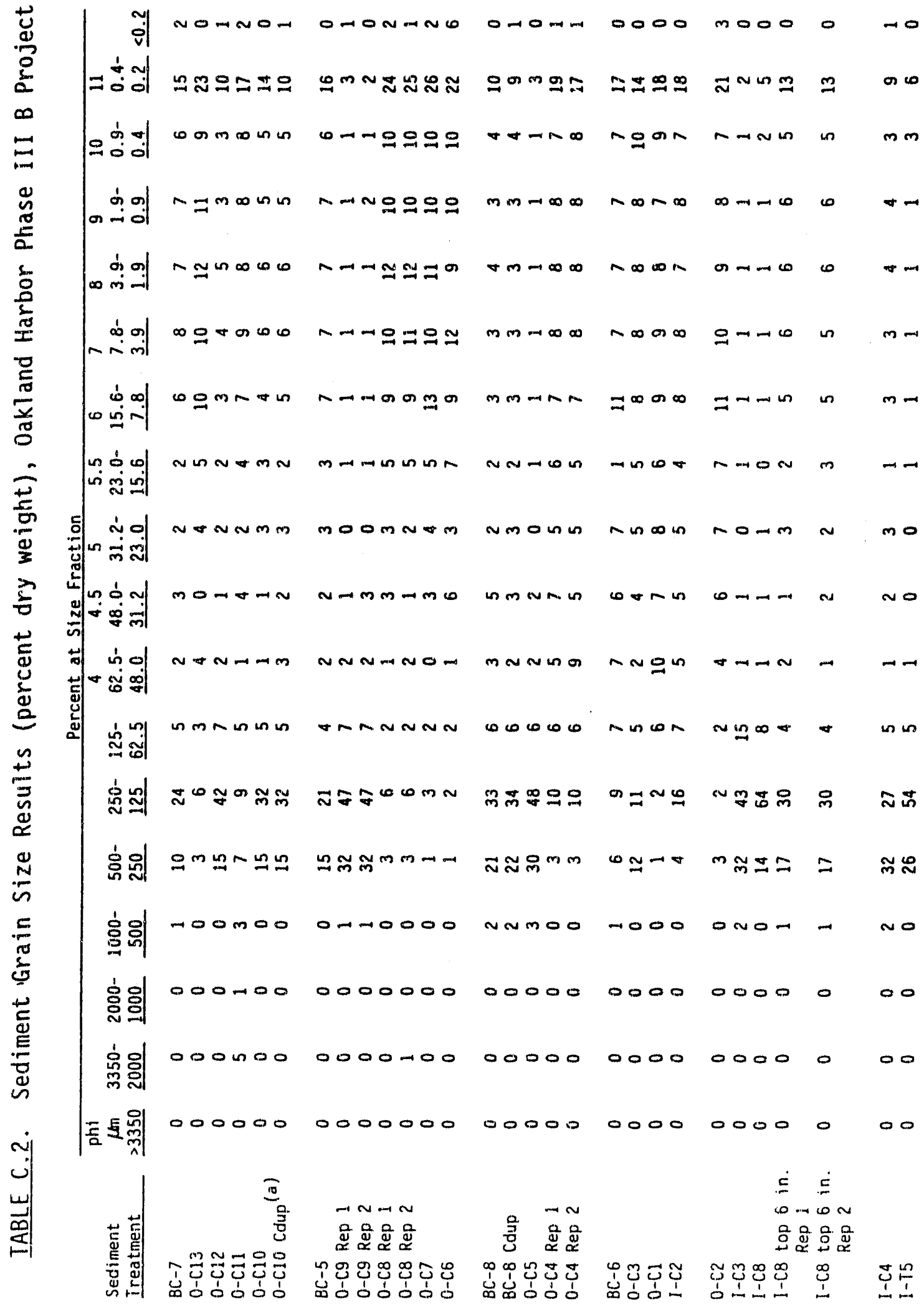

PHASE III B

C. 4 


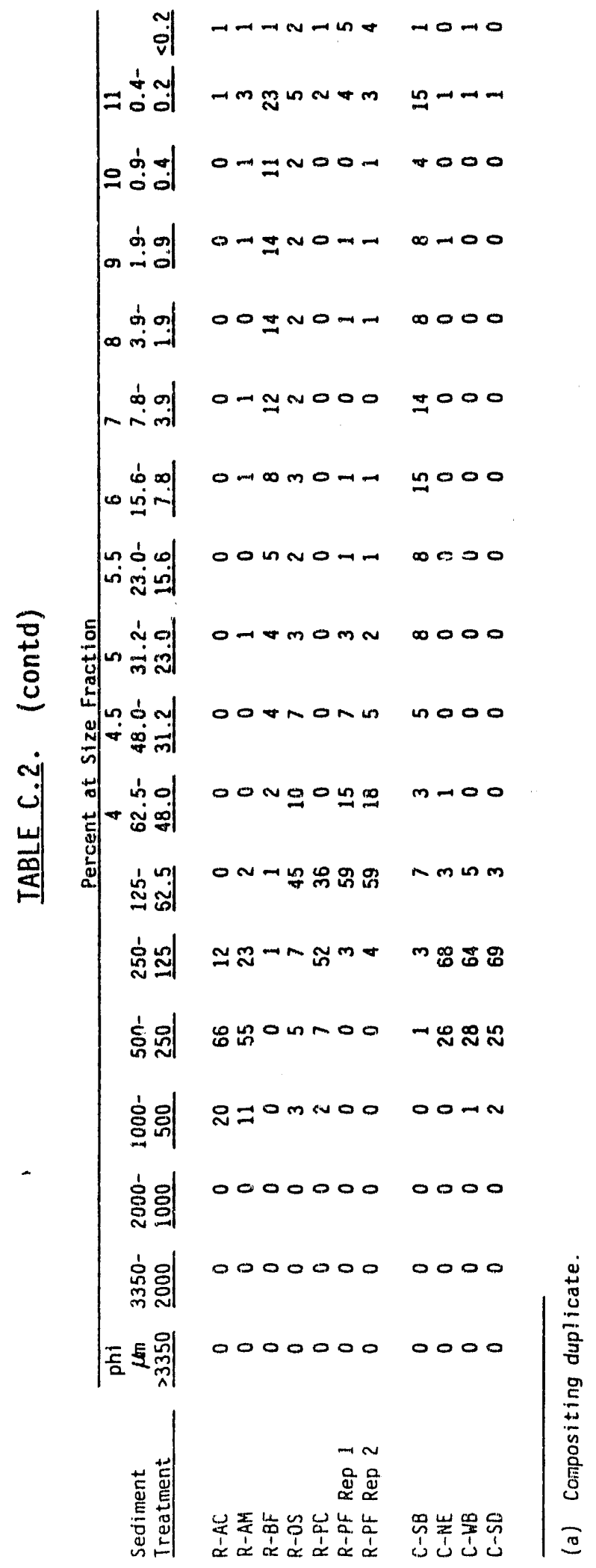




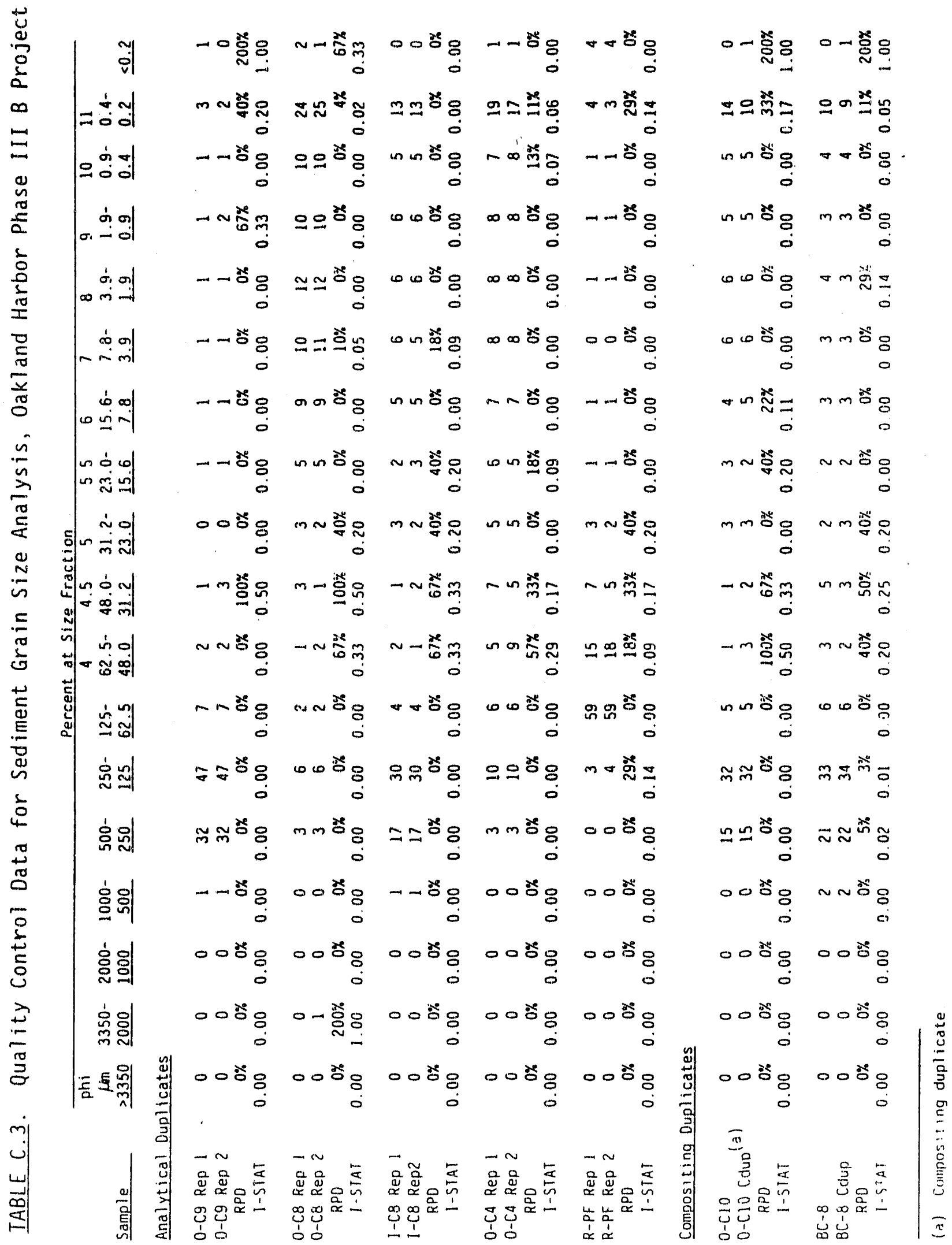

PHASE III B

C. 6 
TABLE C.4. Sediment Total Organic Carbon (TOC) and Total Volatile Solids (TVS) Results, Oakl and Harbor Phase III B Project

\begin{tabular}{|c|c|c|}
\hline $\begin{array}{l}\text { Sediment } \\
\text { Ireatment }\end{array}$ & $\begin{array}{c}\text { TOC } \\
\text { (percent dry weight) }\end{array}$ & $\begin{array}{l}\text { TVS } \\
\text { (percent dry weight) }\end{array}$ \\
\hline $\begin{array}{l}\text { Detection Limit Goal } \\
\text { Detection Limit Achieved }\end{array}$ & $\begin{array}{l}0.01 \\
0.005\end{array}$ & $\begin{array}{l}0.10 \\
0.01\end{array}$ \\
\hline $\begin{array}{l}B-C 7 \\
0-C 13 \\
0-C 12 \\
0-C 11 \\
0-C 10 \\
0-C 10 \text { Cdup }^{(a)}\end{array}$ & $\begin{array}{l}0.55 \\
1.01 \\
0.15 \\
0.65 \\
0.51 \\
0.51\end{array}$ & $\begin{array}{l}4.83 \\
7.41 \\
2.51 \\
5.48 \\
4.23 \\
4.48\end{array}$ \\
\hline $\begin{array}{l}B-C 5 \\
0-C 9 \\
0-C 8 \\
0-C 7 \\
0-C 6\end{array}$ & $\begin{array}{l}0.66 \\
0.03 \\
1.03 \\
1.11 \\
1.15\end{array}$ & $\begin{array}{l}5.35 \\
0.99 \\
6.68 \\
7.15 \\
7.14\end{array}$ \\
\hline $\begin{array}{l}\text { B-C8 } \\
\text { B-C8 rdup } \\
0-C 5 \\
0-C 4\end{array}$ & $\begin{array}{l}0.33 \\
0.34 \\
0.04 \\
0.93\end{array}$ & $\begin{array}{l}2.99 \\
3.20 \\
1.05 \\
5.55\end{array}$ \\
\hline $\begin{array}{l}B-C 6 \\
0-C 3 \\
0-C 1 \\
I-C 2\end{array}$ & $\begin{array}{l}0.75 \\
0.64 \\
0.81 \\
0.87\end{array}$ & $\begin{array}{l}5.25 \\
6.46 \\
6.93 \\
6.39\end{array}$ \\
\hline $\begin{array}{l}\text { O-C2 } \\
\text { I-C3 } \\
\text { I-C8 } \\
\text { I-C8 top } 6 \text { in. }\end{array}$ & $\begin{array}{l}0.75 \\
0.06 \\
0.10 \\
0.48\end{array}$ & $\begin{array}{l}4.62 \\
1.23 \\
2.07 \\
3.93\end{array}$ \\
\hline $\begin{array}{l}I-C 4 \\
I=T 5\end{array}$ & $\begin{array}{l}0.36 \\
0.01\end{array}$ & $\begin{array}{l}3.04 \\
1.38\end{array}$ \\
\hline $\begin{array}{l}R-A C \\
R-A M \\
R-B F \\
R-O S \text { Rep } 1 \\
\text { R-OS Rep } 2 \\
\text { R-OS Rep } 3 \\
R-P C \\
\text { R-PF }\end{array}$ & $\begin{array}{l}0.05 \\
0.19 \\
1.10 \\
0.64 \\
0.62 \\
0.62 \\
0.15 \\
0.41\end{array}$ & $\begin{array}{l}1.20 \\
2.10 \\
9.61 \\
4.28 \\
4.23 \\
4.33 \\
1.67 \\
2.57\end{array}$ \\
\hline
\end{tabular}


TABLE C.4. (contd)

Sediment

Treatment

C-SB Rep 1

C-SB Rep 2

C-SB Rep 3

C-NE

$C-W B$

C-SD
TOC

(percent dry weight)

2.15

2.21

2.15

0.08

0.09

0.05
TVS

(percent dry weight)

8.96

8.99

8.99

1.30

1.08

1.83

(a) Compositing duplicate. 
IABLE C.5. Quality Control Data for Sediment Total Organic Carbon (TOC) and Total Volatile Solids (TVS) Analyses, Oakland Harbor Phase III B Project

Sediment

Treatment

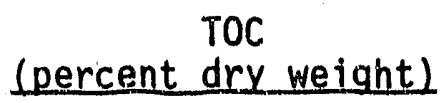

TVS

Method Blanks

Blank 1

0.004

Blank 2

0.006

0.00

$\mathrm{ND}^{(\mathrm{a})}$

Analytical Replicates

R-OS Rep 1

R-OS Rep 2

0.64

4.28

R-OS Rep 3

0.62

4.23

RSD

$2 \%$

4.33

C-SB Rep 1

C-SB Rep 2

C-SB Rep 3

RSD

2.15

8.96

2.21

2.15

8.99

$2 \%$

8.99

$0 \%$

Compositing Duplicates
$0-\mathrm{C} 10$
0.51
4.23
$0-$ C10 Cdup $^{(b)}$
0.51
RPD
$0 \%$
4.48
I -STAT
0.00
0.03
B-C8
0.33
0.34
RPD
I-STAT
0.01
2.99
3.20
0.03

(a) No data - only one method blank analysed for TVS.

(i) Compositing duplicate. 
TABLE C.6. Sediment $0 i 1$ and Grease and Totai Petroleum Hydrocarbons (TPH) Results, Oakland Harbor Phase III B Project

\begin{tabular}{|c|c|c|c|c|}
\hline $\begin{array}{l}\text { Sediment } \\
\text { Ireatment }\end{array}$ & $\begin{array}{l}\text { Analytical } \\
\text { Batch } \\
\end{array}$ & $\begin{array}{l}0 i 1 \text { and Grease }{ }^{(a)} \\
(\mathrm{mg} / \mathrm{kg} \mathrm{dry} \text { weight })\end{array}$ & $\begin{array}{c}T P H(a) \\
(m g / k q d r y \text { weight })\end{array}$ & $\begin{array}{l}\text { Petroleum } \\
\text { Fraction } x\end{array}$ \\
\hline $\begin{array}{l}\text { DL Goal } \\
\text { DL Achieved }\end{array}$ & & $\begin{array}{r}20 \\
0.5\end{array}$ & $\begin{array}{r}20 \\
0.5\end{array}$ & $\begin{array}{l}N A(b) \\
N A\end{array}$ \\
\hline $\begin{array}{l}B-C 7 \\
0-C 13 \\
0-C 13 \quad 41.5 \mathrm{ft} \text { MLLW } \\
0-C 12 \\
0-C 11 \\
0-C 10 \\
0-C 10 \text { Coup (d) }\end{array}$ & $\begin{array}{l}F \\
B \\
E \\
B \\
C \\
C \\
E\end{array}$ & $\begin{array}{r}150 \\
95 \\
8 \\
24 \\
65 \\
89 \\
92\end{array}$ & $\begin{array}{r}87 \\
84 \\
3 \\
31 \\
51 \\
64 \\
68\end{array}$ & $\begin{array}{l}58 \\
88 \\
38 \\
\mathrm{NC}(\mathrm{C}) \\
78 \\
72 \\
74\end{array}$ \\
\hline $\begin{array}{l}B C-5 \\
0-C 9 \\
0-C 8 \\
0-C 7 \\
0-C 6\end{array}$ & $\begin{array}{l}E \\
D \\
D \\
D \\
D\end{array}$ & $\begin{array}{r}116 \\
12 \\
304 \\
251 \\
183\end{array}$ & $\begin{array}{r}78 \\
3 \\
252 \\
159 \\
135\end{array}$ & $\begin{array}{l}67 \\
2.5 \\
83 \\
63 \\
74\end{array}$ \\
\hline $\begin{array}{l}\text { B-C8 } \\
\text { 8-C8 Cdup } \\
0-C 5 \text { Rep } 1 \\
0-C 5 \text { Rep } 2 \\
0-C 5 \text { Rep } 3 \\
0-C 4\end{array}$ & $\begin{array}{l}E \\
G \\
0 \\
D \\
D \\
D\end{array}$ & $\begin{array}{r}62 \\
63 \\
24 \\
10 \\
1 \\
116\end{array}$ & $\begin{array}{r}42 \\
35 \\
3 \\
5 \\
4 \\
70\end{array}$ & $\begin{array}{l}68 \\
56 \\
13 \\
50 \\
N C \\
60\end{array}$ \\
\hline $\begin{array}{l}B-C 6 \\
0-C .3 \\
0-C 1 \\
1-C .2\end{array}$ & $\begin{array}{l}E \\
E \\
E \\
D\end{array}$ & $\begin{array}{r}109 \\
84 \\
80 \\
138\end{array}$ & $\begin{array}{l}61 \\
39 \\
29 \\
99\end{array}$ & $\begin{array}{l}56 \\
46 \\
36 \\
72\end{array}$ \\
\hline $\begin{array}{l}0-C 2 \\
1-C 3 \\
I-C 8 \\
1-C 8 \text { Top } 6 \text { in. }\end{array}$ & $\begin{array}{l}D \\
D \\
D \\
F\end{array}$ & $\begin{array}{r}58 \\
1 \\
42 \\
137\end{array}$ & $\begin{array}{r}32 \\
5 \\
26 \\
97\end{array}$ & $\begin{array}{l}55 \\
N C \\
62 \\
71\end{array}$ \\
\hline $\begin{array}{l}I-C 4 \\
I-75\end{array}$ & $\begin{array}{l}B \\
B\end{array}$ & $\begin{array}{r}2 \\
130\end{array}$ & $\begin{array}{r}12 \\
106\end{array}$ & $\begin{array}{l}N C \\
82\end{array}$ \\
\hline $\begin{array}{l}R-A C \\
R-A M \\
R-B F \\
R-O S \text { Rep } 1 \\
R-O S \text { Rep } 2 \\
R-O S \text { ReP } 3 \\
R-P C \\
R-P F\end{array}$ & $\begin{array}{l}B \\
B \\
A \\
B \\
B \\
B \\
B \\
A\end{array}$ & $\begin{array}{l}7 \\
0.7 u^{(e)} \\
92 \\
12 \\
28 \\
0.8 U \\
0.7 u \\
27\end{array}$ & $\begin{array}{r}5 \\
8 \\
40 \\
10 \\
5 \\
5 \\
0.70 \\
9\end{array}$ & $\begin{array}{l}71 \\
N A \\
43 \\
83 \\
18 \\
N A \\
N A \\
33\end{array}$ \\
\hline $\begin{array}{l}C-S B \\
C-S D \\
C-N E \\
C-W B\end{array}$ & $\begin{array}{l}A \\
A \\
A \\
A\end{array}$ & $\begin{array}{r}147 \\
1 \\
3 \\
72\end{array}$ & $\begin{array}{lll}1.4 & U \\
0.7 & U \\
0 & 6 & U \\
\therefore & 1 & U\end{array}$ & $\begin{array}{l}1 \\
70 \\
20 \\
!\end{array}$ \\
\hline
\end{tabular}

(a) Concentrations are corrected for the amount of anaiyte found in corresponding batch blank.

(b) Not applicable.

(c) Calculation is not applicable--oil and grease is likely $100 \%$ iPH

(d) Compositing duplicate.

(e) Undetected above given concentrations.
PHASt : : B
C. 10 
TABLE C.7. Quality Control Data for Sediment 0 il and Grease and Total Petroleum Hydrocarbons (TPH) Analys is, Dakland Harbor Phase III B Project

Sample

Analytical

$0 i]$ and Grease(a) Batch

( $\mathrm{mg} / \mathrm{kg}$ dry weight)

$\mathrm{TPH}^{(\mathrm{a})}$

$(\mathrm{mg} / \mathrm{kg}$ dry weight)

Method Blanks

Bl ank
Bl ank
Bl ank
Bl ank
Bl ank
Bl ank
Bl ank

$A$
$B$
$C$
$D$
$E$
$F$
$G$

8
15
7
7
2
6
3

2
3
4
2
$0.5 u^{(b)}$
2
$0.5 U$

Matrix Spikes

$B C-8$

BC-8 Cdup(c) MS

G $\quad 63$

E 133

35

Amount spiked

133
45

115

Amount Recovered

70

45

Percent Recovery

$156 \%$

69

$B C-8$

BC- 8 Cdup MSD

G

63

130

$153 \%$

Amount Spiked

Amount Recovered

Percent Recovery

E

35

110

47

67

47

$143 \%$

64

MS/MSD RPD

MS/MSD I-STAT

$9 \%$

$136 \%$

$R-P C$

R-PC MS

Amount Spiked Amount Recovered Percent Recovery

0.04

$12 \%$

$B$

$0.7 U$

0.06

$R-P C$

R-PC MSD

Amount Spiked Amount Recovered Percent Recovery

C

37

$0.7 \mathrm{U}$

32

27

32

37

$116 \%$

27

$84 \%$

$\stackrel{8}{C}$

$0.7 \mathrm{U}$

$0.7 U$

35

32

23

32

35

$109 \%$

23

$72 \%$

MS/MSD RPD

MS/MSD I-STAT

$0.03 \%$

$15 \%$

0.08 
TABLE C.I. (contd)

$\begin{array}{llll}\text { Sample } & \begin{array}{c}\text { Analytical } \\ \text { Batch }\end{array} & \begin{array}{c}0 i 1 \text { and Grease(a) } \\ \text { (mg/kg dryweight) }\end{array} & \begin{array}{c}\text { Total Petroleum } \\ \text { Hydrocarbons } \\ \text { (mg/kg dry weight) }\end{array}\end{array}$

\section{Analytical Replicates}

$\begin{array}{cccc}0-C 5 \text { Rep } 1 & D & 24 & 3 \\ 0-C 5 \text { Rep 2 } & D & 10 & 5 \\ 0-C 5 \text { Rep } 3 & D & 1 & 4 \\ \text { RSD } & & 99 \% & 25 \% \\ \text { R-OS Rep 1 } & B & 12 & 10 \\ \text { R-OS Rep 2 } & B & 28 & 5 \\ \text { R-OS Rep 3 } & B & 0.8 \text { U } & 43 \% \\ \text { RSD } & & \text { NA } & \text { (d) }\end{array}$

Compositing Duplicates

$\begin{array}{lccc}0-C 10 & C & 89 & 64 \\ 0-C 10 \text { Cdup } & \text { E } & 92 & 68 \\ \text { RPD } & & 3 \% & 6 \% \\ \text { I-STAT } & & 0.02 & 0.03 \\ \text { BC-B } & E & 62 & 42 \\ \text { BC-8 Cdup } & G & 63 & 35 \\ \text { RPD } & & 2 \% & 18 \% \\ \text { I-STAT } & & 0.01 & 0.09\end{array}$

(a) Concentrations other than in blanks are corrected for the anount of analyte found in corresponding batch blank.

(b) Undetected above given concentration.

(c) Compositing duplicate.

(d) Not applicable; no calculation performed due to one or more non-detected values. 
TABLE C.8. Total Polynuclear Aromatic Hydrocarbon (PAH) Found in Sedinent Treatments, Oakland Harbor Phase III B Project

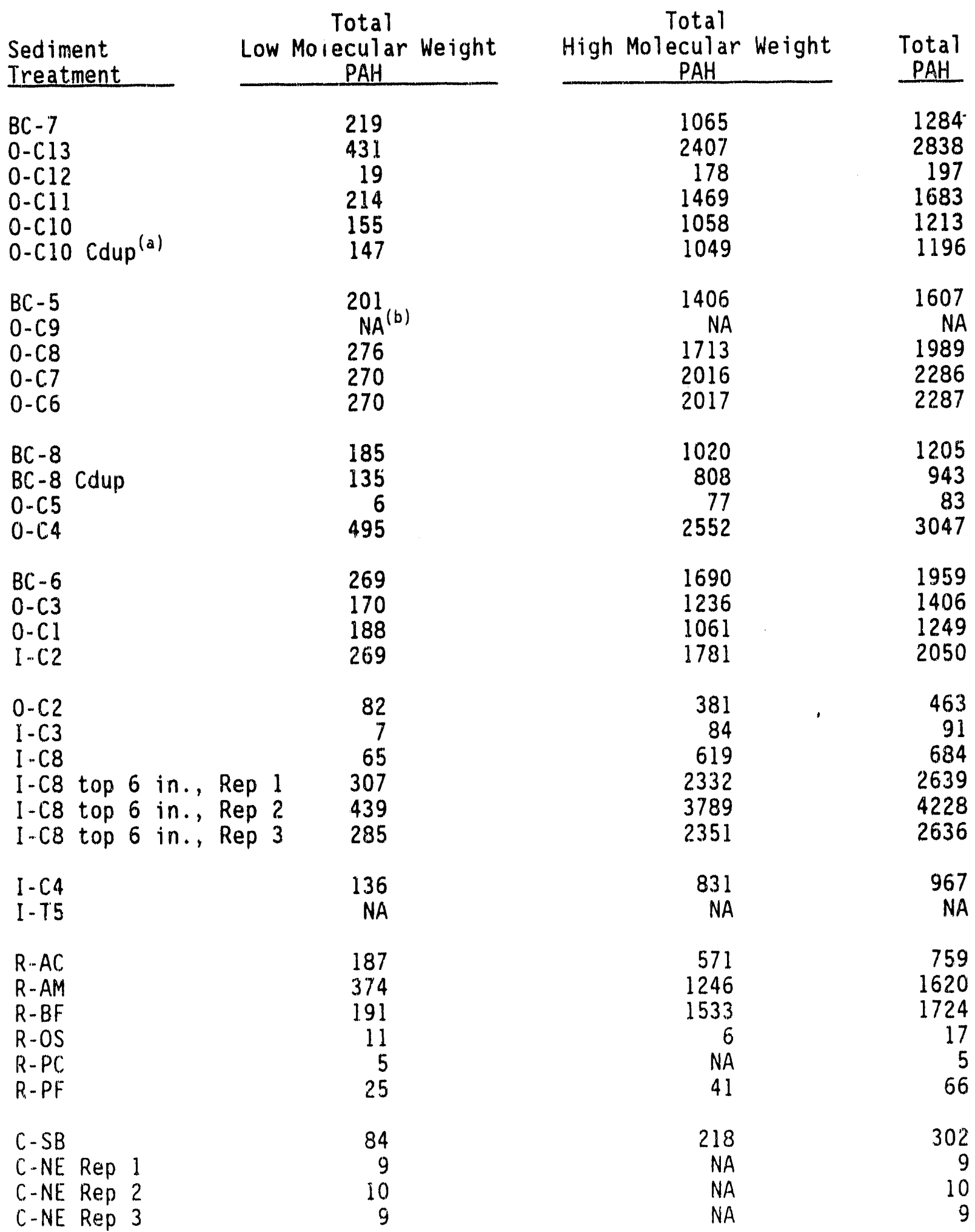


TABLE C.8. (contd)

Sediment

Total

Low Molecular Weight

Treatment PAH

$\begin{gathered}\text { Total } \\ \text { High Molecular Weight } \\ \text { PAH }\end{gathered}$
NA
NA $\begin{array}{r}\text { Total } \\ \text { PAH }\end{array}$

(a) Compositing duplicate.

(b) Not applicable; no PAHs detected. 
IABLE C.9. Sediment Low Molecular Weight Polynuclear Aromatic Hydrocarbon (PAH) Results, Oakland Harbor Phase III B Project

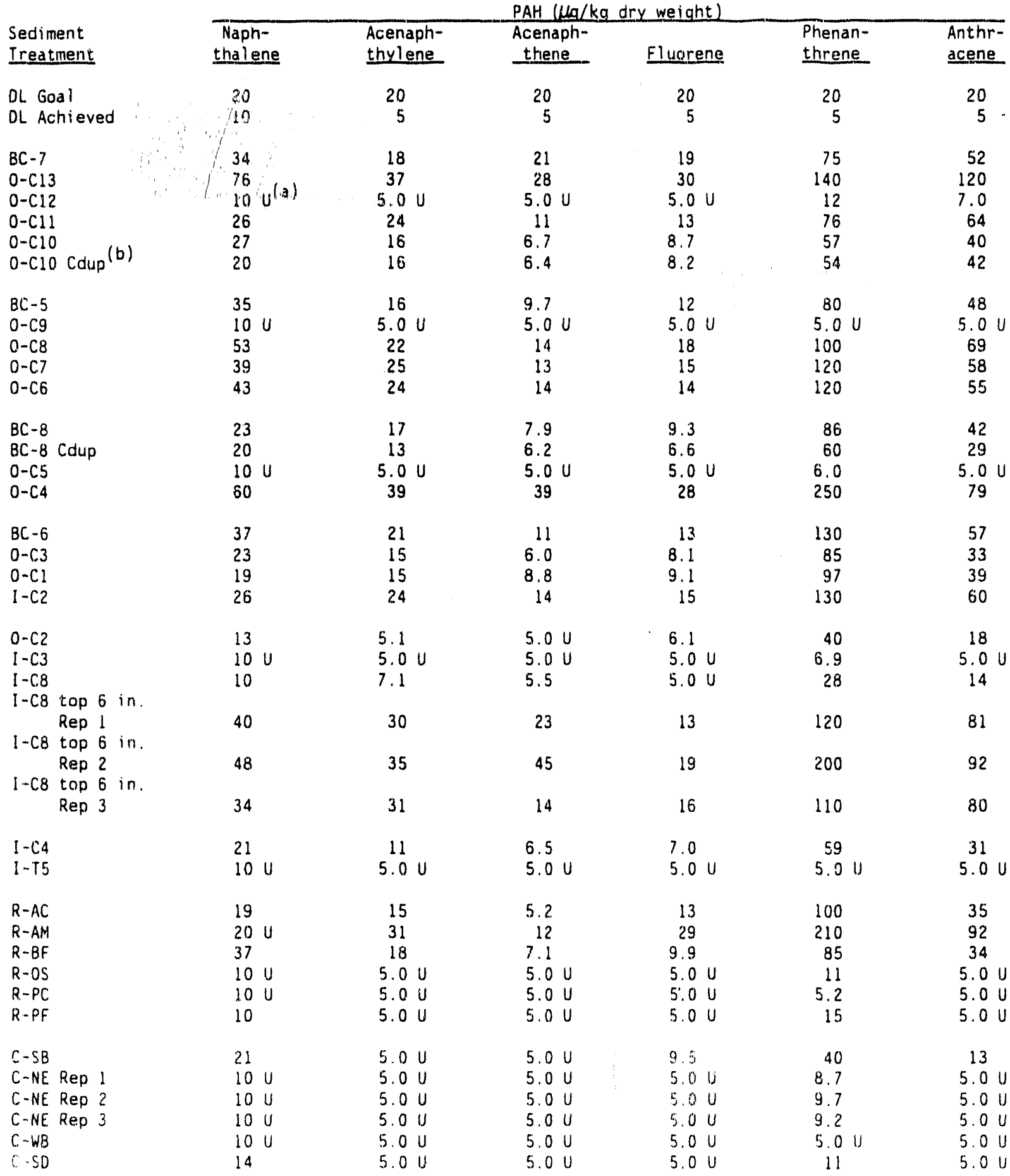

(a) Undetected above given concentration.

(b) Compositing duplicate.

PHASE III B

C. 15 


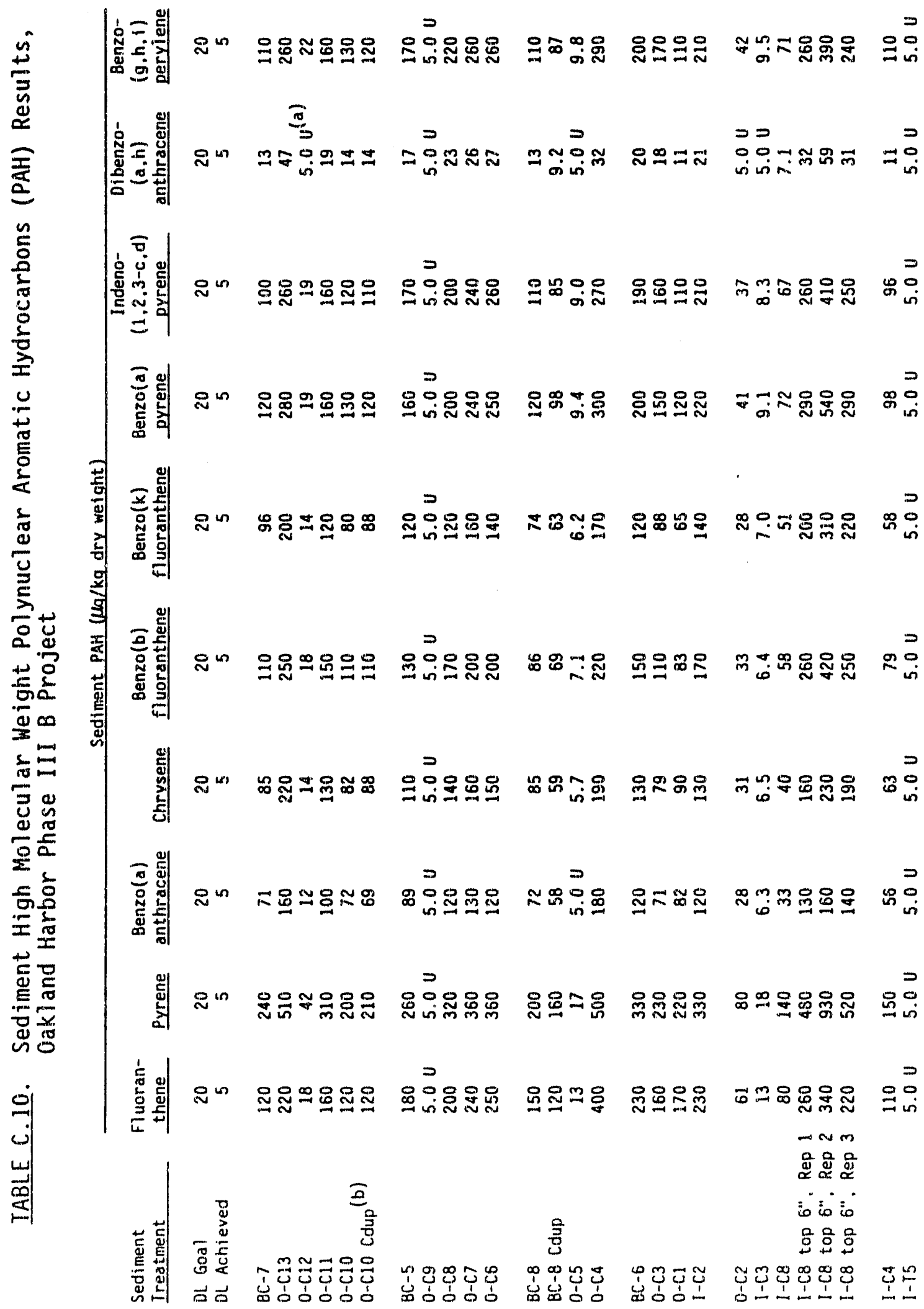

PHASE III $B$

C. 16 


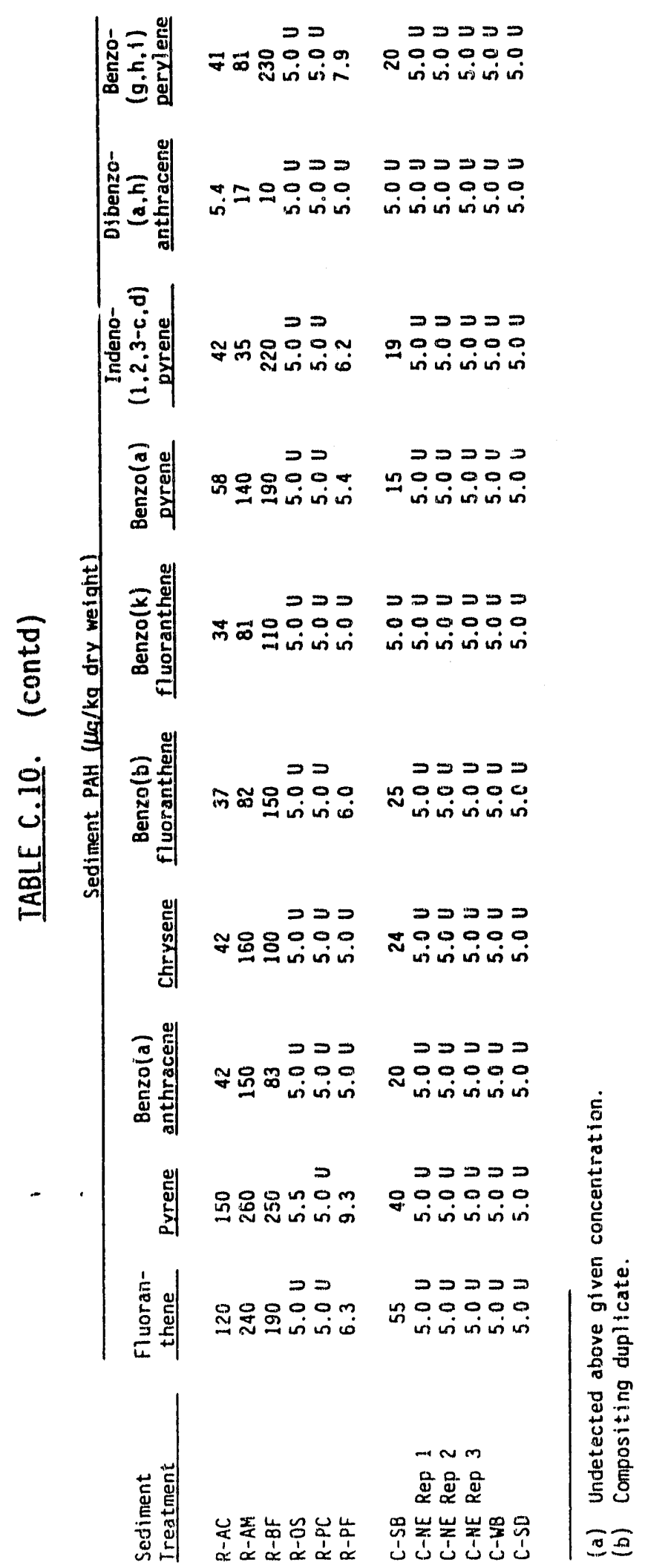


TABLE C.11. Quality Control Data for Sediment Low Molecular Weight Polynuclear Aromatic Hydrocarbon (LPAH) Analysis, Oakland Harbor Phase III B Project

Sample

\begin{tabular}{|c|c|c|c|c|c|}
\hline $\begin{array}{l}\text { Naph- } \\
\text { thalene }\end{array}$ & $\begin{array}{l}\text { Acenaph- } \\
\text { thylene }\end{array}$ & $\begin{array}{l}\text { Acenaph- } \\
\text { thene }\end{array}$ & Fluorene & $\begin{array}{l}\text { Phenan- } \\
\text { threne }\end{array}$ & $\begin{array}{l}\text { Anthr- } \\
\text { acene }\end{array}$ \\
\hline
\end{tabular}

Method Blanks

\section{Blank 1}

$10 u^{(a)}$

Blank?

$10 U(a)$
$10 U$

$5.0 \mathrm{U}$

5.011

$5.0 \mathrm{U}$

$5.0 \mathrm{U}$

5.00

Matrix Spikes

C-WB
C-WB MS
Amount Recovered
Amount Spiked
Recovery

C-WB

C-WB MSD

Amount Recovered

Amount Spiked Recovery

$10 U$
17
17
18
$94 \%$

$5.0 u$
22
22
18
$122 \%$

$5.0 \mathrm{U}$
22
22
18
$122 \%$

$5.0 \mathrm{U}$

$10 \mathrm{U}$

16

16

18

$89 \%$

MS/MSD RPD

MS/MSD I-Stat

$6 \%$

$0-C 12$
$0-C 12$
MS

Amount Recovered

Amount Spiked

Recovery

0.03

22
22
18
$122 \%$

$5.0 \mathrm{U}$

22

22

18

$122 \%$

$5.0 \mathrm{U}$

$5.0 \mathrm{U}$

$5.0 \mathrm{U}$

0-C12

$0-\mathrm{Cl}^{\mathrm{MSO}}{ }^{(\mathrm{b})}$

Amount Recọvered

Amount Spiked

Recovery

$10 U$
27
27
18
$150 \%$

0.00

0.00

$5.0 \mathrm{U}$

22

22
18

$122 \%$

$5.0 \mathrm{U}$

22

22

18

$10 \mathrm{U}$

29

29

18

$161 \%$

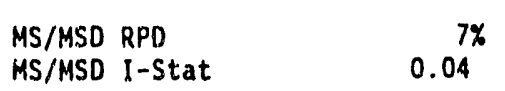

$5.0 \mathrm{U}$

22

22

18
$122 \%$

$122 \%$

$5.0 u$
22
22
18
$122 \%$

$5.0 \mathrm{U}$

$5.0 \mathrm{U}$

22

22

18

$122 \%$

23

18

$5.0 \mathrm{U}$

$5.0 \mathrm{U}$

$128 \%$

MS/MSD I-Stat

$0.04^{7 \%}$

$0 \%$

0.00

$5.0 \mathrm{U}$

22

22

18
$122 \%$

23

23

18

22

22

18

$5.0 \mathrm{U}$

$128 \%$

$122 \% \quad 128 \%$

Standard Reference Materials

HS-4 Rep 1

78

HS-4 Rep 2

0.00

0.02

0.00

0.00

$5.0 \mathrm{U}$

23

23

18

12
32

32

20
18

$128 \%$

$111 \%$

7

7
29

22

18

HS-4 Certified Value

$<150$

$<150$

$5.0 \mathrm{U}$

22

22

18
$122 \%$

$\begin{array}{cc}12 & 7 \\ 34 & 32 \\ 22 & 25 \\ 18 & 18 \\ 122 \% & 139 \%\end{array}$

Analytical Replicates

1-C8 top 6 in.. Rep 1

1-C8 top 6 in. . Rep 2

I-C8 top 6 in. . Rep 3 RSD

$\begin{array}{ll}40 & 30 \\ 48 & 35 \\ 34 & 31 \\ 17 \% & 8 \%\end{array}$

33
30
$<150$


23
45
14
$58 \%$

0.02

$10 \%$

$13 \%$

0.05

0.06

PHASE III B

C. 18

$\begin{array}{rrr}42 & 550(c) & 330^{(c)} \\ 40 & 540^{(c)} & 220^{(c)} \\ <150 & 680 \pm 80 & 140 \pm 70\end{array}$


IABLE C.11. (contd)

\begin{tabular}{|c|c|c|c|c|c|c|}
\hline \multirow[b]{2}{*}{ Sample } & \multicolumn{6}{|c|}{ LPAH $\left(\mu_{\mathrm{g}} / \mathrm{kg}\right.$ dry weight $)$} \\
\hline & $\begin{array}{l}\text { Naph- } \\
\text { thalene }\end{array}$ & $\begin{array}{l}\text { Acenaph- } \\
\text { thylene }\end{array}$ & $\begin{array}{l}\text { Acenaph- } \\
\text { thene }\end{array}$ & Eluorene & $\begin{array}{l}\text { Phenan- } \\
\text { threne }\end{array}$ & $\begin{array}{l}\text { Anthr- } \\
\text { acene }\end{array}$ \\
\hline Analytical & td) & & & & & \\
\hline $\begin{array}{c}\text { C-NE Rep } 1 \\
\text { C-NE Rep } 2 \\
\text { C-NE Rep } 3 \\
\text { RSD }\end{array}$ & $\begin{array}{l}10 U \\
10 U \\
10 U \\
0 \%\end{array}$ & $\begin{array}{l}5.0 U \\
5.0 U \\
5.0 U \\
0 \%\end{array}$ & $\begin{array}{c}5.0 U \\
5.0 U \\
5.0 U \\
0 \%\end{array}$ & $\begin{array}{l}5.0 U \\
5.0 U \\
5.0 U \\
0 x\end{array}$ & $\begin{array}{l}8.7 \\
9.7 \\
9.2 \\
5 \%\end{array}$ & $\begin{array}{l}5.0 U \\
5.0 U \\
5.0 U \\
0 \%\end{array}$ \\
\hline
\end{tabular}

Compositing Duplicates

$\begin{array}{ccccccc}\text { O-C10 Rep 1 } & 27 & 16 & 6.7 & 8.7 & 57 & 40 \\ \text { O-C10 Rep 2 } & 20 & 16 & 6.4 & 8.2 & 54 & 42 \\ \text { RPD } & 30 \% & 0 \% & 5 \% & 6 \% & 5 \% & 5 \% \\ \text { I-STAT } & 0.15 & 0.00 & 0.02 & 0.03 & 0.03 & 0.02 \\ \text { BC-8 Rep 1 } & 23 & 17 & 7.9 & 9.3 & 86 & 42 \\ \text { BC-8 Rep 2 } & 20 & 13 & 6.2 & 6.6 & 60 & 29 \\ \text { RPD } & 14 \% & 27 \% & 24 \% & 34 \% & 36 \% & 37 \% \\ \text { I-STAT } & 0.07 & 0.13 & 0.12 & 0.17 & 0.18 & 0.18\end{array}$

(a) Undetected above given concentration.

(b) Multiple target compounds in sample may have interfered with matrix spike quantitation.

(c) Outside certified range. 


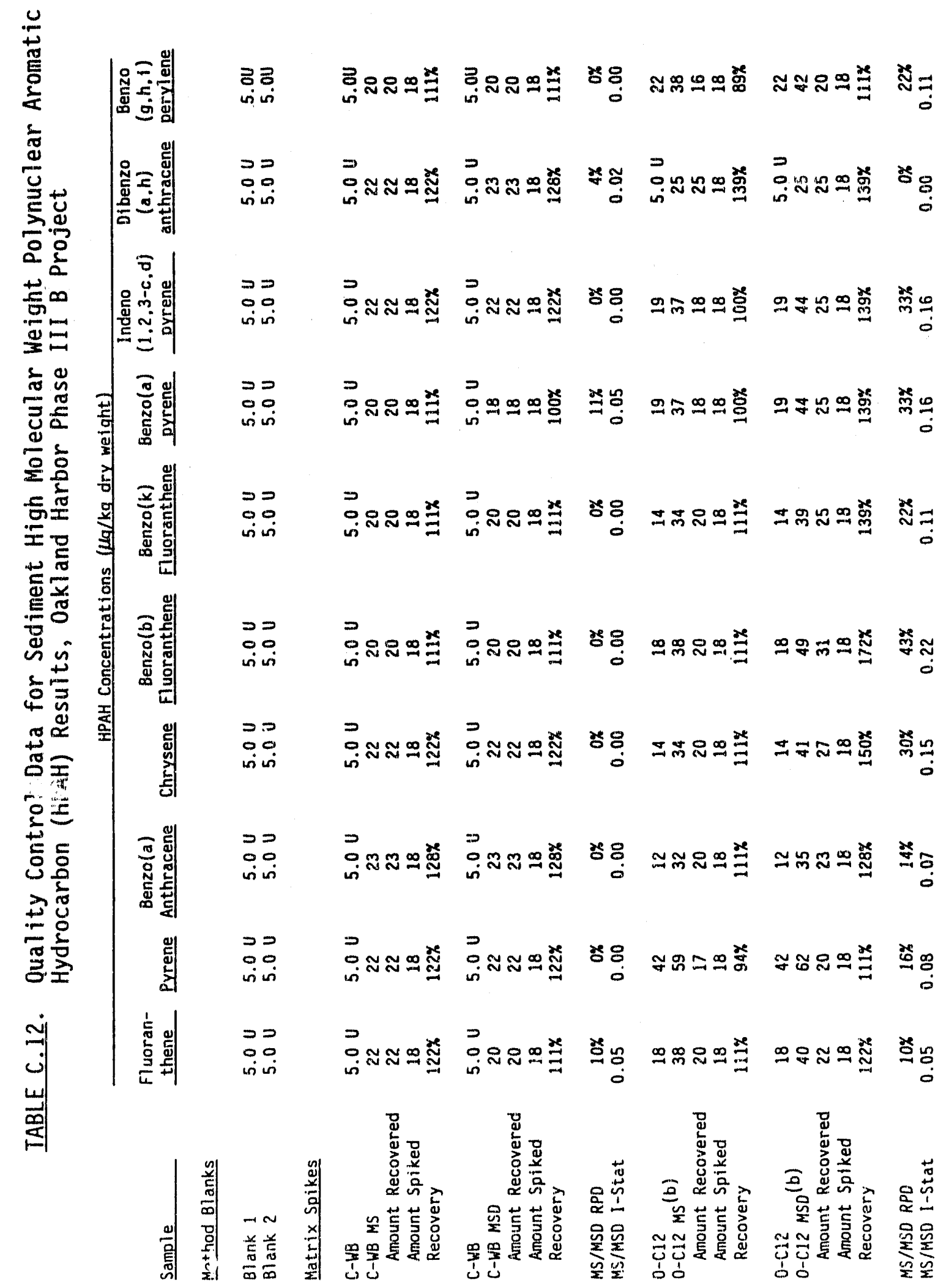

PHASE III B 


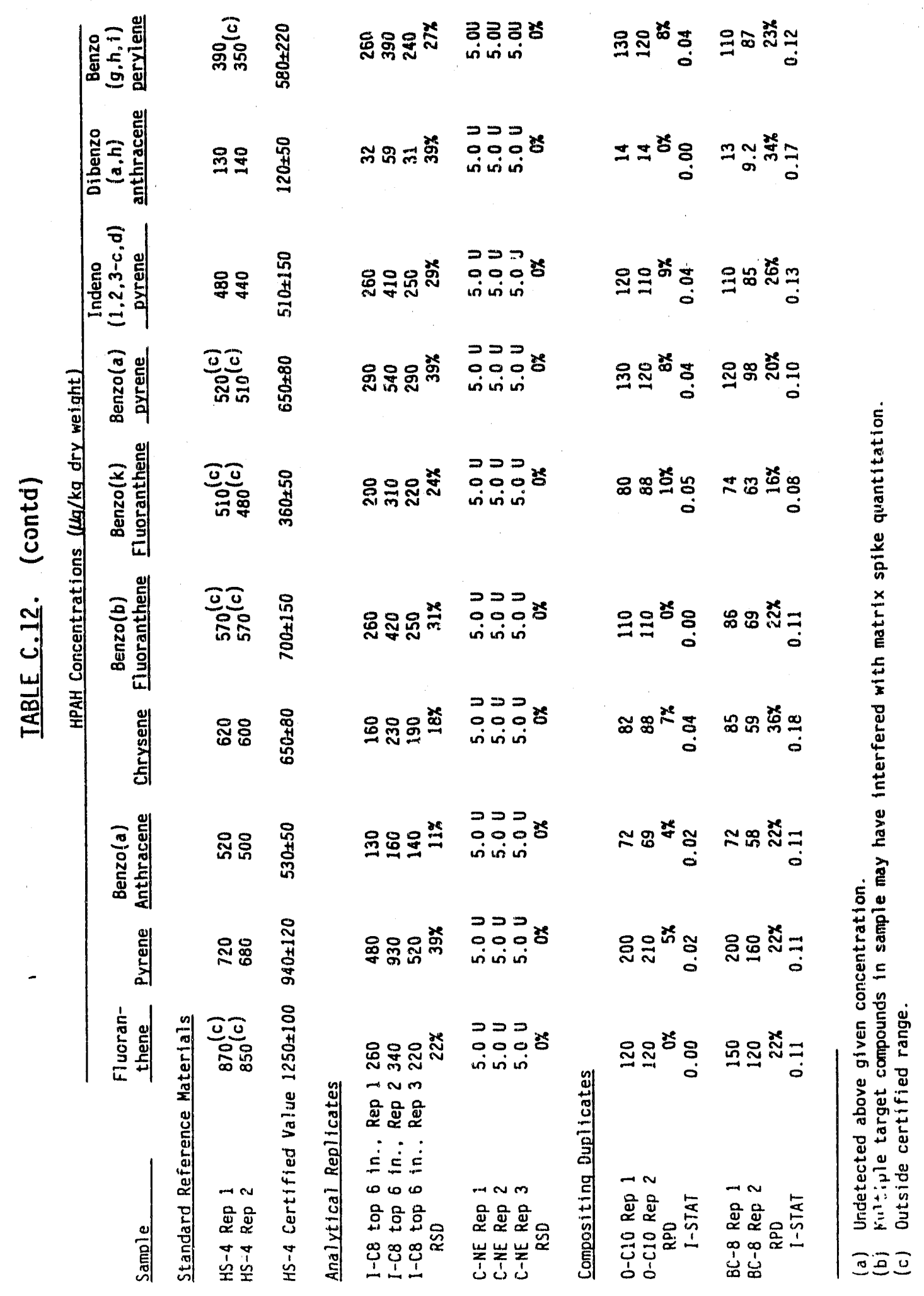


IABLE C.13. Surrogate Recoveries for Sediment Polynuclear Aromatic Hydrocarbons (PAH) Results, Oakland Harbor Phase III B Project

Sediment

Ireatment

$\mathrm{BC}-7$

$0-\mathrm{C} 13$

$0-\mathrm{C} 12$

$0-C 11$

$0-\mathrm{C} 10$

$0-$ C10 Cdup ${ }^{(a)}$

$\mathrm{BC}-5$

$0-C 9$

$0-\mathrm{CB}$

$0-C 7$

$0-\mathrm{C} 6$

$B C-8$

$\mathrm{BC}-8$ Cdup

$0-C 5$

$0-C 4$

$B C-6$

$0-\mathrm{C3}$

$0-C 1$

$\mathrm{I}-\mathrm{C} 2$

$0-C 2$

$\mathrm{I}-\mathrm{C} 3$

$\mathrm{I}-\mathrm{C} 8$

I-C8 top 6 in., Rep I

I-C8 top 6 in., Rep 2

I-C8 top 6 in., Rep 3

I $-\mathrm{C} 4$

I-T5

$R-A C$

$R-A M$

$R-B F$

$R-O S$

$R-P C$

$R-P F$
Surrogate Percent Recoveries

\begin{tabular}{|c|c|c|c|}
\hline $\begin{array}{l}\text { Naph- } \\
\text { thalene } \\
\text { D8 } \\
\end{array}$ & $\begin{array}{l}\text { Acenaph- } \\
\text { thene } \\
\text { D10 } \\
\end{array}$ & $\begin{array}{l}\text { Phenan- } \\
\text { threne } \\
\text { D10 } \\
\end{array}$ & $\begin{array}{c}\text { Benzo(a) - } \\
\text { pyrene } \\
\text { D12 }\end{array}$ \\
\hline $\begin{array}{l}95 \\
80 \\
99 \\
74 \\
94 \\
95\end{array}$ & $\begin{array}{l}96 \\
73 \\
89 \\
85 \\
84 \\
98\end{array}$ & $\begin{array}{l}87 \\
60 \\
81 \\
72 \\
73 \\
88\end{array}$ & $\begin{array}{l}120 \\
100 \\
110 \\
100 \\
100 \\
120\end{array}$ \\
\hline $\begin{array}{r}110 \\
95 \\
90 \\
69 \\
78\end{array}$ & $\begin{array}{r}100 \\
84 \\
88 \\
76 \\
79\end{array}$ & $\begin{array}{l}90 \\
87 \\
74 \\
68 \\
73\end{array}$ & $\begin{array}{r}120 \\
110 \\
110 \\
97 \\
100\end{array}$ \\
\hline $\begin{array}{l}94 \\
93 \\
89 \\
51\end{array}$ & $\begin{array}{l}88 \\
98 \\
84 \\
45^{(b)}\end{array}$ & $\begin{array}{l}82 \\
91 \\
88 \\
42^{(b)}\end{array}$ & $\begin{array}{r}120 \\
120 \\
120 \\
60\end{array}$ \\
\hline
\end{tabular}

$92 \quad 86$

$91 \quad 81$

$68 \quad 82$

$81 \quad 94$

$83 \quad 80$

$100 \quad 90$

$88 \quad 83$

$100 \quad 100$

$100 \quad 100$

$95 \quad 93$

$89 \quad 78$

$92 \quad 77$

$94 \quad 78$

$120 \quad 150$

$71 \quad 67$

$84 \quad 77$

$76 \quad 76$

$86 \quad 76$
78

77

82

84

75

91

75

86

94

82

63

81

85

160

61

78

74

68
110

100

110

120

110

120

100

120

120

120

100

100

120

160

100

100

100

100 
IABLE C.13. (contd)

Sediment

\begin{tabular}{|c|c|c|c|}
\hline $\begin{array}{c}\text { Naph- } \\
\text { thalene } \\
\text { D8 } \\
\end{array}$ & $\begin{array}{l}\text { Acenaph- } \\
\text { thene } \\
\text { D10 } \\
\end{array}$ & $\begin{array}{l}\text { Phenan- } \\
\text { threne } \\
\text { D10 }\end{array}$ & $\begin{array}{c}\text { Benzo(a)- } \\
\text { pyrene } \\
012 \\
\end{array}$ \\
\hline $\begin{array}{l}64 \\
65 \\
79 \\
90 \\
61 \\
87\end{array}$ & $\begin{array}{l}70 \\
71 \\
79 \\
82 \\
70 \\
76\end{array}$ & $\begin{array}{l}70 \\
70 \\
71 \\
72 \\
77 \\
82\end{array}$ & $\begin{array}{l}100 \\
100 \\
110 \\
110 \\
100 \\
110\end{array}$ \\
\hline
\end{tabular}

(a) Compositing duplicate.

(b) Surrogate $>20 \%$; QC requirements met. 


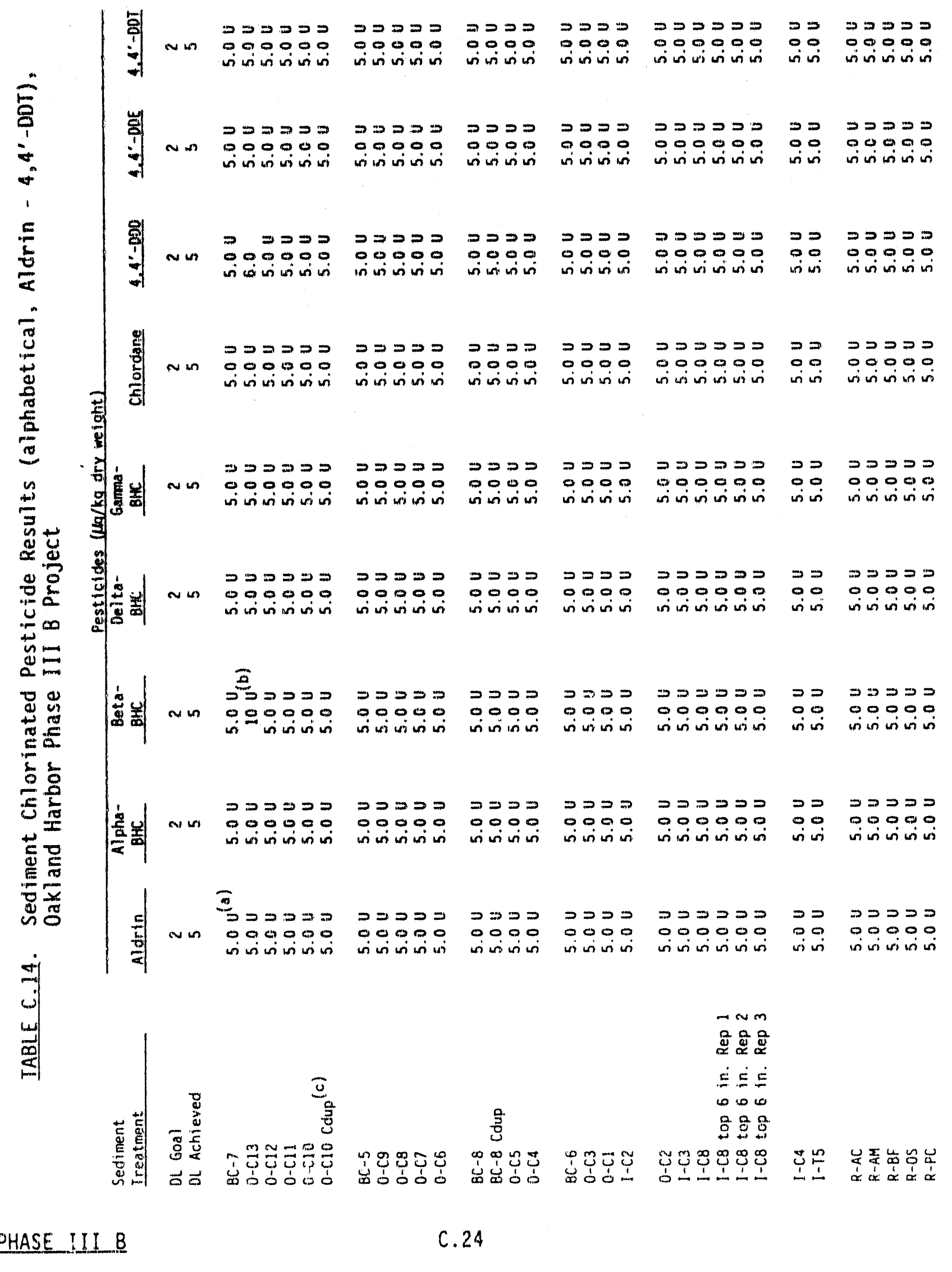




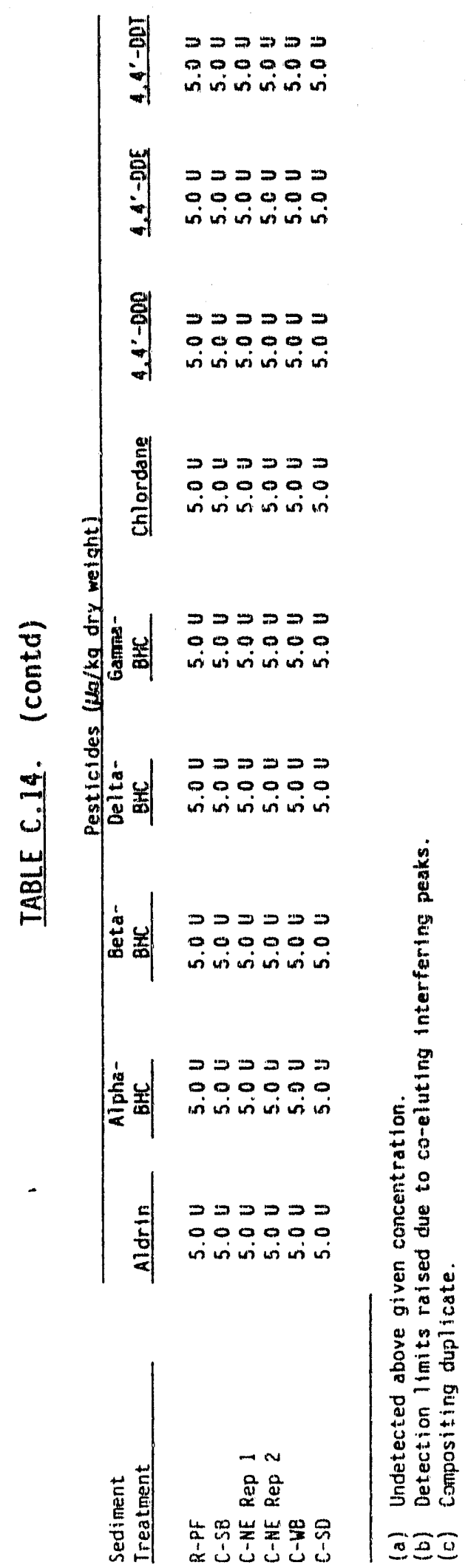

PHASE III B 


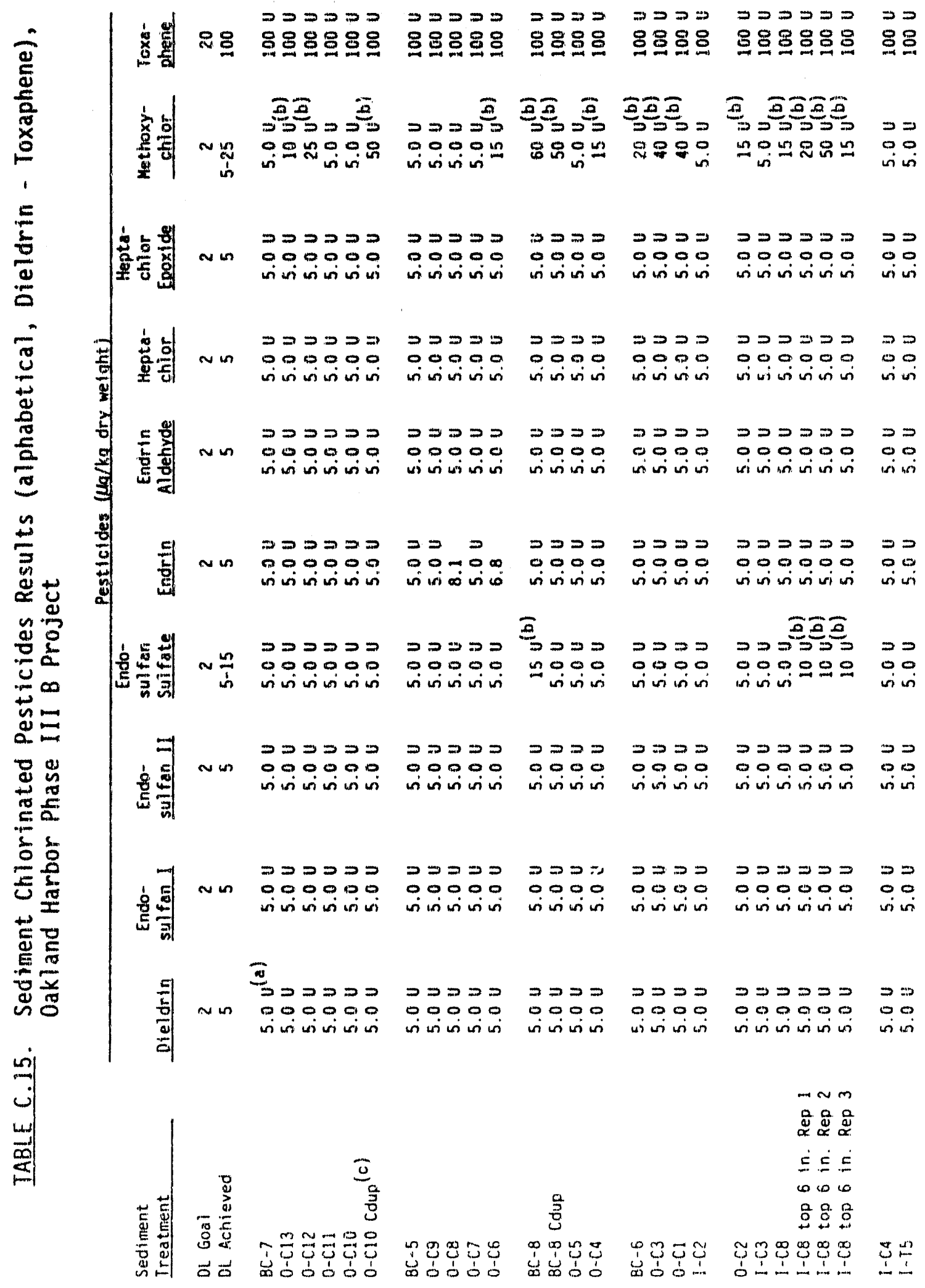

PHASE III B 


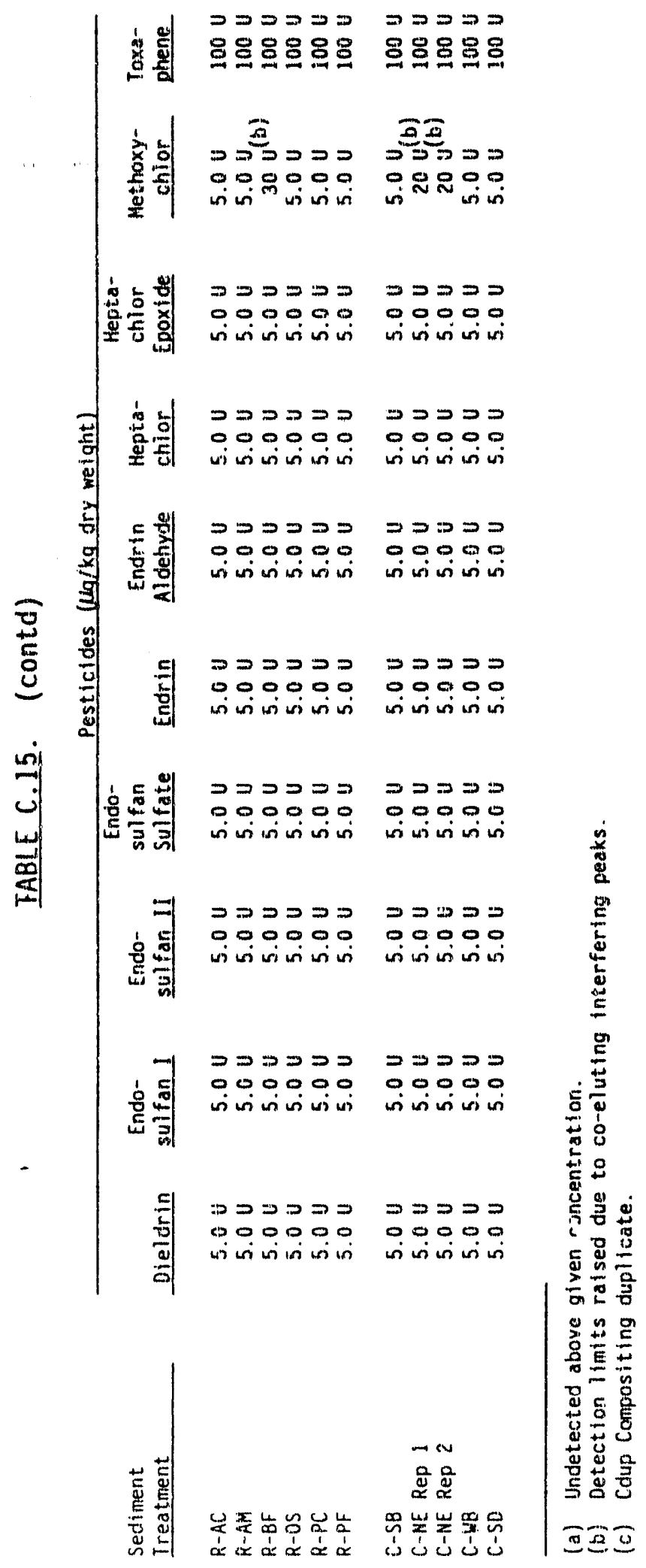




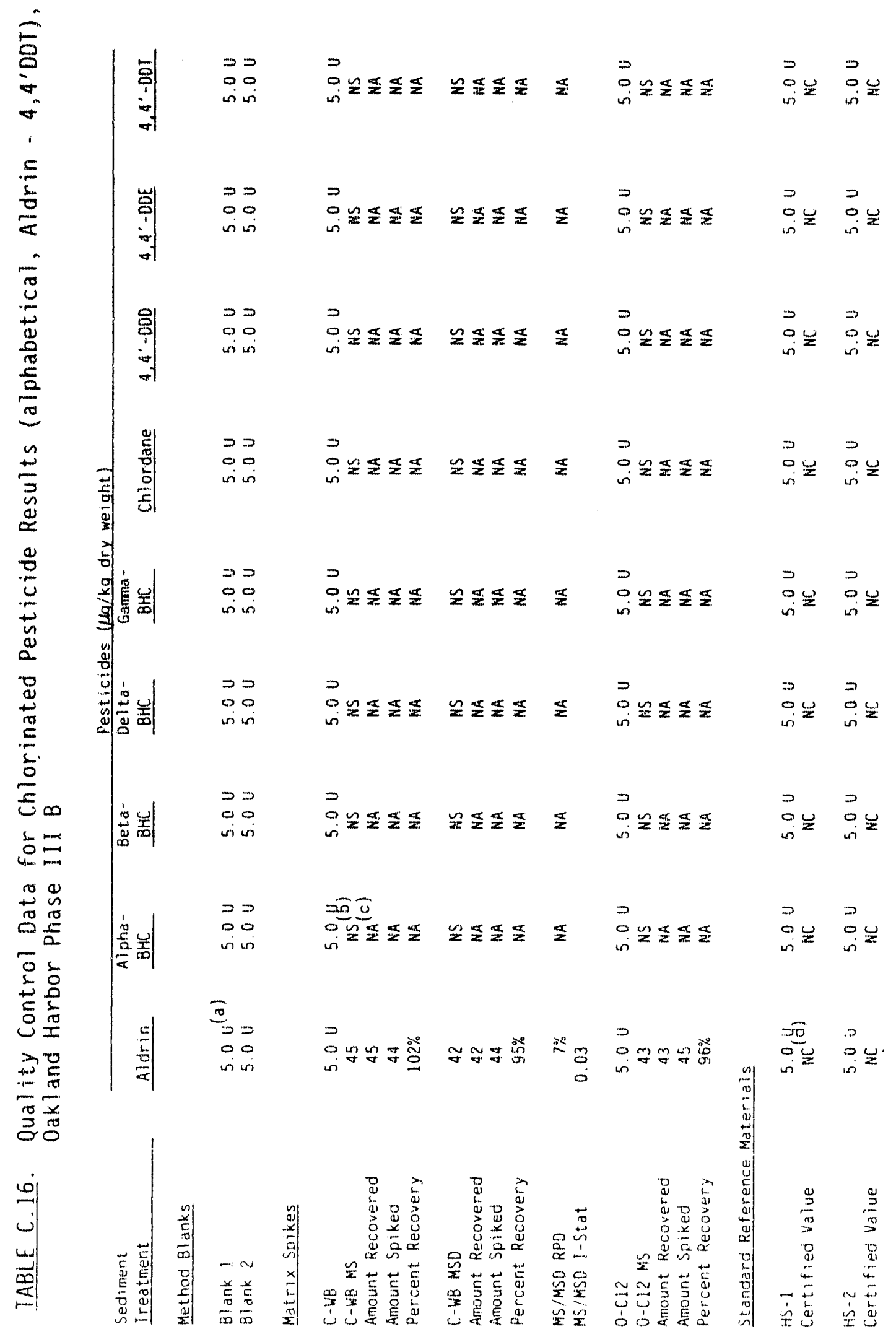

PHASE $111 \mathrm{~B}$ 


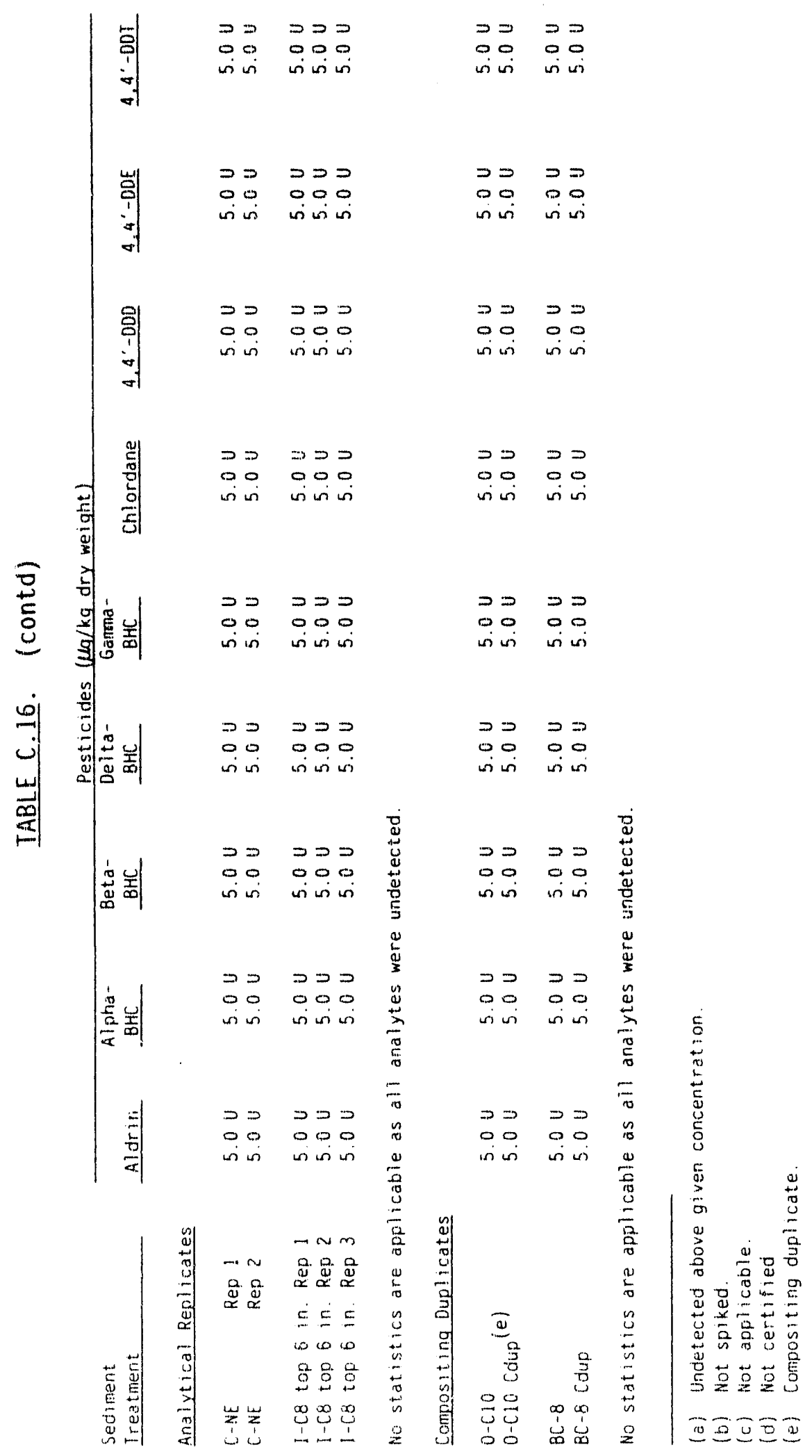




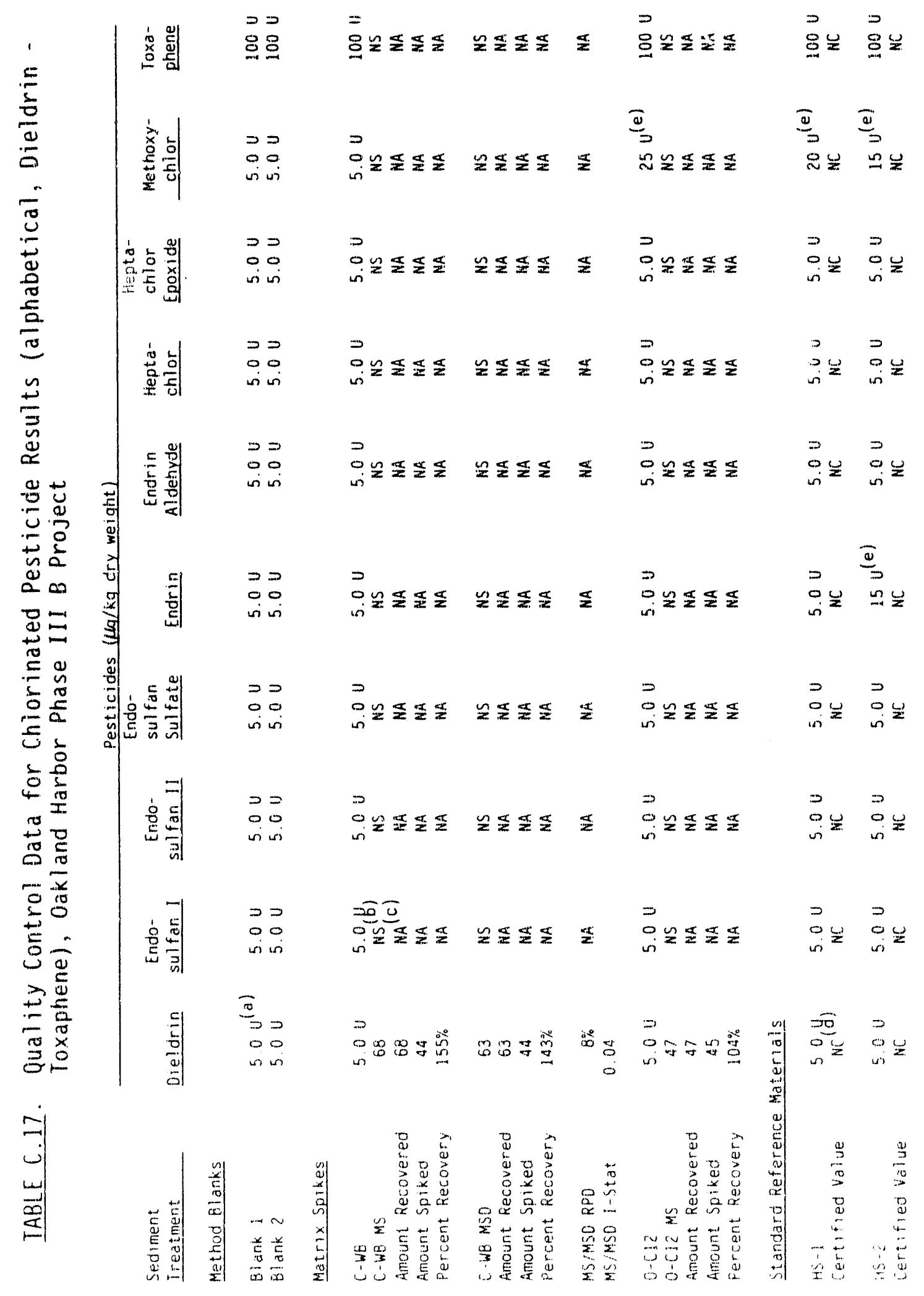




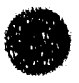

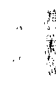
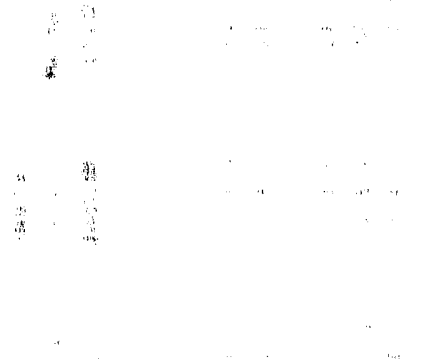
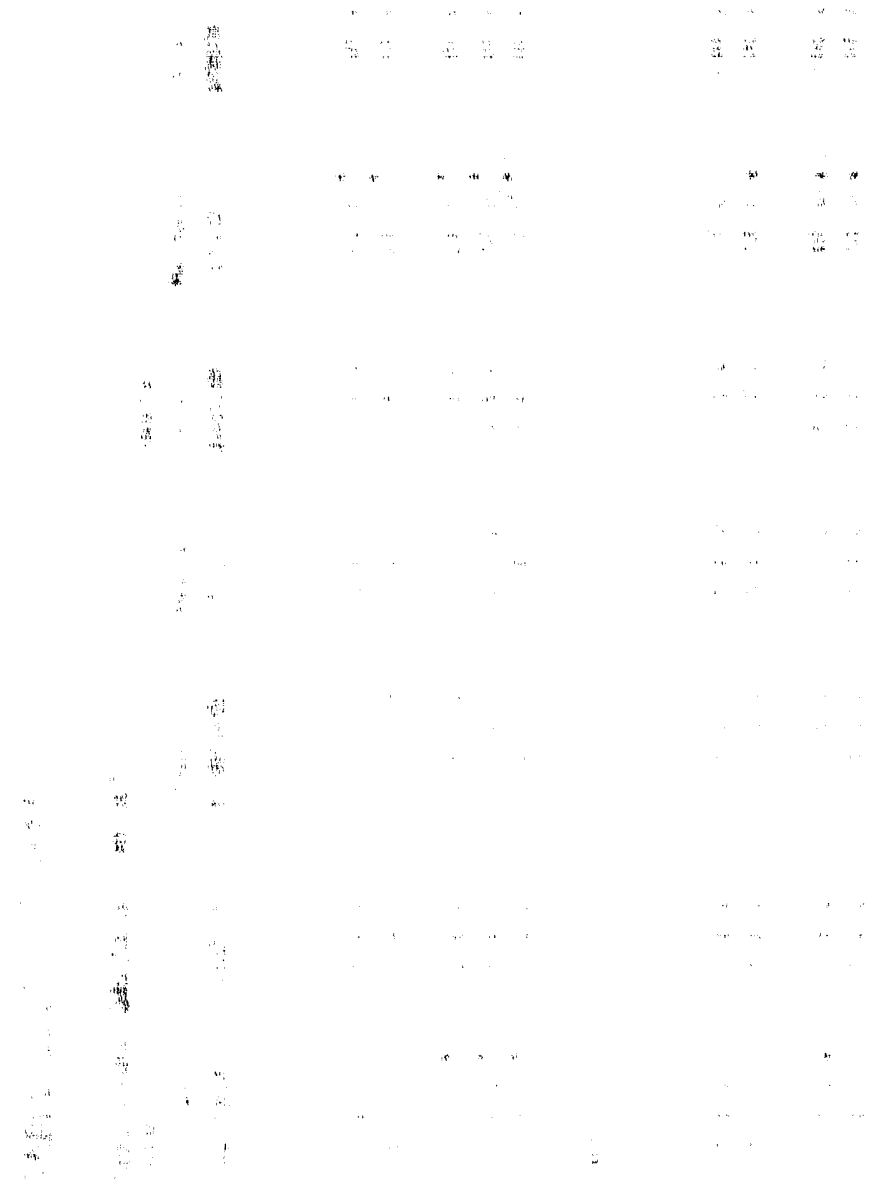

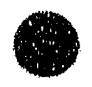


TABLE C.18. Sediment Polychlorinated Biphenyl (PCB) Results, Oakland Harbor Phase III B Project

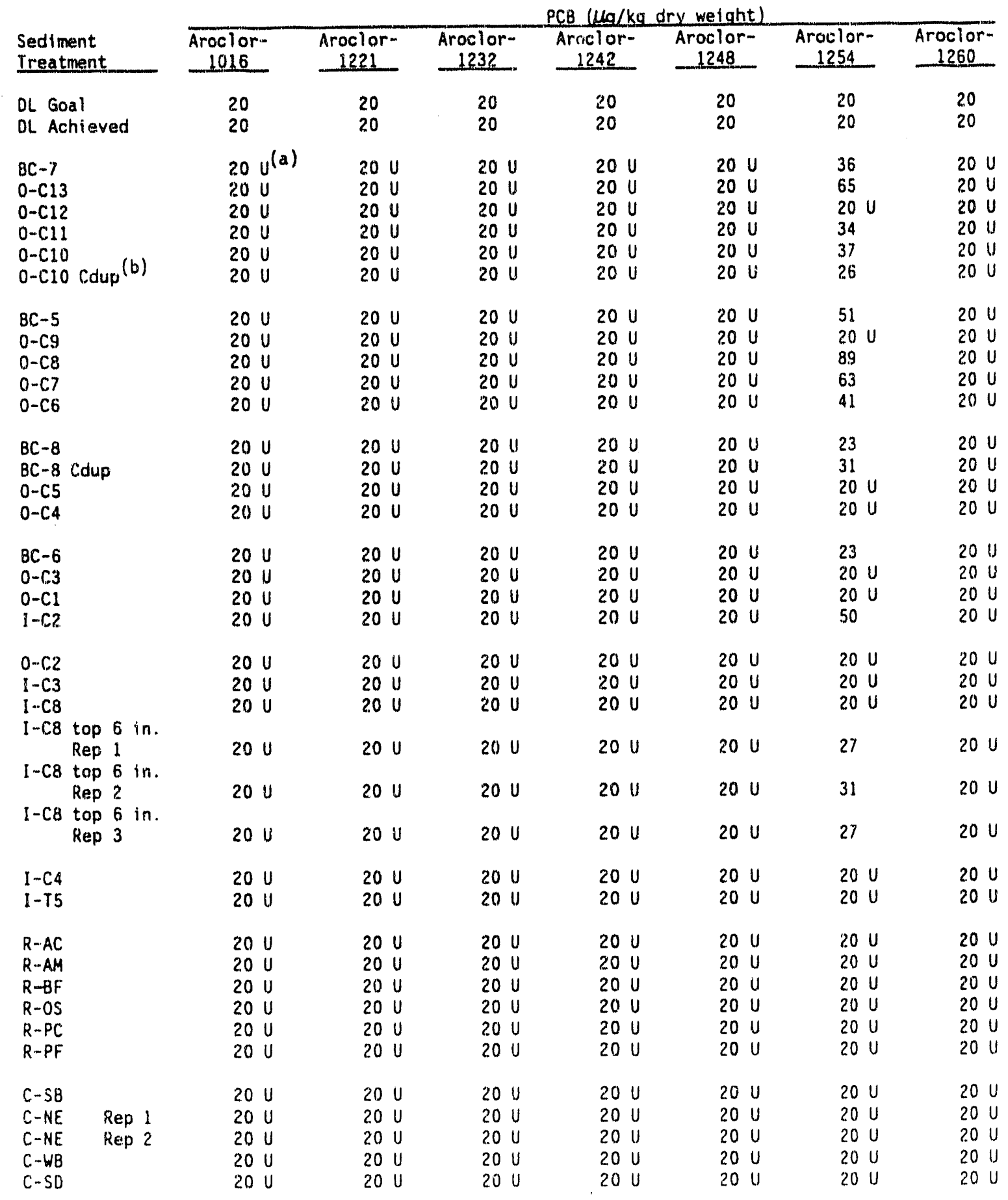

(a) Undetected above given concentration.

(b) Compositing duplicate. 
TABLE C.19. Quality Control Data for Polychlorinated Biphenyl (PCB) Analysis, Oakland Harbor Phase III B Project

\begin{tabular}{|c|c|c|c|c|c|c|c|}
\hline \multirow{2}{*}{ Sample } & \multicolumn{7}{|c|}{ PCB $\left(\mu_{y} / \mathrm{kg}\right.$ dry meight $)$} \\
\hline & $\begin{array}{l}\text { Aroclor- } \\
1016 \\
\end{array}$ & $\begin{array}{l}\text { Araclor- } \\
1221 \\
\end{array}$ & $\begin{array}{l}\text { Aroclor- } \\
1232 \\
\end{array}$ & $\begin{array}{l}\text { Aroclor } \\
1242 \\
\end{array}$ & $\begin{array}{l}\text { Aroclor- } \\
1248 \\
\end{array}$ & $\begin{array}{l}\text { Aroclor- } \\
1254 \\
\end{array}$ & $\begin{array}{l}\text { Aroclor- } \\
1260 \\
\end{array}$ \\
\hline \multicolumn{8}{|l|}{ Method Blanks } \\
\hline $\begin{array}{l}\text { Blank } 1 \\
\text { Blank } 2\end{array}$ & $\begin{array}{l}20 u^{(a)} \\
20 U^{(a)}\end{array}$ & $\begin{array}{l}20 U \\
20 U\end{array}$ & $\begin{array}{l}20 \mathrm{U} \\
20 \mathrm{U}\end{array}$ & $\begin{array}{l}20 \mathrm{U} \\
20 \mathrm{U}\end{array}$ & $\begin{array}{l}20 \mathrm{U} \\
20 \mathrm{U}\end{array}$ & $\begin{array}{l}20 \mathrm{U} \\
20 \mathrm{U}\end{array}$ & $\begin{array}{l}20 \mathrm{U} \\
20 \mathrm{U}\end{array}$ \\
\hline \multicolumn{8}{|c|}{ Standard Reference Material } \\
\hline $\begin{array}{l}\text { HS-1 } \\
\text { Certified Value }\end{array}$ & ${ }_{\mathrm{NC}}^{20}(\mathrm{~b})$ & $\begin{array}{l}20 \mathrm{U} \\
\mathrm{NC}\end{array}$ & $\begin{array}{l}20 U \\
\text { NC }\end{array}$ & $\begin{array}{l}20 \mathrm{U} \\
\mathrm{NC}\end{array}$ & $\begin{array}{l}20 \mathrm{U} \\
\mathrm{NC}\end{array}$ & $\begin{array}{r}73 \\
21.8\end{array}$ & $\begin{array}{l}20 \mathrm{U} \\
\mathrm{NC}\end{array}$ \\
\hline $\begin{array}{l}\text { HS-2 } \\
\text { Certified Value }\end{array}$ & $\begin{array}{l}20 \mathrm{U} \\
\mathrm{NC}\end{array}$ & $\begin{array}{l}20 \mathrm{U} \\
\mathrm{NC}\end{array}$ & $\begin{array}{l}20 \mathrm{U} \\
\mathrm{NC}\end{array}$ & $\begin{array}{l}20 U \\
\text { NC }\end{array}$ & $\begin{array}{l}20 \mathrm{U} \\
\text { NC }\end{array}$ & $\begin{array}{r}200 \\
111.8\end{array}$ & $\begin{array}{l}20 \mathrm{U} \\
\mathrm{NC}\end{array}$ \\
\hline \multicolumn{8}{|c|}{ Analytical Replicates } \\
\hline $\begin{array}{ll}\text { C-NE } & \text { Rep } 1 \\
\text { C-NE } & \text { Rep } 2\end{array}$ & $\begin{array}{l}20 U \\
20 U\end{array}$ & $\begin{array}{l}20 U \\
20 U\end{array}$ & $\begin{array}{l}20 U \\
20 U\end{array}$ & $\begin{array}{l}20 \mathrm{U} \\
20 \mathrm{U}\end{array}$ & $\begin{array}{l}20 \mathrm{U} \\
20 \mathrm{U}\end{array}$ & $\begin{array}{l}20 \mathrm{U} \\
20 \mathrm{U}\end{array}$ & $\begin{array}{l}20 U \\
20 U\end{array}$ \\
\hline I-C8 top 6 in. & $20 \mathrm{U}$ & $20 \mathrm{U}$ & $20 \mathrm{U}$ & $20 \mathrm{U}$ & $20 \mathrm{U}$ & $20 U$ & $20 \mathrm{U}$ \\
\hline $\begin{array}{r}\text { Rep } 2 \\
\text { I-c8 top } 6 \text { in. }\end{array}$ & $20 \mathrm{U}$ & $20 \mathrm{U}$ & $20 u$ & $20 \mathrm{U}$ & $20 \mathrm{U}$ & $20 \mathrm{U}$ & $20 \mathrm{U}$ \\
\hline Rep 3 & $20 \mathrm{U}$ & $20 \mathrm{U}$ & $20 \mathrm{U}$ & $20 U$ & $20 \mathrm{U}$ & $20 \mathrm{U}$ & $20 \mathrm{U}$ \\
\hline \multicolumn{8}{|c|}{ Compositing Ouplicates } \\
\hline $\begin{array}{l}\text { O-C10 } \\
\text { O-C10 Cdup }(c) \\
\text { RPD } \\
\text { I-Stat }\end{array}$ & $\begin{array}{l}20 U \\
20(y) \\
N A \\
N A\end{array}$ & $\begin{array}{l}20 U \\
20 U \\
N A \\
N A\end{array}$ & $\begin{array}{l}20 U \\
20 \mathrm{U} \\
\mathrm{NA} \\
\mathrm{NA}\end{array}$ & $\begin{array}{l}20 U \\
20 U \\
H A \\
N A\end{array}$ & $\begin{array}{l}20 \mathrm{U} \\
20 \mathrm{U} \\
\mathrm{NA} \\
\mathrm{NA}\end{array}$ & $\begin{array}{l}20 U \\
20 U \\
N A \\
N A\end{array}$ & $\begin{array}{l}20 U \\
20 U \\
N A \\
N A\end{array}$ \\
\hline $\begin{array}{l}B C-8 \\
B C-8 \text { Cdup } \\
\text { KPD } \\
{[-5 \text { tat }}\end{array}$ & $\begin{array}{l}20 U \\
20 U \\
M A \\
N A\end{array}$ & $\begin{array}{l}20 U \\
20 U \\
N A \\
N A\end{array}$ & $\begin{array}{l}20 U \\
20 U \\
N A \\
N A\end{array}$ & $\begin{array}{l}20 U \\
20 U \\
N A \\
N A\end{array}$ & $\begin{array}{l}20 U \\
20 U \\
N A \\
N A\end{array}$ & $\begin{array}{l}20 U \\
20 U \\
\text { NA } \\
\text { NA }\end{array}$ & $\begin{array}{c}23 \\
31 \\
30 \% \\
0.15\end{array}$ \\
\hline
\end{tabular}

(a) Undetected above given concentration.

(b) SRM not certified for this analyte.

(c) Compositing duplicate.

(d) Not applicable. 
IABLE C.20. Surrogate Percent Recoveries for Sediment Polychlorinated Biphenyls (PCBs) and Chlorinated Pesticide Results, Oakland Harbor Phase III 8 Project

Sediment
Treatment

$\mathrm{BC}-7$

$0-\mathrm{C} 13$

$0-\mathrm{C} 12$

$0-C 11$

$0-\mathrm{C} 10$

$0-$ C10 Cdup ${ }^{(a)}$

$\mathrm{BC}-5$

$0-C 9$

$0-\mathrm{CB}$

$0-C .7$

$0-16$

$B C-8$

$B C-8$ Cdup

$0-C 5$

$0-C 4$

$B C-6$

O-C3

$0-\mathrm{Cl}$

I-C2

$0-C 2$

$1-\mathrm{C3}$

I $-\mathrm{C} 8$

I-C8 top 6 in.

Rep 1

I-C8 top 6 in.

Rep 2

I-C8 top 6 in.

Rep 3

I $-\mathrm{C} 4$

I $-\mathrm{TS}$

$R-A C$

$R-A M$

$R-B F$

$R-O S$

$R-P C$

$R-P F$
Dibuty1 -

chlorendate

Percent

Recovery

87

117

78

77

81

94

89

98

79

70

74

92

102

32

34

88

81

83

49

63

103

69

97

83

91

82

104

114

114

75

97

95

82 
TABLE C.20. (contd)

Sediment

Treatment

$C-S B$

C-NE Rep 1

C-NE Rep 2

$C-W B$

C-SD

Method Blanks

Blank 1

Blank 2

Matrix Spikes

C-WB

C-WB MS

Amount Recovered

Amount Spiked

Percent Recovery

$0-\mathrm{C} 12$

$0-\mathrm{C} 12 \mathrm{MS}$

Amount Recovered

Amount Spiked

Percent Recovery

Standard Reference Materials

HS- 1

Certified Value

HS-2

Certified Value

Analytical Replicates

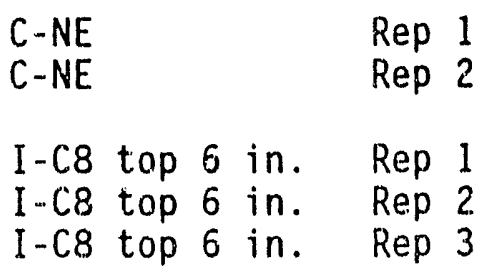

Dibutyl-

chlorendate

Percent

Recovery

68

66

74

112

100

$100 \%$

$98 \%$

$112 \%$

$78 \%$

$100 \%$

$80 \%$

$66 \%$

$74 \%$

$97 \%$

$83 \%$

$91 \%$ 
IABLE C.20. (contd)

Sediment

Dibutyl -

chlorendate

Treatment

Percent

Recovery

Compositing Duplicates

$0-c 10$

0-c10 cdup

$81 \%$

$B C-8$

$94 \%$

BC-8 Cdup

$92 \%$

$102 \%$

(a) Compositing duplicate. 
IABLE C.21. Sediment Metals Results, Oakland Harbor Phase III B Project

\begin{tabular}{|c|c|c|c|c|c|c|c|c|c|c|}
\hline Sediment & & & & & 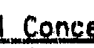 & cetion & $\mathrm{ag} / \mathrm{kg} \mathrm{dr}$ & deight & & \\
\hline Ireatment & $\mathrm{Aq}$ & As & $\mathrm{Cd}$ & Cr & $\mathrm{Cu}_{4}$ & $\mathrm{Ha}$ & $\mathrm{Ni}$ & $\mathrm{Pb}$ & Se & $2 n$ \\
\hline $\begin{array}{l}\text { OL Goal } \\
\text { OL Achieved }\end{array}$ & $\begin{array}{l}1.000 \\
0.004\end{array}$ & $\begin{array}{l}1.00 \\
1.00\end{array}$ & $\begin{array}{l}0.10 \\
0.05\end{array}$ & $\begin{array}{l}1.00 \\
1.00\end{array}$ & $\begin{array}{l}1.00 \\
1.00\end{array}$ & $\begin{array}{l}0.020 \\
0.016\end{array}$ & $\begin{array}{l}1.00 \\
1.00\end{array}$ & $\begin{array}{l}1.00 \\
1.00\end{array}$ & $\begin{array}{l}0.10 \\
0.11\end{array}$ & $\begin{array}{l}1.00 \\
1.00\end{array}$ \\
\hline $\begin{array}{l}8 c-7 \\
0-c 13 \\
0-c 12 \\
0-c 11 \\
0-c 10 \\
0-c 10 \quad c d u p(b)\end{array}$ & $\begin{array}{l}0.280 \\
0.503 \\
0.084 \\
0.291 \\
0.267 \\
0.303\end{array}$ & $\begin{array}{l}7.20 \\
11.6 \\
1.89 \\
9.70 \\
5.78 \\
8.21\end{array}$ & $\begin{array}{l}0.38 \\
0.60 \\
0.25 \\
0.38 \\
0.37 \\
0.34\end{array}$ & $\begin{array}{l}306 \\
230 \\
427 \\
330 \\
332 \\
308\end{array}$ & $\begin{array}{l}46.5 \\
69.1 \\
23.8 \\
51.3 \\
40.3 \\
39.4\end{array}$ & $\begin{array}{l}0.828 \\
1.955 \\
0.213 \\
0.895 \\
0.582 \\
0.858\end{array}$ & $\begin{array}{r}107.3 \\
114.8 \\
86.7 \\
112.2 \\
88.9 \\
88.4\end{array}$ & $\begin{array}{l}25.5 \\
48.0 \\
14.1 \\
29.0 \\
26.2 \\
24.1\end{array}$ & $\begin{array}{l}0.12 \\
0.25 \\
0.12 \\
0.19 \\
0.11 u^{(a)} \\
0.12\end{array}$ & $\begin{array}{r}111.0 \\
165.0 \\
73.7 \\
124.6 \\
104.6 \\
99.7\end{array}$ \\
\hline $\begin{array}{l}B C \sim 5 \\
0-C 9 \\
0-C 8 \\
0-C 7 \\
0-C 6\end{array}$ & $\begin{array}{l}0.395 \\
0.029 \\
0.518 \\
0.480 \\
0.433\end{array}$ & $\begin{array}{l}5.86 \\
1.65 \\
1 ? .4 \\
118 \\
10.8\end{array}$ & $\begin{array}{l}0.31 \\
0.08 \\
0.57 \\
0.42 \\
0.37\end{array}$ & $\begin{array}{l}411 \\
478 \\
233 \\
208 \\
221\end{array}$ & $\begin{array}{l}43.7 \\
12.8 \\
70.1 \\
65.4 \\
64.9\end{array}$ & $\begin{array}{l}0.717 \\
0.056 \\
2.204 \\
1.502 \\
1.018\end{array}$ & $\begin{array}{r}94.2 \\
60.1 \\
: 15.2 \\
114.7 \\
115.7\end{array}$ & $\begin{array}{r}32.8 \\
8.9 \\
47.1 \\
49.8 \\
47.5\end{array}$ & $\begin{array}{l}0.114 \\
0.110 \\
0.24 \\
0.30 \\
0.24\end{array}$ & $\begin{array}{r}115.1 \\
43.5 \\
175.0 \\
170.2 \\
167.1\end{array}$ \\
\hline $\begin{array}{l}B C-8 \\
B C-8 \text { Cdup } \\
0-C 5 \\
0-C 4\end{array}$ & $\begin{array}{l}0.156 \\
0.160 \\
0.032 \\
0.313\end{array}$ & $\begin{array}{l}4.74 \\
5.74 \\
2.78 \\
10.3\end{array}$ & $\begin{array}{l}0.19 \\
0.18 \\
0.10 \\
0.30\end{array}$ & $\begin{array}{l}398 \\
375 \\
531 \\
252\end{array}$ & $\begin{array}{l}26.6 \\
27.8 \\
12.9 \\
51.8\end{array}$ & $\begin{array}{l}0.283 \\
0.548 \\
0.110 \\
1.660\end{array}$ & $\begin{array}{l}73.0 \\
71.3 \\
57.1 \\
99.5\end{array}$ & $\begin{array}{r}17.9 \\
13.1 \\
7.5 \\
30.3\end{array}$ & $\begin{array}{l}0.110 \\
0.110 \\
0.110 \\
0.18\end{array}$ & $\begin{array}{r}70.0 \\
73.0 \\
39.1 \\
145.1\end{array}$ \\
\hline $\begin{array}{l}B C-6 \\
D-C .3 \\
0-C 1 \\
I-C 2 \\
0-C 2 \\
I-C 3 \\
1-C B \\
I-C B \text { top } 6 \text { in. } \\
I-C 4 \\
I-T S \\
R-A C \\
R-A M \\
R-B F \\
R-O S \text { Rep } 1 \\
R-O S \text { Rep } 2 \\
R-O S \text { Rep } 3 \\
R-P C \\
R-P F \\
C-S B \text { Rep } 1 \\
C-S B \text { Rep } 2 \\
C-S B \text { Rep } 3 \\
C-W E \\
C-W B \\
C-S D\end{array}$ & $\begin{array}{l}0.255 \\
0.177 \\
0.166 \\
0.414 \\
0.062 \\
0.031 \\
0.064 \\
0.278 \\
0.145 \\
0.026 \\
0.026 \\
0.033 \\
0.395 \\
0.193 \\
0.191 \\
0.185 \\
0.005 \\
0.033 \\
0.207 \\
0.193 \\
0.203 \\
0.015 \\
0.012 \\
0.005\end{array}$ & $\begin{array}{l}9.64 \\
10.9 \\
10.15 \\
7.7 \\
9.13 \\
4.06 \\
4.68 \\
4.74 \\
5.43 \\
3.12 \\
6.92 \\
6.55 \\
9.2 \\
4.67 \\
3.40 \\
3.69 \\
4.62 \\
4.30 \\
13.2 \\
10.41 \\
11.47 \\
3.26 \\
2.40 \\
5.09\end{array}$ & $\begin{array}{l}0.31 \\
0.33 \\
0.29 \\
0.40 \\
0.31 \\
0.11 \\
0.15 \\
0.31 \\
0.19 \\
0.09 \\
0.07 \\
0.09 \\
0.23 \\
0.30 \\
0.32 \\
0.30 \\
0.21 \\
2.48 \\
0.98 \\
0.99 \\
0.96 \\
0.13 \\
0.12 \\
0.05\end{array}$ & $\begin{array}{r}276 \\
204 \\
175 \\
276 \\
221 \\
476 \\
458 \\
343 \\
455 \\
314 \\
171 \\
156 \\
197 \\
223 \\
238 \\
215 \\
236 \\
390 \\
94 \\
113 \\
114 \\
51 \\
180 \\
148\end{array}$ & $\begin{array}{r}49.5 \\
43.4 \\
43.4 \\
55.7 \\
48.6 \\
12.1 \\
19.8 \\
42.1 \\
27.6 \\
14.5 \\
10.0 \\
12.4 \\
52.6 \\
20.8 \\
20.7 \\
19.5 \\
9.0 \\
10.4 \\
39.3 \\
39.7 \\
41.9 \\
7.5 \\
11.2 \\
7.0\end{array}$ & $\begin{array}{l}0.963 \\
1.310 \\
1.790 \\
0.949 \\
0.778 \\
0.155 \\
0.167 \\
0.543 \\
0.552 \\
0.045 \\
0.080 \\
0.156 \\
1.700 \\
0.222 \\
0.210 \\
0.219 \\
0.084 \\
0.157 \\
1.830 \\
1.790 \\
1.790 \\
0.163 \\
0.087 \\
0.057\end{array}$ & $\begin{array}{r}101.7 \\
92.1 \\
98.2 \\
96.3 \\
114.7 \\
35.7 \\
56.2 \\
84.7 \\
73.6 \\
68.7 \\
37.6 \\
40.7 \\
107.9 \\
66.7 \\
63.5 \\
60.2 \\
29.7 \\
43.0 \\
45.6 \\
47.6 \\
46.5 \\
24.4 \\
43.4 \\
28.0\end{array}$ & $\begin{array}{r}22.5 \\
15.0 \\
17.1 \\
33.7 \\
17.8 \\
8.0 \\
10.8 \\
28.2 \\
15.4 \\
5.3 \\
14.7 \\
14.4 \\
31.8 \\
7.7 \\
11.8 \\
7.8 \\
8.0 \\
8.8 \\
11.0 \\
13.8 \\
12.9 \\
6.3 \\
6.5 \\
4.2\end{array}$ & $\begin{array}{l}0.19 \\
0.11 U \\
0.24 \\
0.24 \\
0.18 \\
0.11 U \\
0.11 U \\
0.11 U \\
0.11 U \\
0.11 U \\
0.11 U \\
0.11 U \\
0.18 \\
0.56 \\
0.62 \\
0.50 \\
0.11 U \\
0.11 U \\
0.85 \\
0.77 \\
0.77 \\
0.11 U \\
0.11 U \\
0.11 U\end{array}$ & $\begin{array}{r}112.9 \\
96.6 \\
102.5 \\
126.0 \\
109.2 \\
31.6 \\
56.2 \\
100.2 \\
78.2 \\
43.5 \\
34.1 \\
37.7 \\
143.4 \\
81.2 \\
85.6 \\
80.6 \\
35.3 \\
52.9 \\
96.3 \\
93.6 \\
95.0 \\
20.3 \\
43.3 \\
24.4\end{array}$ \\
\hline
\end{tabular}

(a) Undetected above given concentration.

(b) Compositing duplicate. 
TABLE C.22. Quality Control Data for Sediment Metals Analyses, Oaklani Harbor Phase III B Project

Metal Concentratio: 2 (ma/kg dry weight)

Somple

$\mathrm{Ag} \quad \mathrm{As}-\mathrm{Cd} \mathrm{Cr} \mathrm{CH} \mathrm{Hg} \mathrm{Hi} \mathrm{Pb}$ Se $\mathrm{Cr}$

Method Blanks

B. ank 1

Blank 2

0.012

0.012

$\mathrm{NA}^{\text {(a) }} 0.049 \mathrm{U}^{(\mathrm{b})} \mathrm{NA}$

NA

0.013

NA

NA $0.11 \mathrm{U}$

$0.11 \mathrm{U}$

Matrix Spikes

$\begin{array}{lrrr}\text { C-SG mean } & 0.201 & \text { NA } & 0.98 \\ \text { C-SB MS } & 2.080 & \text { NA } & 3.00 \\ \text { Spike Recovery } & 1.879 & \text { NA } & 2.02 \\ \text { Amount Spiked } & 2.000 & \text { NA } & 2.00 \\ \text { \% Recovery } & 94 \% & \text { NA } & 101 \% \\ & & & \\ \text { R-OS mean } & 0.190 & \text { NA } & 0.31 \\ \text { R-OS NS } & 1.880 & \text { NA } & 2.40 \\ \text { Spike Recovery } & 1.690 & \text { NA } & 2.09 \\ \text { Amount Spiked } & 2.000 & \text { NA } & 2.00 \\ \text { \% Recovery } & 85 \% & \text { NA } & 105 \%\end{array}$

Standard Reference Materials

\begin{tabular}{|c|c|c|c|c|c|c|c|c|c|c|}
\hline $\begin{array}{l}\text { MESS-1 } \\
\text { MESS-1 } \\
\text { MESS-1 } \\
\text { MESS-1 Certified Value }\end{array}$ & $\begin{array}{l}0.098 \\
N A \\
H A \\
N C(C)\end{array}$ & $\begin{array}{l}11.1 \\
10.1 \\
11.8 \\
10.6 \\
11.2\end{array}$ & $\begin{array}{r}0.69 \\
\mathrm{NA} \\
\mathrm{NA} \\
0.59 \\
\pm 0.10\end{array}$ & $\begin{array}{r}58 \\
64 \\
67 \\
71 \\
\times 11\end{array}$ & $\begin{array}{l}28.0 \\
26.9 \\
27.8 \\
25.1 \\
\pm 3.8\end{array}$ & $\begin{array}{l}0.184 \\
N A \\
N A \\
0.171 \\
\pm 0.014\end{array}$ & $\begin{array}{l}31.7 \\
31.5 \\
28.6 \\
29.5 \\
\pm 2.7\end{array}$ & $\begin{array}{l}32.1 \\
34.4 \\
33.9 \\
34.0 \\
\pm 6.1\end{array}$ & $\begin{array}{l}0.3 \\
0.3 \\
0.3 \\
0.34 \\
\pm 0.06\end{array}$ & $\begin{array}{l}178.2 \\
185.1 \\
178.4 \\
191.0 \\
\pm 17.0\end{array}$ \\
\hline $\begin{array}{l}\text { BCSS-1 } \\
\text { BCSS-1 Certified Value }\end{array}$ & $\begin{array}{r}0.07 \\
\text { NC }\end{array}$ & $\begin{array}{l}\text { MA } \\
\text { NC }\end{array}$ & $\begin{array}{r}0.32 \\
0.25 \\
\pm 0.04\end{array}$ & $\begin{array}{r}\text { NA } \\
0.36 \\
\pm 0.07\end{array}$ & $\begin{array}{l}\text { NA } \\
\text { NC }\end{array}$ & $\begin{array}{l}0.137(d) \\
0.129(d) \\
\pm 0.012\end{array}$ & $\begin{array}{l}\text { NA } \\
\text { KC }\end{array}$ & $\begin{array}{l}\text { NA } \\
\text { NC }\end{array}$ & $\begin{array}{l}0.3 \\
0.43 \\
\pm 0.06\end{array}$ & $\begin{array}{l}\text { NA } \\
\text { NC }\end{array}$ \\
\hline $\begin{array}{l}\text { SRH } 1646 \\
\text { SRM } 1646 \\
\text { SRM } 1646 \\
\text { SRM Certified Value }\end{array}$ & $\begin{array}{r}0.092 \\
N A \\
N A \\
N C\end{array}$ & $\begin{array}{l}10.1 \\
10.5 \\
12.0 \\
11.6 \\
\pm 1.3\end{array}$ & $\begin{array}{r}0.44 \\
\text { NA } \\
N A \\
N C\end{array}$ & $\begin{array}{l}84 \\
78 \\
89 \\
76 \\
23\end{array}$ & $\begin{array}{l}21.4 \\
20.3 \\
23.3 \\
18.0 \\
+3.0\end{array}$ & $\begin{array}{r}0.079 \\
\text { KA } \\
\text { NA } \\
\text { HC }\end{array}$ & $\begin{array}{l}33.1 \\
32.5 \\
32.8 \\
32.0 \\
\pm 3.0\end{array}$ & $\begin{array}{l}30.2 \\
30.0 \\
28.6 \\
28.2 \\
+1.8\end{array}$ & $\begin{array}{l}\text { NA } \\
\text { NA } \\
\text { NA } \\
\text { NC }\end{array}$ & $\begin{array}{r}138.0 \\
140.4 \\
136.7 \\
138.0 \\
\pm 6.0\end{array}$ \\
\hline $\begin{array}{l}\text { BEST-1 } \\
\text { BEST-1 Certified Value }\end{array}$ & $\begin{array}{l}N A \\
\text { NC }\end{array}$ & $\begin{array}{l}\text { NA } \\
M C\end{array}$ & $\begin{array}{l}\text { HA } \\
\text { NC }\end{array}$ & $\begin{array}{l}\text { NA } \\
\text { NC }\end{array}$ & $\begin{array}{l}\text { NA } \\
\text { NC }\end{array}$ & $\begin{array}{r}0.086 \\
0.092 \\
+0.009\end{array}$ & $\begin{array}{l}\text { NA } \\
\text { NC. }\end{array}$ & $\begin{array}{l}\text { NA } \\
\text { NC }\end{array}$ & $\begin{array}{l}\text { NA } \\
\text { NC }\end{array}$ & $\begin{array}{l}\text { NA } \\
\text { NC }\end{array}$ \\
\hline \multicolumn{11}{|l|}{ Analytical Replicates } \\
\hline 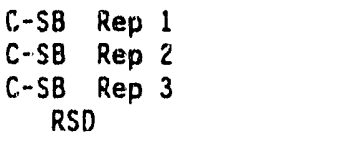 & $\begin{array}{r}0.207 \\
0.193 \\
0.203 \\
4 \%\end{array}$ & $\begin{array}{r}13.2 \\
10.41 \\
11.47 \\
12 \%\end{array}$ & $\begin{array}{r}0.98 \\
0.99 \\
0.96 \\
2 \%\end{array}$ & $\begin{array}{r}94 \\
113 \\
114 \\
11 \%\end{array}$ & $\begin{array}{r}39.3 \\
39.7 \\
41.9 \\
3 \%\end{array}$ & $\begin{array}{r}1.830 \\
1.790 \\
1.790 \\
1 \%\end{array}$ & $\begin{array}{r}45.6 \\
47.6 \\
46.5 \\
2 x\end{array}$ & $\begin{array}{r}11.0 \\
13.8 \\
12.9 \\
11 \%\end{array}$ & $\begin{array}{r}0.85 \\
0.77 \\
0.77 \\
6 \%\end{array}$ & $\begin{array}{r}96.3 \\
93.6 \\
95.0 \\
1 \%\end{array}$ \\
\hline $\begin{array}{l}R-O S \text { Rep } 1 \\
R-O S \text { Rep } 2 \\
\text { R-OS Rep } 3 \\
\text { RSO }\end{array}$ & $\begin{array}{r}0.193 \\
0.191 \\
0.185 \\
2 \%\end{array}$ & $\begin{array}{l}4.67 \\
3.40 \\
3.69 \\
17 \%\end{array}$ & $\begin{array}{r}0.30 \\
0.32 \\
0.30 \\
4 \%\end{array}$ & $\begin{array}{r}223 \\
238 \\
215 \\
5 \%\end{array}$ & $\begin{array}{r}20.8 \\
20.7 \\
19.5 \\
4 \%\end{array}$ & $\begin{array}{r}0.222 \\
0.210 \\
0.219 \\
3 \%\end{array}$ & $\begin{array}{r}66.7 \\
63.5 \\
60.2 \\
5 \%\end{array}$ & $\begin{array}{r}7.7 \\
11.8 \\
7.8 \\
26 \%\end{array}$ & $\begin{array}{r}0.56 \\
0.62 \\
0.50 \\
11 \%\end{array}$ & $\begin{array}{r}81.2 \\
85.6 \\
80.6 \\
3 \%\end{array}$ \\
\hline
\end{tabular}

$\begin{array}{lrrr}\text { NA } & \text { NA } & 0.80 & \text { NA } \\ \text { NA } & \text { NA } & 4.90 & \text { NA } \\ \text { NA } & \text { NA } & 4.10 & \text { NA } \\ \text { NA } & \text { NA } & 5.00 & \text { NA } \\ \text { NA } & \text { NA } & 82 \% & \text { NA } \\ \text { NA } & \text { NA } & 0.56 & \text { NA } \\ \text { NA } & \text { NA } & 4.61 & \text { NA } \\ \text { NA } & \text { NA } & 4.05 & \text { NA } \\ \text { NA } & \text { NA } & 5.0 & \text { NA } \\ \text { NA } & \text { NA } & 81 \% & \text { NA }\end{array}$

NA 
TABLE C.22. (contd)

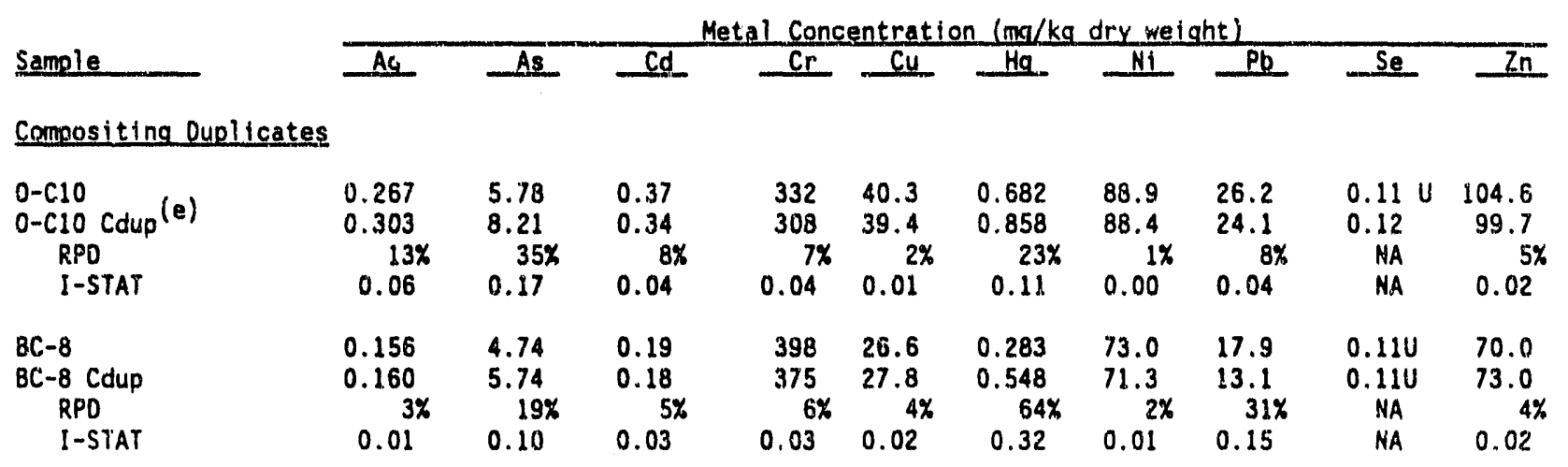

\footnotetext{
(a) Not applicable.

(b) Undetected above given concentration.

(c) Not certified.

(d) The certification of MESS-1 and BCSS-1 for mercury was withdrawn in April 1990 due to potential mercury contamination. Their values are reported for comparisons only and cannot be used to establish accuracy.

(e) Compositing duplicate.
} 
IABLE C.23. Sediment Butyltin Results, Oakland Harbor Phase III B Project

Sediment

Ireatment

DL Goal

DL Achieved

$\mathrm{BC}-7$

$0-\mathrm{C} 13$

$0-C 1341.5 \mathrm{ft} \mathrm{MLLW}$

$0-\mathrm{C} 12$

$0-C 11$

$0-\mathrm{C} 10$

$0-$ C10 Cdup $^{(c)}$

$B C-5$

$0-C 9$

$0-C 8$

$0-C 7$

$0-C 6$

$B C-8$

$B C-8$ Cdup

$0-C 5$

$0-C 4$

$B C-6$

$0-\mathrm{C} 3$

$0-\mathrm{Cl}$

$I-C 2$

$0-C 2$

I $-\mathrm{C} 3$

I $-\mathrm{CB}$

I-C8 Cdup

I $-\mathrm{C} 4$

$\mathrm{I}=\mathrm{T5}$

$R-A C$

$R-A C$

$R-A M$

$R-B F$

$R-B F$

$R-O S$

$R-P C$

$R-P F$

$R-P F$

Rep 1

Rep 2

Rep 1

Rep 2

Rep 1

Rep 2
Tripentyltin

Surrogate

Recovery $(\%)$

$N A^{(a)}$

NA

81

111

73

85

88

77

90

82

78

$198^{(d)}$

112

98

75

46

84

76

87

76

75

83

61

77

75

66

89

73
75

67

67

69

68

56

72

58

7]

\section{Butylt in Species ( $\mu \mathrm{g} / \mathrm{kg}$ dry weight)}

Iributyl Dibutyl Monobutyl

$\begin{array}{lll}10.0 & 10.0 & 10.0\end{array}$

1.0

1.0

1.0

$\begin{array}{rll}6.1 & 1.3 & 1.4 \\ 9.1 & 7.1 & 2.8 \\ 1.0 U^{(b)} & 1.0 \mathrm{U} & 1.0 \mathrm{U} \\ 1.3 & 1.2 & 1.0 \mathrm{U} \\ 5.4 & 4.5 & 3.1 \\ 11.5 & 7.4 & 1.6 \\ 5.0 & 1.6 & 1.0 \mathrm{U}\end{array}$

3.3

$1.0 \mathrm{U}$

8.3

1.7

1.1

$1.0 \mathrm{U}$

1.0

6.5

9.9

3.7

5.9

5.7

8.4

2.4

2.1

1.5

$1.0 \mathrm{U}$

1.1

$1.0 \mathrm{U}$

1.0

2.3

4.8

5.6

1.5

$1.0 \mathrm{U}$

1.3

2.1

6.7

5.5

2.6

$1.0 \mathrm{U}$

$1.0 \mathrm{U}$

$1.0 \mathrm{U}$

1.6

$1.0 \mathrm{U}$

$1.0 \mathrm{U}$

$1.0 \mathrm{U}$

$1.0 \mathrm{U}$

1.4

4.4

$1.0 \mathrm{U}$

$1.0 \mathrm{U}$

$1.0 \mathrm{U}$

1.8

2.3

9.5

3.3

4.0

$1.0 \mathrm{U}$

$1.0 \mathrm{U}$

$1.0 \mathrm{U}$

1.6

1. 3

2.2

$1.0 \mathrm{U}$

1.1

$1.0 \mathrm{U}$

$1.0 \mathrm{U}$

4.8

3.2

1.5

2.4

$1.0 \mathrm{U}$

$1.0 \mathrm{U}$

1.9

$1.0 \mathrm{U}$

$1.0 \mathrm{U}$

$1.0 \mathrm{U}$

$1.0 \mathrm{U}$

$1.0 \mathrm{U}$
$1.0 \mathrm{U}$

$1.0 \mathrm{U}$

$1.0 \mathrm{U}$

2.0

3.8

$1.0 \mathrm{U}$

$1.0 \mathrm{~V}$

$1.0 \mathrm{U}$

3.3 
TABLE C.23. (contd)

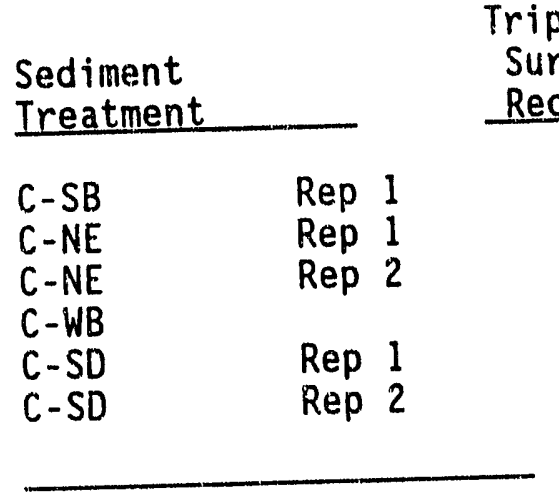

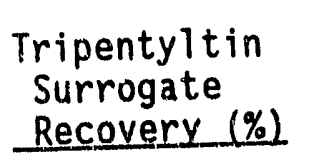

77
67
58
70
70
78

Butyltin Species $(\mu \mathrm{g} / \mathrm{kg}$, dry weight)
Iributyi - Dibutyl Monobutyl

3.5
$1.0 U$
$1.0 U$
$1.0 U$
$1.0 U$
$1.0 U$

1.7

$1.0 \mathrm{U}$

$1.0 \mathrm{U}$

3.2

$1.0 \mathrm{U}$

$1.0 \mathrm{U}$
2.8

$1.0 \mathrm{U}$

$1.0 \mathrm{U}$

$1.0 \mathrm{U}$

$1.0 \mathrm{U}$

1.1

(a) Not applicable.
(b) Undetected above concentration.
(c) Compositing duplicate.
(d) Surrogate compound inadvertantly added two times. 
TABLE C.24. Quality Control Data for Sediment Butyltin Analysis, Oakland Harbor Phase III B Project

Sample

Tripentyltin

Surrogate

Butyltin Species $(\mu \mathrm{g} / \mathrm{kg}$ dry weight)

Recovery (\%)

Iributy 1 Dibuty] Monobutyl

Method BIanks

Blank 1

Blank 2

Blank 3

Blank 4

$\begin{array}{llll}40 & 1.0 U^{(a)} & 1.3 & 1.0 \mathrm{U} \\ 68 & 1.0 \mathrm{U} & 1.0 \mathrm{U} & 1.0 \mathrm{U} \\ 69 & 1.0 \mathrm{U} & 1.0 \mathrm{U} & 1.0 \mathrm{U} \\ 55 & 1.0 \mathrm{U} & 1.0 \mathrm{U} & 1.0 \mathrm{U}\end{array}$

\section{Matrix Spikes}

$B C-5$

$B C-5 M S$

Amount Recovered

Percent Recovery

Spiked with $120 \mu \mathrm{g} / \mathrm{kg}$

$0-c 9$

0 -C9 MS

Amount Recovered

Percent Recovery

Spiked with $82 \mu \mathrm{g} / \mathrm{kg}$

$R$-PF Rep 2

R-PF Rep 2 MS

Amount Recovered

Percent Recovery

Spiked with $102 \mu \mathrm{g} / \mathrm{kg}$

78

64

82
70

3.3
73.8

70.5

$59 \%$

$1.0 U$

41.1

41.1

$50 \%$

71

82

$1.0 \mathrm{U}$

72.7

72.7

$71 \%$

$R-B F$
$R-B F$

RPD

$R-P F$

$R-P F$

RPD

I - STAT
75

67

69

68

I-STAT

Rep 1

Rep 2

Rep 1

Rep 2

RPD

I - STAT

Rep 1

Rep 2

58

71

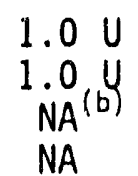

5.5

5.6

0.01

$1.0 \mathrm{U}$

$1.0 \mathrm{U}$

NA

$N A$
1.7

37.0

35.3

$29 \%$

1.1

$1.0 \mathrm{U}$

NA

NA

4.8

3.2

$40 \%$

0.20

1.1

52.6

51.5

$43 \%$

1.0

34.9

33.9

$41 \%$

3.3

29.0

25.7

$25 \%$

$1.0 \mathrm{U}$

$1.0 \mathrm{U}$

NA

NA

2.0

3.8

$62 \%$

0.31

PHASE III B

C. 42

$\begin{array}{ll}1.0 U & 1.0 U \\ 1.0 U & 3.3 \\ \text { NA } & \text { NA } \\ \text { NA } & \text { NA }\end{array}$

$1.0 \mathrm{U}$

NA

NA 
TABLE C.24. (contd)

Tripentyltin

Surrogate

Sample

?igcovery $(\%)$

Butyltin Species ( $\mu \mathrm{g} / \mathrm{kg}$ dry weight)

$\begin{array}{ll}\text { C-NE } & \text { Rep } 1 \\ \text { C-NE } & \text { Rep } 2 \\ \text { RPD } & \\ \text { I-STAT } & \end{array}$

67

58

0.7

0.4

$55 \%$

0.27

Dibuty 7 Monobuty

$C-S D$

C-SD

Rep 1

Rep 2

RPD

I-STAT

70

78

$1.0 \mathrm{U}$

$1.0 \mathrm{U}$

NA

$N A$

$1.0 \mathrm{U}$

$1.0 \mathrm{U}$

NA

$1.0 \mathrm{U}$

$1.0 \mathrm{U}$

NA

NA

Compositing Duplicates

$B C-8$

$B C-8$ Cdup $^{(c)}$

75

RPD

I-STAT

46

2.1

1.5

$33 \%$

0.17

$1.0 \mathrm{U}$

$1.0 \mathrm{U}$

NA

NA

$1.0 \mathrm{U}$

1.1

NA

NA

$0-C 10$

0-C10 Cdup

RPD

I-STAT

77
90

11.5

5.0

$79 \%$

0.39

1.1

$1.0 \mathrm{U}$

$1.0 \mathrm{U}$

NA

$1.0 \mathrm{U}$

NA

NA

NA

1.6

1.6

0.9

$129 \%$

$56 \%$

0.28

Standard Reference Materials

258-PACS - 1

73

568.8

478.8

108.6

258-PACS-2

65

518.0

473.4

370.2

PACS-1 Corrected for $66.7 \%$ Propyltin

PACS-2 Corrected for $63.9 \%$ Propyltin

$853^{(d)}$
$811^{(d)}$

$718^{(\mathrm{d})}$

163

PACS Certified Value

$1270 \pm 220$

$1160 \pm 180$

$579^{(d)}$

$280 \pm 170$
(a) Undetected above given concentration.
(b) Not applicable.
(c) Compositing duplicate.
(d) Surrogate compound inadvertantly added $2 x$. 
APPENDIX D

10-DAY ELOW-THROUGH SOLD-PHASE TEST WITH N CAECOIDES 
IABLE 0.1. Test Results for All Replicates in 10-Day N. caecoides Solid-Phase Test, Oakl and Harbor Phase III B Project

\begin{tabular}{|c|c|c|c|c|}
\hline \multirow[b]{2}{*}{$\begin{array}{l}\text { Sediment } \\
\text { Ireatment }\end{array}$} & \multirow[b]{2}{*}{ Replicate } & \multicolumn{2}{|c|}{ N. caecoides } & \multirow{2}{*}{$\begin{array}{c}\text { Mean } \\
\text { Proportion } \\
\text { Surviving } \\
\end{array}$} \\
\hline & & Live & $\begin{array}{c}\text { Dead } \\
\text { or Missing } \\
\end{array}$ & \\
\hline $\begin{array}{l}0-C 13 \\
0-C 13 \\
0-C 13 \\
0-C 13 \\
0-C 13\end{array}$ & $\begin{array}{l}1 \\
2 \\
3 \\
4 \\
5\end{array}$ & $\begin{array}{l}18 \\
18 \\
19 \\
14 \\
16\end{array}$ & $\begin{array}{l}2 \\
2 \\
1 \\
6 \\
4\end{array}$ & 0.85 \\
\hline $\begin{array}{l}0-C 12 \\
0-C 12 \\
0-C 12 \\
0-C 12 \\
0-C 12\end{array}$ & $\begin{array}{l}1 \\
2 \\
3 \\
4 \\
5\end{array}$ & $\begin{array}{l}17 \\
18 \\
17 \\
20 \\
19\end{array}$ & $\begin{array}{l}3 \\
2 \\
3 \\
0 \\
1\end{array}$ & 0.91 \\
\hline $\begin{array}{l}0-C 11 \\
0-C 11 \\
0-C 11 \\
0-C 11 \\
0-C 11\end{array}$ & $\begin{array}{l}1 \\
2 \\
3 \\
4 \\
5\end{array}$ & $\begin{array}{l}20 \\
18 \\
16 \\
15 \\
17\end{array}$ & $\begin{array}{l}0 \\
2 \\
4 \\
5 \\
3\end{array}$ & 0.86 \\
\hline $\begin{array}{l}0-C 10 \\
0-C 10 \\
0-C 10 \\
0-C 10 \\
0-C 10\end{array}$ & $\begin{array}{l}1 \\
2 \\
3 \\
4 \\
5\end{array}$ & $\begin{array}{l}20 \\
20 \\
20 \\
16 \\
17\end{array}$ & $\begin{array}{l}0 \\
0 \\
0 \\
4 \\
3\end{array}$ & 0.93 \\
\hline $\begin{array}{l}0-C 9 \\
0-C 9 \\
0-C 9 \\
0-C 9 \\
0-C 9\end{array}$ & $\begin{array}{l}1 \\
2 \\
3 \\
4 \\
5\end{array}$ & $\begin{array}{l}20 \\
20 \\
20 \\
20 \\
19\end{array}$ & $\begin{array}{l}0 \\
0 \\
0 \\
0 \\
1\end{array}$ & 0.99 \\
\hline $\begin{array}{l}0-C 8 \\
0-C 8 \\
0-C 8 \\
0-C 8 \\
0-C 8\end{array}$ & $\begin{array}{l}1 \\
2 \\
3 \\
4 \\
5\end{array}$ & $\begin{array}{l}18 \\
16 \\
18 \\
18 \\
20\end{array}$ & $\begin{array}{l}2 \\
4 \\
2 \\
2 \\
0\end{array}$ & 0.90 \\
\hline $\begin{array}{l}0-C 7 \\
0-C 7 \\
0-C 7 \\
0-C 7 \\
0-C 7\end{array}$ & $\begin{array}{l}1 \\
2 \\
3 \\
4 \\
5\end{array}$ & $\begin{array}{l}16 \\
18 \\
13 \\
17 \\
18\end{array}$ & $\begin{array}{l}4 \\
2 \\
7 \\
3 \\
2\end{array}$ & 0.82 \\
\hline PHASE III B & & D. 1 & & \\
\hline
\end{tabular}


IABLE 0.1. (contd)

Sediment

Ireatment

$0-16$

$0-\mathrm{C} 6$

$0-\mathrm{C} 6$

$0-\mathrm{C} 6$

$0-C 6$

$0-C 5$

$0-C 5$

$0-C 5$

$0-C 5$

$0-C 5$

$0-C 4$

$0-C 4$

$0-C 4$

$0-C 4$

$0-C 4$

$0-c 3$

$0-C_{3}$

$0-C 3$

$0-C 3$

$0-C 3$

$0-c 2$

$0-C 2$

$0-\mathrm{C2}$

$0-c 2$

$0-\mathrm{C2}$

$0-C 1$

$0-C 1$

$0-C 1$

$0-C 1$

$0-C 1$

I $-\mathrm{C} 2$

I-C2

I-C2

I-C2

I-C2 \begin{tabular}{r} 
\\
Live \\
\hline 19 \\
18 \\
18 \\
20 \\
18
\end{tabular}

20

20

20

19

19

19

20

19

17

20

20

17

20

20

18

19

20

19

20

20

19

18

15

16
19

20

20

19

20

19
Mean

Proportion

Surviving

0.93

0.98

0.95

0.95

0.98

0.87

0.98

PHASE III B

D. 2 
TABLE D.1. (contd)

Sediment

Treatment

I $-\mathrm{C} 3$

$\mathrm{I}-\mathrm{C3}$

I-C3

I-C3

I-C3

I $-\mathrm{C} 8$

I $-\mathrm{CB}$

I $-\mathrm{CB}$

I $-\mathrm{C} 8$

I-C8

I $-\mathrm{C} 4$

I-C4

I-C4

I-C4

I-C4

I -T5

I-T5

I $-\mathrm{T} 5$

I-TS

I - T 5

$R-A C$

$R-A C$

$R-A C$

$R-A C$

$R-A C$

R-AM

R-AM

R-AM

R-AM

$\mathrm{R}-\mathrm{AM}$

$R-B F$

$R-B F$

$R-B F$

$R-B F$

$R-B F$

\section{Replicate}

1
2

3

4
5

1

2

3

4
5

19

1

2

3

4
5

1

2

3

4
5

5

1
2
3
4
5

1

3

4

5

1
2
3
4
5

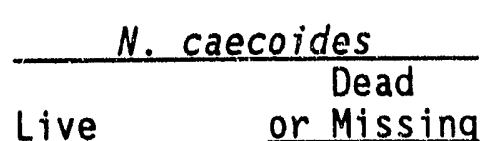

Live

20

20

20

20

19

19

19

17

19

20

20

18

18

19

19

16

19

19

20

20

20

20

20

20

20

20

19

20

20

20

20

18

19

19
Mean

Proportion

Surviving

1.00

0.93

0.95

0.93

1.00

0.99

0.96

PHASE III B

D. 3 
TABLE D.1. (contd)

Sediment

Treatment

R-OS

$R-O S$

$R-O S$

$R-O S$

R-OS

$R-P C$

$R-P C$

$R-P C$

$R-P C$

$R-P C$

$R-P F$

$R-P F$

$R-P F$

$R-P F$

$R-P F$

C-SB

C-SB

C-SB

C-SB

C-SB

C-NE

C-NE

$C-N E$

$C-N E$

C-NE

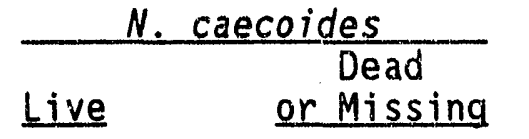

Replicate

1
2
3
4
5

20

20

20

20

19

17

19

20

19

17

19

20

20

20

19

$28^{(a)}$

20

19

18

20

20

20

20

20

20
Mean

Proportion

Surviving

0
0
0
0
1

0.99

0.92

0.98

0.95

1.00

(a) $30 \mathrm{~N}$. caecoides initiated in this replicate. 
TABLE D.2. Rank Order Based on Mean Proportion Surviving 10-Day N. caecoides Solid-Phase Test, Oakl and Harbor Phase III B Project

\begin{tabular}{|c|c|}
\hline $\begin{array}{l}\text { Sediment } \\
\text { Ireatment }\end{array}$ & $\begin{array}{l}\text { Mean } \\
\text { Proportion } \\
\text { Surviving }\end{array}$ \\
\hline $\begin{array}{l}0-C 7 \\
0-C I 3 \\
0-C 11 \\
0-C 1 \\
0-C 8 \\
0-C 12 \\
R-P C \\
I-C 8 \\
I-T 5 \\
0-C 6 \\
0-C 10 \\
C-S B \\
I-C 4 \\
0-C 3 \\
0-C 4 \\
R-B F \\
I-C 2 \\
0-C 2 \\
0-C 5 \\
R-P F \\
0-C 9 \\
R-A M \\
R-O S \\
C-N E \\
I-C 3 \\
R-A C\end{array}$ & $\begin{array}{l}0.82 \\
0.85 \\
0.86 \\
0.87 \\
0.90 \\
0.91 \\
0.92 \\
0.93 \\
0.93 \\
0.93 \\
0.93 \\
0.95 \\
0.95 \\
0.95 \\
0.95 \\
0.96 \\
0.98 \\
0.98 \\
0.98 \\
0.98 \\
0.99 \\
0.99 \\
0.99 \\
1.00 \\
1.00 \\
1.00\end{array}$ \\
\hline
\end{tabular}


TABLE D.3. Water Quality Summary for 10-Day N. caecoides Solid-Phase Test, Oakland Harbor Phase III B Project

\begin{tabular}{|c|c|c|c|c|c|c|c|c|c|c|}
\hline \multirow{2}{*}{$\begin{array}{l}\text { Sadimant } \\
\text { Irostanens? }\end{array}$} & \multicolumn{2}{|c|}{$\begin{array}{c}\text { Temperature } \\
\text { ('C) }\end{array}$} & \multicolumn{2}{|c|}{ ofl } & \multicolumn{2}{|c|}{$\begin{array}{c}\text { Dissol ved } \\
\text { oxysen } \\
\text { (ma/L) } \\
\end{array}$} & \multicolumn{2}{|c|}{$\begin{array}{c}\text { sulinity } \\
(0 / \infty)\end{array}$} & \multicolumn{2}{|c|}{$\begin{array}{c}\text { Flow Rates } \\
(\underline{m} / \min )\end{array}$} \\
\hline & Min & & Ain & $\operatorname{sax}$ & min & Max & Min & $\underline{M x}$ & Min & Max \\
\hline $\begin{array}{l}\text { Acceptable } \\
\text { Rewe }\end{array}$ & 13.0 & 17.0 & 6.90 & 7.90 & 6.0 & $m a^{(a)}$ & 29.0 & 33.0 & 115 & 135 \\
\hline 0.613 & 13.9 & 18.7 & 7.80 & 7.97 & 7.5 & 8.2 & 30.0 & 32.0 & 116 & $136^{(b)}$ \\
\hline 0.012 & $\$ 4.0$ & 15.0 & 7.82 & 8.00 & 7.5 & 8.3 & 30.0 & 32.0 & 116 & 134 \\
\hline 0.011 & 13.8 & 14.8 & 7.81 & 7.98 & 7.4 & 8.3 & 30.0 & 32.0 & 116 & 134 \\
\hline 0.610 & 13.7 & 14.9 & 7.81 & 7.95 & 7.1 & 8.6 & 29.5 & 32.0 & 116 & 134 \\
\hline $0 . c 9$ & 13.8 & 15.9 & 7.80 & 7.90 & 7.4 & 8.3 & 30.0 & 32.0 & 116 & $140^{(b)}$ \\
\hline $0 . c 8$ & 13.1 & $18.3^{(b)}$ & 7.78 & 7.92 & 7.3 & 8.2 & 30.0 & 32.0 & 116 & 132 \\
\hline$a \cdot c y$ & 93.6 & 14.8 & 7.79 & 7.97 & 7.4 & 8.2 & 20.0 & 32.0 & 116 & 132 \\
\hline $0 . \mathrm{Cs}$ & 13.7 & 15.0 & 7.59 & 7.98 & 7.5 & 8.2 & 30.0 & 32.0 & 116 & 132 \\
\hline 0.05 & 13.8 & $17.6^{(b)}$ & 7.66 & 7.97 & 7.3 & 8.3 & 29.5 & 32.0 & 116 & 134 \\
\hline $0 . C_{4}$ & 13,8 & 15.3 & 7.60 & 7.95 & 7.2 & 8.2 & 30.0 & 32.0 & 116 & 132 \\
\hline $0 . c x$ & 13.9 & 15.2 & 7.58 & 8.01 & 7.3 & 8.2 & 30.0 & 32.0 & 116 & 132 \\
\hline 0.62 & 13.5 & 15.3 & 7.63 & 7.97 & 7.3 & 8.2 & 30.0 & 32.0 & 116 & 132 \\
\hline $0 .-c 1$ & 13.6 & 95.6 & 7.62 & 7.93 & 7.3 & 2.2 & 29.5 & 32.0 & 116 & 132 \\
\hline $1 \cdot 12$ & 13.9 & 16.8 & 7.65 & 8.00 & 7.5 & 8.2 & 30.0 & 32.0 & 116 & 132 \\
\hline $1 \cdot c 3$ & 13.7 & 15.1 & 7.61 & 7.96 & 7.5 & 8.3 & 30.0 & 32.0 & 196 & 132 \\
\hline $1 \cdot c 8$ & 14.0 & 95.2 & 7.60 & 7.99 & 7.4 & 8.3 & 30.0 & 32.0 & $112^{(b)}$ & 132 \\
\hline $1 \cdot C_{4}$ & 13.8 & 15.4 & 7.81 & 7.97 & 7.4 & 8.2 & 30.0 & 32.0 & 116 & 132 \\
\hline 1.15 & 13.7 & 14.9 & 7.83 & 7.98 & 7.5 & 8.3 & 30.0 & 32.0 & 116 & 132 \\
\hline$A \cdot A C$ & 13.7 & 14.9 & 7.81 & 7.97 & 7.5 & 0.3 & 30.0 & 31.5 & 116 & 132 \\
\hline R-AM & 13.8 & 15.0 & 7.78 & 7.96 & 7.6 & 8.2 & 30.0 & 32.0 & 116 & 134 \\
\hline$R-8 F$ & 13.5 & 15.0 & 7.70 & 8.00 & 7.2 & 8.2 & 29.5 & 32.0 & $108^{(b)}$ & 132 \\
\hline$R \cdot \cos$ & 13.8 & 15.0 & 7.78 & 7.98 & 7.4 & 8.2 & 30.0 & 32.0 & 116 & 132 \\
\hline X-PC & 13.8 & 15.0 & 7.78 & 7.96 & 7.4 & 8.3 & 30.0 & 32.0 & 116 & 132 \\
\hline$R \cdot P F$ & 13.6 & $17.9^{(b)}$ & 7.78 & 7.93 & 7.3 & 8.2 & 30.0 & 32.0 & 116 & 132 \\
\hline$C \cdot S B$ & 13.7 & 15.0 & 7.81 & 7.87 & 7.3 & 8.3 & 30.0 & 32.0 & 116 & $156^{(b)}$ \\
\hline C. WE & 13.7 & 15.1 & 7.82 & 7.97 & 7.5 & 8.2 & 30.0 & 32.0 & 116 & 132 \\
\hline
\end{tabular}

(a) Mut applicable.

(b) Parameter mas isopor arily outside of target quality control ranye; outcone of test not affecied. 
TABLE D.4. Daily Observations of $N$. caecoides on Sediment Surface During 10-Day Solid-Phase Test, Oakland Harbor Phase III B Project

Sediment

Treatment

Replicate

N. caecoides on Sediment for Test Davs 1 through 10

$0 .-13$

$0-\mathrm{C} 13$

$0-\mathrm{C} 13$

$0-\mathrm{C} 13$

$0-\mathrm{C} 13$

1
2
3
4
5

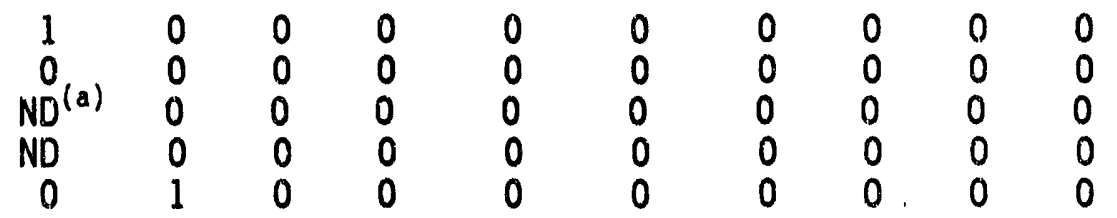

$0-\mathrm{Cl} 2$

$0-\mathrm{C} 12$

$0-\mathrm{C} 12$

$0-\mathrm{C} 12$

$0-\mathrm{C} 12$

1
2
3
4
5

$\begin{array}{llllllllll}0 & 0 & 0 & 0 & 0 & 0 & 0 & 0 & 0 & 0 \\ 1 & 0 & 0 & 0 & 0 & 0 & 0 & 0 & 0 & 0 \\ 0 & 0 & 1 & 1 & 1 & 1 & 1 & 0 & 0 & 0 \\ 0 & 0 & 0 & 0 & 1 & 0 & 0 & 0 & 0 & 0 \\ 0 & 0 & 0 & 0 & 0 & 0 & 0 & 0 & 0 & 0\end{array}$

$0-C 11$

$0-C 11$

0 -C11

$0-\mathrm{C} 11$

0 -C11

$\begin{array}{llllllllll}0 & 0 & 0 & 0 & 1 & 0 & 0 & 0 & 0 & 0 \\ 1 & 0 & 0 & 0 & 0 & 0 & 0 & 0 & 0 & 0 \\ 0 & 2 & 1 & 2 & 1 & 0 & 1 & 0 & 0 & 0 \\ 0 & 0 & 0 & 0 & 0 & 0 & 0 & 0 & 0 & 0 \\ 0 & 0 & 0 & 0 & 0 & 0 & 0 & 0 & 0 & 0\end{array}$

$0-C 10$
$0-C 10$

$0-C 10$

$0-\mathrm{C} 10$

$0-\mathrm{C} 10$

1
2
3
4
5

$0-c 9$

$0-c 9$

$0-C 9$

$0-c 9$

$0-C 9$

1
2
3
4
5

$0-\mathrm{C} 8$

$0-C 8$

$0-C 8$

$0-C 8$

$0-c 8$

$0-\mathrm{C7}$

$0-C 7$

$0-C 7$

$0-C 7$

O-C7

$\begin{array}{rlllllllll}\text { ND } & 0 & 0 & 0 & 0 & 0 & 0 & 0 & 0 & 0 \\ \text { ND } & 0 & 0 & 0 & 0 & 0 & 0 & 0 & 0 & 0 \\ 0 & 0 & 0 & 0 & 0 & 0 & 0 & 0 & 0 & 0 \\ 0 & 0 & 0 & 0 & 0 & 0 & 0 & 0 & 0 & 0 \\ 3 & 0 & 0 & 1 & 1 & 0 & 1 & 0 & 0 & 0\end{array}$

$\begin{array}{rrrllllllll}1 & 0 & 0 & 0 & 0 & 0 & 0 & 0 & 0 & 0 & 0 \\ 2 & 0 & 0 & 0 & 0 & 0 & 0 & 0 & 0 & 0 & 0 \\ 3 & 0 & 0 & 0 & 0 & 0 & 0 & 0 & 0 & 0 & 0 \\ 4 & \text { ND } & 0 & 0 & 0 & 0 & 0 & 0 & 0 & 0 & 0 \\ 5 & 0 & 0 & 0 & 0 & 0 & 0 & 0 & 0 & 0 & 0\end{array}$

$\begin{array}{lrlllllllll}1 & N D & 1 & 1 & 1 & 0 & 0 & 0 & 0 & 0 & 0 \\ 2 & 1 & 1 & 1 & 1 & 0 & 0 & 0 & 0 & 0 & 0 \\ 3 & N D & 0 & 0 & 1 & 0 & 0 & 0 & 0 & 0 & 0 \\ 4 & 1 & 0 & 1 & 1 & 0 & 0 & 0 & 0 & 0 & 0 \\ 5 & 0 & 0 & 0 & 0 & 0 & 0 & 0 & 0 & 0 & 0\end{array}$

$\begin{array}{lrlllllllll}1 & \text { ND } & 0 & 0 & 0 & 0 & 0 & 0 & 0 & 0 & 0 \\ 2 & 0 & 0 & 0 & 0 & 0 & 0 & 0 & 0 & 0 & 0 \\ 3 & 0 & 0 & 0 & 0 & 0 & 0 & 0 & 0 & 0 & 0 \\ 4 & 0 & 0 & 1 & 1 & 0 & 0 & 0 & 0 & 0 & 0 \\ 5 & 1 & 0 & 0 & 0 & 0 & 0 & 0 & 0 & 0 & 0\end{array}$


IABLE D.4. (contd)

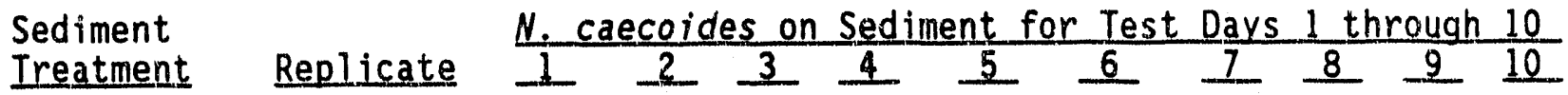

$\begin{array}{llllllllllll}0-C 6 & 1 & 0 & 0 & 0 & 0 & 0 & 0 & 0 & 0 & 0 & 0 \\ 0-C 6 & 2 & 0 & 0 & 0 & 0 & 0 & 0 & 0 & 0 & 0 & 0 \\ 0-C 6 & 3 & 0 & 0 & 0 & 0 & 0 & 0 & 0 & 0 & 0 & 0 \\ 0-C 6 & 4 & 0 & 0 & 0 & 0 & 0 & 0 & 0 & 0 & 0 & 0 \\ 0-C 6 & 5 & 0 & 0 & 0 & 0 & 0 & 0 & 0 & 0 & 0 & 0\end{array}$

$0-C 5$

$0-C 5$

$0-C 5$

$0-C 5$

$\begin{array}{lllllllllll}1 & 0 & 0 & 0 & 0 & 0 & 0 & 0 & 0 & 0 & 0 \\ 2 & 0 & 0 & 0 & 0 & 1 & 0 & 0 & 0 & 0 & 0 \\ 3 & 0 & 0 & 0 & 0 & 0 & 0 & 0 & 0 & 0 & 0 \\ 4 & 0 & 0 & 0 & 0 & 0 & 0 & 0 & 0 & 0 & 0 \\ 5 & 0 & 0 & 0 & 0 & 0 & 0 & 0 & 0 & 0 & 0\end{array}$

$0-C_{4}$

$0-C_{4}$

$0-C_{4}$

$0-C_{4}$

O-C4

$\begin{array}{lllllllllll}1 & 0 & 0 & 0 & 0 & 0 & 0 & 0 & 0 & 0 & 0 \\ 2 & 0 & 0 & 0 & 0 & 1 & 0 & 1 & 0 & 0 & 0 \\ 3 & 0 & 0 & 0 & 0 & 0 & 0 & 0 & 0 & 0 & 0 \\ 4 & 0 & 0 & 0 & 0 & 0 & 0 & 0 & 0 & 0 & 0 \\ 5 & 0 & 0 & 0 & 0 & 0 & 0 & 0 & 0 & 0 & 0\end{array}$

$0-13$

$0-\mathrm{C3}$

$0-C_{3}$

$0-C 3$

$0-\mathrm{C3}$

$\begin{array}{lllllllllll}1 & 0 & 0 & 0 & 0 & 0 & 0 & 0 & 0 & 0 & 0 \\ 2 & 1 & 0 & 0 & 0 & 0 & 0 & 0 & 0 & 0 & 0 \\ 3 & 1 & 0 & 1 & 0 & 0 & 0 & 0 & 0 & 0 & 0 \\ 4 & 0 & 0 & 0 & T^{(b)} & 0 & 0 & 0 & 0 & 0 & 0 \\ 5 & 1 & 0 & 0 & 0 & 0 & 0 & 0 & 0 & 0 & 0 \\ 1 & 0 & 1 & 1 & 0 & 0 & 0 & 0 & 0 & 0 & 0 \\ 2 & 0 & 0 & 0 & 0 & 0 & 0 & 0 & 0 & 0 & 0 \\ 3 & 0 & 0 & 0 & 0 & 0 & 0 & 0 & 0 & 0 & 0 \\ 4 & 0 & 0 & 0 & 0 & 0 & 0 & 0 & 0 & 0 & 0 \\ 5 & 0 & 0 & 0 & 0 & 0 & 0 & 0 & 0 & 0 & 0\end{array}$

$0-C 1$

O-C1

$0-C 1$

$0-C 1$

O-C1

$\begin{array}{lllllllllll}1 & 0 & 1 & 0 & 0 & 0 & 0 & 0 & 0 & 0 & 0 \\ 2 & 2 & 0 & 0 & 0 & 1 & 0 & 0 & 0 & 0 & 0 \\ 3 & 0 & 0 & 0 & 0 & 0 & 0 & 0 & 0 & 0 & 0 \\ 4 & 0 & 0 & 0 & 0 & 0 & 0 & 0 & 0 & 0 & 0 \\ 5 & 1 & 0 & 0 & 0 & 0 & 0 & 0 & 0 & 0 & 0\end{array}$

I $-\mathrm{Cl}$

I-C2

I-C2

I-C2

$\begin{array}{lllllllllll}1 & 0 & 0 & 0 & 0 & 0 & 0 & 0 & 0 & 0 & 0 \\ 2 & 0 & 0 & 0 & 0 & 0 & 0 & 0 & 0 & 0 & 0 \\ 3 & 0 & 0 & 0 & 0 & 0 & 0 & 0 & 0 & 0 & 0 \\ 4 & 0 & 0 & 0 & 0 & 0 & 0 & 0 & 0 & 0 & 0 \\ 5 & 0 & 0 & 0 & 0 & 0 & 0 & 0 & 0 & 0 & 0\end{array}$


TABLE 0.4. (contd)

Sediment
Ireatment Replicate $\frac{N \text {. caecoides on Sediment for Test Days } 1 \text { through } 10}{1}+\frac{3}{9}+10$

I-C3

I $-\mathrm{C} 3$

I- $\mathrm{C} 3$

$\mathrm{I}-\mathrm{C} 3$

$\mathrm{I}-\mathrm{C} 3$

I-C4

I-C4

I-C4

$\mathrm{I}-\mathrm{C} 4$

I-C4

I-C8

I-C8

I $-\mathrm{C} 8$

I-C8

I-C8

I-T5

I-T5

I-T5

I-T5

I-T5

$R-A C$

$R-A C$

$R-A C$

$R-A C$

$\mathrm{R}-\mathrm{AC}$

R-AM

R-AM

$R-A M$

R-AM

R-AM

$R-B F$

$R-B F$

$R-B F$

$R-B F$

$R-B F$
1
2
3
4
5

1
2
3
4
5

1
2
3
4
5

$\begin{array}{lll}1 & 1 \\ 3 & 10 & 0\end{array}$

3
4

5

1
2
3
4
5

1
2
3
4
5

1
2
3
4
5
0
0
1
0
0

$\begin{array}{lllll}0 & 0 & 0 & 0 & 0 \\ 0 & 0 & 0 & 0 & 0 \\ 0 & 1 & 1 & 1 & 1 \\ 0 & 0 & 0 & 0 & 0 \\ 0 & 0 & 0 & 0 & 0\end{array}$

$\begin{array}{llll}0 & 0 & 0 & 0 \\ 0 & 1 & 0 & 0 \\ 0 & 1 & 1 & 0 \\ 3 & 1 & 0 & 0 \\ 0 & 0 & 0 & 0\end{array}$

$\begin{array}{lll}0 & 0 & 1 \\ \text { ND } & 0 & 0\end{array}$

$\begin{array}{llllll}0 & 0 & 0 & 0 & 0\end{array}$

$\begin{array}{rllll}0 & 0 & 0 & 0 & 0 \\ \text { ND } & 0 & 0 & 0 & 0\end{array}$

$\begin{array}{lllll}0 & 0 & 0 & 0 & 0 \\ 0 & 0 & 0 & 0 & 0\end{array}$

$\begin{array}{lllll}0 & 0 & 0 & 0 & 0\end{array}$

$\begin{array}{lllll}0 & 0 & 0 & 0 & 0 \\ 0 & 0 & 0 & 0 & 0\end{array}$

ND $\quad 0 \quad 0 \quad 000$

ND $\quad 0 \quad 00000$

$\begin{array}{lllll}0 & 0 & 0 & 0 & 0 \\ 0 & 0 & 0 & 0 & 0\end{array}$

$\begin{array}{lllll}0 & 0 & 0 & 0 & 0 \\ 0 & 0 & 0 & 0 & 0\end{array}$

$\begin{array}{lllll}0 & 0 & 0 & 0 & 0\end{array}$

$\begin{array}{lllll}1 & 1 & 0 & 0 & 0 \\ 1 & 1 & 0 & 0 & 0\end{array}$

$\begin{array}{rllll}\text { ND } & 0 & 0 & 0 & 0 \\ 0 & 0 & 0 & 0 & 0\end{array}$ $\begin{array}{lllll}0 & 0 & 0 & 0 & 0 \\ 0 & 0 & 0 & 0 & 0 \\ 0 & 0 & 0 & 0 & 0 \\ 0 & 0 & 0 & 0 & 0 \\ 0 & 0 & 0 & 0 & 0\end{array}$

$\begin{array}{lllll}0 & 0 & 0 & 0 & 0 \\ 0 & 0 & 0 & 0 & 0 \\ 0 & 0 & 0 & 0 & 0 \\ 0 & 0 & 0 & 0 & 0 \\ 0 & 0 & 0 & 0 & 0\end{array}$

0
0
0
0
0

$\begin{array}{llll}0 & 0 & 0 & 0 \\ 0 & 0 & 0 & 0 \\ 0 & 0 & 0 & 0 \\ 0 & 0 & 0 & 0 \\ 0 & 0 & 0 & 0\end{array}$ $\begin{array}{lllll}0 & 0 & 0 & 0 & 0\end{array}$ $\begin{array}{llll}0 & 0 & 0 & 0 \\ 0 & 0 & 0 & 0\end{array}$ $\begin{array}{lllll}0 & 0 & 0 & 0 & 0 \\ 0 & 0 & 0 & 0 & 0\end{array}$

$\begin{array}{lllll}0 & 0 & 0 & 0 & 0 \\ 0 & 0 & 0 & 0 & 0 \\ 0 & 0 & 0 & 0 & 0 \\ 0 & 0 & 0 & 0 & 0 \\ 0 & 0 & 0 & 0 & 0\end{array}$

$\begin{array}{lllll}0 & 0 & 0 & 0 & 0 \\ 0 & 0 & 0 & 0 & 0 \\ 0 & 0 & 0 & 0 & 0 \\ 0 & 0 & 0 & 0 & 0 \\ 0 & 0 & 0 & 0 & 0\end{array}$

$\begin{array}{lllll}0 & 0 & 0 & 0 & 0\end{array}$

$\begin{array}{lllll}0 & 0 & 0 & 0 & 0\end{array}$

$\begin{array}{lllll}0 & 0 & 0 & 0 & 0\end{array}$

$\begin{array}{lllll}0 & 0 & 0 & 0 & 0\end{array}$ 
TABLE 0.4. (contd)

Sediment

Ireatment

$$
\text { Replicate } \frac{N . \text { caecoides on Sediment for Test Days } 1 \text { through } 10}{1}+\frac{3}{4}+\frac{1}{5}+10
$$

R-OS

R-OS

$R-O S$

$\mathrm{R}-\mathrm{OS}$

R-OS

$\begin{array}{ll}1 & \\ 2 & \\ 3 & \\ 4 & \\ 5 & \end{array}$

$R-P C$

$R-P C$

$R-P C$

$R-P C$

$R-P C$

$\begin{array}{llllllllll}0 & 0 & 0 & 0 & 0 & 0 & 0 & 0 & 0 & 0 \\ 0 & 0 & 0 & 0 & 0 & 0 & 0 & 0 & 0 & 0 \\ \text { ND } & 0 & 0 & 0 & 0 & 0 & 0 & 0 & 0 & 0 \\ 0 & 0 & 0 & 0 & 0 & 0 & 0 & 0 & 0 & 0 \\ 0 & 0 & 0 & 0 & 0 & 0 & 0 & 0 & 0 & 0\end{array}$

$\begin{array}{llllllllll}0 & 0 & 0 & 0 & 0 & 0 & 0 & 0 & 0 & 0\end{array}$

$\begin{array}{llllllllll}0 & 0 & 0 & 0 & 0 & 0 & 0 & 0 & 0 & 0\end{array}$

$\begin{array}{llllllllll}0 & 0 & 0 & 0 & 0 & 0 & 0 & 0 & 0 & 0\end{array}$

$\begin{array}{llllllllll}0 & 0 & 0 & 0 & 0 & 0 & 1 & 0 & 1 & 0 \\ 0 & 0 & 0 & 0 & 0 & 0 & 0 & 0 & 0 & 0\end{array}$

$R-P F$
$R-P F$

$R-P F$

$R-P F$

$R-P F$

$C-S B$

C-SB

$C-S B$

$C-S B$

C-SB

C-NE

$C-N E$

$\mathrm{C}-\mathrm{NE}$

$\mathrm{C}-\mathrm{NE}$

C-NE

$\begin{array}{lllllllllll}1 & 0 & 0 & 0 & 0 & 0 & 0 & 0 & 0 & 0 & 0 \\ 2 & 0 & 0 & 0 & 0 & 0 & 0 & 0 & 0 & 0 & 0 \\ 3 & 0 & 0 & 0 & 0 & 0 & 0 & 0 & 0 & 0 & 0 \\ 4 & 0 & 0 & 0 & 0 & 0 & 0 & 1 & 0 & 1 & 0 \\ 5 & 0 & 0 & 0 & 0 & 0 & 0 & 0 & 0 & 0 & 0\end{array}$

$\begin{array}{llllllllll}0 & 0 & 0 & 0 & 0 & 0 & 0 & 0 & 0 & 0\end{array}$

$\begin{array}{llllllllll}0 & 0 & 0 & 0 & 0 & 0 & 0 & 0 & 0 & 0\end{array}$

$\begin{array}{llllllllll}0 & 0 & 0 & 0 & 0 & 0 & 0 & 0 & 0 & 0\end{array}$

$\begin{array}{rlllllllll}\text { ND } & 0 & 0 & 0 & 0 & 0 & 0 & 0 & 0 & 0 \\ 0 & 0 & 0 & 0 & 0 & 0 & 0 & 0 & 0 & 0\end{array}$

$\begin{array}{llllllllll}0 & 0 & 0 & 0 & 0 & 0 & 0 & 0 & 0 & 0\end{array}$

$\begin{array}{llllllllll}0 & 0 & 0 & 0 & 0 & 0 & 0 & 0 & 0 & 0\end{array}$

$\begin{array}{rlllllllll}0 & 0 & 0 & 0 & 0 & 0 & 0 & 0 & 0 & 0 \\ \text { ND } & 0 & 0 & 0 & 0 & 0 & 0 & 0 & 0 & 0\end{array}$

$\begin{array}{llllllllll}0 & 0 & 0 & 0 & 0 & 0 & 0 & 0 & 0 & 0\end{array}$

$\begin{array}{llllllllll}0 & 0 & 0 & 0 & 0 & 0 & 0 & 0 & 0 & 0\end{array}$

$\begin{array}{llllllllll}0 & 0 & 0 & 0 & 0 & 0 & 0 & 0 & 0 & 0\end{array}$

$\begin{array}{rlllllllll}0 & 0 & 0 & 0 & 0 & 0 & 0 & 0 & 0 & 0 \\ \text { ND } & 0 & 0 & 0 & 0 & 0 & 0 & 0 & 0 & 0\end{array}$

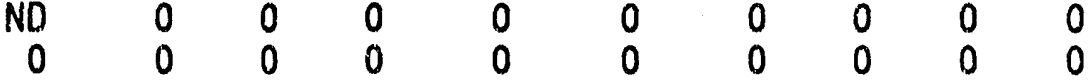

(a) No data-no observation made.

(b) Too turbid to make accurate observation. 
TABLE D.5. Daily Observations of $N$. caecoides Dead and Removed During 10-Day Solid-Phase Test, Oakland Harbor Phase III B Project

Sediment

Ireatment Replicate $1 \quad 2 \quad 3 \quad 4 \quad 5 \quad 6 \quad 1 \quad 8 \quad 96$

$0-\mathrm{C} 13$

$0-\mathrm{C} 13$

$0-\mathrm{C} 13$

$0-\mathrm{C} 13$

$0-\mathrm{C} 13$

$0-\mathrm{C} 12$

$0-\mathrm{C} 12$

$0-\mathrm{C} 12$

$0-\mathrm{C} 12$

$0-\mathrm{C} 12$

$0-C 11$

$0-\mathrm{C} 11$

$0-C 11$

$0-C 11$

$0-C 11$

1
2
3
4
5

$\begin{array}{llll}0 & 0 & 0 & 0 \\ 0 & 0 & 0 & 0 \\ N D^{(a)} & 0 & 0 & 0 \\ N D & 0 & 0 & 0 \\ 0 & 0 & 0 & 0\end{array}$

$\begin{array}{llllll}0 & 0 & 0 & 0 & 0 & 0 \\ 0 & 0 & 0 & 0 & 0 & 0 \\ 0 & 0 & 0 & 0 & 0 & 0 \\ 0 & 0 & 0 & 0 & 0 & 0 \\ 0 & 0 & 0 & 0 & 0 & 0\end{array}$

$0-\mathrm{CIO}$

$0-C 10$

$0-6.10$

$0-\mathrm{C} 10$

$0-\mathrm{C} 10$

1
2
3
4
5

$\begin{array}{llllllllll}0 & 0 & 0 & 0 & 0 & 0 & 0 & 0 & 0 & 0\end{array}$ $\begin{array}{llllllllll}0 & 0 & 0 & 0 & 0 & 0 & 0 & 0 & 0 & 0\end{array}$ $\begin{array}{llllllllll}0 & 0 & 0 & 0 & 0 & 0 & 1 & 0 & 0 & 0\end{array}$ $\begin{array}{llllllllll}0 & 0 & 0 & 0 & 0 & 0 & 0 & 0 & 0 & 0 \\ 0 & 0 & 0 & 0 & 0 & 0 & 0 & 0 & 0 & 0\end{array}$

$0-c 9$

$0-C 9$

$0-c 9$

$0-c 9$

$0-c 9$

$\begin{array}{llllllllll}0 & 0 & 0 & 0 & 0 & 0 & 0 & 0 & 0 & 0 \\ 0 & 0 & 0 & 0 & 0 & 0 & 0 & 0 & 0 & 0 \\ 0 & 0 & 0 & 2 & 0 & 0 & 1 & 0 & 0 & 0 \\ 0 & 0 & 0 & 0 & 0 & 0 & 0 & 0 & 0 & 0 \\ 0 & 0 & 0 & 0 & 0 & 0 & 0 & 0 & 0 & 0\end{array}$

$0-\mathrm{C} 8$

$0-C 8$

$0-C 8$

$0-18$

$0-C 8$

1
2
3
4
5

1
2
3
4
5

$\begin{array}{rl}\text { ND } & 0 \\ \text { ND } & 0 \\ 0 & 0 \\ 0 & 0 \\ 0 & 0\end{array}$

$\begin{array}{llllllll}0 & 0 & 0 & 0 & 0 & 0 & 0 & 0 \\ 0 & 0 & 0 & 0 & 0 & 0 & 0 & 0 \\ 0 & 0 & 0 & 0 & 0 & 0 & 0 & 0 \\ 0 & 0 & 0 & 0 & 0 & 0 & 0 & 0 \\ 0 & 0 & 0 & 0 & 1 & 0 & 0 & 0\end{array}$

$0-\mathrm{Cl}$

$0-C 7$

$0-C 7$

$0-67$

$0-C 7$

$\begin{array}{rlllllllll}0 & 0 & 0 & 0 & 0 & 0 & 0 & 0 & 0 & 0 \\ 0 & 0 & 0 & 0 & 0 & 0 & 0 & 0 & 0 & 0 \\ 0 & 0 & 0 & 0 & 0 & 0 & 0 & 0 & 0 & 0 \\ \text { ND } & 0 & 0 & 0 & 0 & 0 & 0 & 0 & 0 & 0 \\ 0 & 0 & 0 & 0 & 0 & 0 & 0 & 0 & 0 & 0\end{array}$

$\begin{array}{rlllllllll}\text { ND } & 0 & 0 & 1 & 0 & 0 & 0 & 0 & 0 & 0 \\ 0 & 0 & 0 & 1 & 0 & 0 & 0 & 0 & 0 & 0 \\ \text { ND } & 0 & 0 & 1 & 0 & 0 & 0 & 0 & 0 & 0 \\ 0 & 0 & 0 & 1 & 0 & 0 & 0 & 0 & 0 & 0 \\ 0 & 0 & 0 & 0 & 0 & 0 & 0 & 0 & 0 & 0\end{array}$

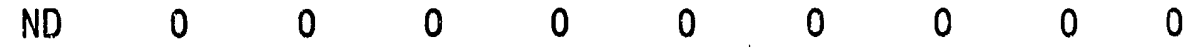
$\begin{array}{llllllllll}0 & 0 & 0 & 0 & 0 & 0 & 0 & 0 & 0 & 0\end{array}$ $\begin{array}{llllllllll}0 & 0 & 0 & 0 & 0 & 0 & 0 & 0 & 0 & 0\end{array}$ $\begin{array}{llllllllll}0 & 0 & 0 & 1 & 0 & 0 & 0 & 0 & 0 & 0 \\ 0 & 0 & 0 & 0 & 0 & 0 & 0 & 0 & 0 & 0\end{array}$ 
IABLE D.5. (contd)

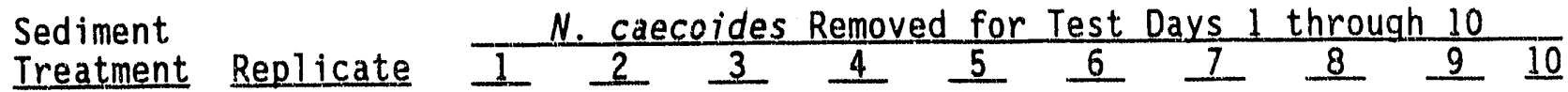

$\begin{array}{llllllllllll}0-C 6 & 1 & 0 & 0 & 0 & 0 & 0 & 0 & 0 & 0 & 0 & 0 \\ 0-C 6 & 2 & 0 & 0 & 0 & 0 & 0 & 0 & 0 & 0 & 0 & 0 \\ 0-C 6 & 3 & 0 & 0 & 0 & 0 & 0 & 0 & 0 & 0 & 0 & 0 \\ 0-C 6 & 4 & 0 & 0 & 0 & 0 & 0 & 0 & 0 & 0 & 0 & 0 \\ 0-C 6 & 5 & 0 & 0 & 0 & 0 & 0 & 0 & 0 & 0 & 0 & 0\end{array}$

$\begin{array}{llllllllllll}0-C 5 & 1 & 0 & 0 & 0 & 0 & 0 & 0 & 0 & 0 & 0 & 0 \\ 0-C 5 & 2 & 0 & 0 & 0 & 0 & 0 & 0 & 0 & 0 & 0 & 0 \\ 0-C 5 & 3 & 0 & 0 & 0 & 0 & 0 & 0 & 0 & 0 & 0 & 0 \\ 0-C 5 & 4 & 0 & 0 & 0 & 0 & 0 & 0 & 0 & 0 & 0 & 0 \\ 0-C 5 & 5 & 0 & 0 & 0 & 0 & 0 & 0 & 0 & 0 & 0 & 0\end{array}$

$\begin{array}{llllllllllll}0-C 4 & 1 & 0 & 0 & 0 & 0 & 0 & 0 & 0 & 0 & 0 & 0 \\ 0-C 4 & 2 & 0 & 0 & 0 & 0 & 1 & 0 & 1 & 0 & 0 & 0 \\ 0-C 4 & 3 & 0 & 0 & 0 & 0 & 0 & 0 & 0 & 0 & 0 & 0 \\ 0-C 4 & 4 & 0 & 0 & 0 & 0 & 0 & 0 & 0 & 0 & 0 & 0 \\ 0-C 4 & 5 & 0 & 0 & 0 & 0 & 0 & 0 & 0 & 0 & 0 & 0\end{array}$

$\begin{array}{llllllllllll}0-C 3 & 1 & 0 & 0 & 0 & 0 & 0 & 0 & 0 & 0 & 0 & 0 \\ 0-C 3 & 2 & 0 & 0 & 0 & 0 & 0 & 0 & 0 & 0 & 0 & 0 \\ 0-C 3 & 3 & 0 & 0 & 0 & 0 & 0 & 0 & 0 & 0 & 0 & 0 \\ 0-C 3 & 4 & 0 & 0 & 0 & 0 & 0 & 0 & 0 & 0 & 0 & 0 \\ 0-C 3 & 5 & 0 & 0 & 0 & 0 & 0 & 0 & 0 & 0 & 0 & 0\end{array}$

$0-\mathrm{C} 2$
$0-\mathrm{C} 2$

$0-\mathrm{C2}$

$0-\mathrm{C} 2$

$0-C 2$

1
2
3
4
5

$\begin{array}{llllllllll}0 & 0 & 0 & 0 & 0 & 0 & 0 & 0 & 0 & 0 \\ 0 & 0 & 0 & 0 & 0 & 0 & 0 & 0 & 0 & 0 \\ 0 & 0 & 0 & 0 & 0 & 0 & 0 & 0 & 0 & 0 \\ 0 & 0 & 0 & 0 & 0 & 0 & 0 & 0 & 0 & 0 \\ 0 & 0 & 0 & 0 & 0 & 0 & 0 & 0 & 0 & 0\end{array}$

$0-C 1$

$0-C 1$

$\mathrm{O}-\mathrm{Cl}$

$0-C 1$

$0-\mathrm{Cl}$

I $-\mathrm{C} 2$

I $-\mathrm{C2}$

I-C2

$\mathrm{I}-\mathrm{C2}$

I-C?

$\begin{array}{llll}0 & 1 & 0 & 0\end{array}$

$\begin{array}{llllllllll}0 & 0 & 0 & 0 & 0 & 0 & 0 & 0 & 0 & 0\end{array}$

$\begin{array}{lllllllllll}2 & 0 & 0 & 0 & 0 & 0 & 0 & 0 & 0 & 0 & 0 \\ 3 & 0 & 0 & 0 & 0 & 0 & 0 & 0 & 0 & 0 & 0\end{array}$

$\begin{array}{lllllllllll}4 & 0 & 0 & 0 & 0 & 0 & 0 & 0 & 0 & 0 & 0 \\ 5 & 0 & 0 & 0 & 0 & 0 & 0 & 0 & 0 & 0 & 0\end{array}$

$\begin{array}{lllllllllll}1 & 0 & 0 & 0 & 0 & 0 & 0 & 0 & 0 & 0 & 0 \\ 2 & 0 & 0 & 0 & 0 & 0 & 0 & 0 & 0 & 0 & 0 \\ 3 & 0 & 0 & 0 & 0 & 0 & 0 & 0 & 0 & 0 & 0 \\ 4 & 0 & 0 & 0 & 0 & 0 & 0 & 0 & 0 & 0 & 0 \\ 5 & 0 & 0 & 0 & 0 & 0 & 0 & 0 & 0 & 0 & 0\end{array}$

PHASE III B

D. 12 
IABLE D.5. (contd)

Sediment

Treatment

I-C3

I $-\mathrm{C} 3$

I-C3

$\mathrm{I}-\mathrm{C} 3$

I $-\mathrm{C.3}$

I $-\mathrm{C}, 4$

I $-\mathrm{C} 4$

I-C4

I $-\mathrm{C4}$

I-C4

I $-\mathrm{CB}$

I $-\mathrm{C} 8$

I $-\mathrm{C} 8$

I $-\mathrm{C} 8$

I-C8

I $-\mathrm{T} 5$

I $-T 5$

I-T5

I-T5

I-T5

$R-A C$

$R-A C$

$\mathrm{R}-\mathrm{AC}$

$R-A C$

$R-A C$

$R-A M$

R-AM

R-AM

$R-A M$

R-AM

$R-B F$

$R-B F$

$R-B F$

$R-B F$

$R-B F$

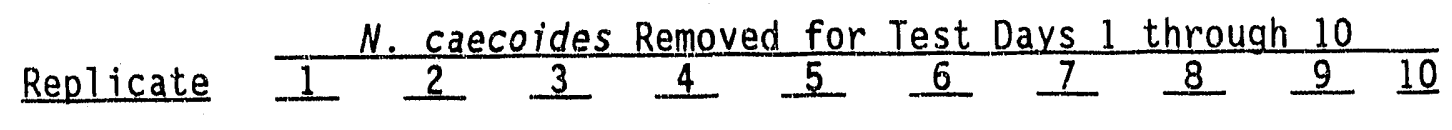

1
2
3
4
5

$\begin{array}{ll}0 & 0 \\ 0 & 0 \\ 0 & 0 \\ 0 & 0 \\ 0 & 0\end{array}$

$\begin{array}{llllllll}0 & 0 & 0 & 0 & 0 & 0 & 0 & 0 \\ 0 & 0 & 0 & 0 & 0 & 0 & 0 & 0 \\ 1 & 0 & 0 & 0 & 0 & 0 & 0 & 0 \\ 0 & 0 & 0 & 0 & 0 & 0 & 0 & 0 \\ 0 & 0 & 0 & 0 & 0 & 0 & 0 & 0\end{array}$

$\begin{array}{llllllllll}0 & 0 & 0 & 0 & 0 & 0 & 0 & 0 & 0 & 0 \\ 0 & 0 & 0 & 0 & 0 & 0 & 0 & 0 & 0 & 0 \\ 0 & 0 & 0 & 0 & 1 & 0 & 0 & 0 & 0 & 0 \\ 0 & 0 & 0 & 0 & 0 & 0 & 0 & 0 & 0 & 0 \\ 0 & 0 & 0 & 0 & 0 & 0 & 0 & 0 & 0 & 0\end{array}$

$\begin{array}{llllllllll}0 & 0 & 0 & 0 & 0 & 0 & 0 & 0 & 0 & 0 \\ 0 & 0 & 0 & 0 & 1 & 0 & 0 & 0 & 0 & 0 \\ 0 & 0 & 0 & 0 & 1 & 0 & 0 & 0 & 0 & 0 \\ 0 & 0 & 0 & 0 & 0 & 0 & 0 & 0 & 0 & 0 \\ 0 & 0 & 0 & 0 & 0 & 0 & 0 & 0 & 0 & 0\end{array}$

$\begin{array}{rlllllllll}0 & 0 & 0 & 0 & 1 & 0 & 0 & 0 & 0 & 0 \\ \text { ND } & 0 & 0 & 0 & 0 & 0 & 0 & 0 & 0 & 0 \\ 0 & 0 & 0 & 0 & 0 & 0 & 0 & 0 & 0 & 0 \\ 0 & 0 & 0 & 0 & 0 & 0 & 0 & 0 & 0 & 0 \\ \text { ND } & 0 & 0 & 0 & 0 & 0 & 0 & 0 & 0 & 0\end{array}$

$\begin{array}{llllllllll}0 & 0 & 0 & 0 & 0 & 0 & 0 & 0 & 0 & 0\end{array}$

$\begin{array}{llllllllll}0 & 0 & 0 & 0 & 0 & 0 & 0 & 0 & 0 & 0 \\ 0 & 0 & 0 & 0 & 0 & 0 & 0 & 0 & 0 & 0\end{array}$

$\begin{array}{llllllllll}0 & 0 & 0 & 0 & 0 & 0 & 0 & 0 & 0 & 0 \\ 0 & 0 & 0 & 0 & 0 & 0 & 0 & 0 & 0 & 0\end{array}$

$\begin{array}{llllllllll}0 & 0 & 0 & 0 & 0 & 0 & 0 & 0 & 0 & 0 \\ 0 & 0 & 0 & 0 & 0 & 0 & 0 & 0 & 0 & 0\end{array}$

$\begin{array}{rlllllllll}\text { ND } & 0 & 0 & 0 & 0 & 0 & 0 & 0 & 0 & 0 \\ \text { ND } & 0 & 0 & 0 & 0 & 0 & 0 & 0 & 0 & 0 \\ 0 & 0 & 0 & 0 & 0 & 0 & 0 & 0 & 0 & 0 \\ 0 & 0 & 0 & 0 & 0 & 0 & 0 & 0 & 0 & 0 \\ 0 & 0 & 0 & 0 & 0 & 0 & 0 & 0 & 0 & 0\end{array}$

$\begin{array}{rlllllllll}0 & 0 & 0 & 0 & 0 & 0 & 0 & 0 & 0 & 0 \\ 0 & 0 & 0 & 0 & 0 & 0 & 0 & 0 & 0 & 0 \\ 0 & 0 & 0 & 0 & 0 & 0 & 0 & 0 & 0 & 0 \\ \text { ND } & 0 & 0 & 0 & 0 & 0 & 0 & 0 & 0 & 0 \\ 0 & 0 & 0 & 0 & 0 & 0 & 0 & 0 & 0 & 0\end{array}$ 
TABLE D.5. (contd)

Sodiment

Treatment

R-OS

R-OS

R-OS

R-OS

R-OS

$R-P C$

$R-P C$

$R-P C$

$R-P C$

$R-P C$

$R-P F$

$R-P F$

$R-P F$

$R-P F$

R-PF

C-SB

$C-S B$

$C-S B$

$C-S B$

C-SB

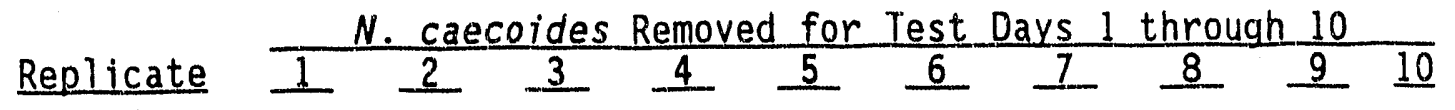

C-NE

C-NE

C-NE

C-NE

C-NE

1
2
3
4
5

$\begin{array}{rlllllllll}0 & 0 & 0 & 0 & 0 & 0 & 0 & 0 & 0 & 0 \\ 0 & 0 & 0 & 0 & 0 & 0 & 0 & 0 & 0 & 0 \\ \text { ND } & 0 & 0 & 0 & 0 & 0 & 0 & 0 & 0 & 0 \\ 0 & 0 & 0 & 0 & 0 & 0 & 0 & 0 & 0 & 0 \\ 0 & 0 & 0 & 0 & 0 & 0 & 0 & 0 & 0 & 0\end{array}$

$\begin{array}{lllllllllll}1 & 0 & 0 & 0 & 0 & 0 & 0 & 0 & 0 & 0 & 0 \\ 2 & 0 & 0 & 0 & 0 & 0 & 0 & 0 & 0 & 0 & 0 \\ 3 & 0 & 0 & 0 & 0 & 0 & 0 & 0 & 0 & 0 & 0 \\ 4 & 0 & 0 & 0 & 0 & 0 & 0 & 0 & 0 & 1 & 0 \\ 5 & 0 & 0 & 0 & 0 & 0 & 0 & 0 & 0 & 0 & 0\end{array}$

$\begin{array}{lrlllllllll}1 & 0 & 0 & 0 & 0 & 0 & 0 & 0 & 0 & 0 & 0 \\ 2 & 0 & 0 & 0 & 0 & 0 & 0 & 0 & 0 & 0 & 0 \\ 3 & 0 & 0 & 0 & 0 & 0 & 0 & 0 & 0 & 0 & 0 \\ 4 & \text { ND } & 0 & 0 & 0 & 0 & 0 & 0 & 0 & 0 & 0 \\ 5 & 0 & 0 & 0 & 0 & 0 & 0 & 0 & 0 & 0 & 0\end{array}$

$\begin{array}{lrlllllllll}1 & 0 & 0 & 0 & 0 & 0 & 0 & 0 & 0 & 0 & 0 \\ 2 & 0 & 0 & 0 & 0 & 0 & 0 & 0 & 0 & 0 & 0 \\ 3 & 0 & 0 & 0 & 0 & 0 & 0 & 0 & 0 & 0 & 0 \\ 4 & \text { ND } & 0 & 0 & 0 & 0 & 0 & 0 & 0 & 0 & 0 \\ 5 & 0 & 0 & 0 & 0 & 0 & 0 & 0 & 0 & 0 & 0\end{array}$

(a) No data--no observation made. 


\section{APPENDIX E}

\section{8-DAY ELOW-THROUGH SOLID-PHASE TEST WTTH}

N. CAECOIDES AND $M$. NASUTA 
TABLE E.1. Test Results for 28-Day M. nasuta Solid-Phase Test, Oakland Harbor Phase III B Project

Sediment

Treatment

$0-C 13$

$0-C 13$

$0-\mathrm{C} 13$

$0-\mathrm{C} 13$

$0-C 13$

$0-C 12$

$0-\mathrm{C} 12$

$0-\mathrm{C} 12$

$0-\mathrm{C} 12$

$0-\mathrm{C} 12$

$0-C 11$

$0-C 11$

$0-C 11$

$0-C 11$

$0-C 11$

$0-C 10$

$0-C 10$

$0-C 10$

$0-C 10$

$0-C 10$

$0-c 9$

$0-c 9$

$0-C 9$

$0-C 9$

$0-C 9$

$0-c 8$

$0-C 8$

$0-C 8$

$0-c 8$

$0-C 8$

$0-C 7$

$0-C 7$

$0-C 7$

$0-c 7$

$0-C 7$
Replicate

1
2
3
4
5

1

2

3

4

5

1
2
3
4
5

1
2
3
4
5

1
2
3
4
5

1
2
3
4
5

1
2
3
4
5

\begin{tabular}{cr} 
& M. nasuta \\
\hline Live & or Mis \\
24 & \\
25 & \\
25 & \\
23 & \\
23 &
\end{tabular}

23

24

25

24

24

24

24

25

25

24

Mean

Proportion

Surviving

1
0
0
2
2

0.96

0.96

24

23

24

25

24

24

24

24

25

24

24

25

25

24

23

23

25

24

25

25
0.96

0.97

0.97

0.98

$$
\begin{aligned}
& 1 \\
& 2 \\
& 1 \\
& 0 \\
& 1
\end{aligned}
$$

1
1
1
0
1

1

0

1

0.98 
TABLE E.1. (contd)

Sediment

Treatment

$0-C 6$

$0-C 6$

$0-C 6$

$0-C 6$

$0-\mathrm{C} 6$

$0-C 5$

$0-C 5$

$0-C 5$

$0-C 5$

$0-C 5$

$0-C 4$

$0-C 4$

$0-C 4$

$0-C 4$

$0-C 4$

$0-C 3$

$0-\mathrm{C} 3$

$0-C 3$

$0-\mathrm{C3}$

$0-\mathrm{C3}$

$0-C 2$

$0-C 2$

$0-\mathrm{C} 2$

$0-c 2$

$0-C 2$

$0-C 1$

0-C1

$0-C 1$

$0-\mathrm{Cl}$

$0-\mathrm{Cl}$

I-C2

$\mathrm{I}-\mathrm{C} 2$

$\mathrm{I}-\mathrm{C} 2$

I-C2

$\mathrm{I}-\mathrm{C} 2$

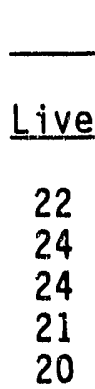

M. nasuta

\section{Replicate}

1
2
3
4
5

1

2

3

4
5

\section{1}

1

3

4

1
2
3
4
5

1
2
3
4
5

1

2

3

4
5

$\begin{array}{ll}1 & 25 \\ 2 & 26 \\ 3 & 25 \\ 4 & 25 \\ 5 & 24\end{array}$

25

23

23

25

25

25

25

23

25

24

24

25

\section{6}

25

25

25

25

25

24

25

25

25

25

26
Mean

Proportion

Surviving

or Missing

0.89

3
1
1
4
5

0

2

2
0

0

0.97

0.98

0

0

2

0
0.98

0.99

1.00

1
1
0
0
0

0

0

0

1

0

0

0

0

0

0

0

0

0.99 
TABLE E.1. (contd)

Sediment

Treatment

I $-\mathrm{C} 3$

$\mathrm{I}-\mathrm{C} 3$

I $-\mathrm{C} 3$

$\mathrm{I}-\mathrm{C} 3$

$\mathrm{I}-\mathrm{C} 3$

I $-\mathrm{C} 8$

I $-\mathrm{CB}$

I $-\mathrm{C} 8$

$\mathrm{I}-\mathrm{C} 8$

I-C8

I $-\mathrm{C} 4$

$\mathrm{I}-\mathrm{C4}$

I $-\mathrm{C} 4$

I $-\mathrm{C} 4$

$\mathrm{I}-\mathrm{C} 4$

$1-T 5$

$I-T 5$

I $-\mathrm{T} 5$

I-T5

I-T5

$R-A C$

$R-A C$

$R-A C$

$R-A C$

$R-A C$

R-AM

$R-A M$

$R-A M$

$R-A M$

R-AM

$R-B F$

$R-B F$

$R-B F$

$R-B F$

$R-B F$

\begin{tabular}{cr} 
& M. nasuta \\
\hline & Dea \\
Live & or Mis \\
25 & 0 \\
25 & 0 \\
25 & 0 \\
26 & 0 \\
25 &
\end{tabular}

25

23

25

25

23

25

24

24

24

25

25

25

24

24

25

25

24

25

25

24

$24 \quad 1$

25

25

25

25

23
25
25
23
25
0.98

0.98

0.98

0.99

Mean

Proportion

Surviving

1.00

0.97

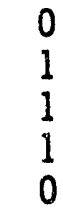

0

0

0

0

1

0

1

0

0

2

0

0

2

0.97 
IABLE E.1. (contd)

Sediment

Ireatment

R-OS

$R-O S$

R-OS

$\mathrm{R}-\mathrm{OS}$

R-OS

$R-P C$

$R-P C$

$R-P C$

$R-P C$

$R-P C$

$R-P F$

$R-P F$

$R-P F$

$R-P F$

$R-P F$

C-SB

$C-S B$

$C-S B$

$C-S B$

C-SB

C-NE

$C-N E$

$\mathrm{C}-\mathrm{NE}$

C. -NE

$\mathrm{C}-\mathrm{NE}$

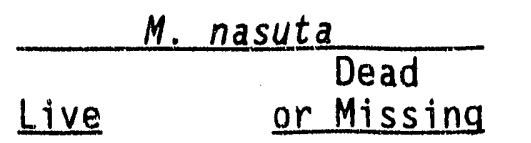

Replicate

1

2

3

4

5

1

2

3

4

5

1

2

5

1

2

4

5

1
2
3
4
5
25

25

24

24

Mean

Proportion

Surviving

232

$\begin{array}{ll}24 & 1 \\ 25 & 0\end{array}$

23

25

25
22

23

23

25

24

24

25

25

24

25

25

25

25

24
0.99

0.98

0.96

0.94

0.98

0

0
0

0
1

0

0
0

1 
IABLE E.2. Rank Order Based on Mean Proportion Surviving 28-Day M. nasuta Solid-Phase Test, Dakland Harbor Phase III B Project

Sediment

Treatment

$0-C 6$

$R-P C$

$0-C 10$

$0 . C 12$

$0-C 13$

$R-O S$

I-C8

$0-C 5$

$0-C 8$

$0-C 9$

$\mathrm{R}-\mathrm{BF}$

$\mathrm{I}-\mathrm{C} 4$

$0-C 11$

$0-C_{4}$

$0-C 7$

$R=P F$

$C-N E$

$I-T 5$

$R-A C$

$0-C 3$

C-SB

$0-C 2$

R-AM

$I-C 2$

$1-\mathrm{C} 3$

$0-C 1$
Mean

Proportion

Surviving

0.89

0.94

0.96

0.96

0.96

0.96

0.97

0.97

0.97

0.97

0.97

0.98

0.98

0.98

0.98

0.98

0.98

0.98

0.98

0.98

0.99

0.99

0.99

0.99

1.00

1.00 
TABLEE.3. Test Results for 28-Day N. caecoides Solid-Phiase Test, Oakl and Harbor Phase III B Project

\begin{tabular}{|c|c|c|c|c|}
\hline \multirow[b]{2}{*}{$\begin{array}{l}\text { Sediment } \\
\text { Treatment }\end{array}$} & \multirow[b]{2}{*}{ Replicate } & \multicolumn{2}{|c|}{ N. caecoides } & \multirow{2}{*}{$\begin{array}{c}\text { Mean } \\
\text { Proportion } \\
\text { Surviving } \\
\end{array}$} \\
\hline & & Live & $\begin{array}{c}\text { Dead } \\
\text { or Missing }\end{array}$ & \\
\hline $\begin{array}{l}0-C 13 \\
0-C 13 \\
0-C 13 \\
0-C 13 \\
0-C 13\end{array}$ & $\begin{array}{l}1 \\
2 \\
3 \\
4 \\
5\end{array}$ & $\begin{array}{l}24 \\
25 \\
29 \\
25 \\
26\end{array}$ & $\begin{array}{l}6 \\
5 \\
1 \\
5 \\
4\end{array}$ & 0.86 \\
\hline $\begin{array}{l}0-C 12 \\
0-C 12 \\
0-C 12 \\
0-C 12 \\
0-C 12\end{array}$ & $\begin{array}{l}1 \\
2 \\
3 \\
4 \\
5\end{array}$ & $\begin{array}{l}25 \\
20 \\
25 \\
28 \\
29\end{array}$ & $\begin{array}{r}5 \\
10 \\
5 \\
2 \\
1\end{array}$ & 0.85 \\
\hline $\begin{array}{l}0-C 11 \\
0-C 11 \\
0-C 11 \\
0-C 11 \\
0-C 11\end{array}$ & $\begin{array}{l}1 \\
2 \\
3 \\
4 \\
5\end{array}$ & $\begin{array}{l}25 \\
27 \\
29 \\
26 \\
26\end{array}$ & $\begin{array}{l}5 \\
3 \\
1 \\
4 \\
4\end{array}$ & 0.89 \\
\hline $\begin{array}{l}0-C 10 \\
0-C 10 \\
0-C 10 \\
0-C 10 \\
0-C 10\end{array}$ & $\begin{array}{l}1 \\
2 \\
3 \\
4 \\
5\end{array}$ & $\begin{array}{l}28 \\
28 \\
26 \\
25 \\
29\end{array}$ & $\begin{array}{l}2 \\
c \\
4 \\
5 \\
1\end{array}$ & 0.91 \\
\hline $\begin{array}{l}0-c 9 \\
0-c 9 \\
0-c 9 \\
0-c 9 \\
0-c 9\end{array}$ & $\begin{array}{l}1 \\
2 \\
3 \\
4 \\
5\end{array}$ & $\begin{array}{l}28 \\
28 \\
28 \\
28 \\
28\end{array}$ & $\begin{array}{l}2 \\
2 \\
2 \\
2 \\
2\end{array}$ & 0.93 \\
\hline $\begin{array}{l}0-c 8 \\
0-c 8 \\
0-c 8 \\
0-c 8 \\
0-c 8\end{array}$ & $\begin{array}{l}1 \\
2 \\
3 \\
4 \\
5\end{array}$ & $\begin{array}{l}29 \\
29 \\
27 \\
27 \\
20\end{array}$ & $\begin{array}{r}1 \\
1 \\
3 \\
3 \\
10\end{array}$ & 0.88 \\
\hline $\begin{array}{l}0-C 7 \\
0-C 7 \\
0-C 7 \\
0-C 7 \\
0-C 7\end{array}$ & $\begin{array}{l}1 \\
2 \\
3 \\
4 \\
5\end{array}$ & $\begin{array}{l}22 \\
30 \\
29 \\
29 \\
28\end{array}$ & $\begin{array}{l}8 \\
0 \\
1 \\
1 \\
2\end{array}$ & 0.92 \\
\hline PHASE III B & & E.6 & & \\
\hline
\end{tabular}


TABLE E.3. (contd)

Sediment

Treatment

$0-\mathrm{C} 6$

$0-\mathrm{C} 6$

$0-C 6$

$0-\mathrm{C} 6$

$0-\mathrm{C} 6$

$0-C 5$

$0-C 5$

$0-C 5$

$0-C 5$

$0-C 5$

$0-C 4$

$0-C 4$

$0-C 4$

$0-C_{4}$

$0-C_{4}$

$0-C 3$

$0-C 3$

$0-\mathrm{C} 3$

$0-C 3$

$0-C 3$

$0-\mathrm{C2}$

$0-\mathrm{C2}$

$0-\mathrm{C2}$

$0-C 2$

$\mathrm{O}-\mathrm{C2}$

$\mathrm{O}-\mathrm{Cl}$

$0-C 1$

$0-C 1$

$0-C 1$

$\mathrm{O}-\mathrm{Cl}$

IC -2

IC -2

IC- 2

IC -2

IC-2

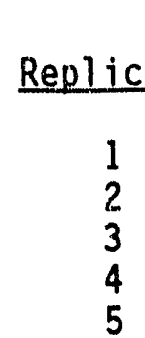

1
2
3
4
5

1

3

4

5

1

2

3

4
5

1

2

3

4
5

1

2

3

4
5

1
2
3
4
5

1
2
3
4
5

$N$.
Live
28
30
28
25
29

27

27

29

28

28

29

28

30

28

25

30

27

27

26

28

28

23

30

28

23

26

28

30

24

26

30

27

26

28

29
Mean

Proportion

Surviving

0.93

0.93

0.93

0.92

0.88

0.89

0.93

PHASE III B

E.7 
TABLE E.3. (contd)

Sediment

Treatment

IC -3

IC -3

IC -3

IC -3

IC -3

IC -8

IC -8

IC -8

IC -8

IC -8

IC -4

IC -4

IC -4

IC -4

IC -4

IT -5

IT -5

IT -5

IT -5

IT -5

$R-A C$

$R-A C$

$R-A C$

$R-A C$

$R-A C$

R-AM

$R-A M$

R-AM

$R-A M$

R-AM

$R-B F$

$R-B F$

$R-B F$

$R-B F$

$R-B F$

\begin{tabular}{ll}
\multicolumn{2}{c}{ N. Caecoides } \\
Live & or Mi \\
29 & \\
29 & \\
30 & \\
30 & \\
29 &
\end{tabular}

Mean

Proportion

Surviving

16

29

30

27

28

28

30

28

30

23

30

26

28

26

29

28

26

30

30

28

30

30

28

29

30

30

28

29

25

30
0.87

0.95

0.98

1
1
0
0
1

0.98

14

1

3

2

0

2

0

0.93

0.93

2

4

0

2

0

0

0

1

0.95

PHASE III B

E. 8 
IABLE E.3. (contd)

Sediment

Trea:ment

R-OS

R-OS

R-OS

R-OS

R-OS

$R-P C$

$R-P C$

$R-P C$

$R-P C$

$R-P C$

$R-P F$

$R-P F$

$R-P F$

$R-P F$

$R-P F$

$C-S B$

C-SB

C-SB

$C-S B$

C-SB

$C-N E$

$C-N E$

C-NE

$C-N E$

C-NE

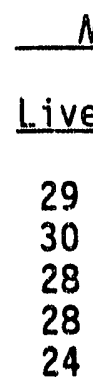

\section{Replicate}

1

2

4

5

1

2

4

5

1

2

3

4
5

30

1

2

3

5

1
2
3
4
5
N. caecoides Dead

or Missing

1

2

2

29

29

30

29

30

29

28

30

30

30

27

25

29

29

29

29

30

29

1
1
0
1
0

1

0

0
Mean Proportion

Surviving

0.93

0.98

0.98

0.93

0.97

E. 9 
TABLE E.4. Rank Order Based on Mean Proportion Surviving 28-Day N. caecoides Solid-Phase Test, Oakl and Harbor Phase III B Project

\section{Sediment \\ Treatment}

$0-C 12$

$0-\mathrm{C} 13$

$\mathrm{I}-\mathrm{C} 8$

$0-\mathrm{C8}$

$0-\mathrm{C2}$

$0-C 11$

$0-\mathrm{ClO}$

$\mathrm{O}-\mathrm{Cl}$

$0-\mathrm{C} 3$

$0-\mathrm{C7}$

$\mathrm{I}-\mathrm{C} 4$

$\mathrm{I}-\mathrm{T} 5$

$0-C 5$

R-OS

C-SB

I $-\mathrm{C} 2$

$0-C 4$

$0-C 6$

O-C9

$R-A C$

$R-B F$

$C-N E$

$I-C 3$

$R-P C$

$R-P F$

$R-A M$
Mean

Proportion

Surviving

0.85

0.86

0.87

0.88

0.89

0.89

0.91

0.92

0.92

0.92

0.93

0.93

0.93

0.93

0.93

0.93

0.93

0.93

0.93

0.95

0.95

0.97

0.98

0.98

0.98

0.99 
IABLEE.5. Water Quality Summary for 28-Day M. nasuta/N. caecoides Solid-Phase Test, Oakl and Harbor Phase III B Project

\begin{tabular}{|c|c|c|c|c|c|c|c|c|c|c|}
\hline \multirow{2}{*}{$\begin{array}{l}\text { Sediment } \\
\text { Treatment } \\
\end{array}$} & \multicolumn{2}{|c|}{$\begin{array}{c}\text { Temperature } \\
\left({ }^{\circ} \mathrm{C}\right)\end{array}$} & \multicolumn{2}{|c|}{$\mathrm{pH}$} & \multicolumn{2}{|c|}{$\begin{array}{l}\text { Dissolved } \\
\text { Oxygen } \\
\text { (mg/L) }\end{array}$} & \multicolumn{2}{|c|}{$\begin{array}{c}\text { Salinity } \\
(0 / 00)\end{array}$} & \multicolumn{2}{|c|}{$\begin{array}{c}\text { Flow Rates } \\
(\mathrm{mL} / \mathrm{min})\end{array}$} \\
\hline & Min & Max & Min & $\operatorname{Max}$ & Min & Max & Min & $\operatorname{Max}$ & $\mathrm{Min}$ & $\operatorname{Max}$ \\
\hline $\begin{array}{l}\text { Acceptable } \\
\text { Range }\end{array}$ & 13.0 & 17.0 & 7.34 & 8.34 & 4.0 & $N A(a)$ & 28.6 & 32.6 & 115 & 135 \\
\hline $\begin{array}{l}0-C 13 \\
0-C 12 \\
0-C 11 \\
0-C 10 \\
0-C 9 \\
0-C 8 \\
0-C 7 \\
0-C 6 \\
0-C 5 \\
0-C 4 \\
0-C 3 \\
0-C 2 \\
0-C 1\end{array}$ & $\begin{array}{l}13.6 \\
13.6 \\
11.8 \\
13.5 \\
13.4 \\
13.0 \\
13.0 \\
13.6 \\
13.0 \\
11.9 \\
13.6 \\
13.5 \\
13.4\end{array}$ & $\begin{array}{l}16.3 \\
16.4 \\
16.3 \\
16.4 \\
16.3 \\
16.4 \\
16.2 \\
16.2 \\
16.3 \\
16.4 \\
16.2 \\
16.4 \\
16.3\end{array}$ & $\begin{array}{l}7.54 \\
7.58 \\
7.57 \\
7.44 \\
7.14 \\
7.52 \\
7.51 \\
7.39 \\
7.62 \\
7.57 \\
7.18 \\
7.61 \\
7.03\end{array}$ & $\begin{array}{l}7.95 \\
7.97 \\
7.92 \\
7.93 \\
7.99 \\
7.94 \\
7.92 \\
7.91 \\
7.95 \\
7.99 \\
8.05 \\
8.06 \\
8.07\end{array}$ & $\begin{array}{l}6.9 \\
7.0 \\
6.4 \\
7.0 \\
7.3 \\
6.0 \\
7.0 \\
6.9 \\
7.0 \\
6.6 \\
7.0 \\
6.4 \\
7.1\end{array}$ & $\begin{array}{l}8.4 \\
8.4 \\
8.4 \\
8.4 \\
8.3 \\
8.4 \\
8.1 \\
8.2 \\
8.6 \\
8.4 \\
8.3 \\
8.3 \\
8.4\end{array}$ & $\begin{array}{l}29.5 \\
30.0 \\
30.0 \\
30.0 \\
29.5 \\
30.0 \\
30.0 \\
30.0 \\
30.0 \\
30.0 \\
30.0 \\
29.5 \\
29.5\end{array}$ & $\begin{array}{l}32.0 \\
32.0 \\
32.0 \\
32.0 \\
32.0 \\
32.0 \\
32.0 \\
32.0 \\
32.0 \\
32.0 \\
32.0 \\
32.0 \\
32.0\end{array}$ & $\begin{array}{l}116 \\
116 \\
114 \\
116 \\
114 \\
88(b) \\
116 \\
116 \\
116 \\
116 \\
116 \\
116 \\
116\end{array}$ & $\begin{array}{l}134 \\
134 \\
132 \\
132 \\
132 \\
132 \\
135 \\
135 \\
132 \\
132 \\
134 \\
132 \\
136(b)\end{array}$ \\
\hline $\begin{array}{l}I-C 2 \\
I-C 3 \\
I-C 8 \\
I-C 4 \\
I-T 5\end{array}$ & $\begin{array}{l}13.6 \\
13.6 \\
13.5 \\
13.4 \\
13.5\end{array}$ & $\begin{array}{l}16.3 \\
16.4 \\
16.3 \\
16.3 \\
16.1\end{array}$ & $\begin{array}{l}7.57 \\
7.47 \\
7.06 \\
7.64 \\
7.63\end{array}$ & $\begin{array}{l}8.05 \\
8.07 \\
8.07 \\
7.97 \\
7.98\end{array}$ & $\begin{array}{l}7.1 \\
7.0 \\
6.4 \\
7.1 \\
6.8\end{array}$ & $\begin{array}{l}8.3 \\
8.2 \\
8.4 \\
8.3 \\
8.4\end{array}$ & $\begin{array}{l}29.5 \\
29.5 \\
30.0 \\
29.5 \\
29.5\end{array}$ & $\begin{array}{l}32.0 \\
32.0 \\
32.0 \\
32.0 \\
32.0\end{array}$ & $\begin{array}{l}116 \\
116 \\
116 \\
116 \\
112(b)\end{array}$ & $\begin{array}{l}132 \\
132 \\
134 \\
138 \\
132\end{array}$ \\
\hline $\begin{array}{l}R-A C \\
R-A M \\
R-B F \\
R-O S \\
R-P C \\
R-P F\end{array}$ & $\begin{array}{l}13.2 \\
13.6 \\
13.4 \\
13.1 \\
13.4 \\
13.3\end{array}$ & $\begin{array}{l}16.6 \\
16.4 \\
16.3 \\
16.5 \\
16.6 \\
16.6\end{array}$ & $\begin{array}{l}7.30 \\
7.68 \\
7.67 \\
7.67 \\
7.58 \\
7.63\end{array}$ & $\begin{array}{l}7.98 \\
7.97 \\
7.98 \\
8.03 \\
7.94 \\
7.99\end{array}$ & $\begin{array}{l}6.8 \\
6.6 \\
6.9 \\
7.2 \\
7.0 \\
7.1\end{array}$ & $\begin{array}{l}8.2 \\
8.3 \\
8.4 \\
8.5 \\
8.5 \\
8.2\end{array}$ & $\begin{array}{l}29.0 \\
30.0 \\
29.5 \\
30.0 \\
30.0 \\
30.0\end{array}$ & $\begin{array}{l}32.0 \\
32.0 \\
32.0 \\
32.0 \\
32.0 \\
32.0\end{array}$ & $\begin{array}{l}116 \\
114 \\
116 \\
112 \\
116 \\
116\end{array}$ & $\begin{array}{l}132 \\
134 \\
136 \\
132 \\
132 \\
134\end{array}$ \\
\hline $\begin{array}{l}C-S B \\
C-N E\end{array}$ & $\begin{array}{l}13.5 \\
13.3\end{array}$ & $\begin{array}{l}16.3 \\
16.3\end{array}$ & $\begin{array}{l}7.65 \\
7.46\end{array}$ & $\begin{array}{l}9.92 \\
7.99\end{array}$ & $\begin{array}{l}6.2 \\
6.6\end{array}$ & $\begin{array}{l}8.1 \\
8.5\end{array}$ & $\begin{array}{l}30.0 \\
29.5\end{array}$ & $\begin{array}{l}32.0 \\
32.0\end{array}$ & $\begin{array}{l}106^{(b)} \\
116^{(b)}\end{array}$ & $\begin{array}{l}132 \\
133\end{array}$ \\
\hline
\end{tabular}

(a) Not applicable.

(b) Outside target quality control range. 


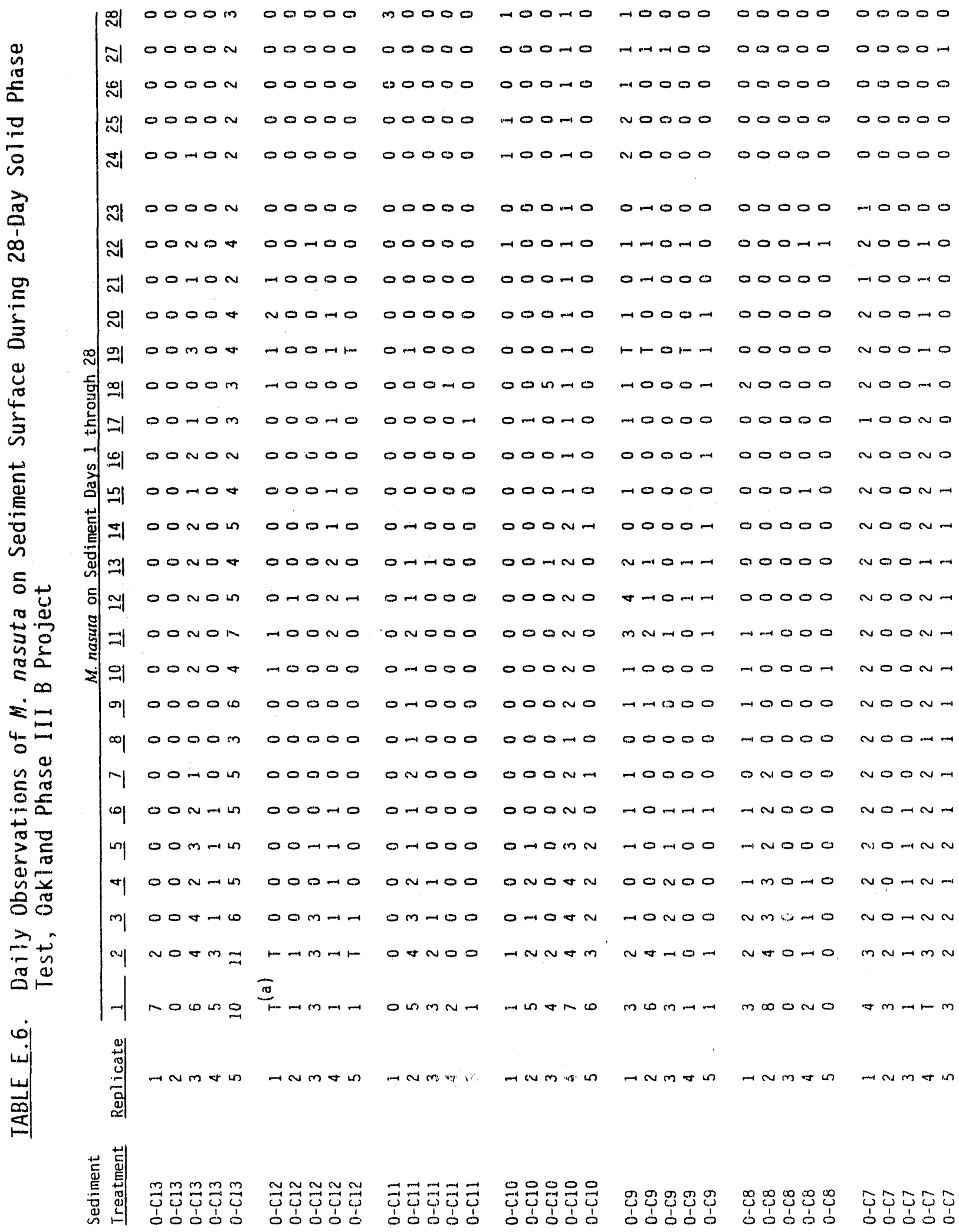




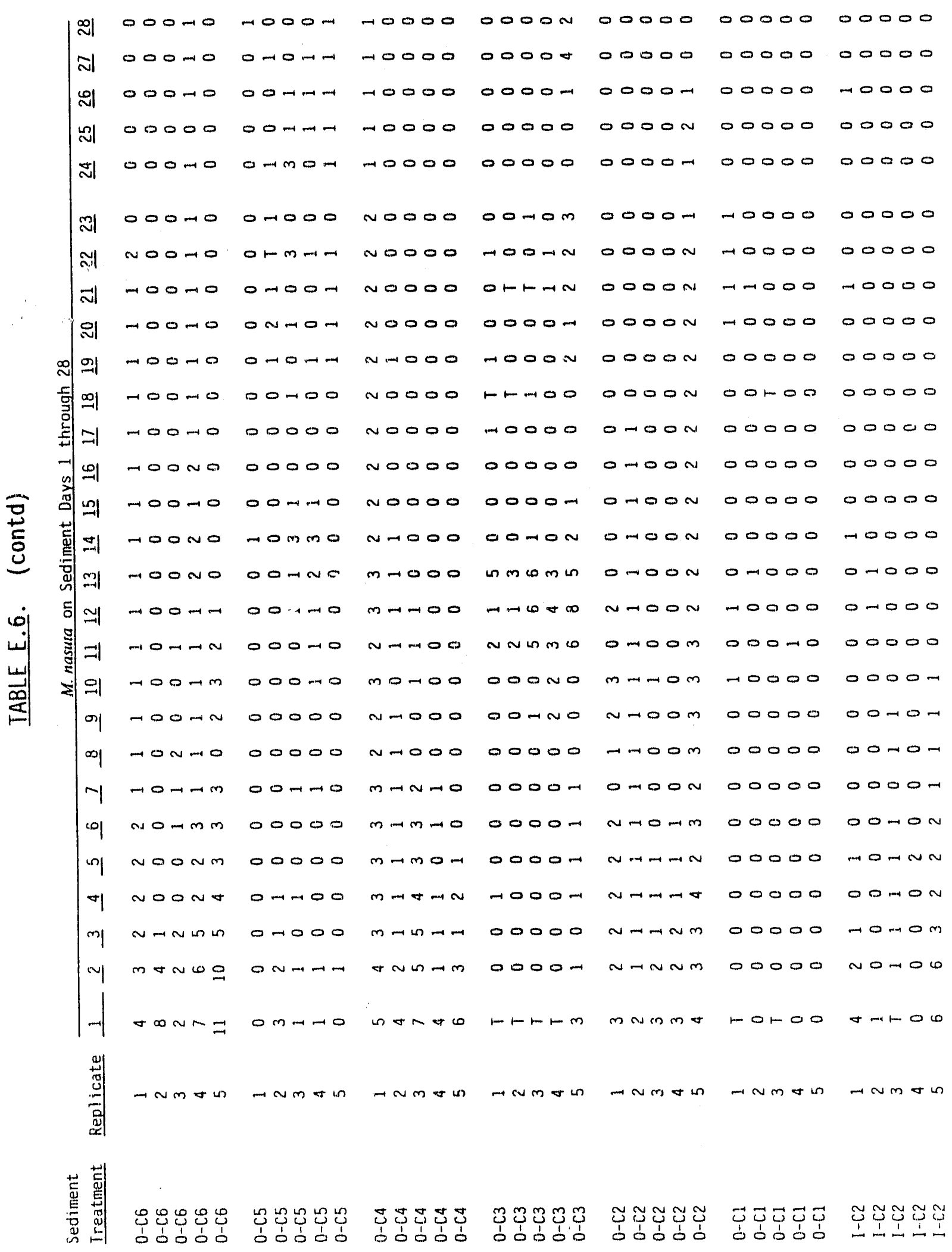




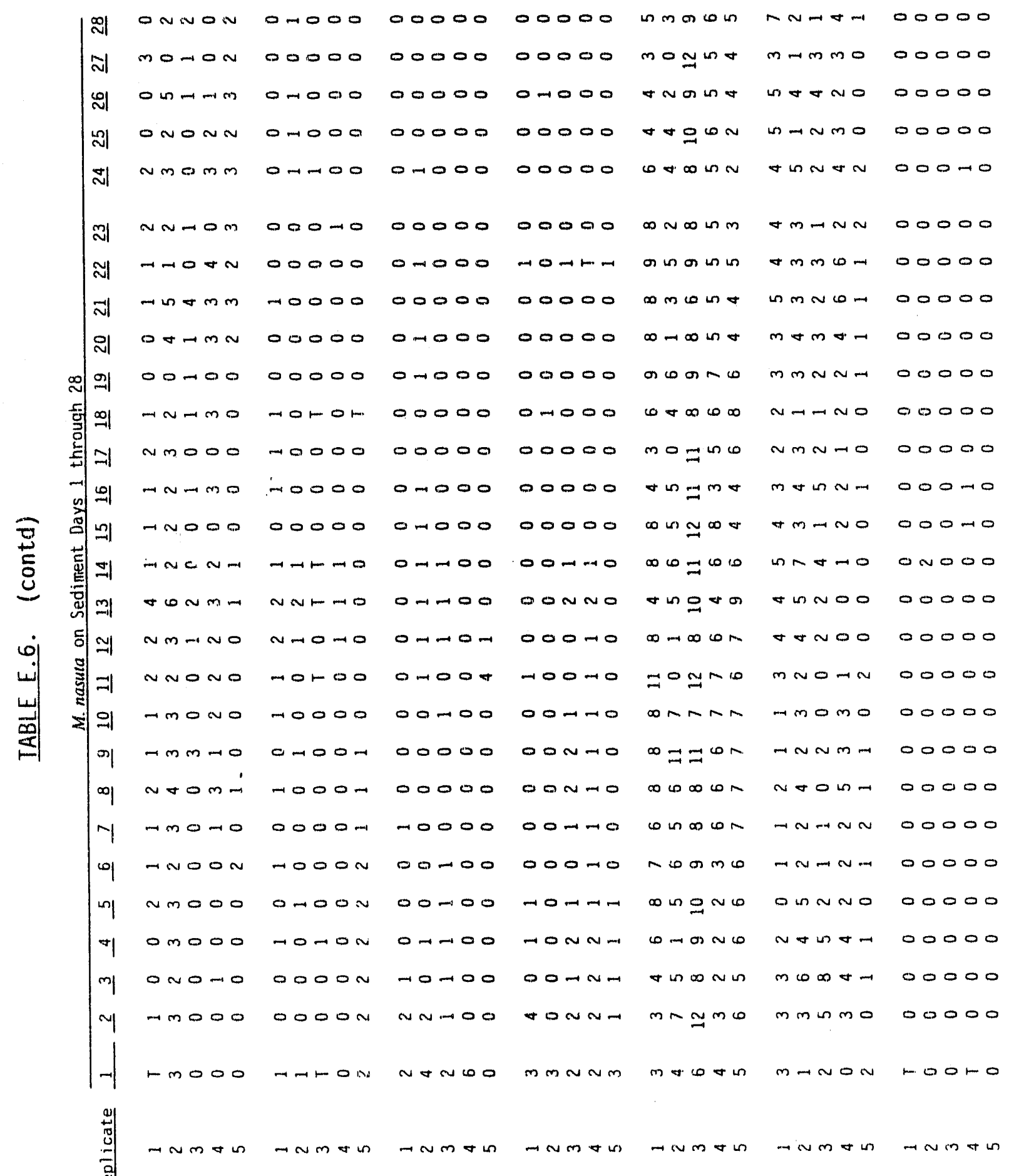

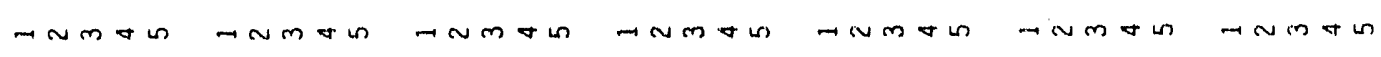

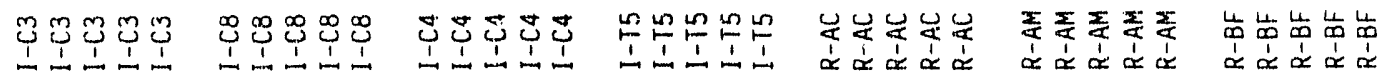




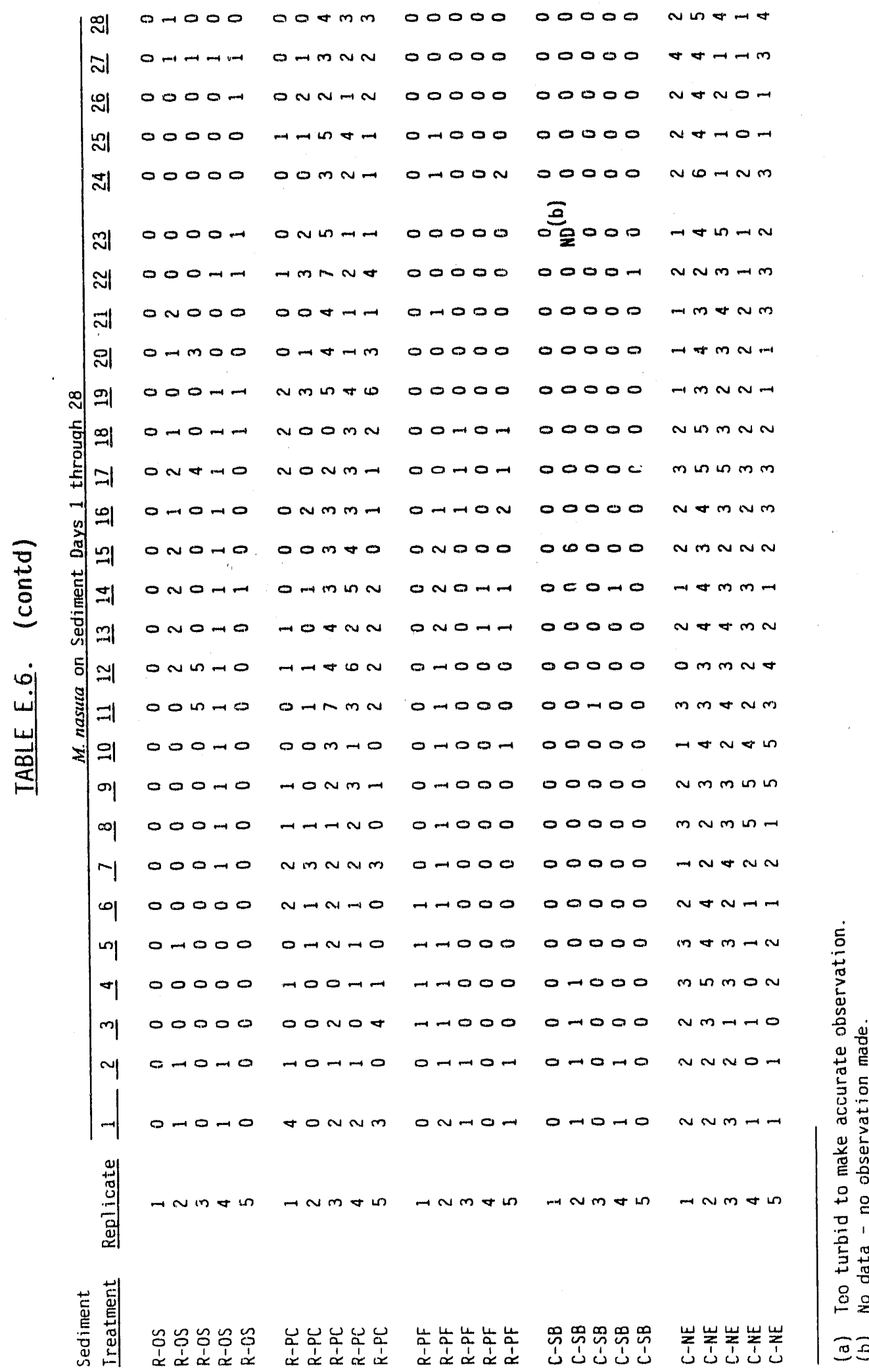




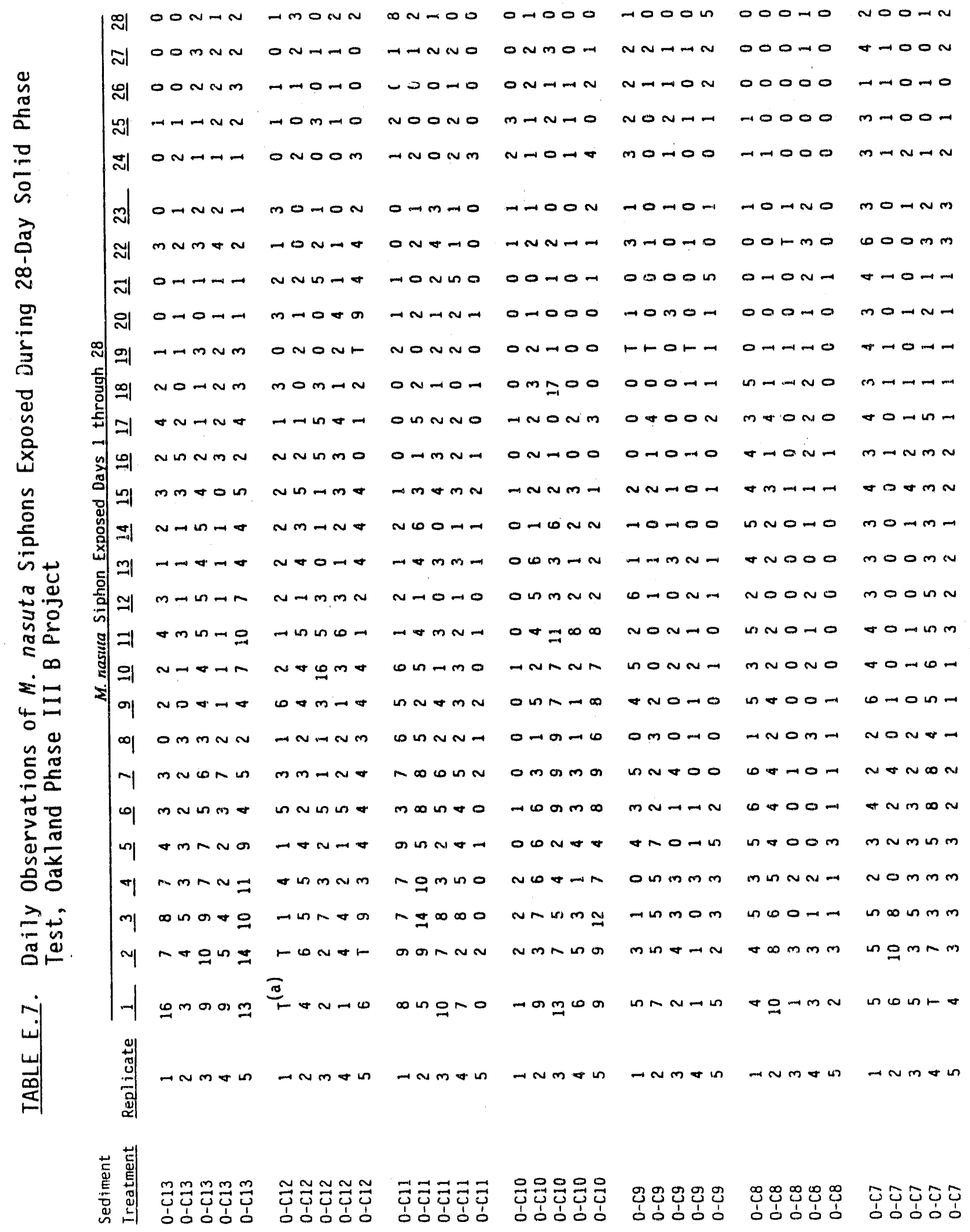




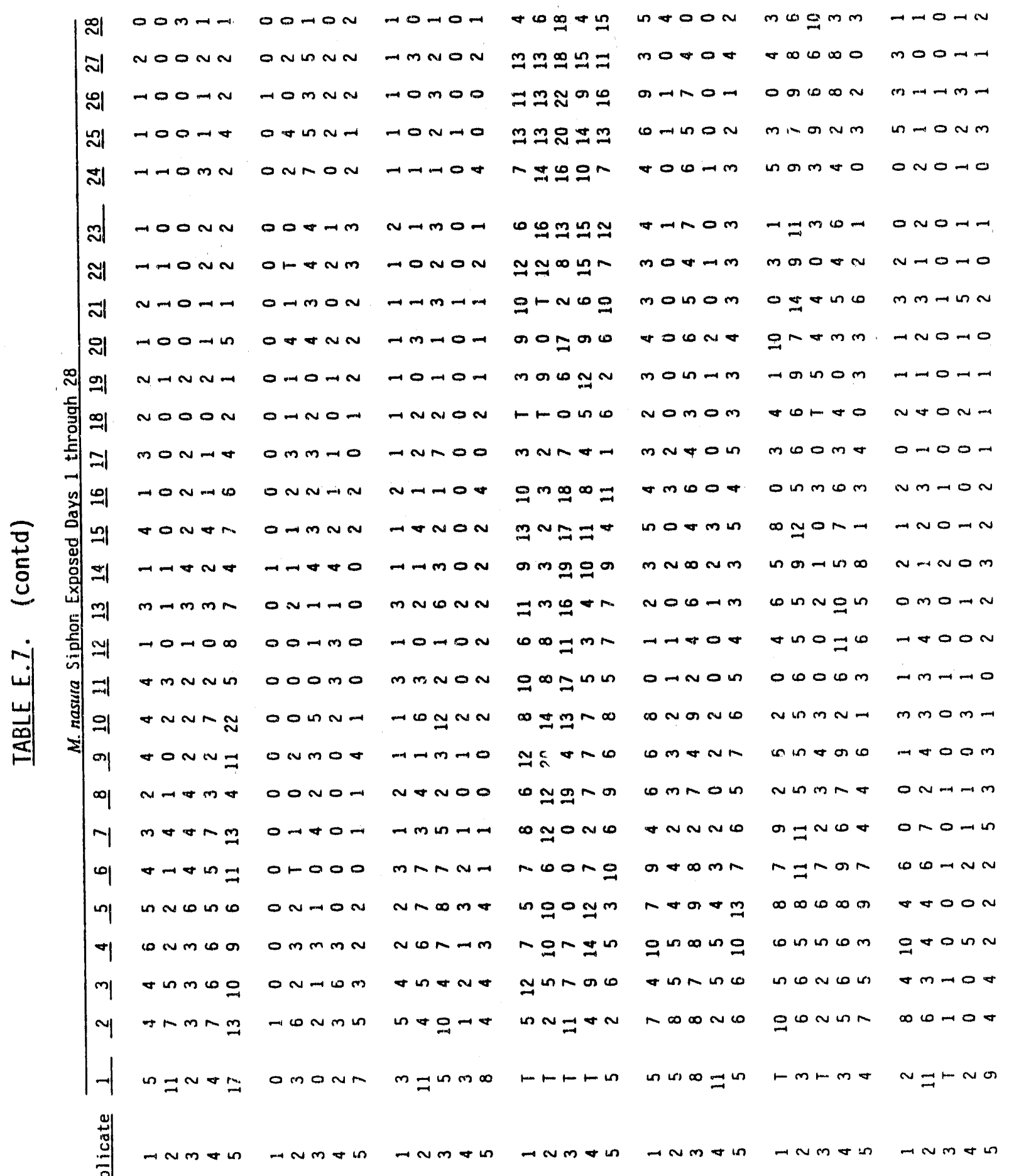

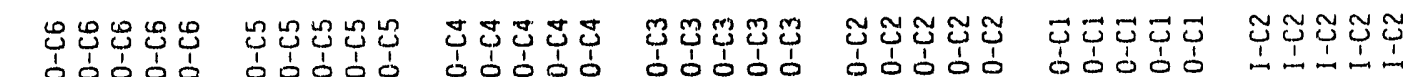
PHASE III B
E. 17 


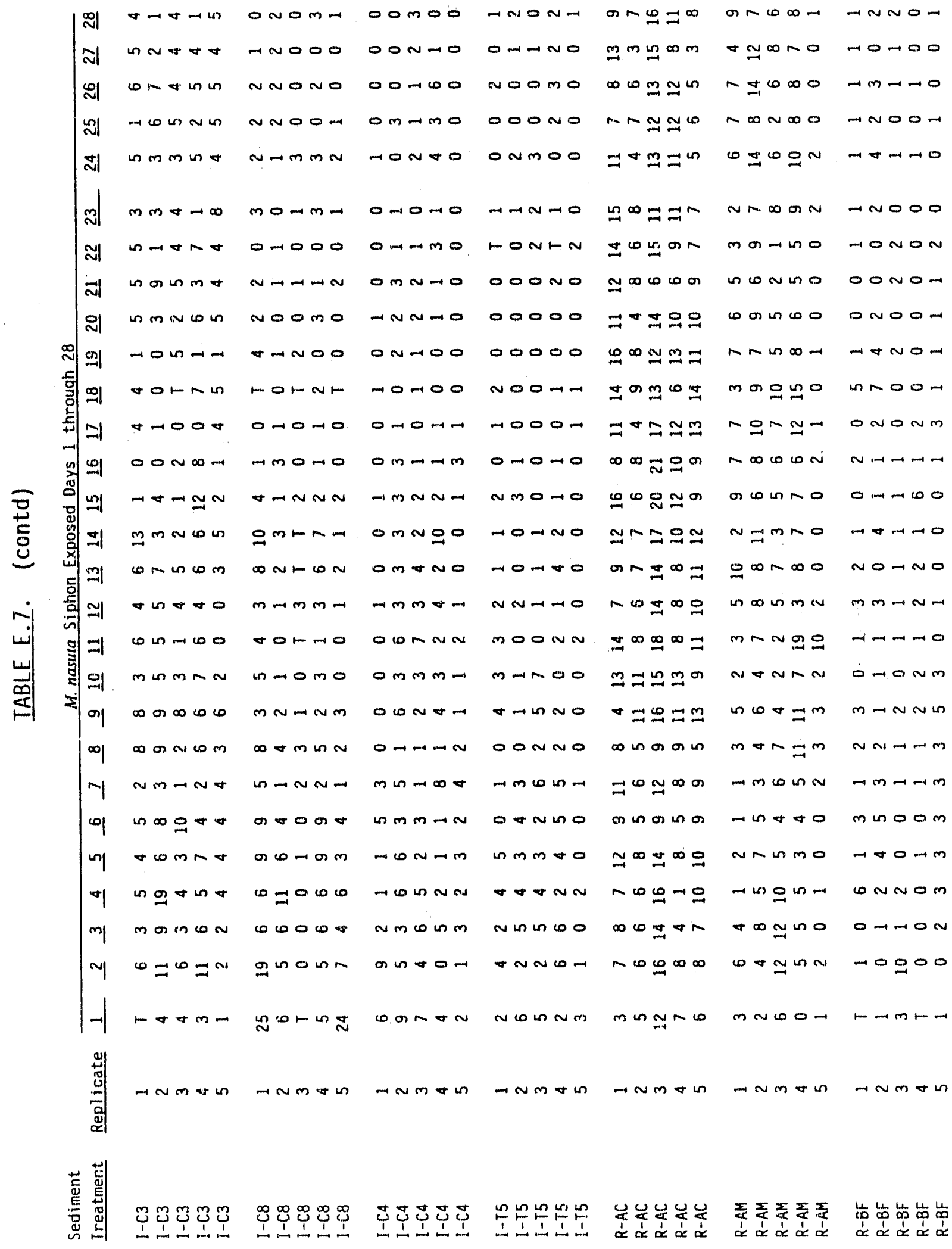




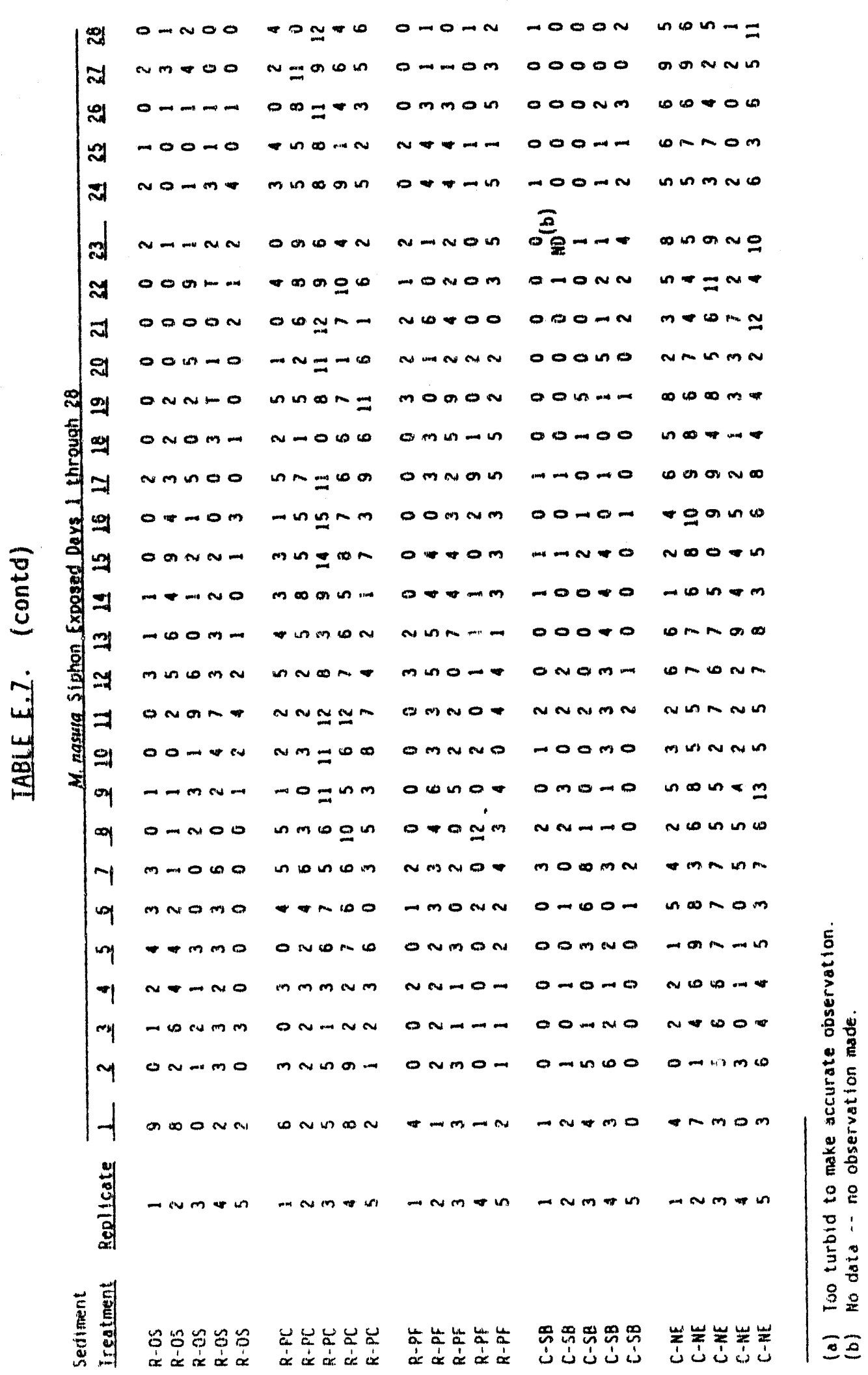




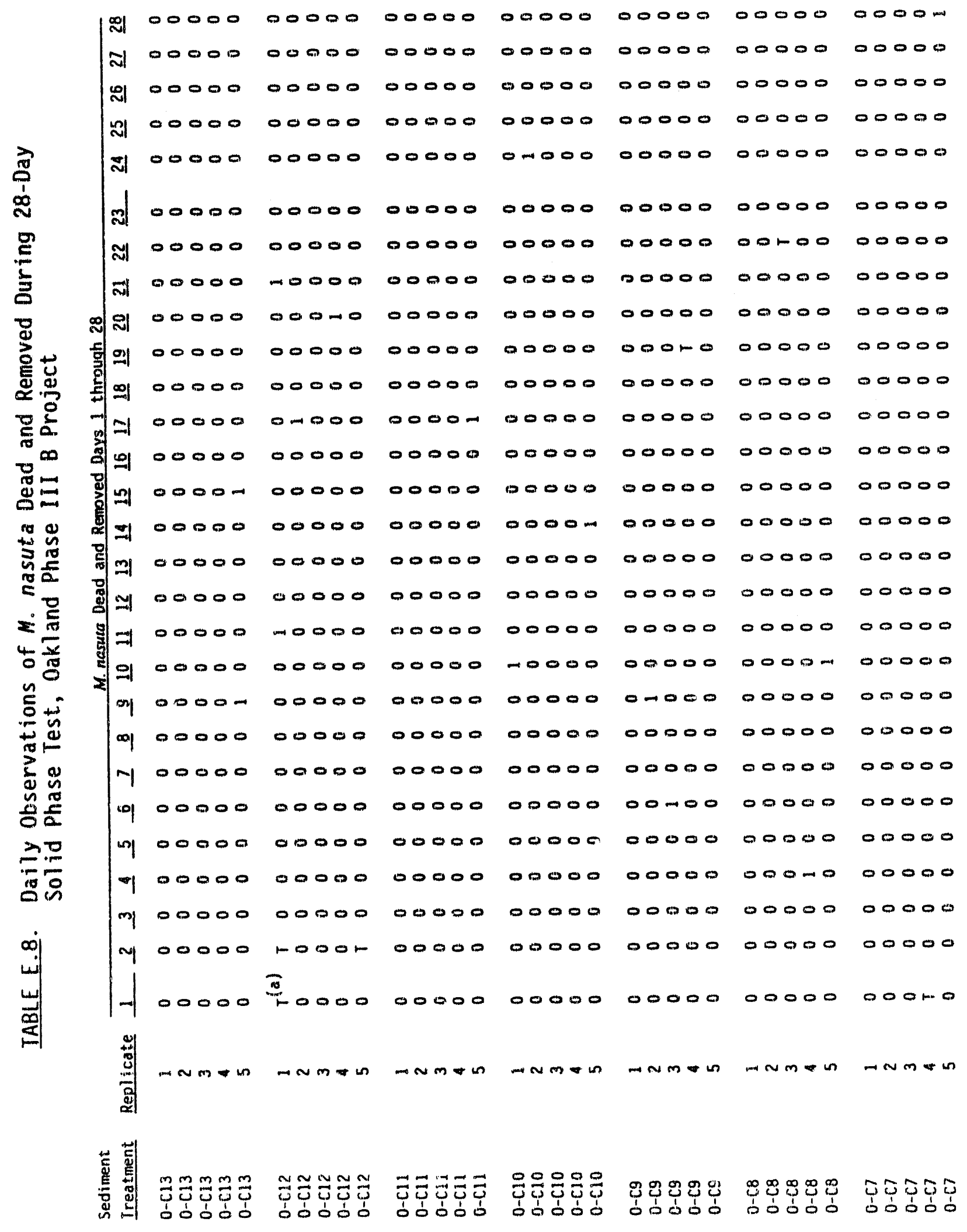




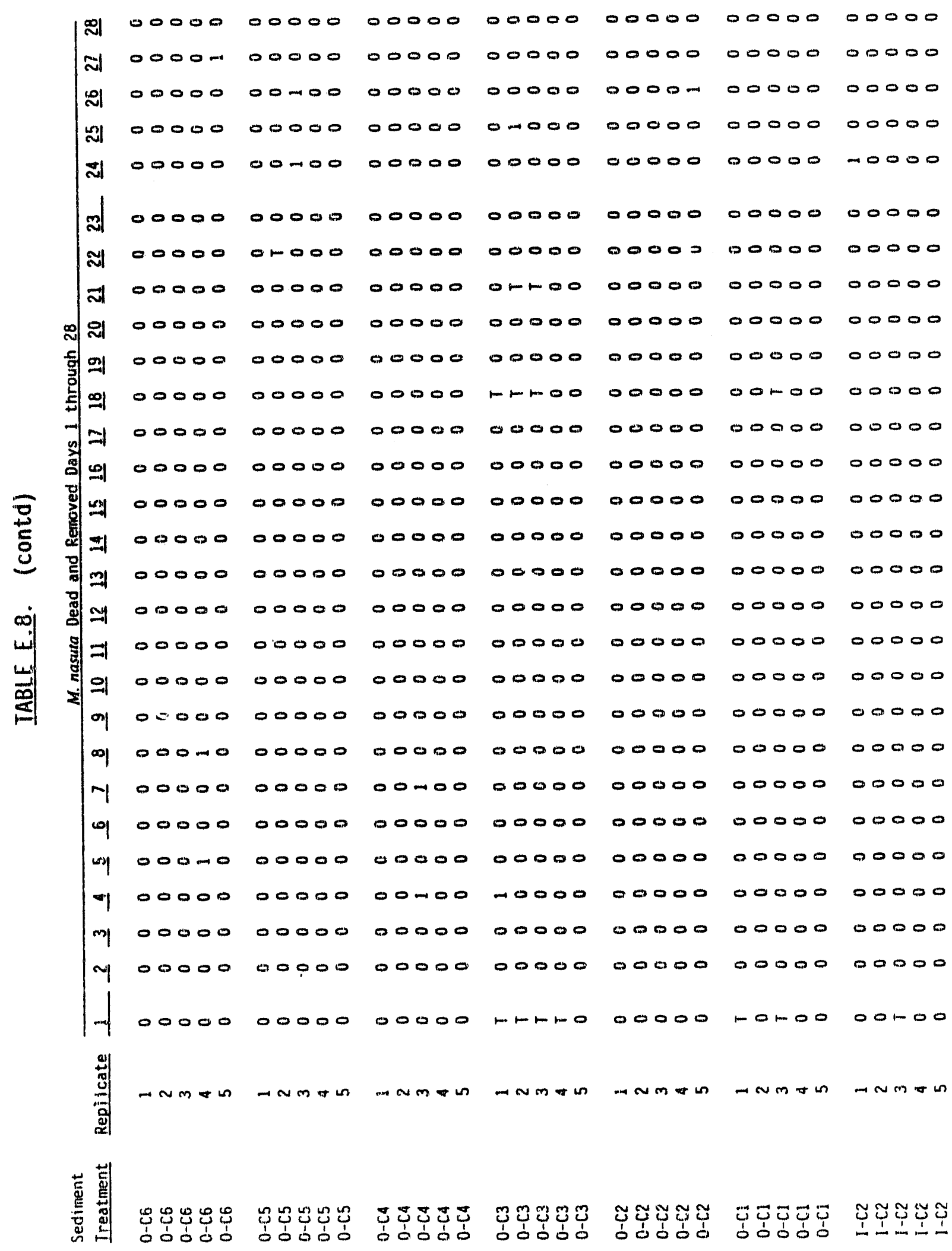




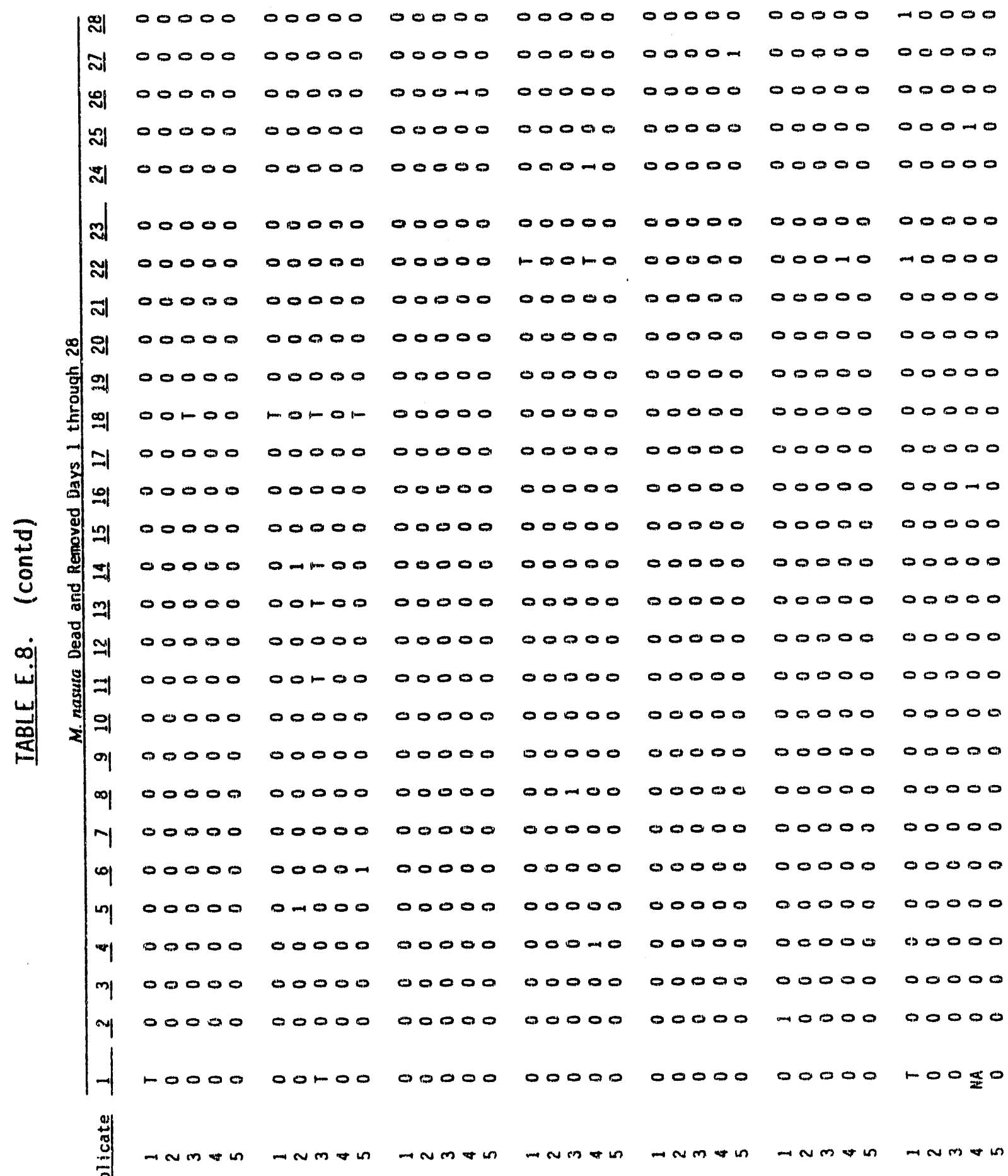




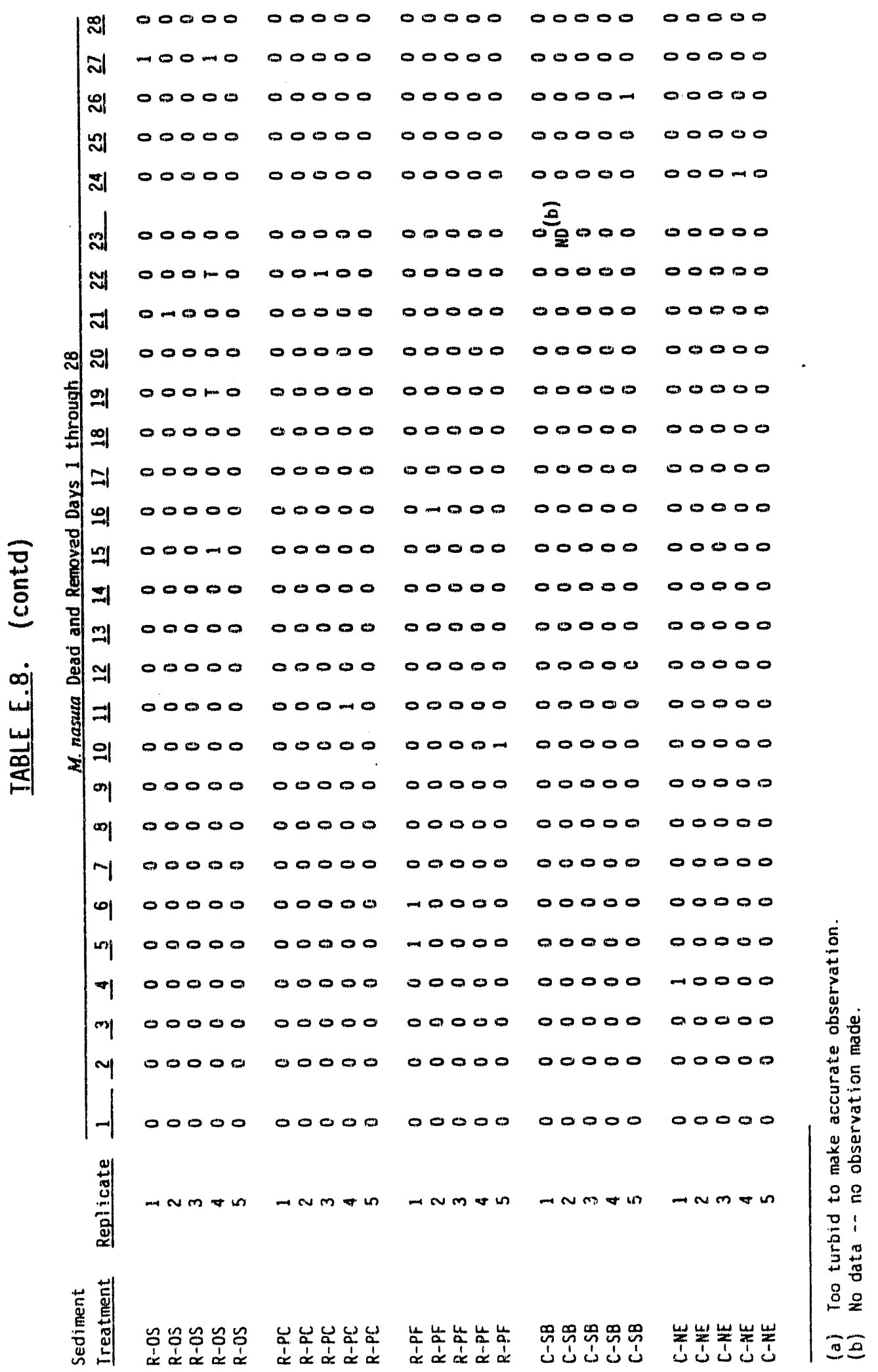




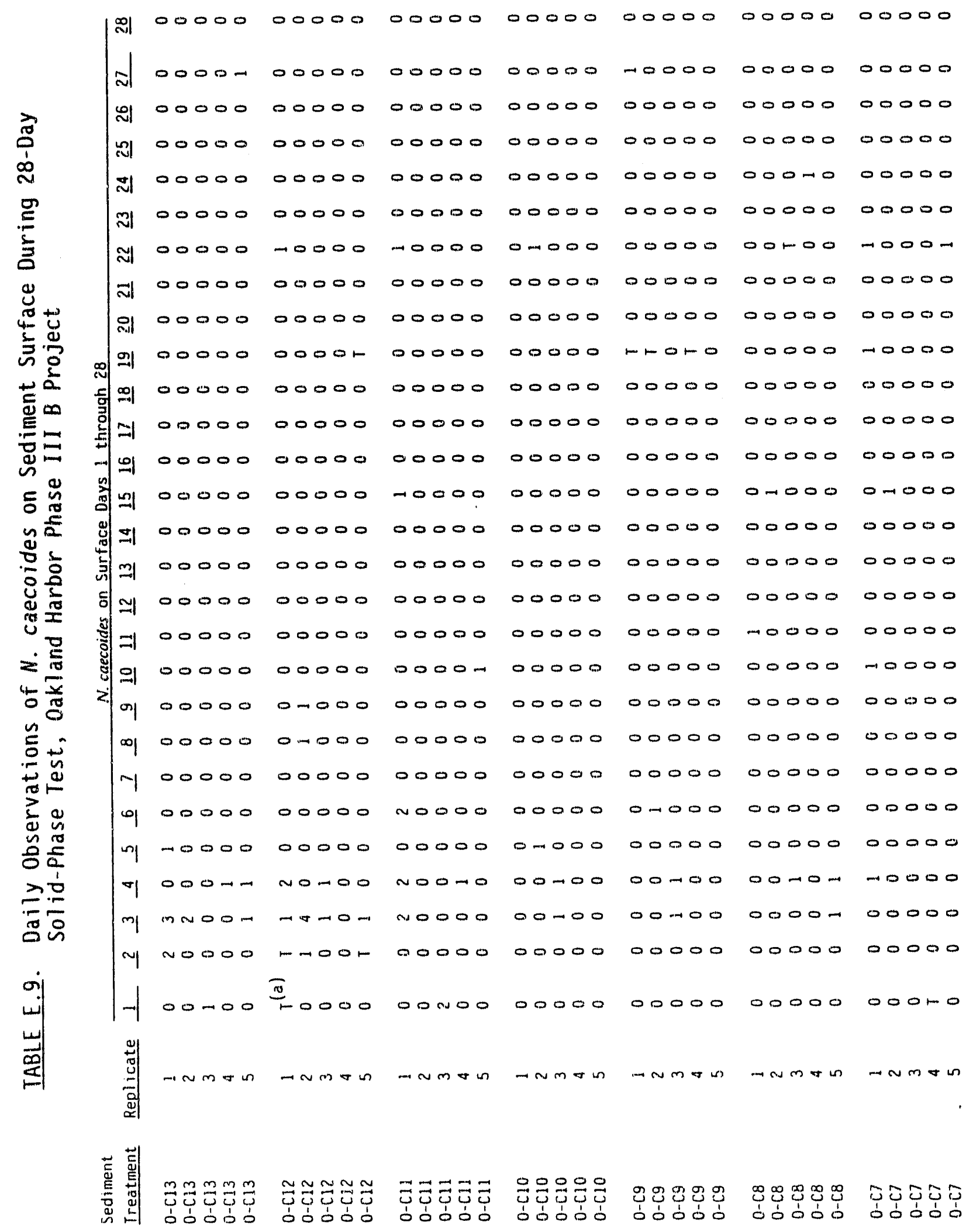




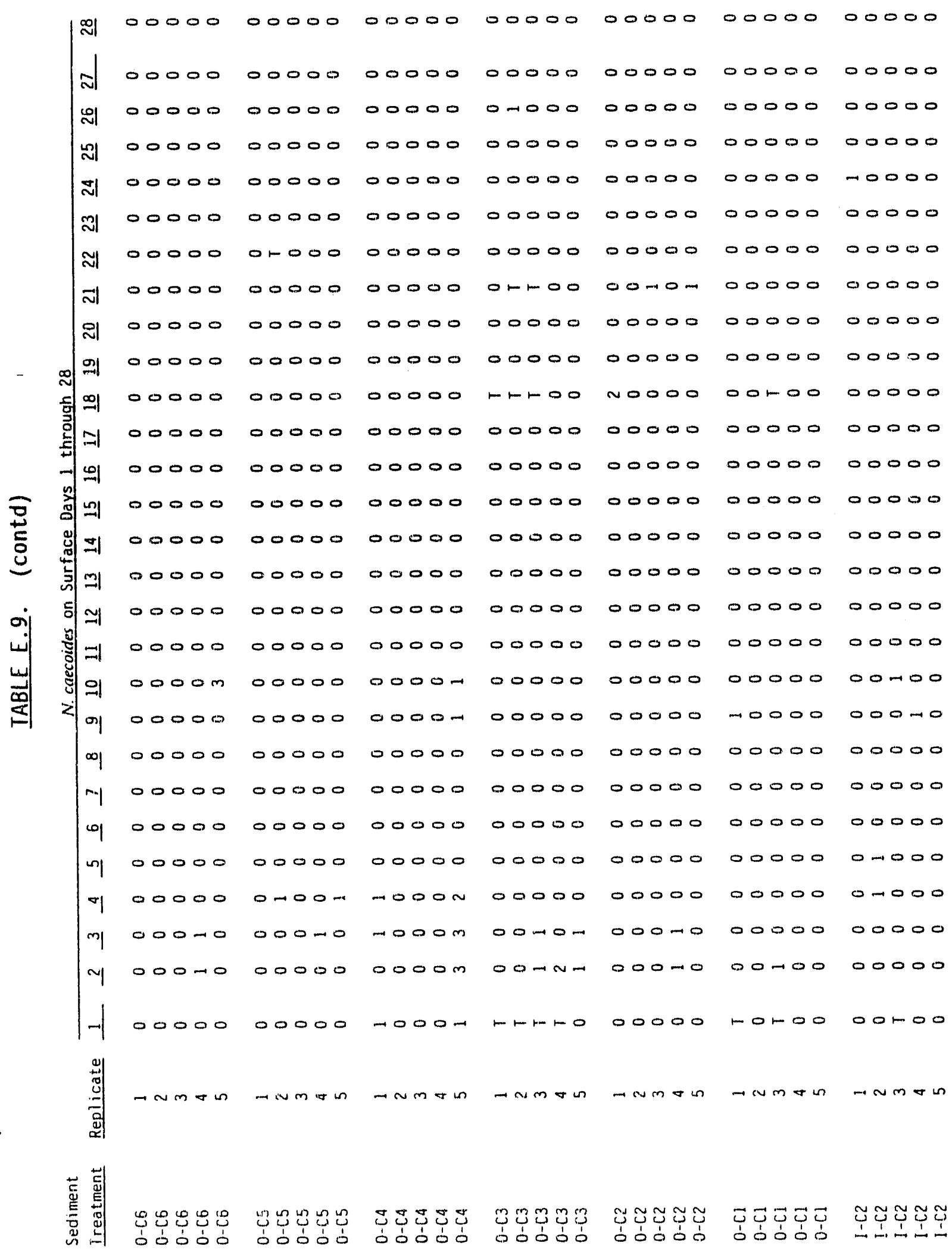




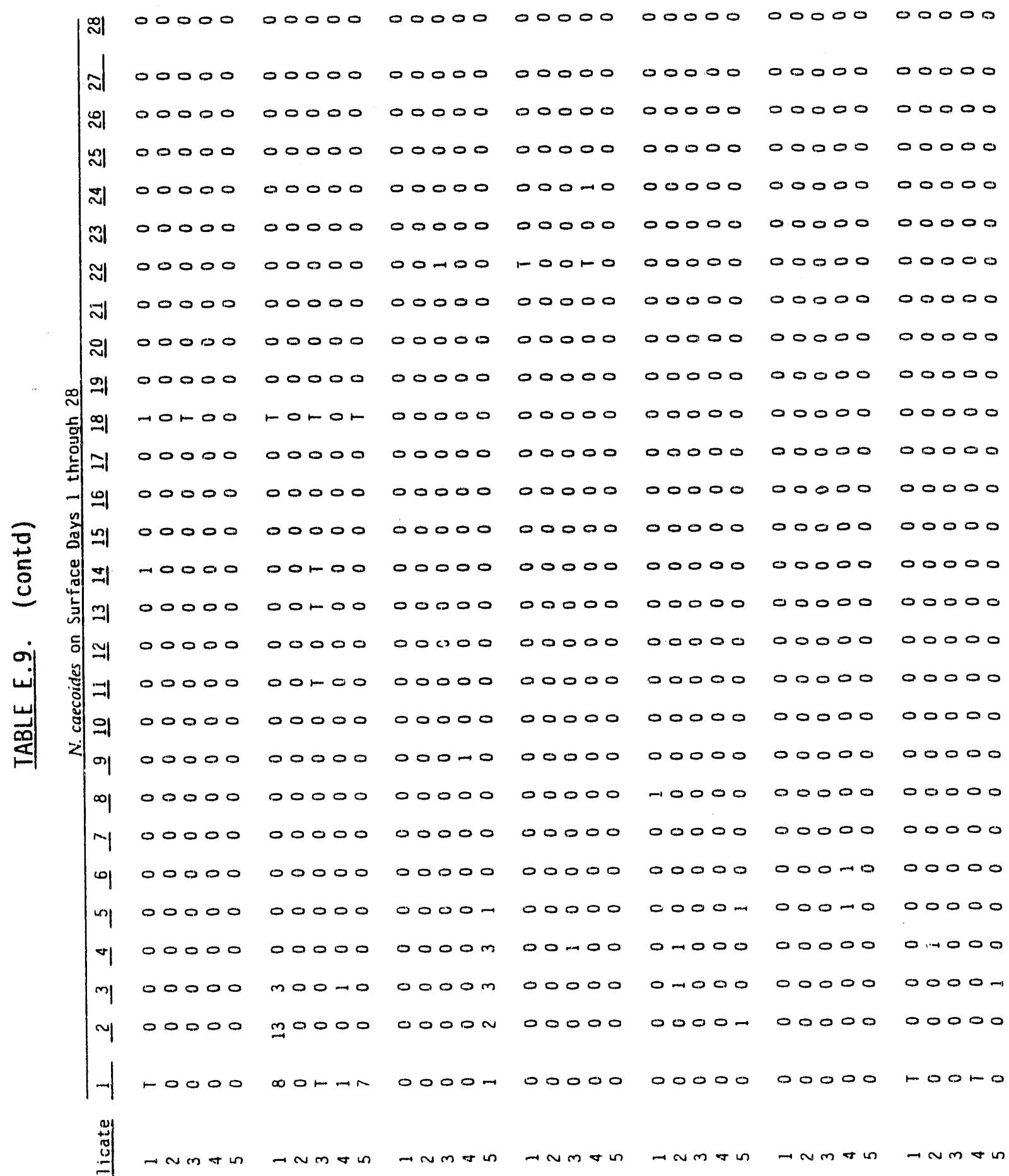

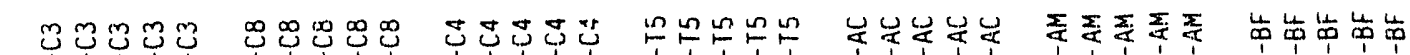

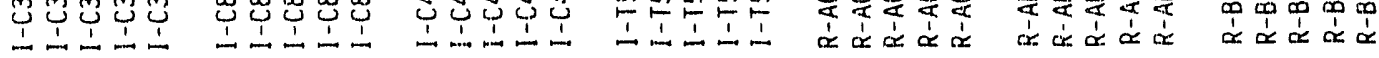




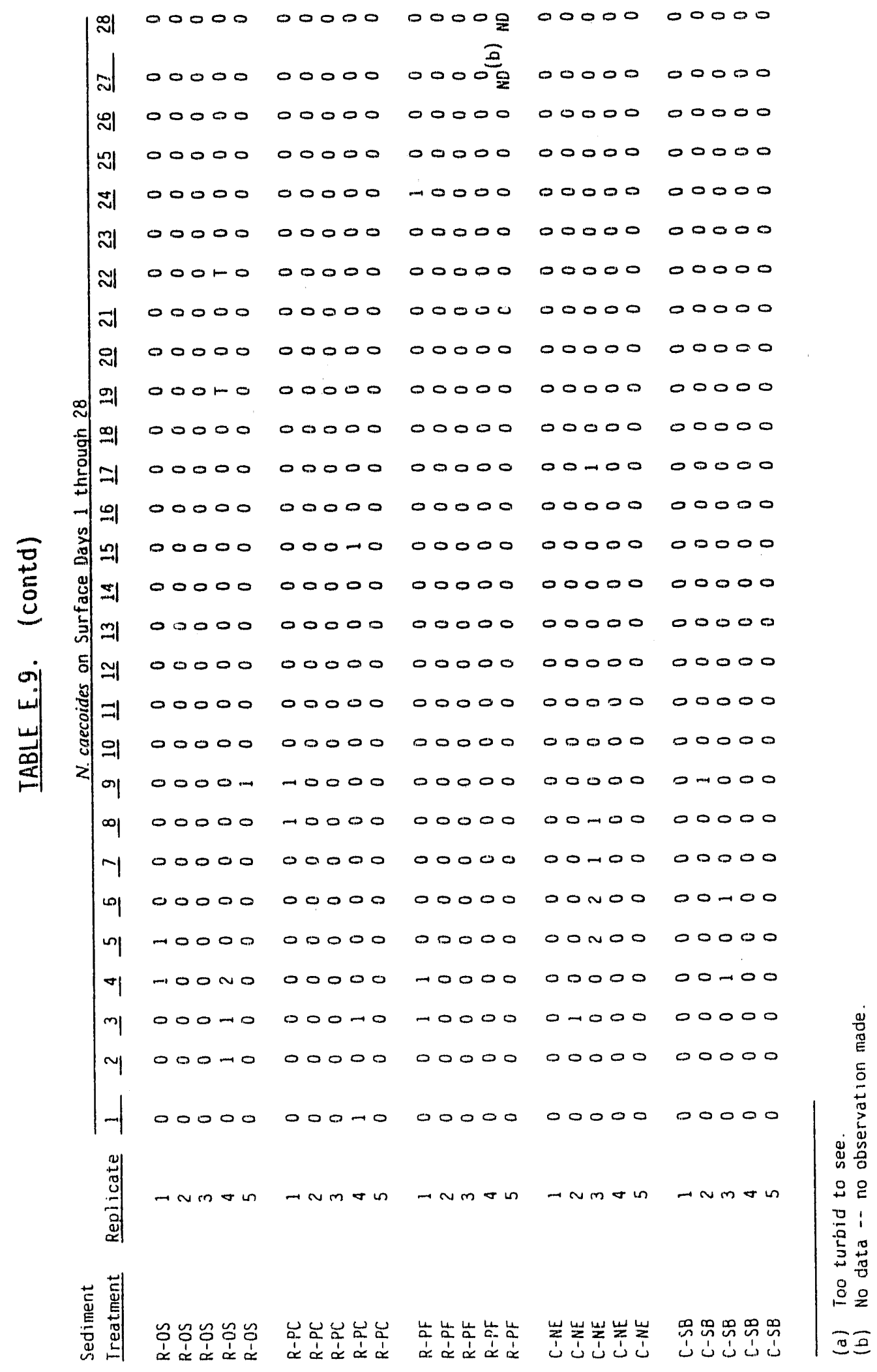

PHASE III B

E. 27 


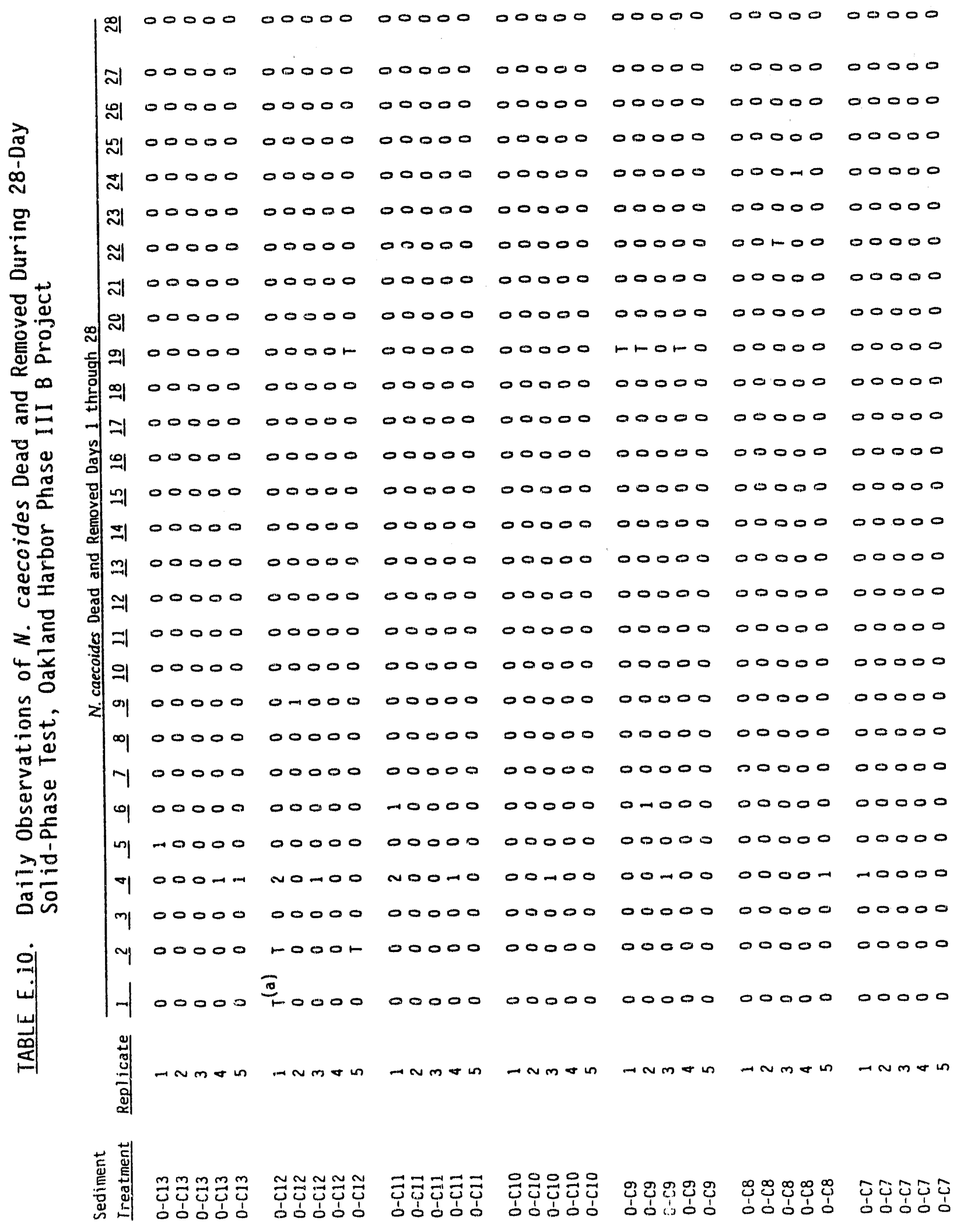




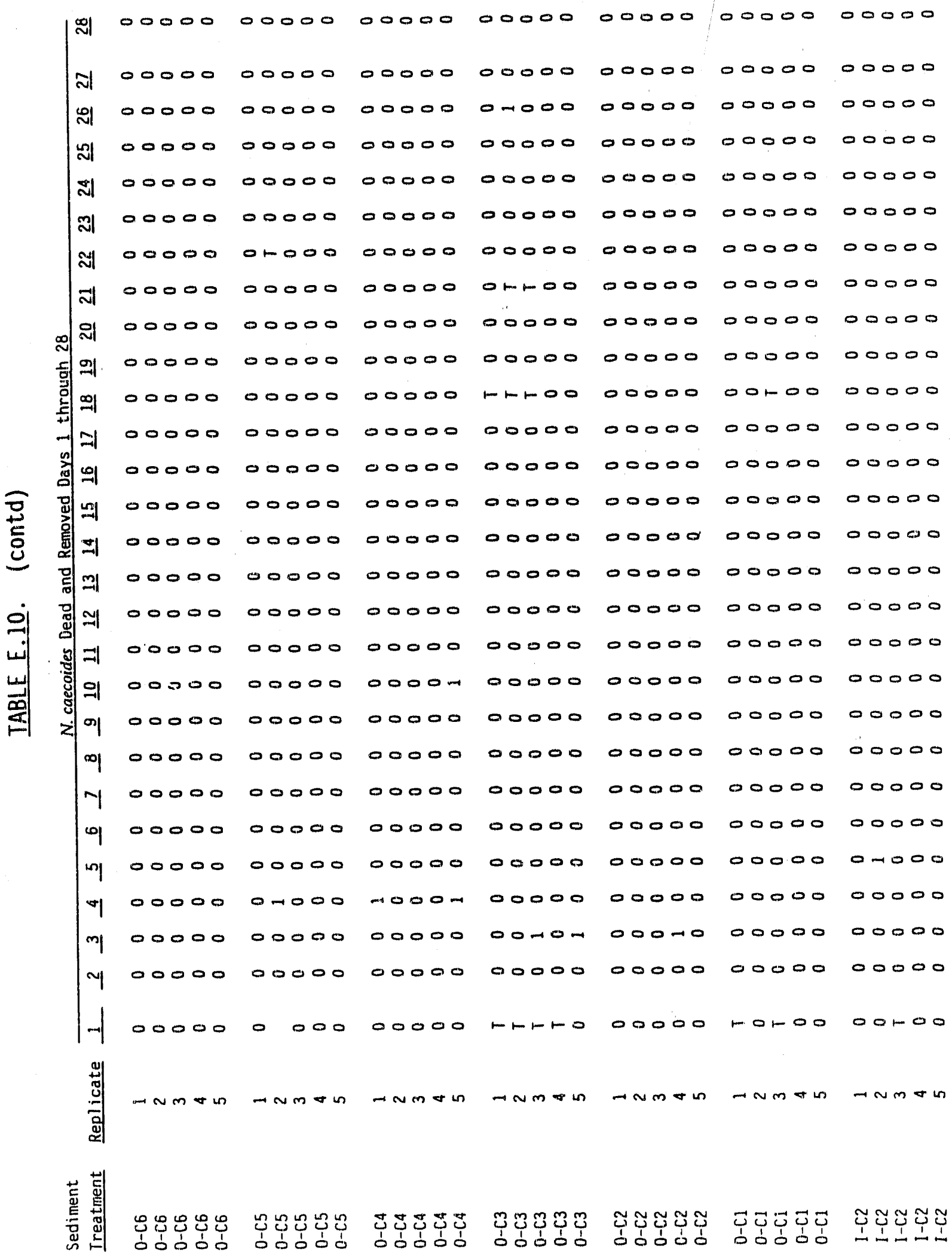




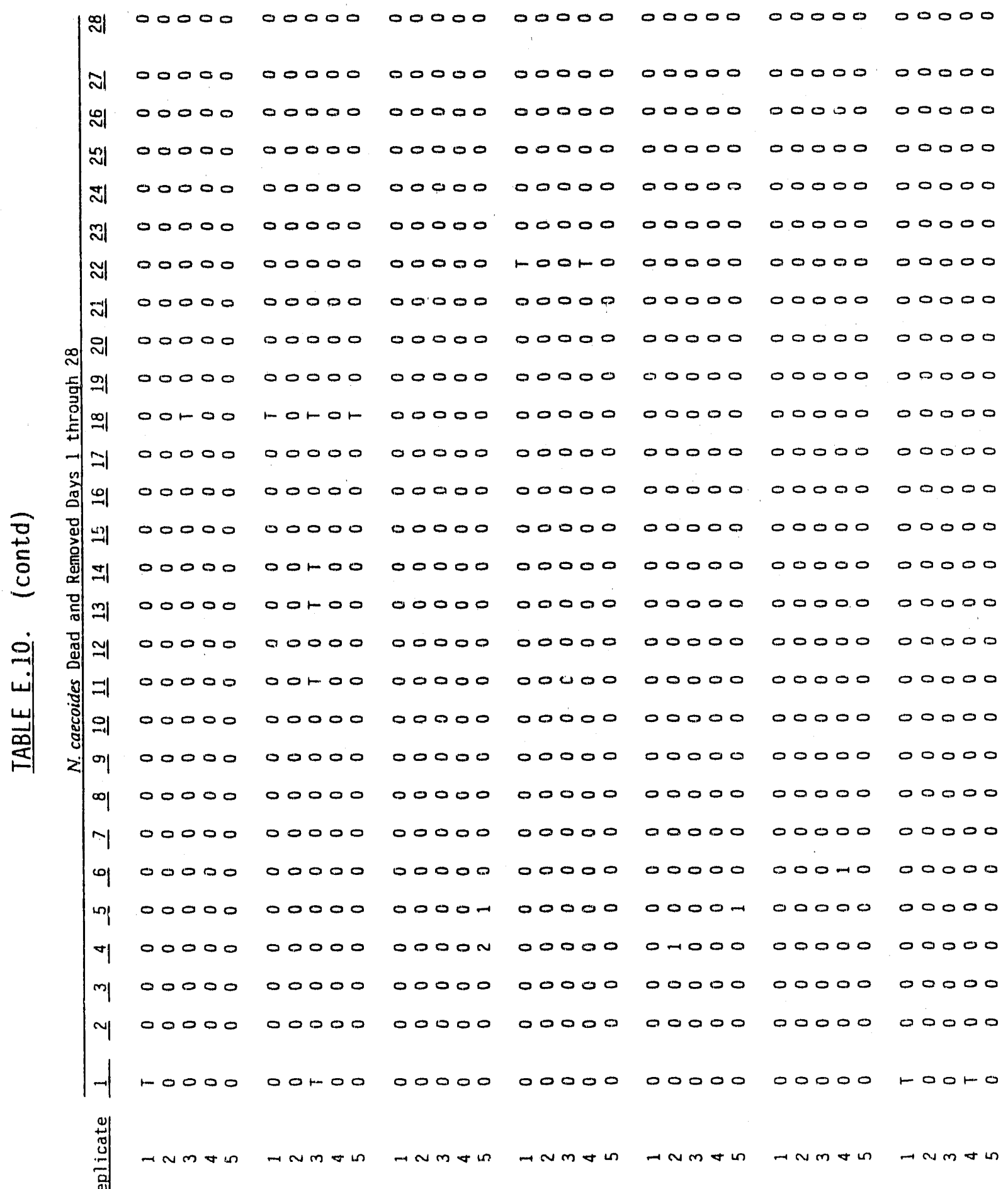

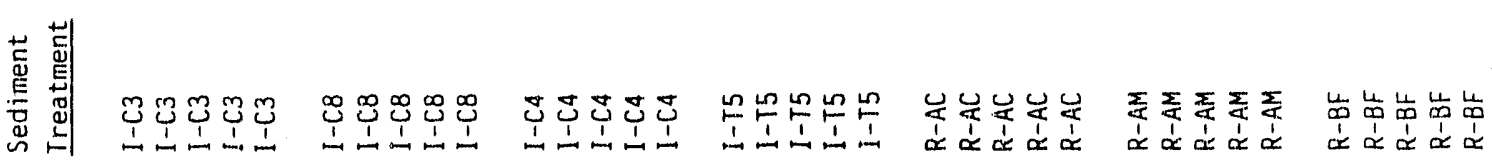




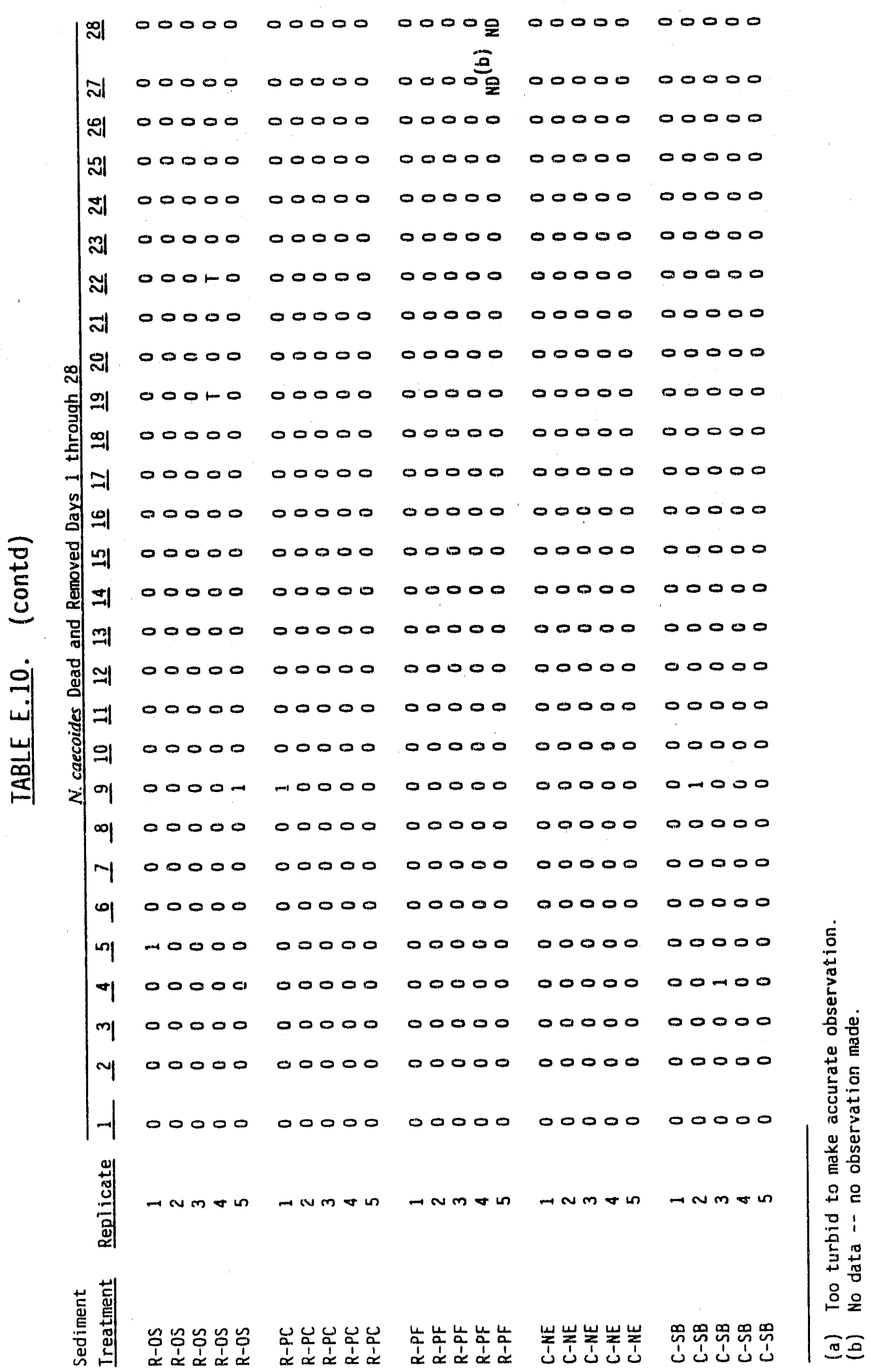


APPENDIX F

10-DAY STATIC SOLID-PHASE TEST WITH R. ABRONIUS 
IABLE F.1. Test Results for All Replicates in 10-Day R. abronius Solid-Phase Static Test, Oakland Harbor Phase III B Project

Sediment

Ircatment,

$0-C 13$

$0 .-C 13$

$0 \cdot-13$

$0-.13$

$0-C 13$

$0 .-12$

$0-\mathrm{Cl}$

$0-\mathrm{C} 12$

$0-C 12$

$0-C 12$

$0-C 11$

$0-C 11$

$0-C 11$

$0-C 11$

$0-C 11$

$0-C 10$

$0-C 10$

$0-C 10$

$0-C 10$

$0-C 10$

$0-6.9$

$0-C 9$

$0-c 9$

$0-C 9$

$0-6.9$

$0-c 8$

$0 .-18$

$0-C B$

$0-C 8$

$0-c 8$

$0 . C 7$

$0-C 7$

$0-67$

$0 .-57$

$0-C 7$

\section{Replicate}

1

2

3

4
5

1

2

3

4
5

1

2

3

4
5

5

1

2

3

4
5

1

2

3

4
5

1

2

3

4
5

1
2
3
4
5

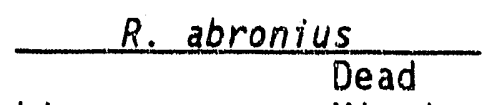

Live

18

18

19

19

20

12

11

17

13

15

18

19

19

20

17

18

19

18

19

18

13

12

16

14

15

18

17

17

17

19

20

18

17

17

14
Mean

Proportion

Surviving

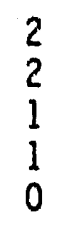

0.94

0.68

0.93

0.92

0.70

0.88

0.86 
IABLE F.1. (contd)

Sediment

Treatment

$0-C 6$

$0-C 6$

$0-\mathrm{C} 6$

$0-\mathrm{C} 6$

$0-\mathrm{C} 6$

$0-C 5$

$0-C 5$

$0-C 5$

$0-C 5$

$0-C 5$

$0-14$

$0-C 4$

$0-C 4$

$0-C$

$0-C 4$

$0-c 3$

$0-C 3$

$0-\mathrm{C3}$

$0-C 3$

0.53

$0-C .2$

$0-C 2$

$0-\mathrm{C2}$

$0-C 2$

$\mathrm{O}-\mathrm{C2}$

$0-C 1$

$0-C 1$

$\mathrm{O}-\mathrm{Cl}$

$\mathrm{O}-\mathrm{Cl}$

$0-C 1$

I-C2

$1-\mathrm{C} 2$

I-C2

$\mathrm{I}-\mathrm{C} 2$

I-C?

\section{Replicate}

1
2
3
4
5

1

2

3

4
5

1
2
3
4
5

1
2
3
4
5

1

2

3
4

5

1

2

3
4

5

1
2
3
4
5

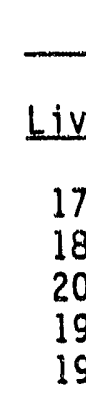

18

18

14

19

16

16

17

17

15

19

19

15

19

8
20

1

11

15

13

17

6

11

10

13

6
11
14
17
11
Mean

Proportion

Surviving

0.93

0.85

0.84

0.81

0.67

0.47

14

9

6

9

0.59

PHASE 1118

F.2 
TABLE F.1. (contd)

Sediment

Treatment

$\mathrm{I}-\mathrm{C} 3$

I $-\mathrm{C} 3$

I $-\mathrm{C} 3$

I-C3

I-C3

I $-\mathrm{C} 8$

I $-\mathrm{C} 8$

I $-\mathrm{C} 8$

I- $-\mathrm{CB}$

I $-\mathrm{C} 8$

$R-A C$

$R-A C$

$R-A C$

$R-A C$

$R-A C$

$R-A M$

$R-A M$

$R-A M$

$R-A M$

$R-A M$

$R-B F$

$R-B F$

$R-B F$

$R-B F$

$R-B F$

R-OS

$R-O S$

$R-O S$

$R-O S$

$R-O S$

$R-P C$

$R-P C$

$R-P C$

$R-P C$

$R-P C$

\section{Replicate}

1

2

4

5

1

2

3

4
5

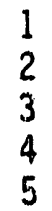

20

20

20

20

19

18

20

20

20

20

16

19

15

18

12

13

17

19

17

17

20

20

20

20

20
Mean

Proportion

Surviving

or Missing

0.96

0.89

0.99

0.98

0.80

0.83

1.00 
IABLE F.1. (contd)

Sediment

Ireatment

$R-P F$

$R-P F$

$R-P F$

$R-P F$

$R-P F$

C-SB

C-SB

$C-S B$

$C-S B$

C-SB

$C-W B$

$C-W B$

C.WB

$C-W B$

$C-W B$

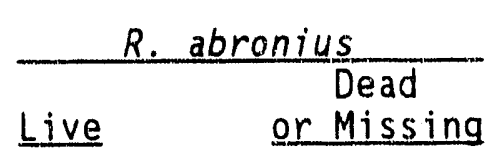

Replicate

1

2

3

4
5

5

1

2

3

4

5

1
2
3
4
5
20

18

18

20

20

19

19

15

19

20

19

20

19

20

20
Mean

Proportion

Surviving

0

2

2

0

0.96

0.92

0.98 
TABLE F.2. Rank Order Based on Mean Proportion Surviving for 10-Day R. abronius Solid-Phase Test, Oakl and Harbor Phase III B Project

\begin{tabular}{|c|c|}
\hline $\begin{array}{l}\text { Sediment } \\
\text { Treatment }\end{array}$ & $\begin{array}{l}\text { Mean } \\
\text { Proportion } \\
\text { Surviving } \\
\end{array}$ \\
\hline $\begin{array}{l}0-C 1 \\
I-C 2 \\
0-C 2 \\
0-C 12 \\
0-C 9 \\
R-B F \\
0-C 3 \\
0-C 4 \\
R-O S \\
0-C 5 \\
0-C 7 \\
0-C 8 \\
I-C 8 \\
C-S B \\
0-C 10 \\
0-C 11 \\
0-C 6 \\
0-C 13 \\
I-C 3 \\
R-P F \\
C-W B \\
R-A M \\
R-A C \\
R-P C\end{array}$ & $\begin{array}{l}0.47 \\
0.59 \\
0.67 \\
0.68 \\
0.70 \\
0.80 \\
0.81 \\
0.82 \\
0.83 \\
0.85 \\
0.86 \\
0.88 \\
0.89 \\
0.92 \\
0.92 \\
0.93 \\
0.93 \\
0.94 \\
0.96 \\
0.96 \\
0.98 \\
0.98 \\
0.99 \\
1.00\end{array}$ \\
\hline
\end{tabular}


TABLE F.3. Water Quality Summary for 10-Day R. abronius Solid-Phase Test, Oakland Harbor Phase III B Project

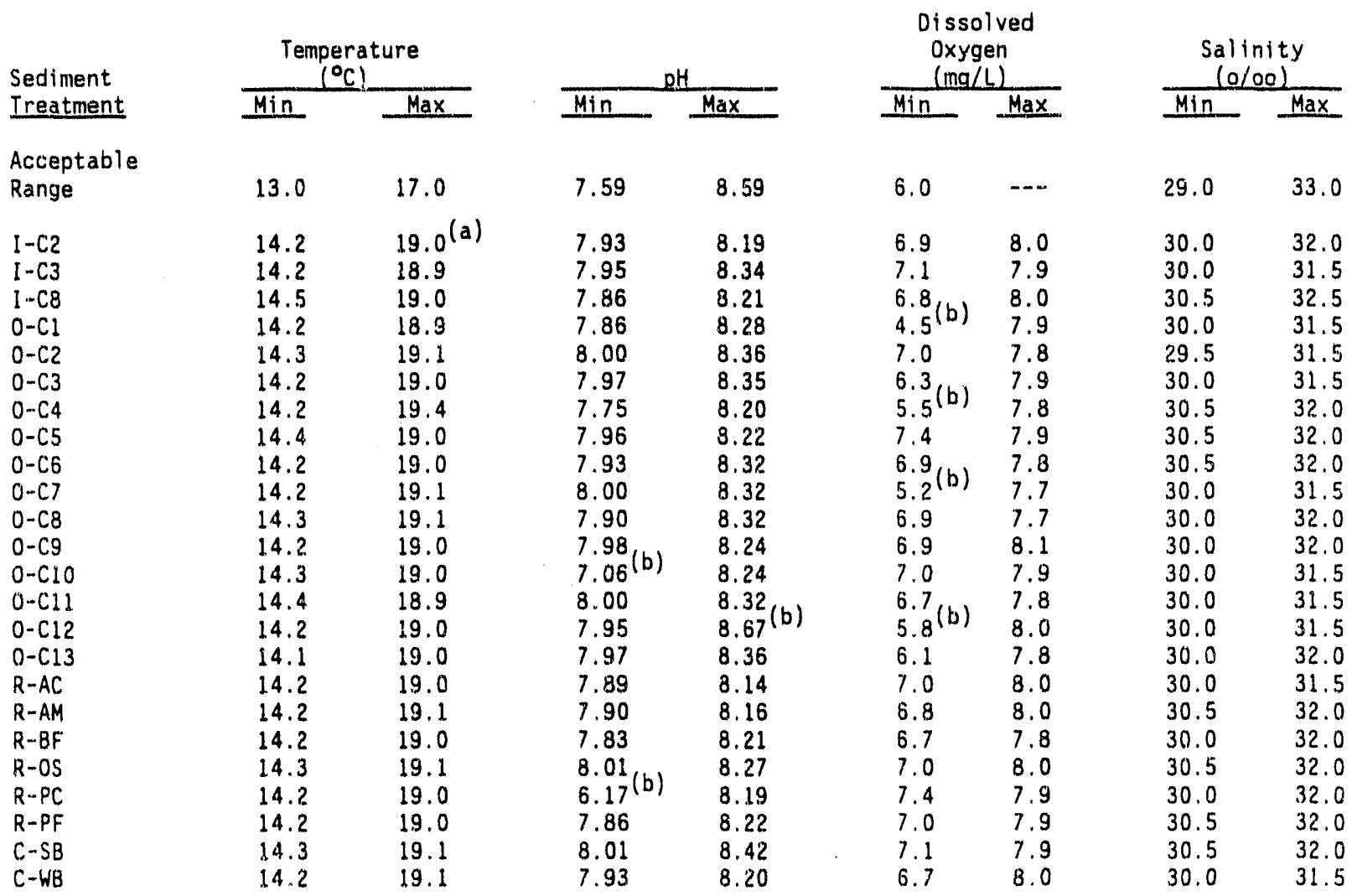

(a) Temperatures were out of range on Days 7 and 8 due to solenoid mal function. Temperatures returned to normal Day 9

(b) Parameter temporarily outside of target quality control range: outcome of test not affected. 
TABLE F.4. Daily Observations of $R$. abronius on Sediment Surface During 10-Day Solid Phase Test, Oakland Harbor

Phase III B Project

Sediment

Treatinent

$0-\mathrm{C} 13$

$0-\mathrm{C} 13$

$0-\mathrm{C} 13$

$0-\mathrm{C} 13$

$0-\mathrm{C} 13$

$0-\mathrm{C} 12$

$0-\mathrm{C} 12$

$0-\mathrm{C} 12$

$0-\mathrm{C} 12$

$0-\mathrm{C} 12$

$0-C 11$

$0-C 11$

$0-C 11$

0 -C11

0-C11

$0-C 10$

$0-C 10$

$0-C 10$

$0-C 10$

$0-C 10$

$0-C 9$

$0-C 9$

$0-C 9$

$0-C 9$

$0-C 9$

$0-18$

$0-C 8$

$0-c 8$

$0-C 8$

$0-C 8$

0-C7

O-C7

O-C7

O-C7

O-C7

1
2
3
4
5

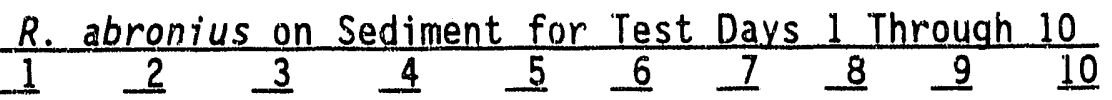

\section{Replicate}

$\begin{array}{llllllllll}0 & 0 & 1 & 0 & 0 & 0 & 0 & 0 & 0 & 0 \\ 0 & 0 & 0 & 0 & 0 & 0 & 0 & 0 & 0 & 2 \\ 0 & 0 & 1 & 0 & 0 & 0 & 0 & 0 & 0 & 0 \\ 0 & 0 & 0 & 0 & 0 & 0 & 0 & 0 & 0 & 0 \\ 0 & 0 & 0 & 1 & 0 & 0 & 0 & 0 & 0 & 0\end{array}$

1
2
3
4
5

$\begin{array}{llllllllll}0 & 0 & 0 & 0 & 0 & 0 & 0 & 0 & 0 & 1 \\ 1 & 1 & 0 & 1 & 0 & 1 & 0 & 0 & 0 & 0 \\ 0 & 0 & 0 & 0 & 0 & 0 & 0 & 0 & 0 & 0 \\ 0 & 0 & 0 & 0 & 0 & 0 & 2 & 1 & 2 & 4 \\ 0 & 0 & 0 & 0 & 0 & 0 & 0 & 2 & 0 & 1\end{array}$

1
2
3
4
5

$\begin{array}{llllllllll}1 & 0 & 0 & 0 & 0 & 0 & 0 & 0 & 0 & 0\end{array}$

$\begin{array}{llllllllll}0 & 0 & 0 & 0 & 0 & 0 & 0 & 0 & 0 & 0\end{array}$

$\begin{array}{llllllllll}0 & 0 & 0 & 0 & 0 & 0 & 0 & 0 & 0 & 0 \\ 0 & 1 & 0 & 0 & 0 & 0 & 0 & 1 & 0 & 0\end{array}$

4

1
2
3
4
5

$0 \quad 0$

(1)

$\begin{array}{llll}0 & 0 & 0 & 0 \\ 0 & 0 & 0 & 0 \\ 1 & 0 & 0 & 0\end{array}$

$\frac{1}{2}$

3

$\begin{array}{ll}0 & 0 \\ 0 & 0\end{array}$

$\begin{array}{lll}0 & 0 & 0 \\ 0 & 0 & 0\end{array}$

$0 \quad 0$

$\begin{array}{llllllll}0 & 0 & 0 & 0 & 0 & 0 & 0 & 0 \\ 0 & 1 & 0 & 0 & 0 & 0 & 1 & 0 \\ 0 & 0 & 0 & 0 & 0 & 0 & 0 & 0 \\ 0 & 0 & 0 & 0 & 0 & 0 & 0 & 0 \\ 0 & 0 & 0 & 0 & 0 & 0 & 1 & 1\end{array}$

$\begin{array}{llllllllll}0 & 0 & 0 & 1 & 0 & 0 & 0 & 0 & 0 & 0 \\ 0 & 0 & 0 & 0 & 0 & 0 & 0 & 0 & 0 & 0 \\ 0 & 0 & 0 & 1 & 0 & 0 & 0 & 1 & 1 & 0 \\ 0 & 0 & 0 & 0 & 0 & 0 & 0 & 0 & 0 & 0 \\ 0 & 0 & 0 & 0 & 0 & 0 & 0 & 0 & 0 & 1\end{array}$

1
2
3
4
5

$\begin{array}{ll}0 & 0 \\ 0 & 0 \\ 0 & 0 \\ 0 & 0 \\ 1 & 0\end{array}$

0
0
0
0
0

$\begin{array}{ll}0 & 0 \\ 0 & 0 \\ 0 & 0 \\ 0 & 0 \\ 0 & 0\end{array}$

$\begin{array}{lllll}0 & 0 & 0 & 1 & 0 \\ 0 & 0 & 0 & 1 & 0 \\ 0 & 0 & 0 & 0 & 0 \\ 0 & 0 & 0 & 0 & 0 \\ 0 & 0 & 0 & 0 & 0\end{array}$ 
TABLE F.4. (contd)

Sediment

Treatment

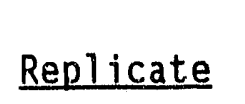

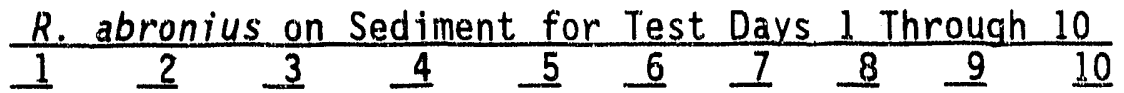

$\begin{array}{ll}0-C 6 & 1 \\ 0-C 6 & 2 \\ 0-C 6 & 3 \\ 0-c 6 & 4 \\ 0-C 6 & 5\end{array}$

$\begin{array}{lll}1 & 0 & 0 \\ 2 & 1 & 0 \\ 3 & 0 & 0 \\ 4 & 0 & 0 \\ 5 & 0 & 0\end{array}$

$0-C 5$

$0-C 5$

$0-C 5$

$0-C 5$

$0-C 5$

1
2
3
4
5

$0-C_{4}$

$0-C 4$

$0-C 4$

$0-C 4$

$0-C 4$

$\begin{array}{ll}0 & 0 \\ 0 & 0 \\ 0 & 0 \\ 0 & 0 \\ 0 & 0\end{array}$

1
2
3
4
5

$0 .-13$

$0-\mathrm{C3}$

$0-\mathrm{C3}$

$0-\mathrm{C} 3$

$0-C 3$

$0-\mathrm{C} 2$

$0-c 2$

$0-\mathrm{C2}$

$0-\mathrm{C2}$

$0-\mathrm{C} 2$

$\begin{array}{llllllllll}1 & 0 & 1 & 0 & 0 & 0 & 0 & 0 & 0 & 0 \\ 0 & 1 & 0 & 0 & 1 & 0 & 0 & 0 & 0 & 0 \\ 0 & 0 & 0 & 0 & 0 & 0 & 0 & 0 & 0 & 0 \\ 1 & 1 & 0 & 0 & 0 & 0 & 0 & 0 & 0 & 0 \\ 0 & 1 & 1 & 0 & 1 & 0 & 0 & 0 & 0 & 0\end{array}$

1
2
3
4
5

$\begin{array}{lll}0 & 0 & 0 \\ 0 & 0 & 0 \\ 0 & 0 & 0 \\ 0 & 0 & 0 \\ 0 & 0 & 0\end{array}$

$\begin{array}{lllllll}0 & 0 & 0 & 0 & 0 & 1 & 0 \\ 0 & 0 & 1 & 0 & 0 & 0 & 0 \\ 0 & 0 & 0 & 0 & 0 & 0 & 0 \\ 0 & 0 & 0 & 1 & 0 & 0 & 1 \\ 0 & 0 & 0 & 0 & 0 & 0 & 0\end{array}$

O-C1

$0-C 1$

$0-C 1$

$0-C 1$

$\mathrm{O}-\mathrm{Cl}$

1
2
3
4
5

0
0

0

0

$\begin{array}{ll}0 & 0 \\ 0 & 0 \\ 0 & 0 \\ 0 & 0 \\ 0 & 0\end{array}$

0
0
0
0
0
0
0
0
0
0

$\begin{array}{llllll}0 & 0 & 0 & 0 & 1 & 0 \\ 0 & 0 & 0 & 0 & 0 & 1 \\ 0 & 0 & 0 & 0 & 0 & 0 \\ 0 & 0 & 0 & 0 & 0 & 0 \\ 0 & 1 & 1 & 1 & 0 & 0\end{array}$

I-C2

$\mathrm{I}-\mathrm{C2}$

$\mathrm{I}-\mathrm{C} 2$

I-C2

I $-\mathrm{C} 2$

1
2
3
4
5
0
0
0
0

$\mathrm{I}-\mathrm{C} 3$

I-C3

I-C3

I-C3

I $-\mathrm{CB}$

1
2
3
4
5

$\begin{array}{llllllllll}0 & 0 & 0 & 0 & 0 & 0 & 1 & 1 & 2 & 1 \\ 0 & 0 & 0 & 0 & 0 & 0 & 0 & 0 & 0 & 0 \\ 0 & 0 & 1 & 1 & 0 & 0 & 0 & 0 & 0 & 0 \\ 0 & 0 & 0 & 0 & 0 & 0 & 0 & 0 & 1 & 0 \\ 0 & 0 & 0 & 0 & 0 & 0 & 0 & 0 & 0 & 0\end{array}$

PHASE III B

1
2
3
4
5

$\begin{array}{ll}0 & 0 \\ 0 & 0 \\ 0 & 0 \\ 1 & 1 \\ 0 & 0\end{array}$

$\begin{array}{lll}0 & 0 & 0 \\ 0 & 0 & 0 \\ 0 & 0 & 0 \\ 0 & 0 & 0 \\ 0 & 0 & 0\end{array}$

$\begin{array}{lllll}0 & 0 & 0 & 0 & 0 \\ 0 & 0 & 0 & 0 & 0 \\ 0 & 0 & 0 & 0 & 0 \\ 0 & 1 & 1 & 1 & 1 \\ 1 & 0 & 0 & 0 & 0\end{array}$

F. 8 
TABLE F.4. (contd)

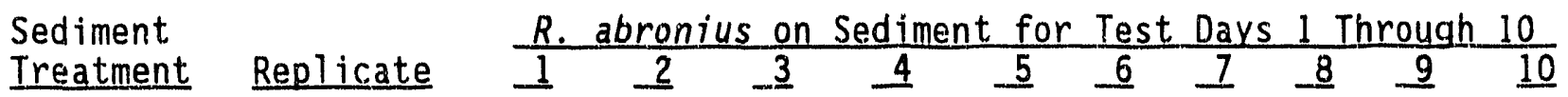

$\begin{array}{llllllllllll}I-C 8 & 1 & 0 & 0 & 0 & 0 & 0 & 0 & 0 & 0 & 0 & 0 \\ I-C B & 2 & 1 & 0 & 0 & 0 & 0 & 0 & 0 & 0 & 0 & 0 \\ I-C 8 & 3 & 0 & 1 & 0 & 0 & 0 & 0 & 0 & 0 & 0 & 0 \\ \text { I-C8 } & 4 & 0 & 0 & 0 & 0 & 0 & 0 & 0 & 0 & 0 & 0 \\ \text { I-CB } & 5 & 0 & 0 & 0 & 0 & 0 & 0 & 0 & 0 & 0 & 0 \\ R-A C & 1 & 0 & 0 & 0 & 0 & 0 & 0 & 0 & 0 & 0 & 0 \\ R-A C & 2 & 0 & 0 & 0 & 0 & 0 & 0 & 0 & 0 & 0 & 0 \\ \text { R-AC } & 3 & 0 & 0 & 0 & 0 & 0 & 0 & 0 & 0 & 0 & 0 \\ R-A C & 4 & 0 & 0 & 0 & 0 & 0 & 0 & 0 & 0 & 0 & 0 \\ \text { R-AC } & 5 & 0 & 0 & 0 & 0 & 0 & 0 & 0 & 0 & 0 & 0\end{array}$

$\begin{array}{llllllllllll}R-A M & 1 & 0 & 0 & 0 & 0 & 1 & 1 & 1 & 1 & 1 & 1 \\ R-A M & 2 & 0 & 0 & 0 & 1 & 0 & 0 & 0 & 0 & 0 & 0 \\ R-A M & 3 & 0 & 0 & 0 & 0 & 0 & 0 & 0 & 0 & 0 & 0 \\ R-A M & 4 & 0 & 0 & 0 & 0 & 1 & 0 & 0 & 0 & 0 & 0 \\ \text { R-AM } & 5 & 0 & 1 & 0 & 0 & 0 & 0 & 0 & 0 & 0 & 0\end{array}$

$\begin{array}{llllllllllll}R-B F & 1 & 1 & 1 & 0 & 0 & 0 & 0 & 0 & 0 & 0 & 1 \\ R-B F & 2 & 0 & 0 & 0 & 0 & 0 & 0 & 0 & 0 & 0 & 0 \\ R-B F & 3 & 0 & 0 & 0 & 0 & 0 & 0 & 0 & 0 & 0 & 0 \\ R-B F & 4 & 0 & 0 & 0 & 0 & 0 & 0 & 0 & 0 & 0 & 0 \\ R-B F & 5 & 0 & 0 & 0 & 0 & 0 & 0 & 0 & 0 & 0 & 0\end{array}$

$\mathrm{R}-\mathrm{OS}$

R-OS

$\mathrm{R}-\mathrm{OS}$

R-OS

R-OS

$\begin{array}{lllllllllll}1 & 0 & 0 & 0 & 1 & 0 & 0 & 0 & 0 & 0 & 0 \\ 2 & 0 & 0 & 0 & 0 & 0 & 0 & 0 & 0 & 0 & 0 \\ 3 & 0 & 0 & 0 & 0 & 0 & 0 & 0 & 0 & 0 & 0 \\ 4 & 0 & 0 & 0 & 0 & 0 & 0 & 0 & 0 & 1 & 1 \\ 5 & 0 & 0 & 0 & 0 & 0 & 1 & 0 & 1 & 0 & 0\end{array}$

$R-P C$

$R-P C$

$R-P C$

$R-P C$

$R-P C$

1
2
3
4
5

$R-P F$

$R-P F$

$R-P F$

$R-P F$

$R-P F$

1
2
3
4
5

$\begin{array}{llllllllll}0 & 0 & 0 & 0 & 0 & 0 & 0 & 0 & 0 & 0\end{array}$ $\begin{array}{llllllllll}0 & 0 & 0 & 0 & 0 & 0 & 0 & 0 & 0 & 0\end{array}$ $\begin{array}{llllllllll}0 & 0 & 0 & 0 & 0 & 0 & 0 & 0 & 0 & 0\end{array}$ $\begin{array}{llllllllll}0 & 0 & 0 & 0 & 0 & 0 & 0 & 0 & 0 & 0 \\ 0 & 0 & 0 & 0 & 0 & 0 & 0 & 0 & 0 & 0\end{array}$

C-SB

C-SB

$C-S B$ $\begin{array}{lllllllllll}1 & 0 & 0 & 0 & 0 & 0 & 0 & 0 & 0 & 0 & 0 \\ 2 & 0 & 0 & 0 & 0 & 0 & 0 & 0 & 0 & 0 & 0\end{array}$ $\begin{array}{lllllllllll}3 & 0 & 0 & 0 & 0 & 2 & 2 & 0 & 0 & 0 & 0\end{array}$ $\begin{array}{lllllllllll}4 & 0 & 0 & 0 & 0 & 0 & 0 & 0 & 0 & 0 & 0 \\ 5 & 0 & 0 & 0 & 0 & 0 & 0 & 0 & 0 & 0 & 0\end{array}$ $C-S B$

C-SB

$\begin{array}{llllllllll}0 & 0 & 0 & 0 & 0 & 0 & 0 & 0 & 0 & 0 \\ 0 & 0 & 0 & 0 & 0 & 0 & 0 & 0 & 0 & 0 \\ 0 & 0 & 0 & 0 & 0 & 0 & 0 & 0 & 0 & 0 \\ 0 & 0 & 0 & 0 & 0 & 0 & 1 & 1 & 1 & 1 \\ 0 & 0 & 0 & 0 & 0 & 0 & 0 & 0 & 0 & 0\end{array}$


TABLE F.4. (contd)

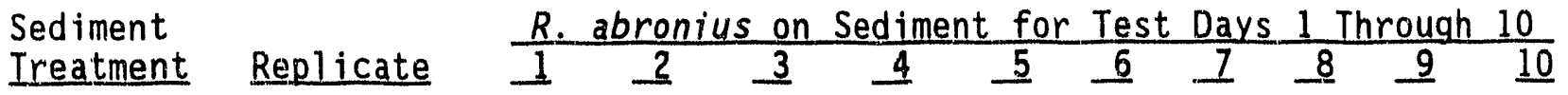

C-WB

C-WB

C-WB

C-WB

C-WB

1
2
3
4
5

0
0
0
0
0

$\begin{array}{ll}1 & 1 \\ 1 & 0 \\ 0 & 1 \\ 0 & 0 \\ 0 & 0\end{array}$

$\begin{array}{lllllll}1 & 1 & 1 & 0 & 0 & 0 & 0 \\ 1 & 0 & 0 & 0 & 0 & 1 & 0 \\ 1 & 1 & 1 & 1 & 1 & 0 & 1 \\ 0 & 0 & 0 & 0 & 0 & 0 & 0 \\ 0 & 0 & 0 & 0 & 0 & 0 & 0\end{array}$


IABLE F.5. Daily Observations of $R$. abronius Floating on Water Surface During 10-Day Solid-Phase Test, Oakland Harbor Phase III B Project

Sediment

Treatiment Replicate

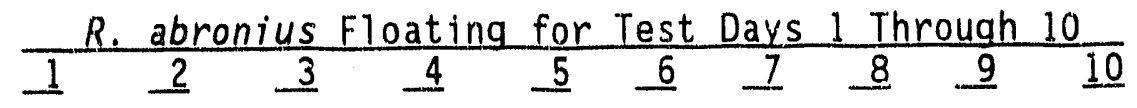

$0-\mathrm{C} 13$

$0-C 13$

$0-\mathrm{C} 13$

$0-\mathrm{Cl3}$

$0-\mathrm{C} 13$

1
2
3
4
5

$\begin{array}{llllllllll}0 & 0 & 1 & 2 & 1 & 3 & 1 & 0 & 1 & 3 \\ 1 & 2 & 3 & 0 & 3 & 2 & 3 & 6 & 6 & 1 \\ 0 & 0 & 0 & 0 & 0 & 1 & 1 & 0 & 3 & 0 \\ 1 & 2 & 2 & 5 & 6 & 3 & 3 & 2 & 0 & 3 \\ 1 & 3 & 2 & 0 & 3 & 3 & 2 & 2 & 2 & 2\end{array}$

$0-\mathrm{C} 12$

$0-\mathrm{Cl} 2$

$0-\mathrm{C} 12$

$0-\mathrm{C} 12$

$0-\mathrm{C} 12$

1
2
3
4
5

$0-C 11$

$0-C 11$

$0-C 11$

$0-C 11$

0-C11

2
0
0
0
0

$\begin{array}{lllll}0 & 2 & 2 & 0 & 1 \\ 1 & 0 & 1 & 1 & 1 \\ 1 & 0 & 0 & 1 & 1 \\ 0 & 1 & 2 & 0 & 4 \\ 2 & 3 & 3 & 0 & 3\end{array}$

$\begin{array}{llll}1 & 3 & 1 & 0 \\ 0 & 0 & 0 & 1 \\ 0 & 0 & 0 & 1 \\ 0 & 4 & 2 & 4 \\ 3 & 1 & 3 & 0\end{array}$

$0-C 10$

$0-C 10$

$0-C 10$

$0-C 10$

$0-\mathrm{C} 10$

1
2
3
4
5

1
2
3
4
5

$\begin{array}{ll}0 & 1 \\ 0 & 3 \\ 0 & 0 \\ 0 & 0 \\ 2 & 0\end{array}$

$\begin{array}{ll}0 & 2 \\ 4 & 4 \\ 1 & 4 \\ 0 & 3 \\ 2 & 0\end{array}$

$\begin{array}{lll}3 & 2 & 2 \\ 1 & 6 & 4 \\ 1 & 3 & 2 \\ 3 & 2 & 3 \\ 1 & 1 & 0\end{array}$

$\begin{array}{lll}5 & 4 & 2 \\ 2 & 1 & 2 \\ 1 & 4 & 5 \\ 1 & 3 & 4 \\ 0 & 0 & 1\end{array}$

$0-19$

$0-C 9$

$0-C 9$

$0-c 9$

$0-C 9$

$0-18$

$0-c 8$

$0-C 8$

$0-C 8$

$0-C 8$

$\begin{array}{llll}0 & 1 & 4 & 1 \\ 0 & 1 & 1 & 3 \\ 2 & 2 & 3 & 4 \\ 0 & 1 & 2 & 2 \\ 0 & 1 & 3 & 4\end{array}$

$\begin{array}{llllll}0 & 2 & 1 & 3 & 4 & 3 \\ 3 & 5 & 1 & 6 & 3 & 5 \\ 1 & 3 & 4 & 3 & 2 & 2 \\ 6 & 3 & 6 & 2 & 3 & 3 \\ 2 & 4 & 3 & 3 & 3 & 1\end{array}$

$0-C 7$

$0-C .7$

$0-\mathrm{C} 7$

O-C7

$0-C 7$

1
2
3
4
5

$\begin{array}{llllllllll}0 & 3 & 1 & 2 & 1 & 3 & 1 & 1 & 1 & 3 \\ 1 & 0 & 1 & 1 & 2 & 1 & 3 & 5 & 3 & 1 \\ 0 & 0 & 0 & 2 & 1 & 0 & 0 & 5 & 2 & 3 \\ 0 & 1 & 0 & 1 & 1 & 1 & 0 & 1 & 3 & 0 \\ 3 & 1 & 2 & 1 & 1 & 0 & 0 & 2 & 0 & 0\end{array}$

$\begin{array}{llllllllll}1 & 2 & 3 & 2 & 2 & 5 & 4 & 4 & 3 & 4 \\ 1 & 0 & 2 & 2 & 2 & 3 & 3 & 2 & 2 & 5 \\ 0 & 2 & 2 & 6 & 5 & 6 & 3 & 2 & 2 & 6 \\ 0 & 0 & 0 & 0 & 0 & 3 & 1 & 2 & 1 & 3 \\ 1 & 2 & 3 & 2 & 4 & 3 & 3 & 3 & 5 & 4\end{array}$

$\begin{array}{llllllllll}0 & 2 & 0 & 4 & 3 & 2 & 4 & 5 & 1 & 4 \\ 0 & 1 & 3 & 3 & 3 & 3 & 2 & 4 & 3 & 8 \\ 0 & 1 & 0 & 1 & 2 & 1 & 3 & 0 & 3 & 2 \\ 0 & 1 & 1 & 1 & 2 & 4 & 4 & 1 & 0 & 1 \\ 0 & 1 & 1 & 4 & 0 & 5 & 1 & 2 & 0 & 1\end{array}$ 
TABLE F.5. (contd)

Sediment

Treatment

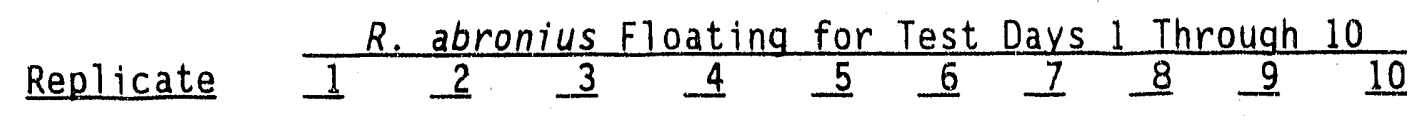

$0-C 6$

1
2
3
4
5
1
2
3
4
5

$\begin{array}{lll}1 & 5 \\ 0 & 1\end{array}$

$0-\mathrm{C} 6$

$0-\mathrm{C} 6$

$0-C 6$

$0-C 5$

$0-C 5$

$0-C 5$

$0-C 5$

$0-C 5$

$0-C 4$

$0-C 4$

$0-C 4$

$0-\mathrm{C} 4$

$0-C 4$

$0-\mathrm{C} 3$

$0-C 3$

$0-\mathrm{C} 3$

$0-C 3$

$0-\mathrm{C} 3$

$0-\mathrm{C2}$

$0-c 2$

$0-\mathrm{C} 2$

$0-\mathrm{C2}$

$0-\mathrm{C2}$

O-C1

$0-C 1$

$0-\mathrm{Cl}$

$0-C 1$

$0-C 1$

I $-\mathrm{C} 2$

$\mathrm{I}-\mathrm{C} 2$

$\mathrm{I}-\mathrm{C} 2$

I-C2

I-C2

1
2
3
4
5

$0 \quad 1$

$\begin{array}{lllll}0 & 1 & 4 & 3 & 3\end{array}$

$\begin{array}{llllllll}3 & 6 & 2 & 4 & 3 & 3 & 4 & 3 \\ 0 & 2 & 1 & 2 & 1 & 4 & 2 & 3\end{array}$

$\begin{array}{lllll}3 & 2 & 2 & 0 & 3 \\ 4 & 1 & 2 & 3 & 4\end{array}$

$\begin{array}{llllllllll}0 & 0 & 0 & 2 & 0 & 3 & 3 & 1 & 4 & 3\end{array}$

$\begin{array}{llllllllll}0 & 0 & 0 & 0 & 0 & 1 & 2 & 1 & 2 & 0 \\ 0 & 1 & 0 & 1 & 0 & 0 & 2 & 0 & 1 & 0\end{array}$

$\begin{array}{llllllllll}0 & 1 & 0 & 1 & 0 & 0 & 2 & 0 & 1 & 0 \\ 1 & 0 & 1 & 0 & 1 & 1 & 0 & 0 & 0 & 0\end{array}$

$\begin{array}{llllllllll}1 & 0 & 1 & 0 & 1 & 1 & 0 & 0 & 0 & 0 \\ 0 & 0 & 0 & 0 & 0 & 1 & 2 & 2 & 0 & 0\end{array}$

$\mathrm{I}-\mathrm{C} 3$

$\mathrm{I}-\mathrm{C3}$

$1-C 3$

I $-\mathrm{C3}$

I $-\mathrm{C3}$

1
2
3
4
5

$\begin{array}{llllllllll}0 & 1 & 5 & 0 & 2 & 0 & 2 & 4 & 4 & 4 \\ 0 & 0 & 2 & 3 & 2 & 3 & 4 & 3 & 4 & 3 \\ 0 & 0 & 0 & 1 & 0 & 3 & 4 & 1 & 0 & 0 \\ 1 & 1 & 2 & 2 & 2 & ? & 2 & 3 & 1 & 3 \\ 1 & 0 & 1 & 1 & 1 & 3 & 1 & 2 & 3 & 2\end{array}$

$\begin{array}{llllllllll}0 & 1 & 0 & 0 & 0 & 0 & 0 & 2 & 0 & 0 \\ 0 & 0 & 0 & 0 & 0 & 1 & 0 & 0 & 0 & 0 \\ 0 & 0 & 0 & 0 & 0 & 1 & 1 & 1 & 2 & 0 \\ 0 & 0 & 0 & 0 & 0 & 0 & 0 & 0 & 0 & 0 \\ 0 & 0 & 0 & 2 & 0 & 0 & 0 & 1 & 0 & 0\end{array}$

$\begin{array}{lllllllllll}1 & 0 & 1 & 4 & 1 & 3 & 3 & 3 & 3 & 3 & 2 \\ 2 & 0 & 0 & 1 & 1 & 3 & 0 & 1 & 3 & 0 & 0 \\ 3 & 0 & 0 & 1 & 2 & 3 & 3 & 1 & 3 & 1 & 1 \\ 4 & 0 & 0 & 3 & 3 & 3 & 4 & 4 & 4 & 2 & 3 \\ 5 & 0 & 1 & 2 & 2 & 2 & 1 & 3 & 1 & 1 & 1\end{array}$

$\begin{array}{lllllllllll}1 & 0 & 0 & 0 & 0 & 0 & 0 & 0 & 0 & 0 & 0 \\ 2 & 0 & 0 & 0 & 0 & 0 & 1 & 0 & 0 & 0 & 0 \\ 3 & 1 & 0 & 1 & 1 & 0 & 0 & 1 & 0 & 0 & 1 \\ 4 & 0 & 0 & 0 & 0 & 0 & 0 & 0 & 2 & 0 & 0 \\ 5 & 0 & 0 & 0 & 1 & 1 & 0 & 0 & 0 & 0 & 1\end{array}$

PHASE III B

$\begin{array}{lllllllllll}1 & 0 & 0 & 0 & 0 & 1 & 1 & 1 & 0 & 0 & 1 \\ 2 & 0 & 1 & 0 & 0 & 0 & 3 & 2 & 0 & 1 & 0 \\ 3 & 0 & 1 & 0 & 0 & 0 & 0 & 1 & 0 & 0 & 2 \\ 4 & 1 & 0 & 1 & 0 & 1 & 1 & 2 & 2 & 2 & 1 \\ 5 & 0 & 0 & 1 & 0 & 0 & 0 & 1 & 0 & 0 & 0\end{array}$

$\begin{array}{lllllllllll}1 & 0 & 0 & 0 & 0 & 0 & 0 & 0 & 0 & 0 & 0 \\ 2 & 0 & 0 & 0 & 3 & 0 & 0 & 0 & 0 & 0 & 0 \\ 3 & 0 & 0 & 0 & 0 & 0 & 0 & 0 & 0 & 0 & 0 \\ 4 & 0 & 0 & 0 & 0 & 0 & 0 & 1 & 0 & 0 & 0 \\ 5 & 0 & 1 & 0 & 0 & 0 & 0 & 0 & 0 & 0 & 0\end{array}$

F.12 
TABLE F.5. (contd)

Sediment

Treatment Replicate

R. abronius Floating for Test Days 1 Through 10

I - C8

I-C8

I $-\mathrm{CB}$

I $-\mathrm{C} 8$

I $-\mathrm{CB}$

$\mathrm{R}-\mathrm{AC}$

$R-A C$

$R-A C$

$R-A C$

$R-A C$

1
2
3
4
5

R-AM

$R-A M$

R-AM

R-AM

R-AM

1
2
3
4
5

$R=B F$

$R-B F$

$R-B F$

$R-B F$

$R-B F$

R-OS

$R-0 S$

$\mathrm{R}-\mathrm{OS}$

$\mathrm{R}-\mathrm{OS}$

R-OS

$R-P C$

$R-P C$

$R-P C$

$R-P C$

R-PC

$R-P F$

$R-P F$

$R-P F$

$R-P F$

$R-P F$

$C-S B$

$C-S B$

$C-S B$

$C-S B$

$C-S B$

1
2
3
4
5

1
2
3
4
5

$\begin{array}{lll}0 & 0 & 0 \\ 0 & 0 & 0 \\ 0 & 0 & 0 \\ 0 & 0 & 0 \\ 0 & 0 & 0\end{array}$

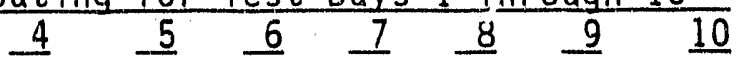

$\begin{array}{llllllllll}0 & 1 & 0 & 0 & 0 & 1 & 0 & 0 & 1 & 0 \\ 0 & 0 & 0 & 1 & 0 & 0 & 0 & 0 & 0 & 0 \\ 0 & 0 & 0 & 1 & 1 & 0 & 1 & 0 & 1 & 0 \\ 0 & 0 & 0 & 1 & 0 & 0 & 0 & 0 & 0 & 0 \\ 0 & 0 & 0 & 0 & 0 & 1 & 0 & 0 & 0 & 0\end{array}$

$\begin{array}{llllllllll}0 & 0 & 0 & 0 & 0 & 0 & 0 & 0 & 0 & 0 \\ 0 & 0 & 0 & 0 & 0 & 0 & 0 & 0 & 0 & 0 \\ 0 & 0 & 0 & 0 & 0 & 0 & 0 & 0 & 0 & 0 \\ 1 & 0 & 0 & 0 & 0 & 0 & 0 & 0 & 0 & 0 \\ 0 & 0 & 0 & 0 & 0 & 0 & 0 & 0 & 0 & 0\end{array}$

$\begin{array}{llllllllll}0 & 0 & 0 & 0 & 0 & 0 & 1 & 0 & 0 & 0\end{array}$

$\begin{array}{llllllllll}0 & 0 & 0 & 0 & 0 & 0 & 0 & 0 & 0 & 0\end{array}$

$\begin{array}{llllllllll}0 & 0 & 0 & 0 & 0 & 0 & 0 & 0 & 0 & 0\end{array}$

$\begin{array}{llllllllll}0 & 0 & 0 & 0 & 0 & 0 & 0 & 0 & 0 & 0 \\ 0 & 0 & 0 & 0 & 1 & 0 & 0 & 0 & 0 & 0\end{array}$ PHASE III B

$\begin{array}{llllllllll}1 & 0 & 1 & 0 & 0 & 0 & 0 & 1 & 0 & 0\end{array}$ $\begin{array}{llllllllll}1 & 0 & 1 & 0 & 0 & 0 & 0 & 1 & 0 & 0\end{array}$ $\begin{array}{lllllllllll}3 & 0 & 1 & 0 & 0 & 0 & 1 & 1 & 1 & 0 & 0 \\ 4 & 0 & 0 & 0 & 1 & 0 & 1 & 1 & 0 & 0 & 0 \\ 5 & 0 & 0 & 1 & 3 & 1 & 0 & 1 & 0 & 0 & 0\end{array}$

5
$\begin{array}{lllllllllll}1 & 0 & 0 & 0 & 0 & 0 & 0 & 0 & 0 & 0 & 0 \\ 2 & 0 & 0 & 0 & 0 & 0 & 0 & 0 & 0 & 0 & 0 \\ 3 & 0 & 1 & 0 & 0 & 0 & 0 & 0 & 0 & 0 & 0 \\ 4 & 0 & 0 & 0 & 0 & 0 & 0 & 0 & 0 & 0 & 0 \\ 5 & 0 & 0 & 0 & 0 & 0 & 0 & 0 & 0 & 0 & 0\end{array}$ $\begin{array}{lllllllllll}1 & 0 & 0 & 0 & 0 & 1 & 0 & 0 & 0 & 0 & 0 \\ 2 & 0 & 0 & 0 & 1 & 0 & 0 & 0 & 0 & 0 & 0 \\ 3 & 0 & 0 & 0 & 0 & 0 & 0 & 0 & 0 & 0 & 0 \\ 4 & 0 & 0 & 0 & 0 & 0 & 0 & 0 & 0 & 0 & 0 \\ 5 & 0 & 0 & 0 & 0 & 0 & 0 & 0 & 0 & 0 & 0\end{array}$ 1
2
3
4
5 $\begin{array}{llllllllll}0 & 0 & 0 & 0 & 0 & 0 & 0 & 0 & 0 & 0 \\ 0 & 1 & 1 & 2 & 1 & 0 & 1 & 0 & 0 & 1 \\ 0 & 1 & 1 & 0 & 0 & 1 & 0 & 1 & 0 & 1 \\ 0 & 0 & 0 & 0 & 0 & 2 & 2 & 1 & 1 & 0 \\ 0 & 0 & 0 & 1 & 0 & 0 & 0 & 0 & 0 & 1\end{array}$

$$
\text { F.13 }
$$


TABLE F.5. (contd)

Sediment

Treatment

$C-W B$

$C-W B$

$C-W B$

$C-W B$

C-WB

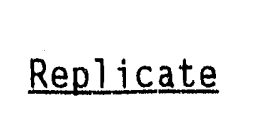

\begin{tabular}{|c|c|c|c|c|c|c|c|c|c|c|}
\hline & R. $a b r$ & ius & at & tin & & est & Days & 1 Th & 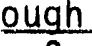 & \\
\hline 1 & $\underline{2}$ & 3 & 4 & & $\underline{5}$ & $\underline{6}$ & 7 & $\underline{8}$ & $\underline{9}$ & $\underline{10}$ \\
\hline 0 & 0 & 0 & 0 & & 0 & 0 & 0 & 0 & 0 & \\
\hline & 0 & 0 & & & 0 & 0 & 0 & & 0 & \\
\hline 0 & 0 & 0 & 0 & 0 & 0 & 0 & 0 & 0 & 3 & \\
\hline 0 & 0 & 0 & & 0 & 0 & 0 & 0 & 0 & 0 & \\
\hline 0 & 0 & 0 & & & 0 & 0 & 0 & 0 & 0 & \\
\hline
\end{tabular}


TABLE F.6. Survival Data for All Replicates in 4-Day R. abronius Reference Toxicant Test, Oakland Harbor Phase III B Project

\begin{tabular}{ccccc}
$\begin{array}{c}\text { Cadmium } \\
\text { Concentration }\end{array}$ & Replicate & Live & $\begin{array}{c}\text { R. abronius } \\
\text { Dead } \\
\text { ar missing }\end{array}$ & $\begin{array}{c}\text { Mean } \\
\text { Proportion } \\
\text { Surviving }\end{array}$ \\
\cline { 5 - 6 } 0 & 1 & 20 & 0 & \\
0 & 2 & 19 & 1 & 0.98 \\
0 & 3 & 20 & 0 & \\
0.5 & 1 & 16 & 4 & 0.75 \\
0.5 & 2 & 15 & 5 & \\
0.5 & 3 & 14 & 6 & 0.40 \\
1 & 1 & 10 & 10 & \\
1 & 2 & 8 & 12 & \\
1 & 3 & 6 & 14 & 0.13 \\
2 & 1 & 2 & 18 & \\
2 & 2 & 4 & 16 & \\
2 & 3 & 2 & 18 & 0.00 \\
4 & 1 & 0 & 20 &
\end{tabular}


IABLEF.7. Water Quality Summary for 4-Day $R$. abronius Reference Toxicant Test, Oakland Harbor Phase III B Project

\begin{tabular}{|c|c|c|c|c|c|c|c|c|}
\hline \multirow{2}{*}{$\begin{array}{l}\text { Cadmi um } \\
\text { Concentration (ma/L) }\end{array}$} & \multicolumn{2}{|c|}{$\begin{array}{l}\text { Temperature } \\
\left({ }^{\circ} \mathrm{C}\right)\end{array}$} & \multicolumn{2}{|c|}{$\mathrm{pH}$} & \multicolumn{2}{|c|}{$\begin{array}{c}\text { Dissolved } \\
\text { Oxygen } \\
\text { (ma/L) } \\
\end{array}$} & \multicolumn{2}{|c|}{$\begin{array}{c}\text { Salinity } \\
(0 / 00)\end{array}$} \\
\hline & Min & $\operatorname{Max}$ & Min & Max & Min & $\operatorname{Max}$ & $\operatorname{Min}$ & Max \\
\hline $\begin{array}{l}\text { Acceptable } \\
\text { Range }\end{array}$ & 13.0 & 17.0 & 7.35 & 8.35 & 6.0 & $N_{A}(a)$ & 28.0 & 32.0 \\
\hline 0 & 15.8 & 16.5 & 7.90 & 8.15 & 7.2 & 8.0 & 29.5 & 31.0 \\
\hline 0.5 & 15.9 & 16.5 & 7.89 & 8.13 & 7.4 & 8.0 & 29.0 & 31.0 \\
\hline 1 & 158 & 16.4 & 7.90 & 8.10 & 7.2 & 8.0 & 30.0 & 32.0 \\
\hline 2 & 15.9 & 16.5 & 7.80 & 8.07 & 7.3 & 8.0 & 30.0 & 31.0 \\
\hline 4 & 15.8 & 16.4 & 7.74 & 8.01 & 7.3 & 8.0 & 30.0 & 31.0 \\
\hline
\end{tabular}

(a) Not applicable. 
TABLEF.8. Daily Observations of $R$. abronius Floating on Water Surface During 4-Day Reference Toxicant Test, Oakland Harbor Phase III B Project

\begin{tabular}{cc}
$\begin{array}{c}\text { Cadmium } \\
\text { Concentration }(m g / L)\end{array}$ & Replicate \\
\hline 0 & 1 \\
0 & 2 \\
0 & 3 \\
0.5 & 1 \\
0.5 & 2 \\
0.5 & 3 \\
1 & \\
1 & 1 \\
1 & 2 \\
2 & 3 \\
2 & 1 \\
2 & 2 \\
4 & 3 \\
4 & 1 \\
4 & 2 \\
& 3
\end{tabular}

$\frac{R \text {. abronius Test Days } 1 \text { Through } 4}{1}$

$\begin{array}{lll}0 & 3 & 4\end{array}$

1

1

3

$\begin{array}{ll}0 & 2 \\ 1 & 1 \\ 0 & 0\end{array}$

$\begin{array}{ll}2 & 2 \\ 1 & 2 \\ 0 & 0\end{array}$

0

0

$\begin{array}{ll}2 & 1 \\ 1 & 3\end{array}$

4

40

3
5 
APPENDIX G

10-DAY ELOW-THROUGH SOLID-PHASE TEST WITH C. STIGMAEUS 
TABLE G.1. Test Results for All Replicates in 10-Day C. stigmaeus Solid-Phase Test, Oakland Harbor Phase III B Project

\begin{tabular}{|c|c|c|c|c|}
\hline \multirow[b]{2}{*}{$\begin{array}{l}\text { Sediment } \\
\text { Ireatment }\end{array}$} & \multirow[b]{2}{*}{ Replicate } & \multicolumn{2}{|c|}{ C. stigmaeus } & \multirow{2}{*}{$\begin{array}{l}\text { Mean } \\
\text { Proportion } \\
\text { Surviving } \\
\end{array}$} \\
\hline & & Live & $\begin{array}{c}\text { Dead } \\
\text { or Missing }\end{array}$ & \\
\hline $\begin{array}{l}0-C 13 \\
0-C 13 \\
0-C 13 \\
0-C 13 \\
0-C 13\end{array}$ & $\begin{array}{l}1 \\
2 \\
3 \\
4 \\
5\end{array}$ & $\begin{array}{r}9 \\
10 \\
9 \\
10 \\
9\end{array}$ & $\begin{array}{l}1 \\
0 \\
1 \\
0 \\
1\end{array}$ & .94 \\
\hline $\begin{array}{l}0-C 12 \\
0-C 12 \\
0-C 12 \\
0-C 12 \\
0-C 12\end{array}$ & $\begin{array}{l}1 \\
2 \\
3 \\
4 \\
5\end{array}$ & $\begin{array}{l}10 \\
10 \\
10 \\
10 \\
10\end{array}$ & $\begin{array}{l}0 \\
0 \\
0 \\
0 \\
0\end{array}$ & 1.00 \\
\hline $\begin{array}{l}0-C 11 \\
0-C 11 \\
0-C 11 \\
0-C 11 \\
0-C 11\end{array}$ & $\begin{array}{l}1 \\
2 \\
3 \\
4 \\
5\end{array}$ & $\begin{array}{r}9 \\
10 \\
10 \\
10 \\
10\end{array}$ & $\begin{array}{l}1 \\
0 \\
0 \\
0 \\
0\end{array}$ & .98 \\
\hline $\begin{array}{l}0-C 10 \\
0-C 10 \\
0-C 10 \\
0-C 10 \\
0-C 10\end{array}$ & $\begin{array}{l}1 \\
2 \\
3 \\
4 \\
5\end{array}$ & $\begin{array}{r}10 \\
9 \\
10 \\
10 \\
9\end{array}$ & $\begin{array}{l}0 \\
1 \\
0 \\
0 \\
1\end{array}$ & .96 \\
\hline $\begin{array}{l}0-C 9 \\
0-C 9 \\
0-C 9 \\
0-C 9 \\
0-C 9\end{array}$ & $\begin{array}{l}1 \\
2 \\
3 \\
4 \\
5\end{array}$ & $\begin{array}{l}10 \\
10 \\
10 \\
10 \\
10\end{array}$ & $\begin{array}{l}0 \\
0 \\
0 \\
0 \\
0\end{array}$ & 1.00 \\
\hline $\begin{array}{l}0-c 8 \\
0-c 8 \\
0-c 8 \\
0-c 8 \\
0-c 8\end{array}$ & $\begin{array}{l}1 \\
2 \\
3 \\
4 \\
5\end{array}$ & $\begin{array}{l}10 \\
10 \\
10 \\
10 \\
10\end{array}$ & $\begin{array}{l}0 \\
0 \\
0 \\
0 \\
0\end{array}$ & 1.00 \\
\hline $\begin{array}{l}0-C 7 \\
0-C 7 \\
0-C 7 \\
0-C 7 \\
0-C 7\end{array}$ & $\begin{array}{l}1 \\
2 \\
3 \\
4 \\
5\end{array}$ & $\begin{array}{l}10 \\
10 \\
10 \\
10 \\
10\end{array}$ & $\begin{array}{l}0 \\
0 \\
0 \\
0 \\
0\end{array}$ & 1.00 \\
\hline
\end{tabular}


IABLE G.1. (contd)

\section{Sediment \\ Treatment}

$0-C 6$

$0-C E$

0 C. 6

$0-C E$

$0-\mathrm{C \sigma}$

$0-C 5$

$0-C 5$

$0-C 5$

$0-C 5$

$0-C 5$

$0-\mathrm{C} 4$

$0-C 4$

$0-C 4$

$0-\mathrm{C} 4$

$0-C_{4}$

$0-C_{3}$

$0-C 3$

$0-\mathrm{C3}$

$0-\mathrm{C3}$

$0-\mathrm{C} 3$

$0 \cdots C 2$

$0-C 2$

$0-\mathrm{C2}$

$0-\mathrm{C} 2$

$0-\mathrm{C2}$

$0-C 1$

$0-C 1$

$0-C 1$

$0-C]$

$\mathrm{O}-\mathrm{Cl}$

I $-\mathrm{C} 2$

I $-\mathrm{C} 2$

$\mathrm{I}-\mathrm{C} 2$

I-C2

$1-\mathrm{C} 2$

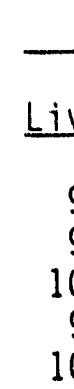

C. stiqmaeus

Dead

or Missing

1

2

3

4
5

1

2

3

4
5

1
2
3
4
5

1

3

4
5

1

2

3

4
5

10

1

3

4

5

1
2
3
4
5

10

10

10

$10^{(a)}$

20

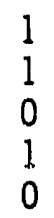

10

10

10

9
10

10

10

10

10

10

10

10

10

10

9

10

10

10

$10 \quad 0$

10

10

9

10
Mean Proportion

Surviving

.94

.98

1.00

1.00

1.00

.98

.98

PHASE III B

G.2 
IABLE G.1. (contd)

\section{Sediment \\ Treatment}

I - C 3

I-C3

I - C3

I $-\mathrm{C} 3$

I $-\mathrm{C} 3$

I - C 8

I $-\mathrm{CB}$

I $-\mathrm{C} 8$

I $-\mathrm{C} 8$

I - C8

$R-A C$

$R-A C$

$R-A C$

$R-A C$

$R-A C$

$R-A M$

$R-A M$

$R-A M$

$R-A M$

$R-A M$

$R-B F$

$R-B F$

$R-B F$

$R-B F$

$R-B F$

$R-O S$

$R-O S$

$R-O S$

$R-O S$

R-OS

$R-P C$

$R-P C$

$R-P C$

$R-P C$

$R-P C$

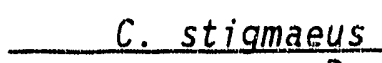

Dead

Replicate

1

2

4
5

1
2
3
4
5

1

2

3

4

5

1

2

3

4
5

1

2

3

5

1

2

3

4

5

1
2
3
4
5

Liv
10
10
9
10
9

$\begin{array}{ll}10 & 0 \\ 10 & 0\end{array}$

$\begin{array}{ll}9 & 1 \\ 10 & 0\end{array}$

91

$10 \quad 0$

$10 \quad 0$

10 (a) 0

9

10

10

10

10

10

10

10

10

10

10

10

10

10

10

10

10

10

10

10

10

10

10

10

10 or Missing
1.00

\begin{tabular}{l}
$\quad$ Mean \\
Proportion \\
Surviving \\
\hline
\end{tabular}

.96

.98

1.00

1.00

1.00

0

0
0

0
0

1.00 
TABLE G.1. (contd)

Sediment

Treatment

$R-P F$

$R-P F$

$R-P F$

$R-P F$

$R-P F$

C-SB

C-SB

C-SB

C-SB

C-SB

C-SD

C-SD

C.SD

$C-S D$

$C-S D$

$\frac{\text { C. stigmaeus }}{\text { Live } \quad \text { or Missing }}$

\section{Replicate}

1

2

3

4

5

1

2

3

4

5

1
2
3
4
5
Mean

Proportion

Surviving

10

10

10

10

10

10

10

10

10

10

10

10

10

10

$20^{(a)}$
1.00

1.00

0

0

0

0

0

0

0

0

0

0

0

0

1.00

(a) 30 organisms were added to the tank at initiation.

(b) Not Initiated. Only 4 replicates of I-C8 were initiated due to lack of sediment. 
IABLE G.2. Water Quality Summary for 10-Day C. stigmaeus Solid-Phase Test, Oaki and Harbor Phase III B Project

\begin{tabular}{|c|c|c|c|c|c|c|c|c|c|c|}
\hline \multirow{3}{*}{$\begin{array}{l}\text { Sediment } \\
\text { Ireatment } \\
\text { Acceptable } \\
\text { Range }\end{array}$} & \multicolumn{2}{|c|}{$\begin{array}{c}\text { Temperature } \\
\left({ }^{\circ} \mathrm{C}\right)\end{array}$} & \multicolumn{2}{|c|}{$\mathrm{pH}$} & \multicolumn{2}{|c|}{$\begin{array}{c}\text { Oissolved } \\
\text { Oxygen } \\
\text { (mg/L) }\end{array}$} & \multicolumn{2}{|c|}{$\begin{array}{c}\text { Salinity } \\
(00 / 0)\end{array}$} & \multicolumn{2}{|c|}{$\begin{array}{c}\text { Flow Rates } \\
\text { (mL/min) }\end{array}$} \\
\hline & Min & $\operatorname{Max}$ & Min & $\operatorname{Max}$ & Min & $\operatorname{Max}$ & Min & Max & Min & Max \\
\hline & 13.0 & 17.0 & 7.35 & 8.35 & 4.0 & NA $(a)$ & 28.5 & 32.5 & 115 & 135 \\
\hline $0-\operatorname{Cl3}$ & 13.6 & 15.0 & 7.77 & 7.97 & 7.3 & 8.0 & 30.0 & 31.5 & $112^{(b)}$ & \\
\hline $0-C 12$ & $\begin{array}{l}10.0 \\
13.6\end{array}$ & $\begin{array}{l}13.0 \\
14.8\end{array}$ & 7.78 & 8.10 & $\begin{array}{l}7.3 \\
7.3\end{array}$ & $\begin{array}{l}0.0 \\
8.1\end{array}$ & 30.0 & 32.0 & $\begin{array}{l}116 \\
116\end{array}$ & $\begin{array}{l}134 \\
132\end{array}$ \\
\hline $0-C 11$ & 13.6 & 14.9 & 7.77 & 7.94 & 7.4 & 8.1 & 30.0 & 32.0 & 116 & 134 \\
\hline $0-C 10$ & 13.6 & 14.9 & 7.75 & 7.93 & 4.4 & 8.2 & 29.5 & 32.0 & 116 & 132 \\
\hline $0-\mathrm{Cg}$ & 13.5 & 14.9 & 2.00 & 7.98 & 7.4 & 8.0 & 30.0 & 32.0 & 116 & 132 \\
\hline $0-c 8$ & 13.7 & 15.0 & 7.60 & 7.96 & 7.0 & 8.0 & 30.0 & 32.0 & 116 & 132 \\
\hline $0-C 7$ & 13.6 & 15.0 & 7.75 & 7.97 & 7.4 & 8.0 & 30.0 & 32.0 & 116 & 132 \\
\hline $0-C 6$ & 14.0 & 15.8 & 7.73 & 7.99 & 7.4 & 8.0 & 30.0 & 32.0 & 116 & 132 \\
\hline $0-C 5$ & 13.8 & 15.2 & 7.76 & 8.03 & 7.5 & 8.1 & 30.0 & 31.5 & $108^{(b)}$ & 132 \\
\hline $0-C 4$ & 13.9 & 15.3 & 7.70 & 7.97 & 7.3 & 8.0 & 30.0 & 32.0 & 116 & 132 \\
\hline $0-C 3$ & 13.8 & 15.0 & 7.71 & 8.03 & 7.4 & 8.0 & 30.0 & 31.5 & 116 & 132 \\
\hline $0-C 2$ & 13.9 & 14.9 & 7.74 & 8.06 & 7.2 & 8.0 & 30.0 & 32.0 & 116 & 132 \\
\hline $0-C 1$ & 13.9 & 15.2 & 7.65 & 8.02 & 6.4 & 8.1 & 30.0 & 32.0 & 116 & 132. \\
\hline$I C-2$ & 13.7 & 15.1 & 7.77 & 8.00 & 7.4 & 8.1 & 30.0 & 32.0 & 116 & 134 \\
\hline IC-3 & 13.9 & 15.4 & 7.70 & 8.00 & 7.4 & 8.0 & 30.0 & 32.0 & 116 & 140 \\
\hline IC-8 & 13.9 & 15.4 & 7.71 & 8.00 & 7.4 & 8.1 & 30.0 & 31.5 & 116 & 132 \\
\hline$R-A C$ & 13.5 & 14.3 & 7.80 & 8.00 & 7.4 & 8.2 & 30.0 & $3 ! 5$ & $106^{(b)}$ & 132 \\
\hline$R-A M$ & 13.7 & 14.9 & 7.79 & 7.94 & 7.0 & 8.3 & 29.5 & 32.0 & 116 & 132 \\
\hline$R-B F$ & 13.7 & 15.0 & 7.78 & 7.99 & 7.3 & 8.2 & 30.0 & 32.0 & 116 & 140 \\
\hline$R-O S$ & 13.5 & 14.9 & 7.61 & 8.00 & 7.5 & 8.2 & 30.0 & 32.0 & 116 & 132 \\
\hline$R-P C$ & 13.7 & 14.9 & 7.75 & 7.97 & 7.4 & 8.0 & 30.0 & 32.0 & 116 & 132 \\
\hline$R-P F$ & 13.3 & 15.0 & 7.76 & 7.97 & 7.3 & 8.1 & 30.0 & 32.0 & $104^{(0)}$ & 132 \\
\hline$C-S B$ & 13.8 & 14.9 & 7.78 & 7.98 & 7.4 & 8.1 & 29.5 & 32.0 & 116 & 132 \\
\hline$C-S D$ & 13.8 & 14.9 & 7.46 & 8.00 & 7.3 & 8.2 & 29.5 & 32.0 & 116 & 132 \\
\hline
\end{tabular}

(a) Parameter temporarily outside of target quality control range; outcome of test not affected. 
APPENDDX H

26-HOUR STATIC SUSPENDED-PARTICULATE-PHASE TEST WITH C. STIGMAEUS 
TABLE H.1. Survival Data for All Replicates in 4-Day C. stigmaeus Suspended-Particulate-Phase Test, Oak1 and Harbor Phase III B Project

\begin{tabular}{|c|c|c|c|c|c|}
\hline $\begin{array}{l}\text { SPP } \\
\text { Ireatment }\end{array}$ & $\begin{array}{l}\text { Concentration } \\
\text { Percent SPP } \\
\end{array}$ & Replicate & Live & $\begin{array}{l}\frac{\text { igmaeus }}{\text { Dead }} \\
\text { or missing }\end{array}$ & $\begin{array}{c}\text { Mean } \\
\text { Proportion } \\
\text { Surviving } \\
\end{array}$ \\
\hline $\begin{array}{l}B C-5 \\
B C-5 \\
B C-5\end{array}$ & $\begin{array}{l}0 \\
0 \\
0\end{array}$ & $\begin{array}{l}1 \\
2 \\
3\end{array}$ & $\begin{array}{l}10 \\
10 \\
10\end{array}$ & $\begin{array}{l}0 \\
0 \\
0\end{array}$ & 1.00 \\
\hline $\begin{array}{l}B C-5 \\
B C-5 \\
B C-5\end{array}$ & $\begin{array}{l}10 \\
10 \\
10\end{array}$ & $\begin{array}{l}1 \\
2 \\
3\end{array}$ & $\begin{array}{l}10 \\
10 \\
10\end{array}$ & $\begin{array}{l}0 \\
0 \\
0\end{array}$ & 1.00 \\
\hline $\begin{array}{l}B C-5 \\
B C-5 \\
B C-5\end{array}$ & $\begin{array}{l}50 \\
50 \\
50\end{array}$ & $\begin{array}{l}1 \\
2 \\
3\end{array}$ & $\begin{array}{l}10 \\
10 \\
10\end{array}$ & $\begin{array}{l}0 \\
0 \\
0\end{array}$ & 1.00 \\
\hline $\begin{array}{l}B C-5 \\
B C-5 \\
B C-5\end{array}$ & $\begin{array}{l}100 \\
100 \\
100\end{array}$ & $\begin{array}{l}1 \\
2 \\
3\end{array}$ & $\begin{array}{l}10 \\
10 \\
10\end{array}$ & $\begin{array}{l}0 \\
0 \\
0\end{array}$ & 1.00 \\
\hline $\begin{array}{l}B C-6 \\
B C-6 \\
B C-6\end{array}$ & $\begin{array}{l}0 \\
0 \\
0\end{array}$ & $\begin{array}{l}1 \\
2 \\
3\end{array}$ & $\begin{array}{l}10 \\
10 \\
10\end{array}$ & $\begin{array}{l}0 \\
0 \\
0\end{array}$ & 1.00 \\
\hline $\begin{array}{l}B C-6 \\
B C-6 \\
B C-6\end{array}$ & $\begin{array}{l}10 \\
10 \\
10\end{array}$ & $\begin{array}{l}1 \\
2 \\
3\end{array}$ & $\begin{array}{l}10 \\
10 \\
10\end{array}$ & $\begin{array}{l}0 \\
0 \\
0\end{array}$ & 1.00 \\
\hline $\begin{array}{l}B C-6 \\
B C-6 \\
B C-6\end{array}$ & $\begin{array}{l}50 \\
50 \\
50\end{array}$ & $\begin{array}{l}1 \\
2 \\
3\end{array}$ & $\begin{array}{l}10 \\
10 \\
10\end{array}$ & $\begin{array}{l}0 \\
0 \\
0\end{array}$ & 1.00 \\
\hline $\begin{array}{l}B C-6 \\
B C,-6 \\
B C-6\end{array}$ & $\begin{array}{l}100 \\
100 \\
100\end{array}$ & $\begin{array}{l}1 \\
2 \\
3\end{array}$ & $\begin{array}{r}9 \\
10 \\
10\end{array}$ & $\begin{array}{l}1 \\
0 \\
0\end{array}$ & 0.97 \\
\hline $\begin{array}{l}B C-7 \\
B C-7 \\
B C-7 \\
B C-7 \\
B C-7 \\
B C-7\end{array}$ & $\begin{array}{r}0 \\
0 \\
0 \\
10 \\
10 \\
10\end{array}$ & $\begin{array}{l}1 \\
2 \\
3 \\
1 \\
2 \\
3\end{array}$ & $\begin{array}{l}10 \\
10 \\
10 \\
10 \\
10 \\
10\end{array}$ & $\begin{array}{l}0 \\
0 \\
0 \\
0 \\
0 \\
0\end{array}$ & 1.00 \\
\hline $\begin{array}{l}B C-7 \\
B C-7 \\
B C-7\end{array}$ & $\begin{array}{l}50 \\
50 \\
50\end{array}$ & $\begin{array}{l}1 \\
2 \\
3\end{array}$ & $\begin{array}{l}10 \\
10 \\
10\end{array}$ & $\begin{array}{l}0 \\
0 \\
0\end{array}$ & 1.00 \\
\hline
\end{tabular}


TABLE H.1. (contd)

\begin{tabular}{|c|c|c|c|c|c|}
\hline \multirow[b]{2}{*}{$\begin{array}{l}\text { SPP } \\
\text { Ireatment }\end{array}$} & \multirow[b]{2}{*}{$\begin{array}{l}\text { Concentration } \\
\text { Percent SPP }\end{array}$} & \multirow[b]{2}{*}{ Replicate } & \multicolumn{2}{|c|}{ C. stigmaeus } & \multirow{2}{*}{$\begin{array}{c}\text { Mean } \\
\text { Proportion } \\
\text { Surviving } \\
\end{array}$} \\
\hline & & & Live & $\begin{array}{c}\text { Dead } \\
\text { or missing } \\
\end{array}$ & \\
\hline $\begin{array}{l}B C-7 \\
B C-7 \\
B C-7\end{array}$ & $\begin{array}{l}100 \\
100 \\
100\end{array}$ & $\begin{array}{l}1 \\
2 \\
3\end{array}$ & $\begin{array}{l}10 \\
10 \\
10\end{array}$ & $\begin{array}{l}0 \\
0 \\
0\end{array}$ & 1.00 \\
\hline $\begin{array}{l}B C-8 \\
B C-8 \\
B C-8\end{array}$ & $\begin{array}{l}0 \\
0 \\
0\end{array}$ & $\begin{array}{l}1 \\
2 \\
3\end{array}$ & $\begin{array}{l}10 \\
10 \\
10\end{array}$ & $\begin{array}{l}0 \\
0 \\
0\end{array}$ & 1.00 \\
\hline $\begin{array}{l}B C-8 \\
B C-8 \\
B C-8\end{array}$ & $\begin{array}{l}10 \\
10 \\
10\end{array}$ & $\begin{array}{l}1 \\
2 \\
3\end{array}$ & $\begin{array}{l}10 \\
10 \\
10\end{array}$ & $\begin{array}{l}0 \\
0 \\
0\end{array}$ & 1.00 \\
\hline $\begin{array}{l}B C-8 \\
B C-8 \\
B C-8\end{array}$ & $\begin{array}{l}50 \\
50 \\
50\end{array}$ & $\begin{array}{l}1 \\
2 \\
3\end{array}$ & $\begin{array}{l}10 \\
10 \\
10\end{array}$ & $\begin{array}{l}0 \\
0 \\
0\end{array}$ & 1.00 \\
\hline $\begin{array}{l}B C-8 \\
B C-8 \\
B C-8\end{array}$ & $\begin{array}{l}100 \\
100 \\
100\end{array}$ & $\begin{array}{l}1 \\
2 \\
3\end{array}$ & $\begin{array}{l}10 \\
10 \\
10\end{array}$ & $\begin{array}{l}0 \\
0 \\
0\end{array}$ & 1.00 \\
\hline $\begin{array}{l}R-A M \\
R-A M \\
R-A M\end{array}$ & $\begin{array}{l}0 \\
0 \\
0\end{array}$ & $\begin{array}{l}1 \\
2 \\
3\end{array}$ & $\begin{array}{l}10 \\
10 \\
10\end{array}$ & $\begin{array}{l}0 \\
0 \\
0\end{array}$ & 1.00 \\
\hline $\begin{array}{l}\text { R-AM } \\
\text { R-AM } \\
\text { R-AM }\end{array}$ & $\begin{array}{l}10 \\
10 \\
10\end{array}$ & $\begin{array}{l}1 \\
2 \\
3\end{array}$ & $\begin{array}{l}10 \\
10 \\
10\end{array}$ & $\begin{array}{l}0 \\
0 \\
0\end{array}$ & 1.00 \\
\hline $\begin{array}{l}R-A M \\
R-A M \\
R-A M \\
R-A M \\
R-A M \\
R-A M\end{array}$ & $\begin{array}{r}50 \\
50 \\
50 \\
100 \\
100 \\
100\end{array}$ & $\begin{array}{l}1 \\
2 \\
3 \\
1 \\
2 \\
3\end{array}$ & $\begin{array}{r}10 \\
10 \\
10 \\
9 \\
10 \\
10\end{array}$ & $\begin{array}{l}0 \\
0 \\
0 \\
1 \\
0 \\
0\end{array}$ & 0.97 \\
\hline $\begin{array}{l}R-B F \\
R-B F \\
R-B F\end{array}$ & $\begin{array}{l}0 \\
0 \\
0\end{array}$ & $\begin{array}{l}1 \\
2 \\
3\end{array}$ & $\begin{array}{l}10 \\
10 \\
10\end{array}$ & $\begin{array}{l}0 \\
0 \\
0\end{array}$ & 1.00 \\
\hline $\begin{array}{l}R-B F \\
R-B F \\
R-B F\end{array}$ & $\begin{array}{l}10 \\
10 \\
10\end{array}$ & $\begin{array}{l}1 \\
2 \\
3\end{array}$ & $\begin{array}{l}10 \\
10 \\
10\end{array}$ & $\begin{array}{l}0 \\
0 \\
0\end{array}$ & 1.00 \\
\hline
\end{tabular}


IABLE H.1. (contd)

\begin{tabular}{|c|c|c|c|c|c|}
\hline \multirow[b]{2}{*}{$\begin{array}{l}\text { SPP } \\
\text { Ireatment }\end{array}$} & \multirow[b]{2}{*}{$\begin{array}{l}\text { Concentration } \\
\text { Percent SPP } \\
\end{array}$} & \multirow[b]{2}{*}{ Replicate } & \multicolumn{2}{|c|}{ C. stigmaeus } & \multirow{2}{*}{$\begin{array}{c}\text { Mean } \\
\text { Proportion } \\
\text { Surviving } \\
\end{array}$} \\
\hline & & & Live & $\begin{array}{c}\text { Dead } \\
\text { or missing }\end{array}$ & \\
\hline $\begin{array}{l}R-B F \\
R-B F \\
R-B F\end{array}$ & $\begin{array}{l}50 \\
50 \\
50\end{array}$ & $\begin{array}{l}1 \\
2 \\
3\end{array}$ & $\begin{array}{l}10 \\
10 \\
10\end{array}$ & $\begin{array}{l}0 \\
0 \\
0\end{array}$ & 1.00 \\
\hline $\begin{array}{l}R-B F \\
R-B F \\
R-B F\end{array}$ & $\begin{array}{l}100 \\
100 \\
100\end{array}$ & $\begin{array}{l}1 \\
2 \\
3\end{array}$ & $\begin{array}{l}10 \\
10 \\
10\end{array}$ & $\begin{array}{l}0 \\
0 \\
0\end{array}$ & 1.00 \\
\hline $\begin{array}{l}R-P F \\
R-P F \\
R-P F\end{array}$ & $\begin{array}{l}0 \\
0 \\
0\end{array}$ & $\begin{array}{l}1 \\
2 \\
3\end{array}$ & $\begin{array}{l}10 \\
10 \\
10\end{array}$ & $\begin{array}{l}0 \\
0 \\
0\end{array}$ & 1.00 \\
\hline $\begin{array}{l}R-P F \\
R-P F \\
R-P F\end{array}$ & $\begin{array}{l}10 \\
10 \\
10\end{array}$ & $\begin{array}{l}1 \\
2 \\
3\end{array}$ & $\begin{array}{l}10 \\
10 \\
10\end{array}$ & $\begin{array}{l}0 \\
0 \\
0\end{array}$ & 1.00 \\
\hline $\begin{array}{l}R-P F \\
R-P F \\
R-P F\end{array}$ & $\begin{array}{l}50 \\
50 \\
50\end{array}$ & $\begin{array}{l}1 \\
2 \\
3\end{array}$ & $\begin{array}{l}10 \\
10 \\
10\end{array}$ & $\begin{array}{l}0 \\
0 \\
0\end{array}$ & 1.00 \\
\hline $\begin{array}{l}R-P F \\
R-P F \\
R-P F\end{array}$ & $\begin{array}{l}100 \\
100 \\
100\end{array}$ & $\begin{array}{l}1 \\
2 \\
3\end{array}$ & $\begin{array}{l}10 \\
10 \\
10\end{array}$ & $\begin{array}{l}0 \\
0 \\
0\end{array}$ & 1.00 \\
\hline
\end{tabular}


IABLE H.2. Rank Order Based on Proportion Survival for 4-Day C. stigmaeus Suspended-Particulate-Phase Test, Oakland Harbor Phase III B Project

\begin{tabular}{|c|c|c|}
\hline $\begin{array}{c}\text { SPP } \\
\text { Ireatment }\end{array}$ & $\begin{array}{l}\text { Concentration } \\
\text { Percent SPP }\end{array}$ & $\begin{array}{l}\text { Proportion } \\
\text { Surviving }\end{array}$ \\
\hline $\begin{array}{l}B C-5 \\
B C-6 \\
B C-7 \\
B C-8 \\
R-A M \\
R-B F \\
R-P F\end{array}$ & $\begin{array}{l}0 \\
0 \\
0 \\
0 \\
0 \\
0 \\
0\end{array}$ & $\begin{array}{l}1.00 \\
1.00 \\
1.00 \\
1.00 \\
1.00 \\
1.00 \\
1.00\end{array}$ \\
\hline $\begin{array}{l}B C-5 \\
B C-6 \\
B C-7 \\
B C-8 \\
R-A M \\
R-B F \\
R-P F\end{array}$ & $\begin{array}{l}10 \\
10 \\
10 \\
10 \\
10 \\
10 \\
10\end{array}$ & $\begin{array}{l}1.00 \\
1.00 \\
1.00 \\
1.00 \\
1.00 \\
1.00 \\
1.00\end{array}$ \\
\hline $\begin{array}{l}B C-5 \\
B C-6 \\
B C-7 \\
B C-8 \\
R-A M \\
R-B F \\
R-P F\end{array}$ & $\begin{array}{l}50 \\
50 \\
50 \\
50 \\
50 \\
50 \\
50\end{array}$ & $\begin{array}{l}1.00 \\
1.00 \\
1.00 \\
1.00 \\
1.00 \\
1.00 \\
1.00\end{array}$ \\
\hline $\begin{array}{l}B C-6 \\
R-A M \\
B C-5 \\
B C-7 \\
B C-8 \\
R-B F \\
R-P F\end{array}$ & $\begin{array}{l}100 \\
100 \\
100 \\
100 \\
100 \\
100 \\
100\end{array}$ & $\begin{array}{l}0.97 \\
0.97 \\
1.00 \\
1.00 \\
1.00 \\
1.00 \\
1.00\end{array}$ \\
\hline
\end{tabular}


IABLE H.3. Water Quality Summary for 4-Day C. stigmaeus

Suspended-Particulate-Phase Test, Oakland

Phase III B Project

\begin{tabular}{|c|c|c|c|c|c|c|c|c|c|}
\hline \multirow{2}{*}{$\begin{array}{l}\text { SPP } \\
\text { Ireatment }\end{array}$} & \multirow{2}{*}{$\begin{array}{l}\text { Concentration } \\
\text { Percent SPP }\end{array}$} & \multicolumn{2}{|c|}{$\begin{array}{c}\text { Temperature } \\
\left({ }^{\circ} \mathrm{C}\right)\end{array}$} & \multicolumn{2}{|c|}{$\mathrm{pH}$} & \multicolumn{2}{|c|}{$\begin{array}{l}\text { Dissolved } \\
\text { Oxygen } \\
(\mathrm{mg} / \mathrm{L})\end{array}$} & \multicolumn{2}{|c|}{$\begin{array}{c}\text { Salinity } \\
(0 / 00)\end{array}$} \\
\hline & & Min & $\operatorname{Max}$ & Min & $\operatorname{Max}$ & Min & $\operatorname{Max}$ & Min & $\operatorname{Max}$ \\
\hline $\begin{array}{l}\text { Acceptable } \\
\text { Range }\end{array}$ & & 13.0 & 17.0 & 7.54 & 8.54 & 4.0 & $N_{A}(a)$ & 28.5 & 32.5 \\
\hline $\begin{array}{l}B C-5 \\
B C-5 \\
B C-5 \\
B C-5\end{array}$ & $\begin{array}{r}0 \\
10 \\
50 \\
100\end{array}$ & $\begin{array}{l}13.1 \\
13.1 \\
13.5 \\
13.1\end{array}$ & $\begin{array}{l}15.5 \\
15.7 \\
15.2 \\
15.4\end{array}$ & $\begin{array}{l}7.85 \\
7.86 \\
7.81 \\
7.77\end{array}$ & $\begin{array}{l}8.20 \\
8.25 \\
8.31 \\
8.38\end{array}$ & $\begin{array}{l}7.4 \\
7.6 \\
7.5 \\
7.5\end{array}$ & $\begin{array}{l}8.3 \\
8.3 \\
8.3 \\
8.3\end{array}$ & $\begin{array}{l}30.0 \\
30.5 \\
30.0 \\
30.0\end{array}$ & $\begin{array}{l}31.5 \\
33.5 \\
32.0 \\
32.0\end{array}$ \\
\hline $\begin{array}{l}B C-6 \\
B C-6 \\
B C-6 \\
B C-6\end{array}$ & $\begin{array}{r}0 \\
10 \\
50 \\
100\end{array}$ & $\begin{array}{l}13.0 \\
14.0 \\
14.2 \\
13.7\end{array}$ & $\begin{array}{l}15.8 \\
15.2 \\
15.3 \\
15.3\end{array}$ & $\begin{array}{l}7.85 \\
7.89 \\
8.00 \\
8.08\end{array}$ & $\begin{array}{l}8.22 \\
8.23 \\
8.26 \\
8.27\end{array}$ & $\begin{array}{l}7.4 \\
7.5 \\
7.4 \\
7.5\end{array}$ & $\begin{array}{l}8.4 \\
8.3 \\
8.1 \\
8.0\end{array}$ & $\begin{array}{l}30.5 \\
30.0 \\
30.0 \\
30.0\end{array}$ & $\begin{array}{l}32.0 \\
32.0 \\
31.5 \\
30.5\end{array}$ \\
\hline $\begin{array}{l}B C-7 \\
B C-7 \\
B C-7 \\
B C-7\end{array}$ & $\begin{array}{r}0 \\
10 \\
50 \\
100\end{array}$ & $\begin{array}{l}14.6 \\
14.3 \\
14.5 \\
14.6\end{array}$ & $\begin{array}{l}15.1 \\
15.2 \\
15.3 \\
15.4\end{array}$ & $\begin{array}{l}7.88 \\
7.91 \\
7.87 \\
7.92\end{array}$ & $\begin{array}{l}8.13 \\
8.12 \\
8.22 \\
8.26\end{array}$ & $\begin{array}{l}7.5 \\
7.5 \\
7.4 \\
7.5\end{array}$ & $\begin{array}{l}8.4 \\
8.4 \\
8.3 \\
8.3\end{array}$ & $\begin{array}{l}30.0 \\
30.0 \\
30.0 \\
30.0\end{array}$ & $\begin{array}{l}31.0 \\
30.5 \\
31.0 \\
30.5\end{array}$ \\
\hline $\begin{array}{l}B C-8 \\
B C-8 \\
B C-8 \\
B C-8\end{array}$ & $\begin{array}{r}0 \\
10 \\
50 \\
100\end{array}$ & $\begin{array}{l}14.7 \\
14.8 \\
14.7 \\
14.7\end{array}$ & $\begin{array}{l}15.6 \\
15.2 \\
15.1 \\
15.3\end{array}$ & $\begin{array}{l}7.81 \\
7.89 \\
7.83 \\
7.80\end{array}$ & $\begin{array}{l}8.12 \\
8.10 \\
8.24 \\
8.27\end{array}$ & $\begin{array}{l}7.5 \\
7.4 \\
7.7 \\
7.4\end{array}$ & $\begin{array}{l}8.1 \\
8.3 \\
8.1 \\
8.0\end{array}$ & $\begin{array}{l}30.0 \\
30.0 \\
30.0 \\
30.0\end{array}$ & $\begin{array}{l}32.0 \\
30.5 \\
31.5 \\
31.0\end{array}$ \\
\hline $\begin{array}{l}R-A M \\
R-A M \\
R-A M \\
R-A M\end{array}$ & $\begin{array}{r}0 \\
10 \\
50 \\
100\end{array}$ & $\begin{array}{l}14.6 \\
14.4 \\
14.6 \\
14.6\end{array}$ & $\begin{array}{l}15.3 \\
15.2 \\
15.2 \\
15.2\end{array}$ & $\begin{array}{l}7.83 \\
7.88 \\
7.91 \\
7.89\end{array}$ & $\begin{array}{l}8.15 \\
8.14 \\
8.15 \\
8.15\end{array}$ & $\begin{array}{l}7.6 \\
7.7 \\
7.6 \\
7.5\end{array}$ & $\begin{array}{l}8.3 \\
8.4 \\
8.3 \\
8.4\end{array}$ & $\begin{array}{l}30.0 \\
30.0 \\
30.0 \\
30.0\end{array}$ & $\begin{array}{l}31.5 \\
31.0 \\
30.5 \\
30.5\end{array}$ \\
\hline $\begin{array}{l}R-B F \\
R-B F \\
R-B F \\
R-B F\end{array}$ & $\begin{array}{r}0 \\
10 \\
50 \\
100\end{array}$ & $\begin{array}{l}13.8 \\
13.8 \\
13.6 \\
13.8\end{array}$ & $\begin{array}{l}15.1 \\
15.1 \\
15.5 \\
15.0\end{array}$ & $\begin{array}{l}7.88 \\
7.90 \\
7.93 \\
7.96\end{array}$ & $\begin{array}{l}8.14 \\
8.21 \\
8.25 \\
8.31\end{array}$ & $\begin{array}{l}7.4 \\
7.0 \\
7.3 \\
7.4\end{array}$ & $\begin{array}{l}8.4 \\
8.4 \\
8.4 \\
8.4\end{array}$ & $\begin{array}{l}30.5 \\
30.5 \\
30.0 \\
30.5\end{array}$ & $\begin{array}{l}32.0 \\
31.5 \\
31.0 \\
32.0\end{array}$ \\
\hline $\begin{array}{l}R-P F \\
R-P F \\
R-P F \\
R=P F\end{array}$ & $\begin{array}{r}0 \\
10 \\
50 \\
100\end{array}$ & $\begin{array}{l}14.0 \\
13.2 \\
13.0 \\
13.5\end{array}$ & $\begin{array}{l}15.5 \\
15.6 \\
15.2 \\
15.6\end{array}$ & $\begin{array}{l}7.90 \\
7.88 \\
7.85 \\
7.83\end{array}$ & $\begin{array}{l}8.16 \\
8.16 \\
8.18 \\
8.17\end{array}$ & $\begin{array}{l}6.1 \\
7.4 \\
7.5 \\
7.4\end{array}$ & $\begin{array}{l}8.4 \\
8.5 \\
8.6 \\
8.6\end{array}$ & $\begin{array}{l}30.0 \\
30.0 \\
30.0 \\
30.0\end{array}$ & $\begin{array}{l}31.0 \\
31.0 \\
31.0 \\
31.5\end{array}$ \\
\hline
\end{tabular}

(a) Not applicabie. 
TABLE H.4. Daily Observations of C. stigmaeus During 4-Day Suspended-Particulate-Phase Test, Oakland Phase III B Project.

\begin{tabular}{|c|c|c|c|c|c|c|c|c|c|c|c|c|c|c|}
\hline$S P P$ & Concentration & & & & stiomaens & SLive & & & & & igmae & us De & & \\
\hline Treatment & Percent SPP & Replicate & $\underline{\mathrm{Oh}}$ & $4 h$ & $24 h$ & $48 \mathrm{~h}$ & $72 h$ & $96 h$ & $\underline{\text { oh }}$ & $4 h$ & $24 h$ & $48 \mathrm{~h}$ & $22 h$ & $96 h$ \\
\hline $\begin{array}{l}B C-5 \\
B C-5 \\
B C-5\end{array}$ & $\begin{array}{l}0 \\
0 \\
0\end{array}$ & $\begin{array}{l}1 \\
2 \\
3\end{array}$ & $\begin{array}{l}10 \\
10 \\
10\end{array}$ & $\begin{array}{l}10 \\
10 \\
10\end{array}$ & $\begin{array}{l}10 \\
10 \\
10\end{array}$ & $\begin{array}{l}10 \\
10 \\
10\end{array}$ & $\begin{array}{l}10 \\
10 \\
10\end{array}$ & $\begin{array}{l}10 \\
10 \\
10\end{array}$ & $\begin{array}{l}0 \\
0 \\
0\end{array}$ & $\begin{array}{l}0 \\
0 \\
0\end{array}$ & $\begin{array}{l}0 \\
0 \\
0\end{array}$ & $\begin{array}{l}0 \\
0 \\
0\end{array}$ & & \\
\hline $\begin{array}{l}B C-5 \\
B C-5 \\
B C-5\end{array}$ & $\begin{array}{l}10 \\
10 \\
10\end{array}$ & $\begin{array}{l}1 \\
2 \\
3\end{array}$ & $\begin{array}{l}10 \\
10 \\
10\end{array}$ & $\begin{array}{l}10 \\
10 \\
10\end{array}$ & $\begin{array}{l}10 \\
10 \\
10\end{array}$ & $\begin{array}{l}10 \\
10 \\
10\end{array}$ & $\begin{array}{l}10 \\
10 \\
10\end{array}$ & $\begin{array}{l}10 \\
10 \\
10\end{array}$ & $\begin{array}{l}0 \\
0 \\
0\end{array}$ & $\begin{array}{l}0 \\
0 \\
0\end{array}$ & $\begin{array}{l}0 \\
0 \\
0\end{array}$ & $\begin{array}{l}0 \\
0 \\
0\end{array}$ & & $\begin{array}{l}0 \\
0 \\
0\end{array}$ \\
\hline $\begin{array}{l}B C-5 \\
B C-5 \\
B C-5\end{array}$ & $\begin{array}{l}50 \\
50 \\
50\end{array}$ & $\begin{array}{l}1 \\
2 \\
3\end{array}$ & $\begin{array}{l}10 \\
10 \\
10\end{array}$ & $\begin{array}{l}10 \\
10 \\
10\end{array}$ & $\begin{array}{l}10 \\
10 \\
10\end{array}$ & $\begin{array}{l}10 \\
10 \\
10\end{array}$ & $\begin{array}{l}10 \\
10 \\
10\end{array}$ & $\begin{array}{l}10 \\
10 \\
10\end{array}$ & $\begin{array}{l}0 \\
0 \\
0\end{array}$ & $\begin{array}{l}0 \\
0 \\
0\end{array}$ & $\begin{array}{l}0 \\
0 \\
0\end{array}$ & $\begin{array}{l}0 \\
0 \\
0\end{array}$ & $\begin{array}{l}0 \\
0 \\
0\end{array}$ & 0 \\
\hline $\begin{array}{l}B C-5 \\
B C-5 \\
B C-5\end{array}$ & $\begin{array}{l}100 \\
100 \\
100\end{array}$ & $\begin{array}{l}1 \\
2 \\
3\end{array}$ & $\begin{array}{l}10 \\
10 \\
10\end{array}$ & $\begin{array}{l}10 \\
10 \\
10\end{array}$ & $\begin{array}{l}10 \\
10 \\
10\end{array}$ & $\begin{array}{l}10 \\
10 \\
10\end{array}$ & $\begin{array}{l}10 \\
10 \\
10\end{array}$ & $\begin{array}{l}10 \\
10 \\
10\end{array}$ & $\begin{array}{l}0 \\
0 \\
0\end{array}$ & $\begin{array}{l}0 \\
0 \\
0\end{array}$ & $\begin{array}{l}0 \\
0 \\
0\end{array}$ & $\begin{array}{l}0 \\
0 \\
0\end{array}$ & $\begin{array}{l}0 \\
0 \\
0\end{array}$ & $\begin{array}{l}0 \\
0 \\
0\end{array}$ \\
\hline $\begin{array}{l}B C-6 \\
B C-6 \\
B C-6\end{array}$ & $\begin{array}{l}0 \\
0 \\
0\end{array}$ & $\begin{array}{l}1 \\
2 \\
3\end{array}$ & $\begin{array}{l}10 \\
10 \\
10\end{array}$ & $\begin{array}{l}10 \\
10 \\
10\end{array}$ & $\begin{array}{l}10 \\
10 \\
10\end{array}$ & $\begin{array}{l}10 \\
10 \\
10\end{array}$ & $\begin{array}{l}10 \\
10 \\
10\end{array}$ & $\begin{array}{l}10 \\
10 \\
10\end{array}$ & $\begin{array}{l}0 \\
0 \\
0\end{array}$ & $\begin{array}{l}0 \\
0 \\
0\end{array}$ & $\begin{array}{l}0 \\
0 \\
0\end{array}$ & $\begin{array}{l}0 \\
0 \\
0\end{array}$ & $\begin{array}{l}0 \\
0 \\
0\end{array}$ & $\begin{array}{l}0 \\
0 \\
0\end{array}$ \\
\hline $\begin{array}{l}B C-6 \\
B C-6 \\
B C-6\end{array}$ & $\begin{array}{l}10 \\
10 \\
10\end{array}$ & $\begin{array}{l}1 \\
2 \\
3\end{array}$ & $\begin{array}{l}10 \\
10 \\
10\end{array}$ & $\begin{array}{l}10 \\
10 \\
10\end{array}$ & $\begin{array}{l}10 \\
10 \\
10\end{array}$ & $\begin{array}{l}10 \\
10 \\
10\end{array}$ & $\begin{array}{l}10 \\
10 \\
10\end{array}$ & $\begin{array}{l}10 \\
10 \\
10\end{array}$ & $\begin{array}{l}0 \\
0 \\
0\end{array}$ & $\begin{array}{l}0 \\
0 \\
0\end{array}$ & $\begin{array}{l}0 \\
0 \\
0\end{array}$ & $\begin{array}{l}0 \\
0 \\
0\end{array}$ & $\begin{array}{l}0 \\
0 \\
0\end{array}$ & 0 \\
\hline $\begin{array}{l}B C-6 \\
B C-6 \\
B C-6\end{array}$ & $\begin{array}{l}50 \\
50 \\
50\end{array}$ & $\begin{array}{l}1 \\
2 \\
3\end{array}$ & $\begin{array}{l}10 \\
10 \\
10\end{array}$ & $\begin{array}{l}10 \\
10 \\
10\end{array}$ & $\begin{array}{l}10 \\
10 \\
10\end{array}$ & $\begin{array}{l}10 \\
10 \\
10\end{array}$ & $\begin{array}{l}10 \\
10 \\
10\end{array}$ & $\begin{array}{l}10 \\
10 \\
10\end{array}$ & $\begin{array}{l}0 \\
0 \\
0\end{array}$ & $\begin{array}{l}0 \\
0 \\
0\end{array}$ & $\begin{array}{l}0 \\
0 \\
0\end{array}$ & $\begin{array}{l}0 \\
0 \\
0\end{array}$ & $\begin{array}{l}0 \\
0 \\
0\end{array}$ & $\begin{array}{l}0 \\
0 \\
0\end{array}$ \\
\hline $\begin{array}{l}B C-6 \\
B C-6 \\
B C-6\end{array}$ & $\begin{array}{l}100 \\
100 \\
100\end{array}$ & $\begin{array}{l}1 \\
2 \\
3\end{array}$ & $\begin{array}{l}10 \\
10 \\
10\end{array}$ & $\begin{array}{l}10 \\
10 \\
10\end{array}$ & $\begin{array}{l}10 \\
T \\
T\end{array}(a)$ & $\begin{array}{l}10 \\
10 \\
10\end{array}$ & $\begin{array}{r}9 \\
10 \\
10\end{array}$ & $\begin{array}{r}9 \\
10 \\
10\end{array}$ & $\begin{array}{l}0 \\
0 \\
0\end{array}$ & $\begin{array}{l}0 \\
0 \\
0\end{array}$ & $\begin{array}{l}0 \\
1 \\
T\end{array}$ & $\begin{array}{l}0 \\
0 \\
0\end{array}$ & $\begin{array}{l}1 \\
0 \\
0\end{array}$ & 1 \\
\hline $\begin{array}{l}B C-7 \\
B C-7 \\
B C-7\end{array}$ & $\begin{array}{l}0 \\
0 \\
0\end{array}$ & $\begin{array}{l}1 \\
2 \\
3\end{array}$ & $\begin{array}{l}10 \\
10 \\
10\end{array}$ & $\begin{array}{l}10 \\
10 \\
10\end{array}$ & $\begin{array}{l}10 \\
10 \\
10\end{array}$ & $\begin{array}{l}10 \\
10 \\
10\end{array}$ & $\begin{array}{l}10 \\
10 \\
10\end{array}$ & $\begin{array}{l}10 \\
10 \\
10\end{array}$ & $\begin{array}{l}0 \\
0 \\
0\end{array}$ & $\begin{array}{l}0 \\
0 \\
0\end{array}$ & $\begin{array}{l}0 \\
0 \\
0\end{array}$ & $\begin{array}{l}0 \\
0 \\
0\end{array}$ & $\begin{array}{l}0 \\
0 \\
0\end{array}$ & 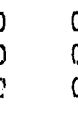 \\
\hline $\begin{array}{l}B C-7 \\
B C-7 \\
B C-7\end{array}$ & $\begin{array}{l}10 \\
10 \\
10\end{array}$ & $\begin{array}{l}1 \\
2 \\
3\end{array}$ & $\begin{array}{l}10 \\
10 \\
10\end{array}$ & $\begin{array}{l}10 \\
10 \\
10\end{array}$ & $\begin{array}{l}10 \\
10 \\
10\end{array}$ & $\begin{array}{l}10 \\
10 \\
10\end{array}$ & $\begin{array}{l}10 \\
10 \\
10\end{array}$ & $\begin{array}{l}10 \\
10 \\
10\end{array}$ & $\begin{array}{l}0 \\
0 \\
0\end{array}$ & $\begin{array}{l}0 \\
0 \\
0\end{array}$ & $\begin{array}{l}0 \\
0 \\
0\end{array}$ & $\begin{array}{l}0 \\
0 \\
0\end{array}$ & $\begin{array}{l}0 \\
0 \\
0\end{array}$ & $\begin{array}{l}0 \\
0\end{array}$ \\
\hline $\begin{array}{l}B C-7 \\
B C-7 \\
B C-7\end{array}$ & $\begin{array}{l}50 \\
50 \\
50\end{array}$ & $\begin{array}{l}1 \\
2 \\
3\end{array}$ & $\begin{array}{l}10 \\
10 \\
10\end{array}$ & $\begin{array}{l}10 \\
10 \\
10\end{array}$ & $\begin{array}{l}10 \\
10 \\
10\end{array}$ & $\begin{array}{r}10 \\
10 \\
10\end{array}$ & $\begin{array}{l}10 \\
10 \\
10\end{array}$ & $\begin{array}{l}10 \\
10 \\
10\end{array}$ & $\begin{array}{l}0 \\
0 \\
0\end{array}$ & $\begin{array}{l}0 \\
0 \\
0\end{array}$ & $\begin{array}{l}0 \\
0 \\
0\end{array}$ & $\begin{array}{l}0 \\
0 \\
0\end{array}$ & & $\begin{array}{l}0 \\
0 \\
0\end{array}$ \\
\hline $\begin{array}{l}B C-7 \\
B C-7 \\
B C-7\end{array}$ & $\begin{array}{l}100 \\
100 \\
100\end{array}$ & $\begin{array}{l}1 \\
2 \\
3\end{array}$ & $\begin{array}{l}10 \\
10 \\
10\end{array}$ & $\begin{array}{l}1 \\
10 \\
10\end{array}$ & $\begin{array}{r}T \\
10 \\
10\end{array}$ & $\begin{array}{l}10 \\
10 \\
10\end{array}$ & $\begin{array}{l}10 \\
10 \\
10\end{array}$ & $\begin{array}{l}10 \\
10 \\
10\end{array}$ & $\begin{array}{l}0 \\
0 \\
0\end{array}$ & $\begin{array}{l}1 \\
0 \\
0\end{array}$ & $\begin{array}{l}T \\
0 \\
0\end{array}$ & $\begin{array}{l}0 \\
0 \\
0\end{array}$ & & $\begin{array}{l}0 \\
0 \\
0\end{array}$ \\
\hline $\begin{array}{l}B C-8 \\
B C-8 \\
B C-8\end{array}$ & $\begin{array}{l}0 \\
0 \\
0\end{array}$ & $\begin{array}{l}1 \\
2 \\
3\end{array}$ & $\begin{array}{l}10 \\
10 \\
10\end{array}$ & $\begin{array}{l}10 \\
10 \\
10\end{array}$ & $\begin{array}{l}10 \\
10 \\
10\end{array}$ & $\begin{array}{l}10 \\
10 \\
10\end{array}$ & $\begin{array}{l}10 \\
10 \\
10\end{array}$ & $\begin{array}{l}10 \\
10 \\
10\end{array}$ & $\begin{array}{l}0 \\
0 \\
0\end{array}$ & $\begin{array}{l}0 \\
0 \\
0\end{array}$ & $\begin{array}{l}0 \\
0 \\
0\end{array}$ & $\begin{array}{l}0 \\
0 \\
0\end{array}$ & & $\begin{array}{l}0 \\
0 \\
0\end{array}$ \\
\hline $\begin{array}{l}B C-8 \\
B C-8 \\
B C-8\end{array}$ & $\begin{array}{l}10 \\
10 \\
10\end{array}$ & $\begin{array}{l}1 \\
2 \\
3\end{array}$ & $\begin{array}{l}10 \\
10 \\
10\end{array}$ & $\begin{array}{l}10 \\
10 \\
10\end{array}$ & $\begin{array}{l}10 \\
10 \\
10\end{array}$ & $\begin{array}{l}10 \\
10 \\
10\end{array}$ & $\begin{array}{l}10 \\
10 \\
10\end{array}$ & $\begin{array}{l}10 \\
10 \\
10\end{array}$ & $\begin{array}{l}0 \\
0 \\
0\end{array}$ & $\begin{array}{l}0 \\
0 \\
0\end{array}$ & $\begin{array}{l}0 \\
0 \\
0\end{array}$ & $\begin{array}{l}0 \\
0 \\
0\end{array}$ & & $\begin{array}{l}0 \\
0 \\
0\end{array}$ \\
\hline
\end{tabular}


TABLE H.4. (contd)

SPP

Preatment Percent SP

$\begin{array}{lr}B C-8 & 50 \\ B C-8 & 50 \\ B C-8 & 50 \\ B C-8 & 100 \\ B C-8 & 100 \\ B C-8 & 100 \\ R-A M & \\ R-A M & 0 \\ R-A M & 0 \\ R-A M & \\ R-A M & \\ R-A M & 10 \\ R-A M & 10 \\ R-A M & 10 \\ R-A M & 50 \\ R-A M & 50 \\ R-A M & 50 \\ R-A M & 100 \\ R-B F & 100 \\ R-B F & 100 \\ R-B F & \end{array}$

$R-B$

$\begin{array}{ll}R-B F & 10 \\ R-B F & 10 \\ R-B F & 10\end{array}$

$R-B F \quad 50$

$R-B F$

$R-B F$

$R-B F$

$R-B F$

$\begin{array}{ll}R-B F & 100\end{array}$

$R-P F$

$R-P F$

$R-P F$

$R-P F$

$R-P F$

$R-P F$

$R \cdot P F \quad 50$

$R-P F$

R WPF

$R-P F$

$R-P F$

$R-P F$

50
50
50

100

100

0
0
0

10

10
10

50

50

100

100

0
0
0

10

10

50

50
50

100

$$
100
$$

0
0

10
10

10

50
50
50 (a) Toc turbid to make accurate observation.

3

1
2
3

3

1
2
3

\section{C. seigmaens live}

Replicate on 4h $24 \mathrm{~h}$ 48h $72 \mathrm{~h}$ 96h

$\begin{array}{lllllll}1 & 10 & 10 & 10 & 10 & 10 & 10\end{array}$

$\begin{array}{llllll}10 & 10 & 10 & 10 & 10 & 10 \\ 10 & 10 & 10 & 10 & 10 & 10\end{array}$

$\begin{array}{rrrrrrr}1 & 10 & 10 & 10 & 10 & 10 & 10 \\ 2 & 10 & 10 & 10 & 10 & 10 & 10 \\ 3 & 10 & T & T & 10 & 10 & 10\end{array}$

$\begin{array}{lllllll}1 & 10 & 10 & 10 & 10 & 10 & 10\end{array}$

$\begin{array}{lllllll}2 & 10 & 10 & 10 & 10 & 10 & 10 \\ 3 & 10 & 10 & 10 & 10 & 10 & 10\end{array}$

$\begin{array}{lllllll}1 & 10 & 10 & 10 & 10 & 10 & 10\end{array}$

$\begin{array}{lllllll}1 & 10 & 10 & 10 & 10 & 10 & 10 \\ 2 & 10 & 10 & 10 & 10 & 10 & 10 \\ 3 & 10 & 10 & 10 & 10 & 10 & 10\end{array}$

$\begin{array}{lllllll}1 & 10 & 10 & 10 & 10 & 10 & 10\end{array}$

$\begin{array}{lllllll}1 & 10 & 10 & 10 & 10 & 10 & 10 \\ 3 & 10 & 10 & 10 & 10 & 10 & 10\end{array}$

$\begin{array}{rrrrrrr}1 & 10 & 10 & 10 & 10 & 10 & 9 \\ 2 & 10 & 10 & 10 & 10 & 10 & 10\end{array}$

$\begin{array}{llllll}10 & 10 & 10 & 10 & 10 & 10\end{array}$

$\begin{array}{llllll}10 & 10 & 10 & 10 & 10 & 10 \\ 10 & 10 & 10 & 10 & 10 & 10 \\ 10 & 10 & 10 & 10 & 10 & 10\end{array}$

$\begin{array}{llllll}10 & 10 & 10 & 10 & 10 & 10\end{array}$

$\begin{array}{llllll}10 & 10 & 10 & 10 & 10 & 10 \\ 10 & 10 & 10 & 10 & 10 & 10\end{array}$

$\begin{array}{llllll}10 & 10 & 10 & 10 & 10 & 10\end{array}$

$\begin{array}{llllll}10 & 10 & 10 & 10 & 10 & 10 \\ 10 & 10 & 10 & 10 & 10 & 10\end{array}$

$\begin{array}{llllll}10 & 10 & 10 & 10 & 10 & 10\end{array}$

$\begin{array}{llllll}10 & 10 & T & T & 10 & 10 \\ 10 & T & T & T & 10 & 10\end{array}$

$\begin{array}{lllllll}1 & 10 & 10 & 10 & 10 & 10 & 10 \\ 2 & 10 & 10 & 10 & 10 & 10 & 10 \\ 3 & 10 & 10 & 10 & 10 & 10 & 10\end{array}$

$1 \quad 10 \quad 10 \quad 10$

$10 \quad 10 \quad 10$

$\begin{array}{llllll}10 & 10 & 10 & 10 & 10 & 10 \\ 10 & 10 & 10 & 10 & 10 & 10 \\ 1 . & 10 & 10 & 10 & 10 & 10\end{array}$

$\begin{array}{llllll}10 & 10 & 10 & 10 & 10 & 10 \\ 10 & 10 & 10 & 10 & 10 & 10\end{array}$

$\begin{array}{llllll}10 & 10 & 10 & 10 & 10 & 10\end{array}$

$\begin{array}{rrrrrr}10 & T & T & T & 10 & 10 \\ 10 & T & T & T & 10 & 10 \\ 10 & 10 & 10 & 10 & 10 & 10\end{array}$ $\frac{c \text { C. scigmaeus Dead }}{\text { oh 4h 24h 48h 72h 96h }}$

$\begin{array}{llllll}0 & 0 & 0 & 0 & 0 & 0 \\ 0 & 0 & 0 & 0 & 0 & 0 \\ 0 & 0 & 0 & 0 & 0 & 0 \\ 0 & 0 & 0 & 0 & 0 & 0 \\ 0 & 0 & 0 & 0 & 0 & 0 \\ 0 & T & T & 0 & 0 & 0\end{array}$

$\begin{array}{llllll}0 & 0 & 0 & 0 & 0 & 0 \\ 0 & 0 & 0 & 0 & 0 & 0 \\ 0 & 0 & 0 & 0 & 0 & 0\end{array}$

$\begin{array}{llllll}0 & 0 & 0 & 0 & 0 & 0 \\ 0 & 0 & 0 & 0 & 0 & 0 \\ 0 & 0 & 0 & 0 & 0 & 0\end{array}$

$\begin{array}{llllll}0 & 0 & 0 & 0 & 0 & 0 \\ 0 & 0 & 0 & 0 & 0 & 0 \\ 0 & 0 & 0 & 0 & 0 & 0\end{array}$

$\begin{array}{llllll}0 & 0 & 0 & 0 & 0 & 1 \\ 0 & 0 & 0 & 0 & 0 & 0 \\ 0 & 0 & 0 & 0 & 0 & 0\end{array}$

$\begin{array}{llllll}0 & 0 & 0 & 0 & 0 & 0 \\ 0 & 0 & 0 & 0 & 0 & 0 \\ 0 & 0 & 0 & 0 & 0 & 0\end{array}$

$\begin{array}{llllll}0 & 0 & 0 & 0 & 0 & 0 \\ 0 & 0 & 0 & 0 & 0 & 0 \\ 0 & 0 & 0 & 0 & 0 & 0\end{array}$

$\begin{array}{llllll}0 & 0 & 0 & 0 & 0 & 0 \\ 0 & 0 & 0 & 0 & 0 & 0 \\ 0 & 0 & 0 & 0 & 0 & 0\end{array}$

$\begin{array}{llllll}0 & 0 & T & 0 & 0 & 0 \\ 0 & 0 & T & T & 0 & 0 \\ 0 & T & T & T & 0 & 0\end{array}$

$\begin{array}{llllll}0 & 0 & 0 & 0 & 0 & 0 \\ 0 & 0 & 0 & 0 & 0 & 0 \\ 0 & 0 & 0 & 0 & 0 & 0\end{array}$

$\begin{array}{llllll}0 & 0 & 0 & 0 & 0 & 0 \\ 0 & 0 & 0 & 0 & 0 & 0 \\ 0 & 0 & 0 & 0 & 0 & 0\end{array}$

$\begin{array}{llllll}0 & 0 & 0 & 0 & 0 & 0 \\ 0 & 0 & 0 & 0 & 0 & 0 \\ 0 & 0 & 0 & 0 & 0 & 0\end{array}$

$\begin{array}{llllll}0 & T & T & T & 0 & 0 \\ 0 & T & T & T & 0 & 0 \\ 0 & 0 & 0 & 0 & 0 & 0\end{array}$

PHASE III B

H. 7 
APPENDIX I

96-HOUR STATIC SUSPENDED-PARTICULATE-PHASE TEST WTTH H. SCULPTA 
TABLE 1.1. Survival Data for All Replicates in 4-Day H. sculpta Suspended-Particulate-Phase Test, 0 akl and Harbor Phase III B Project

\begin{tabular}{|c|c|c|c|c|c|}
\hline \multirow[b]{2}{*}{$\begin{array}{l}\text { Sediment } \\
\text { Ireatment }\end{array}$} & \multirow[b]{2}{*}{$\begin{array}{l}\text { Concentration } \\
\text { Percent SPP }\end{array}$} & \multirow[b]{2}{*}{ Replicate } & \multicolumn{2}{|c|}{ H. sculpta } & \multirow{2}{*}{$\begin{array}{l}\quad \text { Mean } \\
\text { Proportion } \\
\text { Surviving } \\
\end{array}$} \\
\hline & & & Live & $\begin{array}{l}\text { Dead } \\
\text { or Missing }\end{array}$ & \\
\hline$B C-5$ & $\begin{array}{l}0 \\
0 \\
0\end{array}$ & $\begin{array}{l}1 \\
2 \\
3\end{array}$ & $\begin{array}{r}10 \\
8 \\
9\end{array}$ & $\begin{array}{l}0 \\
2 \\
1\end{array}$ & 0.90 \\
\hline$B C-5$ & $\begin{array}{l}10 \\
10 \\
10\end{array}$ & $\begin{array}{l}1 \\
2 \\
3\end{array}$ & $\begin{array}{r}10 \\
8 \\
8\end{array}$ & $\begin{array}{l}0 \\
2 \\
2\end{array}$ & 0.87 \\
\hline$B C-5$ & $\begin{array}{l}50 \\
50 \\
50\end{array}$ & $\begin{array}{l}1 \\
2 \\
3\end{array}$ & $\begin{array}{r}10 \\
9 \\
8\end{array}$ & $\begin{array}{l}0 \\
1 \\
2\end{array}$ & 0.90 \\
\hline$B C-5$ & $\begin{array}{l}100 \\
100 \\
100\end{array}$ & $\begin{array}{l}1 \\
2 \\
3\end{array}$ & $\begin{array}{l}7 \\
8 \\
6\end{array}$ & $\begin{array}{l}3 \\
2 \\
4\end{array}$ & 0.70 \\
\hline$B C-6$ & $\begin{array}{l}0 \\
0 \\
0\end{array}$ & $\begin{array}{l}1 \\
2 \\
3\end{array}$ & $\begin{array}{r}9 \\
10 \\
9\end{array}$ & $\begin{array}{l}1 \\
0 \\
1\end{array}$ & 0.93 \\
\hline$B C-6$ & $\begin{array}{l}10 \\
10 \\
10\end{array}$ & $\begin{array}{r}1 \\
2 \\
. \quad 3\end{array}$ & $\begin{array}{l}7 \\
8 \\
8\end{array}$ & $\begin{array}{l}3 \\
2 \\
2\end{array}$ & 0.77 \\
\hline$B C-6$ & $\begin{array}{l}50 \\
50 \\
50\end{array}$ & $\begin{array}{l}1 \\
2 \\
3\end{array}$ & $\begin{array}{l}7 \\
8 \\
6\end{array}$ & $\begin{array}{l}3 \\
2 \\
4\end{array}$ & 0.70 \\
\hline$B C-6$ & $\begin{array}{l}100 \\
100 \\
100\end{array}$ & $\begin{array}{l}1 \\
2 \\
3\end{array}$ & $\begin{array}{l}7 \\
8 \\
7\end{array}$ & $\begin{array}{l}3 \\
2 \\
3\end{array}$ & 0.73 \\
\hline$B C-7$ & $\begin{array}{l}0 \\
0 \\
0\end{array}$ & $\begin{array}{l}1 \\
2 \\
3\end{array}$ & $\begin{array}{r}9 \\
10 \\
9\end{array}$ & $\begin{array}{l}1 \\
0 \\
1\end{array}$ & 0.93 \\
\hline $\mathrm{BC} \cdots 7$ & $\begin{array}{l}10 \\
10 \\
10\end{array}$ & $\begin{array}{l}1 \\
2 \\
3\end{array}$ & $\begin{array}{r}9 \\
9 \\
10\end{array}$ & $\begin{array}{l}1 \\
1 \\
0\end{array}$ & 0.93 \\
\hline$B C-7$ & $\begin{array}{l}50 \\
50 \\
50\end{array}$ & $\begin{array}{l}1 \\
2 \\
3\end{array}$ & $\begin{array}{l}7 \\
7 \\
8\end{array}$ & $\begin{array}{l}3 \\
3 \\
2\end{array}$ & 0.73 \\
\hline
\end{tabular}


TABLE 1.1. (contd)

\begin{tabular}{|c|c|c|c|c|c|}
\hline \multirow[b]{2}{*}{$\begin{array}{l}\text { Sediment } \\
\text { Ireatment }\end{array}$} & \multirow[b]{2}{*}{$\begin{array}{l}\text { Concentration } \\
\text { Percent SPP } \\
\end{array}$} & \multirow[b]{2}{*}{ Replicate } & \multicolumn{2}{|c|}{ H. sculpta } & \multirow{2}{*}{$\begin{array}{l}\text { Mean } \\
\text { Proportion } \\
\text { Surviving } \\
\end{array}$} \\
\hline & & & Live & $\begin{array}{c}\text { Dead } \\
\text { or Missing } \\
\end{array}$ & \\
\hline$B C-7$ & $\begin{array}{l}100 \\
100 \\
100\end{array}$ & $\begin{array}{l}1 \\
2 \\
3\end{array}$ & $\begin{array}{l}7 \\
6 \\
7\end{array}$ & $\begin{array}{l}3 \\
4 \\
3\end{array}$ & 0.67 \\
\hline$B C-8$ & $\begin{array}{l}0 \\
0 \\
0\end{array}$ & $\begin{array}{l}1 \\
2 \\
3\end{array}$ & $\begin{array}{r}10 \\
10 \\
9\end{array}$ & $\begin{array}{l}0 \\
0 \\
1\end{array}$ & 0.97 \\
\hline$B C-8$ & $\begin{array}{l}10 \\
10 \\
10\end{array}$ & $\begin{array}{l}1 \\
2 \\
3\end{array}$ & $\begin{array}{r}10 \\
8 \\
9\end{array}$ & $\begin{array}{l}0 \\
2 \\
1\end{array}$ & 0.90 \\
\hline$B C-8$ & $\begin{array}{l}50 \\
50 \\
50\end{array}$ & $\begin{array}{l}1 \\
2 \\
3\end{array}$ & $\begin{array}{l}8 \\
9 \\
8\end{array}$ & $\begin{array}{l}2 \\
1 \\
2\end{array}$ & 0.83 \\
\hline$B C-8$ & $\begin{array}{l}100 \\
100 \\
100\end{array}$ & $\begin{array}{l}1 \\
2 \\
3\end{array}$ & $\begin{array}{l}8 \\
8 \\
7\end{array}$ & $\begin{array}{l}2 \\
2 \\
3\end{array}$ & 0.77 \\
\hline R-AM & $\begin{array}{l}0 \\
0 \\
0\end{array}$ & $\begin{array}{l}1 \\
2 \\
3\end{array}$ & $\begin{array}{l}9 \\
9 \\
9\end{array}$ & $\begin{array}{l}1 \\
1 \\
1\end{array}$ & 0.90 \\
\hline$R-A M$ & $\begin{array}{l}10 \\
10 \\
10\end{array}$ & $\begin{array}{l}1 \\
2 \\
3\end{array}$ & $\begin{array}{l}9 \\
9 \\
7\end{array}$ & $\begin{array}{l}1 \\
1 \\
3\end{array}$ & 0.83 \\
\hline$R-A M$ & $\begin{array}{l}50 \\
50 \\
50\end{array}$ & $\begin{array}{l}1 \\
2 \\
3\end{array}$ & $\begin{array}{l}6 \\
8 \\
7\end{array}$ & $\begin{array}{l}4 \\
2 \\
3\end{array}$ & 0.70 \\
\hline$R-A M$ & $\begin{array}{l}100 \\
100 \\
100\end{array}$ & $\begin{array}{l}1 \\
2 \\
3\end{array}$ & $\begin{array}{l}7 \\
7 \\
7\end{array}$ & $\begin{array}{l}3 \\
3 \\
3\end{array}$ & 0.70 \\
\hline$R-B F$ & $\begin{array}{l}0 \\
0 \\
0\end{array}$ & $\begin{array}{l}1 \\
2 \\
3\end{array}$ & $\begin{array}{r}9 \\
10 \\
8\end{array}$ & $\begin{array}{l}1 \\
0 \\
2\end{array}$ & 0.90 \\
\hline$R-B F$ & $\begin{array}{l}10 \\
10 \\
10\end{array}$ & $\begin{array}{l}1 \\
2 \\
3\end{array}$ & $\begin{array}{r}10 \\
9 \\
9\end{array}$ & $\begin{array}{l}0 \\
1 \\
1\end{array}$ & 0.93 \\
\hline PHASE III B & & & & & \\
\hline
\end{tabular}


IABLE 1.1. (contd)

\begin{tabular}{|c|c|c|c|c|c|}
\hline \multirow[b]{2}{*}{$\begin{array}{l}\text { Sediment } \\
\text { Ireatment }\end{array}$} & \multirow[b]{2}{*}{$\begin{array}{l}\text { Concentration } \\
\text { Percent SPP }\end{array}$} & \multirow[b]{2}{*}{ Replicate } & \multicolumn{2}{|c|}{ H. sculpta } & \multirow{2}{*}{$\begin{array}{c}\text { Mean } \\
\text { Proportion } \\
\text { Surviving } \\
\end{array}$} \\
\hline & & & Live & $\begin{array}{c}\text { Dead } \\
\text { or Missing } \\
\end{array}$ & \\
\hline$R-B F$ & $\begin{array}{l}50 \\
50 \\
50\end{array}$ & $\begin{array}{l}1 \\
2 \\
3\end{array}$ & $\begin{array}{l}8 \\
8 \\
9\end{array}$ & $\begin{array}{l}2 \\
2 \\
1\end{array}$ & 0.83 \\
\hline$R-B F$ & $\begin{array}{l}100 \\
100 \\
100\end{array}$ & $\begin{array}{l}1 \\
2 \\
3\end{array}$ & $\begin{array}{l}7 \\
7 \\
6\end{array}$ & $\begin{array}{l}3 \\
3 \\
4\end{array}$ & 0.67 \\
\hline$R-P F$ & $\begin{array}{l}0 \\
0 \\
0\end{array}$ & $\begin{array}{l}1 \\
2 \\
3\end{array}$ & $\begin{array}{r}9 \\
9 \\
10\end{array}$ & $\begin{array}{l}1 \\
1 \\
0\end{array}$ & 0.93 \\
\hline$R-P F$ & $\begin{array}{l}10 \\
10 \\
10\end{array}$ & $\begin{array}{l}1 \\
2 \\
3\end{array}$ & $\begin{array}{l}9 \\
9 \\
9\end{array}$ & $\begin{array}{l}1 \\
1 \\
1\end{array}$ & 0.90 \\
\hline R-PF & $\begin{array}{l}50 \\
50 \\
50\end{array}$ & $\begin{array}{l}1 \\
2 \\
3\end{array}$ & $\begin{array}{l}9 \\
8 \\
8\end{array}$ & $\begin{array}{l}1 \\
2 \\
2\end{array}$ & 0.83 \\
\hline$R-P F$ & $\begin{array}{l}100 \\
100 \\
100\end{array}$ & $\begin{array}{l}1 \\
2 \\
3\end{array}$ & $\begin{array}{l}7 \\
8 \\
6\end{array}$ & $\begin{array}{l}3 \\
2 \\
4\end{array}$ & 0.70 \\
\hline
\end{tabular}


IABLE I.2. Rank Order Based C.1 Mean Proportion Surviving 4-Day H. sculpta Suspended-Particulaie-Phase Test, Oakland Harbor Phase III B Project

\begin{tabular}{|c|c|c|}
\hline $\begin{array}{l}\text { SPP } \\
\text { Treatment }\end{array}$ & $\begin{array}{l}\text { Concentration } \\
\text { Percent SPP }\end{array}$ & $\begin{array}{c}\text { Mean } \\
\text { Proportion } \\
\text { Surviving } \\
\end{array}$ \\
\hline $\begin{array}{l}B C-5 \\
R-B F \\
R-A M \\
B C-6 \\
R-P F \\
B C-7 \\
B C-8\end{array}$ & $\begin{array}{l}0 \\
0 \\
0 \\
0 \\
0 \\
0 \\
0\end{array}$ & $\begin{array}{l}0.90 \\
0.90 \\
0.90 \\
0.93 \\
0.93 \\
0.93 \\
0.97\end{array}$ \\
\hline $\begin{array}{l}B C-6 \\
R-A M \\
B C-5 \\
R-P F \\
B C-8 \\
B C-7 \\
R-B F\end{array}$ & $\begin{array}{l}10 \\
10 \\
10 \\
10 \\
10 \\
10 \\
10\end{array}$ & $\begin{array}{l}0.77 \\
0.83 \\
0.87 \\
0.90 \\
0.90 \\
0.93 \\
0.93\end{array}$ \\
\hline $\begin{array}{l}R-A M \\
B C-6 \\
B C-7 \\
R-B F \\
B C-8 \\
R-P F \\
B C-5\end{array}$ & $\begin{array}{l}50 \\
50 \\
50 \\
50 \\
50 \\
50 \\
50\end{array}$ & $\begin{array}{l}0.70 \\
0.70 \\
0.73 \\
0.83 \\
0.83 \\
0.83 \\
0.90\end{array}$ \\
\hline $\begin{array}{l}R-B F \\
B C-7 \\
B C-5 \\
R-P F \\
R-A M \\
B C-6 \\
B C-8\end{array}$ & $\begin{array}{l}100 \\
100 \\
100 \\
100 \\
100 \\
100 \\
100\end{array}$ & $\begin{array}{l}0.67 \\
0.67 \\
0.70 \\
0.70 \\
0.70 \\
0.73 \\
0.77\end{array}$ \\
\hline
\end{tabular}


TABLE I.3. Water Quality Summary for 4-Day H. sculpta SuspendedParticulate-Phase Test, Oakland Harbor Phase III B Project

\begin{tabular}{|c|c|c|c|c|c|c|c|c|c|}
\hline \multirow{2}{*}{$\begin{array}{l}\text { Sediment } \\
\text { Ireatment }\end{array}$} & \multirow{2}{*}{$\begin{array}{l}\text { Concentration } \\
\text { Percent SPP } \\
\end{array}$} & \multicolumn{2}{|c|}{$\begin{array}{l}\text { Temperature } \\
{ }^{\circ} \mathrm{C}\end{array}$} & \multicolumn{2}{|c|}{$\mathrm{pH}$} & \multicolumn{2}{|c|}{$\begin{array}{c}\text { Dissolved } \\
\text { Oxygen } \\
(\mathrm{mg} / \mathrm{L})\end{array}$} & \multicolumn{2}{|c|}{$\begin{array}{l}\text { Salinity } \\
(0 / 00)\end{array}$} \\
\hline & & Min & Max & Min & Max & Min & $\operatorname{Max}$ & Min. & Max \\
\hline Acceptable & Range & 13.0 & 17.0 & 7.50 & 8.50 & 4.0 & $N A^{(a)}$ & 28.3 & 32.3 \\
\hline $\begin{array}{l}B C-5 \\
B C-5 \\
3 C-5 \\
B C-5\end{array}$ & $\begin{array}{r}0 \\
10 \\
50 \\
100\end{array}$ & $\begin{array}{l}15.1 \\
15.2 \\
15.1 \\
14.9\end{array}$ & $\begin{array}{l}15.6 \\
15.6 \\
15.5 \\
15.3\end{array}$ & $\begin{array}{l}7.86 \\
7.97 \\
7.91 \\
7.61\end{array}$ & $\begin{array}{l}8.12 \\
8.16 \\
8.29 \\
8.31\end{array}$ & $\begin{array}{l}7.2 \\
7.6 \\
7.5 \\
7.5\end{array}$ & $\begin{array}{l}8.5 \\
8.2 \\
8.5 \\
8.4\end{array}$ & $\begin{array}{l}30.0 \\
30.0 \\
30.0 \\
30.0\end{array}$ & $\begin{array}{l}30.5 \\
31.0 \\
30.5 \\
31.0\end{array}$ \\
\hline $\begin{array}{l}B C-6 \\
B C-6 \\
B C-6 \\
B C-6\end{array}$ & $\begin{array}{r}0 \\
10 \\
50 \\
100\end{array}$ & $\begin{array}{l}15.0 \\
15.2 \\
15.1 \\
15.2\end{array}$ & $\begin{array}{l}15.7 \\
15.5 \\
15.5 \\
15.5\end{array}$ & $\begin{array}{r}8.02 \\
7.92 \\
.99 \\
8.05\end{array}$ & $\begin{array}{l}8.20 \\
8.18 \\
8.19 \\
8.22\end{array}$ & $\begin{array}{l}7.6 \\
7.5 \\
7.4 \\
7.3\end{array}$ & $\begin{array}{l}8.5 \\
8.4 \\
8.3 \\
8.4\end{array}$ & $\begin{array}{l}30.0 \\
30.0 \\
30.0 \\
30.0\end{array}$ & $\begin{array}{l}30.5 \\
30.5 \\
30.5 \\
30.5\end{array}$ \\
\hline $\begin{array}{l}B C-7 \\
B C-7 \\
B C-7 \\
B C-7\end{array}$ & $\begin{array}{r}0 \\
10 \\
50 \\
100\end{array}$ & $\begin{array}{l}13.9 \\
14.0 \\
14.0 \\
13.8\end{array}$ & $\begin{array}{l}15.5 \\
15.5 \\
15.5 \\
15.5\end{array}$ & $\begin{array}{l}7.78 \\
7.89 \\
7.88 \\
7.92\end{array}$ & $\begin{array}{l}8.06 \\
8.12 \\
8.14 \\
8.36\end{array}$ & $\begin{array}{l}7.3 \\
7.0 \\
7.5 \\
7.3\end{array}$ & $\begin{array}{l}8.3 \\
8.3 \\
8.3 \\
8.5\end{array}$ & $\begin{array}{l}30.0 \\
30.0 \\
30.0 \\
30.0\end{array}$ & $\begin{array}{l}31.5 \\
32.0 \\
31.0 \\
31.5\end{array}$ \\
\hline $\begin{array}{l}B C-8 \\
B C-8 \\
B C-8 \\
B C-8\end{array}$ & $\begin{array}{r}0 \\
10 \\
50 \\
100\end{array}$ & $\begin{array}{l}14.7 \\
14.5 \\
14.6 \\
14.0\end{array}$ & $\begin{array}{l}15.5 \\
15.5 \\
15.5 \\
15.5\end{array}$ & $\begin{array}{l}7.97 \\
7.58 \\
7.80 \\
7.64\end{array}$ & $\begin{array}{l}8.15 \\
8.00 \\
8.25 \\
8.15\end{array}$ & $\begin{array}{l}7.6 \\
7.5 \\
7.6 \\
7.7\end{array}$ & $\begin{array}{l}8.3 \\
8.3 \\
8.4 \\
8.3\end{array}$ & $\begin{array}{l}30.0 \\
30.0 \\
30.0 \\
30.0\end{array}$ & $\begin{array}{l}31.0 \\
30.5 \\
31.0 \\
30.5\end{array}$ \\
\hline $\begin{array}{l}R-A M \\
R-A M \\
R-A M \\
R-A M\end{array}$ & $\begin{array}{r}0 \\
10 \\
50 \\
100\end{array}$ & $\begin{array}{l}14.9 \\
14.9 \\
14.8 \\
14.8\end{array}$ & $\begin{array}{l}15.5 \\
15.5 \\
15.5 \\
15.5\end{array}$ & $\begin{array}{l}7.84 \\
7.92 \\
7.63 \\
7.74\end{array}$ & $\begin{array}{l}8.08 \\
8.18 \\
8.02 \\
8.11\end{array}$ & $\begin{array}{l}7.3 \\
6.6 \\
5.2 \\
7.1\end{array}$ & $\begin{array}{l}8.3 \\
8.4 \\
8.4 \\
8.3\end{array}$ & $\begin{array}{l}30.0 \\
30.0 \\
30.0 \\
30.0\end{array}$ & $\begin{array}{l}32.0 \\
31.5 \\
32.0 \\
31.5\end{array}$ \\
\hline $\begin{array}{l}R-B F \\
R-B F \\
R-B F \\
R-B F\end{array}$ & $\begin{array}{r}0 \\
10 \\
50 \\
100\end{array}$ & $\begin{array}{l}13.9 \\
13.9 \\
14.0 \\
14.1\end{array}$ & $\begin{array}{l}15.5 \\
15.5 \\
15.7 \\
15.6\end{array}$ & $\begin{array}{l}7.83 \\
7.65 \\
7.81 \\
7.94\end{array}$ & $\begin{array}{l}8.12 \\
8.16 \\
8.21 \\
8.32\end{array}$ & $\begin{array}{l}6.9 \\
7.4 \\
7.4 \\
7.4\end{array}$ & $\begin{array}{l}7.8 \\
7.7 \\
7.6 \\
7.8\end{array}$ & $\begin{array}{l}30.0 \\
30.5 \\
30.0 \\
30.0\end{array}$ & $\begin{array}{l}31.0 \\
32.0 \\
31.5 \\
32.0\end{array}$ \\
\hline $\begin{array}{l}R-P F \\
R-P F \\
R-P F \\
R-P F\end{array}$ & $\begin{array}{r}0 \\
10 \\
50 \\
100\end{array}$ & $\begin{array}{l}14.8 \\
14.7 \\
14.5 \\
14.6\end{array}$ & $\begin{array}{l}15.5 \\
15.5 \\
15.4 \\
15.5\end{array}$ & $\begin{array}{l}7.95 \\
7.89 \\
7.92 \\
7.93\end{array}$ & $\begin{array}{l}8.17 \\
8.14 \\
8.14 \\
8.18\end{array}$ & $\begin{array}{l}7.1 \\
7.4 \\
7.4 \\
7.5\end{array}$ & $\begin{array}{l}8.2 \\
8.1 \\
8.3 \\
8.3\end{array}$ & $\begin{array}{l}30.0 \\
30.5 \\
30.0 \\
30.0\end{array}$ & $\begin{array}{l}31.5 \\
32.0 \\
32.0 \\
30.5\end{array}$ \\
\hline
\end{tabular}

(a) Not applicable. 
IABLE I.4. Daily Observations of 4-Day H. sculpta Suspended-ParticulatePhase Test, Oakland Harbor Phase III B Test

\begin{tabular}{|c|c|c|c|c|c|c|c|c|c|c|c|c|c|c|}
\hline \multirow{2}{*}{$\begin{array}{l}\text { Sediment } \\
\text { Ireaiment }\end{array}$} & \multirow{2}{*}{$\begin{array}{l}\text { Concentration } \\
\text { Percent SPp }\end{array}$} & \multirow[b]{2}{*}{ Replycate } & \multicolumn{6}{|c|}{ H. sculpta Live } & \multicolumn{6}{|c|}{ H. sculpra Dead } \\
\hline & & & $\underline{O W}$ & $\underline{4 h}$ & $24 h$ & $\underline{48 h}$ & $\underline{72 h}$ & $\underline{96 h}$ & oh & $4 h$ & $24 h$ & $48 h$ & $72 h$ & $\underline{96 h}$ \\
\hline $\begin{array}{l}B C-5 \\
B C-5 \\
B C-5\end{array}$ & $\begin{array}{l}0 \\
0 \\
0\end{array}$ & $\begin{array}{l}1 \\
2 \\
3\end{array}$ & $\begin{array}{l}10 \\
10 \\
10\end{array}$ & $\begin{array}{l}10 \\
10 \\
10\end{array}$ & $\begin{array}{l}10 \\
10 \\
10\end{array}$ & $\begin{array}{l}10 \\
10 \\
10\end{array}$ & $\begin{array}{r}10 \\
9 \\
9\end{array}$ & $\begin{array}{r}10 \\
8 \\
9\end{array}$ & $\begin{array}{l}0 \\
0 \\
0\end{array}$ & $\begin{array}{l}0 \\
0 \\
0\end{array}$ & $\begin{array}{l}0 \\
0 \\
0\end{array}$ & $\begin{array}{l}0 \\
0 \\
0\end{array}$ & $\begin{array}{l}0 \\
1 \\
1\end{array}$ & $\begin{array}{l}0 \\
1 \\
0\end{array}$ \\
\hline $\begin{array}{l}B C-5 \\
B C-5 \\
B C-5\end{array}$ & $\begin{array}{l}10 \\
10 \\
10\end{array}$ & $\begin{array}{l}1 \\
2 \\
3\end{array}$ & $\begin{array}{l}10 \\
10 \\
10\end{array}$ & $\begin{array}{l}10 \\
10 \\
10\end{array}$ & $\begin{array}{r}10 \\
10 \\
9\end{array}$ & $\begin{array}{r}10 \\
10 \\
9\end{array}$ & $\begin{array}{r}10 \\
8 \\
9\end{array}$ & $\begin{array}{r}10 \\
8 \\
8\end{array}$ & $\begin{array}{l}0 \\
0 \\
0\end{array}$ & $\begin{array}{l}0 \\
0 \\
0\end{array}$ & $\begin{array}{l}0 \\
0 \\
0\end{array}$ & $\begin{array}{l}0 \\
0 \\
0\end{array}$ & $\begin{array}{l}0 \\
2 \\
0\end{array}$ & $\begin{array}{l}0 \\
0 \\
1\end{array}$ \\
\hline $\begin{array}{l}B C-5 \\
B C-5 \\
B C-5\end{array}$ & $\begin{array}{l}50 \\
50 \\
50\end{array}$ & $\begin{array}{l}1 \\
2 \\
3\end{array}$ & $\begin{array}{l}10 \\
10 \\
10\end{array}$ & $\begin{array}{l}10 \\
10 \\
10\end{array}$ & $\begin{array}{r}10 \\
9 \\
10\end{array}$ & $\begin{array}{r}10 \\
9 \\
10\end{array}$ & $\begin{array}{r}10 \\
9 \\
9\end{array}$ & $\begin{array}{r}10 \\
9 \\
8\end{array}$ & $\begin{array}{l}0 \\
0 \\
0\end{array}$ & $\begin{array}{l}0 \\
? \\
0\end{array}$ & $\begin{array}{l}0 \\
1 \\
0\end{array}$ & $\begin{array}{l}0 \\
0 \\
0\end{array}$ & $\begin{array}{l}0 \\
0 \\
1\end{array}$ & $\begin{array}{l}0 \\
0 \\
1\end{array}$ \\
\hline $\begin{array}{l}8 C-5 \\
B C-5 \\
B C-5\end{array}$ & $\begin{array}{l}100 \\
100 \\
100\end{array}$ & $\begin{array}{l}1 \\
2 \\
3\end{array}$ & $\begin{array}{l}10 \\
10 \\
10\end{array}$ & $\begin{array}{l}10 \\
10 \\
10\end{array}$ & $\begin{array}{r}8 \\
8 \\
10\end{array}$ & $\begin{array}{l}8 \\
8 \\
8\end{array}$ & $\begin{array}{l}8 \\
8 \\
6\end{array}$ & $\begin{array}{l}7 \\
8 \\
6\end{array}$ & $\begin{array}{l}0 \\
0 \\
0\end{array}$ & $\begin{array}{l}0 \\
0 \\
0\end{array}$ & $\begin{array}{l}2 \\
2 \\
0\end{array}$ & $\begin{array}{l}0 \\
0 \\
2\end{array}$ & $\begin{array}{l}0 \\
0 \\
2\end{array}$ & $\begin{array}{l}1 \\
0 \\
0\end{array}$ \\
\hline $\begin{array}{l}B C-6 \\
B C-6 \\
B C-6\end{array}$ & $\begin{array}{l}0 \\
0 \\
0\end{array}$ & $\begin{array}{l}1 \\
2 \\
3\end{array}$ & $\begin{array}{l}10 \\
10 \\
10\end{array}$ & $\begin{array}{l}10 \\
10 \\
10\end{array}$ & $\begin{array}{r}9 \\
10 \\
10\end{array}$ & $\begin{array}{r}9 \\
10 \\
10\end{array}$ & $\begin{array}{r}9 \\
10 \\
10\end{array}$ & $\begin{array}{r}9 \\
10 \\
9\end{array}$ & $\begin{array}{l}0 \\
0 \\
0\end{array}$ & $\begin{array}{l}0 \\
0 \\
0\end{array}$ & $\begin{array}{l}1 \\
0 \\
0\end{array}$ & $\begin{array}{l}0 \\
0 \\
0\end{array}$ & $\begin{array}{l}0 \\
0 \\
0\end{array}$ & $\begin{array}{l}0 \\
0 \\
1\end{array}$ \\
\hline $\begin{array}{l}B C-6 \\
B C-6 \\
B C-6\end{array}$ & $\begin{array}{l}10 \\
10 \\
10\end{array}$ & $\begin{array}{l}1 \\
2 \\
3\end{array}$ & $\begin{array}{l}10 \\
10 \\
10\end{array}$ & $\begin{array}{l}10 \\
10 \\
10\end{array}$ & $\begin{array}{r}10 \\
9 \\
9\end{array}$ & $\begin{array}{l}9 \\
8 \\
9\end{array}$ & $\begin{array}{l}8 \\
8 \\
8\end{array}$ & $\begin{array}{l}7 \\
8 \\
8\end{array}$ & $\begin{array}{l}0 \\
0 \\
0\end{array}$ & $\begin{array}{l}0 \\
0 \\
0\end{array}$ & $\begin{array}{l}0 \\
1 \\
1\end{array}$ & $\begin{array}{l}1 \\
1 \\
0\end{array}$ & $\begin{array}{l}1 \\
0 \\
1\end{array}$ & $\begin{array}{l}1 \\
0 \\
0\end{array}$ \\
\hline $\begin{array}{l}B C-6 \\
8 C-6 \\
8 C-6\end{array}$ & $\begin{array}{l}50 \\
50 \\
50\end{array}$ & $\begin{array}{l}1 \\
2 \\
3\end{array}$ & $\begin{array}{l}10 \\
10 \\
10\end{array}$ & $\begin{array}{l}10 \\
10 \\
10\end{array}$ & $\begin{array}{l}8 \\
9 \\
8\end{array}$ & $\begin{array}{l}8 \\
8 \\
7\end{array}$ & $\begin{array}{l}7 \\
8 \\
6\end{array}$ & $\begin{array}{l}7 \\
8 \\
6\end{array}$ & $\begin{array}{l}0 \\
0 \\
0\end{array}$ & $\begin{array}{l}0 \\
0 \\
0\end{array}$ & $\begin{array}{l}2 \\
1 \\
2\end{array}$ & $\begin{array}{l}0 \\
1 \\
1\end{array}$ & $\begin{array}{l}1 \\
0 \\
1\end{array}$ & $\begin{array}{l}0 \\
0 \\
0\end{array}$ \\
\hline $\begin{array}{l}8 C-6 \\
B C-6 \\
B C-6\end{array}$ & $\begin{array}{l}100 \\
100 \\
100\end{array}$ & $\begin{array}{l}1 \\
2 \\
3\end{array}$ & $\begin{array}{l}10 \\
10 \\
10\end{array}$ & $\begin{array}{l}10 \\
10 \\
10\end{array}$ & $\begin{array}{r}8 \\
10 \\
9\end{array}$ & $\begin{array}{l}8 \\
9 \\
7\end{array}$ & $\begin{array}{l}7 \\
9 \\
7\end{array}$ & $\begin{array}{l}7 \\
8 \\
7\end{array}$ & $\begin{array}{l}0 \\
0 \\
0\end{array}$ & $\begin{array}{l}0 \\
0 \\
0\end{array}$ & $\begin{array}{l}2 \\
0 \\
1\end{array}$ & $\begin{array}{l}0 \\
1 \\
2\end{array}$ & $\begin{array}{l}1 \\
0 \\
0\end{array}$ & $\begin{array}{l}0 \\
1 \\
0\end{array}$ \\
\hline $\begin{array}{l}B C-7 \\
B C-7 \\
B C-7\end{array}$ & $\begin{array}{l}0 \\
0 \\
0\end{array}$ & $\begin{array}{l}1 \\
2 \\
3\end{array}$ & $\begin{array}{l}10 \\
10 \\
10\end{array}$ & $\begin{array}{l}10 \\
10 \\
10\end{array}$ & $\begin{array}{l}10 \\
10 \\
10\end{array}$ & $\begin{array}{r}10 \\
10 \\
9\end{array}$ & $\begin{array}{r}9 \\
10 \\
9\end{array}$ & $\begin{array}{r}9 \\
10 \\
9\end{array}$ & $\begin{array}{l}0 \\
0 \\
0\end{array}$ & $\begin{array}{l}0 \\
0 \\
0\end{array}$ & $\begin{array}{l}0 \\
0 \\
0\end{array}$ & $\begin{array}{l}0 \\
0 \\
1\end{array}$ & $\begin{array}{l}1 \\
0 \\
0\end{array}$ & $\begin{array}{l}0 \\
0 \\
0\end{array}$ \\
\hline $\begin{array}{l}B C-7 \\
B C-7 \\
B C-7\end{array}$ & $\begin{array}{l}10 \\
10 \\
10\end{array}$ & $\begin{array}{l}1 \\
2 \\
3\end{array}$ & $\begin{array}{l}10 \\
10 \\
10\end{array}$ & $\begin{array}{l}10 \\
10 \\
10\end{array}$ & $\begin{array}{l}10 \\
10 \\
10\end{array}$ & $\begin{array}{r}10 \\
9 \\
10\end{array}$ & $\begin{array}{r}9 \\
9 \\
10\end{array}$ & $\begin{array}{r}9 \\
9 \\
10\end{array}$ & $\begin{array}{l}0 \\
0 \\
0\end{array}$ & $\begin{array}{l}0 \\
0 \\
0\end{array}$ & $\begin{array}{l}0 \\
0 \\
0\end{array}$ & $\begin{array}{l}0 \\
1 \\
0\end{array}$ & $\begin{array}{l}i \\
0 \\
0\end{array}$ & $\begin{array}{l}0 \\
0 \\
0\end{array}$ \\
\hline $\begin{array}{l}B C-7 \\
B C-7 \\
B C-7\end{array}$ & $\begin{array}{l}50 \\
50 \\
50\end{array}$ & $\begin{array}{l}1 \\
2 \\
3\end{array}$ & $\begin{array}{l}10 \\
10 \\
10\end{array}$ & $\begin{array}{l}10 \\
1.0 \\
10\end{array}$ & $\begin{array}{r}10 \\
9 \\
10\end{array}$ & $\begin{array}{l}7 \\
8 \\
8\end{array}$ & $\begin{array}{l}7 \\
7 \\
8\end{array}$ & $\begin{array}{l}7 \\
7 \\
8\end{array}$ & $\begin{array}{l}0 \\
0 \\
0\end{array}$ & $\begin{array}{l}0 \\
0 \\
0\end{array}$ & $\begin{array}{l}0 \\
1 \\
0\end{array}$ & $\begin{array}{l}3 \\
1 \\
2\end{array}$ & $\begin{array}{l}0 \\
1 \\
0\end{array}$ & $\begin{array}{l}0 \\
0 \\
0\end{array}$ \\
\hline $\begin{array}{l}B C-7 \\
B C-7 \\
B C-7\end{array}$ & $\begin{array}{l}100 \\
100 \\
100\end{array}$ & $\begin{array}{l}1 \\
2 \\
3\end{array}$ & $\begin{array}{l}10 \\
10 \\
10\end{array}$ & $\begin{array}{l}10 \\
10 \\
10\end{array}$ & $\begin{array}{r}9 \\
10 \\
10\end{array}$ & $\begin{array}{l}8 \\
8 \\
7\end{array}$ & $\begin{array}{l}8 \\
7 \\
7\end{array}$ & $\begin{array}{l}7 \\
6 \\
7\end{array}$ & $\begin{array}{l}0 \\
0 \\
0\end{array}$ & $\begin{array}{l}0 \\
0 \\
0\end{array}$ & $\begin{array}{l}1 \\
0 \\
0\end{array}$ & $\begin{array}{l}1 \\
2 \\
3\end{array}$ & $\begin{array}{l}0 \\
1 \\
0\end{array}$ & $\begin{array}{l}1 \\
2 \\
0\end{array}$ \\
\hline $\begin{array}{l}B C-8 \\
B C-8 \\
B C-8\end{array}$ & $\begin{array}{l}0 \\
0 \\
0\end{array}$ & $\begin{array}{l}1 \\
2 \\
3\end{array}$ & $\begin{array}{l}10 \\
10 \\
10\end{array}$ & $\begin{array}{l}10 \\
10 \\
10\end{array}$ & $\begin{array}{r}10 \\
10 \\
9\end{array}$ & $\begin{array}{r}10 \\
10 \\
9\end{array}$ & $\begin{array}{r}10 \\
10 \\
9\end{array}$ & $\begin{array}{r}10 \\
10 \\
9\end{array}$ & $\begin{array}{l}0 \\
0 \\
0\end{array}$ & $\begin{array}{l}0 \\
0 \\
0\end{array}$ & $\begin{array}{l}0 \\
0 \\
1\end{array}$ & $\begin{array}{l}0 \\
0 \\
0\end{array}$ & $\begin{array}{l}0 \\
0 \\
0\end{array}$ & $\begin{array}{l}0 \\
0 \\
0\end{array}$ \\
\hline $\begin{array}{l}B C-8 \\
B C-8 \\
B C-8\end{array}$ & $\begin{array}{l}10 \\
10 \\
10\end{array}$ & $\begin{array}{l}1 \\
? \\
3\end{array}$ & $\begin{array}{l}10 \\
10 \\
10\end{array}$ & $\begin{array}{l}10 \\
10 \\
10\end{array}$ & $\begin{array}{l}10 \\
10 \\
10\end{array}$ & $\begin{array}{r}10 \\
9 \\
10\end{array}$ & $\begin{array}{r}10 \\
8 \\
9\end{array}$ & $\begin{array}{r}10 \\
8 \\
9\end{array}$ & $\begin{array}{l}0 \\
0 \\
0\end{array}$ & $\begin{array}{l}0 \\
0 \\
0\end{array}$ & $\begin{array}{l}0 \\
0 \\
0\end{array}$ & $\begin{array}{l}0 \\
1 \\
0\end{array}$ & $\begin{array}{l}0 \\
1 \\
1\end{array}$ & $\begin{array}{l}0 \\
0 \\
0\end{array}$ \\
\hline
\end{tabular}


TABLE 1.4. (contd)

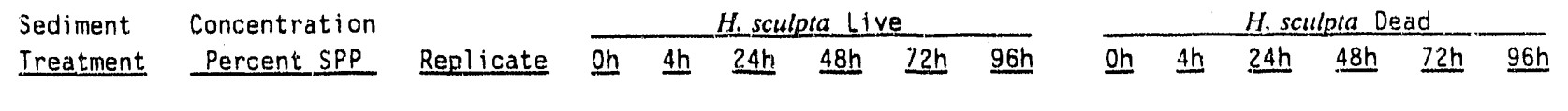

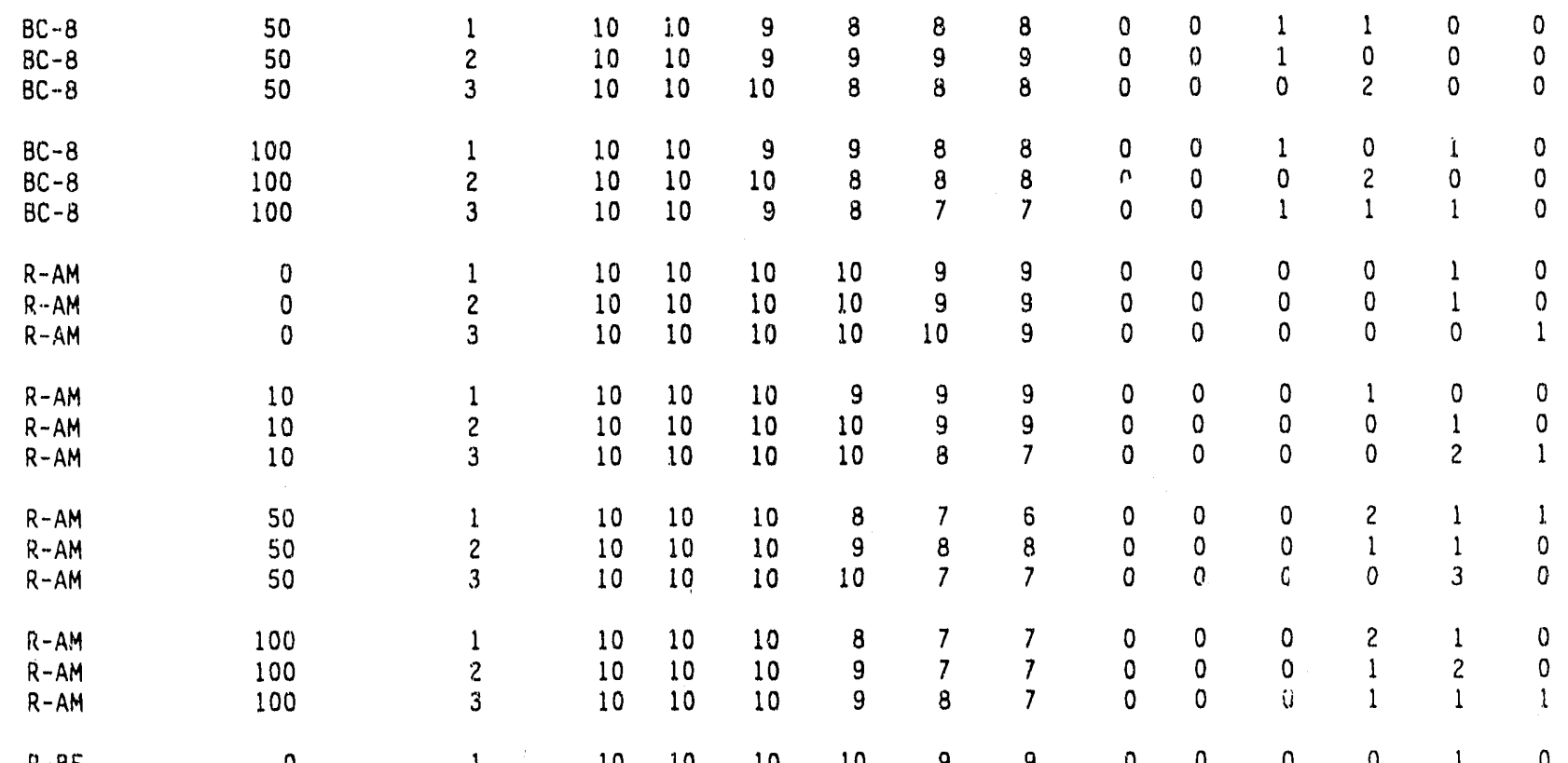

$\begin{array}{lllllllrrrrrrrr}R-B F & 0 & 1 & 10 & 10 & 10 & 10 & 9 & 9 & 0 & 0 & 0 & 0 & 1 & 0 \\ R-t F & 0 & 2 & 10 & 10 & 10 & 10 & 10 & 10 & 0 & 0 & 0 & 0 & 0 & 0 \\ R-B F & 0 & 3 & 10 & 10 & 10 & 10 & 9 & 8 & 0 & 0 & 0 & 0 & 1 & 1\end{array}$

$\begin{array}{rrrrrrrrrrrrrrr}R-B F & 10 & 1 & 10 & 10 & 10 & 10 & 10 & 10 & 0 & 0 & 0 & 0 & 0 & 0 \\ R-B F & 10 & 2 & 10 & 10 & 10 & 10 & 9 & 9 & 0 & 0 & 0 & 0 & 1 & 0 \\ R-B F & 10 & 3 & 10 & 10 & 10 & 9 & 9 & 9 & 0 & 0 & 0 & 1 & 0 & 0\end{array}$

$\begin{array}{lllllllllllllll}R-B F & 50 & 1 & 10 & 10 & 10 & 8 & 8 & 8 & 0 & 0 & 0 & 2 & 0 & 0 \\ R-B F & 50 & 2 & 10 & 10 & 10 & 10 & 9 & 8 & 0 & 0 & 0 & 0 & 1 & 1\end{array}$

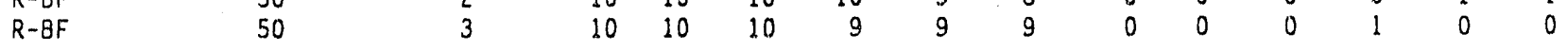

$\begin{array}{lllllllllllllll}R-B F & 100 & 1 & 10 & 10 & 10 & 9 & 8 & 7 & 0 & 0 & 0 & 1 & 1 & 1 \\ R-B F & 100 & 2 & 10 & 10 & 10 & 8 & 8 & 7 & 0 & 0 & 0 & 2 & 0 & 1\end{array}$

$\begin{array}{lllllllllllllll}R-B F & 100 & 3 & 10 & 10 & 10 & 8 & 7 & 6 & 0 & 0 & 0 & 2 & 1 & 1\end{array}$

$\begin{array}{llllllrrrrrrrrr}R-P F & 0 & 1 & 10 & 10 & 10 & 9 & 9 & 9 & 0 & 0 & 0 & 1 & 0 & 0 \\ R-P F & 0 & 2 & 10 & 10 & 10 & 10 & 10 & 9 & 0 & 0 & 0 & 0 & 0 & 1 \\ R-P F & 0 & 3 & 10 & 10 & 10 & 10 & 10 & 10 & 0 & 0 & 0 & 0 & 0 & 0\end{array}$

$\begin{array}{llllllllllllllll}R-P F & 0 & 3 & 10 & 10 & 10 & 10 & 10 & 10 & 0 & 0 & 0 & 0 & 0 & 0 & 0\end{array}$

$\begin{array}{lllllllllllllll}R-P F & 10 & 1 & 10 & 10 & 10 & 10 & 9 & 9 & 0 & 0 & 0 & 0 & 1 & 0 \\ R-P F & 10 & 2 & 10 & 10 & 10 & 9 & 9 & 9 & 0 & 0 & 0 & 1 & 0 & 0 \\ R-P F & 10 & 3 & 10 & 10 & 10 & 9 & 9 & 9 & 0 & 0 & 0 & 1 & 0 & 0\end{array}$

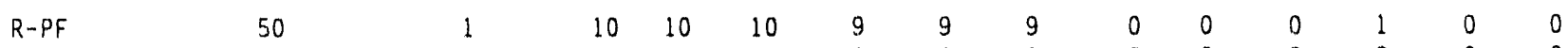

$\begin{array}{lllllllllllllll}R-P F & 50 & 2 & 10 & 10 & 10 & 8 & 8 & 8 & 0 & 0 & 0 & 2 & 0 & 0 \\ R-P F & 50 & 3 & 10 & 10 & 10 & 9 & 8 & 8 & 0 & 0 & 0 & 1 & 1 & 0\end{array}$

$\begin{array}{lllllllllllllll}R-P F & 100 & 1 & 10 & 10 & 10 & 9 & 7 & 7 & 0 & 0 & 0 & 1 & 2 & 0 \\ R-P F & 100 & 2 & 10 & 10 & 10 & 8 & 8 & 8 & 0 & 0 & 0 & 2 & 0 & 0 \\ R-P F & 100 & 3 & 10 & 10 & 10 & 8 & 7 & 6 & 0 & 0 & 0 & 2 & 1 & 1\end{array}$

$\begin{array}{llllllrrrrrrrrr}\mathrm{Zn} & 0 \mathrm{mg} / \mathrm{L} & 1 & 10 & 10 & 10 & 10 & 9 & 9 & 0 & 0 & 0 & 0 & 1 & 0 \\ \mathrm{Zn} & 0 \mathrm{mg} / \mathrm{L} & 2 & 10 & 10 & 10 & 10 & 10 & 10 & 0 & 0 & 0 & 0 & 0 & 0 \\ \mathrm{Zn} & 0 \mathrm{mg} / \mathrm{L} & 3 & 10 & 10 & 10 & 9 & 9 & 9 & 0 & 0 & 0 & 1 & 0 & 0\end{array}$

PHASE III B 
TABLE 1.4. (contd)

\begin{tabular}{|c|c|c|c|c|c|c|c|c|c|c|c|c|c|c|}
\hline \multirow{2}{*}{$\begin{array}{l}\text { Sediment } \\
\text { Ireatment }\end{array}$} & \multirow{2}{*}{$\begin{array}{l}\text { Concentration } \\
\text { Percent SPP }\end{array}$} & \multirow[b]{2}{*}{ Replicate } & \multicolumn{6}{|c|}{ H. sculpta Live } & \multicolumn{6}{|c|}{ H. scuslpta Dead } \\
\hline & & & $\underline{\underline{Q h}}$ & $\underline{4 h}$ & $24 h$ & $48 \mathrm{~h}$ & I2h & $\underline{96 h}$ & $\underline{\text { Oh}}$ & $\underline{4 h}$ & $24 h$ & $48 h$ & $\underline{72 h}$ & $96 h$ \\
\hline $\mathrm{Zn}$ & $0.25 \mathrm{mg} / \mathrm{L}$ & 1 & 10 & 10 & 10 & 8 & 7 & 7 & 0 & 0 & 0 & 2 & 1 & 0 \\
\hline $\mathrm{Zn}$ & $0.25 \mathrm{mg} / \mathrm{L}$ & 2 & 10 & 10 & 10 & 9 & 8 & 7 & 0 & 0 & 0 & 1 & 1 & 1 \\
\hline $\mathrm{Zn}$ & $0.25 \mathrm{mg} / \mathrm{L}$ & 3 & 10 & 10 & 10 & 9 & 7 & 7 & 0 & 0 & 0 & 1 & 2 & 0 \\
\hline $\mathrm{Zn}$ & $0.50 \mathrm{mg} / \mathrm{L}$ & 1 & 10 & 10 & 8 & 6 & 5 & 4 & 0 & 0 & 2 & 2 & 1 & 1 \\
\hline $2 n$ & $0.50 \mathrm{mg} / \mathrm{L}$ & 2 & 10 & 10 & 7 & 7 & 5 & 3 & 0 & 0 & 3 & 0 & 2 & 2 \\
\hline $\mathrm{Zn}$ & $0.50 \mathrm{mg} / \mathrm{L}$ & 3 & 10 & 10 & 8 & 8 & 4 & 3 & 0 & 0 & 2 & 0 & 3 & 1 \\
\hline $\mathrm{Zn}$ & $1.00 \mathrm{mg} / \mathrm{L}$ & 1 & 10 & 10 & 6 & 0 & 0 & 0 & 0 & 0 & 4 & 6 & 10 & 10 \\
\hline $2 n$ & $1.00 \mathrm{mg} / \mathrm{L}$ & 2 & 10 & 10 & 3 & 0 & 0 & 0 & 0 & 0 & 7 & 3 & 10 & 10 \\
\hline $\mathrm{Zn}$ & $1.00 \mathrm{mg} / \mathrm{L}$ & 3 & 10 & 10 & 4 & 0 & 0 & 0 & 0 & 0 & 6 & 4 & 10 & 10 \\
\hline $\mathrm{Zn}$ & $2.00 \mathrm{mg} / \mathrm{L}$ & 1 & 10 & 10 & 0 & 0 & 0 & 0 & 0 & 0 & 10 & 10 & 10 & 10 \\
\hline In & $2.00 \mathrm{mg} / \mathrm{L}$ & 2 & 10 & 10 & 0 & 0 & 0 & 0 & 0 & 0 & 10 & 10 & 10 & 10 \\
\hline $2 n$ & $2.00 \mathrm{mg} / \mathrm{L}$ & 3 & 10 & 10 & 0 & 0 & 0 & 0 & 0 & 0 & 10 & 10 & 10 & 10 \\
\hline
\end{tabular}


TABLE 1.5. Survival Data for All Replicates in 4-Day H. :sulpta Reference Toxicant. Test, Oakl and Harbor Phase III B

\begin{tabular}{|c|c|c|c|c|}
\hline \multirow[b]{2}{*}{$\begin{array}{l}\text { Zinc } \\
\text { Concentration (mg/L) }\end{array}$} & \multirow[b]{2}{*}{ Replicate } & \multicolumn{2}{|c|}{ H. sculpta } & \multirow{2}{*}{\begin{tabular}{l}
\multicolumn{1}{c}{ Mean } \\
Proportion \\
Surviving
\end{tabular}} \\
\hline & & Live & $\begin{array}{l}\text { Deád } \\
\text { or Missing }\end{array}$ & \\
\hline $\begin{array}{l}0 \\
0 \\
0\end{array}$ & $\begin{array}{l}1 \\
2 \\
3\end{array}$ & $\begin{array}{r}9 \\
10 \\
9\end{array}$ & $\begin{array}{l}1 \\
0 \\
1\end{array}$ & 0.93 \\
\hline $\begin{array}{l}0.25 \\
0.25 \\
0.25\end{array}$ & $\begin{array}{l}1 \\
2 \\
3\end{array}$ & $\begin{array}{l}7 \\
7 \\
7\end{array}$ & $\begin{array}{l}3 \\
3 \\
3\end{array}$ & 0.70 \\
\hline $\begin{array}{l}0.50 \\
0.50 \\
0.50\end{array}$ & $\begin{array}{l}1 \\
2 \\
3\end{array}$ & $\begin{array}{l}4 \\
3 \\
3\end{array}$ & $\begin{array}{l}6 \\
7 \\
7\end{array}$ & 0.33 \\
\hline $\begin{array}{l}1.00 \\
1.00 \\
1.00\end{array}$ & $\begin{array}{l}1 \\
2 \\
3\end{array}$ & $\begin{array}{l}0 \\
0 \\
0\end{array}$ & $\begin{array}{l}10 \\
10 \\
10\end{array}$ & 0.00 \\
\hline $\begin{array}{l}2.00 \\
2.00 \\
2.00\end{array}$ & $\begin{array}{l}1 \\
2 \\
3\end{array}$ & $\begin{array}{l}0 \\
0 \\
0\end{array}$ & $\begin{array}{l}10 \\
10 \\
10\end{array}$ & 0.00 \\
\hline
\end{tabular}


TABLE I.6. Water Qual ity Summary for 4-Day H. sci?pta Reference Toxicant Test, Oakland Harbor Phase III B Test

\begin{tabular}{|c|c|c|c|c|c|c|c|c|c|}
\hline \multirow{2}{*}{$\begin{array}{c}\text { Zinc } \\
\text { Concentration }(\mathrm{mg} / L)\end{array}$} & \multirow[b]{2}{*}{ Replicate } & \multicolumn{2}{|c|}{$\begin{array}{c}\text { Temperature } \\
{ }^{\circ} \mathrm{C}\end{array}$} & \multicolumn{2}{|c|}{$\mathrm{pH}$} & \multicolumn{2}{|c|}{$\begin{array}{c}\text { Dissolved } \\
\text { Oxygen } \\
\text { (mg/L) }\end{array}$} & \multicolumn{2}{|c|}{$\begin{array}{c}\text { Salinity } \\
(0 / 00)\end{array}$} \\
\hline & & Min. & $\operatorname{Max}$ & Min. & $\operatorname{Max}$ & Min & $\operatorname{Max}$ & Min & $\operatorname{Max}$ \\
\hline Acceptable Range & & 13.0 & 17.0 & 7.50 & 8.50 & 4.0 & $\mathrm{NA}^{(\mathrm{a})}$ & 28.3 & 32.3 \\
\hline $\begin{array}{l}0 \\
0.25 \\
0.50 \\
1.00 \\
2.00\end{array}$ & $\begin{array}{l}1 \\
1 \\
1 \\
1 \\
1\end{array}$ & $\begin{array}{l}15.1 \\
15.0 \\
15.2 \\
15.3 \\
15.4\end{array}$ & $\begin{array}{l}15.5 \\
15.6 \\
15.5 \\
15.5 \\
15.5\end{array}$ & $\begin{array}{l}7.90 \\
7.89 \\
7.68 \\
7.80 \\
7.99\end{array}$ & $\begin{array}{l}8.19 \\
8.08 \\
8.13 \\
8.05 \\
8.02\end{array}$ & $\begin{array}{l}7.4 \\
7.4 \\
6.5 \\
7.5 \\
7.8\end{array}$ & $\begin{array}{l}8.4 \\
8.1 \\
8.3 \\
8.0 \\
8.1\end{array}$ & $\begin{array}{l}30.0 \\
30.0 \\
30.0 \\
30.0 \\
30.0\end{array}$ & $\begin{array}{l}30.5 \\
31.0 \\
30.5 \\
30.5 \\
30.0\end{array}$ \\
\hline
\end{tabular}

(a) Not applicable.

IABLE 1.7. Daily Observations of 4-Day H. sculpta Reference Toxicant Test, Oakland Harbor Phase III B Project

\begin{tabular}{c} 
Zinc \\
Concentrati \\
\hline 0 \\
0 \\
0 \\
0.25 \\
0.25 \\
0.25 \\
0.50 \\
0.50 \\
0.50 \\
1.00 \\
1.00 \\
1.00 \\
2.00 \\
2.00 \\
2.00
\end{tabular}

Zinc
Replicate

$\begin{array}{rrrrrrr}1 & 10 & 10 & 10 & 10 & 9 & 9 \\ 2 & 10 & 10 & 10 & 10 & 10 & 10 \\ 3 & 10 & 10 & 10 & 9 & 9 & 9 \\ 1 & 10 & 10 & 10 & 8 & 7 & 7 \\ 2 & 10 & 10 & 10 & 9 & 8 & 7 \\ 3 & 10 & 10 & 10 & 9 & 7 & 7 \\ 1 & 10 & 10 & 8 & 6 & 5 & 4 \\ 2 & 10 & 10 & 7 & 7 & 5 & 3 \\ 3 & 10 & 10 & 8 & 8 & 4 & 3 \\ 1 & 10 & 10 & 6 & 0 & 0 & 0 \\ 1 & 10 & 10 & 3 & 0 & 0 & 0 \\ 3 & 10 & 10 & 4 & 0 & 0 & 0 \\ 1 & 10 & 10 & 0 & 0 & 0 & 0 \\ 1 & 10 & 10 & 0 & 0 & 0 & 0 \\ 2 & 10 & 10 & 0 & 0 & 0 & 0 \\ 3 & & & \end{array}$

H. sculpta Dead

\begin{tabular}{cccccc}
\multicolumn{6}{c}{ H. sculpla Dead } \\
\hline$\underline{Q h}$ & $\underline{4 h}$ & $\underline{24 h}$ & $\underline{43 h}$ & $\underline{72 h}$ & $\underline{96 h}$ \\
0 & 0 & 0 & 0 & 1 & 0 \\
0 & 0 & 0 & 0 & 0 & 0 \\
0 & 0 & 0 & 1 & 0 & 0 \\
0 & 0 & 0 & 2 & 1 & 0 \\
0 & 0 & 0 & 1 & 1 & 1 \\
0 & 0 & 0 & 1 & 2 & 0 \\
0 & 0 & 2 & 2 & 1 & 1 \\
0 & 0 & 3 & 0 & 2 & 2 \\
0 & 0 & 2 & 0 & 3 & 1 \\
& & & & & \\
0 & 0 & 4 & 6 & 10 & 10 \\
0 & 0 & 7 & 3 & 10 & 10 \\
0 & 0 & 6 & 4 & 10 & 10 \\
& & & & & \\
0 & 0 & 10 & 10 & 10 & 10 \\
0 & 0 & 10 & 10 & 10 & 10 \\
0 & 0 & 10 & 10 & 10 & 10
\end{tabular}




\section{APPENDIX J}

\section{8-HOUR STATIC SUSPENDED "ARTICULATE-PHASE TEST.}

WITH LARVAL M. EDULIS 
TABLE J.1. Test Results for All Replicates in 48-Hour Larval M. edul is Suspended-Particulate-Phase Test, Oakland Harbor Phase III B Project

\begin{tabular}{|c|c|c|c|c|c|c|c|c|c|}
\hline \multirow[b]{2}{*}{$\begin{array}{l}\text { SPP } \\
\text { Ireatment }\end{array}$} & \multirow[b]{2}{*}{$\begin{array}{l}\text { Concentration } \\
\text { Percent SPP }\end{array}$} & \multirow[b]{2}{*}{ Replicate } & \multicolumn{4}{|c|}{ Number at Development Stage } & \multirow[b]{2}{*}{$\begin{array}{l}\text { Percent } \\
\text { Normal } \\
\end{array}$} & \multirow{2}{*}{$\begin{array}{l}\text { Mean } \\
\text { Percent } \\
\text { Survival (a) } \\
\end{array}$} & \multirow{2}{*}{$\begin{array}{l}\text { Mean } \\
\text { Percent } \\
\text { Survival } \\
\end{array}$} \\
\hline & & & Normal D & $\begin{array}{l}\text { Earlier } \\
\text { Stages }\end{array}$ & $\begin{array}{l}\text { Abnormal } \\
\text { w/ shell }\end{array}$ & $\begin{array}{l}\text { Percent } \\
\text { Normal a) }\end{array}$ & & & \\
\hline$B C-5$ & 0 & 1 & 244 & 10 & 0 & $100.0^{(b)}$ & & $100.0^{(b)}$ & \\
\hline$B C-5$ & 0 & 2 & 210 & 4 & 0 & 90.1 & & 91.8 & \\
\hline$B C-5$ & 0 & 3 & 183 & 19 & 0 & 78.5 & 89.5 & 86.7 & 92.8 \\
\hline$B C-5$ & 10 & 1 & 220 & 6 & 0 & 94.4 & & 97.0 (b) & \\
\hline$B C-5$ & 10 & 2 & 227 & 12 & 0 & $97.4(b)$ & & $100.0(b)$ & \\
\hline$B C-5$ & 10 & 3 & 241 & 19 & 0 & $100.0^{(0)}$ & 97.3 & $100.0^{(B)}$ & 99.0 \\
\hline$B C-5$ & 50 & 1 & 213 & 6 & 0 & 91.4 & & 94.0 & \\
\hline$B C-5$ & 50 & 2 & 200 & 0 & 0 & 85.8 & & 85.8 & \\
\hline$B C-5$ & 50 & 3 & 2.18 & 3 & 0 & 93.6 & 90.3 & 94.8 & 91.5 \\
\hline$B C-5$ & 100 & 1 & 204 & 14 & 0 & 87.6 & & 93.6 & \\
\hline$B C-5$ & 100 & 2 & 149 & 49 & 0 & 63.9 & & 85.0 & \\
\hline$B C-5$ & 100 & 3 & 167 & 47 & 0 & 71.7 & 74.4 & 91.8 & 90.1 \\
\hline$B C-6$ & 0 & 1 & 212 & 16 & 0 & 91.0 & & 97.9 & \\
\hline$B C-6$ & 0 & 2 & 201 & 14 & 0 & 86.3 & & 92.3 & \\
\hline$B C-6$ & 0 & 3 & 222 & 2 & 0 & 95.3 & 90.9 & 96.1 & 95.4 \\
\hline$B C-6$ & 10 & 1 & 214 & 0 & 0 & 91.8 & & 91.8 & \\
\hline $8 C-6$ & 10 & 2 & 227 & 0 & 0 & 97.4 & & 97.4 & \\
\hline$B C-6$ & 10 & 3 & 216 & 0 & 0 & 32.7 & 94.0 & 92.7 & 94.0 \\
\hline$B C-6$ & 50 & 1 & 197 & 2 & 0 & 84.5 & & 85.4 & \\
\hline$B C-6$ & 50 & 2 & 214 & 0 & 0 & 91.8 & & 91.8 & \\
\hline$B C-\hat{\sigma}$ & 50 & 3 & 199 & 6 & 0 & 85.4 & 87.2 & 88.0 & 88.4 \\
\hline$B C-6$ & 100 & 1 & 215 & 3 & 0 & 92.3 & & 93.6 & \\
\hline$B C-6$ & 100 & 2 & 208 & 0 & 0 & 89.3 & & 89.3 & \\
\hline$B C-6$ & 100 & 3 & 198 & 0 & 0 & 85.0 & 88.9 & 85.0 & 89.3 \\
\hline$B C-7$ & 0 & 1 & 217 & 5 & 0 & 93.1 & & 95.3 & \\
\hline$B C-7$ & 0 & 2 & 198 & 2 & 0 & 85.0 & & 85.8 & \\
\hline$B C-7$ & 0 & 3 & 197 & 0 & 0 & 84.5 & 87.5 & 34.5 & 88.5 \\
\hline$B C-7$ & 10 & 1 & 257 & 0 & 0 & $100.0^{(b)}$ & & $100.0^{(b)}$ & \\
\hline$B C-7$ & 10 & 2 & 205 & 6 & 0 & 88.0 & & 90.6 & \\
\hline$B C-i$ & 10 & 3 & 218 & 0 & 0 & 93.6 & 93.9 & 93.6 & 94.7 \\
\hline$B C-7$ & 50 & 1 & 221 & 0 & 0 & 94.8 & & 94.8 & \\
\hline$B C-7$ & 50 & 2 & 210 & 9 & 0 & 90.1 & & 94.0 & \\
\hline$B C-7$ & 50 & 3 & 209 & 4 & 0 & 89.7 & 91.5 & 91.4 & 93.4 \\
\hline$B C-7$ & 100 & 1 & 69 & 110 & 0 & 29.6 & & 76.8 & \\
\hline$B C-?$ & 100 & 2 & 69 & 116 & 0 & 29.6 & & 79.4 & \\
\hline$B C-7$ & 100 & 3 & 89 & 28 & 0 & 38.2 & 32.5 & 50.2 & 68.8 \\
\hline$B C-B$ & 0 & 1 & 199 & 3 & 0 & 85.4 & & 86.7 & \\
\hline$B C-8$ & 0 & 2 & 180 & 8 & 0 & 77.3 & & 80.7 & \\
\hline$B C-8$ & 0 & 3 & 222 & 0 & 0 & 95.3 & 86.0 & 95.3 & 87.6 \\
\hline$B C-8$ & 10 & 1 & 217 & 2 & 0 & 93.1 & & 94.0 & \\
\hline$B C-8$ & 10 & 2 & 207 & b & 0 & 88.8 & & 91.4 & \\
\hline$\overline{B C}-8$ & 10 & 3 & 21.1 & 0 & 0 & 90.6 & 90.8 & 90.6 & 92.0 \\
\hline
\end{tabular}


IABLE J.1. (contd)

SPP Concentration

Treatment Percent SPP Replicate

$\begin{array}{lr}B C-8 & 50 \\ B C-8 & 50 \\ B C-8 & 50 \\ B C-8 & 100 \\ B C-8 & 100 \\ B C-8 & 100 \\ R-A M & 0 \\ R-A M & 0 \\ R-A M & 0 \\ R-A M & 10 \\ R-A M & 10 \\ R-A M & 10\end{array}$

R-AM $\quad 50$

R-AM $\quad 50$

R-AM 50

$R-A M \quad 100$

R-AM $\quad 100$

$R-A M \quad 100$

$R-B F$

$R-B F$

$R-B F$

$R-B F$

$R-B F$

$R-B F$

$R=B F$

$R-B F$

$R-B F$

$R-B F$

$R-B F$

$R-B F$

$R-P F$

$R-P F$

$R-P F$

$R \sim P F$

$R-P F$

$R-P F$

$R-P F$

$R-P F$

$R-P F$
Number at Development Stage Earlier Abnornal Percent Normal D Stages Abnornal Percental
w/ shell Normal a)

Percent Normal

Mean

Percent

Survival (a)

Mean Percent

187
187
241
181
236
229
200
199
247
194
183
205

$\begin{array}{lll}2 & \\ 0 & 0 \\ 0 & 0\end{array}$

$\begin{array}{ll}0 & 80.3 \\ 0 & 80.3(b) \\ 0 & 100.0(b)\end{array}$

81.1

$86.9 \quad \begin{aligned} & 80.3 \\ & \quad 100.0\end{aligned}(b)$

87.1

$\begin{array}{rrr}3 & 0 & 77.7(b) \\ 8 & 0 & 100.0(b) \\ 8 & 0 & 98.3\end{array}$

79.0
$100.0(b)$

98.3

92.0

101.7

93.6

85.8

85.4 (b)

91.0

$90.4 \quad \begin{array}{r}87.6 \\ 100.0\end{array}(\mathrm{~b})$

92.8

83.3

78.5

88.0

87.1
81.5

$83.3 \quad 92.3$

87.0

80.3

91.0

32.3

89.3

86.7

95.3

92.7

80.3

95.3

87.6

96.6

90.1

88.4

75.1

82.4

68.2

961

85.4

$100.0^{(b)}$

94.8
100.0

94.8

91.8
$100.0(b)$

97.4

86.7

85.4

81.1
91.0

$87.9 \quad 93.6$

88.6

$90.4 \quad \begin{array}{r}86.7 \\ \quad 100.0^{(b)}\end{array}$

92.0

97.4

$89.4 \quad \begin{array}{r}88.0 \\ \quad 100.0^{(b)}\end{array}$

95.1

$100.1(b)$

$91.4 \quad 93.1$

95.4

82.7

83.7

68.2

99.1

$83.2 \quad 88.8$

85.4

$100.0^{(b)}$

$98.3 \quad \begin{array}{r}98.7 \\ \quad 100.0^{(b)}\end{array}$

99.6

94.8

$95.5 \quad \begin{aligned} & 94.4 \\ & 100.0\end{aligned}$

96.4

$100.0^{(b)}$

86.7

86.3

91.0 
TABLE J.1. (contd)

SPP Concentration

Number at Development Stage

Earlier

Abnormal Percental
w/ shell Normal

Mean
Percent Percent

Normal

Survival (a)

Mean

Treatment Percent SPP Replicate Normal D Stages

$$
\begin{array}{cc} 
& 100.0^{(b)} \\
& 100.0^{(b)} \\
95.6 \quad & y 0.2
\end{array}
$$

$\begin{array}{ll}Q-P F & 100 \\ Q-P F & 100\end{array}$

$R-P F \quad 100$

226
232
210

13
7
0

97.0

90.1

96.7

(a) Percent normal and percent survival based on stocking density of 2.33 larvae per $10 \mathrm{inL}$

(b) A value of $100 \%$ was used when number of normal 0 or number surviving was greater than the stocking density. 
TABLE J.2. Survival Data for All Replicates in 48-Hour Larval M. edulis Reference Toxicant Test, Oakland Harbor Phase III B Project

\begin{tabular}{|c|c|c|c|c|c|c|c|c|}
\hline \multirow[b]{2}{*}{$\begin{array}{l}\text { Copper } \\
\text { Concentration }(\mathrm{mg} / \mathrm{L})\end{array}$} & \multirow[b]{2}{*}{ Replicate } & \multicolumn{4}{|c|}{ Number at Development Stage } & \multirow{2}{*}{$\begin{array}{l}\text { Mean } \\
\text { Percent } \\
\text { Normal } \\
\end{array}$} & \multirow[b]{2}{*}{$\begin{array}{l}\text { Percent } \\
\text { Survival }\end{array}$} & \multirow{2}{*}{$\begin{array}{c}\text { Mean } \\
\text { Percent } \\
\text { Survival }\end{array}$} \\
\hline & & Normal 0 & $\begin{array}{l}\text { Earlier } \\
\text { Stages }\end{array}$ & $\begin{array}{l}\text { Abnormal } \\
\text { W/ shell }\end{array}$ & $\begin{array}{l}\text { Percent } \\
\text { Normal }\end{array}$ & & & \\
\hline $\begin{array}{l}0 \\
0\end{array}$ & $\begin{array}{l}1 \\
2\end{array}$ & $\begin{array}{l}232 \\
226\end{array}$ & $\begin{array}{r}7 \\
18\end{array}$ & $\begin{array}{l}0 \\
0\end{array}$ & $\begin{array}{l}99.6 \\
97.0\end{array}$ & 98.3 & $\begin{array}{l}100.0^{(b)} \\
100.0^{(b)}\end{array}$ & 100.0 \\
\hline $\begin{array}{l}1 \\
1\end{array}$ & $\begin{array}{l}1 \\
2\end{array}$ & $\begin{array}{l}181 \\
243\end{array}$ & $\begin{array}{l}17 \\
11\end{array}$ & $\begin{array}{l}0 \\
0\end{array}$ & $\begin{aligned} 77.7 & (b)\end{aligned}$ & 88.8 & $\begin{array}{r}85.0 \\
100.0^{(b)}\end{array}$ & 92.5 \\
\hline $\begin{array}{l}4 \\
4\end{array}$ & $\begin{array}{l}1 \\
2\end{array}$ & $\begin{array}{l}207 \\
191\end{array}$ & $\begin{array}{l}9 \\
5\end{array}$ & $\begin{array}{l}0 \\
0\end{array}$ & $\begin{array}{l}88.8 \\
82.0\end{array}$ & 85.4 & $\begin{array}{l}92.7 \\
84.1\end{array}$ & 88.4 \\
\hline $\begin{array}{l}16 \\
16\end{array}$ & $\begin{array}{l}1 \\
2\end{array}$ & $\begin{array}{r}115 \\
0\end{array}$ & $\begin{array}{l}106 \\
169\end{array}$ & $\begin{array}{l}0 \\
0\end{array}$ & $\begin{array}{r}49.4 \\
0.0\end{array}$ & 24.7 & $\begin{array}{l}94.8 \\
72.5\end{array}$ & 83.7 \\
\hline 64 & 1 & 0 & 14 & 0 & 0.0 & 0.0 & 6.0 & 6.0 \\
\hline
\end{tabular}

(a) Percent normal and percent survival based on stocking density of 233 larvae per $10 \mathrm{~mL}$.

(b) A value of $100 \%$ was used when number of normal $D$ or number surviving was greater than the stocking density. 
TABLE J.3. Water Quality Summary for 48-Hour Larval M. edul is Suspended-Particulate-Phase Test, Oakland Harbor Phase III B Project

\begin{tabular}{|c|c|c|c|c|c|c|c|c|c|}
\hline \multirow{2}{*}{$\begin{array}{l}\text { SPP } \\
\text { Ireatment }\end{array}$} & \multirow{2}{*}{$\begin{array}{l}\text { Concentration } \\
\text { Percent SPP }\end{array}$} & \multicolumn{2}{|c|}{$\begin{array}{c}\text { Temperature } \\
\left({ }^{\circ} \mathrm{C}\right)\end{array}$} & \multicolumn{2}{|c|}{$\mathrm{pH}$} & \multicolumn{2}{|c|}{$\begin{array}{c}\text { Oxygen } \\
(\mathrm{mg} / \mathrm{L}) \\
\end{array}$} & \multicolumn{2}{|c|}{$\begin{array}{c}\text { Salinity } \\
(0 / 00) \\
\end{array}$} \\
\hline & & Min. & $\operatorname{Max}$ & Min & Max & Min & Max & Min & Max \\
\hline Acceptable & Range & 13.0 & 17.0 & 7.44 & 8.44 & 4.0 & $N A^{(a)}$ & 28.3 & 32.3 \\
\hline $\begin{array}{l}B C-5 \\
B C-5 \\
B C-5 \\
B C-5\end{array}$ & $\begin{array}{r}0 \\
10 \\
50 \\
100\end{array}$ & $\begin{array}{l}15.7 \\
15.6 \\
15.5 \\
15.6\end{array}$ & $\begin{array}{l}16.0 \\
15.8 \\
15.8 \\
15.8\end{array}$ & $\begin{array}{l}7.86 \\
7.92 \\
7.89 \\
7.85\end{array}$ & $\begin{array}{l}8.28 \\
8.29 \\
8.36 \\
8.40\end{array}$ & $\begin{array}{l}5.6 \\
7.7 \\
6.8 \\
5.6\end{array}$ & $\begin{array}{l}8.1 \\
7.9 \\
7.8 \\
7.9\end{array}$ & $\begin{array}{l}30.0 \\
30.0 \\
30.0 \\
30.0\end{array}$ & $\begin{array}{l}30.5 \\
30.5 \\
30.5 \\
30.5\end{array}$ \\
\hline $\begin{array}{l}B C-6 \\
B C-6 \\
B C-6 \\
B C-6\end{array}$ & $\begin{array}{r}0 \\
10 \\
50 \\
100\end{array}$ & $\begin{array}{l}15.7 \\
15.6 \\
15.2 \\
15.6\end{array}$ & $\begin{array}{l}15.9 \\
16.0 \\
15.6 \\
15.8\end{array}$ & $\begin{array}{l}7.94 \\
7.92 \\
7.93 \\
8.06\end{array}$ & $\begin{array}{l}8.23 \\
8.29 \\
8.22 \\
8.27\end{array}$ & $\begin{array}{l}7.2 \\
7.7 \\
7.8 \\
7.7\end{array}$ & $\begin{array}{l}8.3 \\
8.0 \\
7.9 \\
8.0\end{array}$ & $\begin{array}{l}30.0 \\
30.0 \\
30.0 \\
30.0\end{array}$ & $\begin{array}{l}30.5 \\
30.5 \\
30.5 \\
30.5\end{array}$ \\
\hline $\begin{array}{l}B C-7 \\
B C-7 \\
B C-7 \\
B C-7\end{array}$ & $\begin{array}{r}0 \\
10 \\
50 \\
100\end{array}$ & $\begin{array}{l}15.7 \\
15.4 \\
15.4 \\
15.6\end{array}$ & $\begin{array}{l}15.8 \\
15.8 \\
15.9 \\
15.6\end{array}$ & $\begin{array}{l}7.93 \\
7.88 \\
7.93 \\
7.92\end{array}$ & $\begin{array}{l}8.28 \\
8.27 \\
8.33 \\
8.46\end{array}$ & $\begin{array}{l}7.5 \\
7.4 \\
7.0 \\
5.3\end{array}$ & $\begin{array}{l}8.2 \\
8.0 \\
7.9 \\
7.9\end{array}$ & $\begin{array}{l}30.0 \\
30.0 \\
30.0 \\
30.0\end{array}$ & $\begin{array}{l}30.5 \\
30.5 \\
30.5 \\
30.5\end{array}$ \\
\hline $\begin{array}{l}B C-8 \\
B C-8 \\
B C-8 \\
B C-8\end{array}$ & $\begin{array}{r}0 \\
10 \\
50 \\
100\end{array}$ & $\begin{array}{l}15.7 \\
15.7 \\
15.6 \\
15.6\end{array}$ & $\begin{array}{l}15.8 \\
15.9 \\
15.8 \\
15.8\end{array}$ & $\begin{array}{r}7.93 \\
7.91 \\
7.9 \\
7.88\end{array}$ & $\begin{array}{l}8.28 \\
8.29 \\
8.23 \\
8.31\end{array}$ & $\begin{array}{l}5.4 \\
5.0 \\
7.4 \\
7.0\end{array}$ & $\begin{array}{l}8.1 \\
8.0 \\
8.0 \\
8.2\end{array}$ & $\begin{array}{l}30.0 \\
30.0 \\
30.0 \\
30.0\end{array}$ & $\begin{array}{l}30.5 \\
30.5 \\
30.5 \\
30.5\end{array}$ \\
\hline $\begin{array}{l}R-A M \\
R-A M \\
R-A M \\
R-A M\end{array}$ & $\begin{array}{r}0 \\
10 \\
50 \\
100\end{array}$ & $\begin{array}{l}15.6 \\
15.5 \\
15.6 \\
15.6\end{array}$ & $\begin{array}{l}15.8 \\
16.0 \\
15.7 \\
15.8\end{array}$ & $\begin{array}{l}7.93 \\
7.92 \\
7.94 \\
7.93\end{array}$ & $\begin{array}{l}8.24 \\
8.28 \\
8.23 \\
8.26\end{array}$ & $\begin{array}{l}7.6 \\
7.6 \\
7.8 \\
7.7\end{array}$ & $\begin{array}{l}8.0 \\
8.1 \\
8.2 \\
8.2\end{array}$ & $\begin{array}{l}30.0 \\
30.5 \\
30.0 \\
30.0\end{array}$ & $\begin{array}{l}30.5 \\
30.5 \\
30.5 \\
30.5\end{array}$ \\
\hline $\begin{array}{l}R-B F \\
R-B F \\
R-B F \\
R-B F\end{array}$ & $\begin{array}{r}0 \\
10 \\
50 \\
100\end{array}$ & $\begin{array}{l}15.7 \\
15.7 \\
15.5 \\
15.3\end{array}$ & $\begin{array}{l}16.1 \\
15.8 \\
15.7 \\
15.7\end{array}$ & $\begin{array}{l}7.93 \\
7.93 \\
7.91 \\
7.91\end{array}$ & $\begin{array}{r}8.26 \\
8.27 \\
8.33 \\
8.4\end{array}$ & $\begin{array}{l}7.7 \\
7.7 \\
6.8 \\
4.7\end{array}$ & $\begin{array}{l}8.2 \\
8.0 \\
7.9 \\
8.1\end{array}$ & $\begin{array}{l}30.0 \\
30.0 \\
30.0 \\
30.0\end{array}$ & $\begin{array}{l}30.5 \\
30.5 \\
30.5 \\
31.0\end{array}$ \\
\hline $\begin{array}{l}R-P F \\
R-P F \\
R-P F \\
R-P F\end{array}$ & $\begin{array}{r}0 \\
10 \\
50 \\
100\end{array}$ & $\begin{array}{l}15.3 \\
15.5 \\
15.6 \\
15.6\end{array}$ & $\begin{array}{l}16.0 \\
16.1 \\
15.9 \\
15.7\end{array}$ & $\begin{array}{l}7.93 \\
7.93 \\
7.93 \\
7.93\end{array}$ & $\begin{array}{l}8.28 \\
8.24 \\
8.27 \\
8.29\end{array}$ & $\begin{array}{l}7.5 \\
7.7 \\
7.0 \\
7.6\end{array}$ & $\begin{array}{l}8.0 \\
8.1 \\
7.9 \\
8.0\end{array}$ & $\begin{array}{l}30.0 \\
30.0 \\
30.0 \\
30.0\end{array}$ & $\begin{array}{l}30.0 \\
30.5 \\
30.5 \\
30.5\end{array}$ \\
\hline
\end{tabular}

(a) Not applicable. 
TABLE J.4. Water Qual ity Summary for 48-Hour Larval M. edul is Reference Toxicant Test, Oakland Phase III B Project

\begin{tabular}{|c|c|c|c|c|c|c|c|c|}
\hline \multirow{2}{*}{$\begin{array}{c}\text { Copper } \\
\text { Concentration }(\mathrm{mg} / \mathrm{L})\end{array}$} & \multicolumn{2}{|c|}{$\begin{array}{l}\text { Temperature } \\
\left({ }^{\circ} \mathrm{C}\right)\end{array}$} & \multicolumn{2}{|c|}{$\mathrm{pH}$} & \multicolumn{2}{|c|}{$\begin{array}{c}\text { Oissolved } \\
\text { Oxygen } \\
(\mathrm{mg} / \mathrm{L})\end{array}$} & \multicolumn{2}{|c|}{$\begin{array}{l}\text { Salinity } \\
(0 / 00) \\
\end{array}$} \\
\hline & Min & Max & Min & $\operatorname{Max}$ & Min & Max & Min & Max \\
\hline Acceptable Range & 13.0 & 17.0 & 7.38 & 8.38 & 4.0 & $N A^{(a)}$ & 28.1 & 32.1 \\
\hline 0 & 15.6 & 15.6 & 7.6 & 7.9 & 8.0 & 8.0 & 30.0 & 30.0 \\
\hline 1 & 15.6 & 15.6 & 7.93 & 7.93 & 8.1 & 8.1 & 30.0 & 30.0 \\
\hline 4 & 15.6 & 15.6 & 7.93 & 7.93 & 8.0 & 8.1 & 30.0 & 30.0 \\
\hline 16 & 15.6 & 15.6 & 7.92 & 7.92 & 8.1 & 8.1 & 30.5 & 30.5 \\
\hline 64 & 15.5 & 15.6 & 7.88 & 7.88 & 8.1 & 8.1 & $30.1)$ & 30.0 \\
\hline
\end{tabular}

(a) Not applicable. 
APPENDIX K

M.NASUTA TISSUE CHEMISTRY AND QUALITY CONTROL DATA 


\section{QA/QC SUMMARY}

PROGRAM:

PARAMETER:

LABORATORY:

MATRIX:

SAMPLE NUMBER:

HOLDING TIMES

DETECTION LIMITS

METHOD BLANKS

SURROGATES

MATRIX SPIKES
Oakland Harbor Phase III B

Polynuclear Aromatic Hydrocarbons (PAHs)

Alden Analytical Laboratories, Inc.

$M$. nasuta Tissue

130 (26 treatments, 5 replicates each)

Samples of $M$. nasuta tissues were held frozen for up to 2 months prior to extraction and analysis. All sample extracts were analyzed within the EPArecommended extract holding time of 40 days (EPA 1986).

Target detection limits (Table 2.2) were achieved for all PAH compounds.

Seven method blanks were analyzed. Naphthalene was detected in one method blank at $190 \mu \mathrm{g} / \mathrm{kg}$. This elevated level was a result of quantitation against the low surrogate recovery of $d_{8}$-naphthalene. The low recovery was the result of the sample overheating during extraction. Naphthalene was not detected in any of the samples associated with this method blank. Therefore, the data were not qualified.

Four radiolabeled PAH compounds were added as surrogates to samples prior to analysis: $d_{8}$ naphthalene, $d_{10}$ acenaphthene, $d_{10}$ phenanthrene, and $d_{12}$ benzo(a)anthracene. The target recovery for surrogates was 50\% to125\%. In 37 of 130 sample analyses, up to three of the surrogates were recovered below the target recovery range. Laboratory protocol requires reextraction only if two or more surrogates are below 20\%. This was the case for only one sample, $\mathrm{O}-\mathrm{C} 3$, Replicate 5, where insufficient sample remained for reextraction. No analytes were detected in this sample. Therefore, the data ivere not qualified.

Fourteen samples were spiked with all 16 PAH compounds of interest. Four of these spikes consisted of spike duplicates of two samples. Because of limited sample volumes, the other spikes were performed on individual samples and no comparison of Relative Percent Difference (RPD) was possible. Spike recoveries for LPAH compounds ranged from $3 \%$ to $60 \%$, and from $16 \%$ to $240 \%$ for HPAH compounds. The majority of the spike recoveries were within the $\mathrm{QC}$ recovery limits listed in the QA plan for this project. Those spike recoveries that were outside of the QC limits all exceeded the limits from $2 \%$ to $38 \%$, with the exception of one compound, benzo(b)fluoranthene, which exceeded the QC recovery limits in one matrix spike, I-C8, by almost $100 \%$. For analytes with high recoveries in both batch spikes, data associated with those batches are flagged with an " $E$ " to indicate possible high bias.

\section{REPLICATES}

SRMs
Six samples were analyzed in triplicate. Precision was measured by Relative Standard Deviation (RSD) between replicates. RSDs were not calculated for analytes that were undetected in one or more of the replicates. RSDs of triplicate analyses for all analytes ranged from 5\% to $24 \%$. 


\section{QA/QC SUMMARY}

PROGRAM:

PARAMETER:

LABORATORY:

MATRIX:

SAMPLE NUMBER:

HOLDING TIMES

DETECTION LIMITS

METHOD BLANKS

SURROGATES

MATRIX SPIKES
Oakland Harbor Phase III B

Chlorinated Pesticides and PCBs

Alden Analytical Laboratories, Inc.

$M$. nasuta Tissues

130 (26 treatments, 5 samples each)

Samples were held frozen prior to extraction. All samples were extracted within 2 months of receipt at Battelle MSL. All sample extracts were analyzed within the EPA-recommended holding time of 40 days (EPA 1986).

Target detection limits for chlorinated pesticides $(2.0 \mu \mathrm{g} / \mathrm{kg}$ wet weight) were achieved for all samples with the following exceptions: in four samples, detection limits for $B-B H C$ ranged from $2.5-5.0 \mu \mathrm{g} / \mathrm{kg}$; in one sample, the detection limit for $4,4^{\prime}-\mathrm{DDD}$ was $2.5 \mu \mathrm{g} / \mathrm{kg}$; in one sample, the detection limit. for $4,4^{\prime}-\mathrm{DDT}$ was $5.0 \mu \mathrm{g} / \mathrm{kg}$; and in three samples, the detection limit for endrin aldehyde was $2.5 \mu \mathrm{g} / \mathrm{kg}$. The detection limit for toxaphene in all sarnples was $100 \mu \mathrm{g} / \mathrm{kg}$ wet weight. These elevated detection limits can be autributed to the coelution of interfering peaks with the analytes of interest. The toxaphene detection limit was set higher to ensure accurate quantitation of the analyte and to avoid false positive detections. Target detection limits $(20 \mu \mathrm{g} / \mathrm{kg}$ wet weight) for PCBs were achieved for all samples.

Seven method blanks were analyzed. No pesticides or PCBs were detected in any of the method blanks.

One compound, dibutylchlorendate (DBC), was added to all samples as a surrogate spike. Recoveries were within the EPA CLP-advisory QC limits of $20 \% 10157 \%$ for soils and sediments (no limits have been derived for tissues) with the folowing exceptions: recoveries of $D B C$ in ten samples ranged from $13 \%$ to $19 \%$. Because no other chlorin. ted pesticides or PCBs were detected in those samples, the data were not qualified.

Fourteen samples were spiked with aldrin and dieldrin as matrix spike samples. Recoveries of aldrin were within the EPA CLP control limits of $42 \%$ to $122 \%$ with the following exceptions: aldrin was recovered from one sample at $148 \%$ and another at $152 \%$. Recoveries of dieldrin were within the EPA CL.P control limits of $36 \%$ to $146 \%$ with the following exceptions: dieldrin recoveries in three samples ranged from $150 \%$ to $164 \%$. Because no other chlorinated pesticides or PCBs were detected in those samples, the data were not qualified.

Five samples were analyzed in triplicate and precision was measured by calculating the Relative Percent Difference (RSD) between the replicates. One sample was analyzed in duplicate (the amount of sample was insufficient for a triplicate anlaysis). Precison was measured by Relative Percent Difference (RPD) between duplicate results. However, because no analytes were detected in the samples, neither the RSD nor RPD values were calculated. 


\section{QA/QC SUMMARY}

PROGRAM:

PARAMETER:

LABORATORY:

MATRIX:

SAMPLE NUMBER:

\author{
HOLDING TIMES
}

DETECTION LIMITS

METHOD BLANKS

\section{SURROGATES}

MATRIX SPIKES

\author{
Oakland Harbor Pliase III B \\ Metals (Ag,As,Cd,Cr,Cu,Hg,Ni, $\mathrm{Pb}, \mathrm{Se}, \mathrm{Zn}$ ) \\ Battelle/Marine Sciences Laboratory \\ $M$. nasuta Tissue \\ 130 (26 treatments, 5 samples each)
}

Based on the EPA Draft Implementation Manual (EPA/USACE 1990), the allowed holding time for tissue samples for analysis of $\mathrm{Ag}_{0} \mathrm{As}, \mathrm{Cd}, \mathrm{Cr}, \mathrm{Cu}, \mathrm{Ni}$, $\mathrm{Pb}, \mathrm{Se}$, and $\mathrm{Zn}$ is 6 months, and 28 days for $\mathrm{Hg}$. Samples must be kept frozen. For this project, $M$. nasuta tissue samples were held frozen for up to 21 days after receipt at Battelle MSL. Samples were then freeze dried and stored for up to 3 months in sealed containers. No holding times have been established for freeze dried samples. However, standard reference material prepared by the U.S. National Institute of Standards and Technology (NIST) such as SRM 1566a (Oyster tissue) have been issued for up to 10 years without changes to the certified concentrations for metals, including those for $\mathrm{Hg}$.

Detection limit goals (Table 2.2), were achieved for all metals.

Nine method blanks were analyzed by $\mathrm{AA}$ for $\mathrm{Ag}, \mathrm{Cd}, \mathrm{Cr}, \mathrm{Hg}$, and $\mathrm{Pb}$. Concentrations of metals in blanks ranged as follows: $\mathrm{Ag}, 0.008$ to $0.010 \mu \mathrm{g} / \mathrm{g}$; Cd, 0.07 to $0.10 \mu \mathrm{g} / \mathrm{g} ; \mathrm{Cr}, 0.110$ to $0.345 \mu \mathrm{g} / \mathrm{g} ; \mathrm{Hg}$, undetected to $0.001 \mu \mathrm{g} / \mathrm{g}$; $\mathrm{Pb}$, undetected. Concentrations reported for samples in which these analytes were detected are corrected for the corcentrations found in the corresponding blanks. Method blanks are not applicable to metals analyzed by XRF.

\section{NOT APPLICABLE}

Seven matrix spike samples (frequency of 1 spike sample per 20 samples). were analyzed by $\mathrm{AA}$ for $\mathrm{Ag}, \mathrm{Cd}, \mathrm{Cr}, \mathrm{Hg}$ and $\mathrm{Pb}$. Spike recoveries ranged as follows: $\mathrm{Ag}, 46 \%$ to $98 \%$; $\mathrm{Cd}, 100 \%$ to $119 \%$; $\mathrm{Cr}, 94 \%$ to $104 \%$; $\mathrm{Hg}, 83 \%$ to $98 \%$; $\mathrm{Pb}$, $84 \%$ to $108 \%$. Recovery goals stated in the QC plan are as follows: Cr, $85 \%$. to $115 \% ; \mathrm{Hg}, 75 \%$ to $125 \%$; $\mathrm{Pb}, 75 \%$ to $125 \%$. Recovery goals for these metals were achieved.

Although no recovery goals are set for silver, three out of the seven spikes were below 75\%. The SRMs associated with two of these samples (Batch 2) were also somewhat low and may indicate that the values reported may be biased. The $\mathrm{Ag}$ data associated with Batch 2 have been flagged with an " $E$ " to indicate a possible bias low. The SRM associated with the third low spike recovery was within the certified value for silver and no qualifications were made for samples associated with this batch.

Seven samples were analyzed in triplicate for all metals. Precision was measured by the Relative Standard Deviation (RSD) between replicate results. RSDs ranged as follows: $\mathrm{Ag}, 1 \%$ to $6 \%$; As, $1 \%$ to $4 \%$; $\mathrm{Cd}, 3 \%$ to $10 \% ; \mathrm{Cr}, 1 \%$ to $52 \%$; $\mathrm{Cu}, 1 \%$ to $5 \%$; $\mathrm{Hg}, 0 \%$ to $33 \%$; $\mathrm{Ni}, 9 \%$ to $39 \% ; \mathrm{Pb}, 2 \%$ to $5 \% ; \mathrm{Se}, 5 \%$ 
to $37 \%, \mathrm{Zn}, 0 \%$ to $2 \%$. The target relative precision for all metals analyses is $15 \%$.

SRMs

SRM 1566a, oyster tissue, was obtained from the U.S. National Institute of Standards and Technology (NIST). This SRM was analyzed by both XRF and AA at a minimum frequency of once per 10 samples. The results of the analyses were within the certified values for the SRM with the following exceptions: $\mathrm{Ag}$ was reported below the certified range in two of eight SRM samples analyzed; data associated with these SRMs were qualified with an "E" as mentioned in the above section on matrix spikes. Two out of 12 SRMs analyzed for $\mathrm{Cd}$ and 3 of 8 analyzed for $\mathrm{Pb}$ were slightly outside the certified range, however, they were well within the $15 \%$ QC guidelines specified in the QAVQC plan. Ni and Se, analyzed by XRF, had 13 of 44 and 14 of 44 (respectively) SRM measurements outside of certified range. Though the majority of SRM measurernents were within range, the variability of the ones that weren't within range could not be explained other than by slight differences in analytical technique. 


\section{QA/QC SUMMARY}

PRUGRAM:

PARAMETER:

LABOK:TORY:

MATRIX:

SAMPLE NITMBER:

HOLDING TIMES

DETECTION LIMITS

METHOD BLANKS

SURROGATES

MATRIX SPIKES

REPLICATES

SRMs

\author{
Oakland Harbor Phase III B \\ Butyltins \\ Battelle/Marine Sciences Laboratory \\ $M$. nasusa Tissue \\ 130 (26 treatments, 5 replicates each)
}

Holding times have not been established for bulyltins in tissues. Samples were kept frozen until extraction and analysis. All samples were inicially extracted and analyzed within 2 months of receipt at Battelle MSL. Because of the contamination of some of the original sample extracts, approximately $25 \%$ of the samples were reextracted and reanalyzed approximately 2.5 months after receiph.

Target detection limits (Table 2.2) for butyltins were achieved.

Nine method blanks were analyzed for mono-, di-, and tributyltin. Batch letters indicate which sample analyses are associated with specific blank samples. Tributyltin was detected in all method blanks at concentrations ranging from 4.7$8.0 \mu \mathrm{g} / \mathrm{kg}$. Sample concentrations are not blank corrected. Dibutyltin was detected in four blanks at concentrations ranging from $3.21013 .6 \mu \mathrm{g} / \mathrm{kg}$. Monobutyltin was detected in seven blanks at concentrations ranging from $2.7 \mathrm{ko}$ $45.8 \mathrm{ug} / \mathrm{kg}$. The one exceptionally high monobutyltin blank value of 45.8 $\mu \mathrm{g} / \mathrm{kg}$ is anamalous and is not reflected in actual sample concentrations.

Tripentyltin was added to each sample prior to extraction as a surrogate to assess extraction efficiency. Target goals for recovery of $40 \%$ w $120 \%$ were achieved; all surrogate recoveries ranged from $45 \%$ to $114 \%$.

Seven marrix spike samples were analyzed. Tri-,di- and monobutyltin were used as spiking compounds for all spiked samples. Tetrabutyltin was used as a spiking comound in five of the seven matrix spike camples. Target goals for necovery of $40 \%$ to $120 \%$ were achieved; recoveries for tetra-, tri-, and dibutyluin ranged from $47 \%$ 10 $83 \%$. Recoveries for monobutyltin were lower, ranging from $14 \%$ to $46 \%$. The lower recovery can be atuributed to the higher volatility of monobutyltin compared to the other butyltin compounds.

Twelve samples were analyzed in duplicate. Precision between duplicate analyses was measured by Relative Percent Difference (RPD). RPDs were not calculated for analytes that were undetocted in one or more of the replicates. RPDs were as follows: tetrabutyltin, $1 \%$ to $21 \%$; tributyltin, $0 \%$ to $35 \%$; dibutyltin, 3\% to 49\%; monobutyltin, 4\% to 112\%. Increased variability observed for monobutyltin can most likely be attributed tothe volatility of the compound.

NOT APPLICABLE 


\section{REEERENCES}

U.S. Environmental Protection Agency (FPA). 1986. Test Methods for Evaluating Solid Waste: Physical/Chemical Methods. EPA-955-001-00000. U.S. Environmental Protection Agency, Government Printing Office, Washington, D.C.

U.S. Environmental Protection Agency/U.S. Army Corps of Engineers (EPA/U'jACE). 1990. Draft Ecolosical Evaluation of Proposed Discharge of Dredged Material inte Oc'san Waters. EPA503-8-90/002. U.S. Environmental Protection Agency, Office of Marine and istuarine Protection, Washington, D.C. 
IABLE K.1. Total Detected Polynuclear Aromatic Hydrocarbons (PAHs), Dry Weight, in Tissue of $M$. nasuta, Oakland Harbor Phase III $B$ Project

\begin{tabular}{|c|c|c|c|c|c|c|}
\hline \multirow[b]{2}{*}{$\begin{array}{l}\text { Sediment } \\
\text { Treatment }\end{array}$} & \multirow[b]{2}{*}{ Replicate } & \multirow[b]{2}{*}{ Batch } & \multirow[b]{2}{*}{$\begin{array}{c}\text { Percent } \\
\text { Dry Veight }\end{array}$} & \multicolumn{3}{|c|}{ M. nasuta PAHs $(\mu \mathrm{kg} / \mathrm{kg}$ dry weight $)$} \\
\hline & & & & $\begin{array}{l}\text { Low Molecular Weight } \\
\text { Iotal PAH } \\
\end{array}$ & $\begin{array}{l}\text { High Molecular Weight } \\
\text { Total PAH }\end{array}$ & $\begin{array}{l}\text { Total } \\
\text { PAH } \\
\end{array}$ \\
\hline $0-C 13$ & 1 & $\mathrm{~J}$ & 12.96 & $N A(a)$ & 1034 & 1034 \\
\hline $0-C 13$ & 2 & j & 12.59 & HA & 945 & 945 \\
\hline $0-C 13$ & 3 & $\mathrm{j}$ & 16.25 & NA & 1138 & 1138 \\
\hline $0-C 13$ & 4 & 3 & 15.22 & NA & 611 & 611 \\
\hline $0-\mathrm{C} 13$ & 5 & $\mathrm{~J}$ & 14.58 & NA & 679 & 679 \\
\hline $0-C 12$ & 1 & J. & 13.38 & MA & 157 & 157 \\
\hline $0-C 12$ & 2 & $\mathrm{~J}$ & 12.41 & NA & 89 & 89 \\
\hline $0-C 12$ & 3 & J & 13.58 & NA & 110 & 110 \\
\hline $0-\mathrm{C12}$ & 4 & $\mathrm{~J}$ & 15.64 & NA & 147 & 147 \\
\hline $0-012$ & 5 & $\mathrm{~J}$ & 14.02 & NA & 143 & 143 \\
\hline $0-C 11$ & 1 & $\mathrm{k}$ & 14.77 & 108 & 630 & 738 \\
\hline 0.0 .11 & 2 & $\mathrm{k}$ & 14.67 & NA & 661 & 661 \\
\hline $0-C 11$ & 3 & $k$ & 15.15 & 86 & 522 & 607 \\
\hline $0-\mathrm{C} 11$ & 4 & k & 15.47 & 71 & 698 & 769 \\
\hline $0-611$ & 5 & k & 12.99 & 77 & 624 & 701 \\
\hline $0-C 10$ & 1 & $k$ & 13.50 & 111 & 178 & 289 \\
\hline $0-010$ & 2 & K & 12.54 & 120 & 766 & 885 \\
\hline $0-c 10$ & 3 Rep 1 & $\mathrm{x}$ & 12.55 & 271 & 422 & 693 \\
\hline $0-c 10$ & $3 \operatorname{Rep} 2$ & $k$ & 12.55 & 263 & 1020 & 1283 \\
\hline $0-c 10$ & $3 \operatorname{Rep} 3$ & K & 12.55 & 151 & 534 & 685 \\
\hline $0-c 10$ & 4 & N & 15.46 & 336 & 298 & 634 \\
\hline $0-c 10$ & 5 & k & 12.87 & 93 & 544 & 637 \\
\hline $0-C 9$ & 1 & K & 15.32 & 176 & 444 & 620 \\
\hline $0-c 9$ & 2 & K & 12.80 & 227 & 172 & 398 \\
\hline $0 .-\mathrm{Cg}$ & 3 & K & 14.38 & 202 & NA & 202 \\
\hline $0-C 9$ & 4 & $x$ & 11.21 & 178 & NA & 178 \\
\hline $0-C 9$ & 5 & K & 12.49 & 120 & NA & 120 \\
\hline $0-C 8$ & 1 & $K$ & 15.99 & 100 & 976 & 1076 \\
\hline $0-C B$ & 2 & $k$ & 15.72 & 95 & 528 & 623 \\
\hline $0-c 8$ & 3 & K & 11.45 & 131 & 454 & 585 \\
\hline $0-\mathrm{cB}$ & 4 & K & 12.32 & 130 & 398 & 528 \\
\hline $0-c 8$ & 5 & $\mathrm{k}$ & 12.94 & 93 & 549 & 642 \\
\hline $0-C 7$ & 1 & L & 13.64 & $N A$ & 264 & 264 \\
\hline $0-C 7$ & 2 & $L$ & 13.17 & NA & 349 & 349 \\
\hline $0-C 7$ & 3 & L & 15.19 & NA & 428 & 428 \\
\hline $0=0.7$ & 4 & $\mathrm{~L}$ & 13.96 & NA & 616 & 616 \\
\hline $0-c 7$ & 5 & $L$ & 13.14 & NA & 251 & 251 \\
\hline $0-\mathrm{C} 6$ & 1 & L & 12.90 & $N A$ & 310 & 310 \\
\hline $0-C 6$ & 2 & $L$ & 14.62 & NA & 226 & 226 \\
\hline $0-\mathrm{Cb}$ & 3 & $L$ & 13.09 & $N A$ & 558 & 558 \\
\hline $0-6.6$ & 4 & $L$ & 13.09 & 290 & 275 & 565 \\
\hline $0-c 6$ & 5 & $i$ & 14.46 & 256 & 505 & $76 !$ \\
\hline $0-.55$ & 1 & $L$ & 9.08 & 418 & 154 & 573 \\
\hline $0-C 5$ & 2 & L & 13.94 & $N A$ & WA & NA \\
\hline $0-C 5$ & 3 & $L$ & 13.58 & 155 & 317 & 471 \\
\hline $0-c 5$ & $4 \operatorname{Rep} 1$ & $L$ & 12.62 & NA & 222 & 222 \\
\hline $0-c 5$ & $4 \operatorname{Rep} 2$ & $L$ & 12.64 & NA & 174 & 174 \\
\hline $0-C .5$ & $4 \operatorname{Rep} 3$ & L. & 12.64 & NA & 166 & 166 \\
\hline $0-c 5$ & 5 & L & 14.93 & NA & $N A$ & NA \\
\hline
\end{tabular}


TABLE K.1. (contd)

\begin{tabular}{|c|c|c|c|c|c|c|}
\hline \multirow[b]{2}{*}{$\begin{array}{l}\text { Sediment } \\
\text { Ireatment }\end{array}$} & \multirow[b]{2}{*}{ Replicate } & \multirow[b]{2}{*}{ Batch } & \multirow[b]{2}{*}{$\begin{array}{c}\text { Percent } \\
\text { Dry Weight }\end{array}$} & \\
\hline & & & & $\begin{array}{l}\text { Low Molecular Weight } \\
\text { Iotal PAH }\end{array}$ & $\begin{array}{l}\text { High Molecular Weight } \\
\text { Total PAH }\end{array}$ & $\begin{array}{l}\text { Total } \\
\text { PAH } \\
\end{array}$ \\
\hline $\begin{array}{l}0-C 4 \\
0-C 4 \\
0-C 4 \\
0-C 4 \\
0-C 4\end{array}$ & $\begin{array}{l}1 \\
2 \\
3 \\
4 \\
5\end{array}$ & $\begin{array}{l}L \\
L \\
L \\
L \\
L\end{array}$ & $\begin{array}{l}13.96 \\
15.86 \\
15.66 \\
14.19 \\
16.58\end{array}$ & $\begin{array}{l}\text { NA } \\
N A \\
N A \\
N A \\
\text { NA }\end{array}$ & $\begin{array}{l}107 \\
277 \\
166 \\
233 \\
277\end{array}$ & $\begin{array}{l}107 \\
277 \\
166 \\
233 \\
277\end{array}$ \\
\hline $\begin{array}{l}0-C 3 \\
0-C 3 \\
0-C 3 \\
0-C 3 \\
0-C 3\end{array}$ & $\begin{array}{l}1 \\
2 \\
3 \\
4 \\
5\end{array}$ & $\begin{array}{l}N \\
N \\
N \\
N \\
N\end{array}$ & $\begin{array}{l}15.38 \\
14.90 \\
13.17 \\
13.60 \\
15.89\end{array}$ & $\begin{array}{l}247 \\
208 \\
190 \\
169 \\
132\end{array}$ & $\begin{array}{l}78 \\
N A \\
N A \\
N A \\
N A\end{array}$ & $\begin{array}{l}325 \\
208 \\
190 \\
169 \\
132\end{array}$ \\
\hline $\begin{array}{l}0-C 1 \\
0-C 1 \\
0-C 1 \\
0-C 1 \\
0-C 1\end{array}$ & $\begin{array}{l}1 \\
2 \\
3 \\
4 \\
5\end{array}$ & $\begin{array}{l}N \\
N \\
N \\
N \\
N\end{array}$ & $\begin{array}{l}13.61 \\
14.45 \\
13.69 \\
15.52 \\
13.32\end{array}$ & $\begin{array}{r}213 \\
N A \\
307 \\
213 \\
188\end{array}$ & $\begin{array}{l}\text { NA } \\
N A \\
N A \\
N A \\
N A\end{array}$ & $\begin{array}{r}213 \\
\text { NA } \\
307 \\
213 \\
188\end{array}$ \\
\hline $\begin{array}{l}I-C 2 \\
I-C 2 \\
I-C 2 \\
I-C 2 \\
I-C 2 \\
I-C 2 \\
I-C 2\end{array}$ & $\begin{array}{lll}1 & \operatorname{Rep} & 1 \\
1 & \operatorname{Rep} 2 \\
1 & \operatorname{Rep} 3 \\
2 & & \\
3 & & \\
4 & & \\
5 & & \end{array}$ & $\begin{array}{l}M \\
M \\
M \\
M \\
M \\
M \\
M\end{array}$ & $\begin{array}{l}14.95 \\
14.95 \\
14.95 \\
15.44 \\
13.68 \\
12.19 \\
14.22\end{array}$ & $\begin{array}{l}\text { NA } \\
\text { NA } \\
\text { NA } \\
\text { NA } \\
\text { NA } \\
\text { NA } \\
\text { NA }\end{array}$ & $\begin{array}{l}\text { NA } \\
74 \\
N A \\
117 \\
117 \\
689 \\
120\end{array}$ & $\begin{array}{l}\text { NA } \\
74 \\
\text { NA } \\
117 \\
117 \\
689 \\
120\end{array}$ \\
\hline $\begin{array}{l}0-C 2 \\
0-C 2 \\
0-C 2 \\
0-C 2 \\
0-C 2\end{array}$ & $\begin{array}{l}1 \\
2 \\
3 \\
4 \\
5\end{array}$ & $\begin{array}{l}M \\
M \\
M \\
M \\
M\end{array}$ & $\begin{array}{l}14.37 \\
14.41 \\
16.05 \\
13.06 \\
15.02\end{array}$ & $\begin{array}{l}\text { NA } \\
\text { NA } \\
N A \\
N A \\
N A\end{array}$ & $\begin{array}{l}\text { NA } \\
N A \\
N A \\
N A \\
\text { NA }\end{array}$ & $\begin{array}{l}\text { NA } \\
\text { NA } \\
\text { NA } \\
\text { NA } \\
\text { NA }\end{array}$ \\
\hline $\begin{array}{l}1-C 3 \\
1-C 3 \\
1-C 3 \\
I-C 3 \\
1-C 3\end{array}$ & $\begin{array}{l}1 \\
2 \\
3 \\
4 \\
5\end{array}$ & $\begin{array}{l}M \\
M \\
M \\
M \\
M\end{array}$ & $\begin{array}{l}15.29 \\
16.98 \\
15.06 \\
15.06 \\
15.80\end{array}$ & $\begin{array}{l}\text { NA } \\
\text { NA } \\
\text { NA } \\
\text { NA } \\
\text { NA }\end{array}$ & $\begin{array}{r}\text { NA } \\
\text { NA } \\
\text { NA } \\
126 \\
\text { NA }\end{array}$ & $\begin{array}{r}\text { NA } \\
\text { NA } \\
\text { NA } \\
126 \\
\text { NA }\end{array}$ \\
\hline $\begin{array}{l}I-C 8 \\
1-C 8 \\
I-C 8 \\
1-C 8 \\
I-C 8\end{array}$ & $\begin{array}{l}1 \\
2 \\
3 \\
4 \\
5\end{array}$ & $\begin{array}{l}M \\
M \\
M \\
M \\
M\end{array}$ & $\begin{array}{l}15.44 \\
15.29 \\
14.04 \\
15.32 \\
14.23\end{array}$ & $\begin{array}{l}\text { NA } \\
N A \\
N A \\
N A \\
N A\end{array}$ & $\begin{array}{r}680 \\
1072 \\
634 \\
725 \\
\text { NA }\end{array}$ & $\begin{array}{r}680 \\
1072 \\
634 \\
725 \\
\text { NA }\end{array}$ \\
\hline $\begin{array}{l}1-C 4 \\
1-C 4 \\
1-C 4 \\
1-C 4 \\
I-C 4\end{array}$ & $\begin{array}{l}1 \\
2 \\
3 \\
4 \\
5\end{array}$ & $\begin{array}{l}j \\
j \\
j \\
j\end{array}$ & $\begin{array}{l}12.76 \\
13.97 \\
14.06 \\
13.47 \\
12.85\end{array}$ & $\begin{array}{l}N A \\
N A \\
N A \\
N A \\
\text { HA }\end{array}$ & $\begin{array}{l}118 \\
265 \\
199 \\
245 \\
241\end{array}$ & $\begin{array}{l}118 \\
265 \\
199 \\
245 \\
241\end{array}$ \\
\hline $\begin{array}{l}1-T 5 \\
1-T 55 \\
1-T 5 \\
1-T 5 \\
1-T 5 \\
1-T 5 \\
1-T 5\end{array}$ & $\begin{array}{lll}1 & & \\
2 & \operatorname{Rep} & 1 \\
2 & \operatorname{Rep} & 2 \\
2 & \operatorname{Rep} & 3 \\
3 & & \\
4 & & \\
5 & & \end{array}$ & $\begin{array}{l}J \\
J \\
j \\
j \\
j \\
j \\
j\end{array}$ & $\begin{array}{l}15.93 \\
12.44 \\
12.44 \\
12.44 \\
16.62 \\
13.74 \\
14.70\end{array}$ & $\begin{array}{r}69 \\
113 \\
N A \\
N A \\
N A \\
N A \\
N A\end{array}$ & $\begin{array}{r}251 \\
322 \\
370 \\
346 \\
205 \\
N A \\
265\end{array}$ & $\begin{array}{r}320 \\
434 \\
370 \\
346 \\
205 \\
\text { NA } \\
255\end{array}$ \\
\hline
\end{tabular}


TABLE K.1. (contd)

Sediment

Ireatment

$R-A C$

$R \sim A C$

$R-A C$

$R-A C$

$R-A C$

$R-A M$

$R-A M$

$R-A M$

$R-A M$

R-AM

$R-B F$

$R-B F$

$R-B F$

$R-B F$

$R-B F$

$R-B F$

$R-B F$

$R-O S$

$R-O S$

$R \sim O S$

$R-O S$

$R \sim O S$

$R-P C$

$R-P C$

$R-P C$

$R-P C$

$R=P C$

$R-P F$

$R-P F$

$R-P F$

$R-P F$

$R-P F$

$C-S B$

$C-S B$

C-SB

$C-S B$

$C-58$

$C-S B$

$C-N E$

$C-N E$

$C-N E$

C.NE

$C-N E$
Replicate Batch

$\begin{array}{ll}1 & 1 \\ 2 & 1 \\ 3 & 1 \\ 4 & 1 \\ 5 & 1\end{array}$

1

$$
2
$$$$
2
$$$$
4
$$

1

1

$3 \operatorname{Rep} 1$

3 Rep 2

3 Rep 3

5

1
2
3
4
5

1

2

3

5

1

2
3
4

5

1

2

$4 \operatorname{Rep} 1$

4 Rep 2$$
5
$$

1
2

2

3
4
5

(a) Not applicable no PAHs detected.

M. nasura PAHs $(\mu \mathrm{g} / \mathrm{kg}$ dry weight $)$

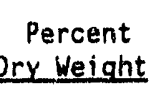

12.99

13.48

12.05

14.33

13.56

13.36

14.78

16.78

17.72

14.87

11.20

15.97

12.86

12.86

12.86

12.33

11.53

14.79

14.41

13.55

14.90

14.49

14.42

13.07

15.69

13.60

16.62

15.37

10.12

11.88

14.03

15.76

14.10

13.29

15.24

12.93

12.93

12.84

16.25

15.51

13.16

11.68

12.50

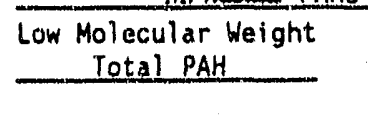

$\begin{array}{rrr}N A & N A & N A \\ N A & 616 & 616 \\ N A & N A & N A \\ N A & N A & N A \\ N A & 258 & 258 \\ & & \\ N A & 329 & 329 \\ 81 & 521 & 602 \\ 72 & 417 & 489 \\ 56 & 214 & 271 \\ 148 & 605 & 753\end{array}$

High Molecular Weight

NA

NA

NA

NA

NA

NA

NA

NA

NA

NA

NA

NA

NA
NA
NA
NA

NA
NA
NA
NA
NA

NA
$N A$
$N A$
$N A$
$M A$
$N A$

NA

NA

NA

NA

$\begin{array}{ll}N A & N A \\ N A & N A \\ N A & N A \\ N A & N A \\ N A & N A \\ N A & N R \\ N A & N A \\ 176 & \\ N A & 176 \\ N A & N A \\ N A & N A \\ N A & N A \\ & N A \\ N A & \\ H A & N A \\ N A & N A \\ N A & N A \\ N A & N A \\ N A\end{array}$

$\begin{array}{ll}N A & N A \\ N A & N A \\ N A & N A \\ N A & N A \\ N A & N A\end{array}$

$\begin{array}{ll}\text { NA } & \text { NA } \\ \text { NA } & \text { NA } \\ \text { NA } & \text { NA } \\ \text { NA } & \text { NA } \\ \text { NA } & \text { NA } \\ \text { NA } & \text { NA }\end{array}$

$\begin{array}{ll}\text { NA } & N A \\ \text { NA } & N A \\ N A & N A \\ N A & N A \\ \text { NA } & N A\end{array}$


TABLE K.2. Low Molecular Weight Polynuclear Aromatic Hydrocarbons (PAHs), Dry Weight, in Tissue of M. nasuta, Oakland Harbor Phase III B Project

\begin{tabular}{|c|c|c|c|c|c|c|c|c|c|}
\hline \multirow[b]{2}{*}{$\begin{array}{l}\text { Sediment } \\
\text { Ireatment }\end{array}$} & \multirow[b]{2}{*}{ Replicate } & \multirow[b]{2}{*}{ Batch } & \multirow[b]{2}{*}{$\begin{array}{c}\text { Percent } \\
\text { Dry Weight } \\
\end{array}$} & \multicolumn{6}{|c|}{ M, nasuta PAHs ( $\mu_{\mathrm{A}} / \mathrm{kg}$ dry weight) } \\
\hline & & & & $\begin{array}{l}\text { Naph- } \\
\text { thalene }\end{array}$ & $\begin{array}{l}\text { Acenaph- } \\
\text { thylene }\end{array}$ & $\begin{array}{l}\text { Acenaph- } \\
\text { thene }\end{array}$ & Fluorene & $\begin{array}{l}\text { Phenan- } \\
\text { threne }\end{array}$ & $\begin{array}{l}\text { Anthr- } \\
\text { acene }\end{array}$ \\
\hline $\begin{array}{l}0-C 13 \\
0-C 13 \\
0-C 13 \\
0-C 13 \\
0-C 13\end{array}$ & $\begin{array}{l}1 \\
2 \\
3 \\
4 \\
5\end{array}$ & $\begin{array}{l}J \\
j \\
j \\
J \\
J\end{array}$ & $\begin{array}{l}12.96 \\
12.59 \\
16.25 \\
15.22 \\
14.58\end{array}$ & $\begin{array}{l}77 U^{(a)} \\
79 U \\
62 U \\
66 U \\
69 U\end{array}$ & $\begin{array}{ll}77 & U \\
79 & U \\
62 & U \\
66 & U \\
69 & U\end{array}$ & $\begin{array}{ll}77 & U \\
79 & U \\
62 & U \\
66 & U \\
69 & U\end{array}$ & $\begin{array}{ll}77 & U \\
79 & U \\
62 & U \\
66 & U \\
69 & U\end{array}$ & $\begin{array}{ll}77 & U \\
79 & U \\
62 & U \\
66 & U \\
69 & U\end{array}$ & $\begin{array}{ll}77 & U \\
79 & U \\
62 & U \\
66 & U \\
69 & U\end{array}$ \\
\hline $\begin{array}{l}0-C 12 \\
0-C 12 \\
0-C 12 \\
0-C 12 \\
0-C 12\end{array}$ & $\begin{array}{l}1 \\
2 \\
3 \\
4 \\
5\end{array}$ & $\begin{array}{l}j \\
j \\
j \\
j \\
j\end{array}$ & $\begin{array}{l}13.38 \\
12.41 \\
13.58 \\
15.64 \\
14.02\end{array}$ & $\begin{array}{ll}75 & U \\
81 & U \\
74 & U \\
64 & U \\
71 & U\end{array}$ & $\begin{array}{ll}75 & U \\
81 & U \\
74 & U \\
64 & U \\
71 & U\end{array}$ & $\begin{array}{ll}75 & U \\
81 & U \\
74 & U \\
64 & U \\
71 & U\end{array}$ & $\begin{array}{ll}75 & U \\
81 & U \\
74 & U \\
64 & U \\
71 & U\end{array}$ & $\begin{array}{ll}75 & U \\
81 & U \\
74 & U \\
64 & U \\
71 & U\end{array}$ & $\begin{array}{ll}75 & U \\
81 & U \\
74 & U \\
64 & U \\
71 & U\end{array}$ \\
\hline $\begin{array}{l}0-C 11 \\
0-C 11 \\
0-C 11 \\
0-C 11 \\
0-C 11\end{array}$ & $\begin{array}{l}1 \\
2 \\
3 \\
4 \\
5\end{array}$ & $\begin{array}{l}K \\
k \\
K \\
K \\
K\end{array}$ & $\begin{array}{l}14.77 \\
14.67 \\
15.15 \\
15.47 \\
12.99\end{array}$ & $\begin{array}{c}108 \\
68 U \\
86 \\
71 \\
77\end{array}$ & $\begin{array}{ll}68 & U \\
68 & U \\
66 & U \\
65 & U \\
77 & U\end{array}$ & $\begin{array}{l}68 U \\
68 U \\
66 U \\
65 U \\
77 U\end{array}$ & $\begin{array}{l}68 \mathrm{U} \\
68 \mathrm{U} \\
66 \mathrm{U} \\
65 \mathrm{U} \\
77 \mathrm{U}\end{array}$ & $\begin{array}{ll}68 & U \\
68 & U \\
66 & U \\
65 & U \\
77 & U\end{array}$ & $\begin{array}{l}68 U \\
68 U \\
66 U \\
65 U \\
77 U\end{array}$ \\
\hline $\begin{array}{l}0-C 10 \\
0-C 10 \\
0-C 10 \\
0-C 10 \\
0-C 10 \\
0-C 10 \\
0-C 10\end{array}$ & $\begin{array}{lll}1 & & \\
2 & & \\
3 & R e p & 1 \\
3 & \text { Rep } & 2 \\
3 & \text { Rep } & 3 \\
4 & & \\
5 & & \end{array}$ & $\begin{array}{l}K \\
K \\
K \\
K \\
K \\
N \\
K\end{array}$ & $\begin{array}{l}13.50 \\
12.54 \\
12.55 \\
12.55 \\
12.55 \\
15.46 \\
12.87\end{array}$ & $\begin{array}{l}111 \\
120 \\
128 \\
159 \\
151 \\
336 \mathrm{E}^{(b)} \\
33\end{array}$ & $\begin{array}{l}74 U \\
80 U \\
80 U \\
80 U \\
80 U \\
65 U \\
78 U\end{array}$ & $\begin{array}{l}74 U \\
80 U \\
80 U \\
80 U \\
80 U \\
65 U \\
78 U\end{array}$ & $\begin{array}{c}74 U \\
80 U \\
143 \\
80 U \\
80 U \\
65 U \\
78 U\end{array}$ & $\begin{array}{r}74 \mathrm{U} \\
80 \mathrm{U} \\
80 \mathrm{U} \\
104 \\
80 \mathrm{U} \\
65 \mathrm{U} \\
78 \mathrm{U}\end{array}$ & $\begin{array}{l}74 U \\
80 U \\
80 U \\
80 U \\
80 U \\
65 U \\
78 U\end{array}$ \\
\hline $\begin{array}{l}0-C 9 \\
0-C 9 \\
0-C .9 \\
0-C 9 \\
0-C 9\end{array}$ & $\begin{array}{l}1 \\
2 \\
3 \\
4 \\
5\end{array}$ & $\begin{array}{l}K \\
K \\
K \\
K \\
K\end{array}$ & $\begin{array}{l}15.32 \\
12.80 \\
14.38 \\
11.21 \\
12.49\end{array}$ & $\begin{array}{l}1.76 \\
227 \\
202 \\
178 \\
120\end{array}$ & $\begin{array}{ll}65 & U \\
78 & U \\
70 & U \\
89 & U \\
80 & U\end{array}$ & $\begin{array}{l}65 \mathrm{U} \\
78 \mathrm{U} \\
70 \mathrm{U} \\
89 \mathrm{U} \\
60 \mathrm{U}\end{array}$ & $\begin{array}{ll}65 & U \\
78 & U \\
70 & U \\
89 & U \\
80 & U\end{array}$ & $\begin{array}{l}65 \mathrm{U} \\
78 \mathrm{U} \\
70 \mathrm{U} \\
89 \mathrm{U} \\
80 \mathrm{U}\end{array}$ & $\begin{array}{l}65 U \\
78 U \\
70 U \\
89 U \\
80 U\end{array}$ \\
\hline $\begin{array}{l}0-C 8 \\
0-C 8 \\
0-C 8 \\
0-C 8 \\
0-C 8\end{array}$ & $\begin{array}{l}1 \\
2 \\
3 \\
4 \\
5\end{array}$ & $\begin{array}{l}K \\
K \\
K \\
k \\
K\end{array}$ & $\begin{array}{l}15.99 \\
15.72 \\
11.45 \\
12.32 \\
12.94\end{array}$ & $\begin{array}{r}100 \\
35 \\
131 \\
130 \\
93\end{array}$ & $\begin{array}{ll}63 & U \\
64 & U \\
87 & U \\
81 & U \\
77 & U\end{array}$ & $\begin{array}{ll}63 & U \\
64 & U \\
87 & U \\
81 & U \\
77 & U\end{array}$ & $\begin{array}{ll}63 & U \\
64 & U \\
87 & U \\
81 & U \\
77 & U\end{array}$ & $\begin{array}{ll}63 & U \\
64 & U \\
87 & U \\
81 & U \\
77 & U\end{array}$ & $\begin{array}{ll}63 & U \\
64 & U \\
87 & U \\
81 & U \\
77 & U\end{array}$ \\
\hline $\begin{array}{l}0-C 7 \\
0-C 7 \\
0=C 7 \\
0-C 7 \\
0-C 7\end{array}$ & $\begin{array}{l}1 \\
2 \\
3 \\
4 \\
5\end{array}$ & $\begin{array}{l}L \\
L \\
L \\
L\end{array}$ & $\begin{array}{l}83.64 \\
13.17 \\
15.19 \\
13.96 \\
13.14\end{array}$ & $\begin{array}{l}147 U \\
152 U \\
132 U \\
143 U \\
152 U\end{array}$ & $\begin{array}{l}73 \mathrm{U} \\
76 \mathrm{U} \\
66 \mathrm{U} \\
72 \mathrm{U} \\
76 \mathrm{U}\end{array}$ & $\begin{array}{ll}73 & U \\
76 & U \\
66 & U \\
72 & U \\
76 & U\end{array}$ & $\begin{array}{ll}73 & U \\
76 & U \\
66 & U \\
72 & U \\
76 & U\end{array}$ & $\begin{array}{ll}73 & U \\
76 & U \\
66 & U \\
72 & U \\
76 & U\end{array}$ & $\begin{array}{ll}73 & U \\
76 & U \\
66 & U \\
72 & U \\
76 & U\end{array}$ \\
\hline $\begin{array}{l}0-C 6 \\
0-C 6 \\
0-C 6 \\
0-C 6 \\
0-C \sigma\end{array}$ & $\begin{array}{l}1 . \\
2 \\
3 \\
4 \\
5\end{array}$ & $\begin{array}{l}L \\
L \\
L \\
L \\
L\end{array}$ & $\begin{array}{l}12.90 \\
14.52 \\
13.09 \\
13.09 \\
14.46\end{array}$ & $\begin{array}{l}155 U \\
137 U \\
153 U \\
191 \\
159\end{array}$ & $\begin{array}{l}78 U \\
68 U \\
76 U \\
76 U \\
69 U\end{array}$ & $\begin{array}{ll}78 & U \\
68 & U \\
76 & U \\
76 & U \\
69 & U\end{array}$ & $\begin{array}{ll}78 & U \\
68 & U \\
76 & U \\
76 & U \\
69 & U\end{array}$ & $\begin{array}{ll}78 & U \\
68 & U \\
76 & U \\
99 & \\
97 & \end{array}$ & $\begin{array}{l}78 U \\
68 U \\
76 U \\
76 U \\
69 U\end{array}$ \\
\hline $\begin{array}{l}0-C 5 \\
0-C 5 \\
0-C 5\end{array}$ & $\begin{array}{l}1 \\
2 \\
3\end{array}$ & $\begin{array}{l}i \\
i\end{array}$ & $\begin{array}{r}9.08 \\
13.94 \\
13.58\end{array}$ & $\begin{array}{l}264 \\
144 U \\
147 U\end{array}$ & $\begin{array}{r}110 \mathrm{U} \\
72 \mathrm{U} \\
74 \mathrm{U}\end{array}$ & $\begin{array}{r}110 \mathrm{U} \\
72 \mathrm{U} \\
74 \mathrm{U}\end{array}$ & $\begin{array}{r}110 \mathrm{U} \\
72 \mathrm{U} \\
74 \mathrm{U}\end{array}$ & $\begin{array}{l}154 \\
720 \\
155\end{array}$ & $\begin{array}{r}110 \mathrm{U} \\
72 \mathrm{U} \\
74 \mathrm{U}\end{array}$ \\
\hline
\end{tabular}


TABLE K.2. (contd)

\begin{tabular}{|c|c|c|c|c|c|c|c|c|c|}
\hline \multirow[b]{2}{*}{$\begin{array}{l}\text { Sediment } \\
\text { Treatment }\end{array}$} & \multirow[b]{2}{*}{ Replicate } & \multirow[b]{2}{*}{ Batch } & \multirow[b]{2}{*}{$\begin{array}{c}\text { Percent } \\
\text { Dry Weight }\end{array}$} & \multicolumn{6}{|c|}{ M. nasusa PAHs ( $(\mathrm{g} / \mathrm{kg}$ dry weight) } \\
\hline & & & & $\begin{array}{l}\text { Naph- } \\
\text { thalene }\end{array}$ & $\begin{array}{l}\text { Acenaph- } \\
\text { thylene }\end{array}$ & $\begin{array}{l}\text { Acenaph- } \\
\text { thene }\end{array}$ & Eluorene & $\begin{array}{l}\text { Phenan- } \\
\text { threne }\end{array}$ & $\begin{array}{l}\text { Anthr- } \\
\text { acene }\end{array}$ \\
\hline $\begin{array}{l}0-C 5 \\
0-C 5 \\
0-C 5 \\
0-C 5\end{array}$ & 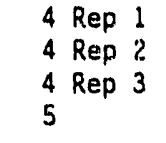 & $\begin{array}{l}L \\
L \\
L \\
L\end{array}$ & $\begin{array}{l}12.62 \\
12.64 \\
12.64 \\
14.93\end{array}$ & $\begin{array}{l}158 U \\
158 U \\
158 U \\
134 U\end{array}$ & $\begin{array}{ll}79 & U \\
79 & U \\
79 & U \\
67 & U\end{array}$ & $\begin{array}{ll}79 & U \\
79 & U \\
79 & U \\
67 & U\end{array}$ & $\begin{array}{ll}79 & U \\
79 & U \\
79 & U \\
67 & U\end{array}$ & $\begin{array}{ll}79 & U \\
79 & U \\
79 & U \\
67 & U\end{array}$ & $\begin{array}{l}79 U \\
79 U \\
79 U \\
67 U\end{array}$ \\
\hline $\begin{array}{l}0-C 4 \\
0-C 4 \\
0-C 4 \\
0-C 4 \\
0-C 4\end{array}$ & $\begin{array}{l}1 \\
2 \\
3 \\
4 \\
5\end{array}$ & $\begin{array}{l}L \\
L \\
L \\
L\end{array}$ & $\begin{array}{l}13.96 \\
15.86 \\
15.66 \\
14.19 \\
16.58\end{array}$ & $\begin{array}{l}143 U \\
126 U \\
128 U \\
141 U \\
121 U\end{array}$ & $\begin{array}{ll}72 & U \\
63 & U \\
64 & U \\
70 & U \\
60 & U\end{array}$ & $\begin{array}{l}72 U \\
63 U \\
64 U \\
70 U \\
60 U\end{array}$ & $\begin{array}{ll}72 & U \\
63 & U \\
64 & U \\
70 & U \\
60 & U\end{array}$ & $\begin{array}{ll}72 & U \\
63 & U \\
64 & U \\
70 & U \\
60 & U\end{array}$ & $\begin{array}{l}72 U \\
63 U \\
64 U \\
70 U \\
60 U\end{array}$ \\
\hline $\begin{array}{l}0-c 3 \\
0-c 3 \\
0-c 3 \\
0-c 3 \\
0-c 3\end{array}$ & $\begin{array}{l}1 \\
2 \\
3 \\
4 \\
5\end{array}$ & $\begin{array}{l}N \\
N \\
N \\
N \\
N\end{array}$ & $\begin{array}{l}15.38 \\
14.90 \\
13.17 \\
13.60 \\
15.89\end{array}$ & $\begin{array}{ll}247 & E \\
208 & E \\
190 & E \\
169 & E \\
132 & E\end{array}$ & 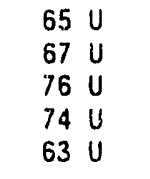 & $\begin{array}{l}65 U \\
67 U \\
76 U \\
74 U \\
63 U\end{array}$ & $\begin{array}{ll}65 & U \\
67 & U \\
76 & U \\
74 & U \\
63 & U\end{array}$ & 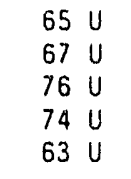 & $\begin{array}{l}65 U \\
67 U \\
76 U \\
74 U \\
63 U\end{array}$ \\
\hline $\begin{array}{l}0-C 1 \\
0-C 1 \\
0-C 1 \\
0-C 1 \\
0-C 1\end{array}$ & $\begin{array}{l}1 \\
2 \\
3 \\
4 \\
5\end{array}$ & $\begin{array}{l}N \\
N \\
N \\
N \\
N\end{array}$ & $\begin{array}{l}13.61 \\
14.45 \\
13.69 \\
15.52 \\
13.32\end{array}$ & $\begin{array}{rl}213 & E \\
69 & U \\
307 & E \\
213 E \\
188 E\end{array}$ & $\begin{array}{l}73 U \\
69 U \\
73 U \\
64 U \\
75 U\end{array}$ & $\begin{array}{l}73 U \\
69 U \\
73 U \\
64 U \\
75 U\end{array}$ & $\begin{array}{l}73 U \\
69 U \\
73 U \\
64 U \\
75 \\
U\end{array}$ & $\begin{array}{l}73 U \\
69 U \\
73 U \\
64 U \\
75 U\end{array}$ & $\begin{array}{l}73 U \\
69 U \\
73 U \\
64 U \\
75 U\end{array}$ \\
\hline $\begin{array}{l}1-\mathrm{C} 2 \\
1-\mathrm{C} 2 \\
1-\mathrm{C} 2 \\
1-\mathrm{C} 2 \\
1-\mathrm{C} 2 \\
1-\mathrm{C} 2 \\
1-\mathrm{C} 2\end{array}$ & $\begin{array}{lll}1 & \operatorname{Rep} & 1 \\
1 & \operatorname{Rep} & 2 \\
1 & \operatorname{Rep} 3 \\
2 & & \\
3 & & \\
4 & & \\
5 & & \end{array}$ & $\begin{array}{l}M \\
M \\
M \\
M \\
M \\
M \\
M\end{array}$ & $\begin{array}{l}14.95 \\
14.95 \\
14.95 \\
15.44 \\
13.68 \\
12.19 \\
14.22\end{array}$ & $\begin{array}{l}134 U \\
134 U \\
134 U \\
130 U \\
146 U \\
164 U \\
141 U\end{array}$ & $\begin{array}{ll}67 & U \\
67 & U \\
67 & U \\
65 & U \\
73 & U \\
82 & U \\
70 & U\end{array}$ & $\begin{array}{l}67 U \\
67 U \\
67 U \\
65 U \\
73 U \\
82 U \\
70 U\end{array}$ & $\begin{array}{l}67 U \\
67 U \\
67 U \\
65 U \\
73 U \\
82 U \\
70 U\end{array}$ & $\begin{array}{ll}67 & U \\
67 & U \\
67 & U \\
65 & U \\
73 & U \\
82 & U \\
70 & U\end{array}$ & $\begin{array}{l}67 U \\
67 U \\
67 U \\
65 U \\
73 U \\
82 U \\
70 U\end{array}$ \\
\hline $\begin{array}{l}0-\mathrm{C} 2 \\
0-\mathrm{C} 2 \\
0-\mathrm{C} 2 \\
0-\mathrm{C2} \\
0-\mathrm{C} 2\end{array}$ & $\begin{array}{l}1 \\
2 \\
3 \\
4 \\
5\end{array}$ & $\begin{array}{l}M \\
M \\
M \\
M \\
M\end{array}$ & $\begin{array}{l}14.37 \\
14.41 \\
16.05 \\
13.06 \\
15.02\end{array}$ & $\begin{array}{l}139 U \\
139 U \\
125 U \\
153 U \\
133 U\end{array}$ & $\begin{array}{l}70 U \\
69 U \\
62 \mathrm{U} \\
77 \\
67 \\
67\end{array}$ & $\begin{array}{l}70 U \\
69 U \\
62 U \\
77 U \\
67 U\end{array}$ & $\begin{array}{l}70 \mathrm{U} \\
69 \mathrm{U} \\
62 \mathrm{U} \\
77 \mathrm{U} \\
67 \mathrm{U}\end{array}$ & $\begin{array}{l}70 \mathrm{U} \\
69 \mathrm{U} \\
62 \mathrm{U} \\
77 \mathrm{U} \\
67 \mathrm{U}\end{array}$ & $\begin{array}{l}70 \mathrm{U} \\
69 \mathrm{U} \\
62 \mathrm{U} \\
77 \mathrm{U} \\
67 \mathrm{U}\end{array}$ \\
\hline $\begin{array}{l}I-C 3 \\
1-C 3 \\
I-C 3 \\
I-C 3 \\
I-C 3 \\
-\end{array}$ & $\begin{array}{l}1 \\
2 \\
3 \\
4 \\
5\end{array}$ & $\begin{array}{l}M \\
M \\
M \\
M \\
M\end{array}$ & $\begin{array}{l}15.29 \\
16.98 \\
15.06 \\
15.06 \\
15.80\end{array}$ & $\begin{array}{l}131 U \\
118 U \\
133 U \\
133 U \\
127 U\end{array}$ & $\begin{array}{ll}65 & U \\
59 & U \\
66 & U \\
66 & U \\
63 & U\end{array}$ & $\begin{array}{ll}65 & U \\
59 & U \\
66 & U \\
66 & U \\
63 & U\end{array}$ & $\begin{array}{ll}65 & \vdots \\
59 & U \\
66 & U \\
66 & U \\
63 & U\end{array}$ & $\begin{array}{ll}65 & U \\
59 & U \\
66 & U \\
66 & U \\
63 & U\end{array}$ & $\begin{array}{l}65 \mathrm{U} \\
59 \mathrm{U} \\
66 \mathrm{U} \\
66 \mathrm{U} \\
63 \mathrm{U}\end{array}$ \\
\hline $\begin{array}{l}1-C 8 \\
I-C 8 \\
I-C 8 \\
I-C 8 \\
I-C 8\end{array}$ & $\begin{array}{l}1 \\
2 \\
3 \\
4 \\
5\end{array}$ & $\begin{array}{l}M \\
M \\
M \\
M \\
M\end{array}$ & $\begin{array}{l}15.44 \\
15.29 \\
14.04 \\
15.32 \\
14.23\end{array}$ & $\begin{array}{l}130 U \\
131 U \\
143 U \\
131 U \\
141 U\end{array}$ & $\begin{array}{ll}65 & U \\
65 & U \\
71 & U \\
65 & U \\
70 & U\end{array}$ & $\begin{array}{ll}65 & U \\
65 & U \\
71 & U \\
65 & U \\
70 & U\end{array}$ & $\begin{array}{ll}65 & U \\
65 & U \\
71 & U \\
65 & U \\
70 & U\end{array}$ & $\begin{array}{ll}65 & U \\
65 & U \\
71 & U \\
65 & U \\
70 & U\end{array}$ & $\begin{array}{ll}65 & U \\
65 & U \\
71 & U \\
65 & U \\
70 & U\end{array}$ \\
\hline $\begin{array}{l}I-C 4 \\
I-C 4 \\
I-C 4 \\
I-C 4 \\
I-C 4\end{array}$ & $\begin{array}{l}1 \\
2 \\
3 \\
4 \\
5\end{array}$ & $\begin{array}{l}j \\
j \\
j \\
j \\
j\end{array}$ & $\begin{array}{l}12.76 \\
13.97 \\
14.06 \\
13.47 \\
12.85\end{array}$ & $\begin{array}{l}78 \mathrm{U} \\
72 \mathrm{U} \\
71 \mathrm{U} \\
74 \mathrm{U} \\
78 \mathrm{U}\end{array}$ & $\begin{array}{l}78 \mathrm{U} \\
72 \mathrm{U} \\
71 \mathrm{U} \\
74 \mathrm{U} \\
78 \mathrm{U}\end{array}$ & $\begin{array}{l}78 \mathrm{U} \\
72 \mathrm{U} \\
71 \mathrm{U} \\
74 \mathrm{U} \\
78 \mathrm{U}\end{array}$ & $\begin{array}{l}78 \mathrm{U} \\
72 \mathrm{U} \\
71 \mathrm{U} \\
74 \mathrm{U} \\
78 \mathrm{U}\end{array}$ & $\begin{array}{l}78 U \\
72 U \\
71 U \\
74 U \\
78 U\end{array}$ & $\begin{array}{l}78 U \\
72 U \\
71 U \\
74 U \\
78 U\end{array}$ \\
\hline
\end{tabular}


IABLE K.2. (contd)

\begin{tabular}{|c|c|c|c|c|c|c|c|c|c|}
\hline \multirow[b]{2}{*}{$\begin{array}{l}\text { Sediment } \\
\text { Ireatment }\end{array}$} & \multirow[b]{2}{*}{ Replicate } & \multirow[b]{2}{*}{ Batch } & \multirow[b]{2}{*}{$\begin{array}{c}\text { Percent } \\
\text { Dry Weight }\end{array}$} & \multicolumn{6}{|c|}{ M. nasula PAATS (ugl $\angle \mathrm{kg}$ ory welant) } \\
\hline & & & & $\begin{array}{c}\text { Naph- } \\
\text { thalene }\end{array}$ & $\begin{array}{l}\text { Acenaph- } \\
\text { thylene }\end{array}$ & $\begin{array}{l}\text { Acenaph- } \\
\text { thene }\end{array}$ & Eluorene & $\begin{array}{l}\text { Phenan- } \\
\text { threne }\end{array}$ & $\begin{array}{l}\text { Anthr- } \\
\text { acene }\end{array}$ \\
\hline $\begin{array}{l}1-T 5 \\
1-T 5 \\
1-T 5 \\
1-T 5 \\
1-T 5 \\
1-T 5 \\
I-T 5\end{array}$ & $\begin{array}{lll}1 & & \\
2 & \operatorname{Rep} & 1 \\
2 & \operatorname{Rep} 2 \\
2 & \operatorname{Rep} 3 \\
3 & & \\
4 & & \\
5 & & \end{array}$ & $\begin{array}{l}\mathrm{J} \\
\mathrm{J} \\
\mathrm{J} \\
\mathrm{J} \\
\mathrm{J} \\
\mathrm{J} \\
\mathrm{J}\end{array}$ & $\begin{array}{l}15.93 \\
12.44 \\
12.44 \\
12.44 \\
16.62 \\
13.74 \\
14.70\end{array}$ & $\begin{array}{c}69 \\
113 \\
80 \mathrm{U} \\
80 \mathrm{U} \\
60 \mathrm{U} \\
73 \mathrm{U} \\
68 \mathrm{U}\end{array}$ & $\begin{array}{l}63 U \\
80 U \\
80 U \\
80 U \\
60 U \\
73 U \\
68 U\end{array}$ & $\begin{array}{l}63 U \\
80 U \\
80 U \\
80 U \\
60 U \\
73 U \\
68 U\end{array}$ & $\begin{array}{l}63 U \\
8 U U \\
80 U \\
80 U \\
60 U \\
73 U \\
68 U\end{array}$ & $\begin{array}{l}63 U \\
80 U \\
80 U \\
80 U \\
60 U \\
73 U \\
68 U\end{array}$ & $\begin{array}{l}63 U \\
80 U \\
80 U \\
80 U \\
60 U \\
73 U \\
68 U\end{array}$ \\
\hline $\begin{array}{l}R-A C \\
R-A C \\
R-A C \\
R-A C \\
R-A C\end{array}$ & $\begin{array}{l}1 \\
2 \\
3 \\
4 \\
5\end{array}$ & $\begin{array}{l}I \\
I \\
I \\
I \\
I\end{array}$ & $\begin{array}{l}12.99 \\
13.48 \\
12.05 \\
14.33 \\
13.56\end{array}$ & $\begin{array}{l}77 U \\
74 U \\
83 U \\
70 U \\
74 U\end{array}$ & $\begin{array}{l}77 U \\
74 U \\
83 U \\
70 U \\
74 U\end{array}$ & $\begin{array}{l}77 U \\
74 U \\
83 U \\
70 U \\
74 U\end{array}$ & $\begin{array}{ll}77 & U \\
74 & U \\
83 & U \\
70 & U \\
74 & U\end{array}$ & $\begin{array}{l}77 U \\
74 U \\
83 U \\
70 U \\
74 U\end{array}$ & $\begin{array}{l}77 U \\
74 U \\
83 U \\
70 U \\
74 U\end{array}$ \\
\hline $\begin{array}{l}R-A M \\
R-A M \\
R-A M \\
R-A M \\
R-A M\end{array}$ & $\begin{array}{l}1 \\
2 \\
3 \\
4 \\
5\end{array}$ & $\begin{array}{l}H \\
H \\
H \\
H \\
H\end{array}$ & $\begin{array}{l}13.36 \\
14.78 \\
16.78 \\
17.72 \\
14.87\end{array}$ & $\begin{array}{l}75 U \\
68 U \\
60 U \\
56 U \\
67 U\end{array}$ & $\begin{array}{l}75 U \\
68 U \\
60 U \\
56 U \\
67 U\end{array}$ & $\begin{array}{l}75 U \\
68 U \\
60 U \\
56 U \\
67 U\end{array}$ & $\begin{array}{l}75 U \\
68 U \\
60 U \\
56 U \\
67 U\end{array}$ & $\begin{array}{l}75 U \\
81 \\
72 \\
56 \\
148\end{array}$ & $\begin{array}{l}75 U \\
68 U \\
60 U \\
56 U \\
67 U\end{array}$ \\
\hline $\begin{array}{l}R-B F \\
R-B F \\
R-B F \\
R-B F \\
R-B F \\
R-B F \\
R-B F\end{array}$ & $\begin{array}{lll}1 & & \\
2 & & \\
3 & \operatorname{Rep} & 1 \\
3 & \operatorname{Rep} & 2 \\
3 & \operatorname{Rep} & 3 \\
4 & & \\
5 & & \end{array}$ & $\begin{array}{l}H \\
H \\
H \\
H \\
H \\
H \\
H\end{array}$ & $\begin{array}{l}11.20 \\
15.97 \\
12.86 \\
12.86 \\
12.86 \\
12.33 \\
11.53\end{array}$ & $\begin{array}{rl}89 & U \\
188 U \\
78 U \\
78 U \\
78 U \\
81 U \\
87 U\end{array}$ & $\begin{array}{rl}89 & U \\
188 & U \\
78 & U \\
78 & U \\
78 & U \\
81 & U \\
87 & U\end{array}$ & $\begin{array}{rl}89 & U \\
188 & U \\
78 & U \\
78 & U \\
78 & U \\
81 & U \\
87 & U\end{array}$ & $\begin{array}{rl}89 & U \\
188 & U \\
78 & U \\
78 & U \\
78 & U \\
81 & U \\
87 & U\end{array}$ & $\begin{array}{rl}89 & U \\
188 & U \\
78 & U \\
78 & U \\
78 & U \\
81 & U \\
87 & U\end{array}$ & $\begin{array}{rl}89 & U \\
188 & U \\
78 & U \\
78 & U \\
78 & U \\
81 & U \\
87 & U\end{array}$ \\
\hline $\begin{array}{l}R-O S \\
R-O S \\
R-O S \\
R-O S \\
R-O S\end{array}$ & $\begin{array}{l}1 \\
2 \\
3 \\
4 \\
5\end{array}$ & $\begin{array}{l}l \\
l \\
I \\
I \\
l\end{array}$ & $\begin{array}{l}14.79 \\
14.41 \\
13.55 \\
14.90 \\
14.49\end{array}$ & $\begin{array}{l}68 U \\
69 U \\
74 U \\
67 U \\
69 U\end{array}$ & $\begin{array}{l}68 U \\
69 U \\
74 U \\
67 U \\
69 U\end{array}$ & $\begin{array}{l}68 U \\
63 U \\
74 U \\
67 U \\
69 U\end{array}$ & $\begin{array}{l}68 U \\
69 U \\
74 U \\
67 \\
69 \\
69\end{array}$ & $\begin{array}{l}68 U \\
69 U \\
74 U \\
67 U \\
69 U\end{array}$ & $\begin{array}{l}68 U \\
69 U \\
74 U \\
67 U \\
69 U\end{array}$ \\
\hline $\begin{array}{l}R-P C \\
R-P C \\
R-P C \\
R-P C \\
R-P C\end{array}$ & $\begin{array}{l}1 \\
2 \\
3 \\
4 \\
5\end{array}$ & $\begin{array}{l}\text { I } \\
\text { I } \\
\text { I } \\
\text { I }\end{array}$ & $\begin{array}{l}14.42 \\
13.07 \\
15.69 \\
13.60 \\
16.62\end{array}$ & $\begin{array}{ll}69 & U \\
77 & U \\
64 & U \\
74 & U \\
60 & U\end{array}$ & $\begin{array}{l}69 U \\
77 U \\
64 U \\
74 U \\
60 \mathrm{U}\end{array}$ & $\begin{array}{l}69 U \\
77 U \\
64 U \\
74 U \\
60 U\end{array}$ & $\begin{array}{l}69 \mathrm{U} \\
77 \mathrm{U} \\
64 \mathrm{U} \\
74 \mathrm{U} \\
60 \mathrm{U}\end{array}$ & $\begin{array}{l}69 U \\
77 U \\
64 U \\
74 U \\
60 U\end{array}$ & $\begin{array}{l}69 U \\
77 U \\
64 U \\
74 U \\
60 U\end{array}$ \\
\hline $\begin{array}{l}R-P F \\
R-P F \\
R-P F \\
R-P F \\
R-P F\end{array}$ & $\begin{array}{l}1 \\
2 \\
3 \\
4 \\
5\end{array}$ & $\begin{array}{l}H \\
H \\
H \\
H \\
H\end{array}$ & $\begin{array}{l}15.37 \\
10.12 \\
11.88 \\
14.03 \\
15.76\end{array}$ & $\begin{array}{ll}65 & U \\
99 & U \\
84 & U \\
71 & U \\
63 & U\end{array}$ & $\begin{array}{l}65 U \\
99 U \\
84 U \\
71 U \\
63 U\end{array}$ & $\begin{array}{l}65 U \\
99 U \\
84 U \\
71 U \\
63 U\end{array}$ & $\begin{array}{ll}65 & U \\
99 & U \\
84 & U \\
71 & U \\
63 & U\end{array}$ & $\begin{array}{ll}65 & U \\
99 & U \\
84 & U \\
71 & U \\
63 & U\end{array}$ & $\begin{array}{l}65 U \\
99 U \\
84 U \\
71 U \\
63 U\end{array}$ \\
\hline $\begin{array}{l}C-S B \\
C-S B \\
C-S B \\
C-S B \\
C-S B \\
C-S B\end{array}$ & $\begin{array}{ll}1 & \\
2 & \\
3 & \\
4 & \operatorname{Rep~} 1 \\
4 & \operatorname{Rep~} 2 \\
5 & \end{array}$ & $\begin{array}{l}1 \\
1 \\
1 \\
1 \\
1 \\
1\end{array}$ & $\begin{array}{l}14.10 \\
13.29 \\
15.24 \\
12.93 \\
12.93 \\
12.84\end{array}$ & $\begin{array}{l}71 \mathrm{U} \\
75 \mathrm{U} \\
66 \mathrm{U} \\
77 \mathrm{U} \\
77 \mathrm{U} \\
78 \mathrm{U}\end{array}$ & $\begin{array}{ll}71 & U \\
75 & U \\
66 & U \\
77 & U \\
77 & U \\
78 & U\end{array}$ & $\begin{array}{ll}71 & U \\
75 & U \\
66 & U \\
77 & U \\
77 & U \\
78 & U\end{array}$ & $\begin{array}{ll}71 & U \\
75 & U \\
66 & U \\
77 & U \\
77 & U \\
78 & U\end{array}$ & $\begin{array}{l}71 \mathrm{U} \\
75 \mathrm{U} \\
66 \mathrm{U} \\
77 \mathrm{U} \\
77 \mathrm{U} \\
78 \mathrm{U}\end{array}$ & $\begin{array}{l}71 \mathrm{U} \\
75 \mathrm{U} \\
66 \mathrm{U} \\
77 \mathrm{U} \\
77 \mathrm{U} \\
78 \mathrm{U}\end{array}$ \\
\hline
\end{tabular}




\section{TABLE K.2. (contd)}

\begin{tabular}{|c|c|c|c|c|c|c|c|c|c|}
\hline \multirow[b]{2}{*}{$\begin{array}{l}\text { Sediment } \\
\text { Treatment }\end{array}$} & \multirow[b]{2}{*}{ Replicate } & \multirow[b]{2}{*}{ Batch } & \multirow[b]{2}{*}{$\begin{array}{c}\text { Percent } \\
\text { Dry Weight }\end{array}$} & \multicolumn{6}{|c|}{ M. nasula PAHs ( $\mu \mathrm{g} / \mathrm{kg}$ dry weight) } \\
\hline & & & & $\begin{array}{l}\text { Naph- } \\
\text { thalene }\end{array}$ & $\begin{array}{l}\text { Acenaph- } \\
\text { thylene }\end{array}$ & $\begin{array}{l}\text { Acenaph- } \\
\text { thene }\end{array}$ & Fluorene & $\begin{array}{l}\text { Phenan- } \\
\text { threne }\end{array}$ & $\begin{array}{l}\text { Anthr- } \\
\text { acene }\end{array}$ \\
\hline $\begin{array}{l}C-N E \\
C-N E \\
C-N E \\
C-N E \\
C-N E\end{array}$ & $\begin{array}{l}1 \\
2 \\
3 \\
4 \\
5\end{array}$ & $\begin{array}{l}H \\
H \\
H \\
H \\
H\end{array}$ & $\begin{array}{l}16.25 \\
15.51 \\
13.16 \\
11.68 \\
12.50\end{array}$ & $\begin{array}{l}62 U \\
64 U \\
76 U \\
86 U \\
80 U\end{array}$ & $\begin{array}{l}62 U \\
64 U \\
76 U \\
86 U \\
80 U\end{array}$ & $\begin{array}{ll}62 & U \\
64 & U \\
76 & U \\
86 & U \\
80 & U\end{array}$ & $\begin{array}{l}62 U \\
64 U \\
76 U \\
86 U \\
80 U\end{array}$ & $\begin{array}{l}62 U \\
64 U \\
76 U \\
86 U \\
80 U\end{array}$ & $\begin{array}{l}62 U \\
64 U \\
76 U \\
86 U \\
80 U\end{array}$ \\
\hline
\end{tabular}

(a) Undetected above given concentration.

(b) Matrix spike recoveries for batch were both above acceptable range: concentration may be biased high. 


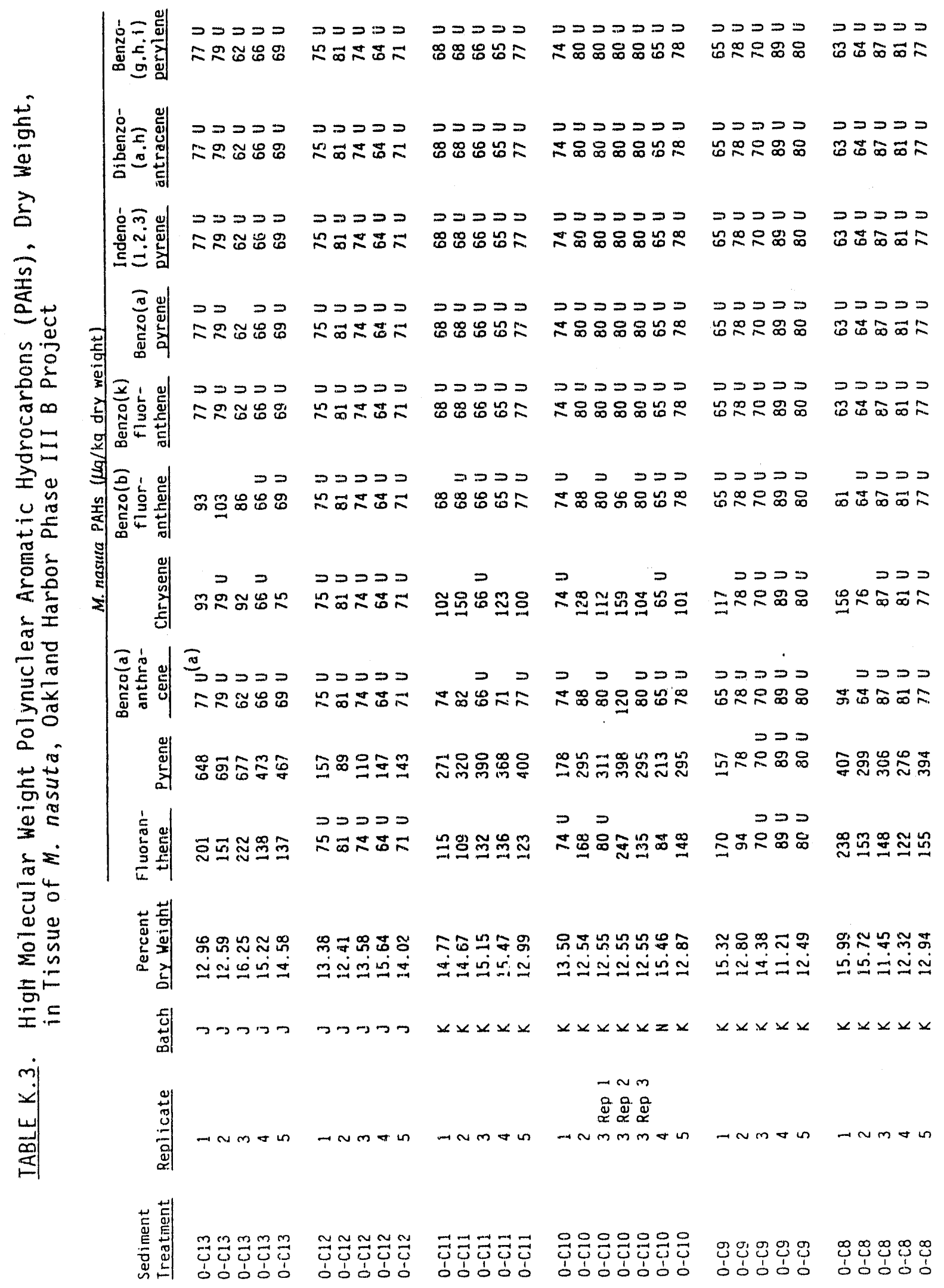




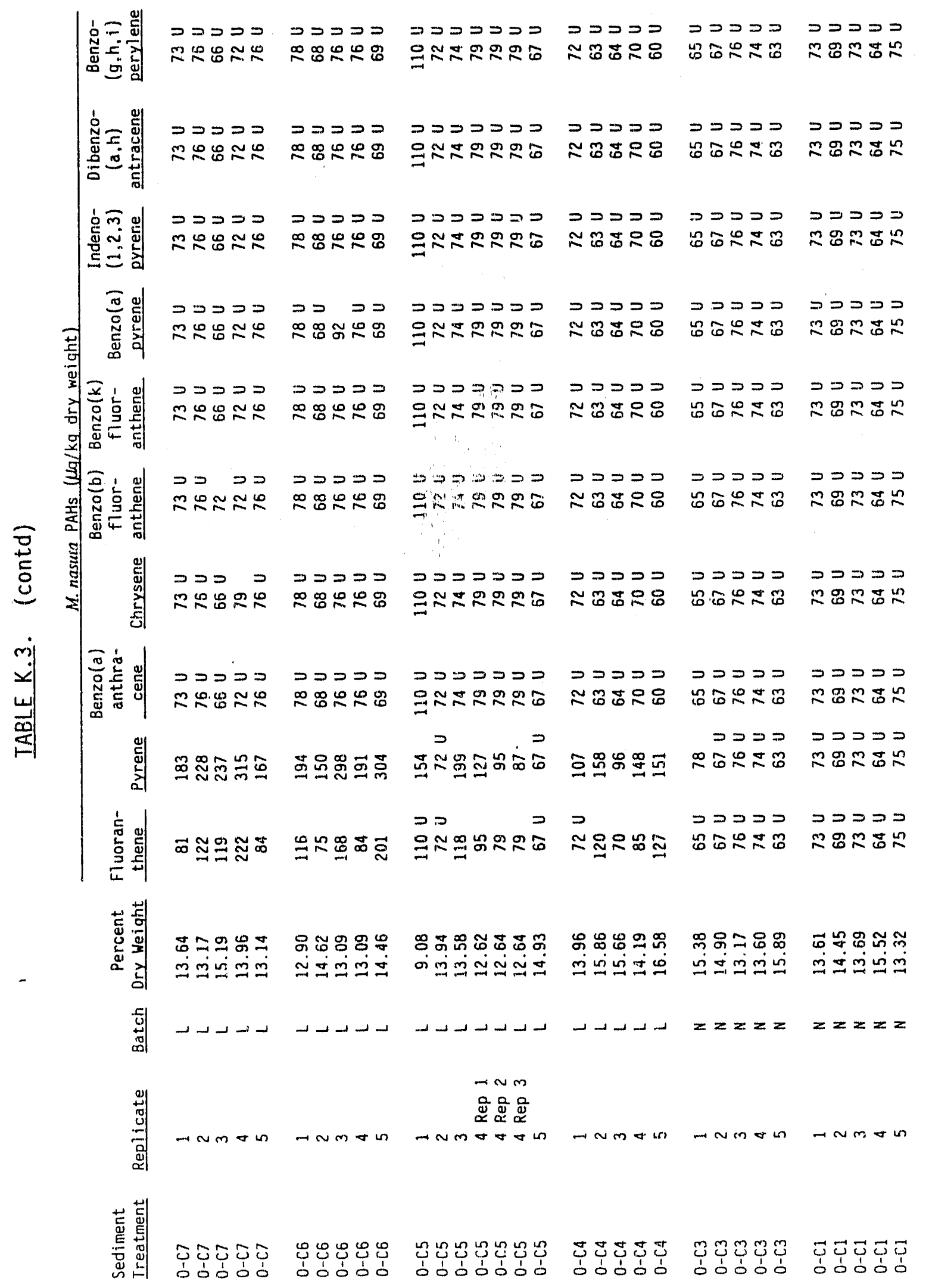




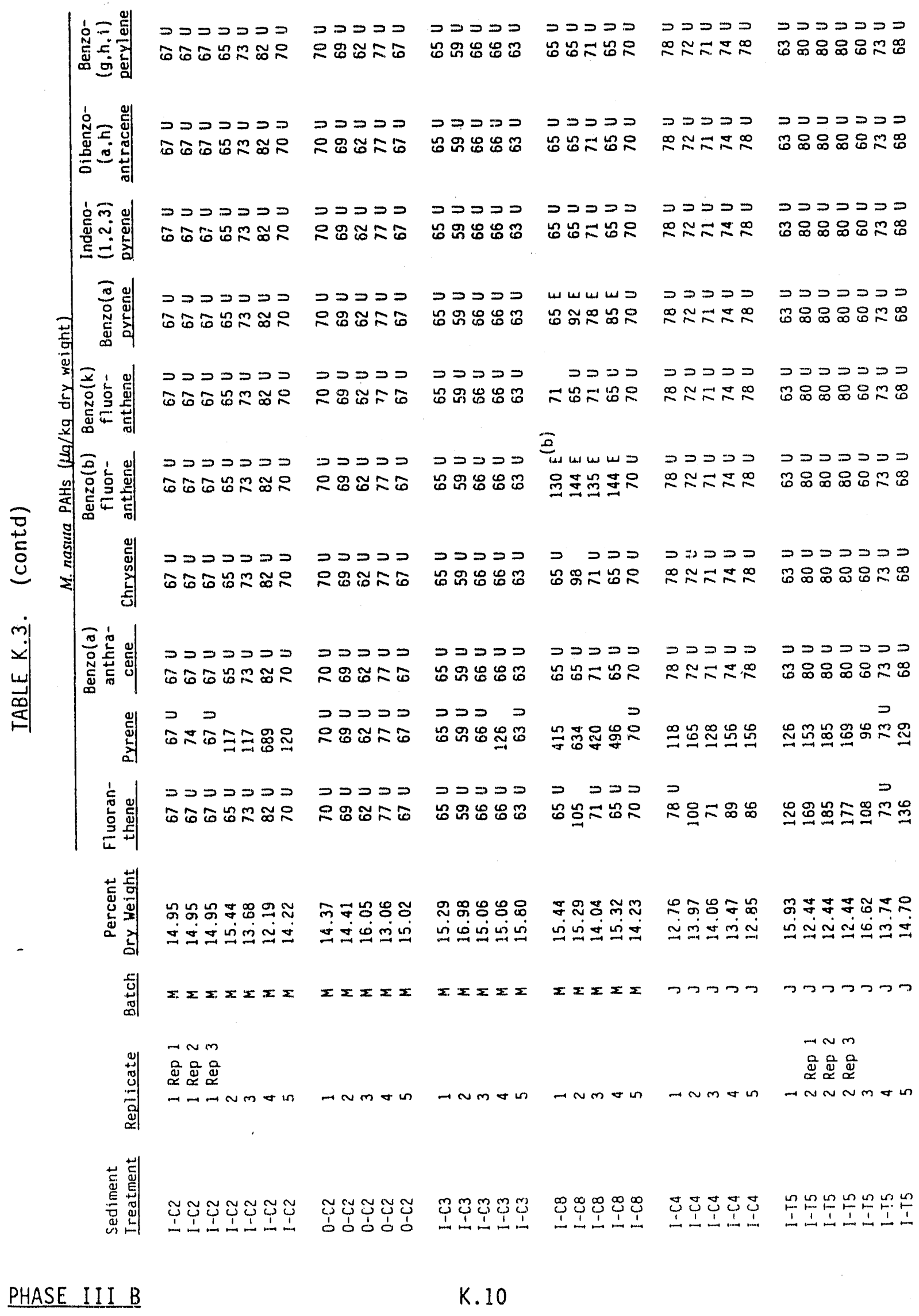




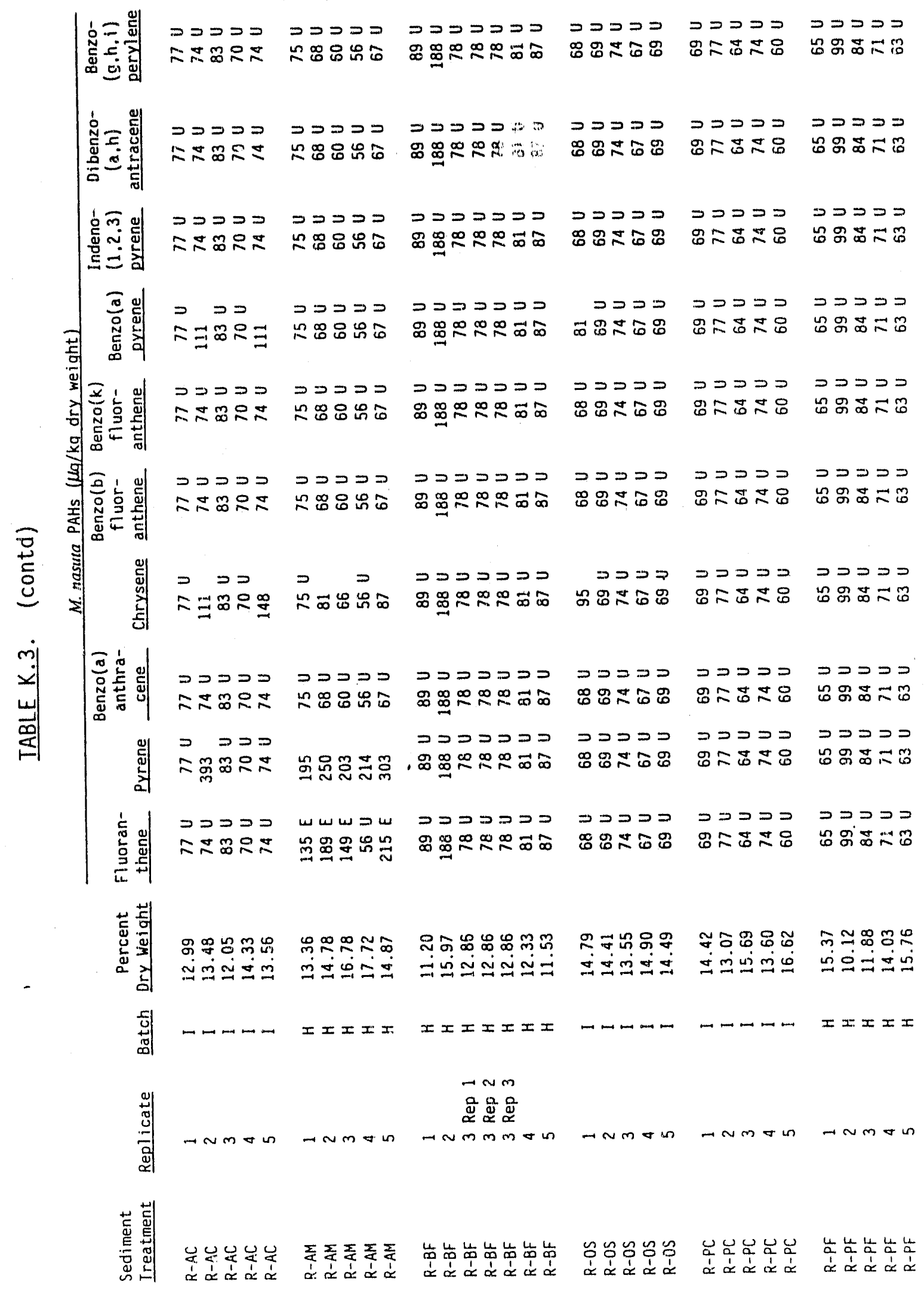




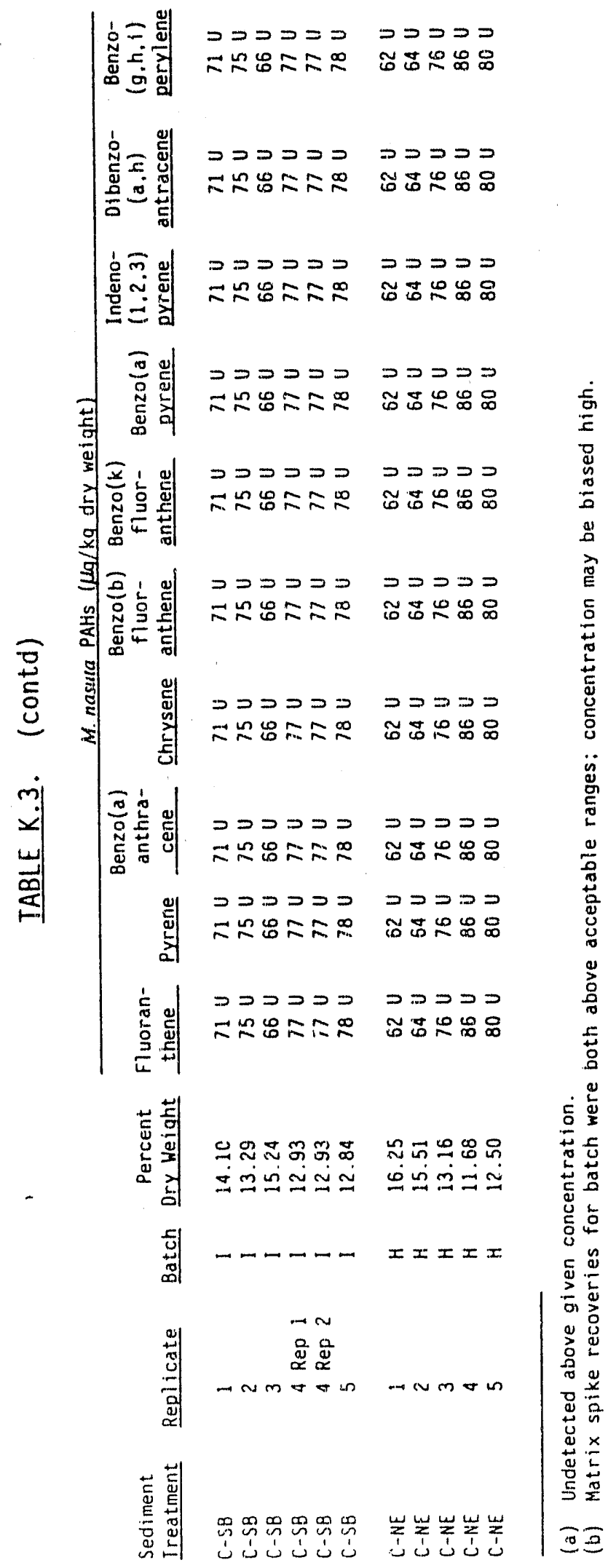


TABLE K.4. Total Detected Polynuclear Aromatic Hydrocarbons (PAilis), Wet Weight, in Tissue of M. nasuta, Oakland Harbor Phase III B Project

\begin{tabular}{|c|c|c|c|c|c|}
\hline \multirow[b]{2}{*}{$\begin{array}{l}\text { Sediment } \\
\text { Ireatment }\end{array}$} & \multirow[b]{2}{*}{ Replicate } & \multirow[b]{2}{*}{ Batch } & \multicolumn{3}{|c|}{ M. nasuta PAHs $(\mu \mathrm{g} / \mathrm{kg}$ wet weight $)$} \\
\hline & & & $\begin{array}{c}\text { Total } \\
\text { Low Molecular Weight } \\
\text { PAH }\end{array}$ & $\begin{array}{c}\text { Total } \\
\text { High Molecular Weight } \\
\text { PAH }\end{array}$ & $\begin{array}{l}\text { Potal } \\
\text { PAH }\end{array}$ \\
\hline $0-C 13$ & 1 & J & $\mathrm{HA}(\mathrm{a})$ & 134 & 134 \\
\hline $0-613$ & $i$ & j & NA & 119 & 119 \\
\hline $0-C 13$ & 3 & J & NA & 185 & 185 \\
\hline 0.613 & 4 & J & NA & 93 & 93 \\
\hline $0-C 13$ & 5 & J & NA & \$.9 & 99 \\
\hline $0-012$ & 1 & J & NA & 21 & 21 \\
\hline $0-012$ & 2 & j & NA & 11 & ii \\
\hline $0-012$ & 3 & J & NA & 15 & 15 \\
\hline 0.612 & 4 & $\mathrm{~J}$ & NA & 23 & 23 \\
\hline $0-0,12$ & 5 & j & WA & 20 & 20 \\
\hline $0-\mathrm{Cll}$ & 1 & $k$ & 16 & 93 & 109 \\
\hline $0-611$ & 2 & $\mathrm{k}$ & HA & 97 & 97 \\
\hline $0-C 11$ & 3 & $\mathrm{k}$ & 13 & 79 & 92 \\
\hline $0-c 11$ & 4 & K & 11 & 108 & 119 \\
\hline $0-c 11$ & 5 & $k$ & 10 & 81 & 91 \\
\hline $0-610$ & 1 & K & 15 & 24 & 39 \\
\hline $0-610$ & 2 & $k$ & 15 & 96 & 111 \\
\hline $0-C 10$ & $3 \operatorname{Rep} 1$ & $k$ & 34 & 53 & 87 \\
\hline $0-C 10$ & 3 Rep 2 & K & 33 & 128 & 161 \\
\hline $0-610$ & $3 \operatorname{Rep} 3$ & $k$ & 19 & 67 & 86 \\
\hline $0-c 10$ & 4 & N & 52 & 46 & 98 \\
\hline $0-610$ & 5 & K & 12 & 70 & 82 \\
\hline $0-\mathrm{cg}$ & 1 & K & 27 & 68 & 95 \\
\hline $0-19$ & ? & $k$ & 29 & 22 & 51 \\
\hline $0-C 9$ & 3 & $k$ & 29 & 0 & 29 \\
\hline $0-c 9$ & 4 & $k^{\prime}$ & 20 & HA & 20 \\
\hline $0 \mathrm{cs}$ & 5 & K & 15 & NA & 15 \\
\hline $0-\mathrm{C} 8$ & 1 & $k$ & 16 & 156 & 172 \\
\hline $0-68$ & 2 & $k$ & 15 & 83 & 98 \\
\hline $0-58$ & 3 & K & 15 & 52. & 67 \\
\hline $0-c 8$ & 4 & $k$ & 16 & 49 & 65 \\
\hline $0 .-18$ & 5 & $k$ & 12 & 71 & 83 \\
\hline $0 .-C 7$ & 1 & L & MA & 36 & 36 \\
\hline $0=07$ & 2 & $L$ & $N A$. & 46 & 46 \\
\hline $0-\mathrm{Cl}^{2}$ & 3 & I. & $N A$ & 65 & 65 \\
\hline $0-67$ & 4 & L & $N A$ & 86 & 86 \\
\hline 0.67 & 5 & $i$ & NA & 33 & 33 \\
\hline $0-\mathrm{C} 6$ & 1 & $L$ & NA & 40 & 40 \\
\hline $0-C 6$ & 2 & $i$ & NA & 33 & 33 \\
\hline $0-c .6$ & 3 & i. & $\mathrm{HA}$ & 73 & 73 \\
\hline $0 . C G$ & 4 & $i$ & 38 & 36 & 74 \\
\hline 0.05 & 5 & $L$ & 37 & 73 & 1.10 \\
\hline $0 .-65$ & 1 & $L$ & 38 & 14 & 52 \\
\hline $0 .-55$ & 2 & $i$ & NA & NA & NA \\
\hline $0-c 5$ & 3 & 1. & 21 & 4.3 & 64 \\
\hline
\end{tabular}


IABLE K.4. (contd)

\begin{tabular}{|c|c|c|c|c|c|}
\hline \multirow[b]{2}{*}{$\begin{array}{l}\text { Seoiment } \\
\text { Ireatment }\end{array}$} & \multirow[b]{2}{*}{ Replicate } & \multirow[b]{2}{*}{ Batch } & \multicolumn{3}{|c|}{$M$. nasuta PAHs $(\mu \mathrm{g} / \mathrm{kg}$ wet weight $)$} \\
\hline & & & $\begin{array}{c}\text { Total } \\
\text { Low Molecular Weight } \\
\text { PAH } \\
\end{array}$ & $\begin{array}{c}\text { Total } \\
\text { High Molecular Weight } \\
\text { PAH }\end{array}$ & $\begin{array}{l}\text { Total } \\
\text { PAH } \\
\end{array}$ \\
\hline $\begin{array}{l}0-C 5 \\
0-C 5 \\
0-C 5 \\
0-C 5\end{array}$ & $\begin{array}{l}4 \text { Rep } 1 \\
4 \operatorname{Rep} 2 \\
4 \operatorname{Rep} 3 \\
5\end{array}$ & $\begin{array}{l}L \\
L \\
L\end{array}$ & $\begin{array}{l}\text { NA } \\
\text { NA } \\
\text { NA } \\
\text { NA }\end{array}$ & $\begin{array}{l}28 \\
22 \\
21 \\
\text { NA }\end{array}$ & $\begin{array}{l}28 \\
22 \\
21 \\
\text { NA }\end{array}$ \\
\hline $\begin{array}{l}0-C 4 \\
0-C 4 \\
0-C 4 \\
0-C 4 \\
0-C 4\end{array}$ & $\begin{array}{l}1 \\
2 \\
3 \\
4 \\
5\end{array}$ & $\begin{array}{l}L \\
L \\
L \\
L \\
L\end{array}$ & $\begin{array}{l}\text { NA } \\
\text { NA } \\
N A \\
N A \\
N A\end{array}$ & $\begin{array}{l}15 \\
44 \\
26 \\
33 \\
46\end{array}$ & $\begin{array}{l}15 \\
44 \\
26 \\
33 \\
46\end{array}$ \\
\hline $\begin{array}{l}0-C 3 \\
0-C 3 \\
0-C 3 \\
0-C 3 \\
0-C 3\end{array}$ & $\begin{array}{l}1 \\
2 \\
3 \\
4 \\
5\end{array}$ & $\begin{array}{l}N \\
N \\
N \\
N \\
N\end{array}$ & $\begin{array}{l}38 \\
31 \\
25 \\
23 \\
21\end{array}$ & $\begin{array}{l}12 \\
N A \\
N A \\
N A \\
N A\end{array}$ & $\begin{array}{l}50 \\
31 \\
25 \\
23 \\
21\end{array}$ \\
\hline $\begin{array}{l}0-C 1 \\
0-C 1 \\
0-C 1 \\
0-C 1 \\
0-C 1\end{array}$ & $\begin{array}{l}1 \\
2 \\
3 \\
4 \\
5\end{array}$ & $\begin{array}{l}M \\
N \\
N \\
N \\
N\end{array}$ & $\begin{array}{l}29 \\
N A \\
42 \\
33 \\
25\end{array}$ & $\begin{array}{l}\text { NA } \\
\text { NA } \\
\text { NA } \\
\text { NA } \\
\text { NA }\end{array}$ & $\begin{array}{l}29 \\
\text { NA } \\
42 \\
33 \\
25\end{array}$ \\
\hline $\begin{array}{l}1-C 2 \\
I-C 2 \\
I-C 2 \\
I-C 2 \\
I-C 2 \\
1-C 2 \\
I-C 2\end{array}$ & $\begin{array}{lll}1 & \operatorname{Rep} & 1 \\
1 & \operatorname{Rep} 2 \\
1 & \operatorname{Rep} 3 \\
2 & & \\
3 & \\
4 & \\
5 & & \end{array}$ & $\begin{array}{l}M \\
M \\
M \\
M \\
M \\
M \\
M\end{array}$ & $\begin{array}{l}\text { NA } \\
\text { NA } \\
\text { NA } \\
\text { NA } \\
\text { NA } \\
\text { NA } \\
\text { NA }\end{array}$ & $\begin{array}{l}N A \\
11 \\
N A \\
18 \\
16 \\
84 \\
17\end{array}$ & $\begin{array}{l}\text { NA } \\
11 \\
N A \\
18 \\
16 \\
84 \\
17\end{array}$ \\
\hline $\begin{array}{l}0-C 2 \\
0-C 2 \\
0-c 2 \\
0-C 2 \\
0-C 2\end{array}$ & $\begin{array}{l}1 \\
2 \\
3 \\
4 \\
5\end{array}$ & $\begin{array}{l}M \\
M \\
M \\
M \\
M\end{array}$ & $\begin{array}{l}\text { NA } \\
\text { HA } \\
\text { NA } \\
\text { NA } \\
\text { NA }\end{array}$ & $\begin{array}{l}\text { NA } \\
\text { NA } \\
\text { NA } \\
\text { WA } \\
\text { NA }\end{array}$ & $\begin{array}{l}\text { NA } \\
N A \\
N A \\
N A \\
N A\end{array}$ \\
\hline $\begin{array}{l}I-C 3 \\
I-C 3 \\
I-C 3 \\
I-C 3 \\
I-C 3\end{array}$ & $\begin{array}{l}1 \\
2 \\
3 \\
4 \\
5\end{array}$ & $\begin{array}{l}M \\
M \\
M \\
M \\
M\end{array}$ & $\begin{array}{l}N A \\
N A \\
N A \\
N A \\
N A\end{array}$ & $\begin{array}{l}\text { WA } \\
\text { WA } \\
\text { NA } \\
19 \\
\text { NA }\end{array}$ & $\begin{array}{l}\text { NA } \\
\text { NA } \\
\text { NA } \\
19 \\
\text { NA }\end{array}$ \\
\hline $\begin{array}{l}I-C 4 \\
I-C 4 \\
I-C 4 \\
I-C 4 \\
I-C 4\end{array}$ & $\begin{array}{l}1 \\
2 \\
3 \\
4 \\
5\end{array}$ & $\begin{array}{l}\mathrm{J} \\
\mathrm{J} \\
\mathrm{J} \\
\mathrm{J} \\
\mathrm{J}\end{array}$ & $\begin{array}{l}\text { NA } \\
N A \\
N A \\
N A \\
N A\end{array}$ & $\begin{array}{l}15 \\
37 \\
28 \\
33 \\
31\end{array}$ & $\begin{array}{l}15 \\
37 \\
28 \\
33 \\
31\end{array}$ \\
\hline $\begin{array}{l}1-C 8 \\
1-C 8 \\
1-C 8 \\
1-C 8 \\
i-C 8\end{array}$ & $\begin{array}{l}1 \\
2 \\
3 \\
4 \\
5\end{array}$ & $\begin{array}{l}M \\
M \\
M \\
M \\
M\end{array}$ & $\begin{array}{l}\text { NA } \\
\text { HA } \\
\text { NA } \\
\text { NA } \\
\text { NA }\end{array}$ & $\begin{array}{r}105 \\
164 \\
89 \\
111 \\
\text { NA }\end{array}$ & $\begin{array}{r}105 \\
164 \\
89 \\
111 \\
\text { NA }\end{array}$ \\
\hline
\end{tabular}


IABLE K.4. (contd)

Sediment

Treatment

$1-T 5$

$1-15$

I-T 5

$1-T 5$

I-T5

$1-75$

I-T5

$R-A C$

$R-A C$

$R-A C$

$R-A C$

$R-A C$.

$R-A M$

$R-A M$

R-AM

$R-A M$

R-AM

$R-B F$

$R-B F$

$R-B F$

$R-B F$

$R-B F$

$R-B F$

$R-B F$

R-OS

$R-O S$

$R-O S$

$R-O S$

$R-O S$

$R-P C$

$R-P C$

$R-P C$

$R-P C$

$R \backsim P C$

$R-P F$

$R-P F$

$R-P F$

$R-P F$

$R+P F$

C-SB

C-SB

C-SB

$C-S B$

$C-S B$

C-SB

\section{Replicate}

1

2 Rep

2 Rep 2

2 Rep 3

3

4

$\begin{array}{ll}1 & ! \\ 2 & 1 \\ 3 & \text { I } \\ 4 & \text { I }\end{array}$

1

1

3

5

2

3 Rep 1

3 Rep 2

3 Rep 3

4
5

1

1

3

4
5

1
2
3
4
5

1

2

3
4
5

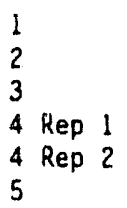

5
M. nasuia PAHs $(\mu \mathrm{g} / \mathrm{kg}$ wet weight)

$$
\text { Total }
$$

Low Molecular Weight
PAH $\quad \begin{gathered}\text { High Molecular Weight } \\ \text { PAH }\end{gathered} \quad \begin{gathered}\text { Total } \\ \text { PAH }\end{gathered}$

$\begin{array}{lll}11 & 40 & 51 \\ 14 & 40 & 54 \\ \text { NA } & 46 & 46 \\ \text { NA } & 43 & 43 \\ \text { NA } & 34 & 34 \\ \text { NA } & \text { NA } & \text { NA } \\ \text { NA } & 39 & 39 \\ \text { NA } & & \\ \text { NA } & \text { NA } & \text { NA } \\ \text { NA } & 83 & 83 \\ \text { NA } & \text { NA } & \text { NA } \\ \text { NA } & \text { NA } & \text { NA }\end{array}$

NA 44

44

77
70

$38 \quad 48$

112

NA

NA

NA

NA

NA

WA

NA

NA

NA

NA

NA

NA

$N A$

NA

NA

NA

NA

NA

NA

NA

NA

NA

NA

NA

NA

NA

\section{1}

46

43

A

A

5

94
8
2

NA
$N A$
$N A$
WA
NA
NA
NA

26

NA

NA

NA

$N A$
$N A$
$H A$
$N A$
$N A$
$N A$
$N A$
$N A$
$N A$
$N A$
$N A$

NA

NA

NA

NA

NA 
TABLE K.4. (contd)

\begin{tabular}{lcc} 
Sediment & & \\
Ireatment & Replicate & Batch \\
\cline { 2 - 3 } C-NE & & \\
C-NE & 1 & $H$ \\
C-NE & 2 & $H$ \\
C-NE & 3 & $H$ \\
C-NE & 4 & $H$ \\
& 5 & $H$
\end{tabular}

\begin{tabular}{ccc}
\multicolumn{3}{c}{ M. nasula PAHs (Ha/kg wet weight) } \\
\hline $\begin{array}{c}\text { Total } \\
\text { Low Molecular Weight }\end{array}$ & $\begin{array}{c}\text { High Molecular Weight } \\
\text { PAH }\end{array}$ & $\begin{array}{c}\text { Total } \\
\text { PAH }\end{array}$ \\
\cline { 2 - 3 } NA & NA & NA \\
NA & NA & NA \\
NA & NA & NA \\
NA & NA & NA \\
NA & NA & NA
\end{tabular}

(a) Not applicable no PAHs detected. 
TABLE K.5. Low Molecular Weight Polynuclear Aromatic Hydrocarbons

(PAHs), Wet Weight, in Tissue of $M$. nasuta, Dakland

Harbor Phase III B Project

\begin{tabular}{|c|c|c|c|c|c|c|c|c|}
\hline \multirow[b]{2}{*}{$\begin{array}{l}\text { Sedinent } \\
\text { Ireatment }\end{array}$} & \multirow[b]{2}{*}{ Replicate } & \multirow[b]{2}{*}{ Batch } & \multicolumn{6}{|c|}{ M. nasure PAHs ( $(\mathrm{g} / \mathrm{kg}$ wet weight) } \\
\hline & & & $\begin{array}{l}\text { Naph- } \\
\text { thalene }\end{array}$ & $\begin{array}{l}\text { Acenaph- } \\
\text { thylene }\end{array}$ & $\begin{array}{l}\text { Acenaph- } \\
\text { thene }\end{array}$ & Eluorene & $\begin{array}{l}\text { Phenan- } \\
\text { threne }\end{array}$ & $\begin{array}{l}\text { Anthr- } \\
\text { acene }\end{array}$ \\
\hline $\begin{array}{l}\text { OL Goal } \\
\text { OL. Achieved }\end{array}$ & & & $\begin{array}{l}20 \\
10\end{array}$ & $\begin{array}{l}20 \\
10\end{array}$ & $\begin{array}{l}20 \\
10\end{array}$ & $\begin{array}{l}20 \\
10\end{array}$ & $\begin{array}{l}20 \\
10\end{array}$ & $\begin{array}{l}20 \\
10\end{array}$ \\
\hline $\begin{array}{l}0-C 13 \\
0-C 13 \\
0-C 13 \\
0-C 13 \\
0-C 13\end{array}$ & $\begin{array}{l}1 \\
2 \\
3 \\
4 \\
5\end{array}$ & $\begin{array}{l}j \\
j \\
j \\
j \\
j\end{array}$ & $\begin{array}{l}10 U^{(a)} \\
10 U \\
10 U \\
10 U \\
10 U\end{array}$ & $\begin{array}{l}10 U \\
10 U \\
10 U \\
10 U \\
10 U\end{array}$ & $\begin{array}{l}10 U \\
10 U \\
10 U \\
10 U \\
10 U\end{array}$ & $\begin{array}{l}10 U \\
10 U \\
10 U \\
10 U \\
10 U\end{array}$ & $\begin{array}{l}10 U \\
10 U \\
10 U \\
10 U \\
10 U\end{array}$ & $\begin{array}{ll}10 & U \\
10 & U \\
10 & U \\
10 & U \\
10 & U\end{array}$ \\
\hline $\begin{array}{l}0-C 12 \\
0-C 12 \\
0-C 12 \\
0-C 12 \\
0-C 12\end{array}$ & $\begin{array}{l}1 \\
2 \\
3 \\
4 \\
5\end{array}$ & $\begin{array}{l}J \\
j \\
j \\
j \\
j\end{array}$ & $\begin{array}{l}10 U \\
10 U \\
10 U \\
10 U \\
10 U\end{array}$ & $\begin{array}{l}10 U \\
10 U \\
10 U \\
10 U \\
10 U\end{array}$ & $\begin{array}{l}10 U \\
10 U \\
10 U \\
10 U \\
10 U\end{array}$ & $\begin{array}{l}10 U \\
10 U \\
10 U \\
10 U \\
10 U\end{array}$ & $\begin{array}{l}10 \mathrm{U} \\
10 \mathrm{U} \\
10 \mathrm{U} \\
10 \mathrm{U} \\
10 \mathrm{U}\end{array}$ & $\begin{array}{l}10 U \\
10 U \\
10 U \\
10 U \\
10 U\end{array}$ \\
\hline $\begin{array}{l}0-C 11 \\
0-C 11 \\
0-C 11 \\
0-C 11 \\
0-c 11\end{array}$ & $\begin{array}{l}1 \\
2 \\
3 \\
4 \\
5\end{array}$ & $\begin{array}{l}k \\
k \\
k \\
k \\
k\end{array}$ & $\begin{array}{l}16 \\
10 U \\
13 \\
11 \\
10\end{array}$ & $\begin{array}{l}10 U \\
10 U \\
10 U \\
10 U \\
10 U\end{array}$ & $\begin{array}{l}10 U \\
10 U \\
10 U \\
10 U \\
10 U\end{array}$ & $\begin{array}{l}10 U \\
10 U \\
10 U \\
10 U \\
10 U\end{array}$ & $\begin{array}{l}10 U \\
10 U \\
10 U \\
10 U \\
10 U\end{array}$ & $\begin{array}{ll}10 & U \\
10 & U \\
10 U \\
10 U \\
10 U\end{array}$ \\
\hline $\begin{array}{l}0-C 10 \\
0-C 10 \\
0-C 10 \\
0-C 10 \\
0-C 10 \\
0-C 10 \\
0-C 10\end{array}$ & $\begin{array}{lll}1 & & \\
2 & & \\
3 & \operatorname{Rep} & 1 \\
3 & \operatorname{Rep} & 2 \\
3 & \operatorname{Rep} & 3 \\
4 & & \\
5 & & \end{array}$ & $\begin{array}{l}K \\
K \\
K \\
K \\
K \\
N \\
K\end{array}$ & $\begin{array}{l}15 \\
15 \\
16 \\
20 \\
19 \\
52 \mathrm{~F}^{(b)} \\
12\end{array}$ & $\begin{array}{l}10 U \\
10 U \\
10 U \\
10 U \\
10 U \\
10 U \\
10 U\end{array}$ & $\begin{array}{l}10 U \\
10 U \\
10 U \\
10 U \\
10 U \\
10 U \\
10 U\end{array}$ & $\begin{array}{l}10 \mathrm{U} \\
10 \mathrm{U} \\
18 \\
10 \mathrm{U} \\
10 \mathrm{U} \\
10 \mathrm{U} \\
10 \mathrm{U}\end{array}$ & $\begin{array}{l}10 U \\
10 U \\
10 U \\
13 \\
10 U \\
10 U \\
10 U\end{array}$ & $\begin{array}{l}10 U \\
10 U \\
10 U \\
10 U \\
10 U \\
10 U \\
10 U\end{array}$ \\
\hline $\begin{array}{l}0-C 9 \\
0-C 9 \\
0-C 9 \\
0-C 9 \\
0-C 9\end{array}$ & $\begin{array}{l}1 \\
2 \\
3 \\
4 \\
5\end{array}$ & $\begin{array}{l}K \\
K \\
K \\
K \\
K\end{array}$ & $\begin{array}{l}27 \\
29 \\
29 \\
20 \\
15\end{array}$ & $\begin{array}{l}10 \mathrm{U} \\
10 \mathrm{U} \\
10 \mathrm{U} \\
10 \mathrm{U} \\
10 \mathrm{U}\end{array}$ & $\begin{array}{l}10 \mathrm{U} \\
10 \mathrm{U} \\
10 \mathrm{U} \\
10 \mathrm{U} \\
10 \mathrm{U}\end{array}$ & $\begin{array}{l}10 U \\
10 U \\
10 U \\
10 U \\
10 U\end{array}$ & $\begin{array}{l}10 U \\
10 U \\
10 U \\
10 U \\
10 U\end{array}$ & $\begin{array}{l}10 U \\
10 U \\
10 U \\
10 U \\
10 U\end{array}$ \\
\hline $\begin{array}{l}0-c 8 \\
0-C 8 \\
0-c .8 \\
0-C 8 \\
0-c 8\end{array}$ & $\begin{array}{l}1 \\
2 \\
3 \\
4 \\
5\end{array}$ & $\begin{array}{l}k \\
k \\
k \\
k \\
k\end{array}$ & $\begin{array}{l}16 \\
15 \\
15 \\
16 \\
12\end{array}$ & 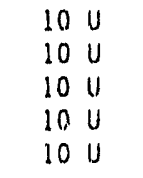 & $\begin{array}{l}10 U \\
10 U \\
10 U \\
10 U \\
10 U\end{array}$ & $\begin{array}{l}10 U \\
10 U \\
10 U \\
10 U \\
10 U\end{array}$ & $\begin{array}{ll}10 & U \\
10 & U \\
10 & U \\
10 & U \\
10 & U\end{array}$ & $\begin{array}{l}10 U \\
10 U \\
10 U \\
10 U \\
10 U\end{array}$ \\
\hline $\begin{array}{l}0-C 7 \\
0-C 7 \\
0-C 7 \\
0-C 7 \\
0-C 7\end{array}$ & $\begin{array}{l}1 \\
2 \\
3 \\
4 \\
5\end{array}$ & $\begin{array}{l}L \\
L \\
L \\
L \\
L\end{array}$ & $\begin{array}{l}20 U \\
20 U \\
20 U \\
20 U \\
20 U\end{array}$ & $\begin{array}{ll}10 & U \\
10 & U \\
10 & U \\
10 & U \\
10 & U\end{array}$ & $\begin{array}{l}10 U \\
10 U \\
10 U \\
10 U \\
10 U\end{array}$ & $\begin{array}{ll}10 & U \\
10 & U \\
10 & U \\
10 & U \\
10 & U\end{array}$ & $\begin{array}{l}10 U \\
10 U \\
10 U \\
10 U \\
10 U\end{array}$ & $\begin{array}{l}10 \mathrm{U} \\
10 \mathrm{U} \\
10 \mathrm{U} \\
10 \mathrm{U} \\
10 \mathrm{U}\end{array}$ \\
\hline $\begin{array}{l}0-C 6 \\
0-C 6 \\
0-C 6 \\
0-C E \\
0-C 6\end{array}$ & $\begin{array}{l}1 \\
2 \\
3 \\
4 \\
5\end{array}$ & $\begin{array}{l}L \\
L \\
L \\
L \\
L .\end{array}$ & $\begin{array}{ll}20 & U \\
20 & U \\
20 & U \\
25 & \\
23 & \end{array}$ & $\begin{array}{ll}10 & U \\
10 & U \\
10 & U \\
10 & U \\
10 & U\end{array}$ & $\begin{array}{l}10 \mathrm{U} \\
10 \mathrm{U} \\
10 \mathrm{U} \\
10 \mathrm{U} \\
10 \mathrm{U}\end{array}$ & $\begin{array}{ll}10 & U \\
10 & U \\
10 & U \\
10 & U \\
10 & U\end{array}$ & $\begin{array}{l}10 \mathrm{U} \\
10 \mathrm{U} \\
10 \mathrm{U} \\
13 \\
14\end{array}$ & $\begin{array}{l}10 U \\
10 U \\
10 U \\
10 U \\
10 U\end{array}$ \\
\hline $\begin{array}{l}0-C 5 \\
0-c 5 \\
0-C 5\end{array}$ & $\begin{array}{l}1 \\
2 \\
3\end{array}$ & $\begin{array}{l}L \\
L \\
L\end{array}$ & $\begin{array}{l}24 \\
20 \mathrm{U} \\
20 \mathrm{U}\end{array}$ & $\begin{array}{l}10 \mathrm{U} \\
10 \mathrm{U} \\
10 \mathrm{U}\end{array}$ & $\begin{array}{l}10 \mathrm{U} \\
10 \mathrm{U} \\
10 \mathrm{U}\end{array}$ & $\begin{array}{l}10 u \\
10 U \\
10 U\end{array}$ & $\begin{array}{l}14 \\
10 u \\
21\end{array}$ & $\begin{array}{l}10 u \\
10 U \\
10 U\end{array}$ \\
\hline
\end{tabular}


TABLE K.5. (contd)

\begin{tabular}{|c|c|c|c|c|c|c|c|c|}
\hline \multirow[b]{2}{*}{$\begin{array}{l}\text { Sediment } \\
\text { Ireatment }\end{array}$} & \multirow[b]{2}{*}{ Replicate } & \multirow[b]{2}{*}{ Batch } & \multicolumn{6}{|c|}{ M. nasuta PAHs $(\mu \mathrm{g} / \mathrm{kg}$ wet weight $)$} \\
\hline & & & $\begin{array}{c}\text { Naph- } \\
\text { thalene }\end{array}$ & $\begin{array}{l}\text { Acenaph- } \\
\text { thylene }\end{array}$ & $\begin{array}{l}\text { Acenaph- } \\
\text { thene }\end{array}$ & Eluorene & $\begin{array}{l}\text { Phenan- } \\
\text { threne }\end{array}$ & $\begin{array}{l}\text { Anthr- } \\
\text { acene }\end{array}$ \\
\hline $\begin{array}{l}0-C 5 \\
0-C 5 \\
0-C .5 \\
0-C 5\end{array}$ & $\begin{array}{lll}4 & \operatorname{Rep} 1 \\
4 & \operatorname{Rep} 2 \\
4 & \operatorname{Rep} 3 \\
5 & & \end{array}$ & $\begin{array}{l}L \\
L \\
L\end{array}$ & $\begin{array}{l}20 U \\
20 U \\
20 U \\
20 U\end{array}$ & $\begin{array}{l}10 U \\
10 U \\
10 U \\
10 U\end{array}$ & $\begin{array}{l}10 U \\
10 U \\
10 U \\
10 U\end{array}$ & $\begin{array}{l}10 U \\
10 U \\
10 U \\
10 U\end{array}$ & $\begin{array}{l}10 U \\
10 U \\
10 U \\
10 U\end{array}$ & $\begin{array}{l}10 U \\
10 U \\
10 U \\
10 U\end{array}$ \\
\hline $\begin{array}{l}0-c 4 \\
0-C 4 \\
0-C 4 \\
0-C 4 \\
0-C 4\end{array}$ & $\begin{array}{l}1 \\
2 \\
3 \\
4 \\
5\end{array}$ & $\begin{array}{l}L \\
L \\
L \\
L \\
L\end{array}$ & $\begin{array}{l}20 U \\
20 U \\
20 U \\
20 U \\
20 U\end{array}$ & $\begin{array}{l}10 U \\
10 U \\
10 U \\
10 U \\
10 U\end{array}$ & $\begin{array}{l}10 U \\
10 U \\
10 U \\
10 U \\
10 U\end{array}$ & $\begin{array}{l}10 U \\
10 U \\
10 U \\
10 U \\
10 U\end{array}$ & $\begin{array}{l}10 U \\
10 U \\
10 U \\
10 U \\
10 U\end{array}$ & $\begin{array}{l}10 U \\
10 U \\
10 U \\
10 U \\
10 U\end{array}$ \\
\hline $\begin{array}{l}0-c 3 \\
0-c 3 \\
0-c 3 \\
0-C 3 \\
0-c 3\end{array}$ & $\begin{array}{l}1 \\
2 \\
3 \\
4 \\
5\end{array}$ & $\begin{array}{l}N \\
N \\
N \\
N \\
N\end{array}$ & $\begin{array}{ll}38 & E \\
31 & E \\
25 & E \\
23 & E \\
21 & E\end{array}$ & $\begin{array}{ll}10 & U \\
10 & U \\
10 & U \\
10 & U \\
10 & U\end{array}$ & $\begin{array}{l}10 U \\
10 U \\
10 U \\
10 U \\
10 U\end{array}$ & $\begin{array}{l}10 U \\
10 U \\
10 U \\
10 U \\
10 U\end{array}$ & $\begin{array}{l}10 U \\
10 U \\
10 U \\
10 U \\
10 U\end{array}$ & $\begin{array}{l}10 U \\
10 U \\
10 U \\
10 U \\
10 U\end{array}$ \\
\hline $\begin{array}{l}0-C 1 \\
0-C 1 \\
0-C 1 \\
0-C 1 \\
0-C 1\end{array}$ & $\begin{array}{l}1 \\
2 \\
3 \\
4 \\
5\end{array}$ & $\begin{array}{l}N \\
N \\
N \\
N \\
N\end{array}$ & $\begin{array}{l}29 E \\
10 U \\
42 E \\
33 E \\
25 E\end{array}$ & $\begin{array}{l}10 U \\
10 U \\
10 U \\
10 U \\
10 U\end{array}$ & $\begin{array}{l}10 U \\
10 U \\
10 U \\
10 U \\
10 U\end{array}$ & $\begin{array}{l}10 U \\
10 U \\
10 U \\
10 U \\
10 U\end{array}$ & $\begin{array}{l}10 U \\
10 U \\
10 U \\
10 U \\
10 U\end{array}$ & $\begin{array}{l}10 U \\
10 U \\
10 U \\
10 U \\
10 U\end{array}$ \\
\hline $\begin{array}{l}1-C 2 \\
1-C 2 \\
1-C 2 \\
1-C 2 \\
1-C 2 \\
1-C 2 \\
1-C 2\end{array}$ & $\begin{array}{lll}1 & \operatorname{Rep} & 1 \\
1 & \operatorname{Rep} & 2 \\
1 & \operatorname{Rep} & 3 \\
2 & & \\
3 & & \\
4 & & \\
5 & & \end{array}$ & $\begin{array}{l}M \\
M \\
M \\
M \\
M \\
M \\
M\end{array}$ & $\begin{array}{l}20 U \\
20 U \\
20 U \\
20 U \\
20 U \\
20 U \\
20 U\end{array}$ & $\begin{array}{l}10 U \\
10 U \\
10 U \\
10 U \\
10 U \\
10 U \\
10 U\end{array}$ & $\begin{array}{l}10 U \\
10 U \\
10 U \\
10 U \\
10 U \\
10 U \\
10 U\end{array}$ & $\begin{array}{l}10 U \\
10 U \\
10 U \\
10 U \\
10 U \\
10 U \\
10 U\end{array}$ & $\begin{array}{l}10 U \\
10 U \\
10 U \\
10 U \\
10 U \\
10 U \\
10 U\end{array}$ & $\begin{array}{ll}10 U \\
10 U \\
10 U \\
10 U \\
10 U \\
10 U \\
10 U\end{array}$ \\
\hline $\begin{array}{l}0-C 2 \\
0-C 2 \\
0-C 2 \\
0-C 2 \\
0-C 2\end{array}$ & $\begin{array}{l}1 \\
2 \\
3 \\
4 \\
5\end{array}$ & $\begin{array}{l}M \\
M \\
M \\
M \\
M\end{array}$ & $\begin{array}{l}20 U \\
20 U \\
20 U \\
20 U \\
20 U\end{array}$ & $\begin{array}{l}10 \mathrm{U} \\
10 \mathrm{U} \\
10 \mathrm{U} \\
10 \mathrm{U} \\
10 \mathrm{U}\end{array}$ & $\begin{array}{l}10 \mathrm{U} \\
10 \mathrm{U} \\
10 \mathrm{U} \\
10 \mathrm{U} \\
10 \mathrm{U}\end{array}$ & $\begin{array}{l}10 U \\
10 U \\
10 U \\
10 U \\
10 U\end{array}$ & $\begin{array}{l}10 \mathrm{U} \\
10 \mathrm{U} \\
10 \mathrm{U} \\
10 \mathrm{U} \\
10 \mathrm{U}\end{array}$ & $\begin{array}{l}10 U \\
10 U \\
10 U \\
10 U \\
10 U\end{array}$ \\
\hline $\begin{array}{l}1-C 3 \\
1-C 3 \\
1-C 3 \\
1-C 3 \\
1-C 3\end{array}$ & $\begin{array}{l}1 \\
2 \\
3 \\
4 \\
5\end{array}$ & $\begin{array}{l}M \\
M \\
M \\
M \\
M\end{array}$ & $\begin{array}{l}20 U \\
20 U \\
20 U \\
20 U \\
20 U\end{array}$ & $\begin{array}{l}10 U \\
10 U \\
10 U \\
10 U \\
10 U\end{array}$ & $\begin{array}{l}10 U \\
10 U \\
10 U \\
10 U \\
10 U\end{array}$ & $\begin{array}{l}10 \mathrm{U} \\
10 \mathrm{U} \\
10 \mathrm{U} \\
10 \mathrm{U} \\
10 \mathrm{U}\end{array}$ & $\begin{array}{l}10 \mathrm{U} \\
10 \mathrm{U} \\
10 \mathrm{U} \\
10 \mathrm{U} \\
10 \mathrm{U}\end{array}$ & $\begin{array}{l}10 \mathrm{U} \\
10 \mathrm{U} \\
10 \mathrm{U} \\
10 \mathrm{U} \\
10 \mathrm{U}\end{array}$ \\
\hline $\begin{array}{l}1-C 8 \\
1-C 8 \\
1-C 8 \\
1-C 8 \\
1-C 8\end{array}$ & $\begin{array}{l}1 \\
2 \\
3 \\
4 \\
5\end{array}$ & $\begin{array}{l}M \\
M \\
M \\
M \\
M\end{array}$ & $\begin{array}{l}20 U \\
20 U \\
20 U \\
20 U \\
20 U\end{array}$ & $\begin{array}{l}10 U \\
10 U \\
10 U \\
10 U \\
10 U\end{array}$ & $\begin{array}{l}10 U \\
10 U \\
10 U \\
10 U \\
10 U\end{array}$ & $\begin{array}{l}10 \mathrm{U} \\
10 \mathrm{U} \\
10 \mathrm{U} \\
10 \mathrm{U} \\
10 \mathrm{U}\end{array}$ & $\begin{array}{l}10 \mathrm{U} \\
10 \mathrm{U} \\
10 \mathrm{U} \\
10 \mathrm{U} \\
10 \mathrm{U}\end{array}$ & $\begin{array}{l}10 \mathrm{U} \\
10 \mathrm{U} \\
10 \mathrm{U} \\
10 \mathrm{U} \\
10 \mathrm{U}\end{array}$ \\
\hline $\begin{array}{l}1-C 4 \\
1-C 4 \\
1-C 4 \\
1-C 4 \\
1-C 4\end{array}$ & $\begin{array}{l}1 \\
2 \\
3 \\
4 \\
5\end{array}$ & $\begin{array}{l}j \\
j \\
j \\
j \\
j\end{array}$ & $\begin{array}{l}10 u \\
10 U \\
10 U \\
10 u \\
10 u\end{array}$ & $\begin{array}{l}10 U \\
10 U \\
10 U \\
10 U \\
10 U\end{array}$ & $\begin{array}{l}10 \mathrm{U} \\
10 \mathrm{U} \\
10 \mathrm{U} \\
10 \mathrm{U} \\
10 \mathrm{U}\end{array}$ & $\begin{array}{ll}10 & U \\
10 & U \\
10 & U \\
10 & U \\
10 & 1\end{array}$ & $\begin{array}{l}10 U \\
10 U \\
10 U \\
10 U \\
1 .\end{array}$ & $\begin{array}{l}10 U \\
10 U \\
10 U \\
10 U \\
10 U\end{array}$ \\
\hline
\end{tabular}


IABLE K.5. (contd)

M. nasura PAHs $(\mu \mathrm{g} / \mathrm{kg}$ wet weight)

Sediment

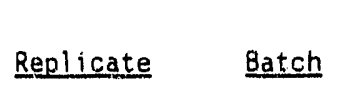

\begin{tabular}{|c|c|c|c|c|c|}
\hline $\begin{array}{c}\text { Naph- } \\
\text { thalene } \\
\end{array}$ & $\begin{array}{l}\text { Acenaph- } \\
\text { thylene }\end{array}$ & $\begin{array}{l}\text { Acenaph- } \\
\text { thene }\end{array}$ & Eluorene & $\begin{array}{l}\text { Phenan- } \\
\text { threne }\end{array}$ & $\begin{array}{l}\text { Anthr } \\
\text { acene }\end{array}$ \\
\hline $\begin{array}{l}11 \\
14 \\
10 U \\
10 U \\
10 U \\
10 U \\
10 U\end{array}$ & $\begin{array}{l}10 U \\
10 U \\
10 U \\
10 U \\
10 U \\
10 U \\
10 U\end{array}$ & $\begin{array}{l}10 U \\
10 U \\
10 U \\
10 U \\
10 U \\
10 U \\
10 U\end{array}$ & $\begin{array}{l}10 U \\
10 U \\
10 U \\
10 U \\
10 U \\
10 U \\
10 U\end{array}$ & $\begin{array}{l}10 U \\
10 U \\
10 U \\
10 U \\
10 U \\
10 U \\
10 U\end{array}$ & $\begin{array}{ll}10 U \\
10 U \\
10 U \\
10 U \\
10 U \\
10 U \\
10 U\end{array}$ \\
\hline $\begin{array}{l}10 U \\
10 U \\
10 U \\
10 U \\
10 U\end{array}$ & $\begin{array}{l}10 U \\
10 U \\
10 U \\
10 U \\
10 U\end{array}$ & $\begin{array}{l}10 U \\
10 U \\
10 U \\
10 U \\
10 U\end{array}$ & $\begin{array}{l}10 U \\
10 U \\
10 U \\
10 U \\
10 U\end{array}$ & $\begin{array}{l}10 U \\
10 U \\
10 U \\
10 U \\
10 U\end{array}$ & $\begin{array}{ll}10 & U \\
10 & U \\
10 & U \\
10 & U \\
10 & U\end{array}$ \\
\hline $\begin{array}{l}10 U \\
10 U \\
10 U \\
10 U \\
10 U\end{array}$ & $\begin{array}{l}10 \mathrm{U} \\
10 \mathrm{U} \\
10 \mathrm{U} \\
10 \mathrm{U} \\
10 \mathrm{U}\end{array}$ & $\begin{array}{l}10 U \\
10 U \\
10 U \\
10 U \\
10 U\end{array}$ & $\begin{array}{l}10 U \\
10 U \\
10 U \\
10 U \\
10 U\end{array}$ & $\begin{array}{l}10 U \\
12 \\
12 \\
10 \\
22\end{array}$ & $\begin{array}{ll}10 & U \\
10 & U \\
10 & U \\
10 & U \\
10 & U\end{array}$ \\
\hline
\end{tabular}

$1-T 5$
$1-T 5$

I-T5

$1-T 5$

$1-15$

$1-T 5$

I- $T 5$

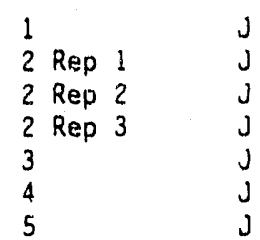

$J$
$J$
$J$
$j$
$j$
$j$
$j$

$R-A C$

$R-A C$

$R-A C$

$R-A C$

1

$R-A C$

$R-A M$

$R-A M$

$R \sim A M$

R-AM

$10 \mathrm{U}$

$R-B F$

$R-B F$

$R-B F$
$R-B F$

$R-B F$

$R-B F$

$R-B F$

$10 \mathrm{U}$

$10 \mathrm{U}$

$30 \mathrm{U}$

$10 \mathrm{U}$

$10 \mathrm{U}$

$10 U$

$10 \mathrm{U}$

$\therefore$

$R-O S$.

$R-O S$

$R-O S$

$R-O S$

R-OS

$R-P C$

$R-P C$

$R-P C$

$R-P C$

$R-P C$

$10 \mathrm{U}$

$10 \mathrm{U}$

$10 \mathrm{U}$

$10 \mathrm{U}$

$10 \mathrm{U}$

$10 \mathrm{U}$

$10 \mathrm{U}$
$10 \mathrm{U}$

$10 \mathrm{U}$
$10 \mathrm{U}$

$10 \mathrm{U}$

$10 \mathrm{U}$

$30 \mathrm{~V}$

$10 \mathrm{U}$

$10 \mathrm{U}$

$10 \mathrm{U}$

$10 \mathrm{U}$

$10 \mathrm{U}$

$10 \mathrm{U}$

$10 \mathrm{U}$

$10 \mathrm{U}$

$10 \mathrm{U}$

$10 \mathrm{~V}$

$10 \mathrm{~V}$

$10 \mathrm{~V}$

$10 \mathrm{U}$

$10 \mathrm{U}$

$10 \mathrm{U}$

$10 \mathrm{U}$

$10 \mathrm{U}$

$10 \mathrm{U}$

$10 \mathrm{U}$

$10 \mathrm{U}$

$10 \mathrm{U}$

$10 \mathrm{U}$

$10 \mathrm{U}$

$10 \mathrm{U}$

$10 \mathrm{U}$

$10 \mathrm{U}$

$10 \mathrm{U}$

$10 \mathrm{U}$

$10 \mathrm{U}$

$10 \mathrm{~V}$

$30 \mathrm{U}$

$10 \mathrm{U}$

$10 \mathrm{U}$

$10 \mathrm{U}$

$10 \mathrm{U}$

$10 \mathrm{U}$

$10 \mathrm{U} \quad 10 \mathrm{U}$

$30 \mathrm{U} \quad 30 \mathrm{U}$

$10 \mathrm{U} \quad 10 \mathrm{U}$

$10 \mathrm{U} \quad 10 \mathrm{U}$

$10 \mathrm{U} \quad 10 \mathrm{U}$

$10 \mathrm{U} \quad 10 \mathrm{U}$

$10 \mathrm{U} \quad 10 \mathrm{U}$

$R-P F$

$R-P F$

$R=P F$

$R-P F$

$10 \mathrm{U}$

$10 \mathrm{U}$

$10 \mathrm{U}$

$10 \mathrm{U}$

$10 \mathrm{U}$

$10 \mathrm{U}$

$10 \mathrm{U} \quad 10 \mathrm{U}$

$10 \mathrm{U} \quad 10 \mathrm{U} \quad 10 \mathrm{U}$

$10 \mathrm{U} \quad 10 \mathrm{U} \quad 10 \mathrm{U}$

$10 \mathrm{U} \quad 10 \mathrm{U} \quad 10 \mathrm{U}$

$10 \mathrm{U} \quad 10 \mathrm{U} \quad 10 \mathrm{U}$

$R-P F \quad 5$

$10 \mathrm{U}$

$C-S B$

$C-S B$

C-SB

$C-S B$

$C-S B$

$C-S B$

$10 \mathrm{U}$

$10 \mathrm{U}$

$10 \mathrm{U}$

101

$10 \mathrm{U}$

$10 \mathrm{U}$

$10 \mathrm{U} \quad 10 \mathrm{U}$

$10 \mathrm{U}$

$10 \mathrm{U}$

$10 \mathrm{U} \quad 10 \mathrm{U}$

$10 \mathrm{U} \quad 10 \mathrm{U}$

$10 \mathrm{U} \quad 10 \mathrm{U}$

$10 \mathrm{U} \quad 10 \mathrm{U} \quad 10 \mathrm{U}$

$10 \mathrm{U}$

$10 \mathrm{U}$

$10 \mathrm{U}$

$10 \mathrm{U}$

$10 \mathrm{U}$

100

$10 \mathrm{U}$

$10 \mathrm{U}$

$10 \mathrm{U}$

$10 \mathrm{U}$

$10 \mathrm{U}$

$10 \mathrm{U}$

$10 \mathrm{U}$

$10 \mathrm{U} \quad 10 \mathrm{U}$

$10 \mathrm{U} \quad 10 \mathrm{U}$

$\begin{array}{lll}10 U & 10 U & 10 U \\ 10 U & 10 U & 10 U\end{array}$

$10 \mathrm{U} \quad 10 \mathrm{U} \quad 10 \mathrm{U}$

4 Rep 2

$10 \mathrm{U}$

$10 \mathrm{U}$

$10 U$
$10 U$
$10 U$
$10 U$
$10 U$
$10 U$

$10 \mathrm{U} \quad 10 \mathrm{U}$

$10 \mathrm{U} \quad 10 \mathrm{U}$

$10 \mathrm{U} \quad 10 \mathrm{U}$

$10 \mathrm{U} \quad 10 \mathrm{U}$

$10 \mathrm{U} \quad 10 \mathrm{U}$

PHASE III B

K. 19 
TABLE K.5. (contd)

Sediment Treatment

$\begin{array}{ll}\text { C-NE } & 1 \\ \text { C-NE } & 2 \\ \text { C-NE } & 3 \\ \text { C-NE } & 4 \\ \text { C-NE } & 5\end{array}$

M. nasula PAHs $(\mu \mathrm{g} / \mathrm{kg}$ wet weight $)$

Naph- Acenaph- Acenaph- Phenan- Anthr-
thalene thylene thene Fluorene

enanacene

(a) Undetected above given concentration.

(b) Matrix spike recoveries for batch were both above acceptable range: concentration may be biased high.

$\begin{array}{lllll}10 U & 10 U & 10 U & 10 U & 10 U \\ 10 U & 10 U & 10 U & 10 U & 10 U \\ 10 U & 10 U & 10 U & 10 U & 10 U \\ 10 U & 10 U & 10 U & 10 U & 10 U \\ 10 U & 10 U & 10 U & 10 U & 10 U\end{array}$




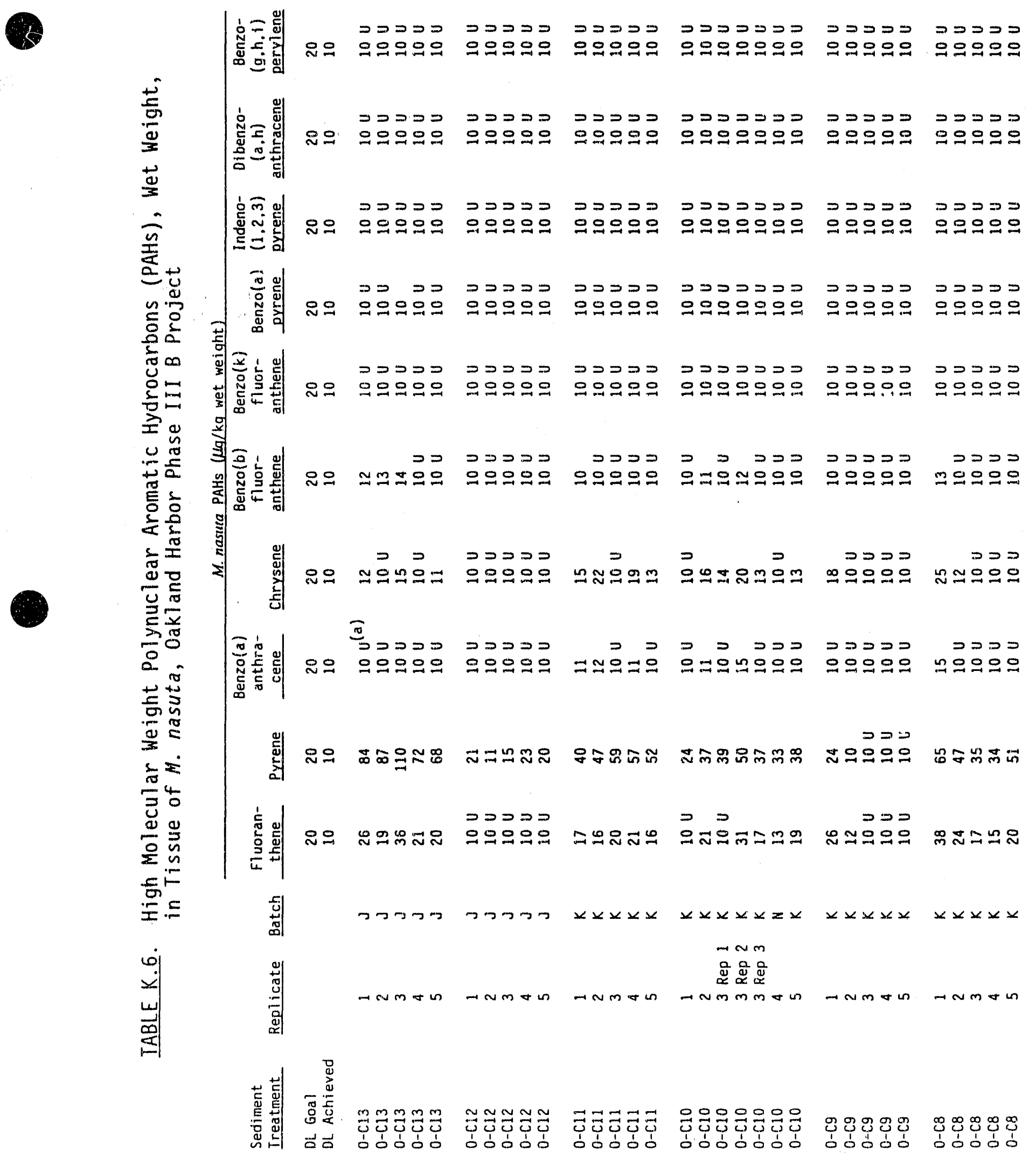




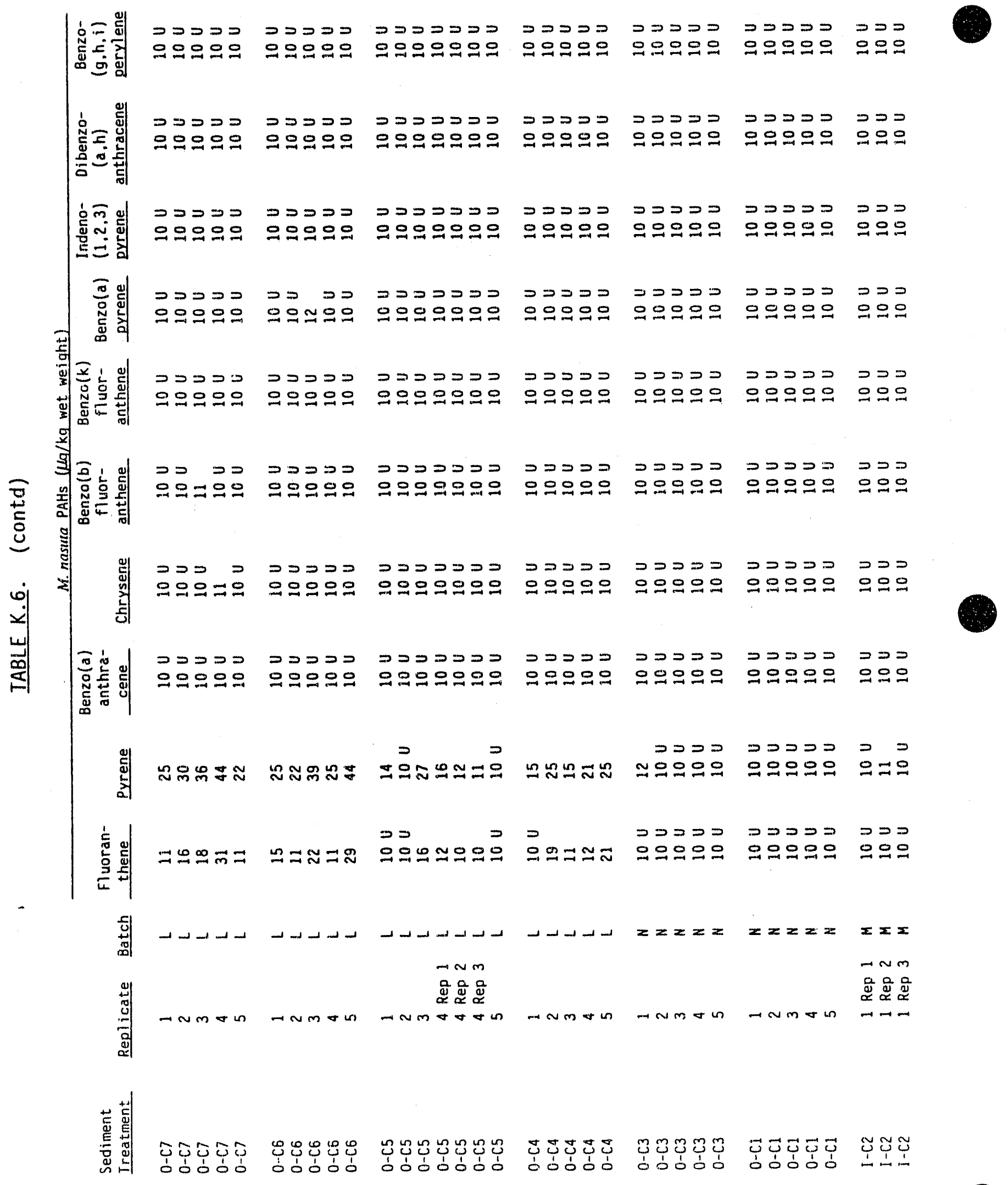




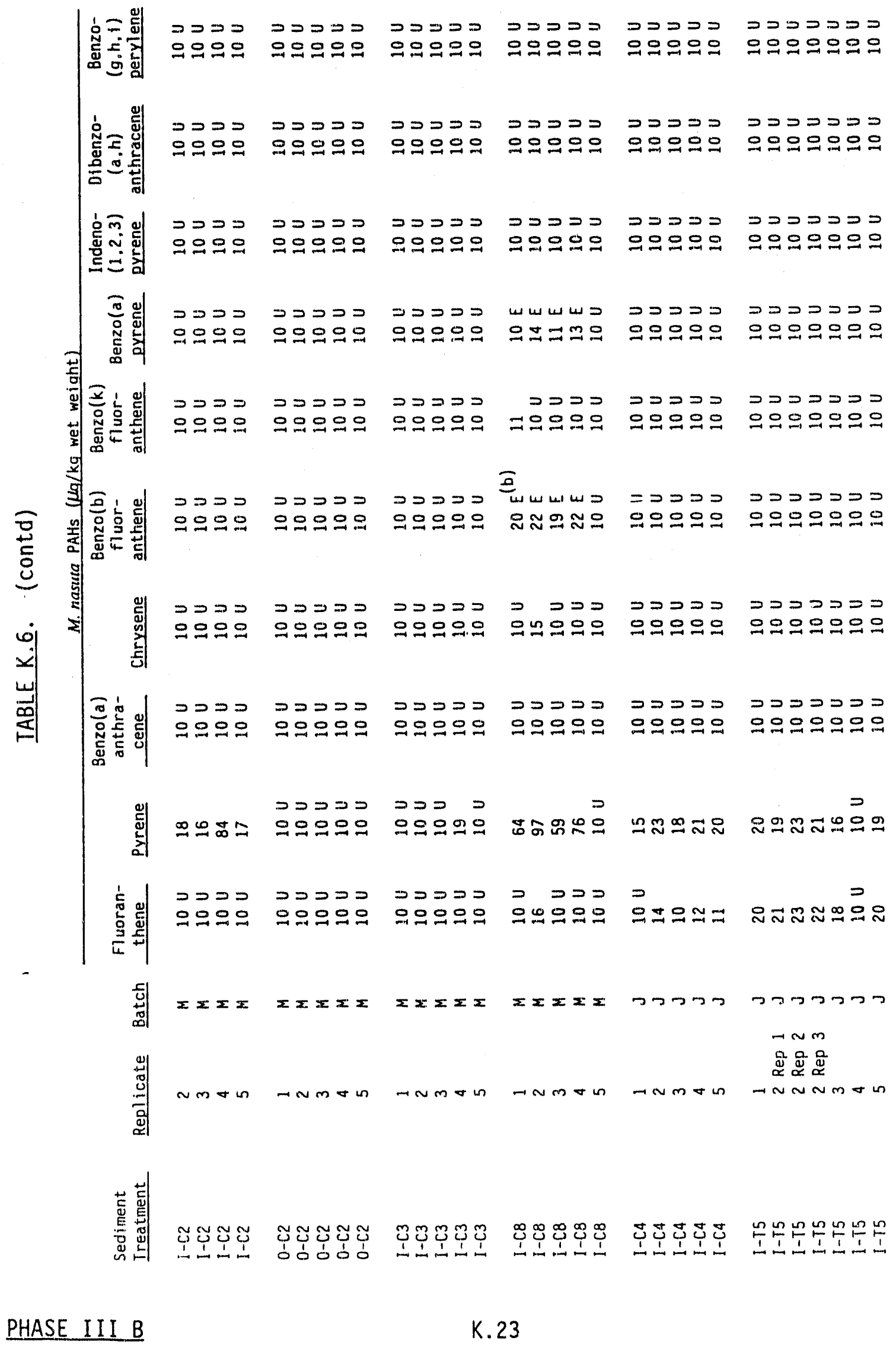




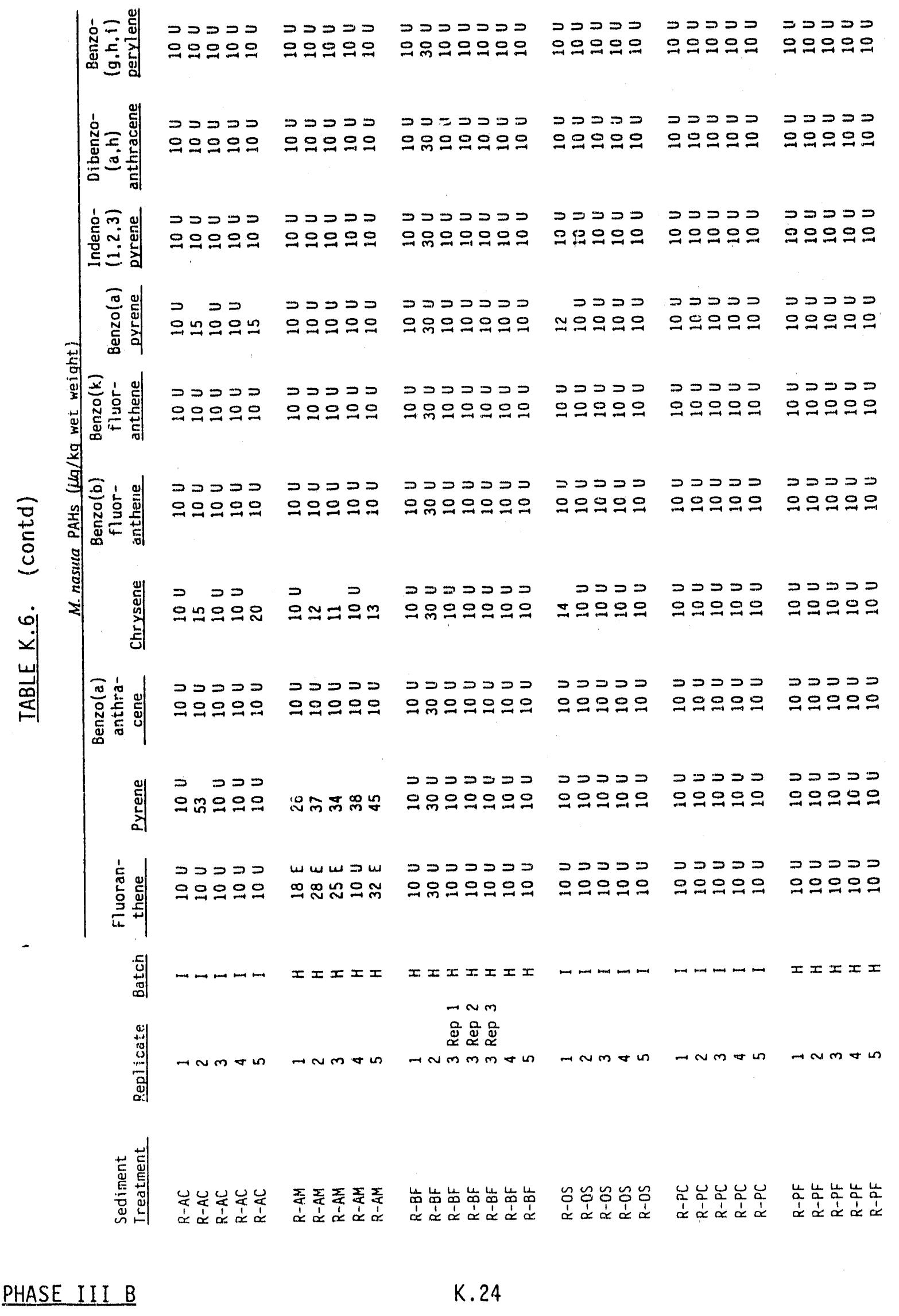




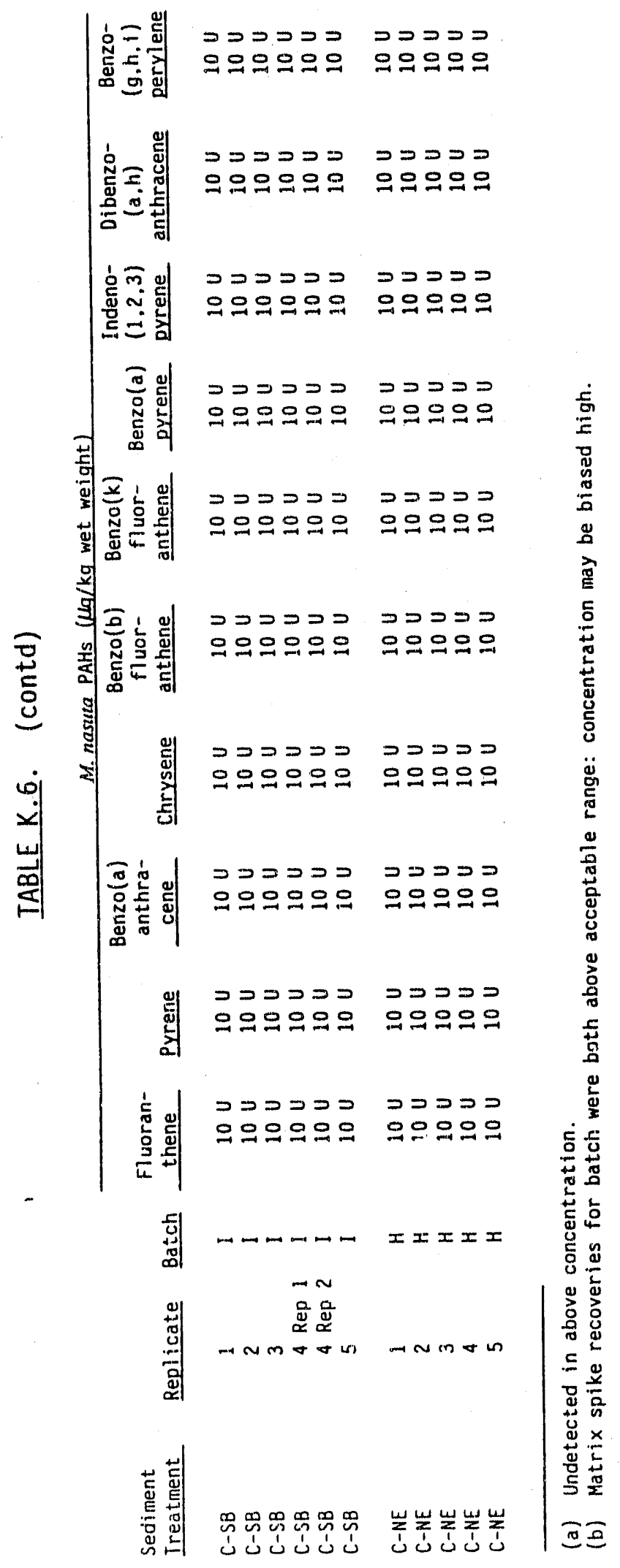


TABLE K.7. Quality Control Data for Low Molecular Weight Polynuclear Aromatic Hydrocartons (PAHs), Wet Weight, in Tissue of M. nasuta, Oak1 and Harbor Phase III B Project

Sample
Method 8lank
Blank H
Blank I
Blank J
Blank K
Blank L
Blank M
Blank N

M. nasuta PAHs $(\mu \mathrm{g} / \mathrm{kg}$ wet weicht)

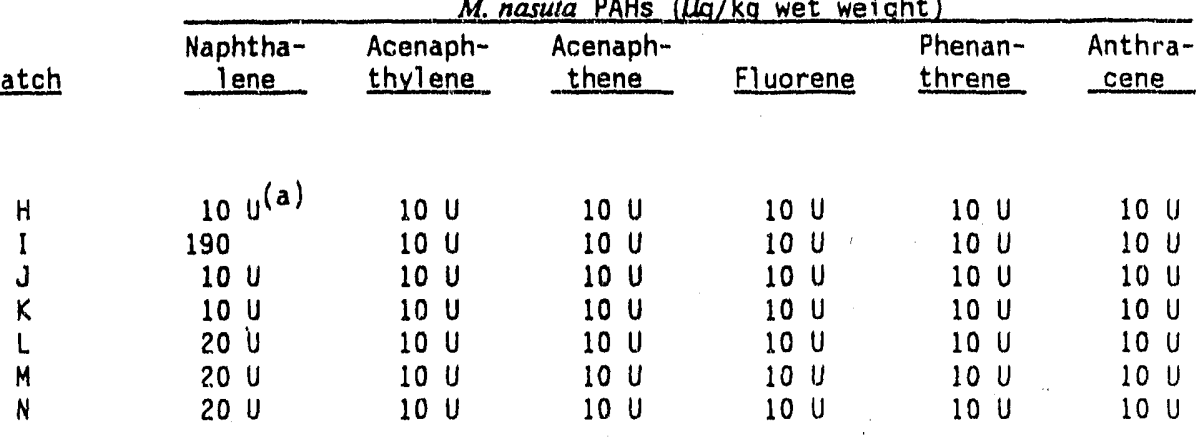

\section{Matrix Spikes}

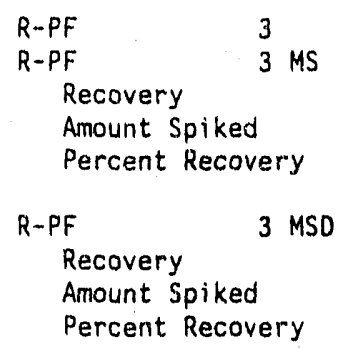

$10 U$
22
22
20
$110 \%$

$10 U$
22
22
20
$110 \%$

$10 U$
24
24
20
$120 \%$

$10 U$
22
22
20
$110 \%$

$\begin{array}{cc}10 \mathrm{U} & 10 \mathrm{U} \\ 24 & 22 \\ 24 & 22 \\ 20 & 20 \\ 120 \% & 110 \%\end{array}$

H

22
22
20
$110 \%$

20
20
20
$100 \%$

22
22
20
$110 \%$

22
22
20
$110 \%$

$\begin{array}{cc}24 & 22 \\ 24 & 22 \\ 20 & 20 \\ 120 \% & 110 \%\end{array}$

$0.0 \%$

I

0.00

$10 u$
19
19
20
$96 \%$

0.05

$29 \%$

$0 \%$

$0 \%$

$0 \%$

$\begin{array}{cc}10 u & 10 u \\ 22 & 22 \\ 22 & 22 \\ 20 & 20 \\ 110 \% & 110 \%\end{array}$

$10 u$
22
22
20
$110 \%$

$\begin{array}{cc}10 U & 10 u \\ 22 & 22 \\ 22 & 22 \\ 20 & 20 \\ 110 \% & 110 \%\end{array}$

I

12

12
18

18
$63 \%$

22

22
18
$110 \%$

22

22

$110 \%$

$110 \%$

22
22

22
18

$42 \%$

0.21

$0.0 \%$

0.00

$122 \%$

0.00

$0.00^{0 \%}$

0.00

$10 U$
14
14
20
$71 \%$
$10 U$
0.6
0.6
20
$3 \%$

$10 U$
22
22
20
$110 \%$
$10 u$
22
22
20
$1.10 \%$

$10 \mathrm{U}$

24

24

20
$120 \%$

$10 \mathrm{U}$

24

24

20

$\begin{array}{cc}22 & 22 \\ 22 & 22 \\ 18 & 18 \\ 110 \% & 110 \%\end{array}$

$120 \%$

$10 u$
22
22
20
$110 \%$

$10 U$
26
26
20
$130 \%$

$\begin{array}{cc}10 \mathrm{U} & 10 \mathrm{U} \\ 24 & 24 \\ 24 & 24 \\ 20 & 20 \\ 120 \% & 120 \% \\ & \\ 10 \mathrm{U} & 10 \mathrm{U} \\ 22 & 22 \\ 22 & 22 \\ 20 & 20 \\ 110 \% & 110 \%\end{array}$


IABLE K.7. (contd)

Sample Replicate Barch

$\begin{array}{lll}0-C d 1 & 1 & k \\ 0-C 11 & I M S & K\end{array}$

Recovery

Anount Spiked

Percent Recovery
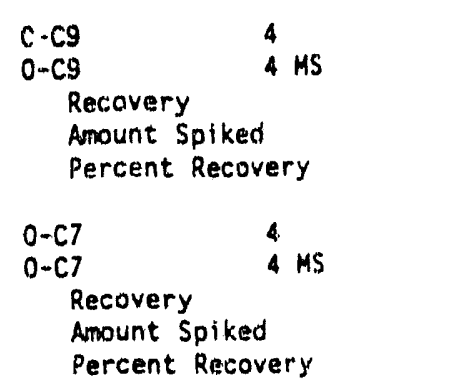
$\begin{array}{ll}0-C 7 & 5 \\ 0-C 7 & 5 \mathrm{MS}\end{array}$
Recovery
Amount Splked
Percent Recovery

$0-C 2$
$0-C 2$
Recovery
Amount Spiked
Percent Recovery
I-CB 1
I $\mathrm{CB}$ I MS
Recovery
Amount Spiked
Percent Recovery
$\begin{array}{ll}0-C l & 1 \\ 0-C l & 1\end{array}$
Recovery
Amount Spiked
Percent Recovery
$\begin{array}{ll}\mathrm{O}=\mathrm{Cl} & 3 \\ \mathrm{O}-\mathrm{Cl} & 3 \mathrm{NS}\end{array}$
Recovery
Amount Spiked
Percent Recovery

Analytical Replicates

$\begin{array}{ll}R-B F & 3 \text { Rep } 1 \\ R-B F & 3 \text { Rep } 2 \\ R-B F & 3 \operatorname{Rep} 3 \\ R S S O & \end{array}$

PHASE III B

$K$
$k$

$L$

$M$
M. nasuea PAHs (ka/kg wet weight)

\begin{tabular}{cccccc}
$\begin{array}{c}\text { Naphtha- } \\
\text { lene }\end{array}$ & $\begin{array}{c}\text { Acenaph- } \\
\text { thylene }\end{array}$ & $\begin{array}{c}\text { Acenaph- } \\
\text { thene }\end{array}$ & Fluorene & $\begin{array}{c}\text { Phenan- } \\
\text { threne }\end{array}$ & $\begin{array}{c}\text { Anthra- } \\
\text { cene }\end{array}$ \\
\cline { 1 - 3 } & & & & & \\
16 & $10 \mathrm{U}$ & $10 \mathrm{U}$ & $10 \mathrm{U}$ & $10 \mathrm{U}$ & $10 \mathrm{U}$ \\
38 & 26 & 26 & 20 & 24 & 32 \\
22 & 26 & 26 & 26 & 24 & 32 \\
20 & 20 & 20 & 20 & 20 & 20 \\
$110 \%$ & $130 \%$ & $130 \%$ & $130 \%$ & $120 \%$ & $160 \%$
\end{tabular}

$\begin{array}{cc}20 & 10 u \\ 42 & 24 \\ 22 & 24 \\ 20 & 20 \\ 110 \% & 120 \%\end{array}$

$10 \mathrm{U}$

26

26

20

$20 \mathrm{U} \quad 10 \mathrm{U}$

$16 \quad 22$

$\begin{array}{ll}16 & 22 \\ 20 & 20\end{array}$

$81 \% \quad 110 \%$

$20 \mathrm{U}$

${ }_{N S}^{0}(b)$

$10 u$
24
24
20
$120 \%$

$10 \mathrm{U}$

10
24
24

24
20

20
$120 \%$

$20 \mathrm{U}$

17

17

20

$86 x$

$10 \mathrm{U}$

22

22

20

$110 \%$

$10 U$
26
26
20
$130 \%$

$10 \mathrm{U} \quad 10 \mathrm{U}$

$26 \quad 26$

$26 \quad 26$

$20 \quad 20$

$130 \% \quad 130 \%$

$\begin{array}{cc} & \\ & 86 x \\ M & 20 \\ & 24 \\ & 24 \\ & 20 \\ & 12.0 \%\end{array}$

$10 \mathrm{U}$

$10 \mathrm{U} \quad 10 \mathrm{U}$

$10 \cup$
19
19
20
$95 \%$

$\begin{array}{cc}10 U & 10 U \\ 18.8 & 28 \\ 18.8 & 28 \\ 20 & 20 \\ 94 \% & 140 \%\end{array}$

$10 \mathrm{U}$

20

20

20

$100 \%$

$\begin{array}{cc}10 U & 10 U \\ 28 & 28 \\ 28 & 28 \\ 20 & 20 \\ 140 \% & 140 \%\end{array}$

$20 \mathrm{U} \quad 10$

$24 \quad 22$

$\begin{array}{lc}20 & 20 \\ 20 \% & 110 \%\end{array}$

29
83
54
32

$10 \mathrm{U}$

32
32

32
32

$170 x$

$100 x$

$\begin{array}{ll}4 ? & 10 \mathrm{U} \\ 83 & 32\end{array}$

32

32

$100 \%$

24

24
20

$120 \%$

22

22

20

$110 \%$

$10 \mathrm{U}$

$10 U \quad 10$

$24 \quad 24$

$24 \quad 24$

$\begin{array}{cc}20 & 20 \\ 120 \% & 120 \%\end{array}$

$10 \mathrm{U} \quad 10 \mathrm{U} \quad 10 \mathrm{U} \quad 10 \mathrm{U}$

$\begin{array}{llll}24 & 24 & 24 & 24\end{array}$

$\begin{array}{llll}24 & 24 & 24 & 24\end{array}$

$20 \quad 20 \quad 20 \quad 20$

$\begin{array}{cccc}20 & 20 & 20 & 20 \% \\ 120 \% & 120 \% & 120 \% & 120 \%\end{array}$

41
32

$230 \%$

38.4

$10 \mathrm{~W}$

$10 \mathrm{U}$

$10 \mathrm{U}$

38.4

32

$120 \%$

35.2

35.2

32

$10 \mathrm{U}$

$110 \%$

38.4

38.4

$10 \mathrm{U}$

35.2

35.2

32

$120 \%$

SO U
100
100
NA

$10 U$
$10 U$

$10 \mathrm{U}$

NA

$10 U$
$10 U$
$10 U$
NA

$10 \mathrm{U}$

$10 \mathrm{U}$

$10 \mathrm{U}$

$10 \mathrm{U}$

$10 \mathrm{U}$

$10 \mathrm{U}$ 
TABLE K.7. (contd)

\begin{tabular}{|c|c|c|c|c|c|c|c|c|}
\hline \multirow[b]{2}{*}{ Sample } & \multirow[b]{2}{*}{ Replicate } & \multirow[b]{2}{*}{ Batch } & \multicolumn{6}{|c|}{ M. nasurg PAHs ( $(\mathrm{kg} / \mathrm{kg}$ wet weight) } \\
\hline & & & $\begin{array}{l}\text { Naphtha- } \\
\text { lene }\end{array}$ & $\begin{array}{l}\text { Acenaph- } \\
\text { thylene }\end{array}$ & $\begin{array}{l}\text { Acenaph- } \\
\text { thene }\end{array}$ & Eluorene & $\begin{array}{l}\text { Phenan- } \\
\text { threne }\end{array}$ & $\begin{array}{l}\text { Anthra- } \\
\text { cenes }\end{array}$ \\
\hline $\begin{array}{l}\text { C-SB } \\
\text { C-SB } \\
\text { RPO } \\
\text { I-Stat }\end{array}$ & $\begin{array}{l}4 \text { Rep } 1 \\
4 \text { Rep } 2\end{array}$ & 1 & $\begin{array}{l}10 U \\
10 \text { UI } \\
\text { HA } \\
\text { HA }\end{array}$ & $\begin{array}{l}10 U \\
10 U \\
N A \\
N A\end{array}$ & $\begin{array}{l}10 \mathrm{U} \\
10 \mathrm{U} \\
\text { NA } \\
\text { NA }\end{array}$ & $\begin{array}{l}10 \mathrm{U} \\
10 \mathrm{U} \\
N A \\
N A\end{array}$ & $\begin{array}{l}10 U \\
10 U \\
N A \\
N A\end{array}$ & $\begin{array}{l}10 U \\
10 U \\
N A \\
N A\end{array}$ \\
\hline $\begin{array}{l}1-Y 5 \\
1-T 5 \\
1-T 5 \\
\text { RSD }\end{array}$ & $\begin{array}{lll}2 & \operatorname{Rep} & 1 \\
2 & \operatorname{Rep} & 2 \\
2 & \operatorname{Rep} & 3\end{array}$ & $\begin{array}{l}J \\
j \\
J\end{array}$ & $\begin{array}{l}14 \\
10 U \\
10 U \\
N A\end{array}$ & $\begin{array}{l}10 U \\
10 U \\
10 U \\
N A\end{array}$ & $\begin{array}{l}10 U \\
10 U \\
10 U \\
\text { NA }\end{array}$ & $\begin{array}{l}10 U \\
10 U \\
10 U \\
N A\end{array}$ & $\begin{array}{l}10 U \\
17 U \\
10 U \\
\text { NA }\end{array}$ & $\begin{array}{l}10 U \\
10 U \\
10 U \\
\text { NA }\end{array}$ \\
\hline $\begin{array}{l}0-C 10 \\
0-C 10 \\
0-C 10 \\
\text { RSD }\end{array}$ & 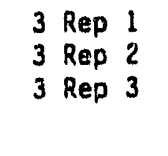 & $\begin{array}{l}k \\
K \\
K\end{array}$ & $\begin{array}{l}16 \\
20 \\
19 \\
11 \%\end{array}$ & $\begin{array}{l}10 U \\
10 U \\
10, U \\
N A\end{array}$ & $\begin{array}{l}10 \mathrm{U} \\
10 \mathrm{U} \\
10 \mathrm{U} \\
\text { NA }\end{array}$ & $\begin{array}{l}18 \\
10 U \\
10 U \\
\text { NA }\end{array}$ & $\begin{array}{l}10 \mathrm{U} \\
13 \\
10 \mathrm{U} \\
\text { NA }\end{array}$ & $\begin{array}{l}10 U \\
10 U \\
10 U \\
\text { NA }\end{array}$ \\
\hline $\begin{array}{l}0-C 5 \\
0-C 5 \\
0-C 5 \\
\text { RSD }\end{array}$ & $\begin{array}{l}4 \operatorname{Rep} 1 \\
4 \operatorname{Rep} 2 \\
4 \operatorname{Rep} 3\end{array}$ & $l$ & $\begin{array}{l}20 U \\
20 U \\
20 U \\
\text { NA }\end{array}$ & $\begin{array}{l}10 U \\
10 U \\
10 U \\
\text { NA }\end{array}$ & $\begin{array}{l}10 U \\
10 U \\
10 U \\
H A\end{array}$ & $\begin{array}{l}10 U \\
10 U \\
10 U \\
N A\end{array}$ & $\begin{array}{l}10 U \\
10 U \\
10 U \\
\text { NA }\end{array}$ & $\begin{array}{l}10 U \\
10 U \\
10 U \\
\text { NA }\end{array}$ \\
\hline $\begin{array}{l}I-C 2 \\
1-C 2 \\
I-C 2 \\
\text { RSD }\end{array}$ & $\begin{array}{lll}1 & \text { Rep } 1 \\
1 & \text { Rep } 2 \\
1 & \text { Rep } 3\end{array}$ & $\begin{array}{l}M \\
M \\
M\end{array}$ & $\begin{array}{l}20 U \\
20 U \\
20 U \\
N A\end{array}$ & $\begin{array}{l}10 U \\
10 U \\
10 U \\
N A\end{array}$ & $\begin{array}{l}10 U \\
10 U \\
10 U \\
K A\end{array}$ & $\begin{array}{l}10 U \\
10 U \\
10 U \\
\text { NA }\end{array}$ & $\begin{array}{l}10 U \\
10 U \\
10 U \\
N A\end{array}$ & $\begin{array}{l}10 U \\
10 U \\
10 U \\
N A\end{array}$ \\
\hline
\end{tabular}

\footnotetext{
(a) Undetected above given concentration.

(b) Analyte not spiked.

(c) Not applicable.
} 


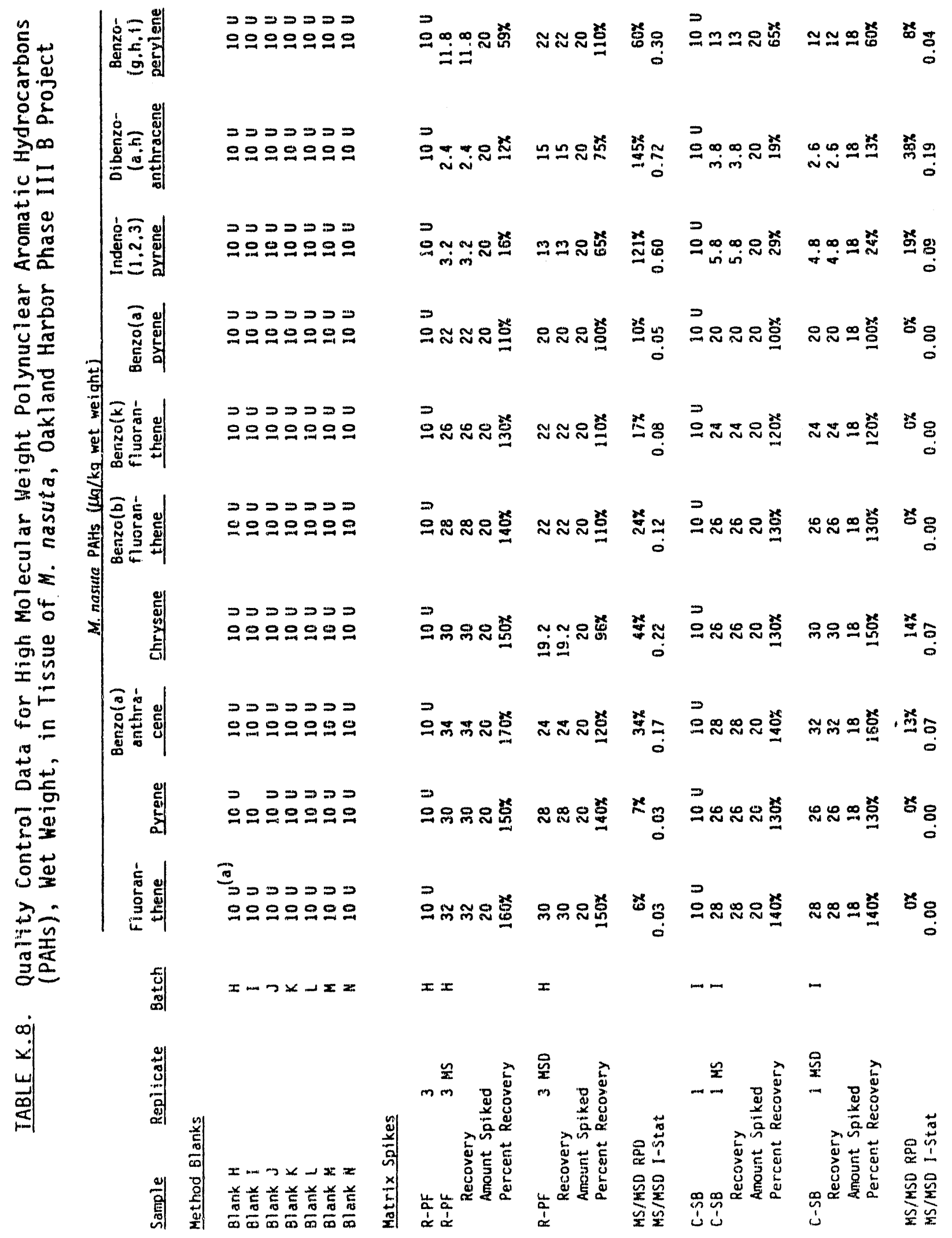




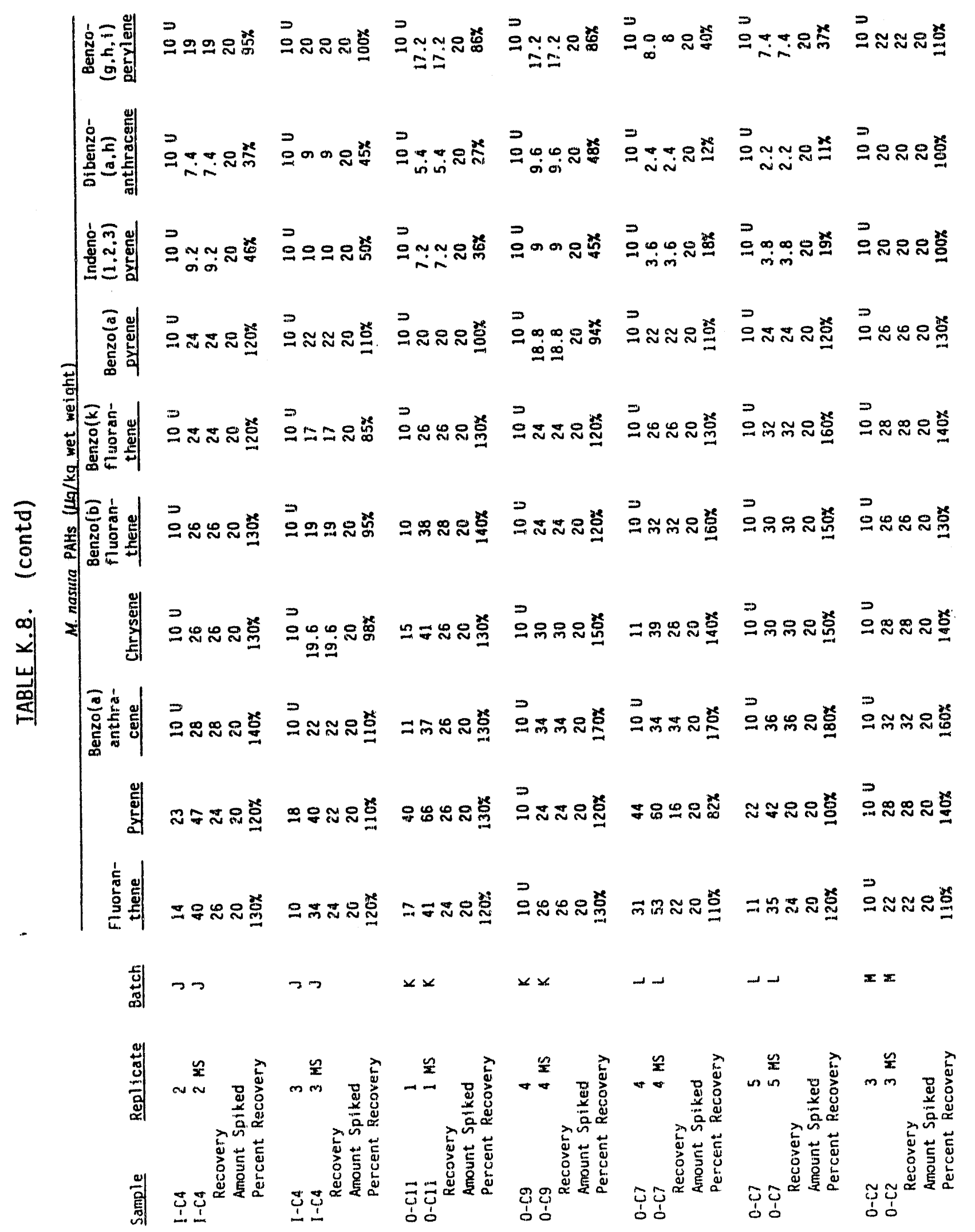

PHASE_III B

K. 30 


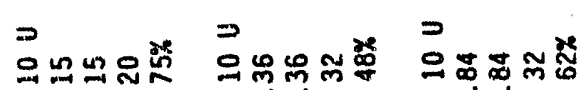
in

in in

总死管

우욤요

음

음 : 용

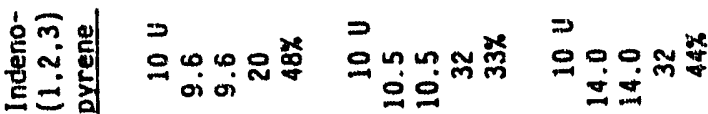

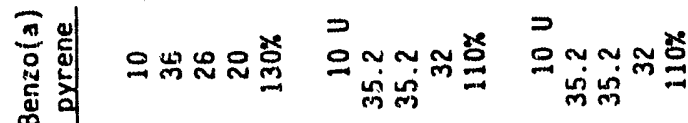

홍 邹全京

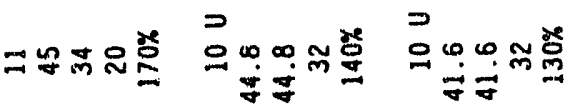

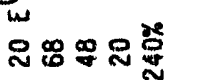

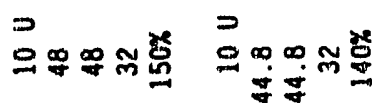

市 迹 $\frac{2}{4}$

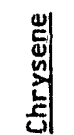

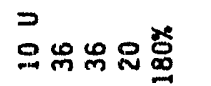

อินกำ

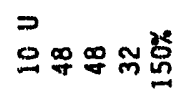

焉点

옹워 응융ㅇㅇ

응 $\stackrel{\infty}{\infty} \underset{0}{\infty}$

ำ ㄸํำ

20
0

50
0

$3=$

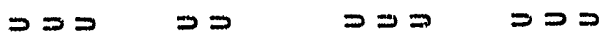

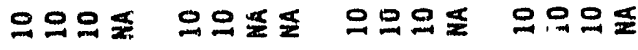

矛

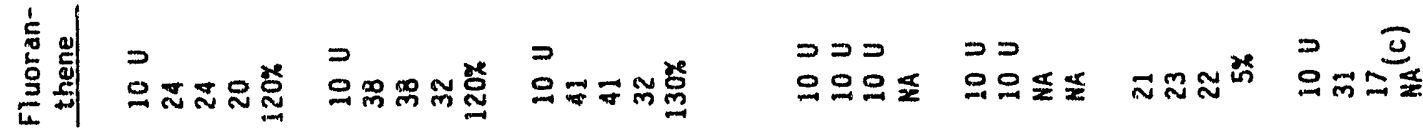

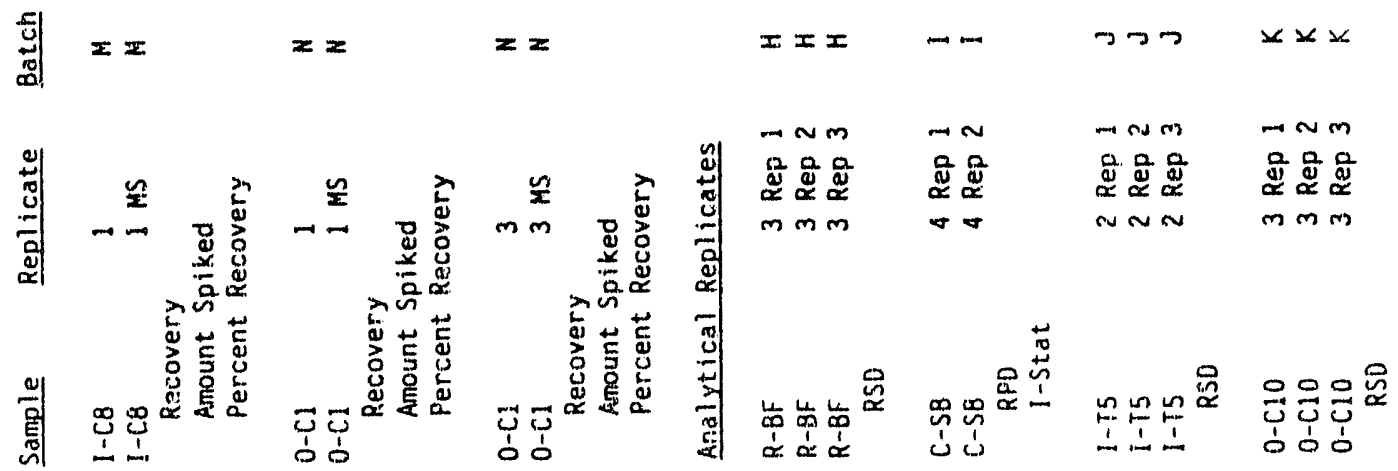




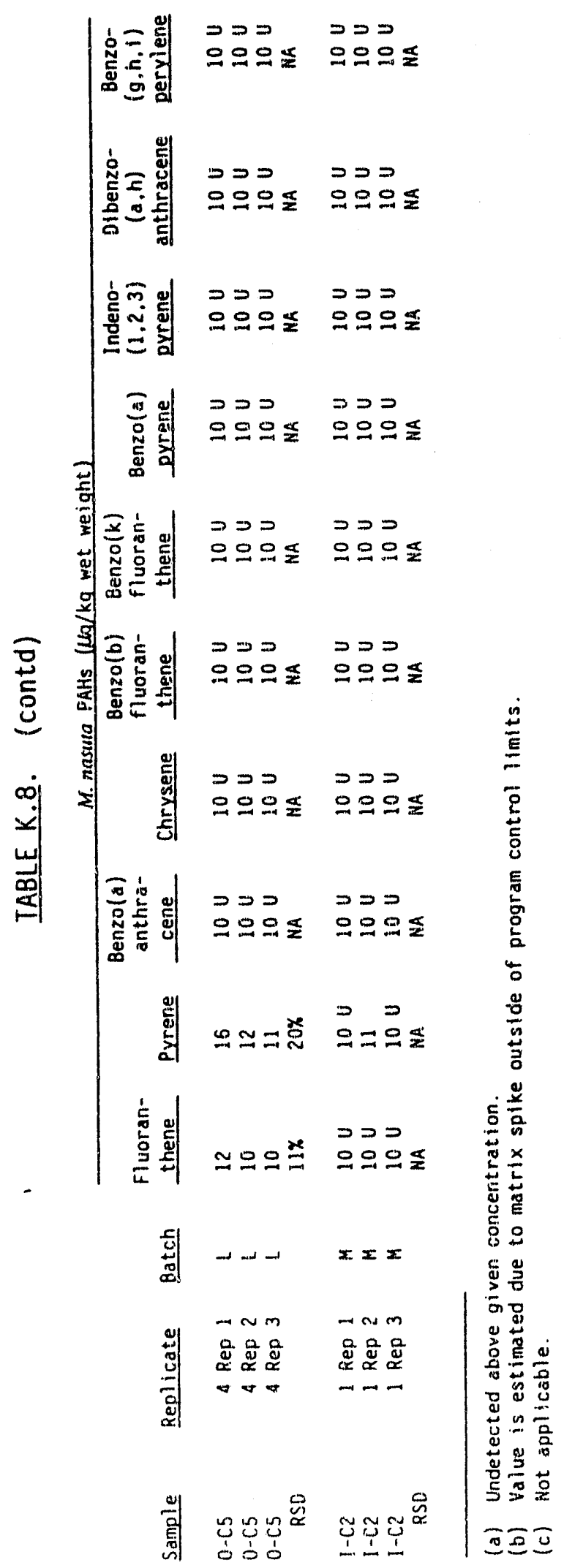


TABLE K.9. Surrogate Recoveries for Polynuclear Aromatic Hydrocarbons (PAHs) in Tissue of M. nasuta, Oakland Harbor Phase III B Project

Sediment

Treatment

$0-\mathrm{C} 13$

$0-\mathrm{Cl3}$

$0-\mathrm{Cl3}$

$0-\mathrm{C} 13$

$0-\mathrm{C} 13$

$0-\mathrm{C} 12$

$0-\mathrm{C} 12$

$0-\mathrm{C} 12$

$0-\mathrm{C} 12$

$0-\mathrm{C} 12$

$0-C 11$

$0-C 11$

$0-C 11$

$0-C 11$

$0-611$

$0-\mathrm{C} 10$

$0-\mathrm{C} 10$

$0-\mathrm{C} 10$

$0-C 10$

$0-\mathrm{C} 10$

$0-\mathrm{C} 10$

$0-C 10$

$0-C 9$

$0-C 9$

$0-C 9$

$0-C .9$

$0-C .9$

$0-C 8$

$0-C B$

$0-C 8$

$0-18$

$0-c 8$

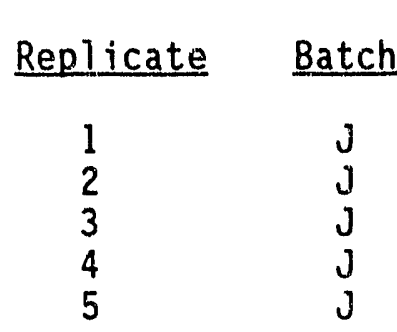

Surrogate Percent Recoveries Naphtha- Acenaph- Phenan- Benzo(a)
lene thene threne Anthra-

D8 D10 010 cene D12

61

77

90

63

68

76

90

90

90

81

74

89

110

97

$100 \quad 90$

100

89

$\begin{array}{rr}87 & 100 \\ 7 \text { (a) } & 65\end{array}$

78

77

100

91

$\begin{array}{ll}1 & \mathrm{~J} \\ 2 & \mathrm{~J} \\ 3 & \mathrm{~J} \\ 4 & \mathrm{~J} \\ 5 & \mathrm{~J}\end{array}$

65
90

120

100

$95 \quad 61$

100

89

58

94

110

95

88

110

110

$\begin{array}{ll}1 & K \\ 2 & K \\ 3 & K \\ 4 & K \\ 5 & K\end{array}$

110

93

110

$\begin{array}{rr}K & 120 \\ K & 41\end{array}$

$\begin{array}{rr}K & 120 \\ K & 41\end{array}$

$87 \quad 89$

40

97
110

98

$94 \quad 79$

$52 \quad 120$

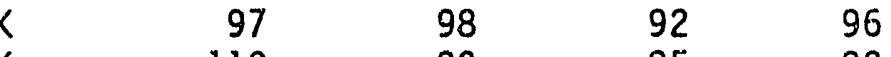

$\begin{array}{llll}1 & & & K \\ 2 & & & K \\ 3 & \operatorname{Rep~} 1 & K \\ 3 & \operatorname{Rep~} 2 & K \\ 3 & \operatorname{Rep~} 3 & K \\ 4 & & & N \\ 5 & & & K\end{array}$

$\begin{array}{lll}K & 100 \quad 88\end{array}$

82

100

85

92

$84 \quad 85$

61.67

$82 \quad 77$

56

59

$\begin{array}{rr}73 & 59 \\ 100 & 87 \\ 37 & 31\end{array}$

$\begin{array}{rrr}K & 100 & 87 \\ N & 37 & 31 \\ K & 110 & 99\end{array}$

82

78

25

$17^{(\mathrm{a})}$

91

98

$\begin{array}{lll}K & 76 & 66\end{array}$

$\mathrm{K} \quad 75$

66

61

60

70

58

63

110

96

56

50

K

53

50

50

5

$\begin{array}{ll}K & 78 \\ K\end{array}$

46

46

80

46

110

89

72

$\begin{array}{rrr}K & 72 & 58 \\ K & 110 & 110\end{array}$

$\begin{array}{rrr}K & 72 & 58 \\ K & 110 & 110\end{array}$

67

92

$55 \quad 55$

97

100 
IABLE K.9. (contd)

Sediment

Treatment

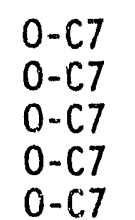

$0-c 6$

$0-C 6$

$0-C 6$

$0-C 6$

$0-C 6$

$0-C 5$

$0-C 5$

$0-C 5$

$0-C 5$

$0-C 5$

$0-C 5$

$0-C 5$

$0-C 4$

$0-C 4$

$0-C 4$

$0-c 4$

$0-C 4$

$0-\mathrm{C3}$

$0-C 3$

$0-C 3$

$0-C 3$

$0-C 3$

$0-\mathrm{Cl}$

$0-C 1$

$0-C 1$

$0-C 1$

$0-C 1$

$\mathrm{I}-\mathrm{C} 2$

$\mathrm{I}-\mathrm{C2}$

I-C2
1
2
3
4
5

1

2

3

4

$5 \quad 1$

$\begin{array}{llll}1 & & & \text { L } \\ 2 & & & \text { L } \\ 3 & & & L \\ 4 & \operatorname{Rep} 1 & L \\ 4 & \operatorname{Rep} 2 & L \\ 4 & \operatorname{Rep} 3 & L \\ 5 & & & L\end{array}$

1
2
3
4
5

1
2
3
4
5

1
2
3
4
5

1 Rep 1

1 Rep 2

$1 \operatorname{Rep} 3$
Surrogate Percent Recoveries Naphtha- Acenaph- Phenan- Benzo(a)
lene thene threne AnthraD8 D10 D10 cene D12

$\begin{array}{rrrr}120 & 120 & 87 & 71 \\ 110 & 110 & 86 & 76 \\ 120 & 120 & 89 & 79 \\ 36 & 33 & 25 & 25 \\ 88 & 95 & 77 & 69\end{array}$

$\begin{array}{rrrr}110 & 110 & 83 & 89 \\ 120 & 110 & 84 & 80 \\ 70 & 77 & 55 & 66 \\ 26 & 24 & 18 & 18 \\ 24 & 22 & 16 & 17\end{array}$

$\begin{array}{llll}31 & 27 & 21 & 17\end{array}$

$\begin{array}{llll}120 & 110 & 81 & 77\end{array}$

$\begin{array}{llll}32 & 28 & 22 & 23\end{array}$

$\begin{array}{llll}40 & 36 & 27 & 26\end{array}$

$\begin{array}{llll}40 & 37 & 28 & 24\end{array}$

$\begin{array}{rrrr}110 & 120 & 92 & 92\end{array}$

$\begin{array}{lllll}43 & 40 & 31 & 24\end{array}$

$\begin{array}{llll}100 & 100 & 81 & 78\end{array}$

$\begin{array}{llll}110 & 120 & 90 & 82\end{array}$

$\begin{array}{llll}95 & 96 & 74 & 71\end{array}$

$\begin{array}{llll}110 & 100 & 76 & 64\end{array}$

$\begin{array}{llll}73 & 70 & 52 & 62\end{array}$

$\begin{array}{llll}37 & 34 & 26 & 25\end{array}$

$\begin{array}{llll}43 & 39 & 30 & 23\end{array}$

$\begin{array}{llll}38 & 36 & 29 & 26\end{array}$

$\begin{array}{llll}53 & 47 & 38 & 21 \\ 14^{(a)} & 14^{(a)} & 13^{(a)} & 32\end{array}$

$29^{(a)} \quad 26 \quad 20 \quad 22$

$\begin{array}{llll}9 & 42 & 42 & 23\end{array}$

$\begin{array}{llll}30 & 30 & 21 & 21\end{array}$

$\begin{array}{llll}45 & 42 & 33 & 33\end{array}$

$\begin{array}{llll}48 & 44 & 33 & 32\end{array}$

$\begin{array}{llll}100 & 97 & 72 & 54\end{array}$

$\begin{array}{llll}110 & 110 & 78 & 73\end{array}$

$\begin{array}{llll}97 & 94 & 73 & 73\end{array}$


TABLE K.9. (contd)

Sediment
Treatment

\begin{tabular}{|c|c|c|c|c|c|}
\hline \multirow[b]{2}{*}{ Replicate } & \multirow[b]{2}{*}{ Batch } & \multicolumn{4}{|c|}{ Surrogate Percent Recoveries } \\
\hline & & $\begin{array}{c}\text { Naphtha- } \\
\text { lene } \\
\text { D8 } \\
\end{array}$ & $\begin{array}{l}\text { Acenaph- } \\
\text { thene } \\
\text { D10 } \\
\end{array}$ & $\begin{array}{l}\text { Phenan- } \\
\text { threne } \\
\text { D10 } \\
\end{array}$ & $\begin{array}{l}\text { Benzo(a) } \\
\text { Anthra- } \\
\text { cene D12 }\end{array}$ \\
\hline $\begin{array}{l}2 \\
3 \\
4 \\
5\end{array}$ & $\begin{array}{l}M \\
M \\
M \\
M\end{array}$ & $\begin{array}{l}79 \\
83 \\
76 \\
69\end{array}$ & $\begin{array}{l}76 \\
82 \\
69 \\
67\end{array}$ & $\begin{array}{l}54 \\
59 \\
53 \\
50\end{array}$ & $\begin{array}{l}51 \\
59 \\
35 \\
42\end{array}$ \\
\hline $\begin{array}{l}1 \\
2 \\
3 \\
4 \\
5\end{array}$ & $\begin{array}{l}M \\
M \\
M \\
M \\
M\end{array}$ & $\begin{array}{r}87 \\
79 \\
83 \\
120 \\
120\end{array}$ & $\begin{array}{r}84 \\
76 \\
93 \\
120 \\
120\end{array}$ & $\begin{array}{l}61 \\
58 \\
68 \\
86 \\
86\end{array}$ & $\begin{array}{l}51 \\
52 \\
53 \\
75 \\
73\end{array}$ \\
\hline $\begin{array}{l}1 \\
2 \\
3 \\
4 \\
5\end{array}$ & $\begin{array}{l}M \\
M \\
M \\
M \\
M\end{array}$ & $\begin{array}{r}120 \\
100 \\
98 \\
98 \\
63\end{array}$ & $\begin{array}{r}110 \\
97 \\
92 \\
96 \\
60\end{array}$ & $\begin{array}{l}81 \\
70 \\
68 \\
73 \\
43\end{array}$ & $\begin{array}{l}74 \\
55 \\
52 \\
65 \\
33\end{array}$ \\
\hline $\begin{array}{l}1 \\
2 \\
3 \\
4 \\
5\end{array}$ & $\begin{array}{l}M \\
M \\
M \\
M \\
M\end{array}$ & $\begin{array}{r}120 \\
91 \\
110 \\
86 \\
86\end{array}$ & $\begin{array}{r}120 \\
85 \\
100 \\
81 \\
78\end{array}$ & $\begin{array}{l}89 \\
61 \\
74 \\
60 \\
59\end{array}$ & $\begin{array}{l}57 \\
55 \\
61 \\
43 \\
39\end{array}$ \\
\hline $\begin{array}{l}1 \\
2 \\
3 \\
4 \\
5\end{array}$ & $\begin{array}{l}\mathrm{J} \\
\mathrm{J} \\
\mathrm{J} \\
\mathrm{J} \\
\mathrm{J}\end{array}$ & $\begin{array}{l}92 \\
71 \\
63 \\
72 \\
81\end{array}$ & $\begin{array}{r}100 \\
94 \\
84 \\
92 \\
94\end{array}$ & $\begin{array}{r}110 \\
110 \\
99 \\
110 \\
110\end{array}$ & $\begin{array}{r}97 \\
100 \\
81 \\
100 \\
95\end{array}$ \\
\hline $\begin{array}{lll}1 & & \\
2 & \operatorname{Rep} & 1 \\
2 & \operatorname{Rep} & 2 \\
2 & \operatorname{Rep} & 3 \\
3 & & \\
4 & & \\
5 & \end{array}$ & $\begin{array}{l}\mathrm{J} \\
\mathrm{J} \\
\mathrm{J} \\
\mathrm{J} \\
\mathrm{J} \\
\mathrm{J} \\
\mathrm{J}\end{array}$ & $\begin{array}{l}65 \\
40 \\
37 \\
81 \\
80 \\
84 \\
13^{(a)}\end{array}$ & $\begin{array}{r}75 \\
44 \\
42 \\
98 \\
100 \\
100 \\
35\end{array}$ & $\begin{array}{r}88 \\
53 \\
51 \\
110 \\
120 \\
120 \\
63\end{array}$ & $\begin{array}{l}67 \\
42 \\
42 \\
97 \\
98 \\
96 \\
8^{(a)}\end{array}$ \\
\hline $\begin{array}{l}1 \\
2 \\
3 \\
4 \\
5\end{array}$ & $\begin{array}{l}\text { I } \\
\text { I } \\
\text { I } \\
\text { I } \\
\text { I }\end{array}$ & $\begin{array}{l}63 \\
74 \\
84 \\
79 \\
77\end{array}$ & $\begin{array}{l}68 \\
87 \\
94 \\
87 \\
93\end{array}$ & $\begin{array}{r}72 \\
96 \\
100 \\
110 \\
100\end{array}$ & $\begin{array}{l}84 \\
92 \\
88 \\
79 \\
92\end{array}$ \\
\hline
\end{tabular}


IABLE K.9. (contd)

Sediment

Ireatment Replicate Batch

$R-A M$

$R-A M$

$\mathrm{R}-\mathrm{AM}$

$R-A M$

$\mathrm{R}-\mathrm{AM}$

$R-B F$

$R-B F$

$R-B F$

$R-B F$

$R-B F$

$R-B F$

$R-B F$

R-OS

$R-O S$

$\mathrm{R}-\mathrm{OS}$

$R-O S$

R-OS

$R-P C$

$R-P C$

$R-P C$

$R-P C$

$R-P C$

$R \sim P F$

$R-P F$

$R-P F$

$R-P F$

$R-P F$

$C \sim S B$

C-SB

$C-S B$

$C-S B$

$C-S B$

C-SB
1

2

3

4

$1 \quad H$

2

3 Rep 1

3 Rep 2

3 Rep 3

4
5

5

1

2

3

4

5 I

$\begin{array}{ll}1 & \text { I } \\ 2 & \text { I } \\ 3 & \text { I } \\ 4 & \text { I } \\ 5 & \text { I }\end{array}$

$1 \quad H$

2

3

4

5

$\begin{array}{llll}1 & & & \text { I } \\ 2 & & & \text { I } \\ 3 & & & \text { I } \\ 4 & \operatorname{Rep~} 1 & & \text { I } \\ 4 & \operatorname{Rep~2} & & \text { I } \\ 5 & & & \text { I }\end{array}$

H

H

H
Surrogate Percent Recoveries

Naphtha- Acenaph- Phenan- Benzo(a)
$08 \quad 010 \quad 010$ cene 012

86

$0^{(b)}$

89

32

92

85
76

82

84

87

$\begin{array}{ll}98 & 95 \\ 72 & 74\end{array}$

$100 \quad 100$

$93 \quad 97$

76

83

48

46

83

65

74

86

82

62

51

89

72

87

98

97

92

83

$14^{(a)}$

58

72

100

96

93

$91 \quad 88$

$74 \quad 76$

$54 \quad 45^{(a)}$

$97 \quad 86$

$79 \quad 65$

$96 \quad 79$

60

70

76

.74

77

91

32

76

81

110

92

100

40

84

97

75

79

86

83

88

89

93

98

95

100

87

65

91

86

84

99

73

99

97

100

81

85

98

86

92

90

94

97

110

100

100

100

81 
TABLE K.9. (contd)

Sediment

Treatment

Replicate Batch

Surrogate Percent Recoveries

\begin{tabular}{cccc}
\hline $\begin{array}{c}\text { Naphtha- } \\
\text { Tene } \\
\text { Tenaph- } \\
\text { thene }\end{array}$ & $\begin{array}{c}\text { Phenan- } \\
\text { threne }\end{array}$ & $\begin{array}{c}\text { Benzo(a) } \\
\text { Anthra- }\end{array}$ \\
08 & 010 & 010 & cene 012 \\
\hline
\end{tabular}

C-NE

$C-N E$

$C-N E$

$C-N E$

$C-N E$

$1 \quad H$

H

45

57

85

69

57

52 (a)

3
4
5

H

H

66

$38^{(a)}$

65
94
74
75
68

65

73

69

69

61

(a) Surrogate recovery below target quality control range.

Inadequate sample mass to re-extract.

(b) Quantitation surrogate recoveries greater than $20 \%$, QC passed. 


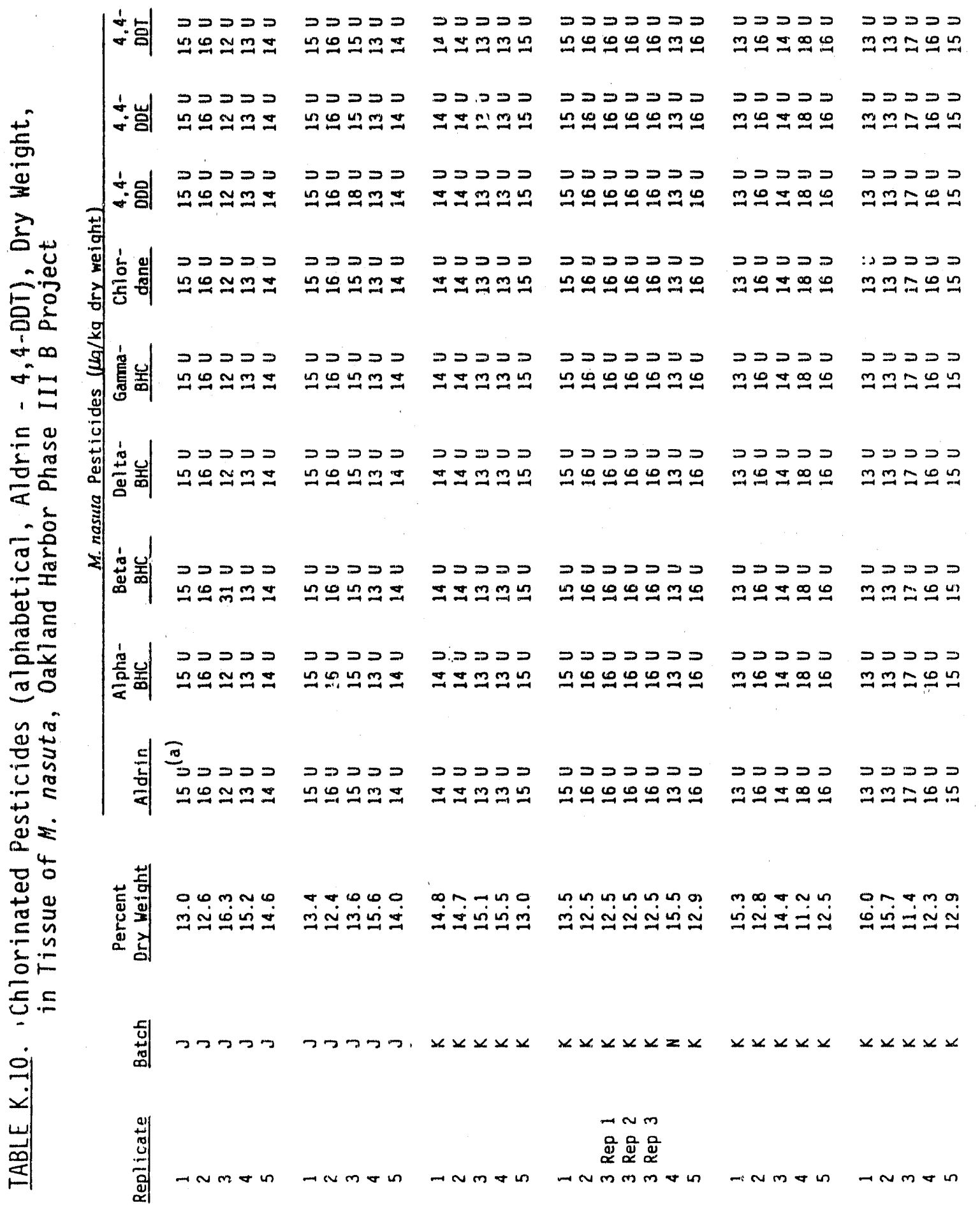

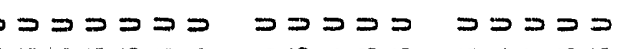

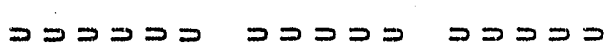

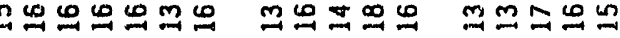

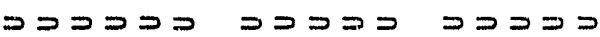

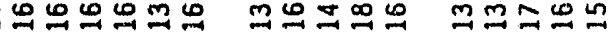

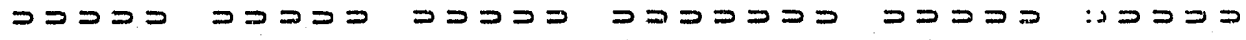
뜨믐

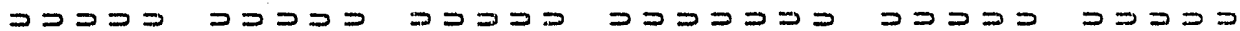
뜨묨

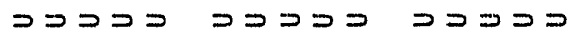

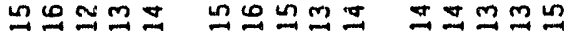

フココつつつつ

つコつコつコつつつ

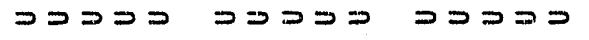

$\neg コ コ コ コ コ つ$

コココココココココ

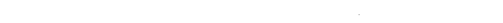

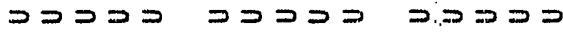

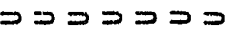

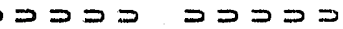

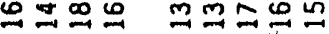

1

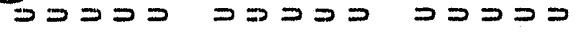

ココココロココ

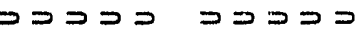

$\underline{m}=0$ 


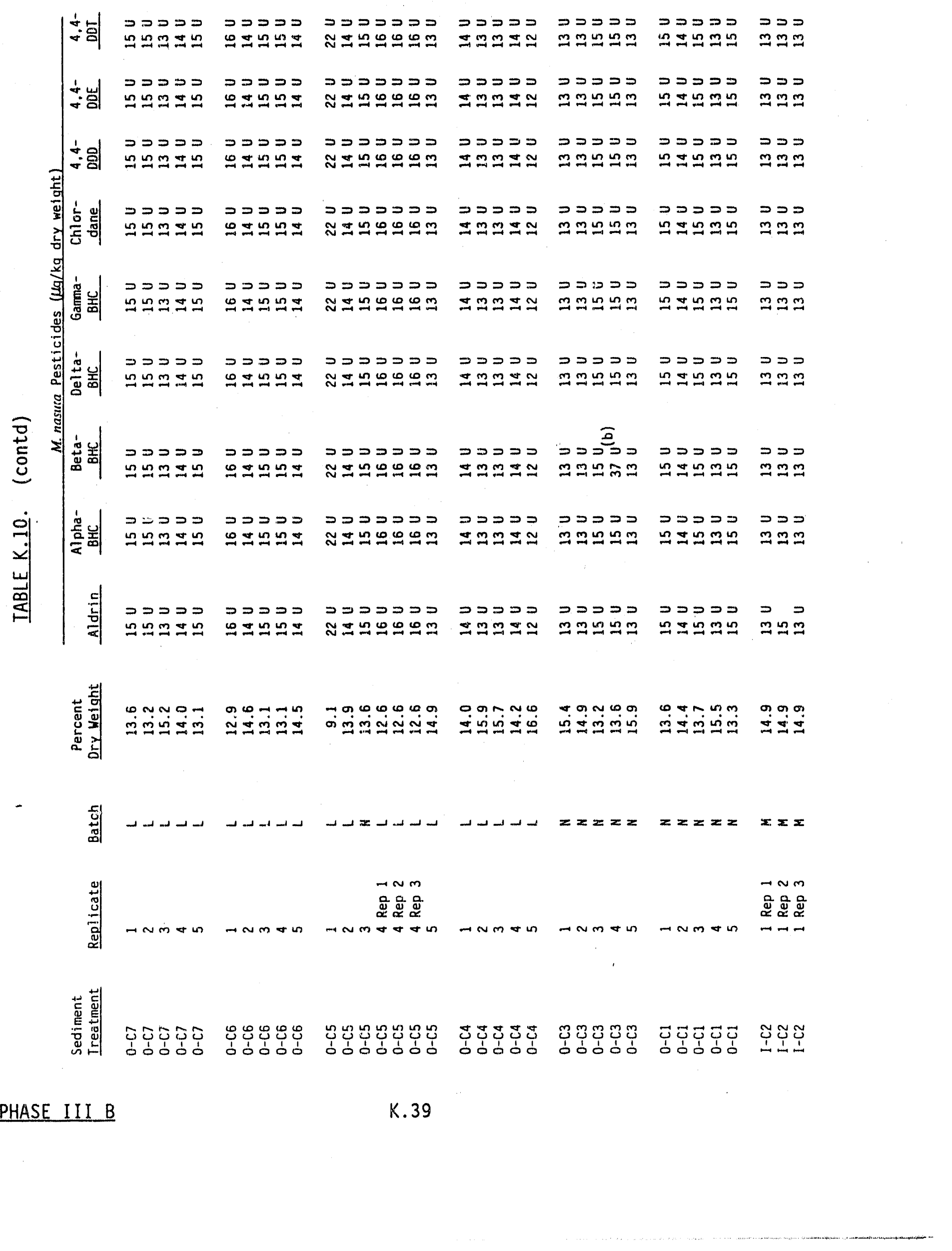


$\Rightarrow \operatorname{lom}^{2}$

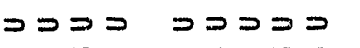

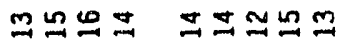

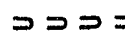

m요요

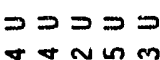

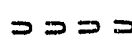

$m \stackrel{9}{2}$

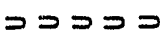

포픔

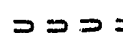

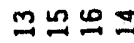

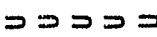

페뇨

$\supset \supset \supset$ $\because 20$

๖コつつ

车 뜬

$\supset \supset$ $m \underset{\sim}{m}$

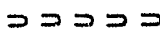

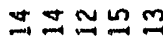

$\supset \supset \supset \supset \supset$

$m \sim m m m$

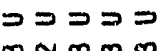

$\underline{a} \boldsymbol{\sim} \boldsymbol{m} \boldsymbol{m}$

$\supset \supset \supset \supset$

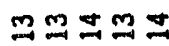

$\neg ロ コ ロ コ$

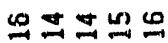

フコココロココ

부ㅂㅜㅛ

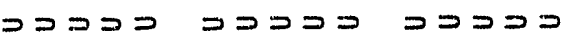

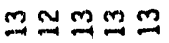

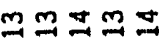

본

ココココロ

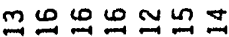

$\supset \supset \supset$

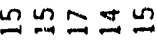

\section{ココつつ

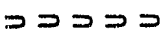

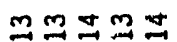

모요

フコココココ

똔묘

$\supset \supset \supset \supset$ nㅡㄴ

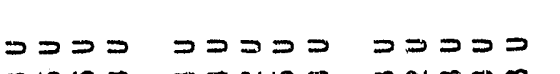

$\stackrel{m}{\rightarrow \infty} \oplus$

$\pm \pm N m$

$\neg \supset \supset \supset$ DDD

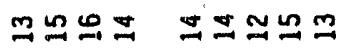

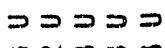

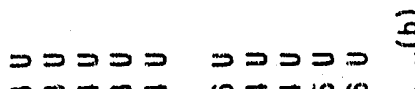

$\underline{a}$

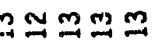

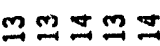

电步出吕

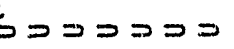

"ె்

$\supset \supset \supset \supset つ$

กะニะณ

$\neg \supset \supset \supset$ จ $⿻ コ$

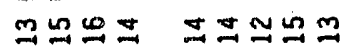

$\supset \supset \supset \supset$

จコココ

$\supset \supset \supset \supset \supset$

วココว ว

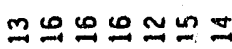

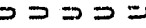

๓กエะ

救

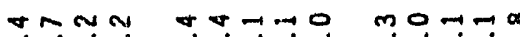

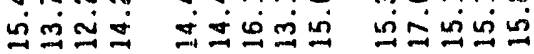

mom

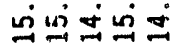

$\infty 0-1$ in $\infty$

of

๖コココロコつ

m요문

$\supset \supset \supset \supset$

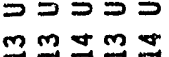

$00 \operatorname{tin}$

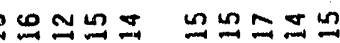

કี

$\sum \sum \sum \sum \Sigma \Sigma \Sigma \Sigma$

$\Sigma \Sigma x \geq$

$x \Sigma x \geq x$

つつつつ

Hnm

웜웜웜

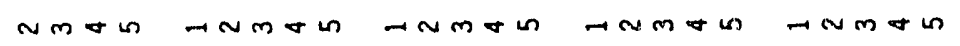

tannmath thmon

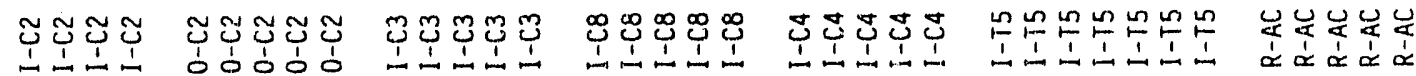




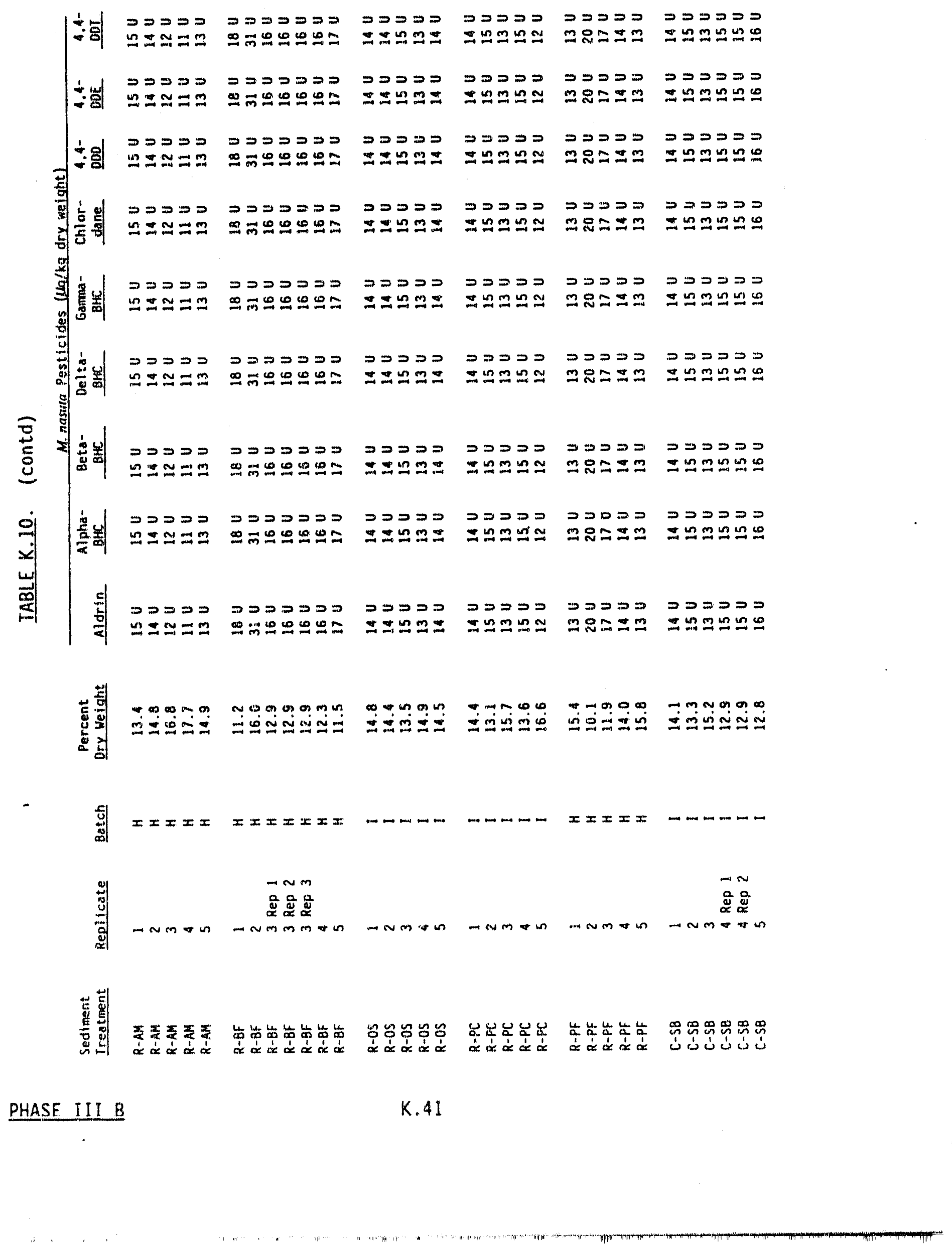




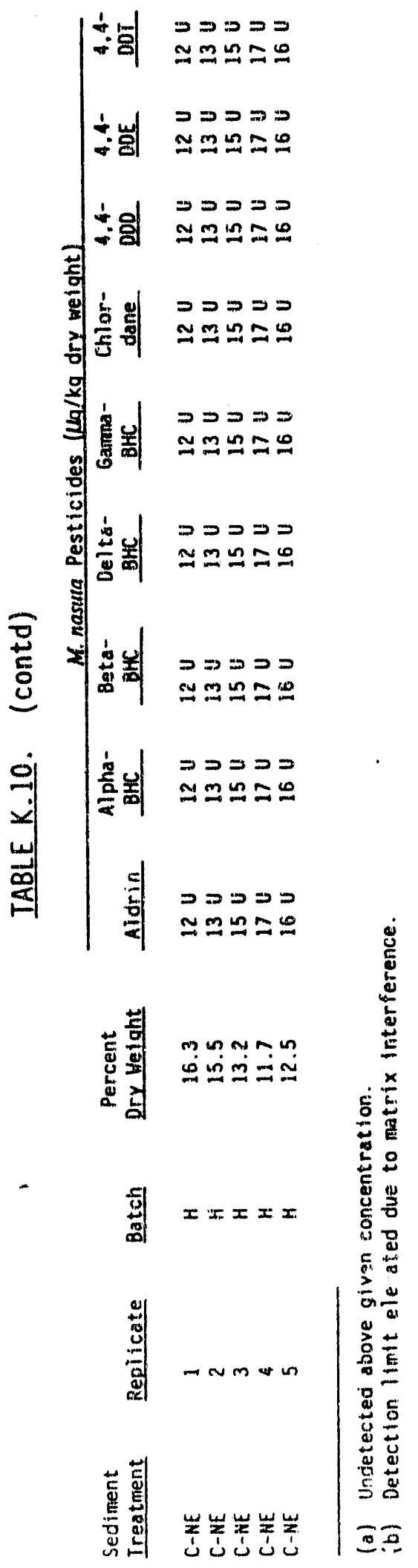

PHASE III B 


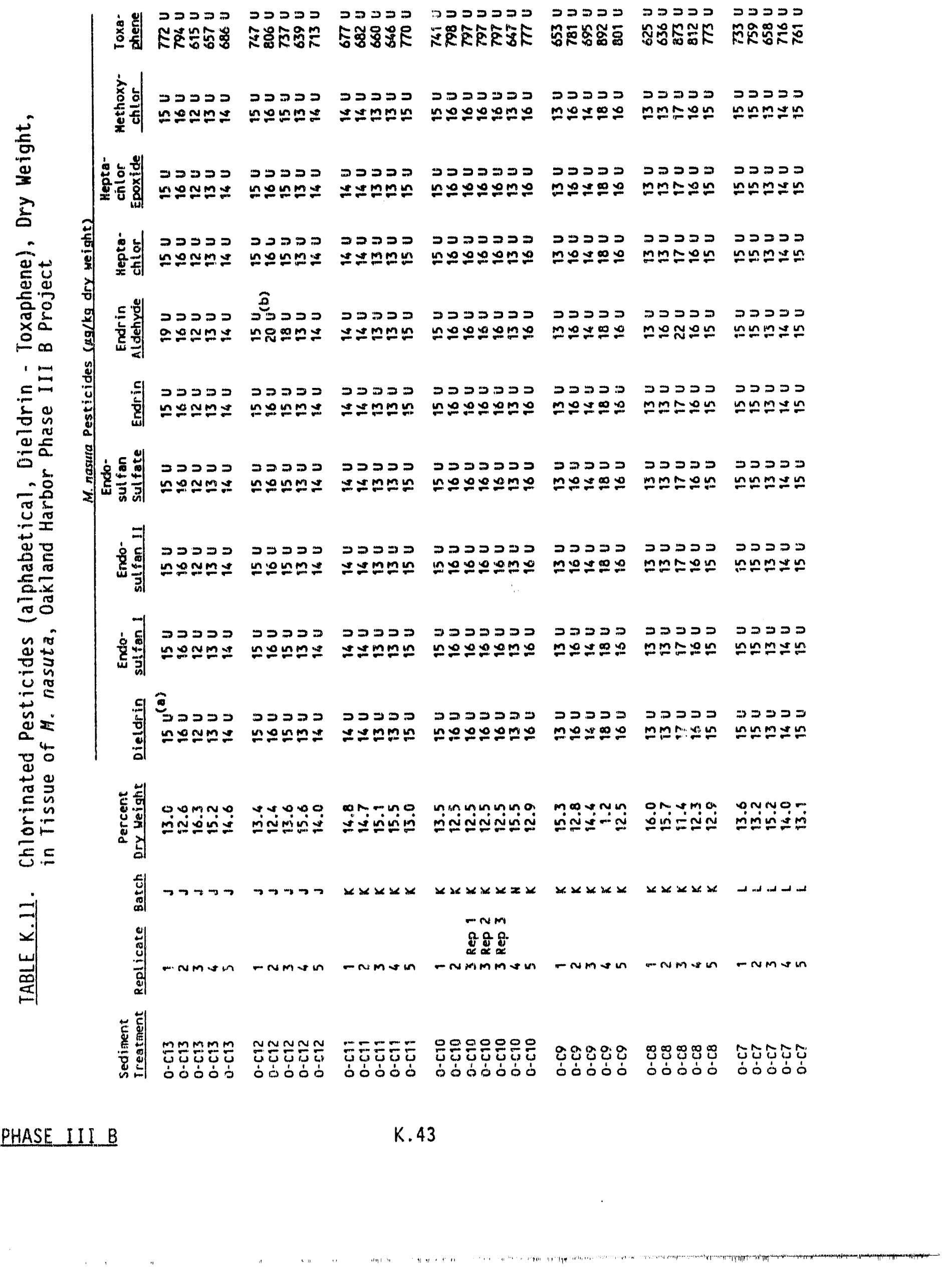




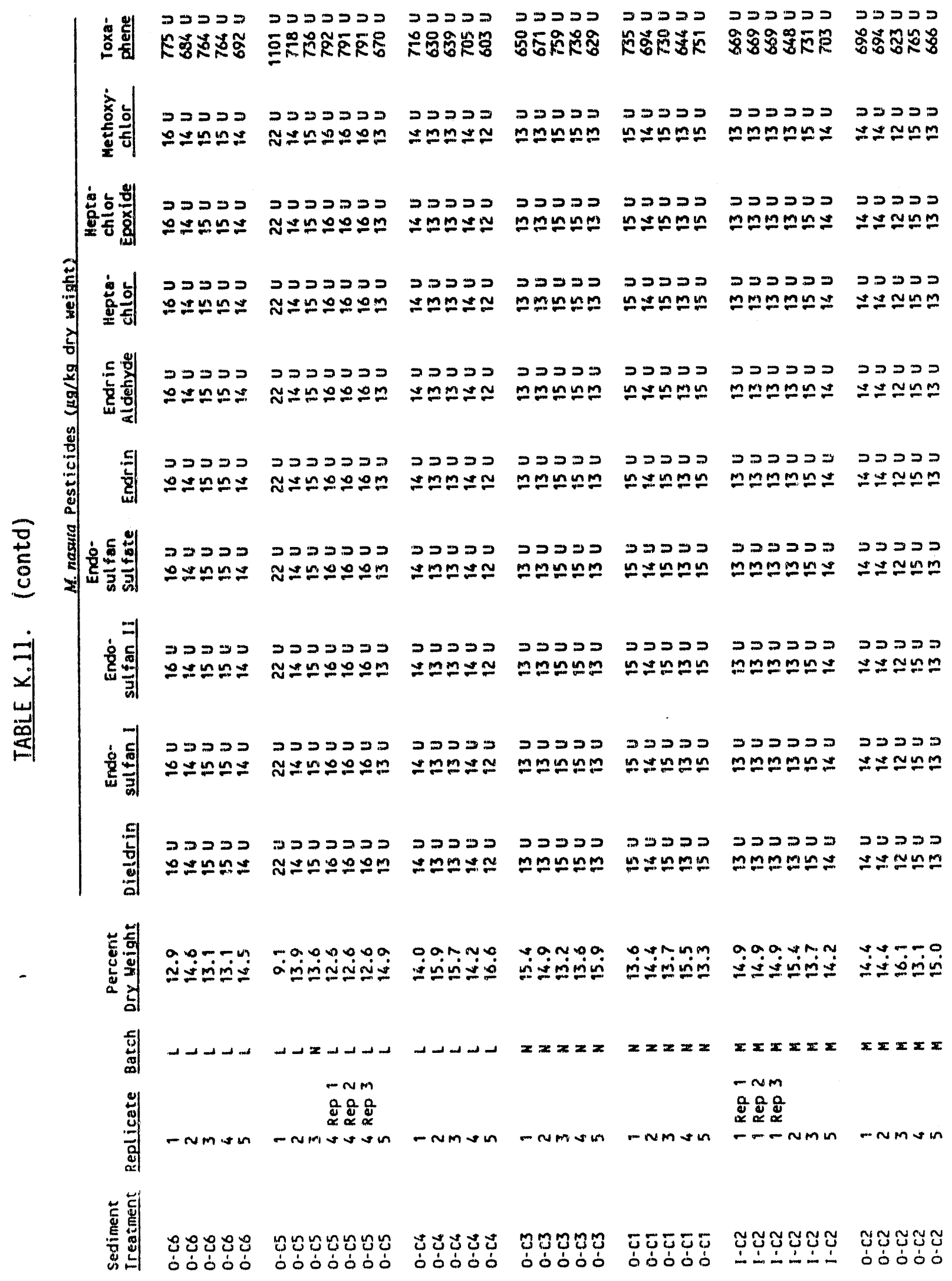




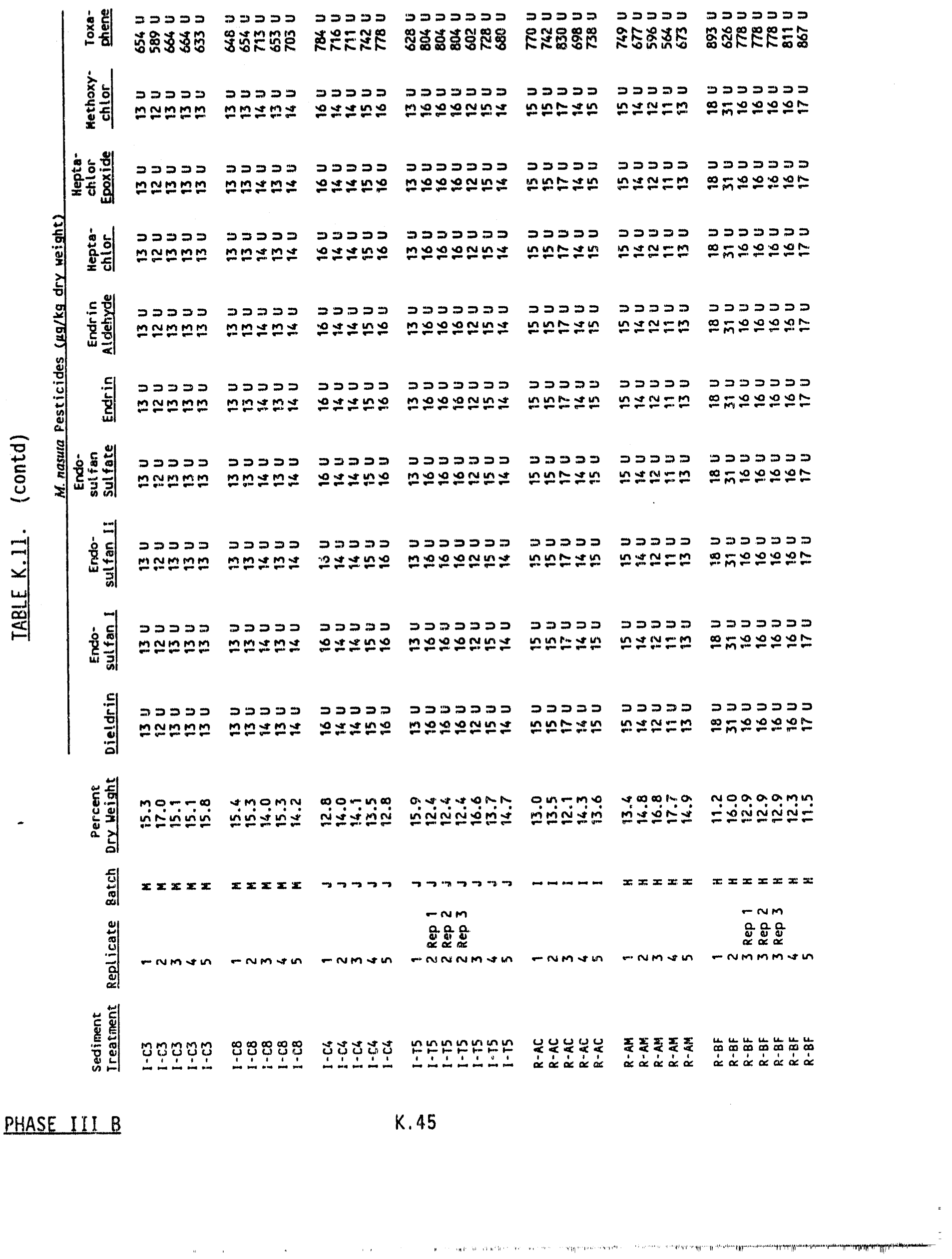




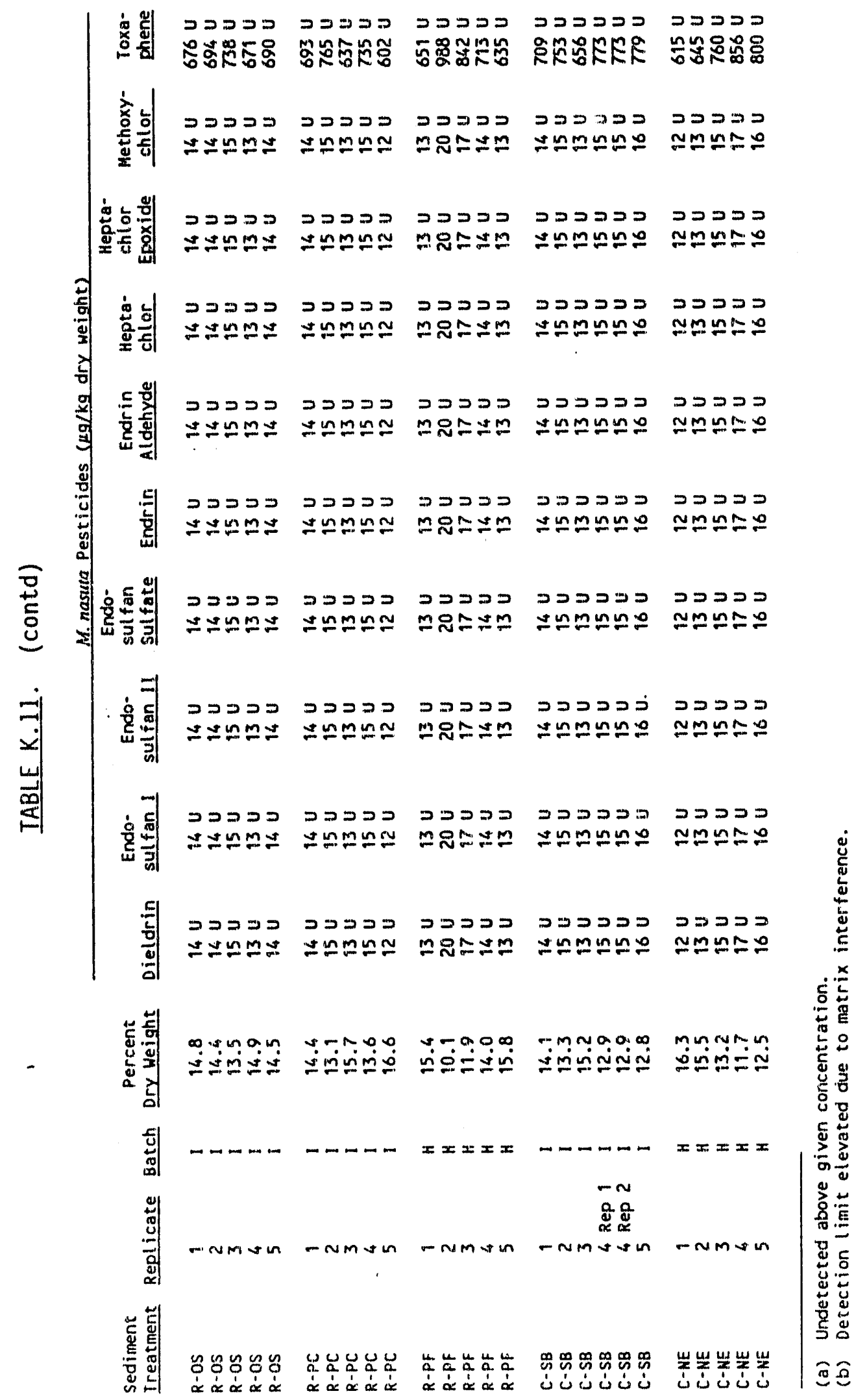




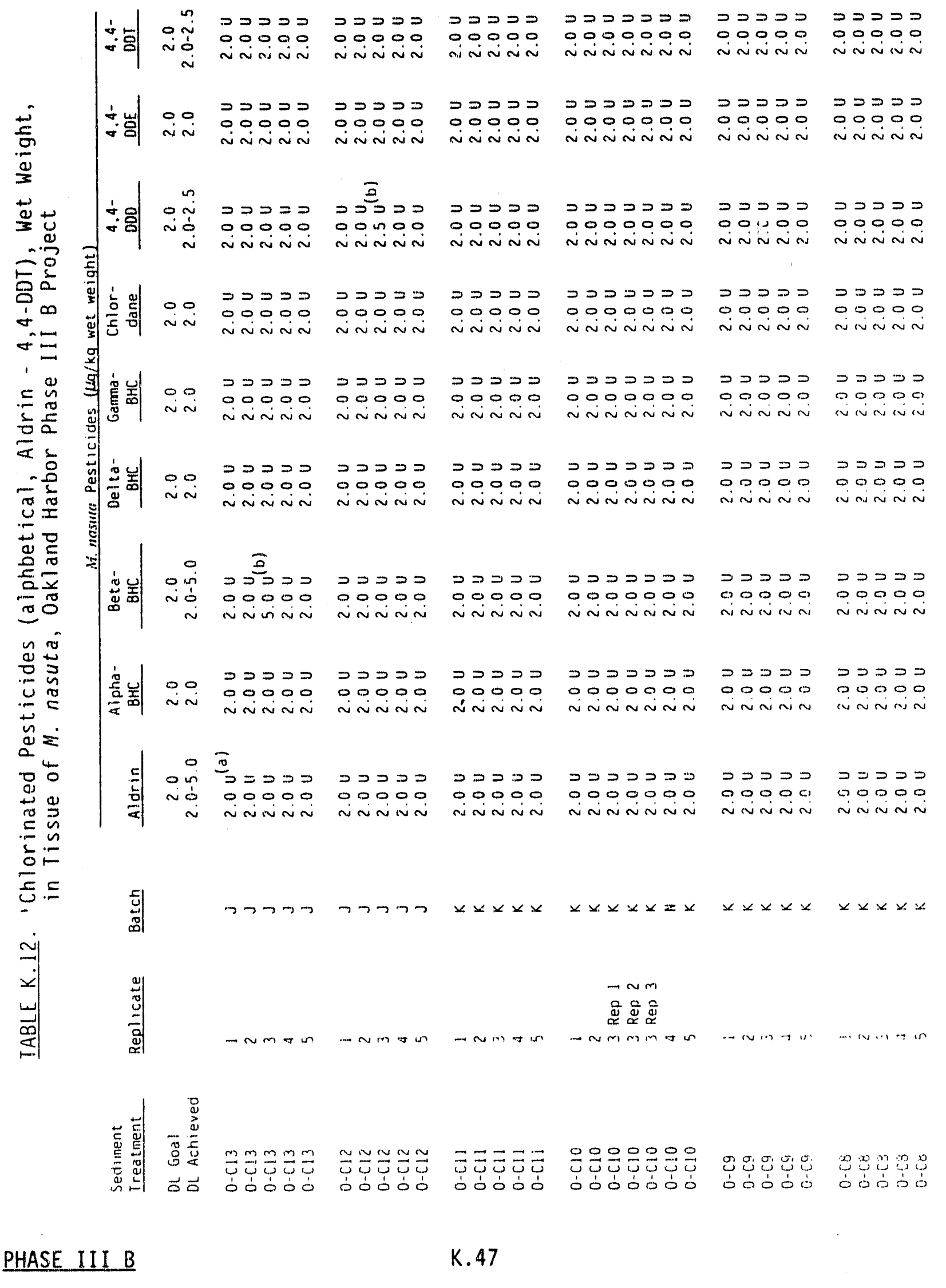




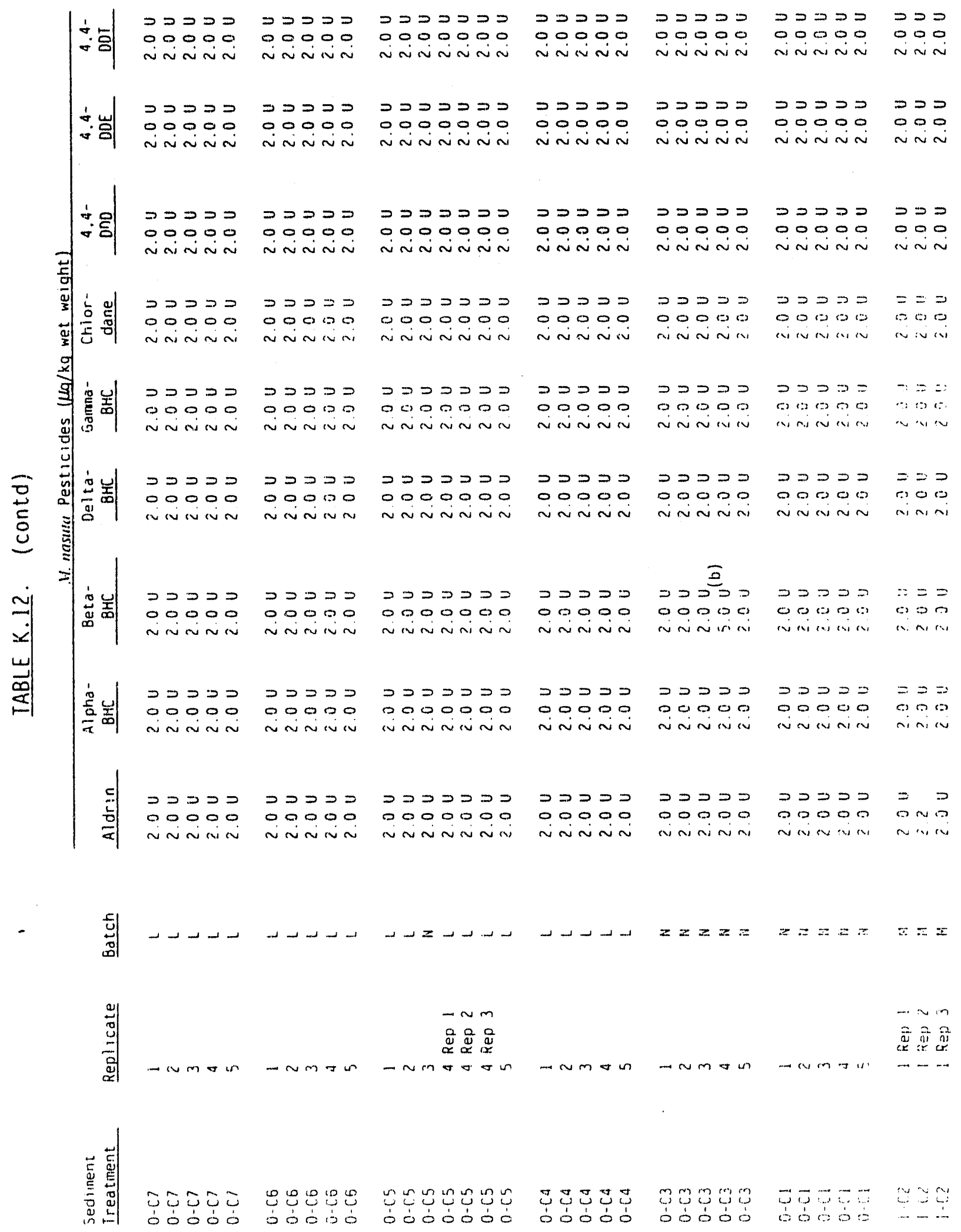

PHASE III B 


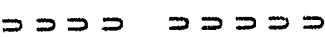
000000000 Nini Ninivi

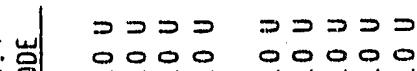

inin ainini

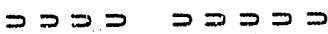

0000 0.0000

viñ viññ

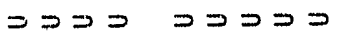

O000 00000

viñ inivin

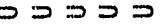

00000

ininin

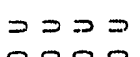

viniv

$\supset \supset \supset \supset$

00000

$\supset \supset \supset \supset \supset$

c) 0000

$\sim N N \sim N$

Niñ

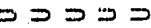

00000

nN $\sim N$

$\supset \supset \supset \supset$

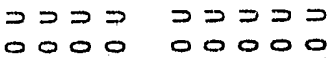

inivinininin

00000

vivini

$\supset \supset \supset \supset \supset$

00000

ininin

$\Rightarrow \supset \supset \supset \supset$ 00000 Ninis

ocoso

ก $\sim \sim N$

つコココ 00000 ininin

$\supset \supset \supset \supset$ 00000 ininin

$\supset \supset \supset \supseteq$ 00000 จ $\sim \sim \sim$

$>\supset \supset \supset \supset$ 00000 N N N

$\Rightarrow D=0 D=$

00.0000

NiniñN

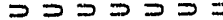
0000000 viñNi

$D \supset \supset \supset$ 00000 ninini

$\supset \supset \supset \supset \supset \supset$ 0000000 inininin

$\supset \supset コ \supset$ 00000 vinin

$\supset \triangleright \supset \supset \supset \supset$ 0000000 ง $\sim \sim N \sim \sim$

$\supset \supset \supset \supset \supset$ 00000 N $\sim \sim \sim$

จフココว 0000000 nivinin

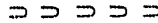
00000 vinivin

$\supset \supset \supset \supset \supset \supset \supset$ 0000000 जininin

$\supset \supset \supset \supset$ 00000 Niñni

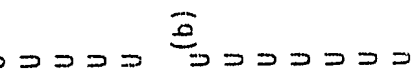
00000 0000000 Niñ viñ $\sim \sim \sim$

$\supset \supset \supset \supset$ on 000 N $\sim \sim N$

$\supset \supset \supset \supset \supset$ 00000 Nivin

$\supset \supset \supset \supset \supset \supset$ 0000000

$\supset \supset \supset \supset$ s0000 00000 o 0000 inini Nivin inini in i 


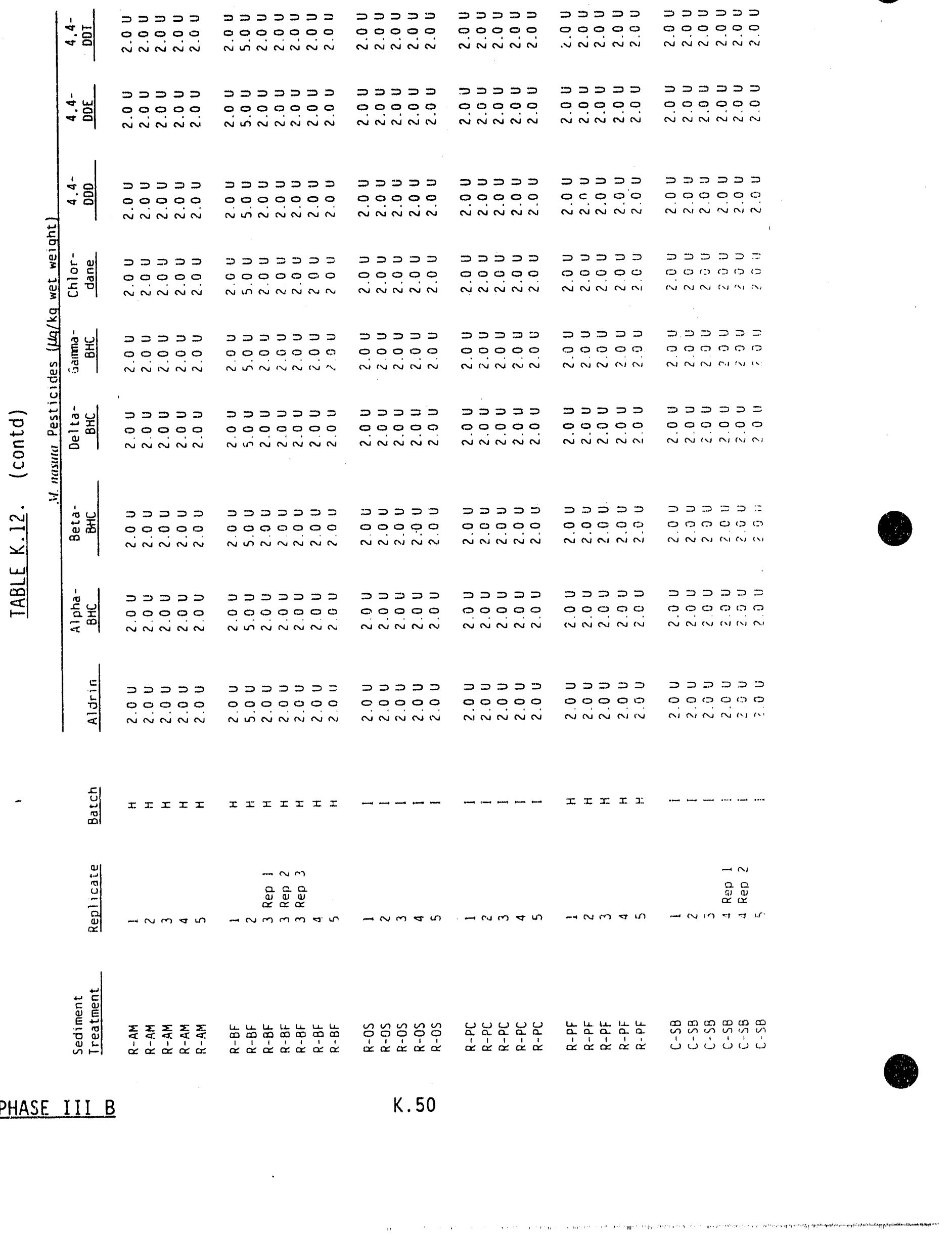




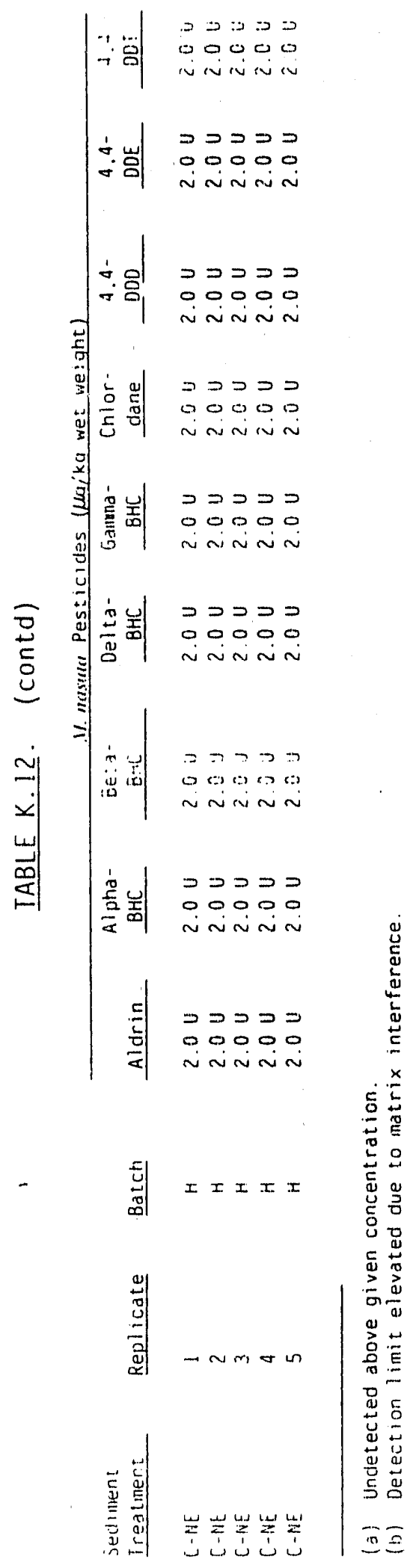

PHASE III B 


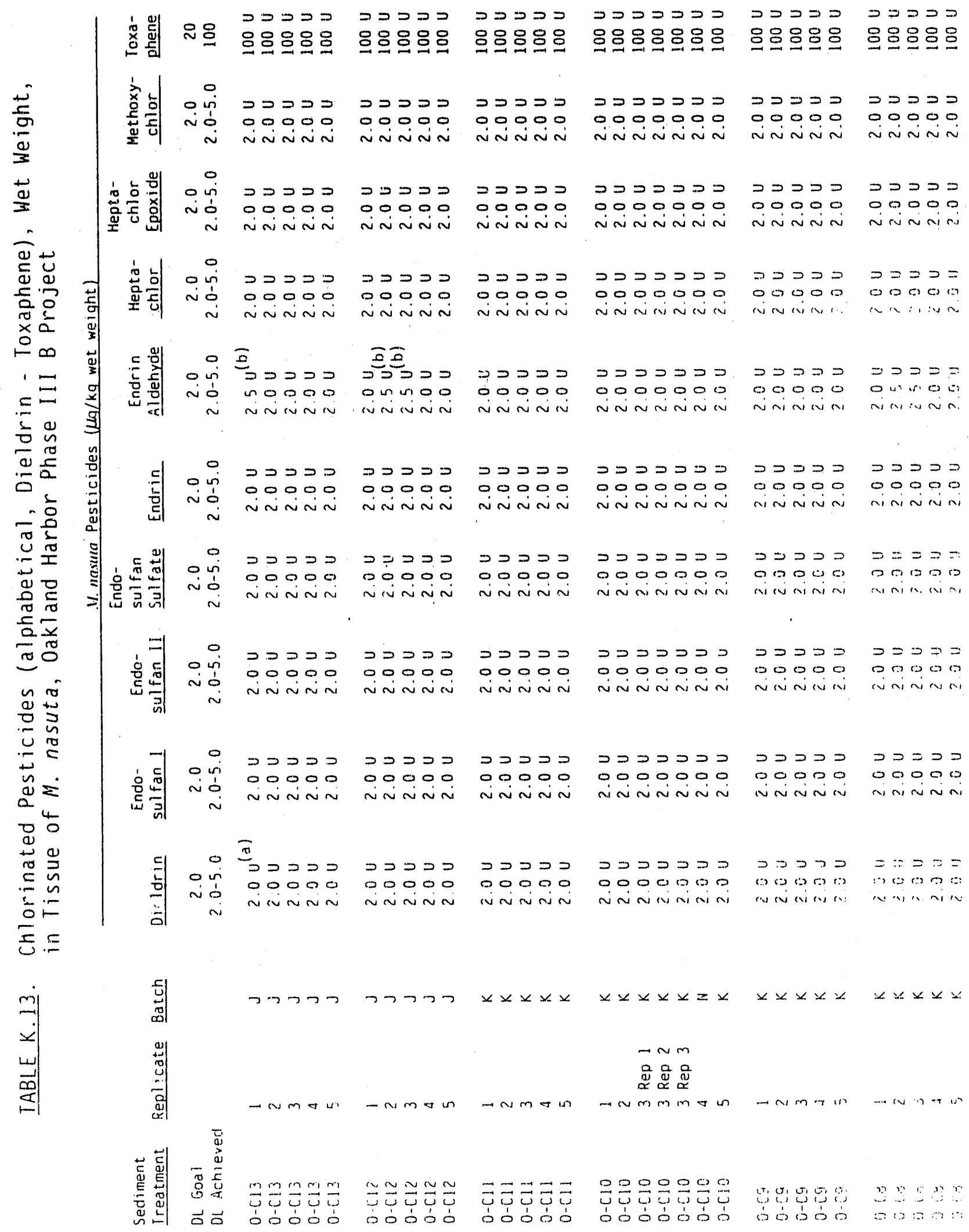




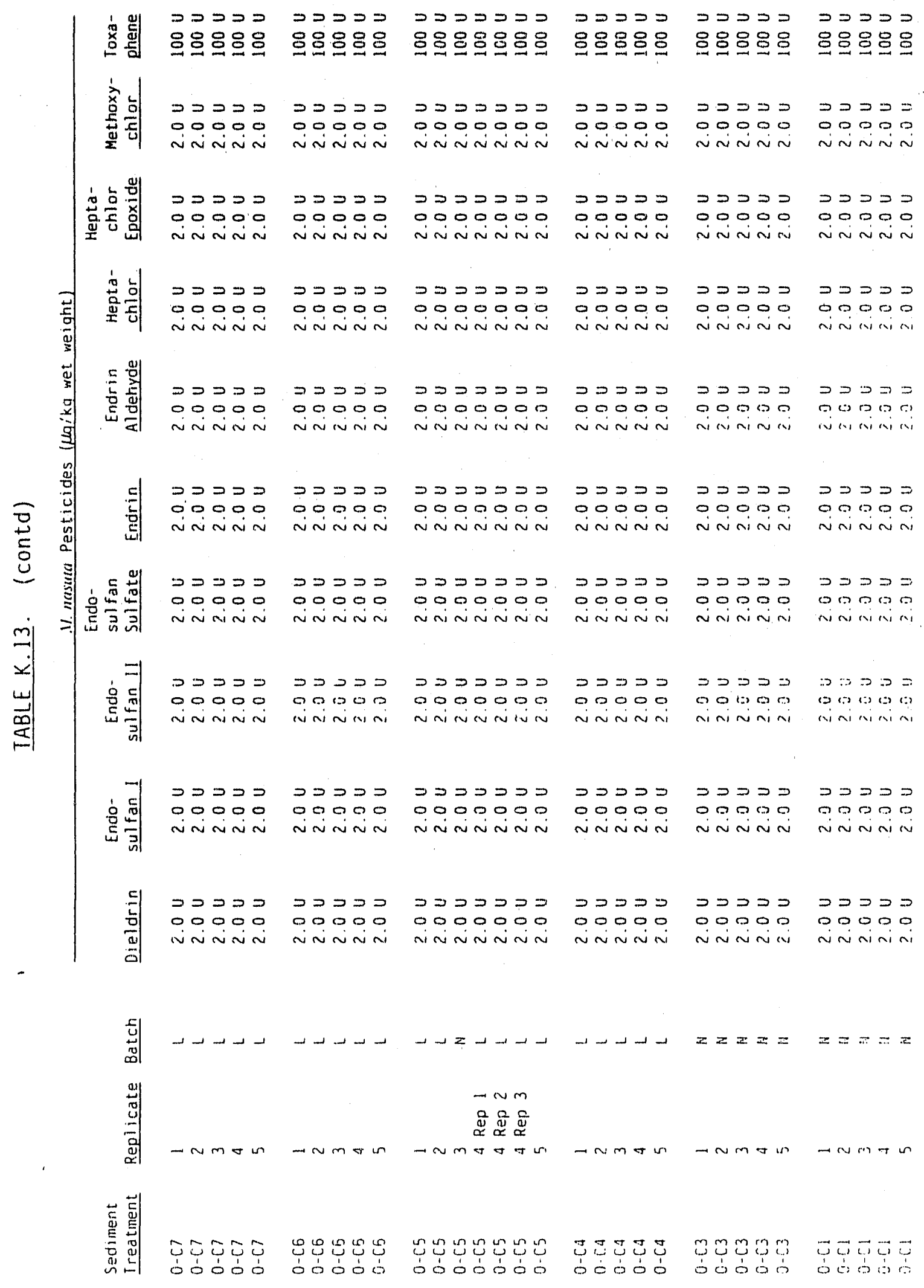




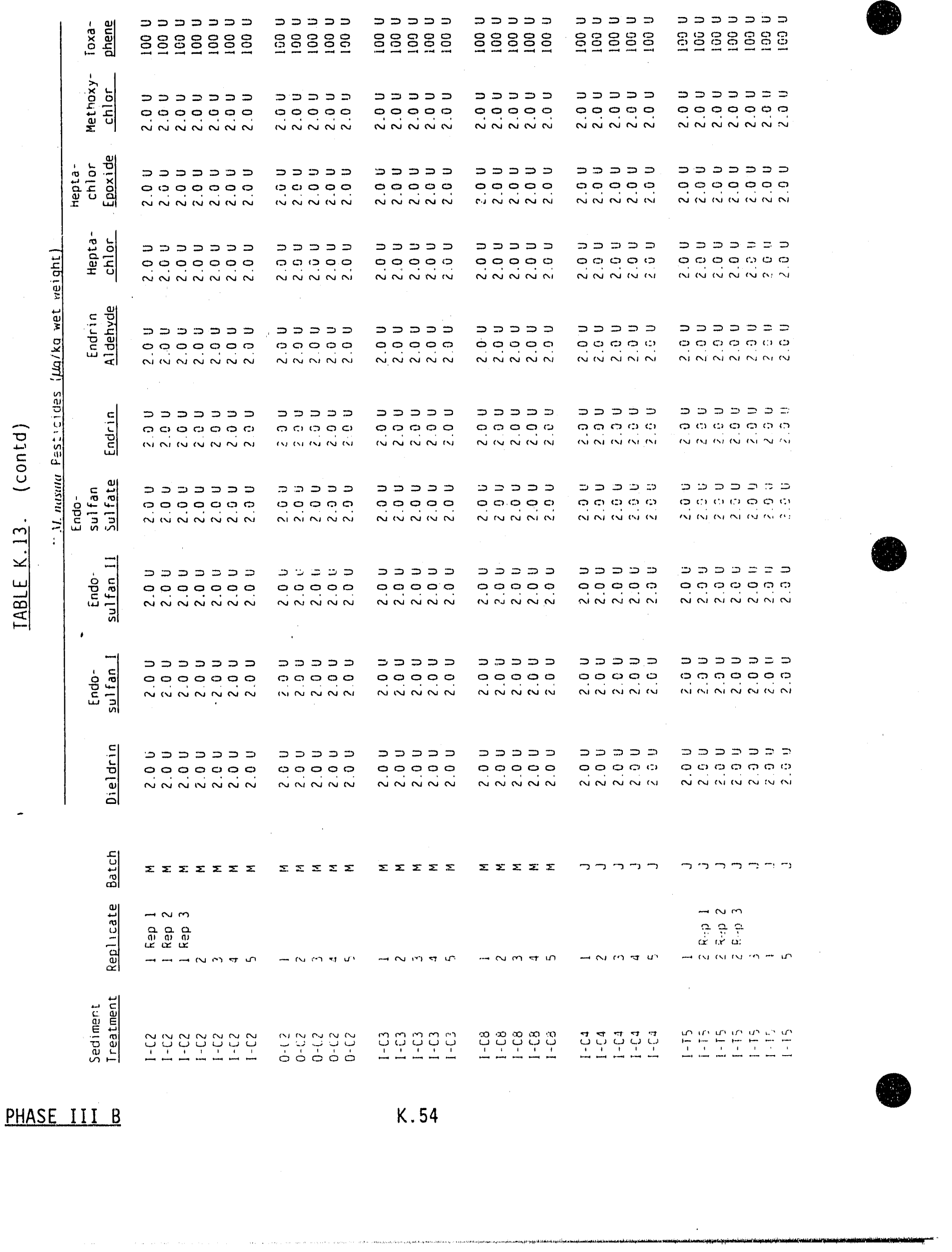




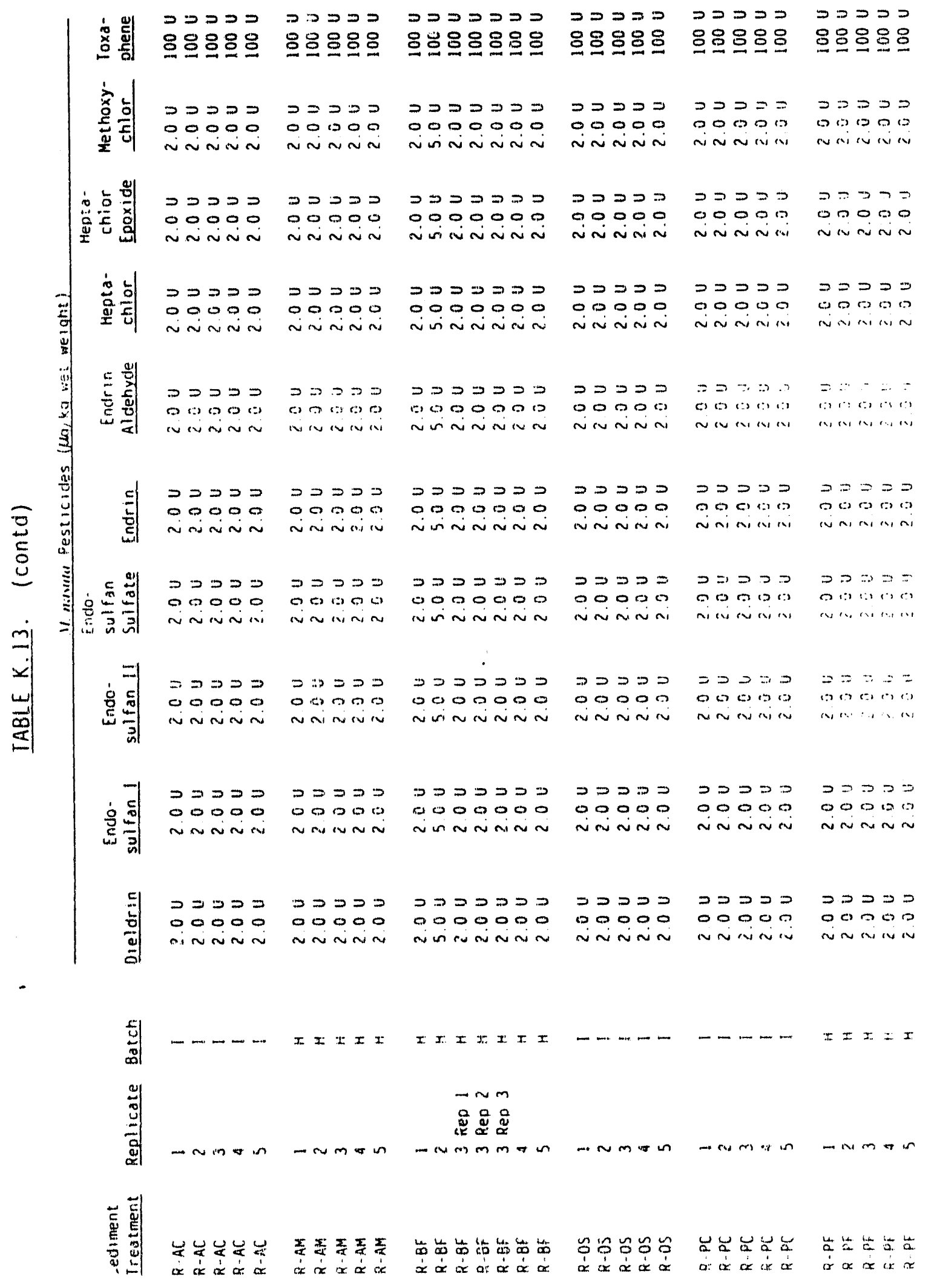




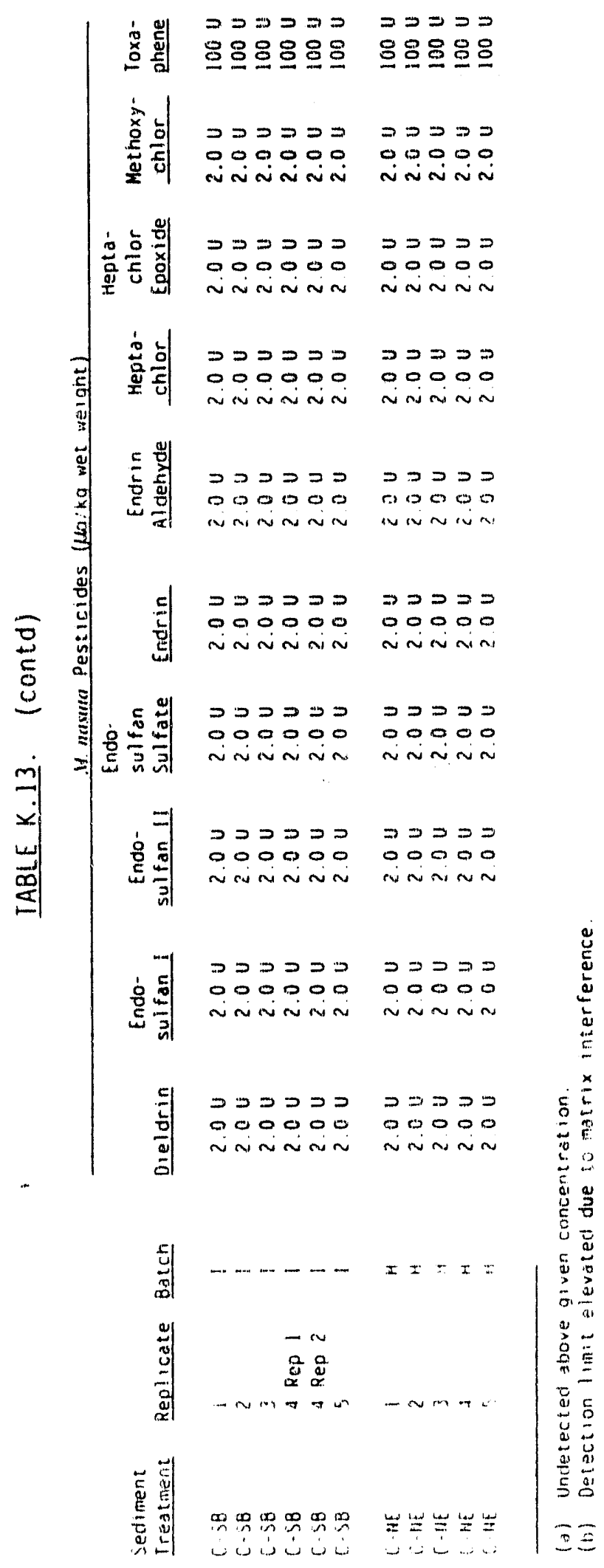




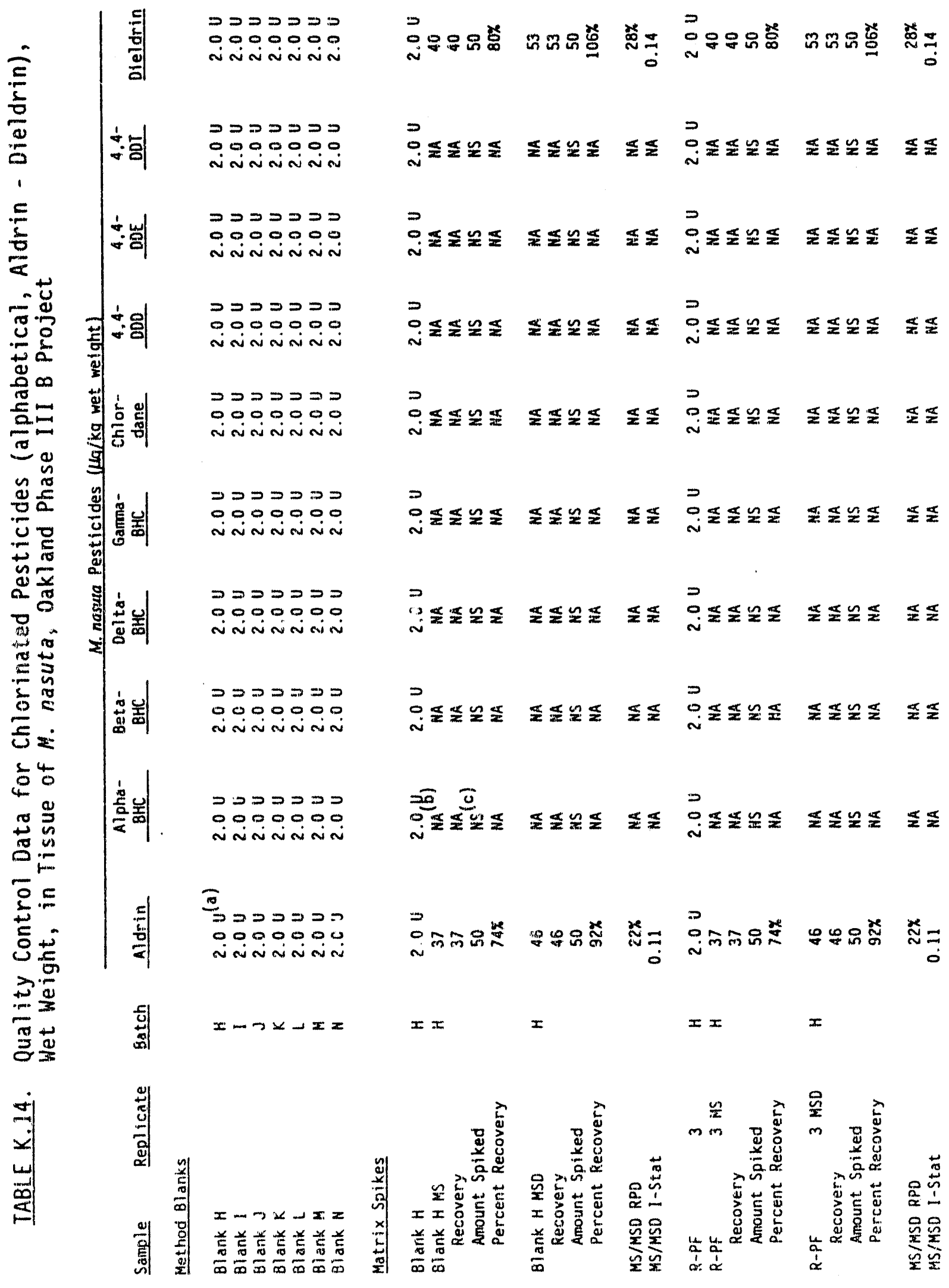




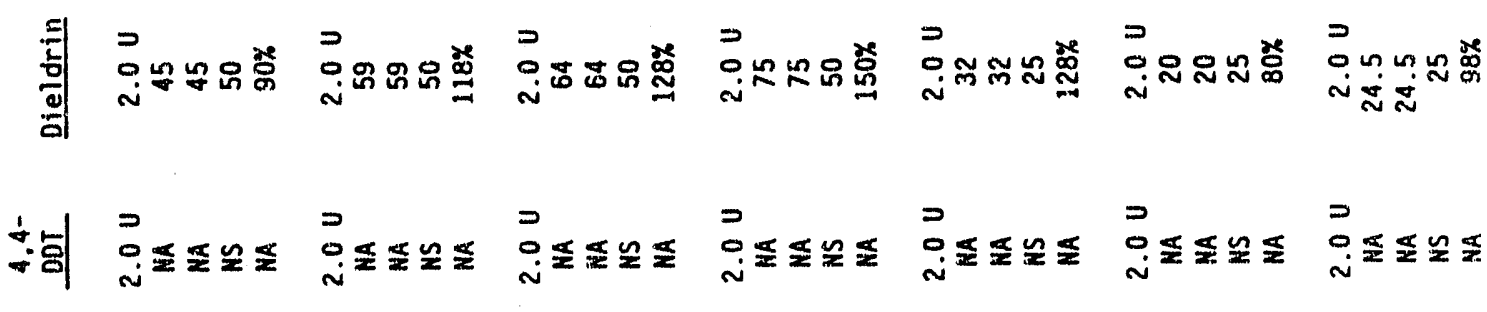

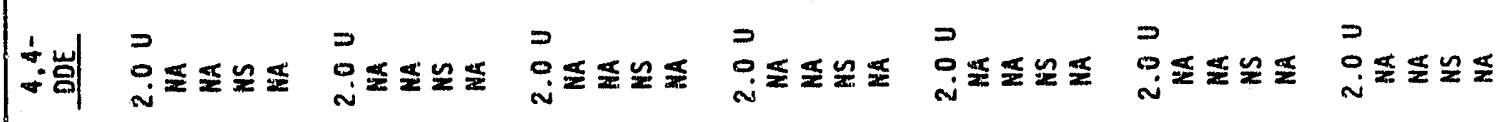

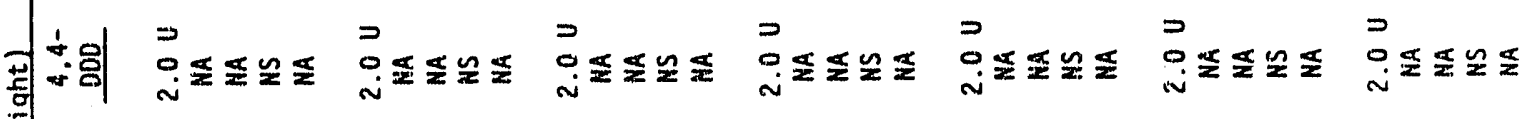

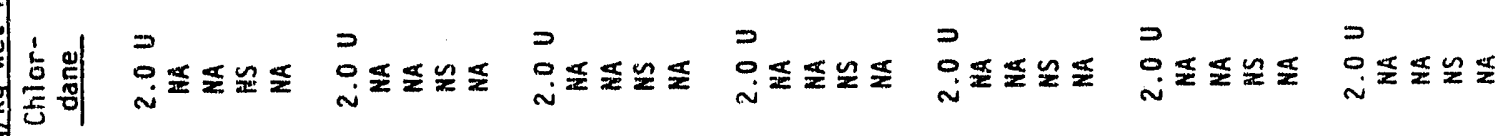

茾

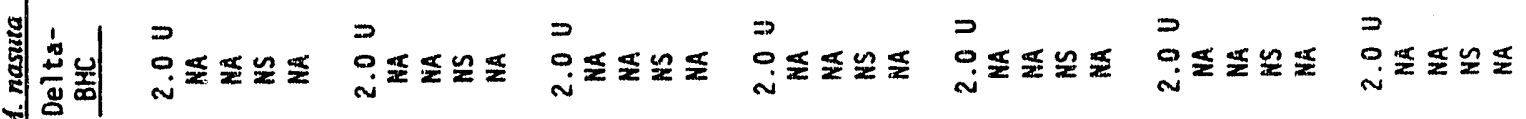

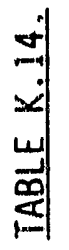

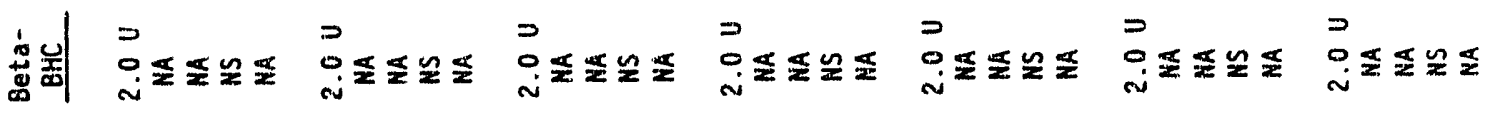

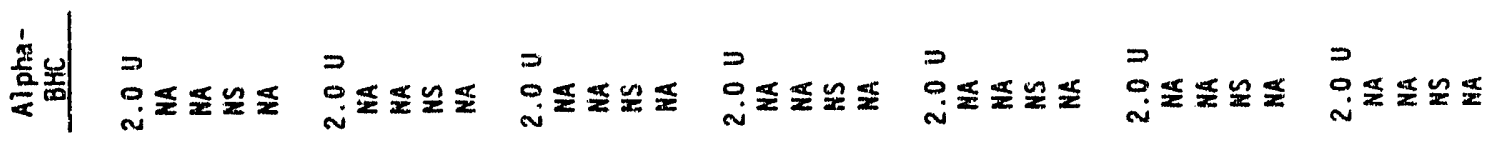

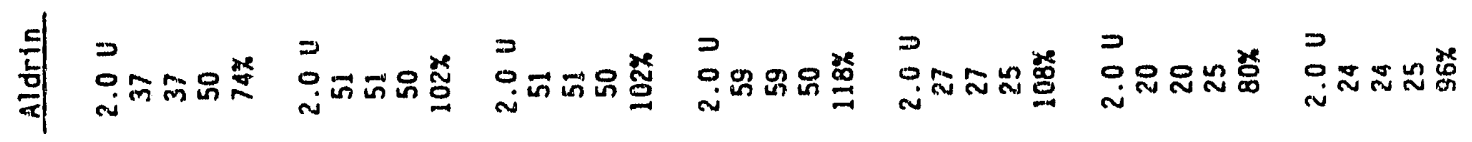

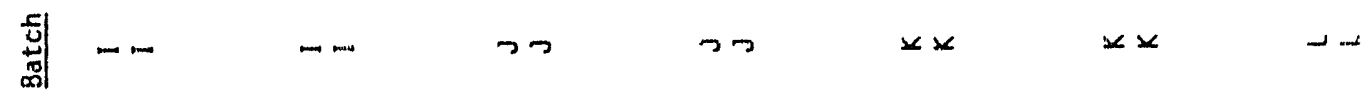

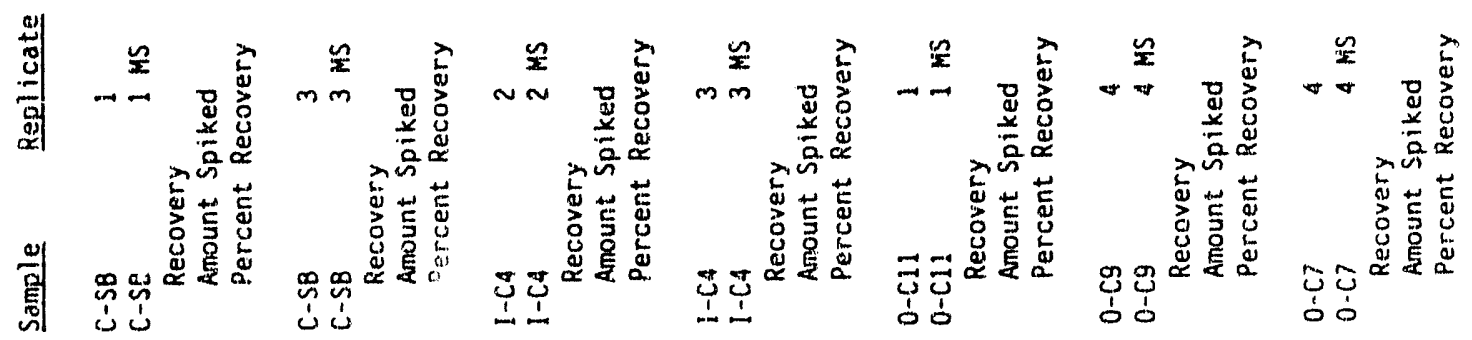
PHASE III B K. 58 


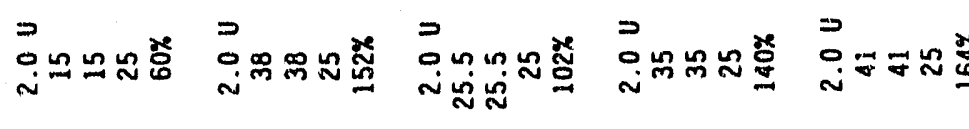
赫 fistat

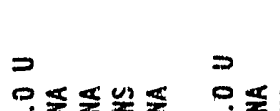

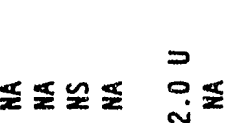

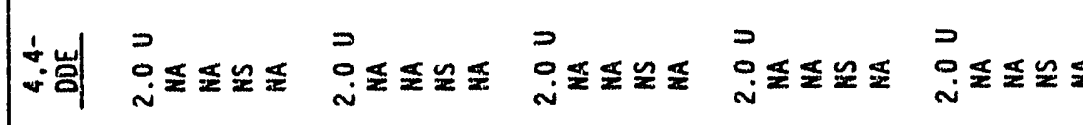

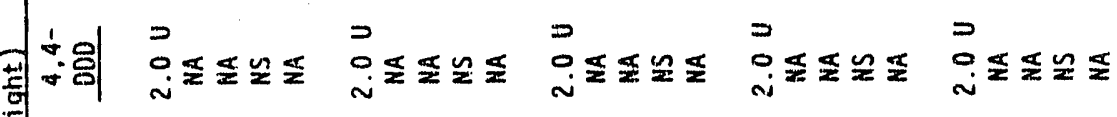
$\begin{array}{llll}D & D & D & D \\ 0 & 0 & 0 & 0\end{array}$ vini vi vin

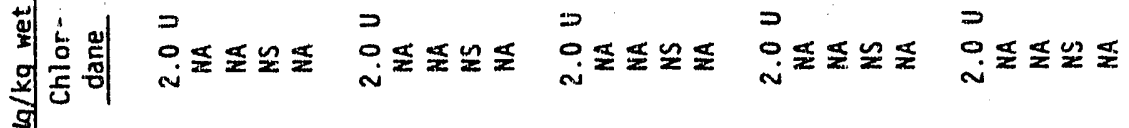

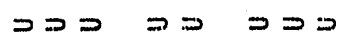
o00 00 000 vin vi nivi

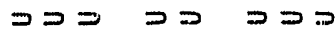
000 ơ 0 in

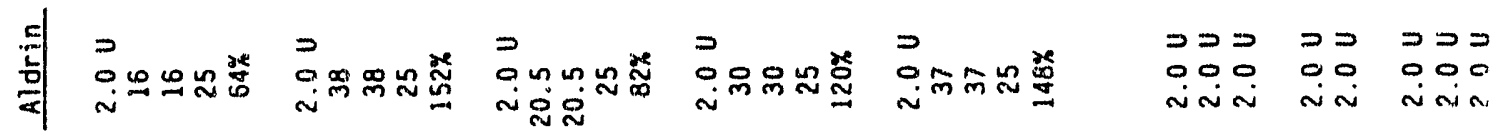

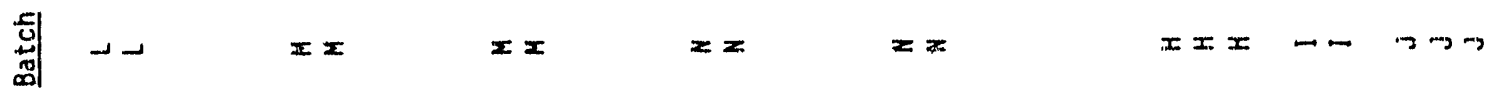

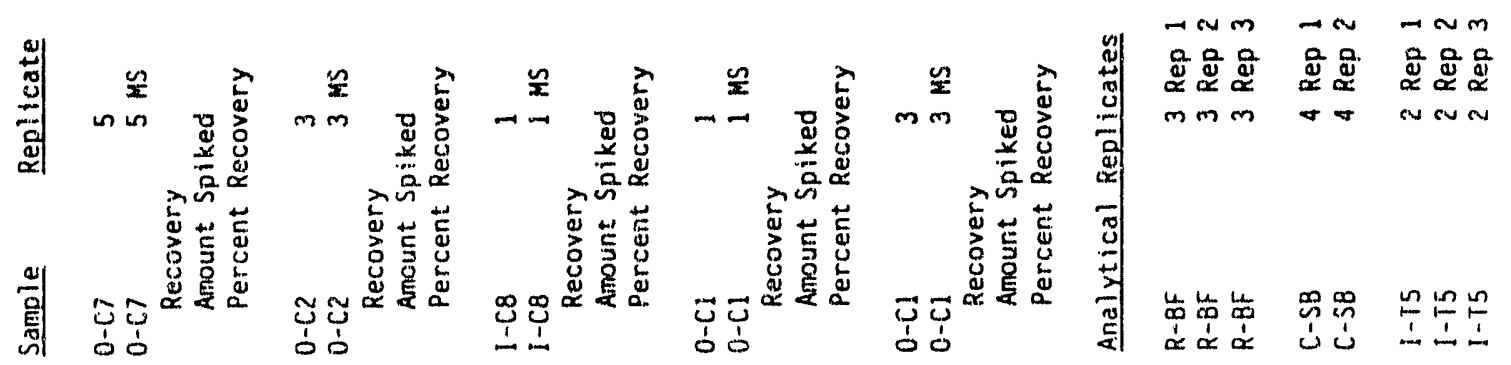




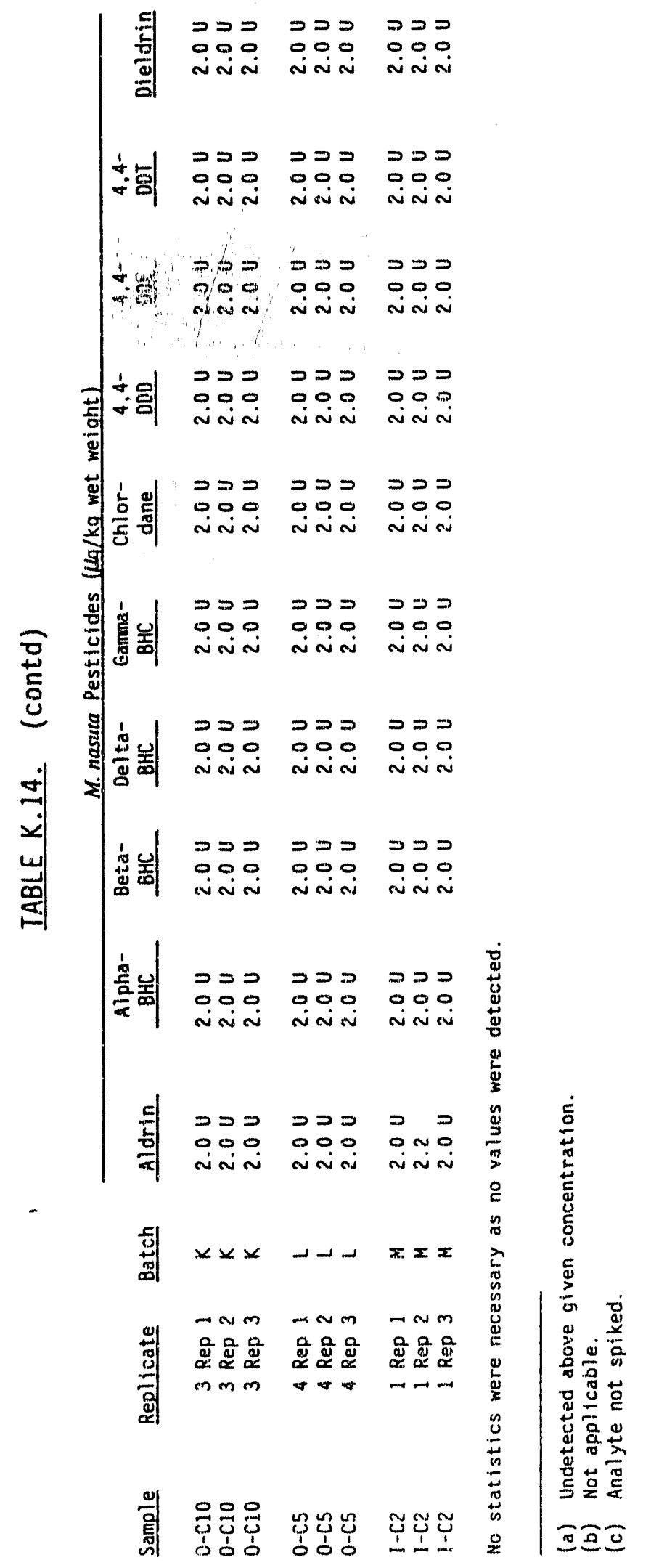

PHASE III B K.60 


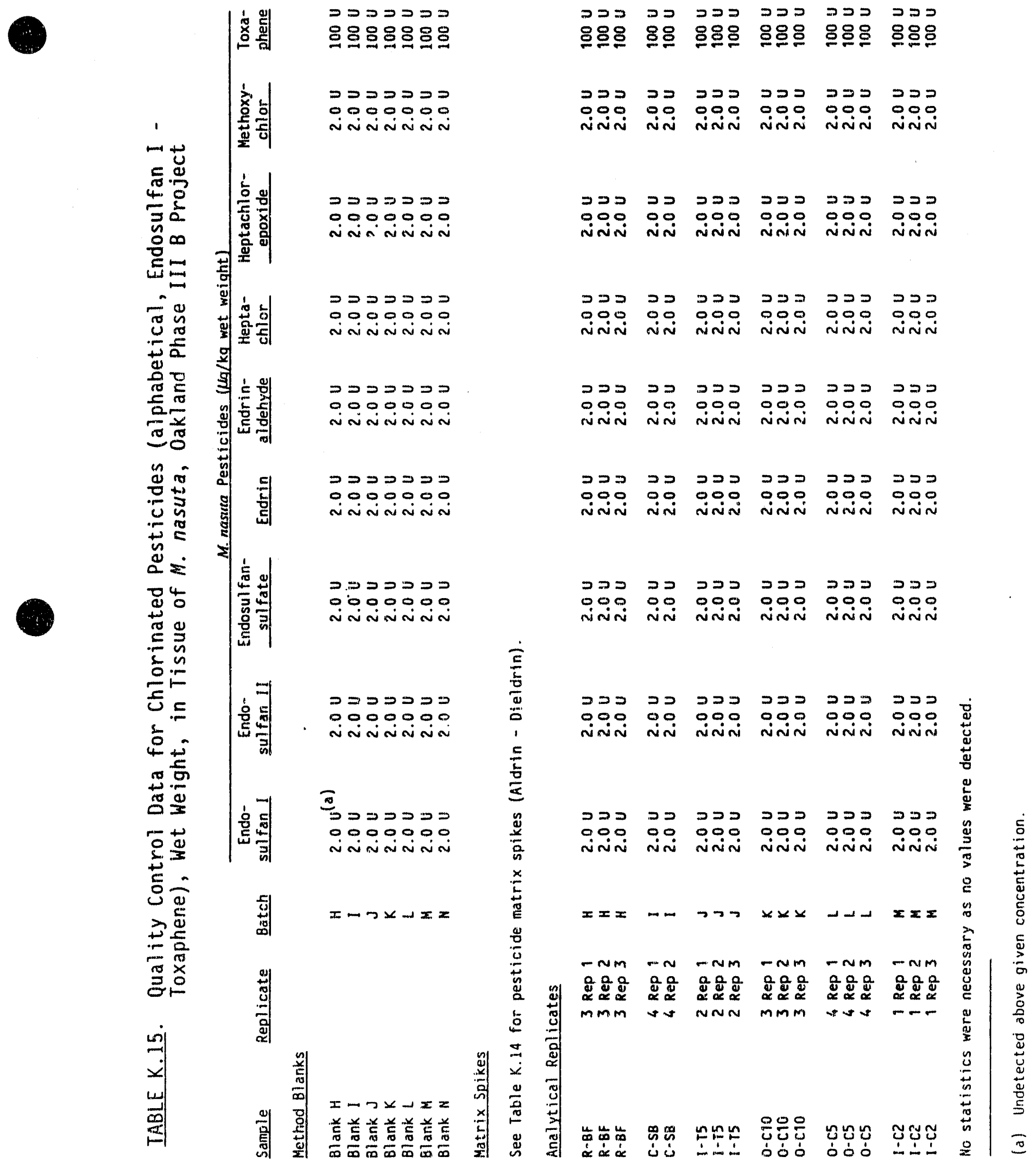




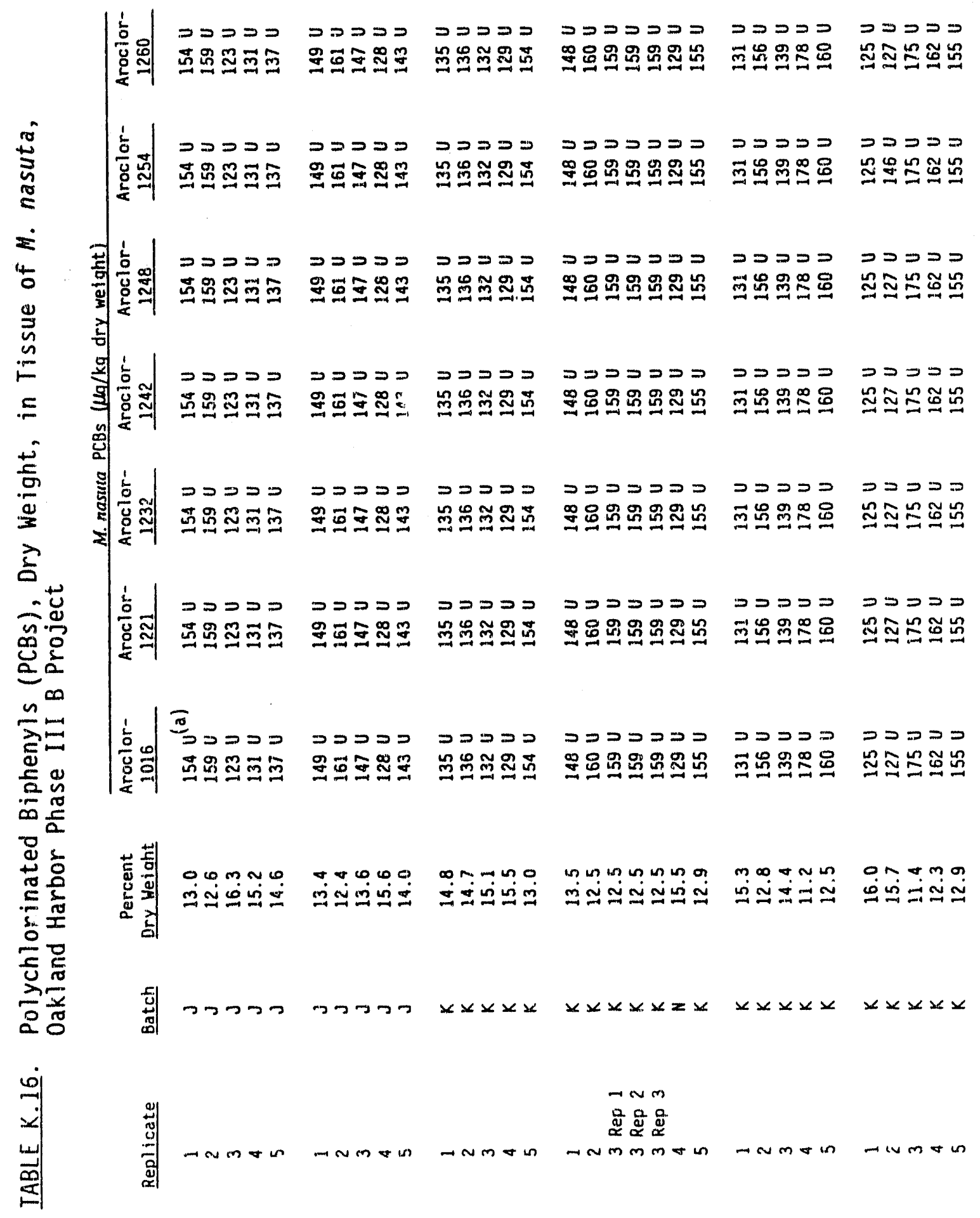

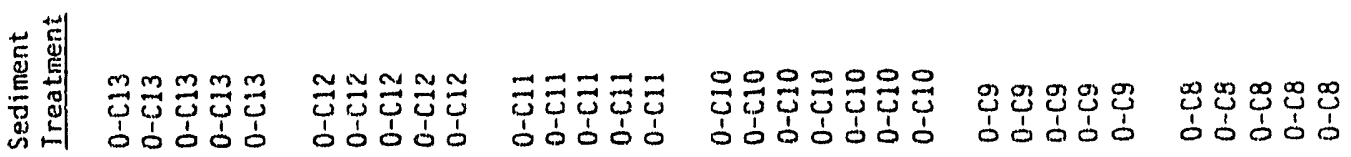




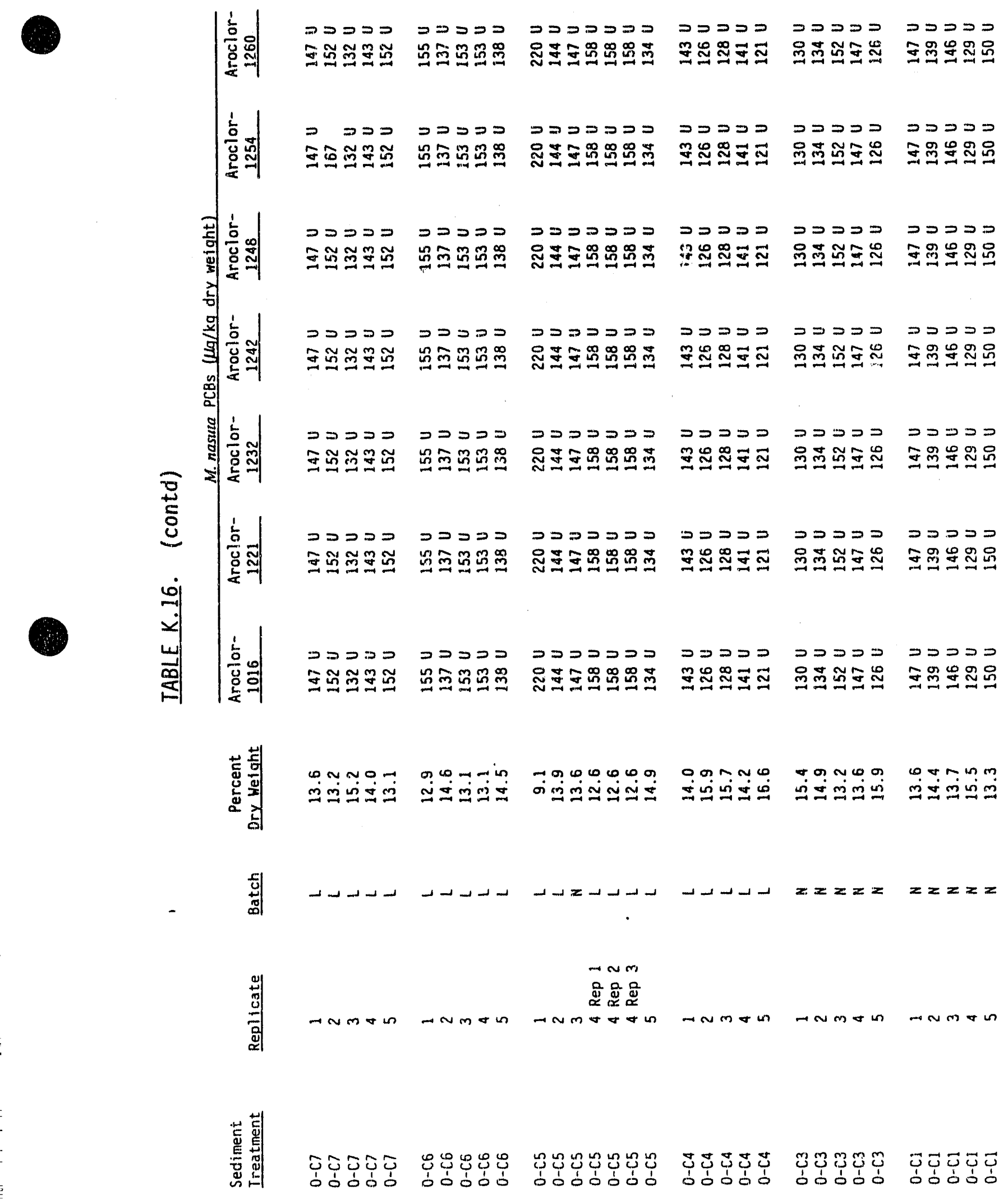




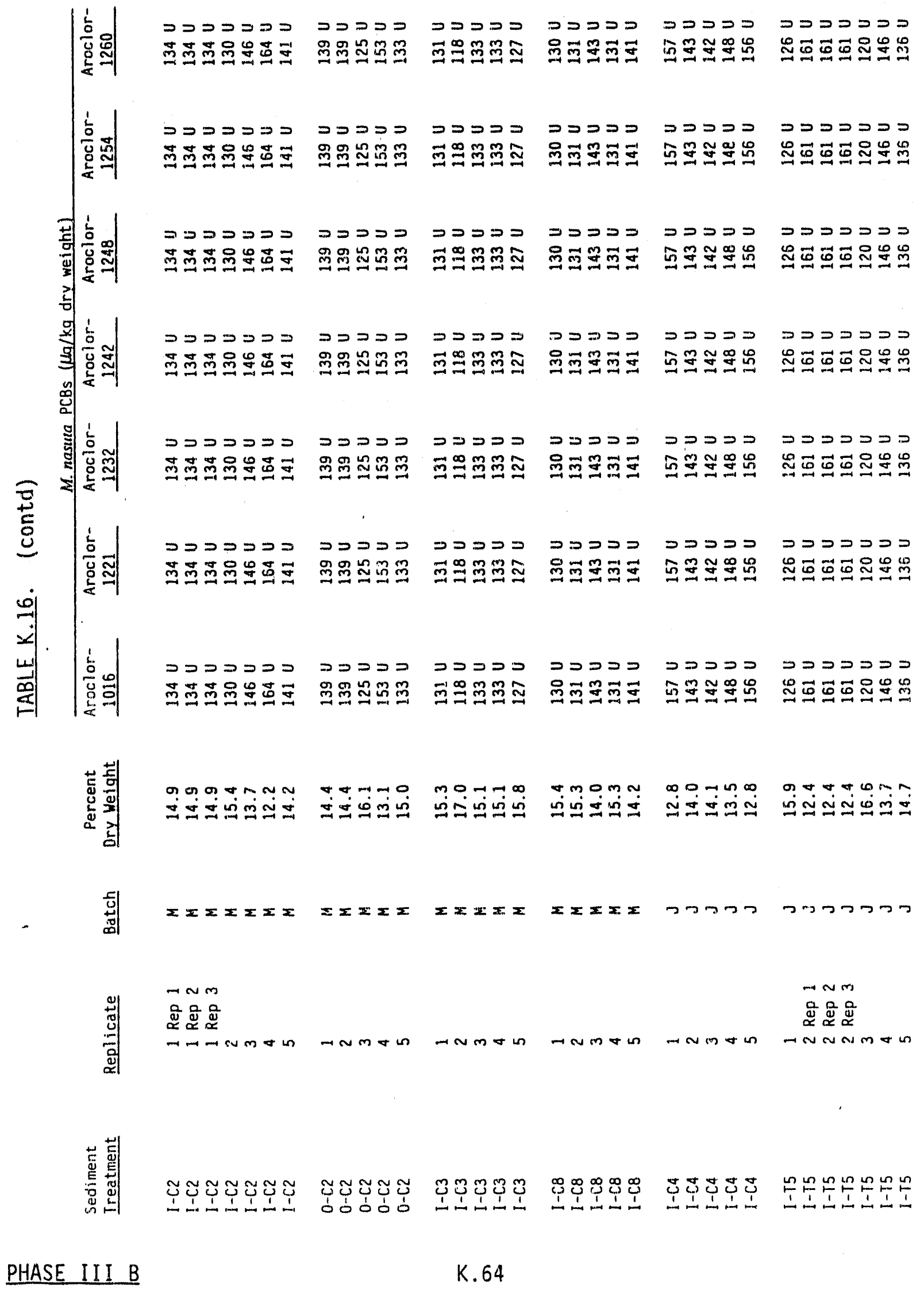




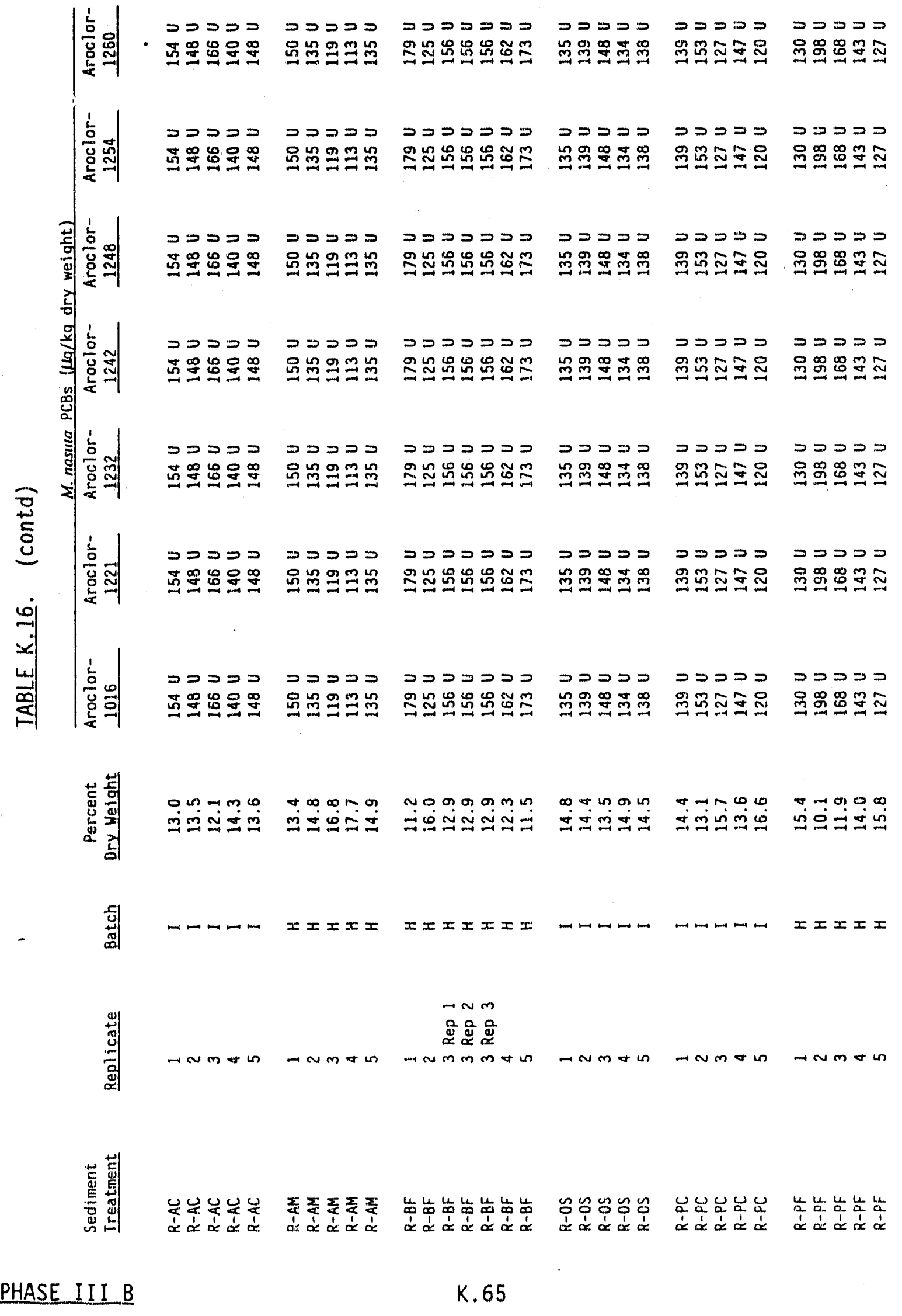




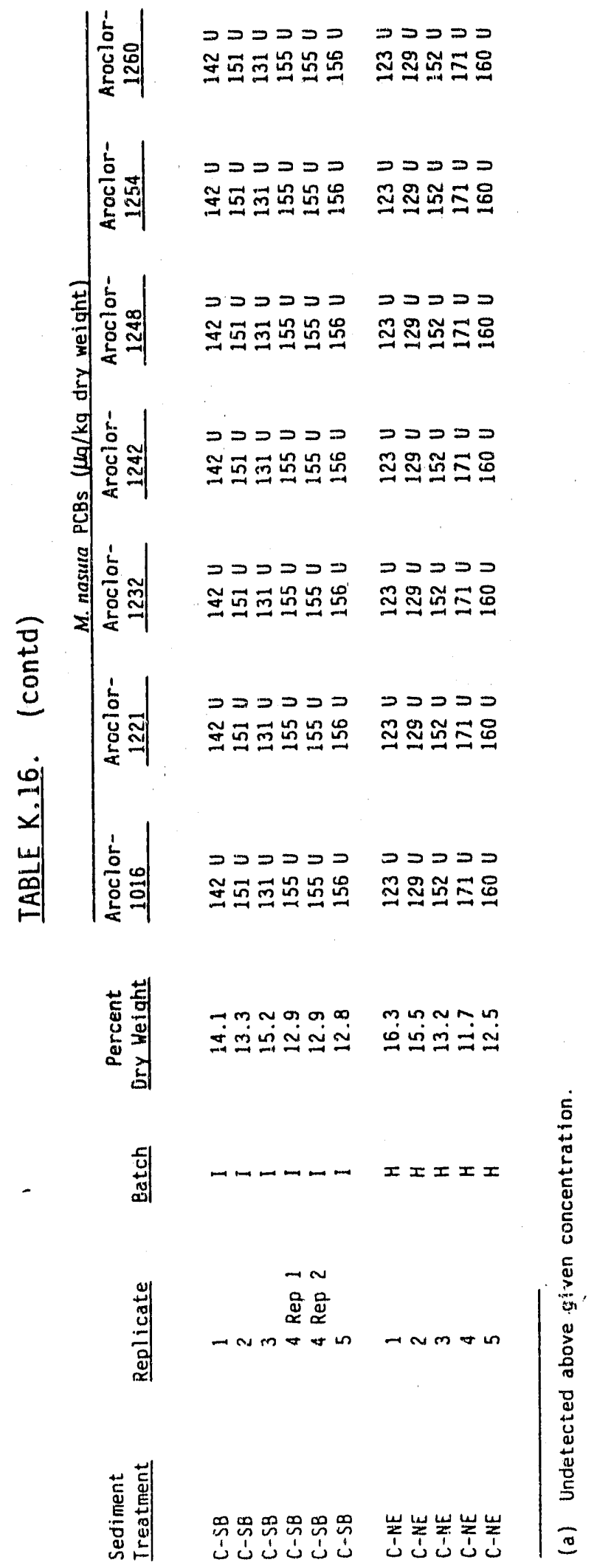




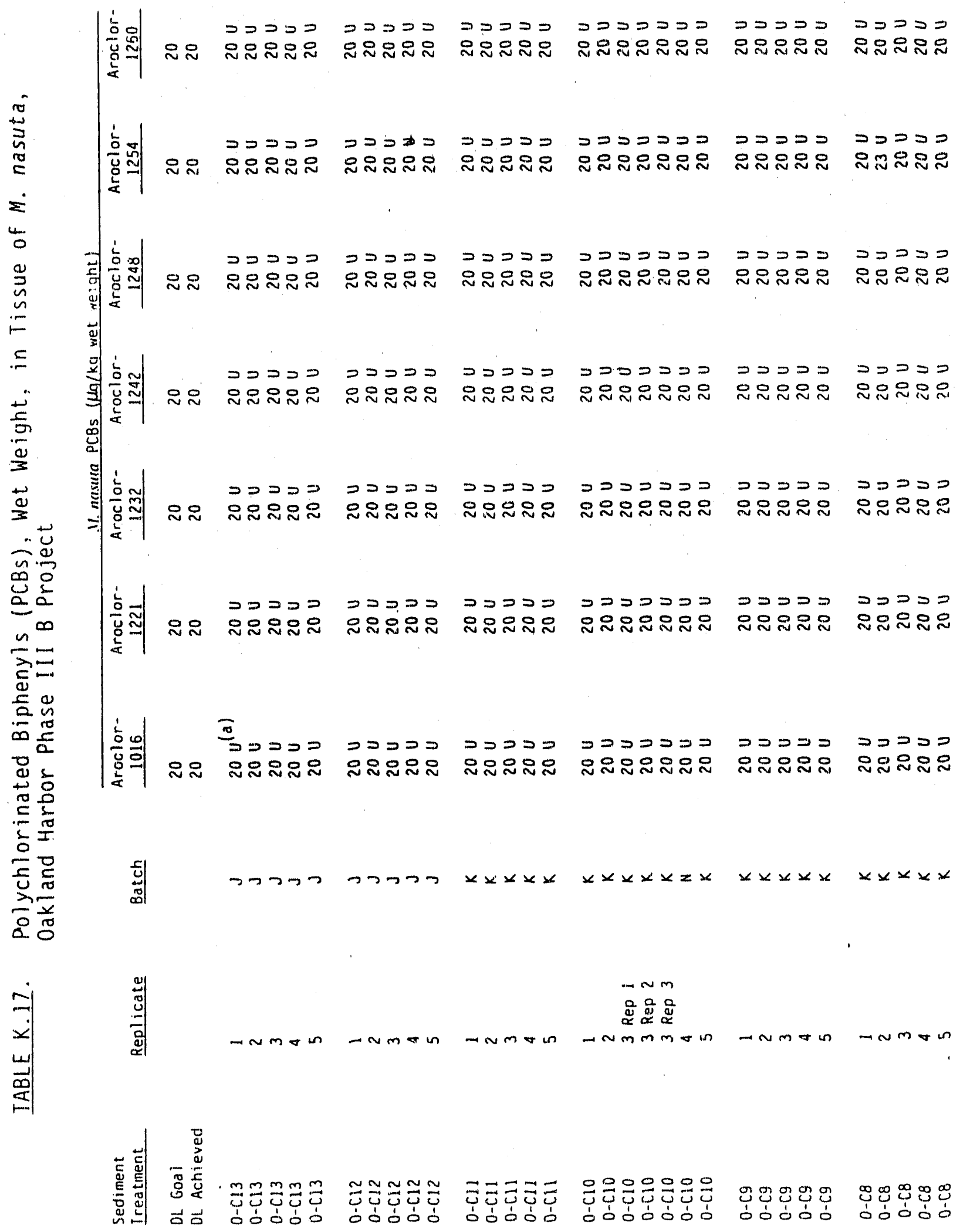




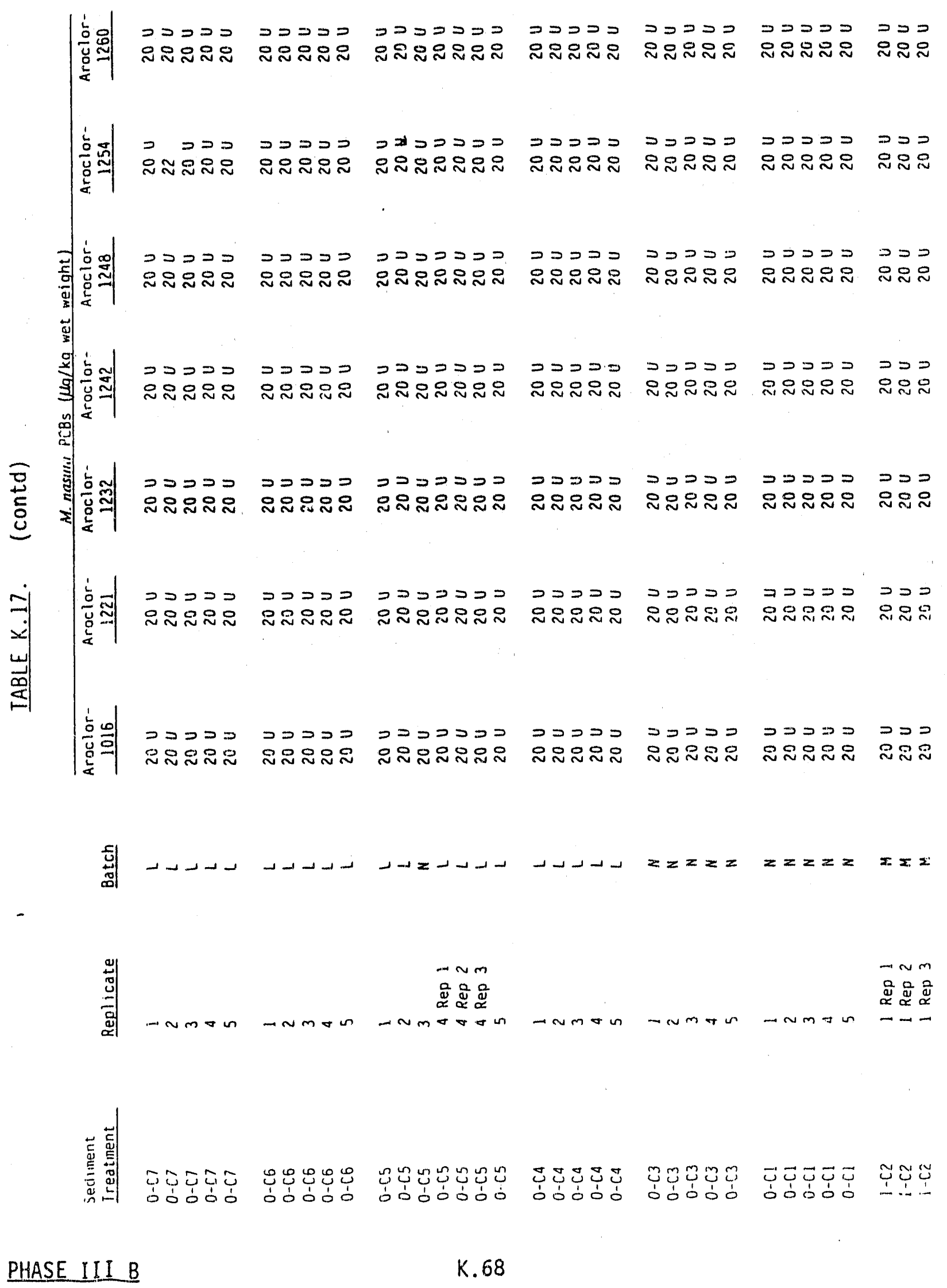




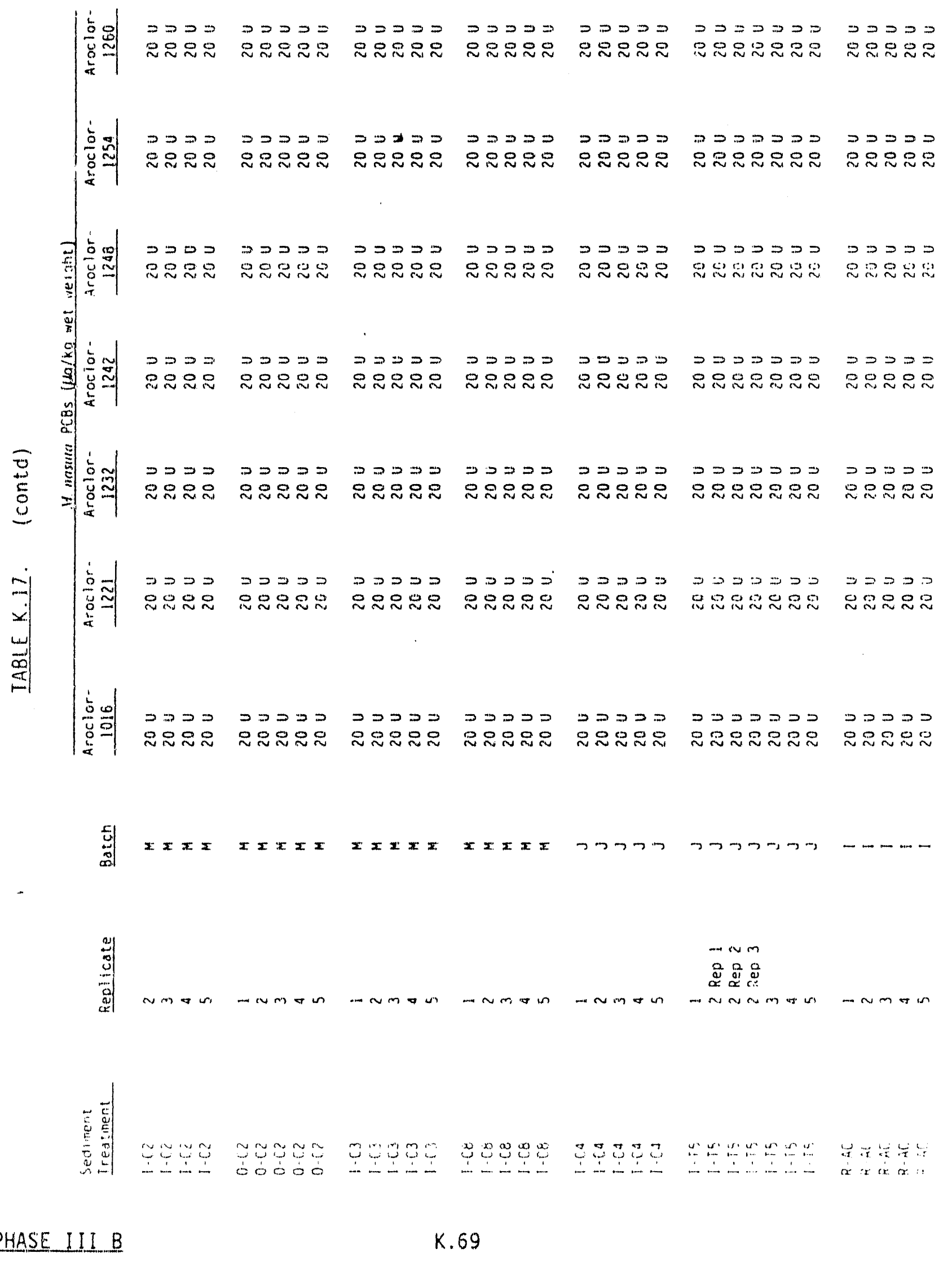




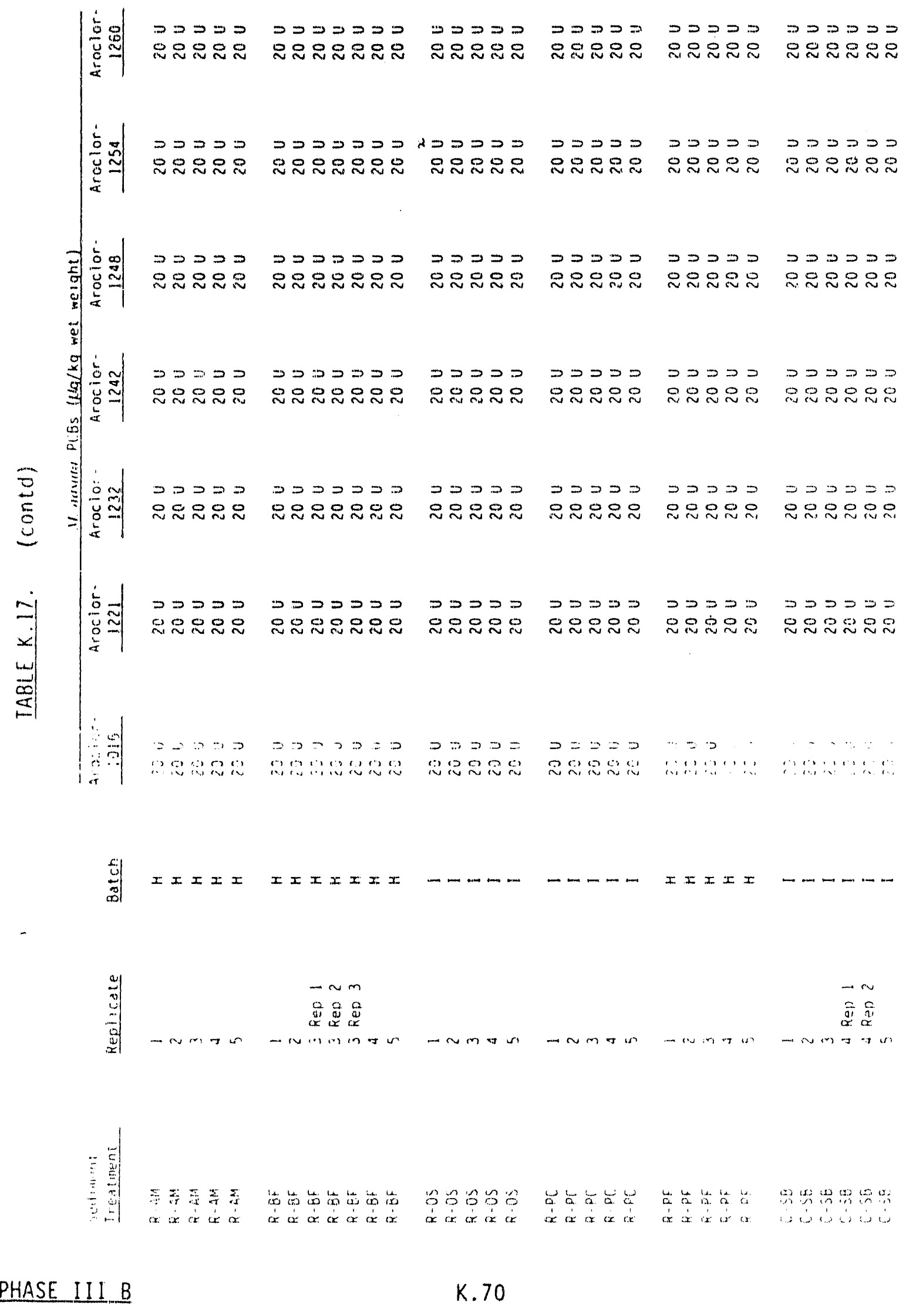




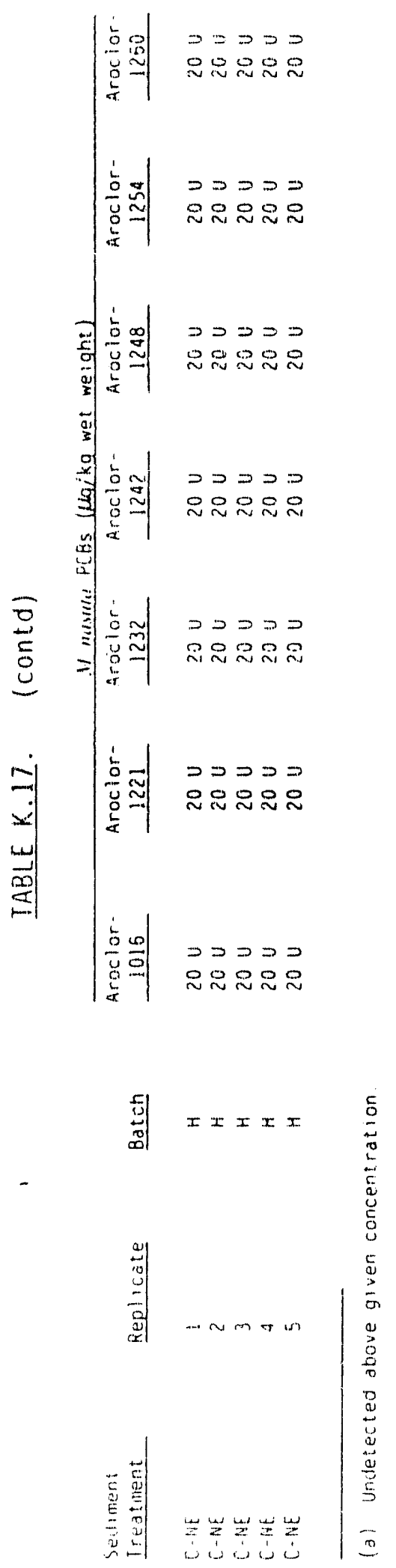




\section{TABLE K.18. Quality Control Data for Polychlorinated Biphenyls (PCBS), Wet Weight, in Tissue of $M$. nasuta, Oakland Phase III $B$ Project}

\begin{tabular}{|c|c|c|c|c|c|c|c|c|c|}
\hline Sample & Replicate & Batch & $\begin{array}{l}\text { Aroclor- } \\
1016 \\
\end{array}$ & $\begin{array}{c}\text { Aroclor- } \\
1221 \\
\end{array}$ & $\begin{array}{c}\text { Aroclor- } \\
1232 \\
\end{array}$ & $\begin{array}{c}\text { Aroclor- } \\
1242 \\
\end{array}$ & $\begin{array}{c}\text { Aroclor - } \\
1248 \\
\end{array}$ & $\begin{array}{l}\text { Aroclor"- } \\
\quad 1254 \\
\end{array}$ & $\begin{array}{r}\text { Arocilor } \\
\quad 1260 \\
\end{array}$ \\
\hline \multicolumn{10}{|c|}{ Method Blanks } \\
\hline Blank H & & $\mathrm{H}$ & $20 u^{(a)}$ & $20 U$ & $20 \mathrm{U}$ & $20 \mathrm{U}$ & $20 \mathrm{U}$ & $20 \mathrm{U}$ & $20 \mathrm{U}$ \\
\hline Blank i & & 1 & $20 \mathrm{U}$ & $20 U$ & $20 \mathrm{U}$ & $20 U$ & $20 \mathrm{U}$ & $20 \mathrm{U}$ & $20 \mathrm{U}$ \\
\hline Blank J & & J & $20 \mathrm{U}$ & $20 \mathrm{U}$ & $20 \mathrm{U}$ & $20 \mathrm{U}$ & 204 & $20 \mathrm{U}$ & $20 \mathrm{U}$ \\
\hline Blank K & & $K$ & $20 \mathrm{U}$ & $20 \mathrm{U}$ & $20 \mathrm{U}$ & $20 \mathrm{U}$ & $20 \mathrm{U}$ & $20 \mathrm{U}$ & $20 \mathrm{U}$ \\
\hline Blank L & & $L$ & $20 \mathrm{U}$ & $20 \mathrm{U}$ & $20 U$ & $20 \mathrm{U}$ & $20 \mathrm{U}$ & $20 \mathrm{U}$ & $20 \mathrm{U}$ \\
\hline Blank. M & & $M$ & $20 \mathrm{U}$ & $20 \mathrm{U}$ & $20 \mathrm{U}$ & $20 \mathrm{U}$ & $20 U$ & $20 \mathrm{U}$ & $20 \mathrm{U}$ \\
\hline Blank $N$ & & $N$ & $20 \mathrm{U}$ & $20 \mathrm{U}$ & $20 \mathrm{U}$ & $20 \mathrm{~N}$ & $20 \mathrm{U}$ & $20 \mathrm{U}$ & 20 \\
\hline
\end{tabular}

\section{Matrix Spikes}

No PCB Aroclors spiked. See Table K.14, pesticide matrix spikes.

\section{Analytical Replicates}

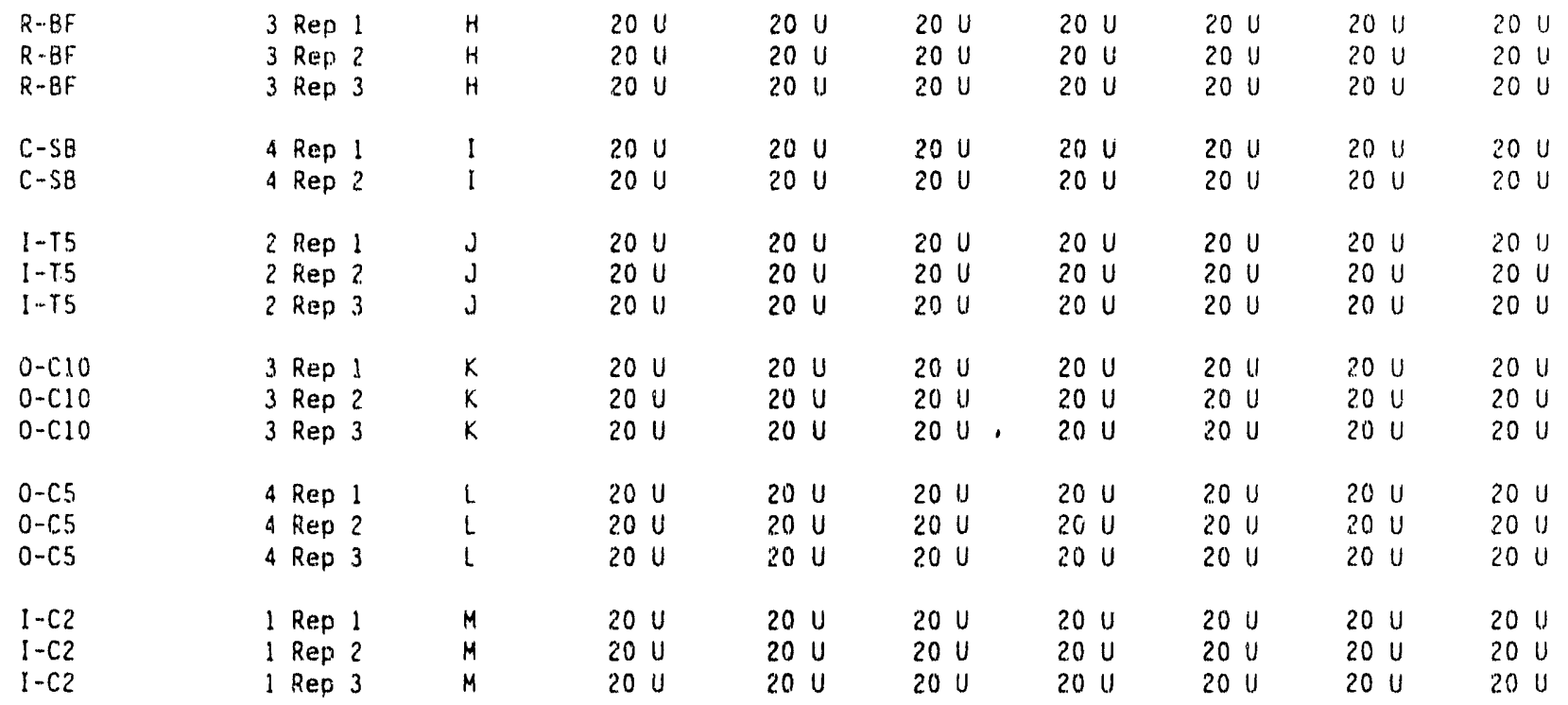

No statistics were necessary as no values were detected.

(a) Undetected above given concentration. 
IABLEK.19. Surrogate Percent Recoveries for Chlorinated Pesticides and Polychlorinated Biphenyls (PCBs) in Tissue of M. nasuta, Oakland Harbor Phase III B Project

Sediment

Treatment

$0-C 13$

$0-\mathrm{C}] 3$

$0-013$

$0-C 13$

$0-C 13$

$0-C 12$

$0-\mathrm{C} 12$

$0-\mathrm{Cl} 2$

$0-\mathrm{C} 12$

$0-\mathrm{C} 12$

$0-C 11$

$0-C 1$ !

$0-C 11$

$0-C 11$

$0-C 11$

$0-C 10$

$0-C 10$

$0-C 10$

$0-C 10$

$0-\mathrm{C} 10$

$0-C 10$

$0-C 10$

$0-c 9$

$0-C 9$

$0-C 9$

$0-c 9$

$0 . C 9$

$0-c 8$

$0 . \mathrm{C} 8$

$0 .-18$

$0-\mathrm{CB}$

$0-\mathrm{C} 8$

$0-C 7$

$0-C 7$

$0-C 7$

$0 . C 7$

$0-C 7$

\section{Replicate Batch}

1

3

3

5

1

2

3

4

5

1

2

3

4

5

1

2

3 Rep 1

3 Rep 2

3 Rep 3

4

5

1

2

3

4

5

1

3

4

5

1
2
3
4
5

J

J

J

J

Dibutyl -

chlorendate

Percent

Recovery

63

58

74

66

62

94

99

73

66

77

30

35

18

25

26

35

21

34

18

33

23

30

21

18

23

25

26

2)

17

18

20

26

$6 ?$

53

76

22

58 
TABLE K.19. (contd)

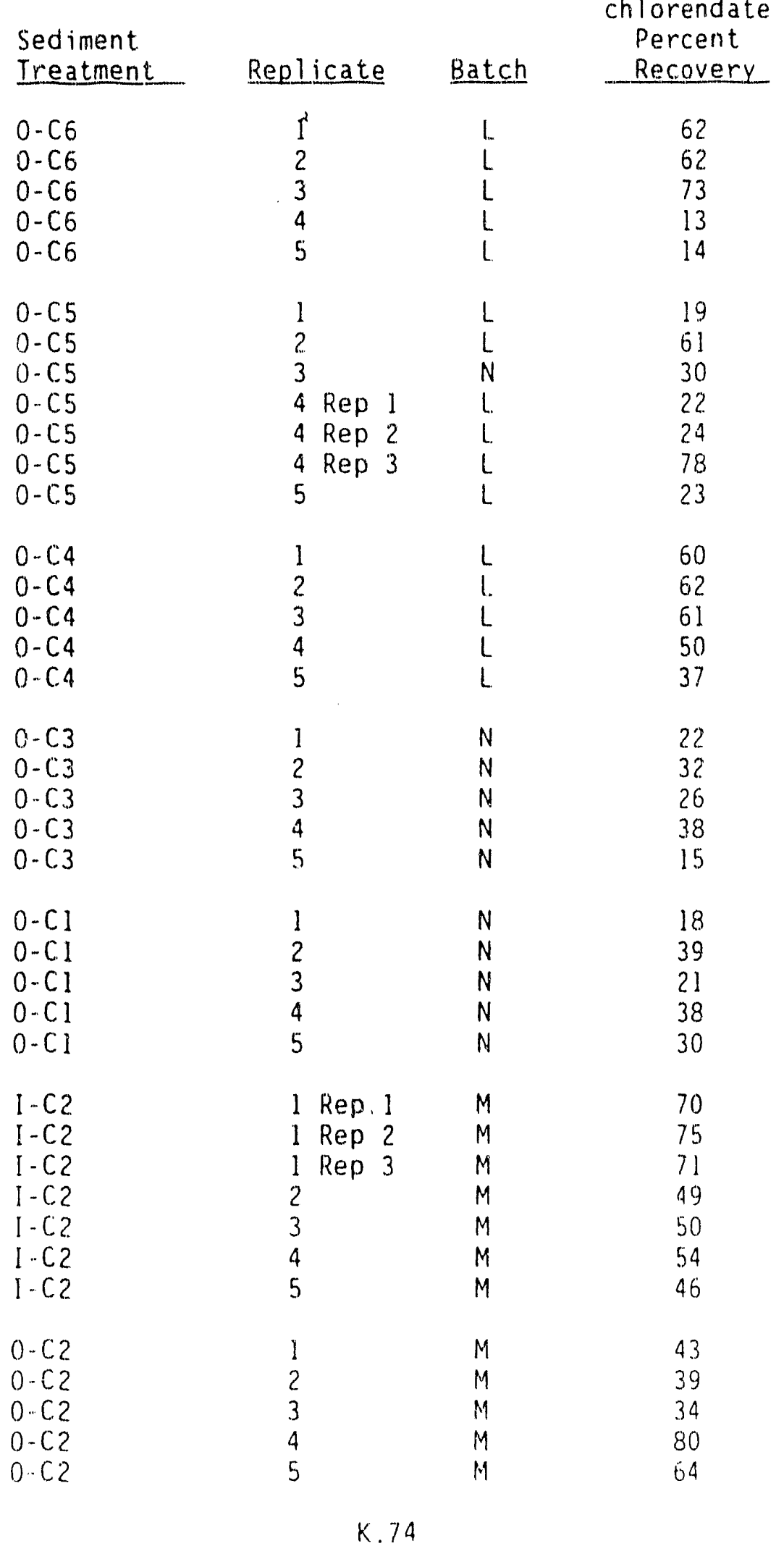


TABLE K.19. (contd)

\begin{tabular}{|c|c|c|c|}
\hline $\begin{array}{l}\text { Sediment } \\
\text { Treatment }\end{array}$ & Replicate & Batch & $\begin{array}{l}\text { Dibuty - } \\
\text { chlorendate } \\
\text { Percent } \\
\text { Recovery } \\
\end{array}$ \\
\hline $\begin{array}{l}I-C 3 \\
I-C 3 \\
I-C 3 \\
I-C 3 \\
I-C 3\end{array}$ & $\begin{array}{l}1 \\
2 \\
3 \\
4 \\
5\end{array}$ & $\begin{array}{l}M \\
M \\
M \\
M \\
M\end{array}$ & $\begin{array}{l}72 \\
57 \\
58 \\
64 \\
39\end{array}$ \\
\hline $\begin{array}{l}I-C 8 \\
I-C 8 \\
I-C 8 \\
I-C 8 \\
I-C 8\end{array}$ & $\begin{array}{l}1 \\
2 \\
3 \\
4 \\
5\end{array}$ & $\begin{array}{l}M \\
M \\
M \\
M \\
M\end{array}$ & $\begin{array}{l}74 \\
51 \\
69 \\
60 \\
61\end{array}$ \\
\hline $\begin{array}{l}I-C 4 \\
I-C 4 \\
I-C 4 \\
I-C 4 \\
I-C 4\end{array}$ & $\begin{array}{l}1 \\
2 \\
3 \\
4 \\
5\end{array}$ & $\begin{array}{l}J \\
J \\
J \\
J \\
J\end{array}$ & $\begin{array}{l}92 \\
75 \\
82 \\
91 \\
86\end{array}$ \\
\hline $\begin{array}{l}I-T 5 \\
1-T 5 \\
1-T 5 \\
1-T 5 \\
1-T 5 \\
I-T 5 \\
I-T 5\end{array}$ & $\begin{array}{lll}1 & & \\
2 & \text { Rep } & 1 \\
2 & \operatorname{Rep} & 2 \\
2 & \operatorname{Rep} & 3 \\
3 & & \\
4 & & \\
5 & & \end{array}$ & $\begin{array}{l}\mathrm{J} \\
\mathrm{J} \\
\mathrm{J} \\
\mathrm{J} \\
\mathrm{J} \\
\mathrm{J} \\
\mathrm{J}\end{array}$ & $\begin{array}{l}50 \\
35 \\
34 \\
99 \\
81 \\
74 \\
46\end{array}$ \\
\hline $\begin{array}{l}R-A C \\
R-A C \\
R-A C \\
R-A C \\
R-A C\end{array}$ & $\begin{array}{l}1 \\
2 \\
3 \\
4 \\
5\end{array}$ & $\begin{array}{l}\text { I } \\
I \\
I \\
I \\
I\end{array}$ & $\begin{array}{r}66 \\
99 \\
130 \\
120 \\
110\end{array}$ \\
\hline $\begin{array}{l}R-A M \\
R-A M \\
R-A M \\
R-A M \\
R-A M\end{array}$ & $\begin{array}{l}1 \\
2 \\
3 \\
4 \\
5\end{array}$ & $\begin{array}{l}H \\
H \\
H \\
H \\
H\end{array}$ & $\begin{array}{r}102 \\
72 \\
120 \\
125 \\
122\end{array}$ \\
\hline $\begin{array}{l}R \cdot B F \\
R-B F \\
R-B F \\
R-B F \\
R-B F \\
R-B F \\
R-B F\end{array}$ & $\begin{array}{lll}1 & & \\
2 & & \\
3 & \operatorname{Rep} & 1 \\
3 & \operatorname{Rep} & 2 \\
3 & \operatorname{Rep} & 3 \\
4 & & \\
5 & & \end{array}$ & $\begin{array}{l}H \\
H \\
H \\
H \\
H \\
H \\
H\end{array}$ & $\begin{array}{r}87 \\
129 \\
52 \\
94 \\
82 \\
91 \\
110\end{array}$ \\
\hline
\end{tabular}


IABLE K.19. (contd)

\begin{tabular}{|c|c|c|c|}
\hline $\begin{array}{l}\text { Sediment } \\
\text { Treatment }\end{array}$ & Replicate & Batch & $\begin{array}{l}\text { Percent } \\
\text { Recovery }\end{array}$ \\
\hline$R-O S$ & 1 & 1 & 104 \\
\hline$R-0 S$ & 2 & I & 111 \\
\hline$R-O S$ & 3 & I & 44 \\
\hline$R-O S$ & 4 & I & 97 \\
\hline R-OS & 5 & I & 98 \\
\hline$R-P C$ & 1 & I & 78 \\
\hline$R-P C$ & 2 & I & 92 \\
\hline$R-P C$ & 3 & I & 92 \\
\hline$R-P C$ & 4 & I & 98 \\
\hline$R-P C$ & 5 & I & 65 \\
\hline$R-P F$ & 1 & $H$ & 121 \\
\hline$R-P F$ & 2 & H & 90 \\
\hline$R-P F$ & 3 & $H$ & 112 \\
\hline$R-P F$ & 4 & $H$ & 91 \\
\hline$R-P F$ & 5 & $H$ & 120 \\
\hline$C-S B$ & 1 & I & 99 \\
\hline$C-S B$ & 2 & I & 111 \\
\hline$C-S B$ & 3 & I & 115 \\
\hline$C-S B$ & 4 Rep 1 & I & 107 \\
\hline$C-S B$ & $4 \operatorname{Rep} 2$ & I & 103 \\
\hline$C-S B$ & 5 & I & 101 \\
\hline$C-N E$ & 1 & H & 101 \\
\hline $\mathrm{C}-\mathrm{NE}$ & 2 & H & 89 \\
\hline$C-N E$ & 3 & H & 97 \\
\hline$C-N E$ & 4 & $H$ & 86 \\
\hline$C-N E$ & 5 & $H$ & 82 \\
\hline
\end{tabular}




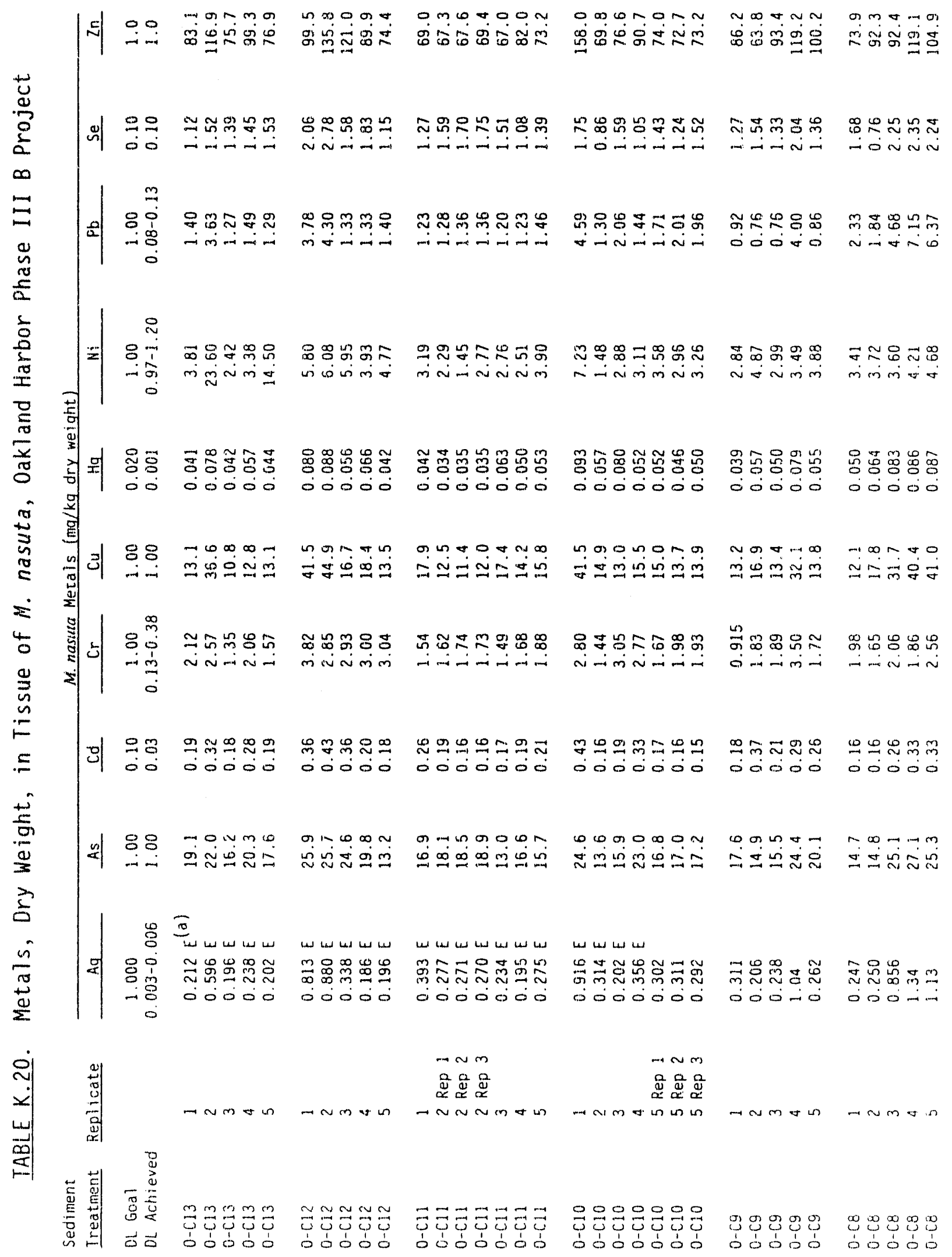




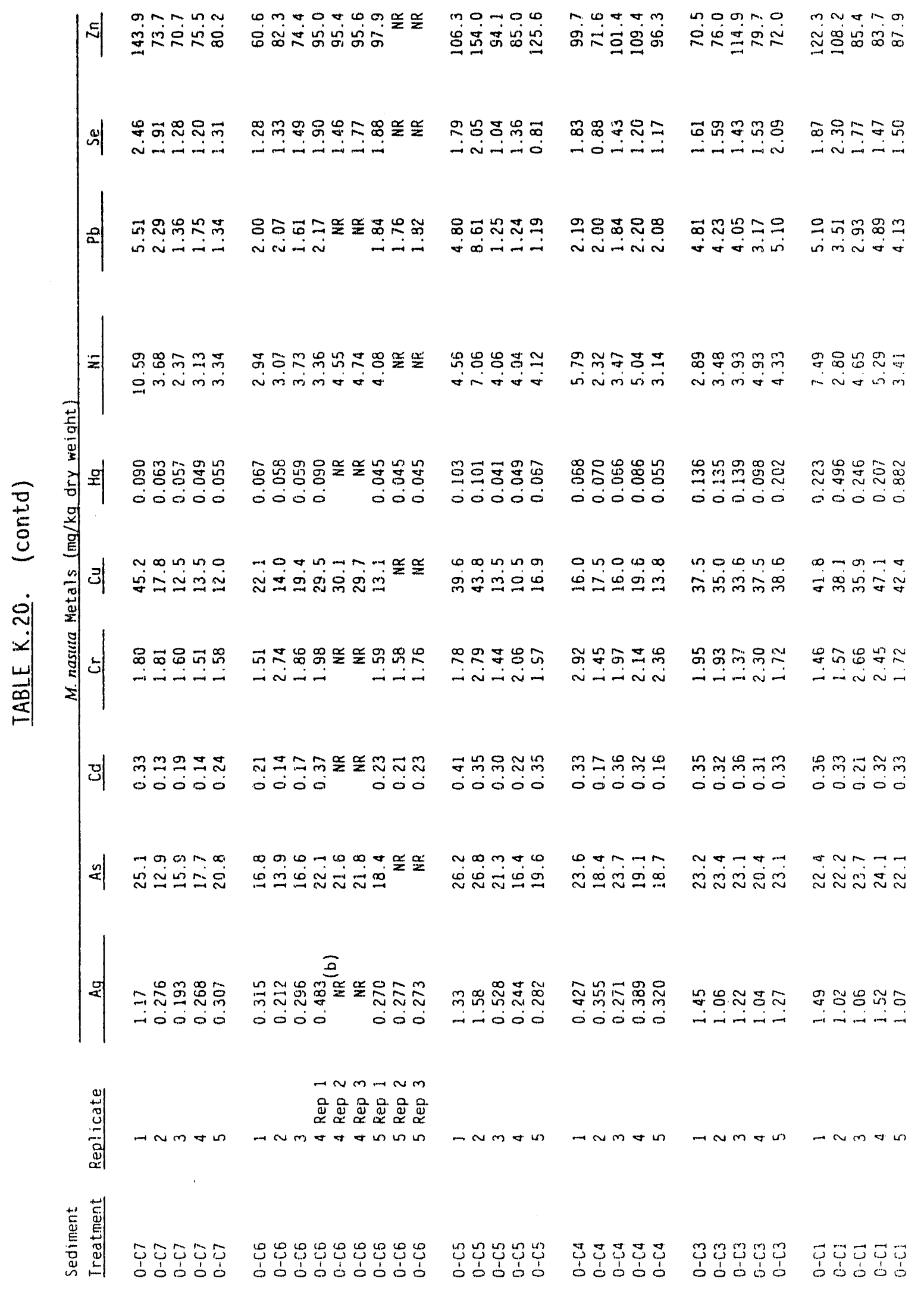




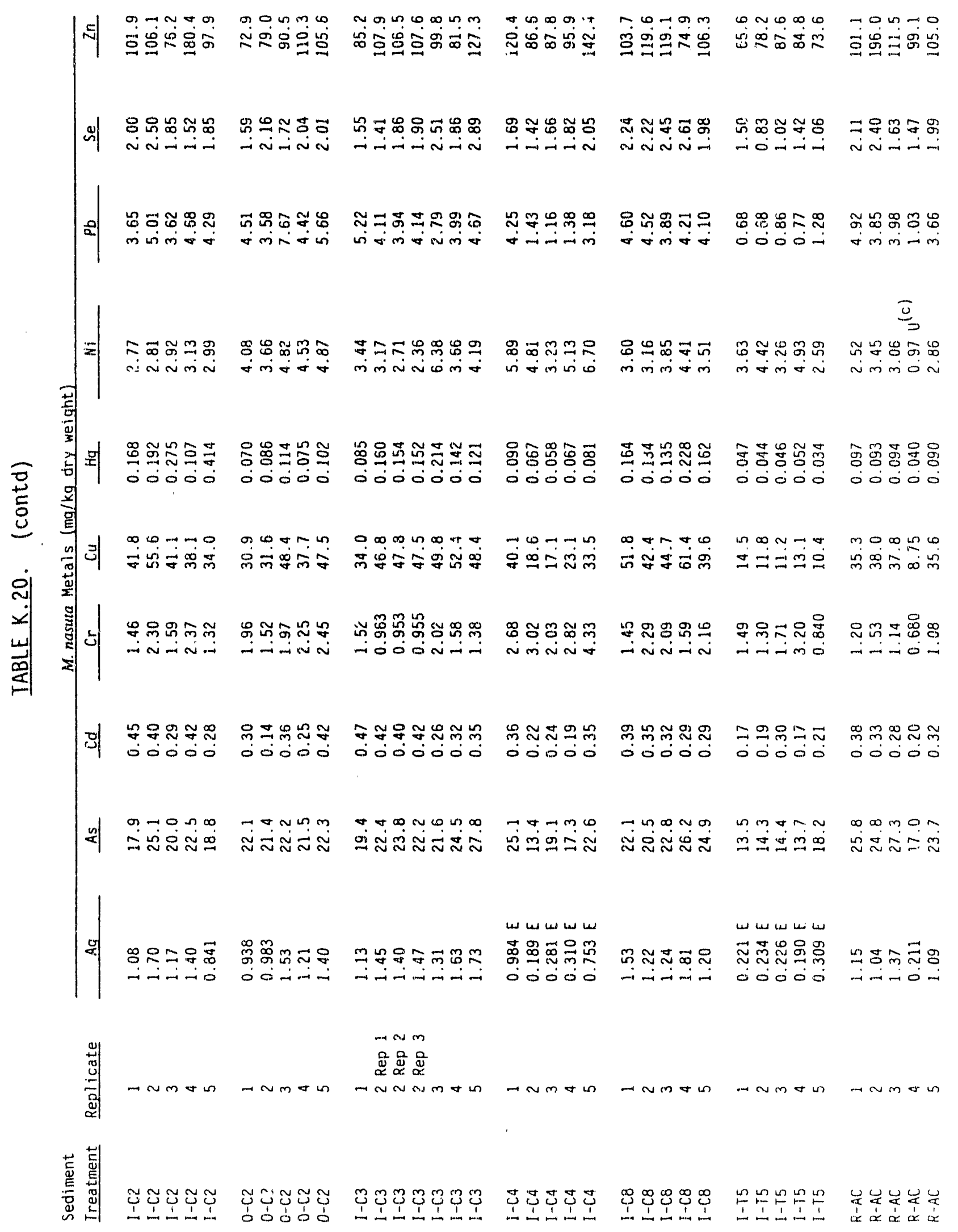




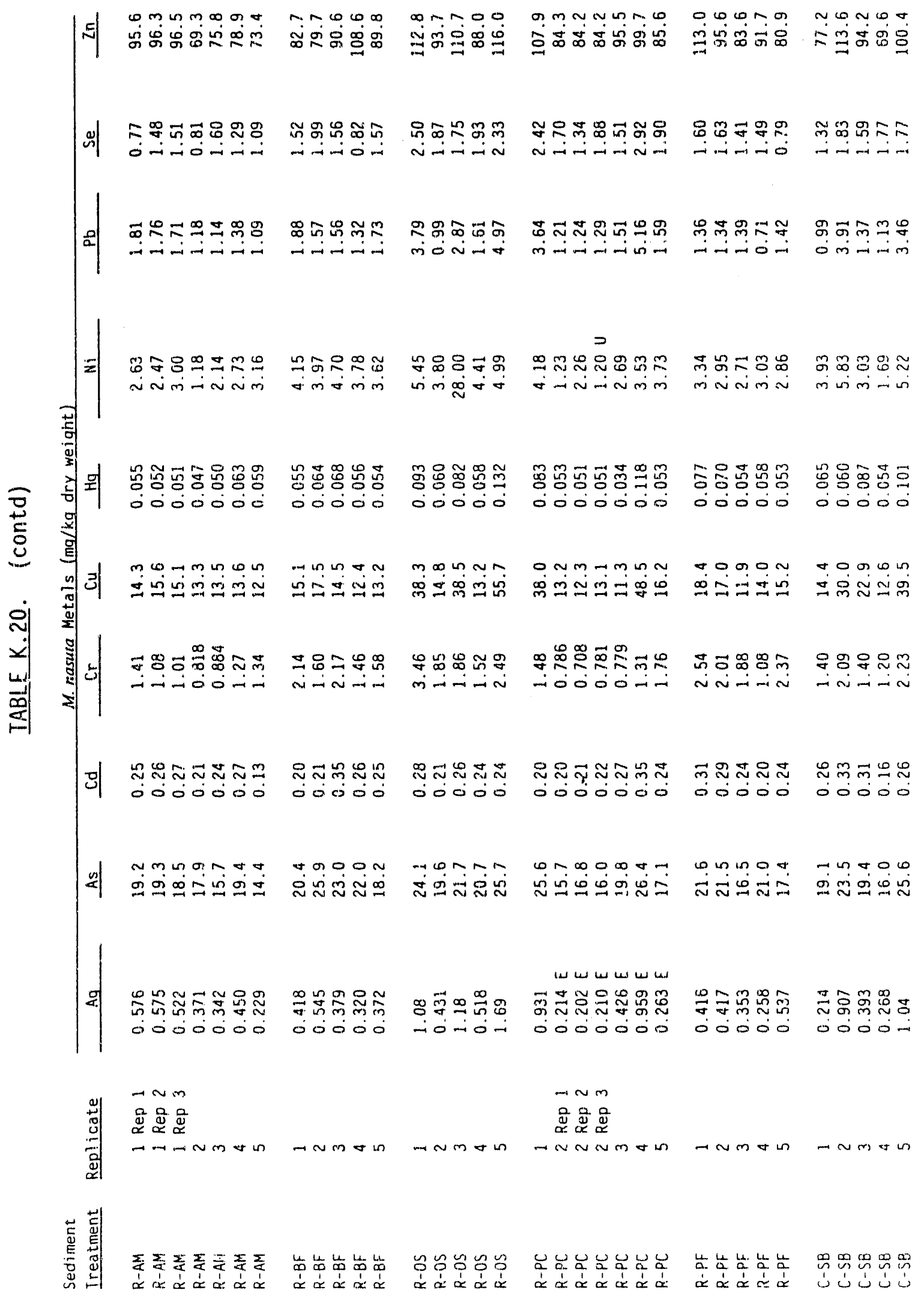




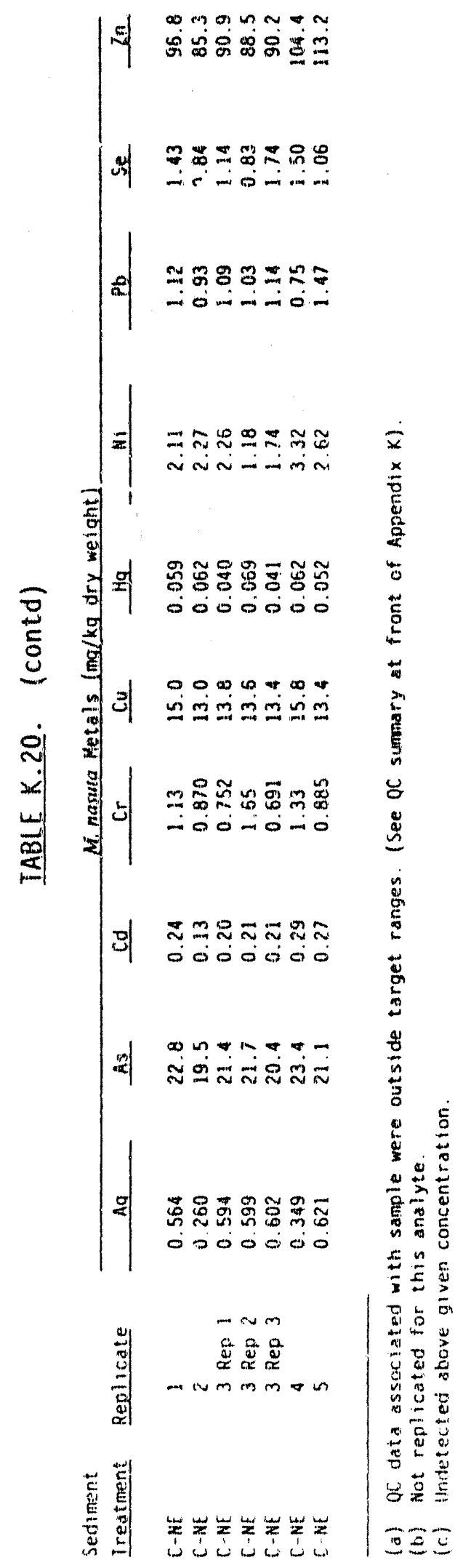




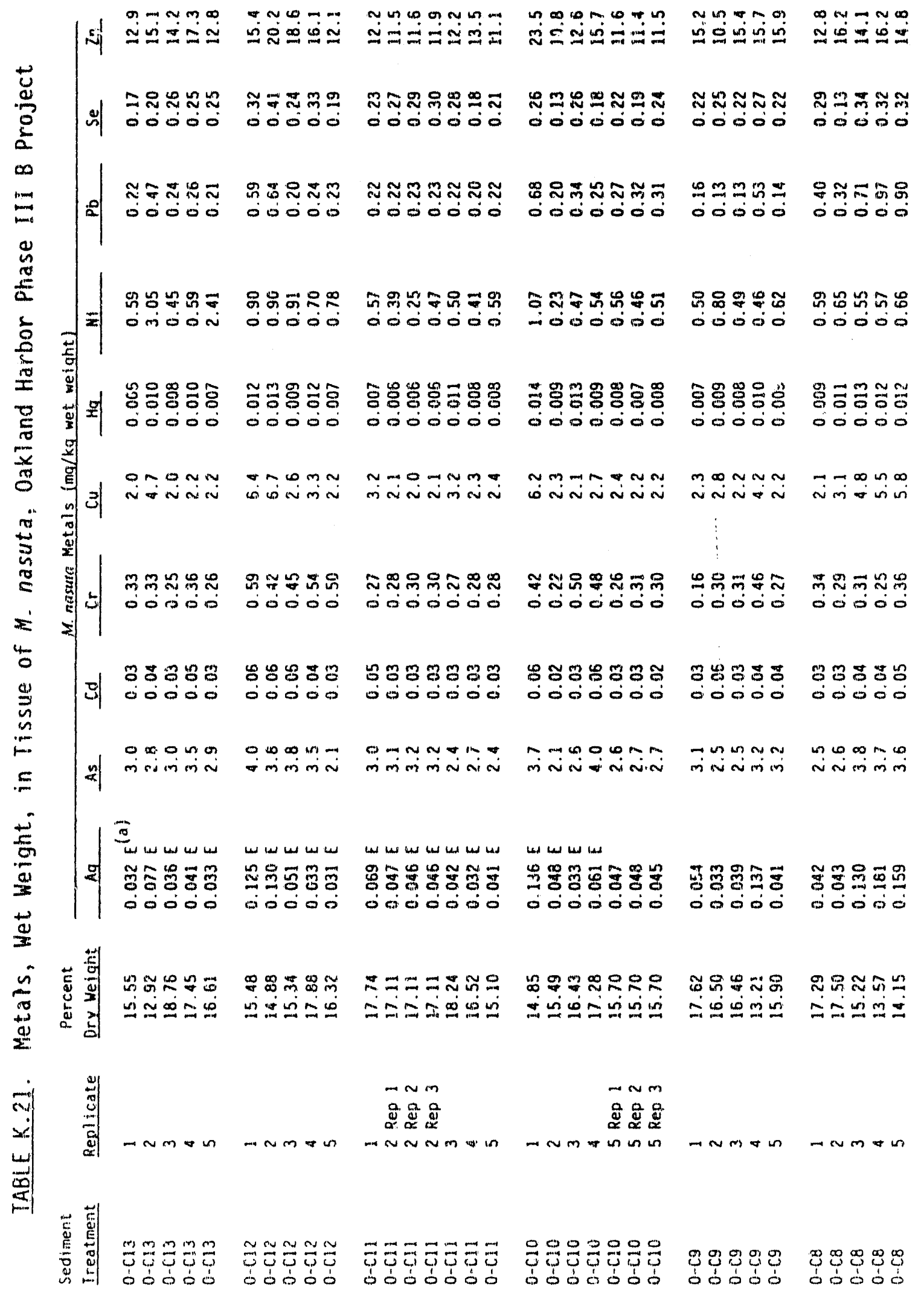




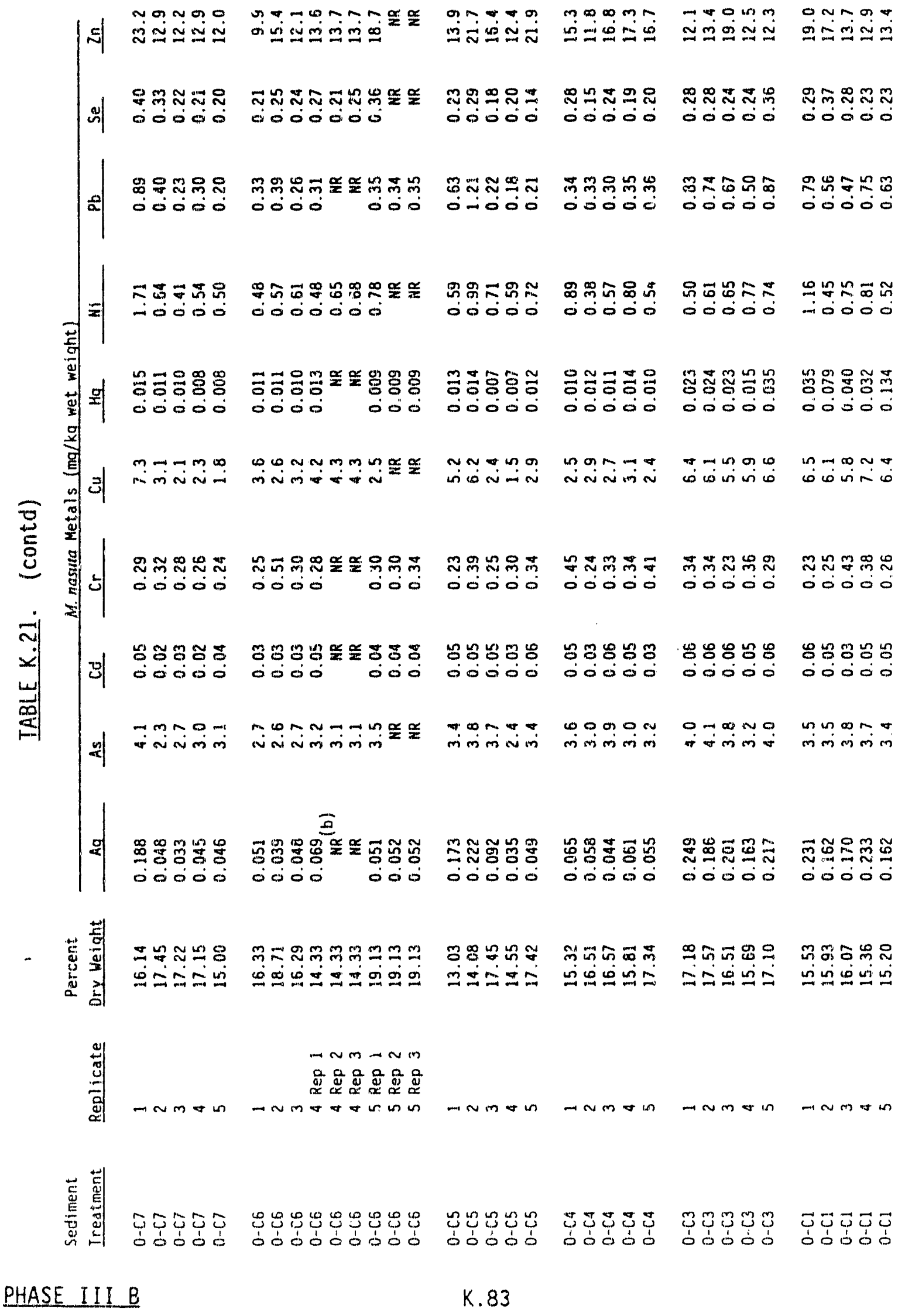




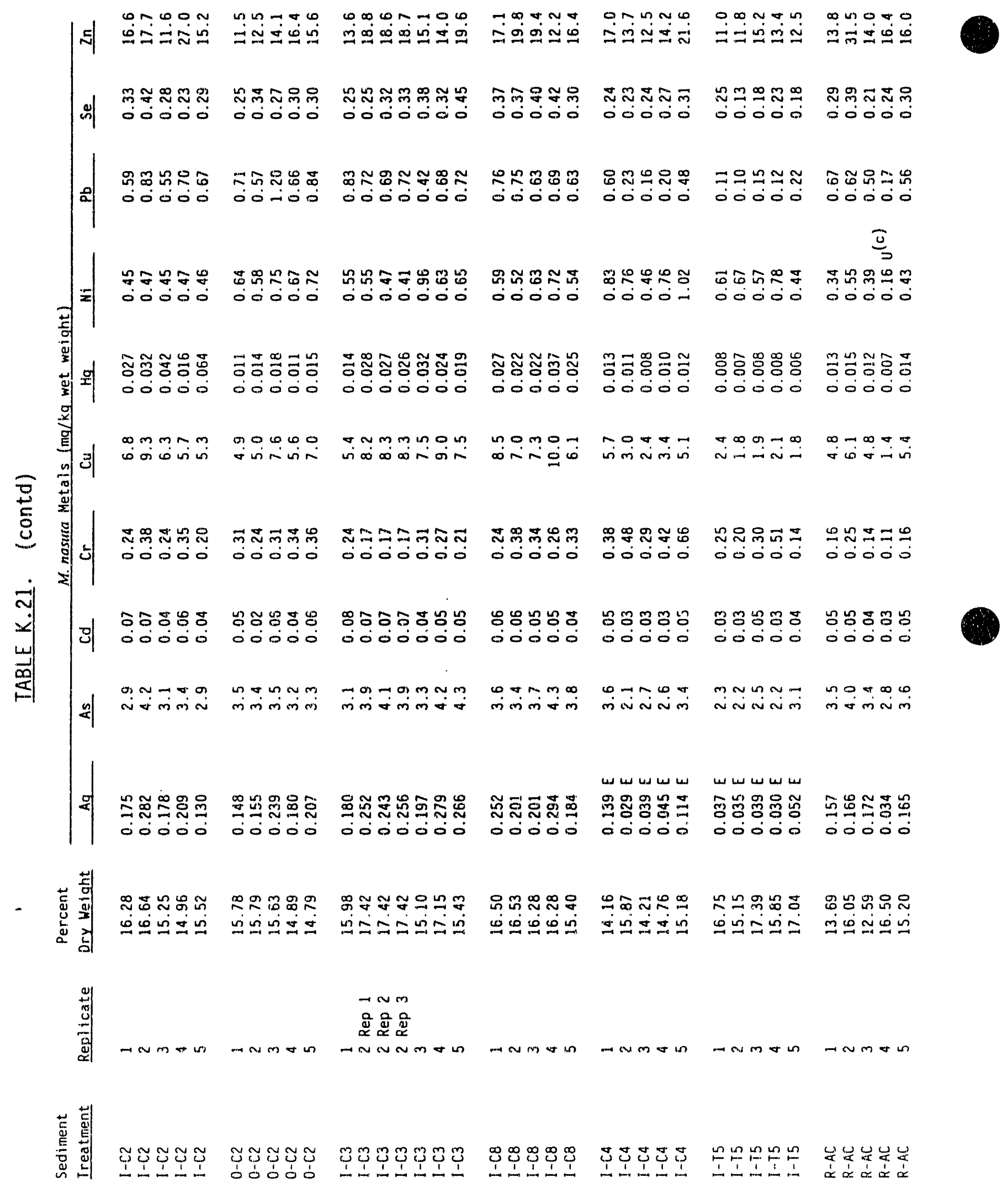




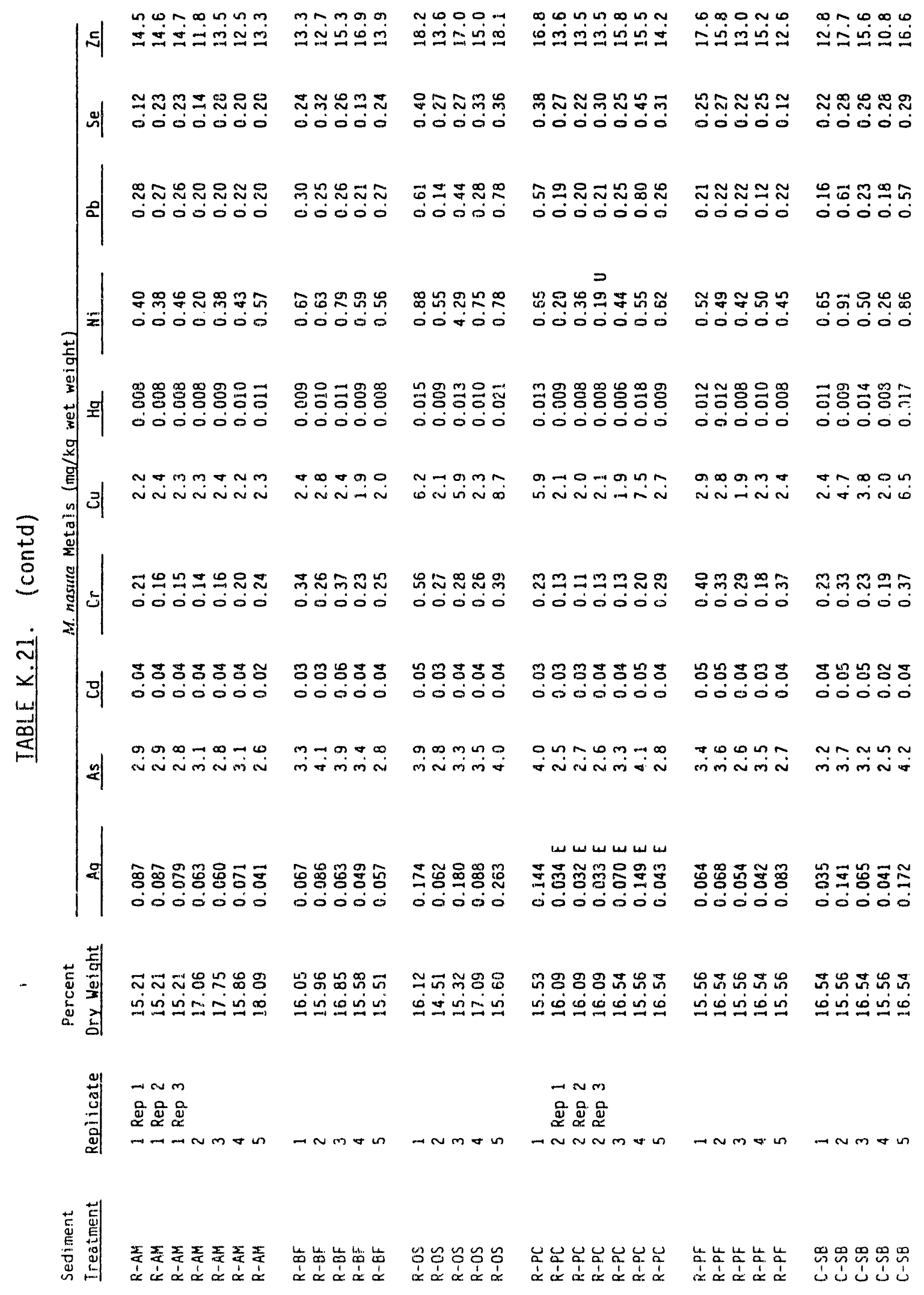




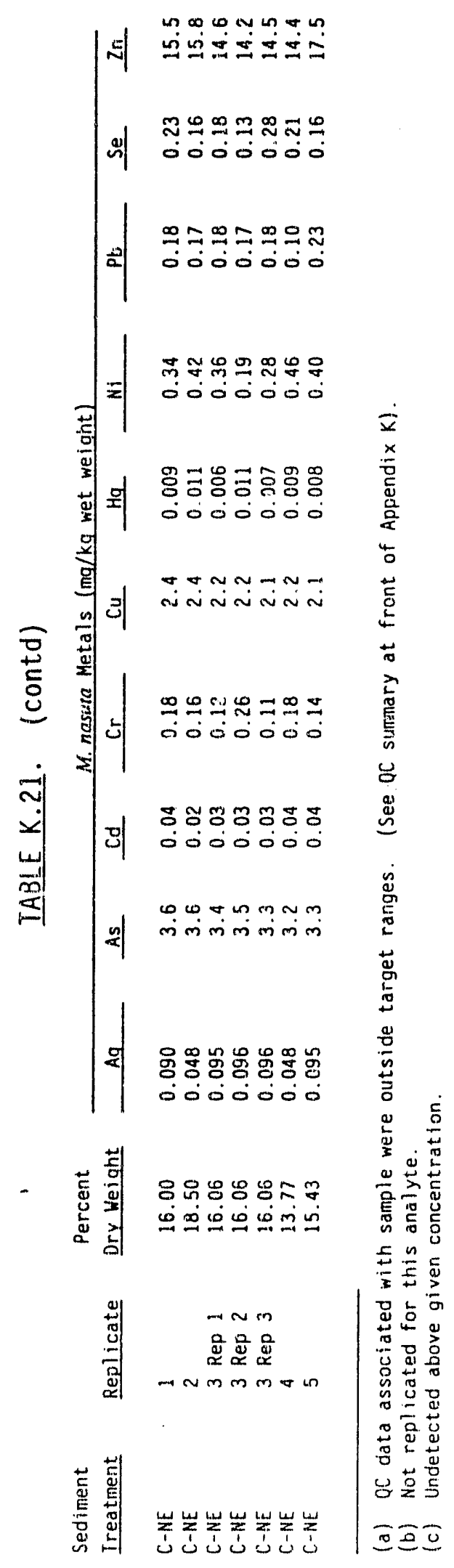

PHASE III B 


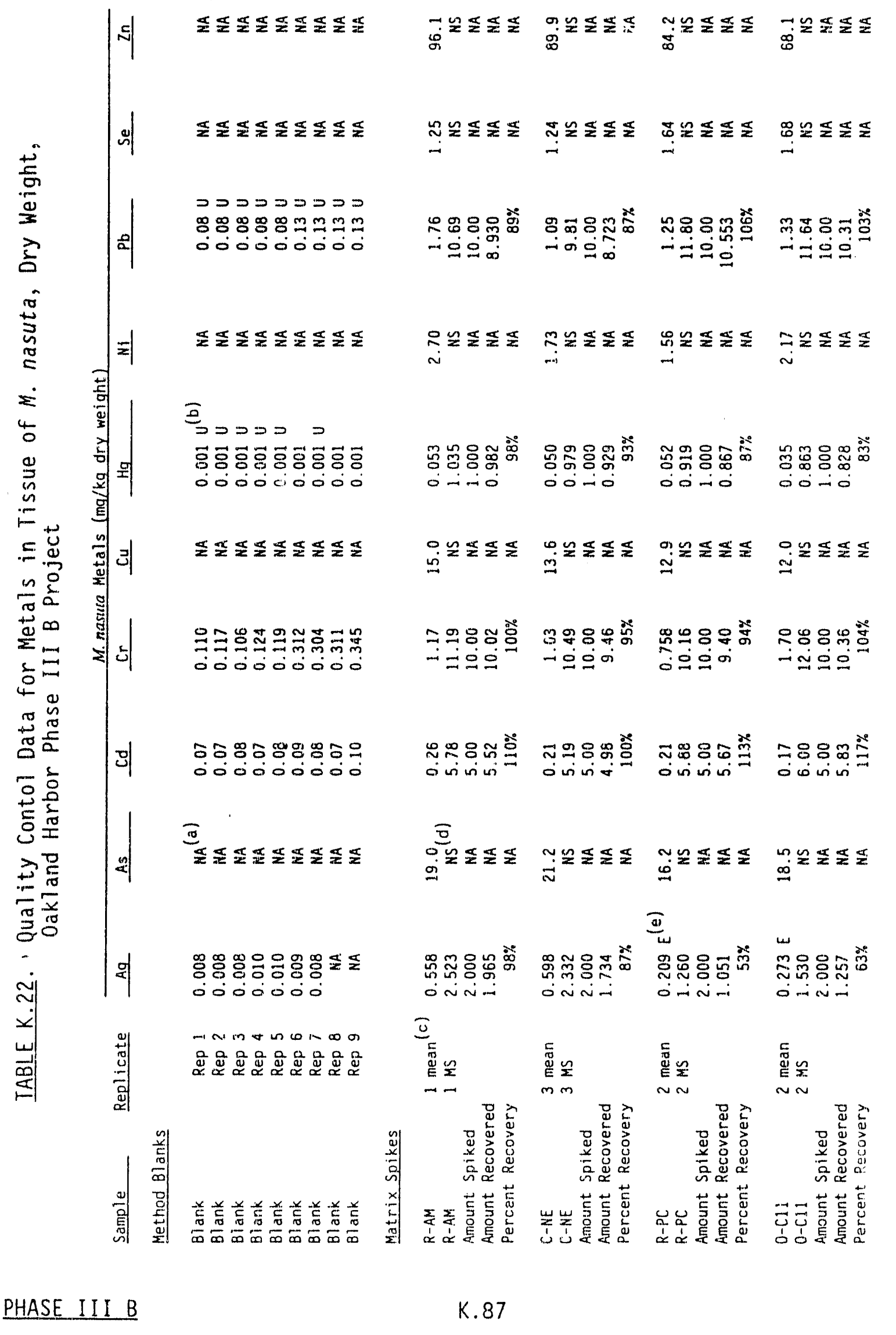




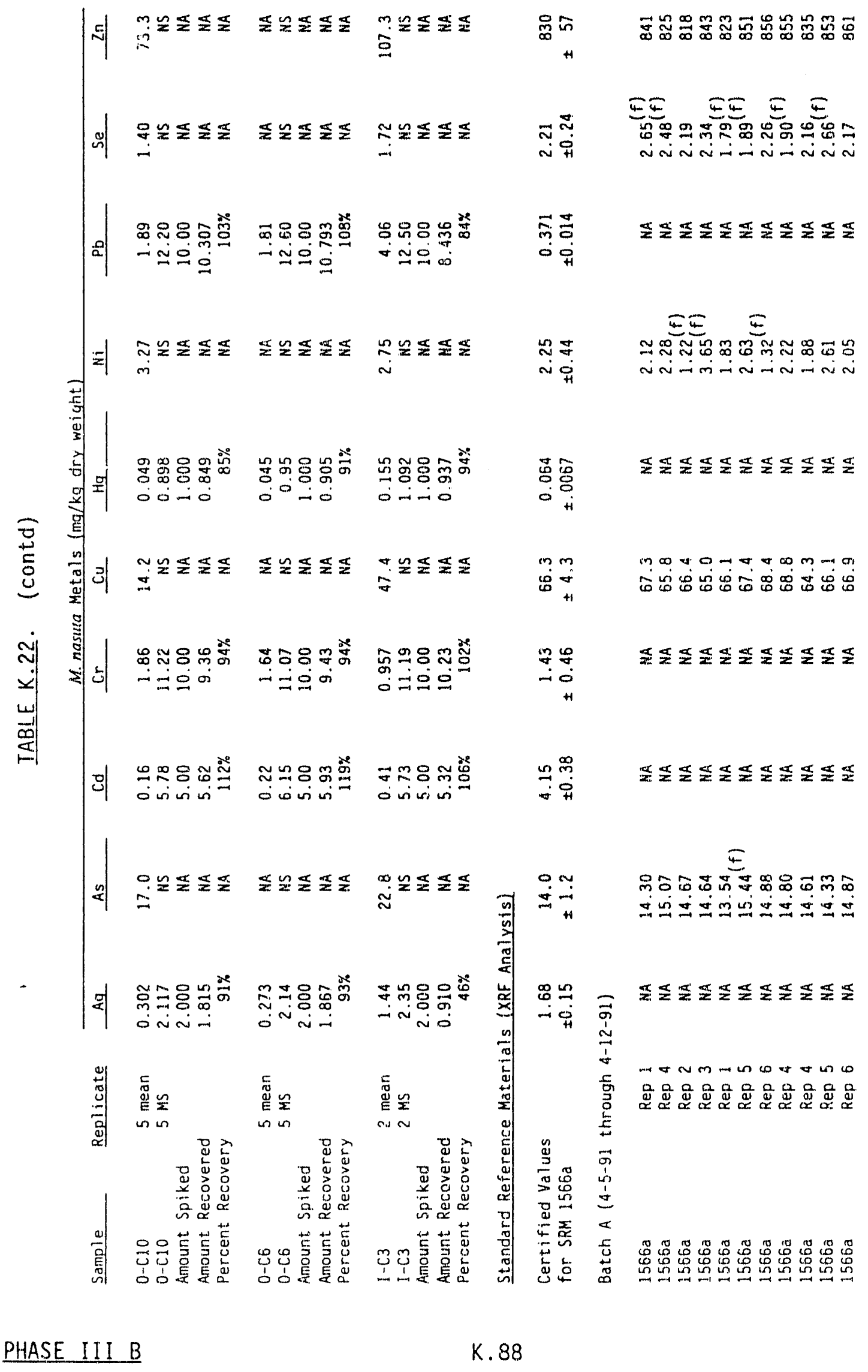




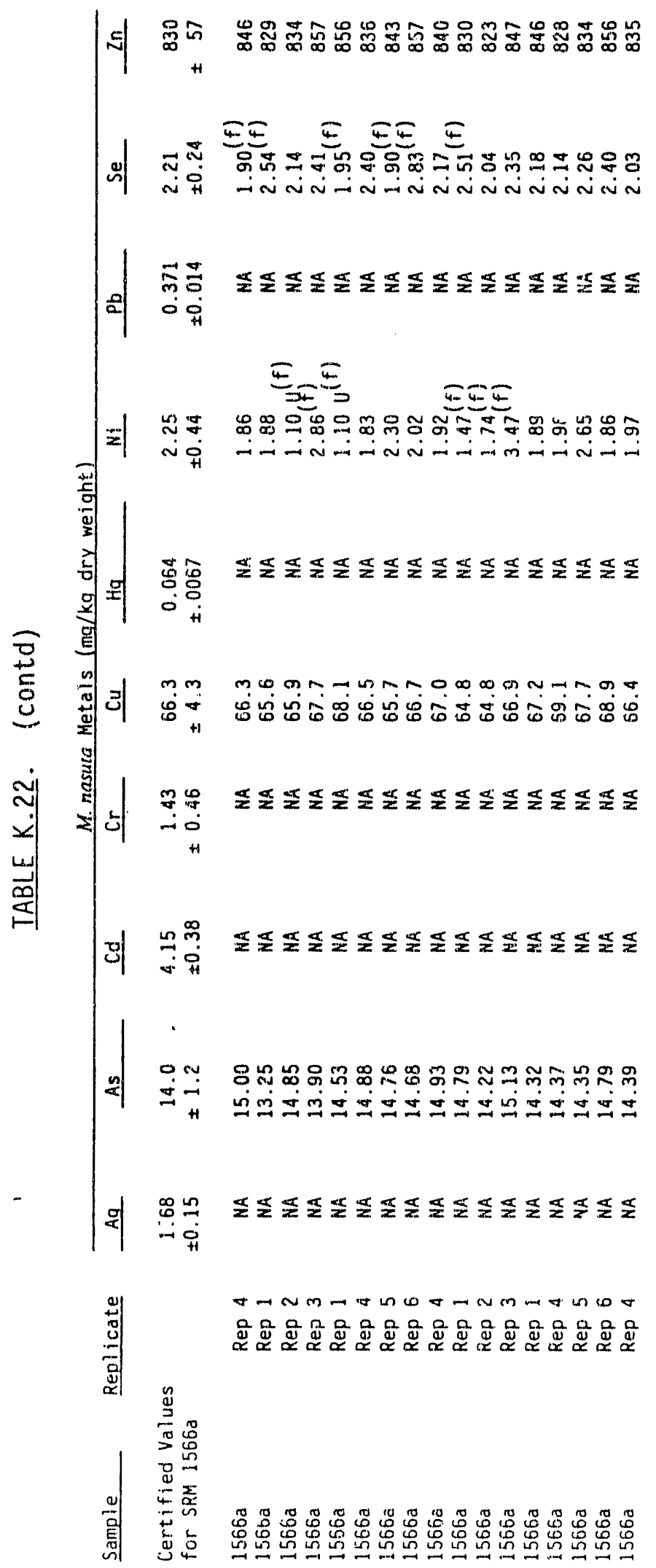

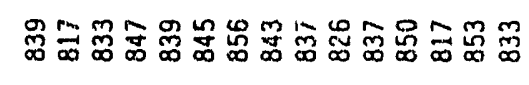

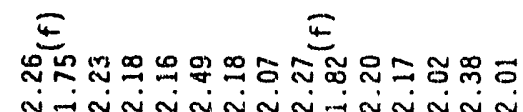

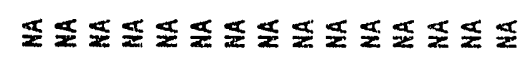

E $4 \pi$

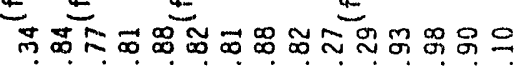

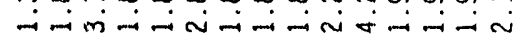

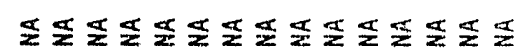

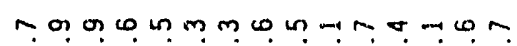

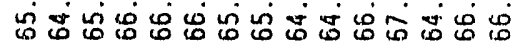

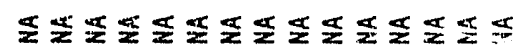

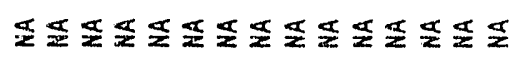

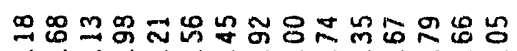

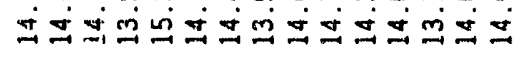

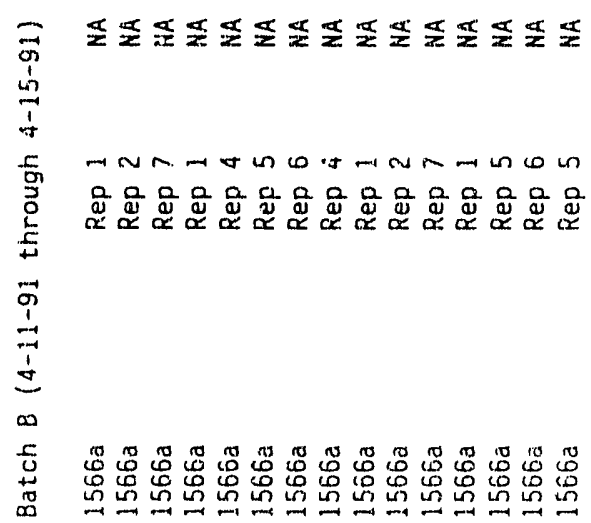




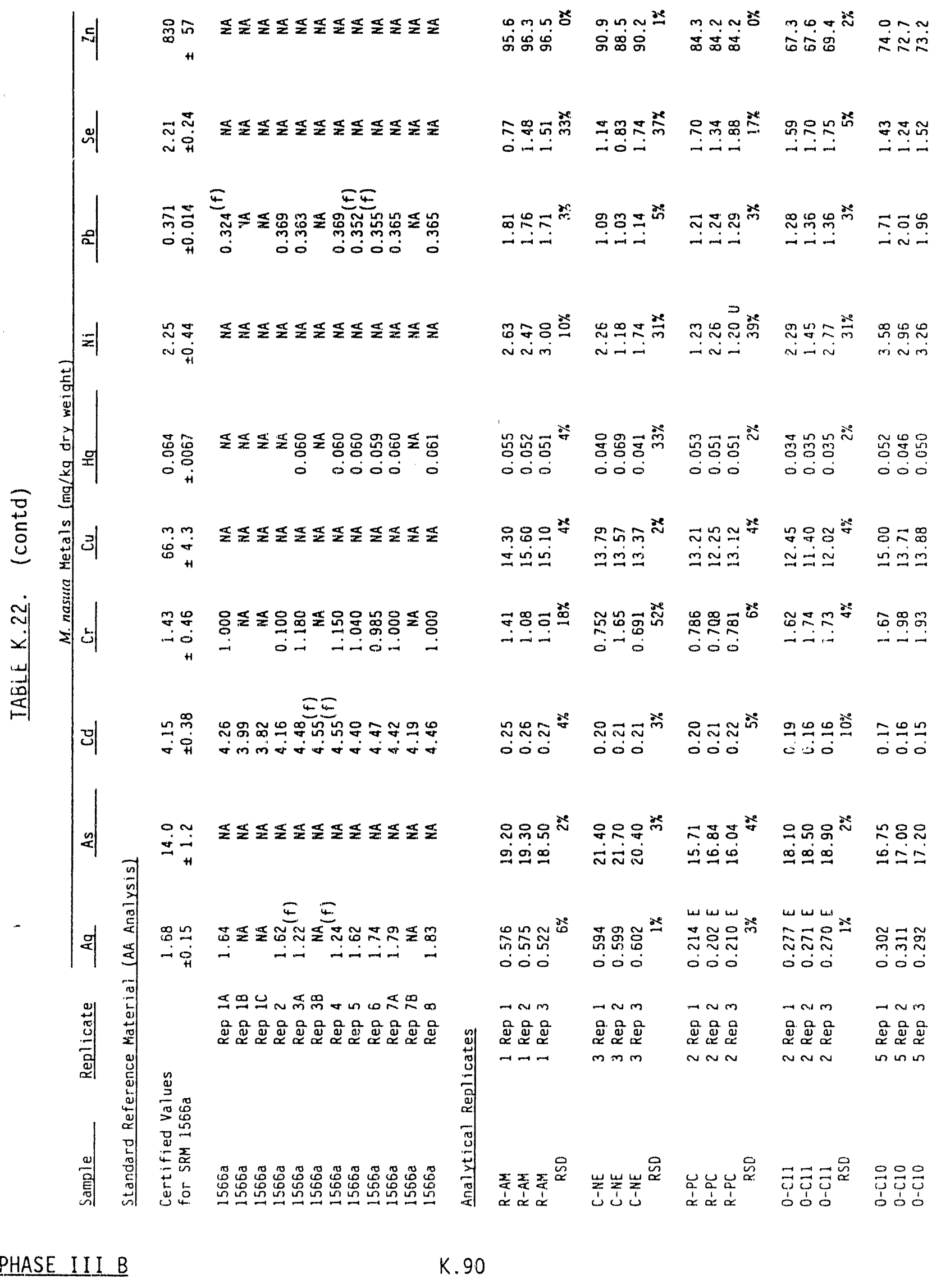




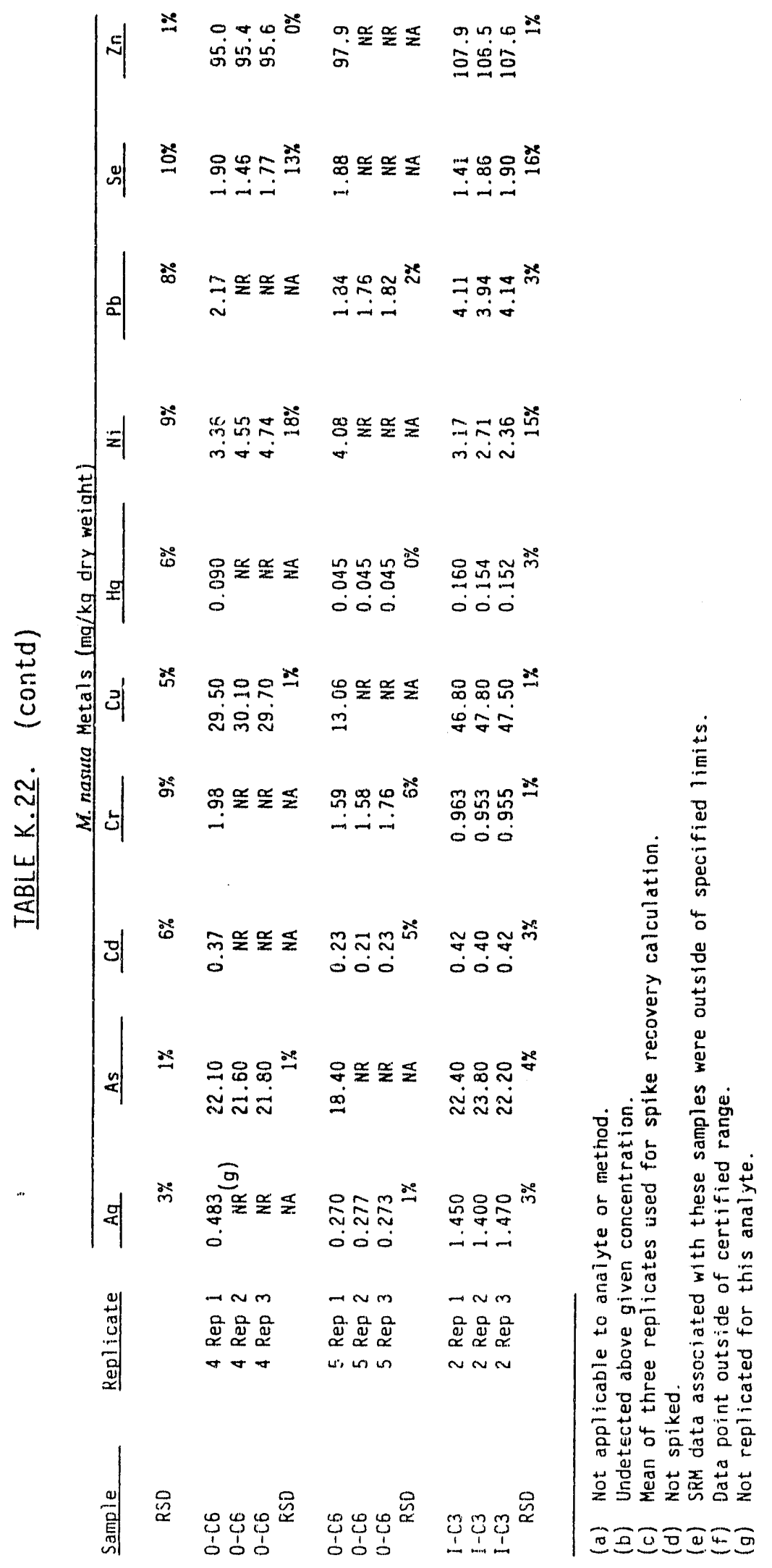




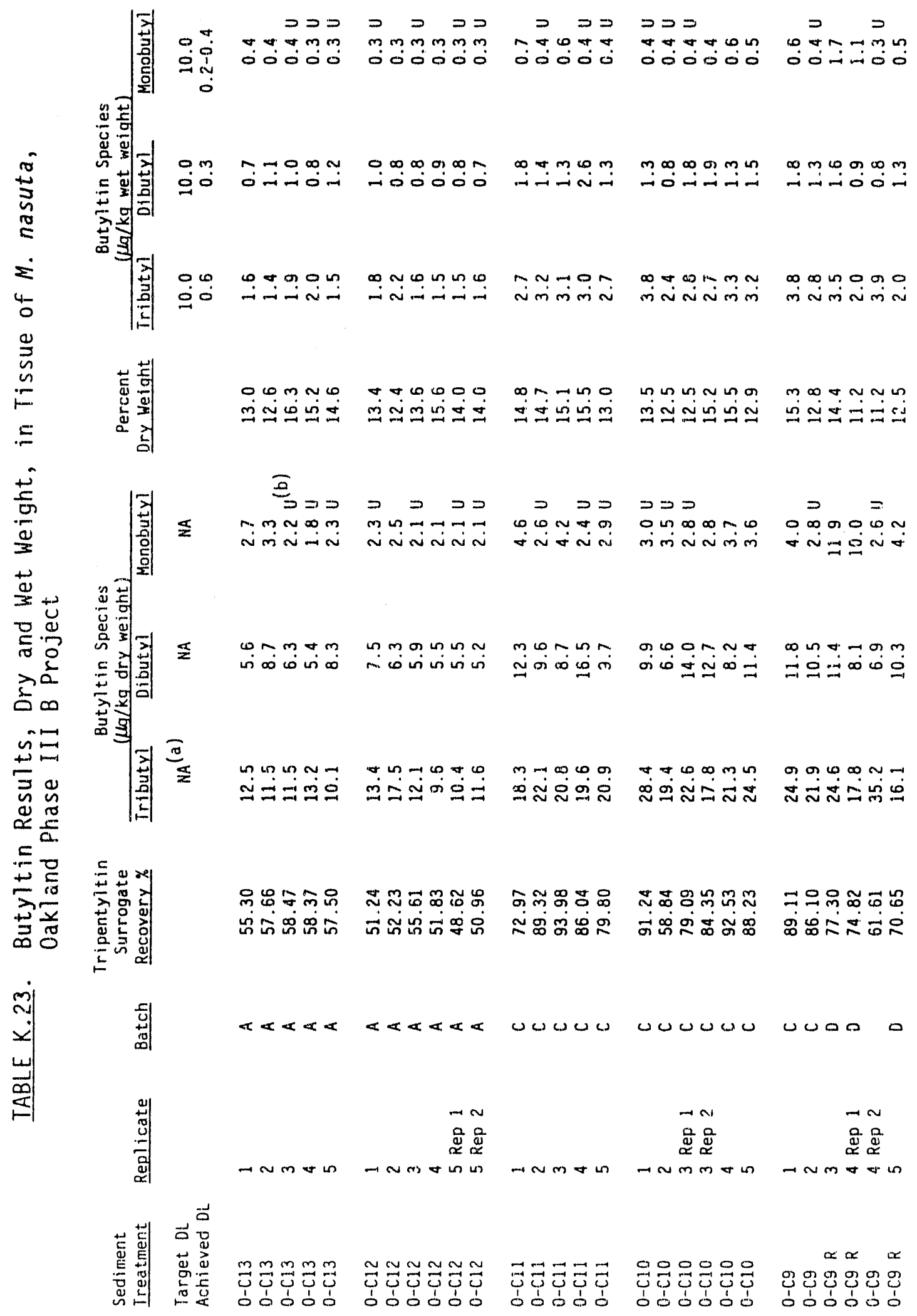

PHASE III B 
ornouso $>$

$P=\geq$

$\omega \dot{4} \mathrm{mmm}$

-íión fó00

-0000 000000

ํำ

$=$

- n $\sin \infty$ ن vió

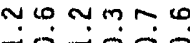

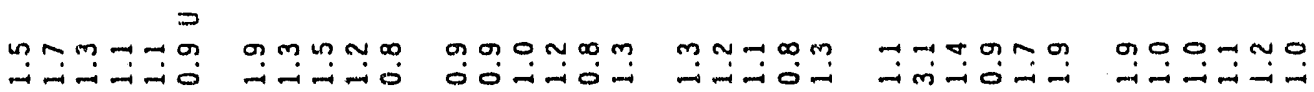

or

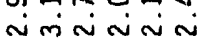

กับ

m

mำ

ต

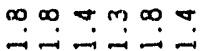

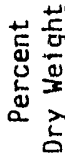

o. ㄱ. Tmon ตำํำำำ

ต พับ

on $007-n$

$-\cos 00$ $\ddot{m} \ddot{m} \underset{\sim}{m} \dot{m}$

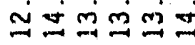

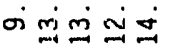

ㅇำ บ 6

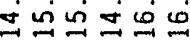

Tก ก พ $m \square m m$

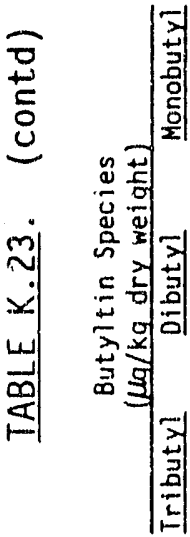

$n+\Delta n-m$

3

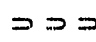

क⿺辶一兀由

NmN No

mos

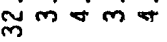

ชnini-

m

$>$

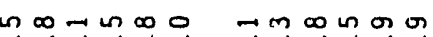

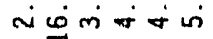

$\infty \dot{\infty} \infty \dot{\sim} \dot{0}$

$\rightarrow \infty m \sigma \infty 0$ 门’ ก

บับ $0=1$

$m+0 \quad 0$

$\neg \infty-\infty-\infty$ लंळं

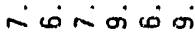

¿ $\infty \infty \omega \infty$

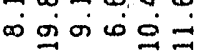

$\forall \infty \infty \sim \pi$ in $\simeq \omega \sim \infty$ i

\section{유.} $\infty 0=0$

पm $m$ 就表

m 0 n in $\infty$ $\pm \cong \dot{\square}=\dot{\square}$

$m \infty n-1 n$ مّذ்

$+\infty$ ot $\infty$ in

con 0 on $\because=000 \infty$

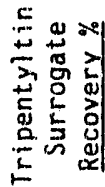

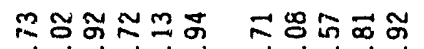

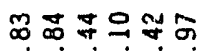

으요

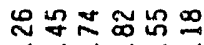

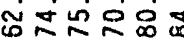

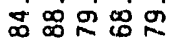

只过的姐品

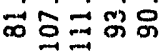

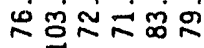

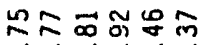

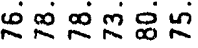

矛

D00000 00س

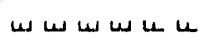

-

ーோடルே

IIIx I

$\rightarrow \sim$

웜 웅

$-\sim$

Ham $\rightarrow$ an $\rightarrow$ n

윰 염

TतNom or

- $ง \mathrm{~m} v \operatorname{Ln}$

$\rightarrow$ N

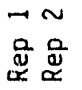

$-\sim$

这虽

十 Nmm

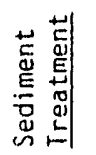

$\propto \propto \alpha \propto \alpha \propto$

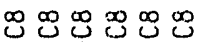

$\propto \alpha \propto \alpha \propto$

$\alpha, \alpha$

$\alpha \alpha$

$x \alpha x \alpha$

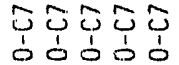

بو

إِ

$\propto x \propto x$

$\propto \propto \alpha \propto \alpha$ 1́ أ́t

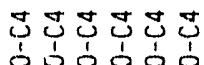

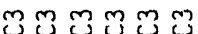

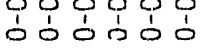


wm n

0000

○ंல்

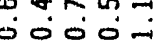

i.00000

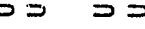

mmmmm

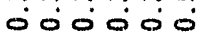

$\infty$ or m in

-

o.

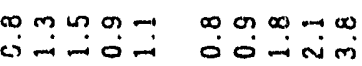

$\mathscr{0}$

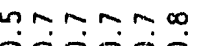

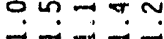

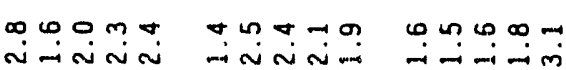

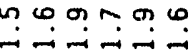

$\infty+i=\infty$ in

$\infty$

ก N $\operatorname{in}$

苍茊

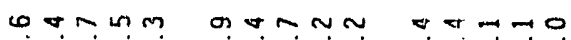

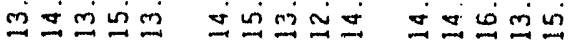

$m 0 \div-\div$

ต่ำ

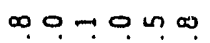
$\cong \pm \Xi \dot{\square} \cong$

-mom 네욤

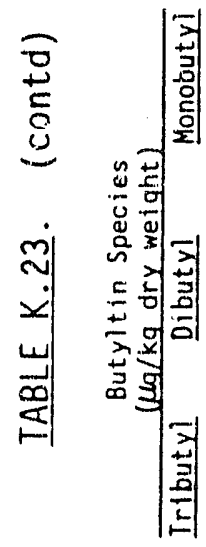

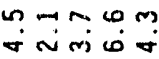

บับขึ

on $\rightarrow 00$

in 0 m n

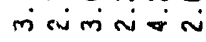

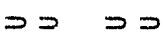

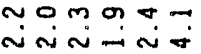

$\because+m \sim$ $\doteq N+\sim m$

$\infty \Rightarrow m \&$ in

inisio

m

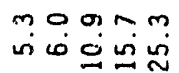

กต ต

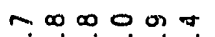
in 10 on

$m+\nabla$ witi

in ம $\dot{\infty} \infty \dot{0}$

m. n 过 $=\dot{\square}$

กตั

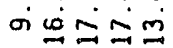

$m m-m$ m $\Rightarrow 0 \circ \dot{0}$

or $\cos$ in $\cos$ कांत्रिक

ชกำ $\sim$ $\forall \cong \simeq m$

$\sim \sim \infty \quad \infty$ $\infty \underset{\infty}{\infty} \infty$

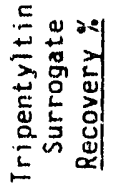

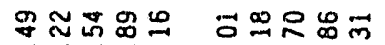

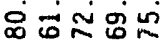
주요요

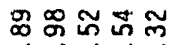
노엥

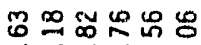
ति용요

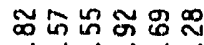

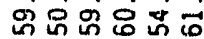

㛡合命

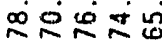

矛

ט

0000

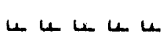

ט ט ט ט ט

$\infty \infty \infty \infty \infty$

ט ט

밈

tnm

- Nm ton

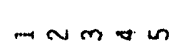

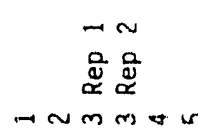

证

ज Nmण in

-1 Nmon

$\rightarrow \sim m$ in

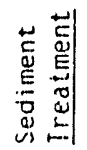

$\propto \propto \alpha \propto$

$\alpha$ a o $\alpha \propto x$

$\alpha \propto \alpha \propto \alpha$

$\alpha \propto \alpha \propto \alpha$

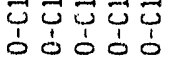

行足足

ํํำ

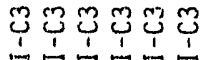

过可可

$\alpha \propto \alpha \propto \alpha$

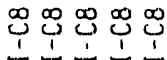


$\Rightarrow v=$

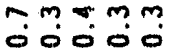

$\cos -\infty$ कं
$5>$

- Dnn mana

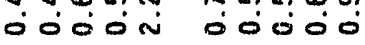

$=0$

10 几 m

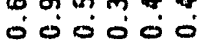

$\Rightarrow=$ 0ं

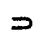
$m \square 0 \sigma$ एं

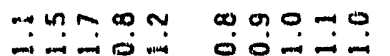
ஊ $\dot{0} \dot{0} \dot{0}$ $\infty \sim \infty m$

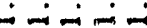

Unण

$\dot{i n} \dot{-i} \dot{\sim} \dot{m}$

जmm on inin - i.

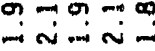

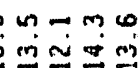

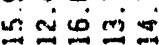

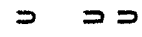

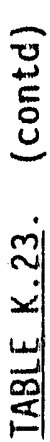

manmmm

$\sim \sim m m$ vim

$\Rightarrow \rightarrow$

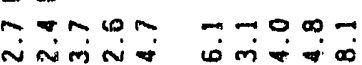

an an m $\rightarrow \infty \omega n$ N

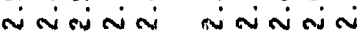

$\forall \infty \infty \pi 0$

Noon m

$\infty+\infty \pi n$

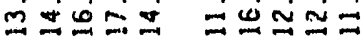

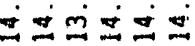

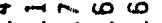

$\pm \dot{m} \operatorname{in} \infty$

$+\operatorname{aro}$

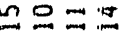

YMon

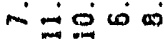

?mo $00 m$

क ⿻心㇒日

$\infty$
$=\infty$
$=\infty$

moim

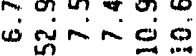

on un?

ம்

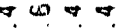

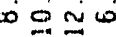

in $\ln +4 \rightarrow$ wi $x$ 메

7 on $\pm n \dot{m}=$

ช พ

सक्षण

a 0 \%

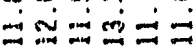

$\because$ 엥 $\infty$ \pm ion

$\Rightarrow \infty$ or 10

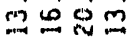

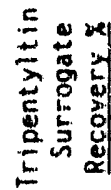

8 ํㅜ용

品芯施

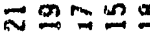

워롱

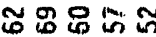

禹禹虫员

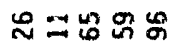

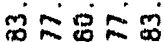

용요 용요

ติำ요응

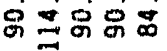

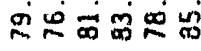

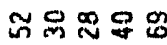

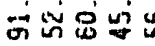

两的界专

휜

$\infty \infty \infty<$
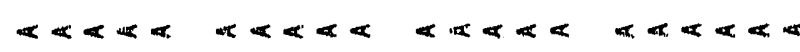

an $\infty$

$x<x<$

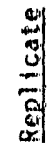

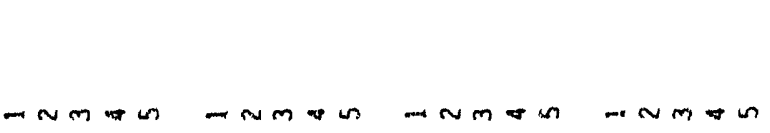

$\rightarrow \infty$

웜

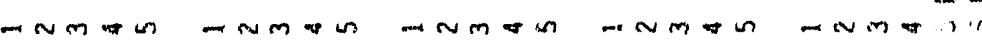

蔗

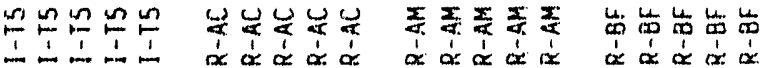

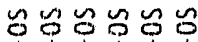

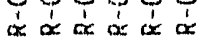

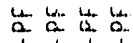
$\frac{1}{x} \alpha \frac{1}{x} \alpha$ 

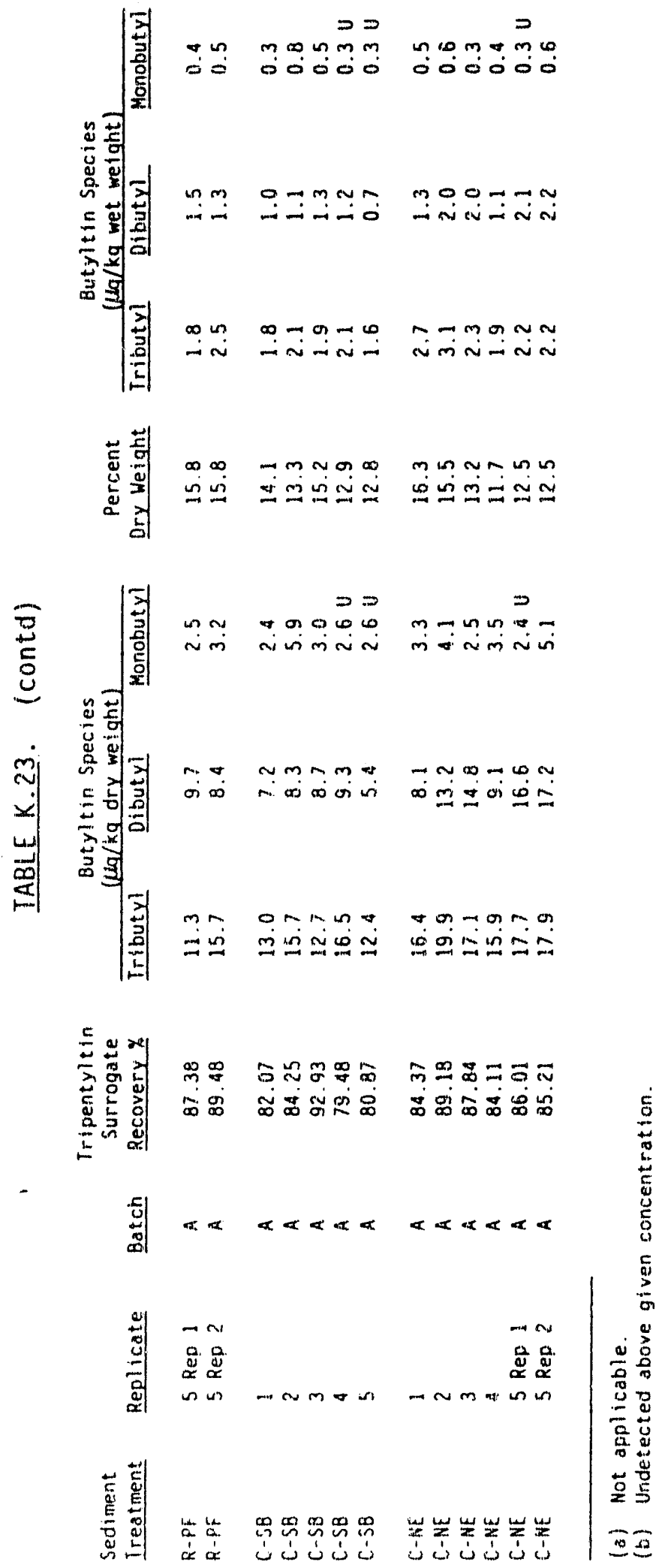

PHASE III B K.96 
TABLE K.24. Quality Control Data for Butyltins Aralyses in Tissue of M. nasuta, Oakland Harbor Phase III B Project

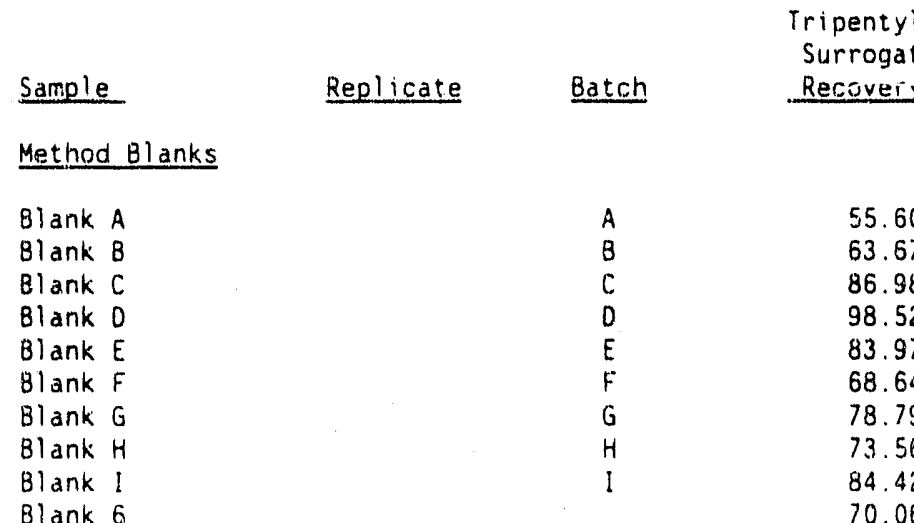

Tripentyltin

rogate

55.60

63.67

86.98

98.52
83.97

68.64

78.79

73.56

70.06

5 mean $(b)$

5 MS

$R-P F$

Anount Spiked

Recovery

Percent Recovery

C-SB 3

$C-S B$

Amount Spiked

Recovery

Percent Recovery

R-PC
$R-P C$
Amount Spiked
Recovery
Percent Recovery

I-TS
I-TS
$\quad$ Amount Spiked
Recovery
Percent Recovery

$\begin{array}{ll}0-C 13 & 1\end{array}$

Anount Spiked

Recovery

Percent Recovery

$0-C 9$

O-C9 ? MS

Amount Spiked

Recovery

Percent Recovery

$\begin{array}{ll}0-C 8 & R \\ 0-C 8 & R\end{array}$

Amount spiked

Recavery

Percent Recovery
A $\quad A^{(c)}$

80.05

13.5
457.0
633.0
443.5

$70 \%$

92.93

78.65

A

12.7

482.3

633.0

469.6

$74 \%$

91.52

87.35

14.1
548.5

685.0

534.4

$78 \%$

69.52

62.35

18.5

402.2

741.0

$383 . ?$

$52 \%$

55.30

53.28

$A$
$A$

12.5

383.8

769.0

3713

$48 \%$

86.10

87.22

2]. 9

669.4

781.0

647.5

$83 \%$

75.92

76.48

174

764.7

10750

7473

$70 \%$

$2.2 U^{(a)}$
$2.1 U$
$2.6 U$
13.6
$3.9 U$
3.2
3.5
3.3
$2.6 U$
3.5

$2.1 \mathrm{U}$

2.7

$2.6 \mathrm{U}$

45.8

6.1

6.2

19.3

18.3

9.1

5.4 
IABLE K.24. (contd)

\begin{tabular}{|c|c|c|c|c|c|c|}
\hline \multirow{2}{*}{ Sampie } & & & \multirow{2}{*}{$\begin{array}{l}\text { Tripentyltin } \\
\text { Surrogate } \\
\text { Recovery } \%\end{array}$} & \multicolumn{3}{|c|}{ Butyltin Species $(\mu \mathrm{g} / \mathrm{kg} d r y$ weight $)$} \\
\hline & Replicate & Batch & & Iributyl & Dibutyl & Monobutyl \\
\hline Analytical & & & & & & \\
\hline $\begin{array}{l}R-P F \\
R-P F \\
\quad R P D \\
\quad 1-S \text { tat }\end{array}$ & $\begin{array}{l}5 \text { Rep } 1 \\
5 \text { Rep ? }\end{array}$ & $\begin{array}{l}A \\
A\end{array}$ & $\begin{array}{l}87.38 \\
89.48\end{array}$ & $\begin{array}{l}11.3 \\
15.7 \\
33 \% \\
0.16\end{array}$ & $\begin{array}{c}9.7 \\
8.4 \\
14 \% \\
0.07\end{array}$ & $\begin{array}{l}2.5 \\
3.2 \\
2.5 \% \\
0.12\end{array}$ \\
\hline $\begin{array}{l}C-N E \\
C-N E \\
\quad R P D \\
\quad \text { I-Stat }\end{array}$ & $\begin{array}{l}5 \operatorname{Rep} 1 \\
5 \operatorname{Rep} 2\end{array}$ & $\begin{array}{l}A \\
A\end{array}$ & $\begin{array}{l}86.01 \\
85.21\end{array}$ & $\begin{array}{l}17.7 \\
17.9 \\
1 \% \\
0.01\end{array}$ & $\begin{array}{l}16.6 \\
17.2 \\
4 \% \\
0.02\end{array}$ & $\begin{array}{l}2.4 U \\
5.1 \\
\text { NA } \\
\text { NA }\end{array}$ \\
\hline $\begin{array}{l}\text { R-OS } \\
\text { R-OS } \\
\text { RPD } \\
\quad 1-S t a t\end{array}$ & $\begin{array}{ll}5 & \operatorname{Rep} 1 \\
5 & \operatorname{Rep}\end{array}$ & $\begin{array}{l}A \\
A\end{array}$ & $\begin{array}{l}78.22 \\
85.07\end{array}$ & $\begin{array}{l}11.1 \\
11.0 \\
1 \% \\
0.00\end{array}$ & $\begin{array}{l}10.9 \\
10.6 \\
3 \% \\
0.01\end{array}$ & $\begin{array}{l}2.6 U \\
2.6 U \\
\text { NA } \\
\text { NA }\end{array}$ \\
\hline $\begin{array}{l}\text { I-C4 } \\
\text { I- }-C 4 \\
\quad R P D \\
\text { I-Stat. }\end{array}$ & 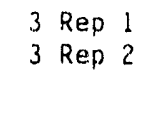 & $\begin{array}{l}B \\
B\end{array}$ & $\begin{array}{l}59.55 \\
60.9 ?\end{array}$ & $\begin{array}{l}12.2 \\
12.2 \\
0 \% \\
0.00\end{array}$ & $\begin{array}{l}4.8 \\
5.0 \\
4 \% \\
0.02\end{array}$ & $\begin{array}{l}2.3 \\
1.9 U \\
N A \\
N A\end{array}$ \\
\hline $\begin{array}{c}0-r .12 \\
0-C 12 \\
\text { RPD } \\
\quad 1-\text { Stat }\end{array}$ & $\begin{array}{l}5 \text { Rep } 1 \\
5 \text { Rep } 2\end{array}$ & $\begin{array}{l}A \\
A\end{array}$ & $\begin{array}{l}48.62 \\
50.96\end{array}$ & $\begin{array}{l}10.4 \\
11.6 \\
11 \% \\
0.05\end{array}$ & $\begin{array}{l}5.5 \\
5.2 \\
6 \% \\
0.03\end{array}$ & $\begin{array}{l}2.1 U \\
2.1 U \\
N A \\
N A\end{array}$ \\
\hline $\begin{array}{l}0-C 10 \\
0-C 10 \\
R P D \\
\text { J-Stat }\end{array}$ & $\begin{array}{l}3 \text { Rep } 1 \\
3 \operatorname{Rep} 2\end{array}$ & $\begin{array}{l}c \\
C\end{array}$ & $\begin{array}{l}79.09 \\
84.35\end{array}$ & $\begin{array}{l}22.6 \\
17.8 \\
24 \% \\
0.12\end{array}$ & $\begin{array}{l}14.0 \\
12.7 \\
10 \% \\
0.05\end{array}$ & $\begin{array}{l}2.8 \mathrm{U} \\
2.8 \\
\mathrm{NA} \\
\mathrm{NA}\end{array}$ \\
\hline $\begin{array}{l}0-C 9 \\
0-c 9 \\
\quad R P D \\
\text { I-stat }\end{array}$ & $\begin{array}{l}4 \text { Rep } 1 \\
4 \text { Rep } 2\end{array}$ & D & $\begin{array}{l}74.82 \\
61.61\end{array}$ & $\begin{array}{l}17.8 \\
35.2 \\
35 \% \\
0.18\end{array}$ & $\begin{array}{l}8.1 \\
6.9 \\
49 \% \\
0.25\end{array}$ & $\begin{array}{l}10.0 \\
2.6 \mathrm{U} \\
\mathrm{NA} \\
\mathrm{NA}\end{array}$ \\
\hline $\begin{array}{c}0-C B R \\
0-C B R \\
R P D \\
1-\text { Stat }\end{array}$ & $\begin{array}{ll}1 & \text { Rep } 1 \\
1 & \text { Rep } 2\end{array}$ & $\begin{array}{l}0 \\
0\end{array}$ & $\begin{array}{l}62.73 \\
74.02\end{array}$ & $\begin{array}{l}18.0 \\
19.3 \\
7 \% \\
0.03\end{array}$ & $\begin{array}{c}9.1 \\
10.6 \\
15 \% \\
0.08\end{array}$ & $\begin{array}{c}6.5 \\
4.4 \\
39 \% \\
0.19\end{array}$ \\
\hline $\begin{array}{l}0-C 6 \\
O-C 6 \\
\quad R P D \\
\quad 1-5 t a t\end{array}$ & $\begin{array}{l}3 \text { Rep } 1 \\
3 \text { Rep } 2\end{array}$ & $\begin{array}{l}E \\
E\end{array}$ & $\begin{array}{l}81.44 \\
78.10\end{array}$ & $\begin{array}{l}14.7 \\
14.5 \\
1 \% \\
0.01\end{array}$ & $\begin{array}{c}7.9 \\
9.1 \\
14 \% \\
0.07\end{array}$ & $\begin{array}{l}2.6 \mathrm{U} \\
2.4 \mathrm{U} \\
\mathrm{NA} \\
\mathrm{NA}\end{array}$ \\
\hline $\begin{array}{l}0-C A R \\
O-C A \\
R P O \\
I-5 t a t\end{array}$ & $\begin{array}{l}5 \text { Rep } 1 \\
5 \text { Rep } 2\end{array}$ & $\begin{array}{l}F \\
F\end{array}$ & $\begin{array}{l}83.55 \\
79 \quad 18\end{array}$ & $\begin{array}{l}19.4 \\
18.9 \\
3 \% \\
0.01\end{array}$ & $\begin{array}{l}10.4 \\
11.6 \\
11 \% \\
0.05\end{array}$ & $\begin{array}{c}4.8 \\
5.0 \\
4 \% \\
0.02\end{array}$ \\
\hline $\begin{array}{l}1-C 3 R \\
1-C 3 \\
R P D \\
\quad 1-5 t a t\end{array}$ & $\begin{array}{l}3 \text { Rep } 1 \\
3 \text { Rep } ~\end{array}$ & $\begin{array}{l}G \\
G\end{array}$ & $\begin{array}{ll}80 & 82 \\
70.76\end{array}$ & $\begin{array}{l}12.7 \\
11.5 \\
10 \% \\
0.05\end{array}$ & $\begin{array}{l}8.6 \\
7.7 \\
11 \% \\
0.06\end{array}$ & $\begin{array}{l}3.0 \\
2.3 \\
26 \% \\
0.13\end{array}$ \\
\hline
\end{tabular}




\section{IABLE K.24. (contd)}

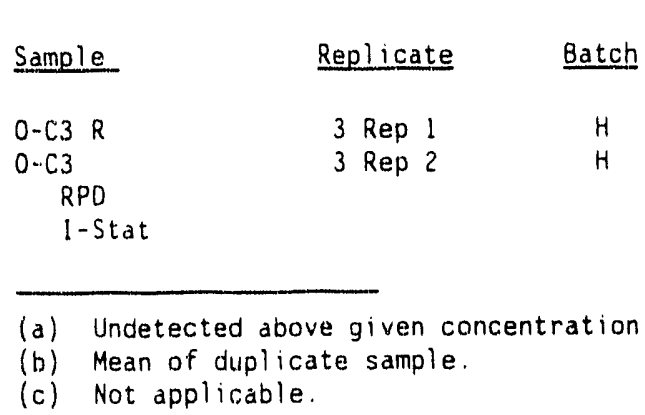

Tripentyltin

Surrogate

Recovery \%

78.81

73.92
Butylt in Species $(\mu \mathrm{g} / \mathrm{kg}$ dry weight)

Iributyl Dibuty

10.7
10.0
$7 \%$
0.03

7.8
8.2
$5 \%$
0.02

8. 8

2.5

$112 \%$

0.56

(b) Mean of duplicate sample.

(c) Not applicable. 
APPENDIX L

N. CAECOIDES TISSUE CHEMISTRY AND QUALITY CONTROL DATA 


\section{QA/QC SUMMARY}

PROGRAM:

PARAMETER:

LABORATORY:

MATRIX:

SAMPLE NUMBER:

HOLDING TIMES

DETECTION LIMITS

METHOD BLANKS

SURROGATES

MATRIX SPIKES

REPLICATES

SRMs
Oakland Harbor Phase III B

Polynuclear Aromatic Hydrocarbons (PAHs)

Alden Analytical Laboratories, Inc.

$N$. caecoides Tisssue

130 (26 treatments, 5 replicates each)

Samples of $N$. caecoides tissues were held frozen for up to 2 months prior to extraction and analysis. All sample extracts were analyzed within the EPArecommended extract holding time of 40 days (EPA 1986).

Target detection limits (Table 2.2) were achieved for all PAH compounds.

Seven method blanks were analyzed. There were no PAHs detected in any of the blanks.

Four radiolabeled PAH compounds were added as surrogates to samples prior to analysis: $d_{8}$ naphthalene, $d_{10}$ acenaphthene, $d_{10}$ phenanthrene, and $d_{12}$

benzo(a)pyrene. The target recovery for surrogates of $50 \%$ to $125 \%$ was achieved with the following exceptions: $d_{8}$ naphthalene was recovered from 19 samples at $1 \%$ to $49 \%$; $d_{10}$ acenaphthene was recovered from two samples at $43 \%$ and $49 \%$. Laboratory protocol requires reextraction only if two or more surrogates are below $20 \%$. This condition did not occur in any samples, therefore, the data were not qualified.

Nineteen samples were spiked with all 16 PAI compounds of interest: one single matrix spiked blank; six matrix spike duplicate blanks, for a total of 12 ; and six samples. Spike recoveries for LPAH compounds were within the limits specified for each analyte in the project QC plan, ranging from 40-120\%. One sample, the duplicate of matrix spiked blank $B$, had a recovery of anthracene at $1410 \%$. The reason for this outlier is unknown. Spike recoveries for HPAH compounds ranged from $42 \%$ to $220 \%$. The matrix spikes corresponding to the Batches A, C, D, E, F, and G exceeded the limits specified in the project QC plan for at least one HPAH analyce. This condition may bias the results of detected concentrations of fluoranthene, pyrene, chrysene, and benzo(b)fluoranthene in those batches (concentrations of these analytes may actually be lower than reported).

Six samples were analyzed in duplicate to assess precision. Precision was measured by Relative Percent Difference (RPD) between replicates. RSDs were not calculated because there were no analytes detected in any of the replicates.

NOT APPLICABLE 


\author{
PROJECT: \\ PARAMETER: \\ LABORATORY: \\ MATRIX: \\ SAMPLE NUMBER:
}

HOLDING TIMES

DETECTION LIMITS

METHOD BLANKS

SURROGATES

MATRIX SPIKES

\section{QA/QC SUMMARY}

Oakland Phase III B

Chlorinated Pesticides and PCBs

Alden Analytical Laboratories, Inc.

N. Caecoides Tissue

130 (78 treatments, 3 replicates each)

Samples were held frozen prior to extraction. All samples were extracted within 2 months of receipt at Battelle MSL. All sample extracts were analyzed within the EPA-recommended holding time of 40 days (EPA 1986).

Target detection limits for pesticides $(2.0 \mu \mathrm{g} / \mathrm{kg}$ wet weight) were not achieved because of limited sample volumes. The smaller sample volumes resulted in detection limits ranging from 10 to $20 \mu \mathrm{g} / \mathrm{kg}$. Detection limits in five samples were as high as $100 \mu \mathrm{g} / \mathrm{kg}$ for $\partial-\mathrm{BHC}$ because of interfering peaks coeluting with these analytes. Detection limits for toxaphene were also elevated, 500 to $1000 \mu \mathrm{g} / \mathrm{kg}$ wet weight. This elevated detection limit can be attributed to multicomponent toxaphene peaks coeluting with with other pesticide and $\mathrm{PCB}$ peaks on the chromatogram. The higher detection limit was set to ensure accurate quantitation of the pesticide and to avoid false positive detections. Target PCB detection limits $(20 \mu \mathrm{g} / \mathrm{kg}$ wet weight) were also exceeded. Detection limits for aroclors ranged from 100 to $200 \mathrm{\mu g} / \mathrm{kg}$ wet weight, because of limited sample volumes. One batch of samples, Batch C, was inadvertantly spiked with the aroclor 1254 instead of the surrogate compound DBC.

Detection limits for these samples were increased (up to $250 \mu \mathrm{g} / \mathrm{kg}$ ) to account for this error.

Seven method blanks were analyzed. No chlorinated pesticides or PCBs were detected in any of the method blanks.

One compound, dibutylchlorendate (DBC), was added to all samples as a surrogate spike, with the exception of samples in Batch $C$ as described above under Detection Limits. Recoveries for all spiked samples ranged from $34 \%$ to $154 \%$, within the EPA CLP advisory QC limits of $20-157 \%$ for soils and sediments (no limits have been derived for tissues). Although no spike recoveries were reported with the 21 samples associated with batch C, no PCBs or pesticides were detected in these samples with the exception of one sample, and therefore, the data were not qualified: B-BHC was detected in I-T5 at a concentration of $13 \mu \mathrm{g} / \mathrm{kg}$, near the detection limits. This value was flagged with an " $E$ " to indicate the value is an estimate.

Fourteen samples were spiked with aldrin and dieldrin and analyzed as matrix spike samples. These samples were either actual samples or blank $M$. nasuta tissue provided by Batelle. Recoveries of aldrin were within the EPA CLP control limits of $42 \%$ to $122 \%$. Recoveries of dieldrin were within the EPA CLP control limits of $36 \%$ to $146 \%$.

Sixteen tissue samples were spiked with aroclor 1254. Recoveries of aroclor 1254 were within the EPA CLP control limits of $29 \%$ to $131 \%$ with one exception: aroclor 1254 was recovered in one sample at $158 \%$. No PCBs were detected in any samples therfore the data were not qualified. 
REPLICATES

SRMs
Seven samples were analyzed in duplicate (there were insufficient quantities of sample for triplicate anlayses) to assess precision. Precison was measured by Relative Percent Difference (RPD) between duplicate results. No RPD values were calculated for any of the samples because no analytes were detected.

NOT APPLICABLE 


\section{QA/QC SUMMARY}

PROGRAM:

PARAMETER:

LABORATORY:

MATRIX:

SAMPLE NUMBER:

NOTE:

HOLDING TIMES

DETECTION LIMITS

ME'THOD BLANKS

SURROGATES

MATRIX SPIKES
Oakland Phase III B

Metals (Ag,As, $\mathrm{Cd}, \mathrm{Cr}, \mathrm{Cu}, \mathrm{Hg}, \mathrm{Ni}, \mathrm{Pb}, \mathrm{Se}, \mathrm{Zn}$ )

Battelle/Marine Sciences Laboratory

$N$. caecoides Tissue

78

Because there was a limited amount of $N$. caecoides tissue available for analyses, the five tissue replicates from each of the 26 sediment treatments were composited. Each of these $N$. caecoides composited samples was analyzed in triplicate by both $\mathrm{AA}$ and $\mathrm{XRF}$ with the followng exception: three treatments (C-SB, R-OS, and O-C13) contained enough tissue for triplicate XRF analysis but only enough for duplicate AA analysis. Therefore, results for these three samples for $\mathrm{Ag}, \mathrm{Cd}, \mathrm{Cr}, \mathrm{Hg}, \mathrm{Pb}$, and $\mathrm{Se}$ are based on duplicate analyses.

Based on the EPA Draft Implementation Manual (EPA/USACE 1990), the allowed holding time for tissue samples for analysis of $\mathrm{Ag}, \mathrm{As}, \mathrm{Cd}, \mathrm{Cr}, \mathrm{Cu}, \mathrm{Ni}$, $\mathrm{Pb}, \mathrm{Se}$, and $\mathrm{Zn}$ is 6 months, and 28 days for $\mathrm{Hg}$. Samples must be kept frozen. For this project, $N$. caecoides tissue samples were held frozen for up to 21 days after receipt at Battelle MSL. Samples were then freeze dried and stored for up to 3 months in sealed containers. No holding times have been established for freezedried samples. However, standard reference material prepared by the U.S. National Institute of Standards and Technology (NIST) such as SRM 1566a (Oyster tissue) have been issued for up to 10 years without changes to the certified concentrations for metals, including those for $\mathrm{Hg}$.

Target detection limits (Table 2.2), based on dry weight concentrations, were achieved for all metals except $\mathrm{Ni}_{\text {. }}$ Detection limits for $\mathrm{Ni}$ ranged from 1.6 to 2.7 $\mu \mathrm{g} / \mathrm{g}$ dry we,ight, slightly exceeding target goal of $1.0 \mu \mathrm{g} / \mathrm{g}$.

Five method blanks were analyzed by $\mathrm{AA}$ for $\mathrm{Ag}, \mathrm{Cd}, \mathrm{Cr}, \mathrm{Hg}, \mathrm{Pb}$, and $\mathrm{Se}$. Concentrations of metals in blanks ranged as follows: Ag, 0.006 to $0.010 \mu \mathrm{g} / \mathrm{g}$; $\mathrm{Cd}, 0.07$ to $0.09 \mu \mathrm{g} / \mathrm{g} ; \mathrm{Cr}, 0.037$ to $0.071 \mu \mathrm{g} / \mathrm{g} ; \mathrm{Hg}, 0.001$ to $0.003 \mu \mathrm{g} / \mathrm{g} ; \mathrm{Pb}$ and $\mathrm{Se}$, andetected. Concentrations reported for samples in which these analytes were ditected are corrected for the concentrations found in the corresponding blanks. Method blanks are not applicable to metals analyzed by XRF.

\section{NOT APPLICABLE}

Five matrix spike samples (frequency of 1 spike sample per 20 samples) were analyzed by $\mathrm{AA}$ for $\mathrm{Ag}, \mathrm{Cd}, \mathrm{Cr}, \mathrm{Hg}, \mathrm{Pb}$, and $\mathrm{Se}$. Spike recoveries ranged as follows: $\mathrm{Ag}, 41 \%$ to $111 \%$; $\mathrm{Cd}, 101 \%$ to $118 \% ; \mathrm{Cr}, 89 \%$ to $109 \% ; \mathrm{Hg}, 90 \%$ to $96 \%, \mathrm{~Pb}, 89 \%$ to $93 \%, \mathrm{Se}, 84 \%$ to $95 \%$. Recovery goals stated in the QC plan are as follows: $\mathrm{Cr}, 85 \%$ to $115 \% ; \mathrm{Hg}, 75 \%$ to $125 \% ; \mathrm{Pb}, 75 \%$ to $125 \%$; Se, $75 \%$ to $115 \%$. Recovery goals for these metals were achieved. Two samples contained at least one metal that was not recovered within $15 \%$ of the amount spiked. 
REPLICATES

SRMs
All samples were analyzed in triplicate by XRF and all but three samples were analyzed in triplicate by $\mathrm{AA}$ to assess precision. (See note above). Precision was measured by the Relative Standard Deviation (RSD) between resulis of replicate analyses. RSDs ranged as follows: $\mathrm{Ag}, 0 \%$ to $20 \%$; $\mathrm{As}, 1 \%$ to $5 \%$; $\mathrm{Cd}, 1 \%$ to $11 \%$; $\mathrm{Cr}, 5 \%$ to $149 \%$; $\mathrm{Cu}, 1 \%$ to $20 \% ; \mathrm{Hg}, 1 \%$ to $22 \% ; \mathrm{Ni}, 3 \%$ to $86 \% ; \mathrm{Pb}, 1 \%$ to $61 \% ; \mathrm{Se}, 1 \%$ to $87 \% ; \mathrm{Zn}, 1 \%$ to $8 \%$.

SRM 1566a, oyster tissue, was obtained from the U.S. National Institute of Standards and Technology (NIST). This SRM was analyzed by both XRF and AA at a minimum frequency of once per 10 samples. The results of samples analyses were within the certified values for the SRM with the following exceptions: 19 analyses for $\mathrm{Ni}$ were exceeded the certified range for this analyte. Because the sample values were all reported close to the detection limits, reanalysts were not performed and data were not qualified. 


\section{QA/QC SUMMARY}

PROGRAM:

PARAMETER:

LABORATORY:

MATRIX:

SAMPLE NUMBER:

HOLDING TIMES

DETECTION LIMITS

METHOD BLANKS

SURROGATES

MATRIX SPIKES
Oakland Phase III B

Butyltins

Battelle Ocean Sciences Laboratory

$N$. caecoides Tisssue

130 (78 treatments, 3 replicates each)

Holding times have not been established for analysis of butyltins in tissues. Samples were held frozen prior to extraction. All samples were extracted within 2 months of receipt in the laboratory.

Target detection limits of $10.0 \mu \mathrm{g} / \mathrm{kg}$ wet weight were achieved for di- and monobutyltins. The detection limit acheived for tributyltin was $19.0 \mu \mathrm{g} / \mathrm{kg}$ wet wt. This higher detection limit can be attributed to the small amount of sample material that was available for analysis.

Seven method blanks were analyzed. Tributyltin was detected in two method blanks at 11.5 and $26.2 \mu \mathrm{g} / \mathrm{kg}$ wet $y / t$. All detected results of sample analyses associated with the respective blanks were corrected for the concentrations in those blanks.

Tripentyltin was added to each sample prior to extraction as a surrogate to assess extraction efficiency. Recoveries of tripentyltin were within the target range of $40 \%$ to $120 \%$ with the following exceptions: 21 samples had tripentyltin recoveries ranging from $19 \%$ to $152 \%$. All detected results of sample analyses associated with these out-of-range surrogates were flagged with an " $E$ " to indicate that the value is estimated because of a potential bias.

Seven samples were spiked with tri-, di-, and monobutyltin and analyzed as matrix spike samples. Recoveries ranged as follows: tributyltin, $61 \%$ to $190 \%$; dibutyltin, $64 \%$ to $184 \%$; monobutyltin, $22 \%$ to $29 \%$. There are no established limits for recovery of butyltin matrix spikes. However, all detected results of sample analyses in batches associated with matrix spikes having recoveries outside of the range of $50 \%$ to $120 \%$ were flagged with an " $E$ " to indicatis that the value is estimated because of a potential bias. The following samples were flagged: tributyltin results for samples in Batches 2 and 7; dibutyltin results for samples in Batches 2 and 7; and monobutyltin results for samples in all batches.

Seven samples were analyzed in duplicate to assess precision. Precision was measured by Relative Percent Difference (RPD) between duplicate analyses. RPDs were calculated only in samples where concentrations of tri-, di-, and n.n nobutyltins were detected and ranged as follows: tributyltin, $10 \%$ to $32 \%$; dibutyltin, $134 \%$. Note that these comparisons were made using estimated sample concentrations detected at or below the detection limit. 


\section{REEERENCES}

U.S. Environunental Protection Agency (EPA). 1986. Test Methods for Evaluating Solid Waste: Physical/Chemical Methods. EPA-955-001-00000. U.S. Environmental Protection Agency, Government Printing Office, Washington, D.C.

U.S. Environmental Protection Agency/U.S. Army Corps of Engineers (EPA/USACE). 1990. Draft Ecological Evaluation of Proposed Discharge of Dredged Material into Ocean Waters. EPA503-8-90/002. U.S. Environmental Protection Agency, Office of Marine and Estuarine Protection, Washington, D.C. 
IABLE L.1. Total Detected Polynuclear Aromatic Hydrocarbons (PAHs), Dry Weight, in Tissue of $N$. caecoides, Oakland Harbor Phase III B Project

\begin{tabular}{|c|c|c|c|c|c|c|}
\hline \multirow[b]{2}{*}{$\begin{array}{l}\text { Sediment } \\
\text { Ireatment }\end{array}$} & \multirow[b]{2}{*}{ Replicate } & \multirow[b]{2}{*}{ Batch } & \multirow[b]{2}{*}{$\begin{array}{c}\text { Percent } \\
\text { Dry Weight }\end{array}$} & \multicolumn{3}{|c|}{$N$. caecoides PAHs $(\mu \mathrm{g} / \mathrm{kg}$ dry weight $)$} \\
\hline & & & & $\begin{array}{c}\text { Total } \\
\text { Low Molecular Weight } \\
\text { PAH } \\
\end{array}$ & $\begin{array}{c}\text { Total } \\
\text { High Molecular Weight } \\
\text { PAH }\end{array}$ & $\begin{array}{l}\text { Total } \\
\text { PAH }\end{array}$ \\
\hline $\begin{array}{l}0-C 13 \\
0-C 13 \\
0-C 13 \\
0-C 13 \\
0-C 13\end{array}$ & $\begin{array}{l}1 \\
2 \\
3 \\
4 \\
5\end{array}$ & $\begin{array}{l}c \\
c \\
c \\
c \\
c\end{array}$ & $\begin{array}{l}13.39 \\
14.72 \\
17.78(b) \\
15.81 \text { (b) } \\
1.7 .34\end{array}$ & $\begin{array}{c}381 \\
1134 \\
N A \\
209 \\
209 \\
265\end{array}$ & $\begin{array}{r}1180 \\
700 \\
703 \\
1043 \\
658\end{array}$ & $\begin{array}{r}1561 \\
1834 \\
703 \\
1252 \\
923\end{array}$ \\
\hline $\begin{array}{l}0-C 12 \\
0-C 12 \\
0-C 12 \\
0-C 12 \\
0-C 12 \\
0-C 12\end{array}$ & $\begin{array}{lll}1 & & \\
2 & \operatorname{Rep} & 1 \\
2 & \operatorname{Rep} & 2 \\
3 & & \\
4 & & \\
5 & & \end{array}$ & $\begin{array}{l}c \\
c \\
c \\
c \\
c \\
c\end{array}$ & $\begin{array}{l}17.76 \\
18.98 \\
18.98 \\
17.17 \\
20.17 \\
20.23\end{array}$ & $\begin{array}{r}N A \\
N A \\
N A \\
216 \\
N A \\
N A\end{array}$ & $\begin{array}{l}\text { NA } \\
\text { NA } \\
\text { NA } \\
\text { NA } \\
\text { NA } \\
89\end{array}$ & $\begin{array}{r}\text { NA } \\
\text { NA } \\
N A \\
216 \\
N A \\
89\end{array}$ \\
\hline $\begin{array}{l}0-C 11 \\
0-C 11 \\
0-C 11 \\
0-C 11 \\
0-C 11\end{array}$ & $\begin{array}{l}1 \\
2 \\
3 \\
4 \\
5\end{array}$ & $\begin{array}{l}D \\
D \\
D \\
0 \\
D\end{array}$ & $\begin{array}{l}20.85 \\
20.52 \\
18.25 \\
18.18 \\
19.56\end{array}$ & $\begin{array}{r}\text { NA } \\
83 \\
\text { NA } \\
187 \\
\text { NA }\end{array}$ & $\begin{array}{l}297 \\
765 \\
290 \\
336 \\
726\end{array}$ & $\begin{array}{l}297 \\
848 \\
290 \\
523 \\
726\end{array}$ \\
\hline $\begin{array}{l}0-C 10 \\
0-C 10 \\
0-C 10 \\
0-C 10 \\
0-C 10\end{array}$ & $\begin{array}{l}1 \\
2 \\
3 \\
4 \\
5\end{array}$ & $\begin{array}{l}D \\
D \\
D \\
D \\
D\end{array}$ & $\begin{array}{l}19.93 \\
19.30 \\
19.95 \\
19.25 \\
17.90\end{array}$ & $\begin{array}{l}\text { NA } \\
N A \\
N A \\
N A \\
N A\end{array}$ & $\begin{array}{r}95 \\
161 \\
190 \\
1018 \\
201\end{array}$ & $\begin{array}{r}95 \\
161 \\
190 \\
1018 \\
201\end{array}$ \\
\hline $\begin{array}{l}0-C 9 \\
0-C 9 \\
0-C 9 \\
0-C 9 \\
0-C 9\end{array}$ & $\begin{array}{l}1 \\
2 \\
3 \\
4 \\
5\end{array}$ & $\begin{array}{l}D \\
D \\
D \\
D \\
D\end{array}$ & $\begin{array}{l}21.01 \\
19.95 \\
14.97 \\
21.03 \\
16.72\end{array}$ & $\begin{array}{l}\text { NA } \\
\text { NA } \\
\text { NA } \\
\text { NA } \\
\text { NA }\end{array}$ & $\begin{array}{l}N A \\
N A \\
N A \\
N A \\
N A\end{array}$ & $\begin{array}{l}\text { NA } \\
\text { NA } \\
\text { NA } \\
\text { NA } \\
\text { NA }\end{array}$ \\
\hline $\begin{array}{l}0-C 8 \\
0-C 8 \\
0-C 8 \\
0-C 8 \\
0-C 8 \\
0-C 8\end{array}$ & $\begin{array}{lll}1 & & \\
2 & & \\
3 & \operatorname{Rep} & 1 \\
3 & \operatorname{Rep} & 2 \\
4 & & \\
5 & & \end{array}$ & $\begin{array}{l}D \\
D \\
D \\
D \\
D \\
D\end{array}$ & $\begin{array}{l}21.38 \\
12.67 \\
15.45 \\
15.45 \\
19.30 \\
15.86\end{array}$ & $\begin{array}{r}N A \\
N A \\
809 \\
N A \\
212 \\
N A\end{array}$ & $\begin{array}{l}285 \\
158 \\
628 \\
369 \\
181 \\
158\end{array}$ & $\begin{array}{r}285 \\
158 \\
1437 \\
369 \\
394 \\
158\end{array}$ \\
\hline $\begin{array}{l}0-C 7 \\
0-C 7 \\
0-C 7 \\
0-C 7 \\
0-C .7\end{array}$ & $\begin{array}{l}1 \\
2 \\
3 \\
4 \\
5\end{array}$ & $\begin{array}{l}E \\
E \\
E \\
E \\
E\end{array}$ & $\begin{array}{l}17.59 \\
18.57 \\
19.84 \\
18.56 \\
21.15\end{array}$ & $\begin{array}{l}\text { NA } \\
\text { NA } \\
111 \\
\text { NA } \\
\text { NA }\end{array}$ & $\begin{array}{r}N A \\
108 \\
96 \\
108 \\
76\end{array}$ & $\begin{array}{r}\text { NA } \\
108 \\
207 \\
108 \\
76\end{array}$ \\
\hline $\begin{array}{l}0-c 6 \\
0-C 6 \\
0-c 6 \\
0-c 6 \\
0-C 6\end{array}$ & $\begin{array}{l}1 \\
2 \\
3 \\
4 \\
5\end{array}$ & $\begin{array}{l}E \\
E \\
E \\
E \\
E\end{array}$ & $\begin{array}{l}20.07 \\
14.78 \\
20.13 \\
20.42 \\
24.45\end{array}$ & $\begin{array}{r}209 \\
\text { NA } \\
\text { NA } \\
98 \\
\text { NA }\end{array}$ & $\begin{array}{r}154 \\
81 \\
70 \\
64 \\
90\end{array}$ & $\begin{array}{r}364 \\
81 \\
70 \\
162 \\
90\end{array}$ \\
\hline $\begin{array}{l}0-C 5 \\
0-C 5 \\
0-C 5 \\
0-C 5 \\
0-C 5\end{array}$ & $\begin{array}{l}1 \\
2 \\
3 \\
4 \\
5\end{array}$ & $\begin{array}{l}E \\
E \\
E \\
E \\
E\end{array}$ & $\begin{array}{l}15.99 \\
14.73 \\
16.98 \\
22.03 \\
20.41\end{array}$ & $\begin{array}{r}125 \\
N A \\
N A \\
113 \\
113\end{array}$ & $\begin{array}{r}\text { NA } \\
\text { NA } \\
\text { NA } \\
141 \\
\text { NA }\end{array}$ & $\begin{array}{r}125 \\
N A \\
N A \\
254 \\
113\end{array}$ \\
\hline
\end{tabular}


IABLE L.1. (contd)

\begin{tabular}{|c|c|c|c|c|c|c|}
\hline \multirow[b]{2}{*}{$\begin{array}{l}\text { Sediment } \\
\text { Treatment }\end{array}$} & \multirow[b]{2}{*}{ Replicate } & \multirow[b]{2}{*}{ Batch } & \multirow[b]{2}{*}{$\begin{array}{c}\text { Percent } \\
\text { Dry Weight }\end{array}$} & \multicolumn{3}{|c|}{ N. caecoides PAHs ( $1 \mathrm{gg} / \mathrm{kg}$ ory weight $)$} \\
\hline & & & & $\begin{array}{c}\text { Total } \\
\text { Low Molecular Weight } \\
\text { PAH }\end{array}$ & $\begin{array}{c}\text { Total } \\
\text { High Molecular Weight } \\
\text { PAH }\end{array}$ & $\begin{array}{l}\text { Total } \\
\text { PAH } \\
\end{array}$ \\
\hline $\begin{array}{l}0-C 4 \\
0-C 4 \\
0-C 4 \\
0-C 4 \\
0-C 4 \\
0-C 4\end{array}$ & 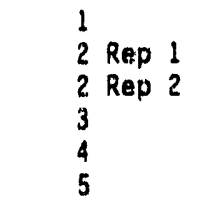 & $\begin{array}{l}E \\
E \\
E \\
E \\
E \\
E\end{array}$ & $\begin{array}{l}18.94 \\
20.92 \\
20.92 \\
15.95 \\
20.32 \\
22.55\end{array}$ & $\begin{array}{r}\text { NA } \\
148 \\
143 \\
144 \\
359 \\
\text { NA }\end{array}$ & $\begin{array}{r}N A \\
1735 \\
81 \\
69 \\
315 \\
71\end{array}$ & $\begin{array}{r}N A \\
1883 \\
225 \\
213 \\
674 \\
71\end{array}$ \\
\hline $\begin{array}{l}0-C 3 \\
0-C .3 \\
0-C .3 \\
0-C 3 \\
0-C .3\end{array}$ & $\begin{array}{l}1 \\
2 \\
3 \\
4 \\
5\end{array}$ & $\begin{array}{l}G \\
G \\
G \\
G \\
G\end{array}$ & $\begin{array}{l}18.10 \\
15.65 \\
17.67 \\
17.28 \\
14.94\end{array}$ & $\begin{array}{l}\text { NA } \\
\text { HA } \\
\text { NA } \\
\text { NA } \\
\text { NA }\end{array}$ & $\begin{array}{l}\text { NA } \\
\text { NA } \\
N A \\
\text { NA } \\
N A\end{array}$ & $\begin{array}{l}\text { NA } \\
\text { NA } \\
\text { NA } \\
\text { NA } \\
\text { NA }\end{array}$ \\
\hline $\begin{array}{l}0-C 1 \\
0-C 1 \\
0-C 1 \\
0-C .1 \\
0-C 1 \\
0-C 1\end{array}$ & $\begin{array}{lll}1 & & \\
2 & \text { Rep } 1 \\
2 & \operatorname{Rep} & 2 \\
3 & & \\
4 & \\
5 & \end{array}$ & $\begin{array}{l}G \\
G \\
G \\
G \\
G \\
G\end{array}$ & $\begin{array}{l}13.07 \\
16.57 \\
16.57 \\
16.02 \\
16.87 \\
19.63\end{array}$ & $\begin{array}{l}\text { NA } \\
N A \\
N A \\
N A \\
N A \\
N A\end{array}$ & $\begin{array}{l}\text { NA } \\
\text { NA } \\
\text { NA } \\
\text { NA } \\
\text { WA } \\
\text { HA }\end{array}$ & $\begin{array}{l}\text { NA } \\
\text { NA } \\
\text { NA } \\
\text { NA } \\
\text { NA } \\
\text { NA }\end{array}$ \\
\hline $\begin{array}{l}I-C 2 \\
I-C 2 \\
I-C 2 \\
I+C 2 \\
I-C 2\end{array}$ & $\begin{array}{l}1 \\
2 \\
3 \\
4 \\
5\end{array}$ & $\begin{array}{l}F \\
F \\
F \\
F \\
F\end{array}$ & $\begin{array}{l}21.37 \\
10.14 \\
18.46 \\
21.10 \\
18.74\end{array}$ & $\begin{array}{l}\text { NA } \\
\text { NA } \\
276 \\
N A \\
\text { NA }\end{array}$ & $\begin{array}{r}\text { NA } \\
\text { NA } \\
103 \\
76 \\
229\end{array}$ & $\begin{array}{r}N A \\
N A \\
379 \\
76 \\
229\end{array}$ \\
\hline $\begin{array}{l}0-C 2 \\
0-C 2 \\
0-C 2 \\
0-C 2 \\
0-C 2\end{array}$ & $\begin{array}{l}1 \\
2 \\
3 \\
4 \\
5\end{array}$ & $\begin{array}{l}F \\
F \\
F \\
F \\
F\end{array}$ & $\begin{array}{l}15.00 \\
16.36 \\
16.17 \\
17.25 \\
13.95\end{array}$ & $\begin{array}{r}\text { NA } \\
397 \\
\text { NA } \\
696 \\
\text { NA }\end{array}$ & $\begin{array}{l}\text { NA } \\
\text { NA } \\
\text { NA } \\
\text { NA } \\
\text { NA }\end{array}$ & $\begin{array}{r}\text { NA } \\
397 \\
\text { NA } \\
696 \\
\text { NA }\end{array}$ \\
\hline $\begin{array}{l}1-C 3 \\
1-C 3 \\
1-C 3 \\
1-C 3 \\
1-C 3 \\
1-C 3\end{array}$ & $\begin{array}{ll}1 & \\
2 & \\
3 & \\
4 & R e p 1 \\
4 & R e p 2 \\
5 & \end{array}$ & $\begin{array}{l}F \\
F \\
F \\
F \\
F \\
F\end{array}$ & $\begin{array}{l}10.72 \\
16.08 \\
19.63 \\
20.49 \\
20.49 \\
12.35\end{array}$ & $\begin{array}{l}\text { NA } \\
W A \\
N A \\
N A \\
220 \\
N A\end{array}$ & $\begin{array}{l}N A \\
N A \\
N A \\
N A \\
N A \\
N A\end{array}$ & $\begin{array}{r}N A \\
N A \\
N A \\
N A \\
220 \\
N A\end{array}$ \\
\hline $\begin{array}{l}I-C 4 \\
I-C 4 \\
I-C 4 \\
1-C 4 \\
I-C 4\end{array}$ & $\begin{array}{l}1 \\
2 \\
3 \\
4 \\
5\end{array}$ & $\begin{array}{l}c \\
c \\
c \\
c \\
c\end{array}$ & $\begin{array}{l}14.71 \\
16.76 \\
14.13 \\
17.72 \\
12.91\end{array}$ & $\begin{array}{r}95 \\
286 \\
N A \\
175 \\
\text { NA }\end{array}$ & $\begin{array}{r}231 \\
N A \\
N A \\
N A \\
N A\end{array}$ & $\begin{array}{r}326 \\
286 \\
N A \\
175 \\
N A\end{array}$ \\
\hline $\begin{array}{l}I-C 8 \\
I-C 8 \\
I-C 8 \\
I-C 8 \\
I-C 8\end{array}$ & $\begin{array}{l}1 \\
2 \\
3 \\
4 \\
5\end{array}$ & $\begin{array}{l}F \\
F \\
F \\
F \\
F\end{array}$ & $\begin{array}{l}21.16 \\
11.85 \\
13.24 \\
22.87 \\
15.47\end{array}$ & $\begin{array}{r}\text { NA } \\
\text { NA } \\
484 \\
\text { NA } \\
\text { HA }\end{array}$ & $\begin{array}{r}539 \\
236 \\
204 \\
66 \\
252\end{array}$ & $\begin{array}{r}539 \\
236 \\
688 \\
66 \\
252\end{array}$ \\
\hline $\begin{array}{l}1-T 5 \\
1-T 5 \\
1-T 5 \\
1-T 5 \\
1-T 5\end{array}$ & $\begin{array}{l}1 \\
2 \\
3 \\
4 \\
5\end{array}$ & $\begin{array}{l}c \\
c \\
c \\
c \\
c\end{array}$ & $\begin{array}{l}16.23 \\
18.78 \\
20.98 \\
14.20 \\
20.39\end{array}$ & $\begin{array}{r}179 \\
W A \\
1125 \\
N A \\
88\end{array}$ & $\begin{array}{l}\text { NA } \\
\text { NA } \\
272 \\
\text { NA } \\
\text { NA }\end{array}$ & $\begin{array}{r}179 \\
N A \\
1297 \\
N A \\
88\end{array}$ \\
\hline
\end{tabular}

PHASE III B 
TABLE L.1. (contd)

\begin{tabular}{|c|c|c|c|c|c|c|}
\hline \multirow[b]{2}{*}{$\begin{array}{l}\text { Sediment } \\
\text { Ireatment: }\end{array}$} & \multirow[b]{2}{*}{ Replicate } & \multirow[b]{2}{*}{ Batch } & \multirow[b]{2}{*}{$\begin{array}{c}\text { Percent } \\
\text { Pry Weight }\end{array}$} & \multicolumn{3}{|c|}{ N. caecoides PAHs ( $(\mu \mathrm{g} / \mathrm{kg}$ dry weight) } \\
\hline & & & & $\begin{array}{c}\text { Total } \\
\text { Low Molecular Weight } \\
\text { PAH }\end{array}$ & $\begin{array}{c}\text { Total } \\
\text { High Molecular Weight } \\
\text { PAH }\end{array}$ & $\begin{array}{l}\text { Total } \\
\text { PAH }\end{array}$ \\
\hline$R-A C$ & 1 & B & 17.69 & NA & 170 & 170 \\
\hline$R-A C$ & 2 & B & 13.48 & 275 & 19.3 & 468 \\
\hline$R-A C$ & 3 & B & 21.41 & NA & NA & NA \\
\hline$R-A C$ & 4 & B & 20.16 & NA & 273 & 273 \\
\hline$R-A C$ & 5 & $\theta$ & 15.84 & NA & 177 & 177 \\
\hline$R-A M$ & 1 & A & 17.43 & 69 & 109 & 178 \\
\hline$R-A M$ & 2 & A & .4 .88 & NA & 94 & 94 \\
\hline$R-A M$ & 3 & A & 17.30 & NA & 127 & 127 \\
\hline R-AM & 4 & A & 15.68 & 69 & 121 & 210 \\
\hline$R=A M$ & 5 & A & 17.78 & 62 & 96 & 158 \\
\hline$R-B F$ & 1 & A & 13.29 & NA & NA & NA \\
\hline$R-B F$ & 2 & A & 19.11 & NA & NA & NA \\
\hline$R-B F$ & 3 & A & 14.85 & NA & NA & NA \\
\hline$R-B F$ & 4 & A & 16.71 & HA & NA & NA \\
\hline$R-B F$ & 5 & A & 18.07 & NA & NA & NA \\
\hline$R-C J$ & 1 & 8 & $17.08^{(b)}$ & NA & $N A$ & NA \\
\hline$R-0 S$ & 2 & 8 & 23.44 & NA & $N A$ & NA \\
\hline$R-O S$ & 3 & B & 10.18 & NA & $N A$ & NA \\
\hline$R \cdots O S$ & 4 & B & 19.93 & $N A$ & NA & NA \\
\hline$R-O S$ & 5 & 8 & 14.76 & NA & NA & NA \\
\hline$R-P C$ & $1 \operatorname{Rep} 1$ & B & 16.00 & $N A$ & NA & NA \\
\hline$R-P C$ & 1 Rep 2 & B & 16.00 & NA & NA & NA \\
\hline$R \sim P C$ & & $B$ & 20.86 & NA & NA & NA. \\
\hline$R-P C$ & 3 & B & 20.52 & NA & NA & NA \\
\hline$R-P C$ & 4 & 8 & 16.36 & NA & $N A$ & NA \\
\hline$R-P C$ & 5 & B & 20.47 & NA & NA & הA \\
\hline R-PF & 1 & A & 14.50 & $N A$ & NA & NA \\
\hline$R-P F$ & 2 & A & 15.40 & $\mathrm{NA}$ & MA & $N A$ \\
\hline$R-P F$ & $3 \operatorname{Rep} 1$ & A & 14.23 & $N A$ & $N A$ & NA \\
\hline$R-P F$ & $3 \operatorname{Rep} 2$ & A & 14.23 & NA & NA & NA \\
\hline$R-P F$ & 4 & A & 14.96 & $N A$ & NA & NA \\
\hline$R-P F$ & 5 & A & 15.08 & NA & $W A$ & NA \\
\hline$C-S B$ & 1 & B & 21.76 & NA & NA & NA \\
\hline C.SB & 2 & B & 13.05 & $N A$ & $N A$ & NA \\
\hline$c-58$ & 3 & B & 20.69 & NA & 63 & 63 \\
\hline C-SB & 4 & B & 20.20 & NA & NA & NA \\
\hline$C-5 B$ & 5 & B & 12.31 & NA & NA & $N A$ \\
\hline C-NE & 1 & A & 17.55 & $N A$ & $N A$ & NA \\
\hline$C+N E$ & 2 & A & 17.85 & NA & NA & NA \\
\hline$C-N E$ & 3 & A & 133.39 & WA & NA & NA \\
\hline C-NE & 4 & A & 16.70 & $N A$ & $N A$ & NA \\
\hline C. - ME & 5 & A & 18.54 & $H A$ & $N A$ & NA \\
\hline
\end{tabular}

(a) Not applicable: No PAHs detected.

(b) No percent dry weight: used mean percent dry weight of other four replicates. 
IABLE L.2. Low Molecular Weight Polynuclear Aromatic Hydrocarbons (PAHS), Dry Weight, in Tissue of $N$. caecuides, Oakland Harbor Phase III B Project

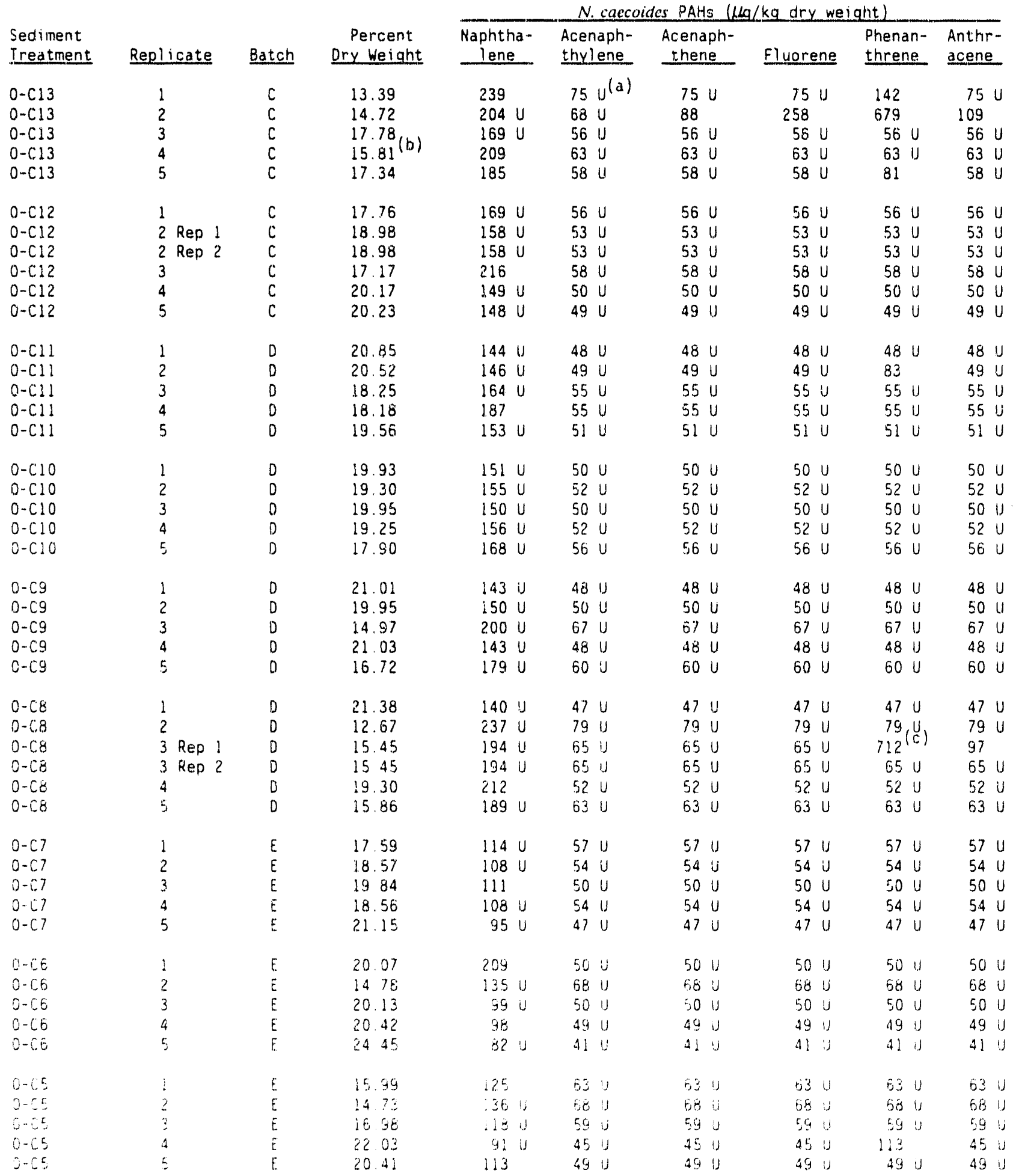




\section{IABLE L.2. (contd)}

\begin{tabular}{|c|c|c|c|c|c|c|c|c|c|}
\hline \multirow{2}{*}{$\begin{array}{l}\text { Sediment } \\
\text { Ireatment }\end{array}$} & \multirow[b]{2}{*}{ Replicate } & \multirow[b]{2}{*}{ Batch } & \multirow[b]{2}{*}{$\begin{array}{c}\text { Percent } \\
\text { Dry Weight }\end{array}$} & \multicolumn{6}{|c|}{$N$. caecoides PAHs $(\mu \mathrm{g} / \mathrm{kg}$ dry we ight $)$} \\
\hline & & & & $\begin{array}{l}\text { Naphtha- } \\
\text { lene } \\
\end{array}$ & $\begin{array}{l}\text { Acenaph- } \\
\text { thylene }\end{array}$ & $\begin{array}{l}\text { Acenaph- } \\
\text { thene }\end{array}$ & Fluorene & $\begin{array}{l}\text { Phenan } \\
\text { threne }\end{array}$ & $\begin{array}{l}\text { Anthr- } \\
\text { acene }\end{array}$ \\
\hline $\begin{array}{l}0-C 4 \\
0-C 4 \\
0-C 4 \\
0-C 4 \\
0-C 4 \\
0-C 4\end{array}$ & $\begin{array}{lll}1 & & \\
2 & \operatorname{Rep} & 1 \\
2 & \operatorname{Rep} & 2 \\
3 & & \\
4 & & \\
5 & & \end{array}$ & $\begin{array}{l}E \\
E \\
E \\
E \\
E \\
E\end{array}$ & $\begin{array}{l}18.94 \\
20.92 \\
20.92 \\
15.95 \\
20.32 \\
22.55\end{array}$ & $\begin{array}{r}106 U \\
96 U \\
143 \\
144 \\
98 U \\
89 U\end{array}$ & $\begin{array}{l}53 U \\
48 U \\
48 U \\
63 U \\
49 U \\
44 U\end{array}$ & $\begin{array}{l}53 U \\
48 U \\
48 U \\
63 U \\
49 U \\
44 U\end{array}$ & $\begin{array}{l}53 U \\
48 U \\
48 U \\
63 U \\
49 U \\
44 U\end{array}$ & $\begin{array}{l}53 \mathrm{U} \\
91 \\
48 \mathrm{U} \\
63 \mathrm{U} \\
310 \\
44 \mathrm{U}\end{array}$ & $\begin{array}{l}53 \mathrm{U} \\
57 \\
48 \mathrm{U} \\
63 \mathrm{U} \\
49 \\
44 \mathrm{U}\end{array}$ \\
\hline $\begin{array}{l}0-c 3 \\
0-c 3 \\
0-C 3 \\
0-c 3 \\
0-c 3\end{array}$ & $\begin{array}{l}1 \\
2 \\
3 \\
4 \\
5\end{array}$ & $\begin{array}{l}G \\
G \\
G \\
G \\
G\end{array}$ & $\begin{array}{l}18.10 \\
15.65 \\
17.67 \\
17.28 \\
14.94\end{array}$ & $\begin{array}{l}332 U \\
639 U \\
340 U \\
579 U \\
402 U\end{array}$ & $\begin{array}{l}55 U \\
64 U \\
57 U \\
58 U \\
67 U\end{array}$ & 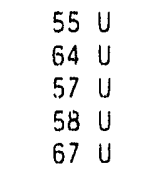 & $\begin{array}{ll}55 & U \\
64 & U \\
57 & U \\
58 & 11 \\
67 & U^{\prime}\end{array}$ & $\begin{array}{l}55 U \\
64 U \\
5 y \\
58 U \\
67 U \\
67\end{array}$ & $\begin{array}{l}55 U \\
64 \\
57 \\
58 \\
58 \\
67\end{array}$ \\
\hline $\begin{array}{l}0-C 1 \\
0-C 1 \\
0-C 1 \\
0-C 1 \\
0-C 1 \\
0-C 1\end{array}$ & $\begin{array}{lll}1 & & \\
2 & R e p & 1 \\
2 & R e p & 2 \\
3 & & \\
4 & & \\
5 & & \end{array}$ & $\begin{array}{l}G \\
G \\
G \\
G \\
G \\
G\end{array}$ & $\begin{array}{l}13.07 \\
16.57 \\
16.57 \\
16.02 \\
16.87 \\
19.63\end{array}$ & $\begin{array}{l}459 U \\
362 U \\
362 U \\
624 U \\
356 U \\
306 U\end{array}$ & $\begin{array}{l}77 U \\
60 U \\
60 U \\
62 U \\
59 U \\
51 U\end{array}$ & $\begin{array}{l}77 U \\
60 U \\
60 U \\
62 U \\
59 U \\
51 U\end{array}$ & $\begin{array}{ll}77 & U \\
60 & U \\
60 & U \\
62 & U \\
59 & U \\
51 & U\end{array}$ & $\begin{array}{ll}77 & U \\
6 J & U \\
60 & U \\
62 & U \\
59 & U \\
51 & U\end{array}$ & $\begin{array}{ll}77 & U \\
60 & U \\
60 & U \\
62 & U \\
59 & U \\
51 & U\end{array}$ \\
\hline $\begin{array}{l}1-C 2 \\
1-C 2 \\
1-C 2 \\
1-C 2 \\
1-C 2\end{array}$ & $\begin{array}{l}1 \\
2 \\
3 \\
4 \\
5\end{array}$ & $\begin{array}{l}F \\
F \\
F \\
F \\
F\end{array}$ & $\begin{array}{l}21.37 \\
10.14 \\
18.46 \\
21.10 \\
18.74\end{array}$ & $\begin{array}{l}187 U \\
394 U \\
276 \\
190 U \\
213 U\end{array}$ & $\begin{array}{ll}47 & U \\
99 & U \\
54 & U \\
47 & U \\
53 & U\end{array}$ & $\begin{array}{ll}47 & U \\
99 & U \\
54 & U \\
47 & U \\
53 & U\end{array}$ & $\begin{array}{l}47 U \\
99 U \\
54 U \\
47 U \\
53 U\end{array}$ & $\begin{array}{ll}47 & U \\
99 & U \\
54 & U \\
47 & U \\
53 & U\end{array}$ & $\begin{array}{l}47 U \\
99 \\
54 \\
47 \\
47 \\
53\end{array}$ \\
\hline $\begin{array}{l}0-C 2 \\
0-C 2 \\
0-C 2 \\
0-C 2 \\
0-C .2\end{array}$ & $\begin{array}{l}1 \\
2 \\
3 \\
4 \\
5\end{array}$ & $\begin{array}{l}F \\
F \\
F \\
F \\
F\end{array}$ & $\begin{array}{l}15.00 \\
16.36 \\
16.17 \\
17.25 \\
13.95\end{array}$ & $\begin{array}{l}267 \mathrm{U} \\
397 \\
247 \mathrm{U} \\
696 \\
287 \mathrm{U}\end{array}$ & $\begin{array}{ll}67 & U \\
61 & U \\
62 & U \\
58 & U \\
72 & U\end{array}$ & $\begin{array}{l}67 \mathrm{U} \\
61 \mathrm{U} \\
62 \mathrm{U} \\
58 \mathrm{U} \\
72 \mathrm{U}\end{array}$ & $\begin{array}{ll}67 & U \\
61 & U \\
62 & U \\
58 & U \\
72 & U\end{array}$ & $\begin{array}{ll}67 & U \\
61 & U \\
62 & U \\
58 & U \\
72 & U\end{array}$ & $\begin{array}{ll}67 & U \\
61 & U \\
62 & U \\
58 & U \\
72 & U\end{array}$ \\
\hline $\begin{array}{l}1-C 3 \\
1-C 3 \\
1-C 3 \\
1-C 3 \\
1-C 3 \\
1-C 3\end{array}$ & $\begin{array}{l}1 \\
2 \\
3 \\
4 \text { Rep } 1 \\
4 \text { Rep 2 } \\
5\end{array}$ & $\begin{array}{l}F \\
F \\
F \\
F \\
F \\
F\end{array}$ & $\begin{array}{l}16.72 \\
16.08 \\
19.63 \\
20.49 \\
20.49 \\
12.35\end{array}$ & $\begin{array}{l}239 U \\
249 U \\
204 U \\
195 U \\
220 \\
324 U\end{array}$ & 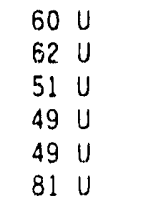 & $\begin{array}{l}60 U \\
62 U \\
51 U \\
49 U \\
49 U \\
81 U\end{array}$ & 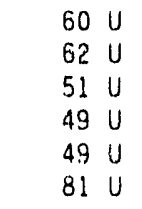 & $\begin{array}{ll}60 & U \\
62 & U \\
51 & U \\
49 & U \\
49 & U \\
81 & U\end{array}$ & $\begin{array}{ll}60 & U \\
62 & U \\
51 & U \\
49 & U \\
49 & U \\
81 & U\end{array}$ \\
\hline $\begin{array}{l}1-C 4 \\
1-C 4 \\
1-C 4 \\
I-C 4 \\
1-C 4\end{array}$ & $\begin{array}{l}1 \\
2 \\
3 \\
4 \\
5\end{array}$ & $\begin{array}{l}c \\
c \\
c \\
c \\
c\end{array}$ & $\begin{array}{l}14.71 \\
16.76 \\
14.13 \\
17.72 \\
12.91\end{array}$ & $\begin{array}{l}204 U \\
286 \\
212 U \\
175 \\
232 U\end{array}$ & $\begin{array}{ll}68 & U \\
60 & U \\
71 & U \\
56 & U \\
77 & U\end{array}$ & $\begin{array}{ll}68 & U \\
60 & U \\
71 & U \\
56 & U \\
77 & U\end{array}$ & $\begin{array}{ll}68 & U \\
60 & U \\
71 & U \\
56 & U \\
77 & U\end{array}$ & $\begin{array}{l}95 \\
60 \\
71 \\
56 \\
47 \\
77\end{array}$ & $\begin{array}{ll}68 & U \\
60 & U \\
71 & U \\
56 & U \\
77 & U\end{array}$ \\
\hline $\begin{array}{l}1-C 8 \\
1-C 8 \\
1-C 8 \\
1-C 8 \\
1-C 8\end{array}$ & $\begin{array}{l}1 \\
2 \\
3 \\
4 \\
5\end{array}$ & $\begin{array}{l}F \\
F \\
F \\
F \\
F\end{array}$ & $\begin{array}{l}21.16 \\
11.85 \\
13.24 \\
22.87 \\
15.47\end{array}$ & $\begin{array}{l}189 \mathrm{U} \\
338 \mathrm{U} \\
484 \\
175 \mathrm{U} \\
259 \mathrm{U}\end{array}$ & $\begin{array}{ll}47 & U \\
84 & U \\
76 & U \\
44 & U \\
65 & U\end{array}$ & $\begin{array}{ll}47 & U \\
84 & U \\
76 & U \\
44 & U \\
65 & U\end{array}$ & $\begin{array}{ll}47 & U \\
84 & U \\
76 & U \\
44 & U \\
55 & U\end{array}$ & $\begin{array}{ll}47 & U \\
84 & U \\
75 & U \\
44 & U \\
65 & U\end{array}$ & $\begin{array}{ll}47 & U \\
84 & U \\
76 & U \\
44 & U \\
65 & U\end{array}$ \\
\hline $\begin{array}{l}1-T 5 \\
1-T 5 \\
1-T 5 \\
1-T 5 \\
1-T E\end{array}$ & $\begin{array}{l}1 \\
2 \\
3 \\
4 \\
5\end{array}$ & $\begin{array}{l}c \\
c \\
c \\
c \\
c\end{array}$ & $\begin{array}{l}16.23 \\
18.78 \\
20.98 \\
14.20 \\
20.39\end{array}$ & $\begin{array}{l}185 \mathrm{U} \\
160 \mathrm{U} \\
143 \mathrm{U} \\
211 \mathrm{U} \\
147 \mathrm{U}\end{array}$ & $\begin{array}{l}62 \mathrm{U} \\
53 \mathrm{U} \\
48 \mathrm{U} \\
70 \mathrm{U} \\
49 \mathrm{U}\end{array}$ & $\begin{array}{r}62 U \\
53 \mathrm{U} \\
176 \\
70 \mathrm{U} \\
49 \mathrm{U}\end{array}$ & $\begin{array}{rl}52 & U \\
53 & U \\
305 & \\
70 & U \\
49 & U\end{array}$ & $\begin{array}{c}179 \\
53 \mathrm{U} \\
572 \\
70 \mathrm{U} \\
88\end{array}$ & $\begin{array}{l}62 \mathrm{U} \\
53 \\
72 \\
70 \\
49\end{array}$ \\
\hline
\end{tabular}


IABLE L.2. (contd)

Sediment

Ireatment

$R-A C$

$R-A C$

$R-A C$

$R-A C$

$R-A C$

$R-A M$

$R-A M$

$R-A M$

$R-A M$

$R-A M$

$R-B F$

$R-B F$

$R-B F$

$R-B F$

$R-B F$

$R-O S$

$R-O S$

$R-O S$

$R-O S$

R-OS

$R-P C$

$R-P C$

$R \sim P C$

$R-P C$

$R-P C$

$R-P C$

$R-P F$

$R-P F$

$R-P F$

$R-P F$

$R-P F$

$R-P F$

$C-S B$

$C-5 B$

C.SB

C.SB

C.SB

$C-N E$

$C-N E$

$C-N E$

$C-N E$

$C-N E$

\section{Replicate Batch}

$B$
$B$
$B$
$B$
$B$

17.69

13.48

21.41

20.16

15.84

17.43

14.88

17.30

15.68

17.78

13. 29

19.11

14.85

16.11

$18.0 \%$

$17.08^{(b)}$

23.44
10.18

10.18

14.76

16.00

16.00

20.86

20.52

16.36

20.47

14.50

15.40

14.23

14.23

14.96

15.08

21.76

13.05

20.69

20.20

12.31

17.55

17. 85

18.39

16.70

18.54
$N$. caecoides PAHs $(\mu \mathrm{kg} / \mathrm{kg}$ dry weight $)$

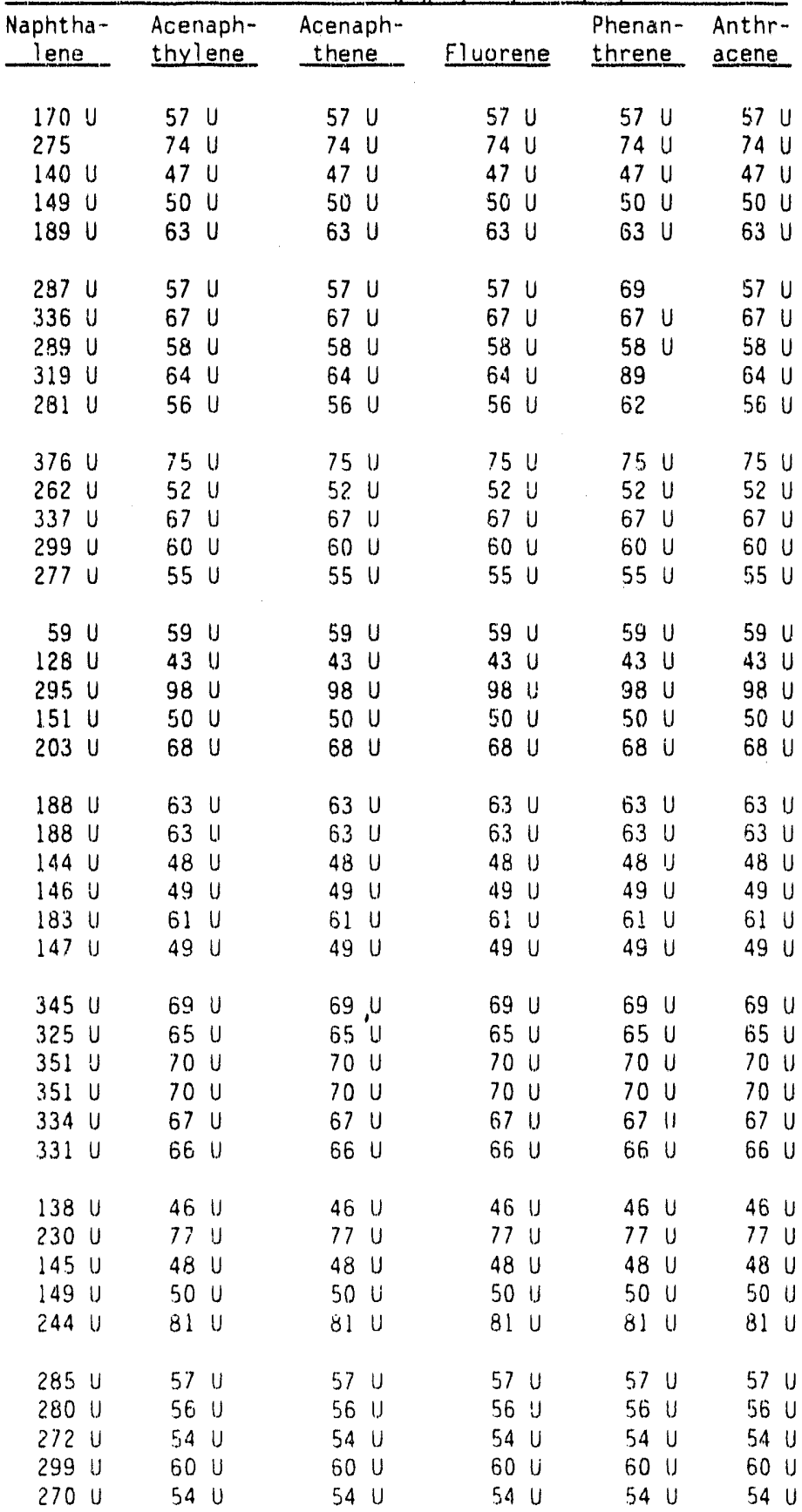

(a) Undetected above given concentration

(b) No percent dry weight: used mean percent dry weight of other four replicates.

(i) Ariomalous datia point not used in statistical analysis. 


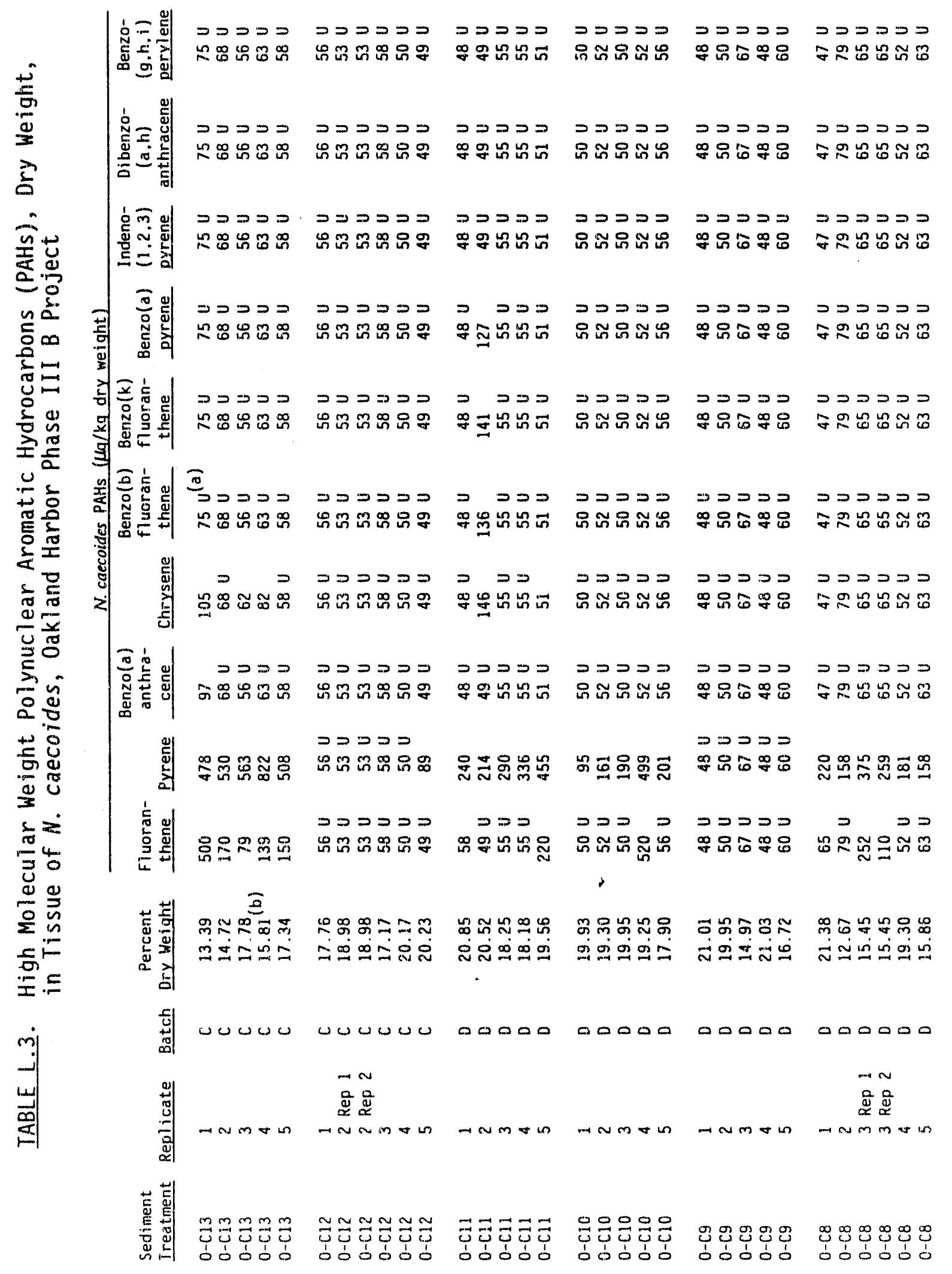




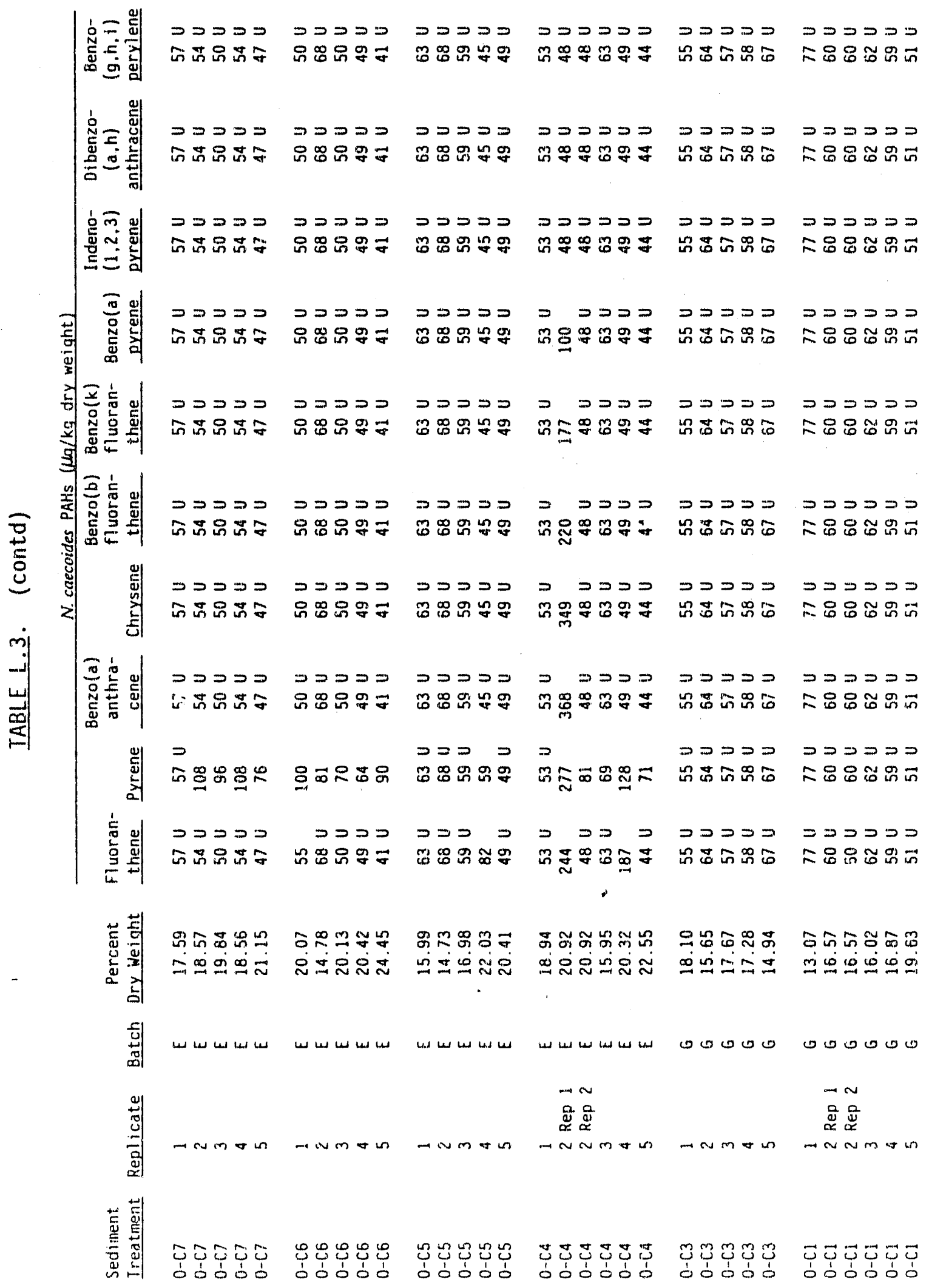




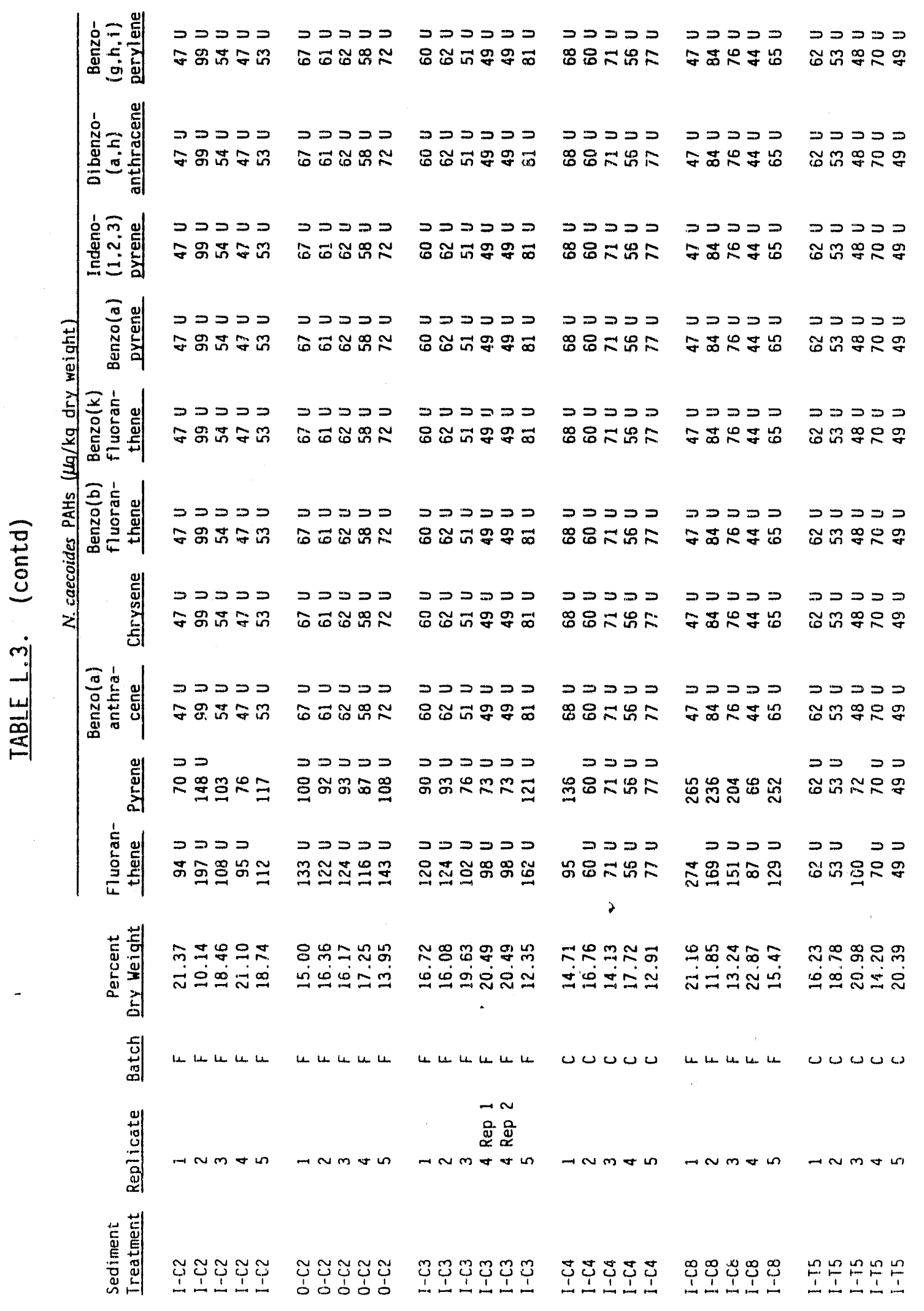




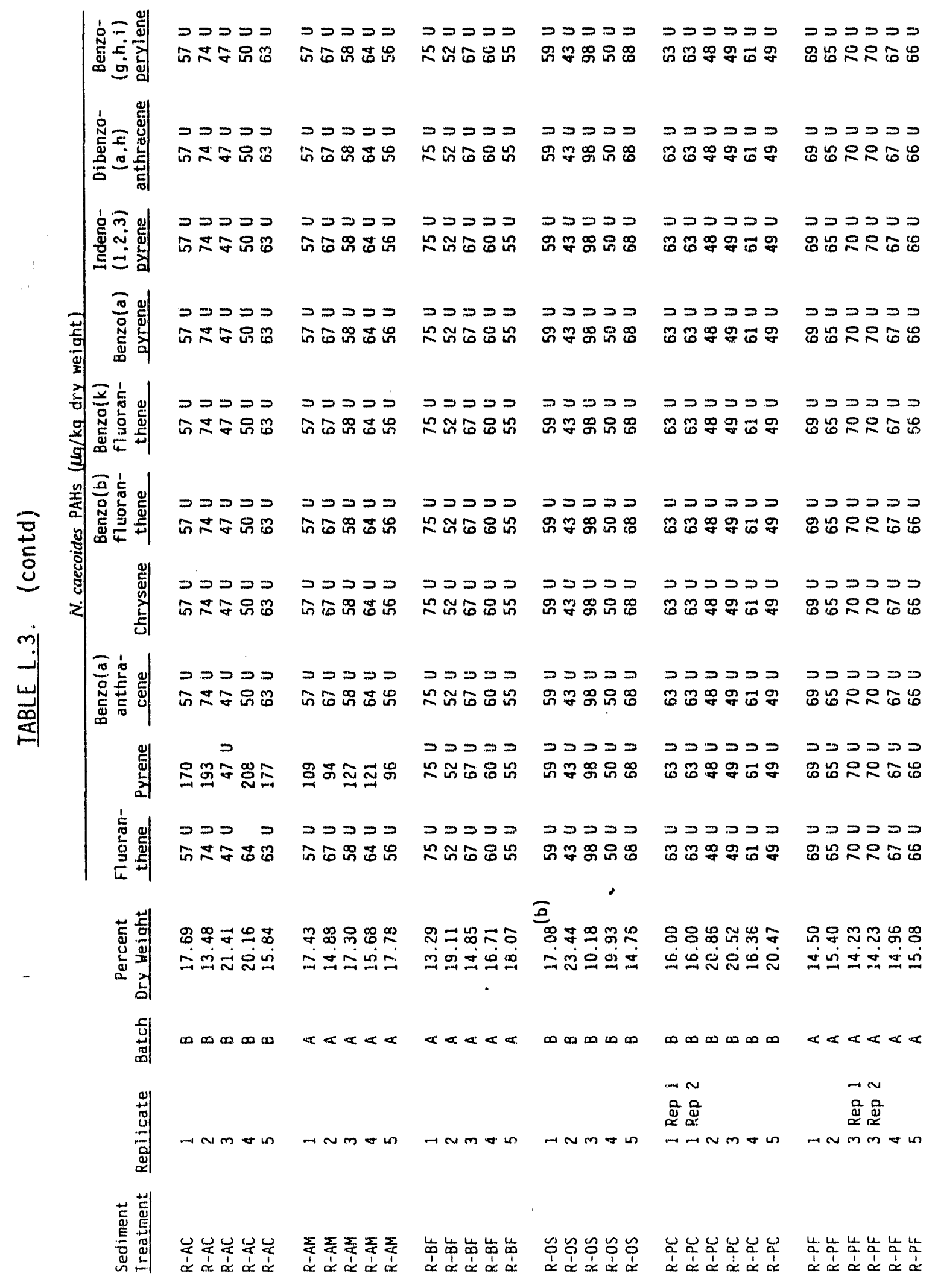

PHASE III B

L. 10 


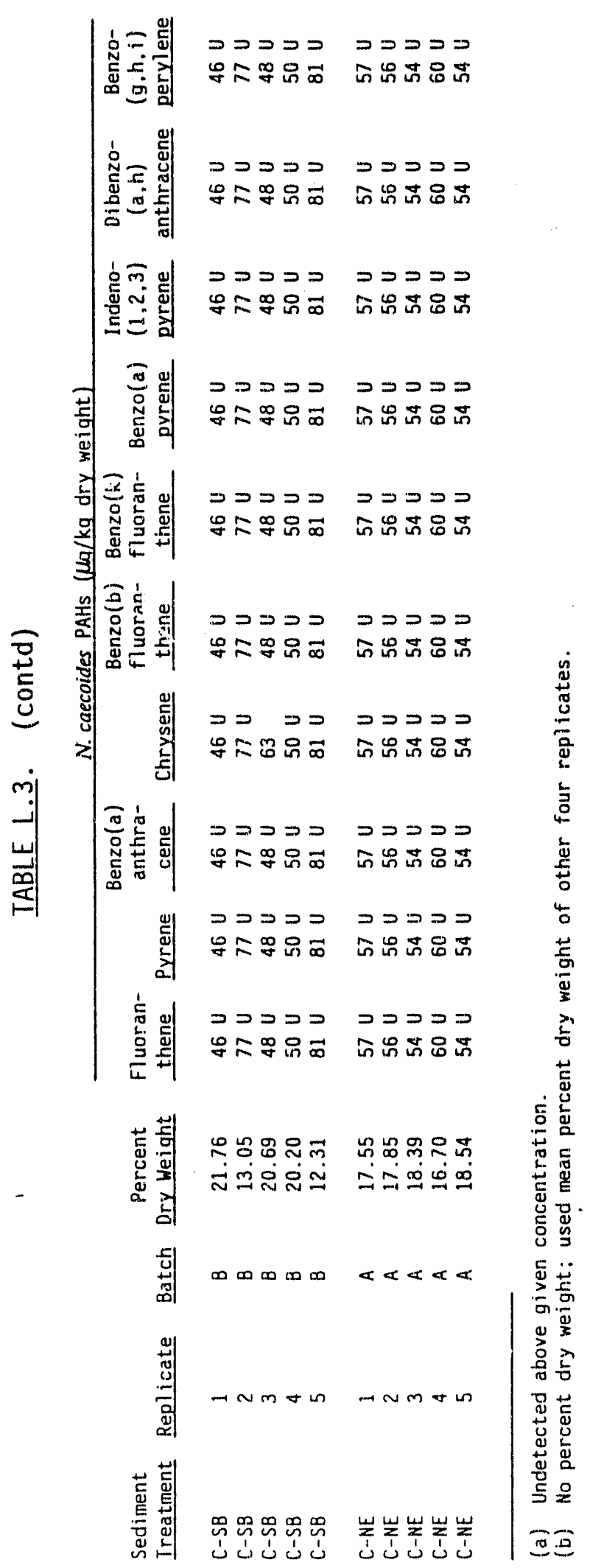


TABLE L.4. Total Detected Polynuclear Aromatic Hydrocarbons (PAHs), Wet Weight, in Tissue of N. caecoides, Oakland Harbor Phase III B Project

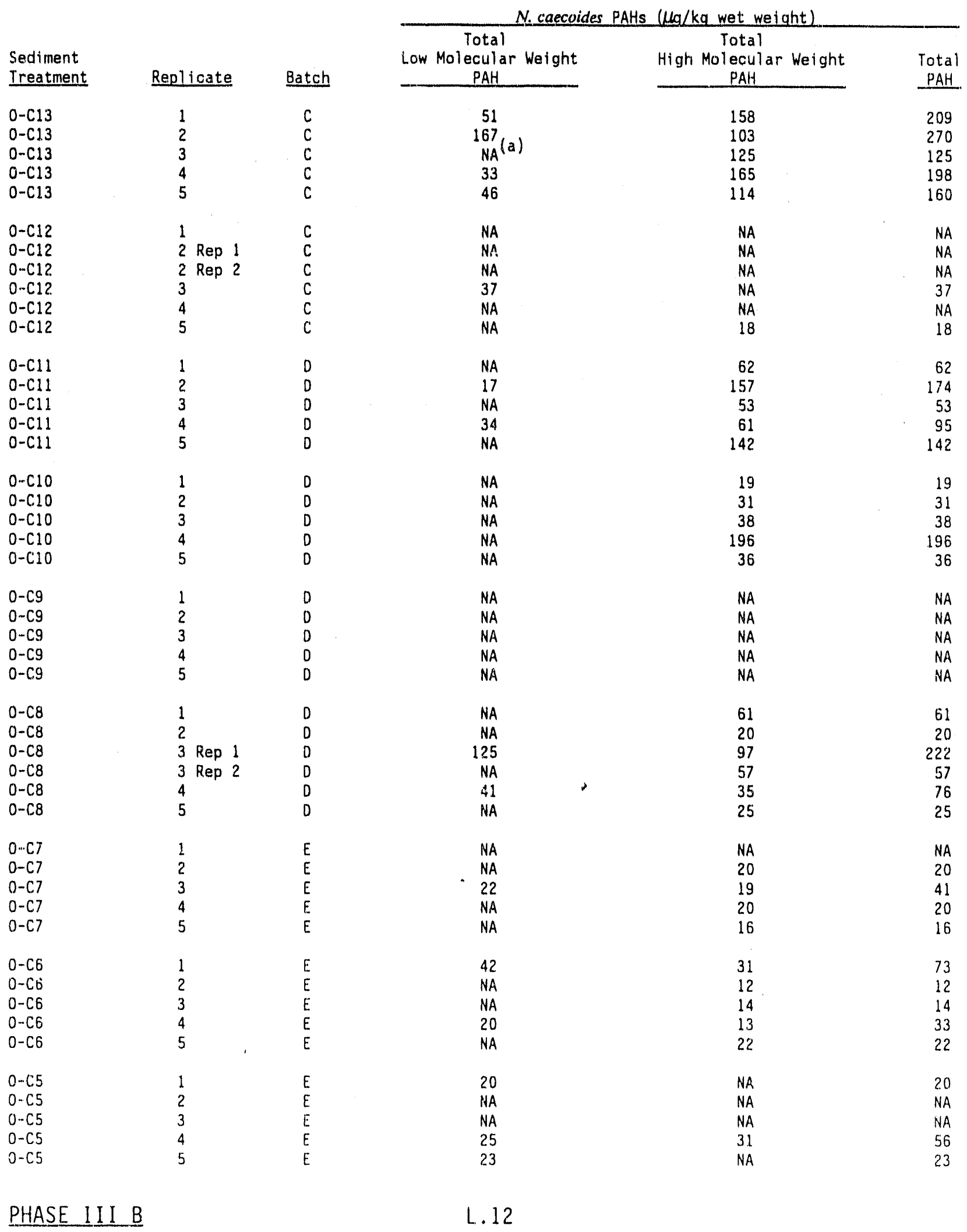


TABLE L.4. (contd)

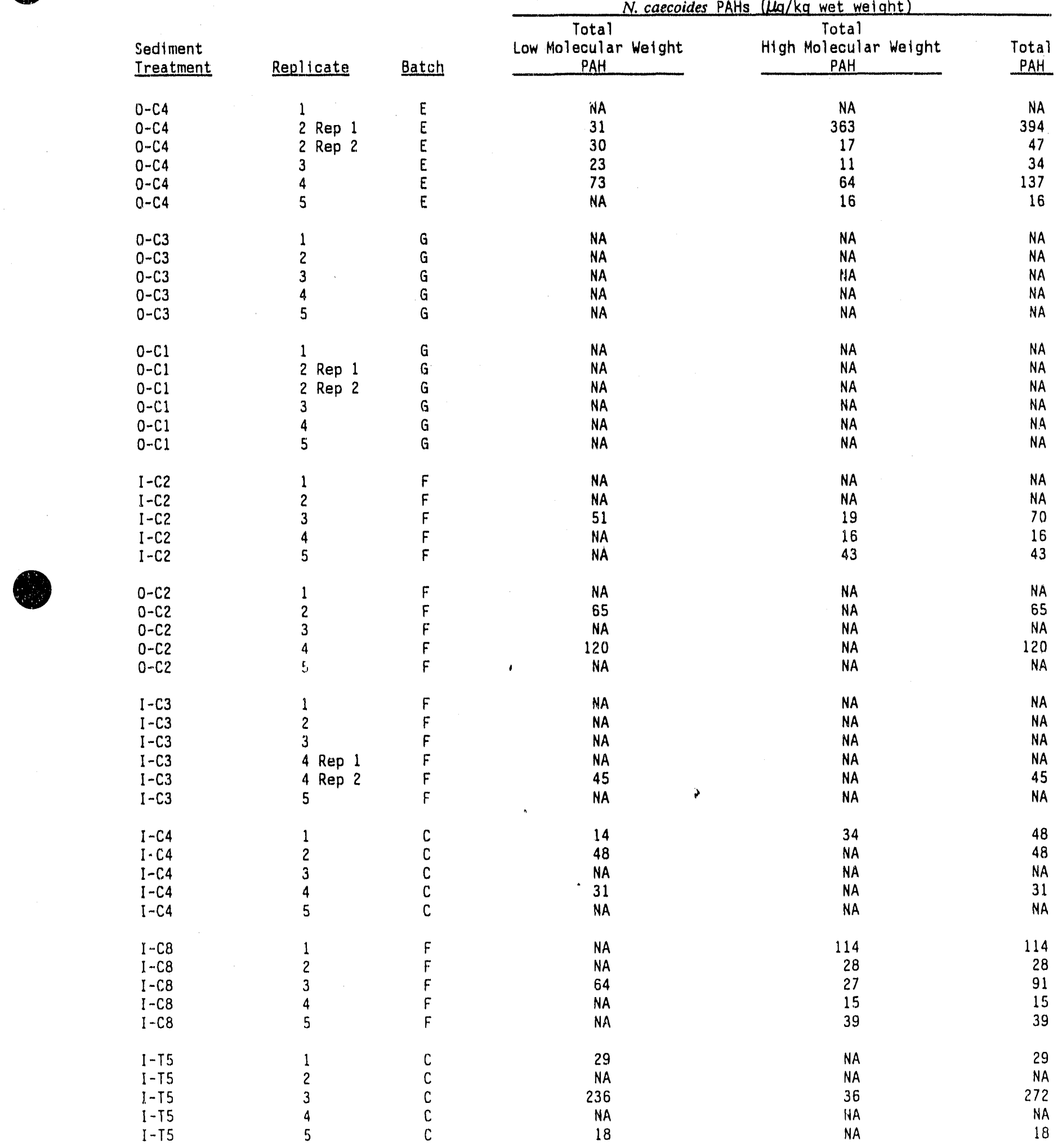

\begin{tabular}{|c|c|}
\hline $\begin{array}{l}\text { Total } \\
\text { Low Molecular Weight }\end{array}$ & $\begin{array}{l}\text { Total } \\
\text { High Molecular Weight }\end{array}$ \\
\hline
\end{tabular}


TABLE L.4. (contd)

Sediment

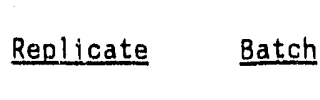

$\frac{N \text { caecoides PAHs (kg/kg wet weight) }}{\text { Total }}$

Treatment

Low Molecular Weight

$\mathrm{PAH}$

High Molecular Weight

$R=A C$

$R-A C$

$R-A C$

$R-A C$

$\begin{array}{ll}1 & B \\ 2 & B\end{array}$

2

$R-A C$

$R-A M$

$R-A M$

$R-A M$

$R-A M$

$R-A M$

$R-8 F$

$R-B F$

$R-B F$

$R-B F$

$R-B F$

$R-O S$

$R-O S$

$R-O S$

R-OS

$R-O S$

$R-P C$

$R-P C$

$R-P C$

$R-P C$

$R-P C$

$R-P C$

$R-P F$

$R-P F$

$R-P F$

$R-P F$

$R-P F$

$R-P F$

C-SB

C-SB

$C-S B$

C-SB

C-SB

C-NE

C-NE

C-NE

C-NE

C-NE

\section{NA}

37

NA

NA

NA

PAH

PAH

$\begin{array}{ll}30 & 30 \\ 26 & 63 \\ \text { NA } & \text { NA } \\ 55 & 55 \\ 28 & 28 \\ & \\ 19 & 31 \\ 14 & 14 \\ 22 & 22 \\ 19 & 33 \\ 17 & 28\end{array}$

1

2

3

5

1

2

3
4

5

1 Rep 1

1 Rep 2

2

3

4

3 Rep 1

3 Rep 2

4

12

NA

NA

14

NA

NA

NA

NA

NF

NA

NA

NA

$N A$

NA

$\begin{array}{lll}N A & N A & N A \\ N A & N A & N A \\ N A & N A & N A \\ N A & N A \\ N A & N A & N A\end{array}$

NA

NA

NA

NA

NA

NA

NA

NA

NA

NA

NA

NA

NA

NA

NA

NA

NA

NA

NA

NA

NA

NA

$\begin{array}{ll}N A & N A \\ N A & N A \\ \text { I'A } & N A \\ N h & N A \\ N A & N A \\ N A & N A\end{array}$

NA

NA NA

NA NA

NA NA

$\begin{array}{ll}N A & N A \\ N A & N A\end{array}$

NA NA

NA NA

13

NA NA

NA NA

NA NA

NA NA

$\begin{array}{ll}N A & N A \\ N A & N A\end{array}$

NA NA

(a) Not applicable: No PAHs detected. 
TABLE L.5. Low Molecular Weight Polynuclear Aromatic Hydrocarbons (PAHs), Wet Weight, in Tissue of N. caecoides, Oakl and Harbor Phase III B Project

\begin{tabular}{|c|c|c|c|c|c|c|c|c|}
\hline \multirow[b]{2}{*}{$\begin{array}{l}\text { Sediment } \\
\text { Ireatment }\end{array}$} & \multirow[b]{2}{*}{ Replicate } & \multirow[b]{2}{*}{ Batch } & \multicolumn{5}{|c|}{$N$. caecoides PAHs $(\mu \mathrm{g} / \mathrm{kg}$ wet weight $)$} & \multirow[b]{2}{*}{$\begin{array}{l}\text { Anthr- } \\
\text { acene }\end{array}$} \\
\hline & & & $\begin{array}{l}\text { Naphtha- } \\
\text { lene }\end{array}$ & $\begin{array}{l}\text { Acerlaph- } \\
\text { thylene }\end{array}$ & $\begin{array}{l}\text { Acenaph- } \\
\text { - Bhene }\end{array}$ & Eluorene & $\begin{array}{l}\text { Phenan- } \\
\text { threne }\end{array}$ & \\
\hline $\begin{array}{l}\text { OL Goal } \\
\text { OL Achieved }\end{array}$ & & & $\begin{array}{c}20 \\
20-100\end{array}$ & $\begin{array}{l}20 \\
10\end{array}$ & $\begin{array}{l}20 \\
10\end{array}$ & $\begin{array}{l}20 \\
10\end{array}$ & $\begin{array}{l}20 \\
10\end{array}$ & $\begin{array}{l}20 \\
10\end{array}$ \\
\hline $\begin{array}{l}0-C 13 \\
0-C 13 \\
0-C 13 \\
0-C 13 \\
0-C 13\end{array}$ & $\begin{array}{l}1 \\
2 \\
3 \\
4 \\
5\end{array}$ & $\begin{array}{l}c \\
c \\
c \\
c \\
c\end{array}$ & $\begin{array}{l}32 \\
30 U \\
30 U \\
33 \\
32\end{array}$ & $\begin{array}{l}10 U^{(a)} \\
10 U^{(a)} \\
10 U \\
10 U \\
10 U\end{array}$ & $\begin{array}{l}10 U \\
13 \\
10 U \\
10 U \\
10 U\end{array}$ & $\begin{array}{l}10 \mathrm{U} \\
38 \\
10 \mathrm{U} \\
10 \mathrm{U} \\
10 \mathrm{U}\end{array}$ & $\begin{array}{l}19 \\
100 \\
10 \mathrm{U} \\
10 \mathrm{U} \\
14\end{array}$ & $\begin{array}{l}10 U \\
16 \\
10 U \\
10 U \\
10 U\end{array}$ \\
\hline $\begin{array}{l}0-C 12 \\
0-C 12 \\
0-C 12 \\
0-C 12 \\
0-C 12 \\
0-C 12\end{array}$ & 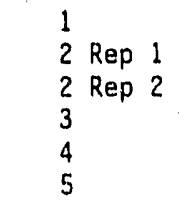 & $\begin{array}{l}c \\
c \\
c \\
c \\
c \\
c\end{array}$ & $\begin{array}{l}30 U \\
30 U \\
30 U \\
37 \\
30 U \\
30 U\end{array}$ & $\begin{array}{l}10 \mathrm{U} \\
10 \mathrm{U} \\
10 \mathrm{U} \\
10 \mathrm{U} \\
10 \mathrm{U} \\
10 \mathrm{U}\end{array}$ & $\begin{array}{l}10 U \\
10 U \\
10 U \\
10 U \\
10 U \\
10 U\end{array}$ & $\begin{array}{l}10 U \\
10 U \\
10 U \\
10 U \\
10 U \\
10 U\end{array}$ & $\begin{array}{l}10 U \\
10 U \\
10 U \\
10 U \\
10 U \\
10 U\end{array}$ & $\begin{array}{ll}10 & U \\
10 & U \\
10 & U \\
10 & U \\
10 & U \\
10 & U\end{array}$ \\
\hline $\begin{array}{l}0-C 11 \\
0-C 11 \\
0-C 11 \\
0-C 11 \\
0-C 11\end{array}$ & $\begin{array}{l}1 \\
2 \\
3 \\
4 \\
5\end{array}$ & $\begin{array}{l}0 \\
0 \\
0 \\
0 \\
0\end{array}$ & $\begin{array}{l}30 U \\
30 U \\
30 U \\
34 \\
30 U\end{array}$ & $\begin{array}{l}10 U \\
10 U \\
10 U \\
10 U \\
10 U\end{array}$ & $\begin{array}{ll}10 & U \\
10 & U \\
10 & U \\
10 U \\
10 U\end{array}$ & $\begin{array}{l}10 U \\
10 U \\
10 U \\
10 U \\
10 U\end{array}$ & $\begin{array}{l}10 \mathrm{U} \\
17 \\
10 \mathrm{U} \\
10 \mathrm{U} \\
10 \mathrm{U}\end{array}$ & $\begin{array}{ll}10 & U \\
10 & U \\
10 U \\
10 U \\
10 U\end{array}$ \\
\hline $\begin{array}{l}0-C 10 \\
0-C 10 \\
0-C 10 \\
0-C 10 \\
0-C 10\end{array}$ & $\begin{array}{l}1 \\
2 \\
3 \\
4 \\
5\end{array}$ & $\begin{array}{l}D \\
D \\
D \\
D \\
D\end{array}$ & $\begin{array}{l}30 U \\
30 U \\
30 U \\
30 U \\
30 U\end{array}$ & $\begin{array}{l}10 U \\
10 U \\
10 U \\
10 U \\
10 U\end{array}$ & $\begin{array}{l}10 U \\
10 U \\
10 U \\
10 U \\
10 U\end{array}$ & $\begin{array}{l}10 U \\
10 U \\
10 U \\
10 U \\
10 U\end{array}$ & $\begin{array}{l}10 U \\
10 U \\
10 U \\
10 U \\
10 U\end{array}$ & $\begin{array}{ll}10 U \\
10 U \\
10 U \\
10 U \\
10 U\end{array}$ \\
\hline $\begin{array}{l}0-c 9 \\
0-c 9 \\
0-c 9 \\
0-c 9 \\
0-c 9\end{array}$ & $\begin{array}{l}1 \\
2 \\
3 \\
4 \\
5\end{array}$ & $\begin{array}{l}0 \\
D \\
D \\
0 \\
D\end{array}$ & $\begin{array}{l}30 U \\
30 U \\
30 U \\
30 U \\
30 U\end{array}$ & $\begin{array}{ll}10 & U \\
10 & U \\
10 & U \\
10 & U \\
10 & U\end{array}$ & $\begin{array}{l}10 U \\
10 U \\
10 U \\
10 U \\
10 U\end{array}$ & $\begin{array}{l}10 U \\
10 U \\
10 U \\
10 U \\
10 U\end{array}$ & $\begin{array}{l}10 U \\
10 U \\
10 U \\
10 U \\
10 U\end{array}$ & $\begin{array}{l}10 U \\
10 U \\
10 U \\
10 U \\
10 U\end{array}$ \\
\hline $\begin{array}{l}0-C 8 \\
0-C 8 \\
0-C 8 \\
0-C 8 \\
0-C 8 \\
0-C 8 \\
-\end{array}$ & 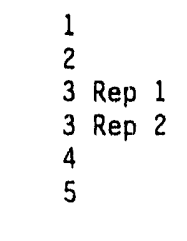 & $\begin{array}{l}D \\
0 \\
0 \\
0 \\
0 \\
D\end{array}$ & $\begin{array}{l}30 \mathrm{U} \\
30 \mathrm{U} \\
30 \mathrm{U} \\
30 \mathrm{U} \\
41 \\
30 \mathrm{U}\end{array}$ & $\begin{array}{l}10 U \\
10 U \\
10 U \\
10 U \\
10 U \\
10 U\end{array}$ & $\begin{array}{l}10 U \\
10 U \\
10 U \\
10 U \\
10 U \\
10 U\end{array}$ & $\begin{array}{ll}10 U \\
10 U \\
10 U \\
10 U \\
10 U \\
10 U\end{array}$ & $\begin{array}{l}10 \mathrm{U} \\
10 \mathrm{U} \\
110 \\
10 \mathrm{U} \\
10 \mathrm{U} \\
10 \mathrm{U}\end{array}$ & $\begin{array}{l}10 U \\
10 U \\
15 \\
10 U \\
10 U \\
10 U\end{array}$ \\
\hline $\begin{array}{l}0-C 7 \\
0-C 7 \\
0-C 7 \\
0-C 7 \\
0-C 7\end{array}$ & $\begin{array}{l}1 \\
2 \\
3 \\
4 \\
5\end{array}$ & $\begin{array}{l}E \\
E \\
E \\
E \\
E\end{array}$ & $\begin{array}{l}20 U \\
20 U \\
22 \\
20 U \\
20 U\end{array}$ & $\begin{array}{ll}10 & U \\
10 & U \\
10 & U \\
10 & U \\
10 & U\end{array}$ & $\begin{array}{ll}10 U \\
10 U \\
10 U \\
10 U \\
10 U\end{array}$ & $\begin{array}{ll}10 U \\
10 U \\
10 U \\
10 U \\
10 U\end{array}$ & $\begin{array}{l}10 U \\
10 U \\
10 U \\
10 U \\
10 U\end{array}$ & $\begin{array}{l}10 U \\
10 U \\
10 U \\
10 U \\
10 U\end{array}$ \\
\hline $\begin{array}{l}0-C 6 \\
0-C 6 \\
0-C 6 \\
0-C 6 \\
0-C 6\end{array}$ & $\begin{array}{l}1 \\
2 \\
3 \\
4 \\
5\end{array}$ & $\begin{array}{l}E \\
E \\
E \\
E \\
E\end{array}$ & $\begin{array}{l}42 \\
20 U \\
20 U \\
20 \\
20 U\end{array}$ & $\begin{array}{ll}10 & U \\
10 & U \\
10 & U \\
10 & U \\
10 & U\end{array}$ & $\begin{array}{ll}10 U \\
10 U \\
10 U \\
10 U \\
10 U\end{array}$ & $\begin{array}{ll}10 U \\
10 U \\
10 U \\
10 U \\
10 U\end{array}$ & $\begin{array}{ll}10 U \\
10 U \\
10 U \\
10 U \\
10 U\end{array}$ & $\begin{array}{l}10 U \\
10 U \\
10 U \\
10 U \\
10 U\end{array}$ \\
\hline
\end{tabular}


IABLE L.5. (contd)

\begin{tabular}{|c|c|c|c|c|c|c|c|c|}
\hline \multirow[b]{2}{*}{$\begin{array}{l}\text { Sediment } \\
\text { Ireatment }\end{array}$} & \multirow[b]{2}{*}{ Replicate } & \multirow[b]{2}{*}{ Batch } & \multicolumn{6}{|c|}{ 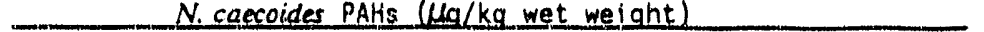 } \\
\hline & & & $\begin{array}{l}\text { Naphtha- } \\
\text { lene }\end{array}$ & $\begin{array}{l}\text { Acenaph- } \\
\text { thylene }\end{array}$ & $\begin{array}{l}\text { Acenaph- } \\
\text { thene }\end{array}$ & Fluorene & $\begin{array}{l}\text { Phenan- } \\
\text { threne }\end{array}$ & $\begin{array}{l}\text { Anthr- } \\
\text { acene }\end{array}$ \\
\hline $\begin{array}{l}0-C 5 \\
0-C 5 \\
0-C 5 \\
0-C 5 \\
0-C 5\end{array}$ & $\begin{array}{l}1 \\
2 \\
3 \\
4 \\
5\end{array}$ & $\begin{array}{l}E \\
E \\
E \\
E \\
E\end{array}$ & $\begin{array}{l}20 \\
20 U \\
20 U \\
20 U \\
23\end{array}$ & $\begin{array}{l}10 \mathrm{U} \\
10 \mathrm{U} \\
10 \mathrm{U} \\
10 \mathrm{U} \\
10 \mathrm{U}\end{array}$ & $\begin{array}{l}10 U \\
10 U \\
10 U \\
10 U \\
10 U\end{array}$ & $\begin{array}{l}10 \mathrm{U} \\
10 \mathrm{U} \\
10 \mathrm{U} \\
10 \mathrm{U} \\
10 \mathrm{U}\end{array}$ & $\begin{array}{l}10 U \\
10 U \\
10 U \\
25 \\
10 U\end{array}$ & $\begin{array}{ll}10 & U \\
10 & U \\
10 & U \\
10 & U \\
10 U\end{array}$ \\
\hline $\begin{array}{l}0-C 4 \\
0-C 4 \\
0-C 4 \\
0-C 4 \\
0-C 4 \\
0-C 4\end{array}$ & $\begin{array}{lll}1 & & \\
2 & \text { Rep } 1 \\
2 & \operatorname{Rep} 2 \\
3 & & \\
4 & \\
5 & \end{array}$ & $\begin{array}{l}E \\
E \\
E \\
E \\
E \\
E\end{array}$ & $\begin{array}{l}20 \mathrm{U} \\
20 \mathrm{U} \\
30 \\
23 \\
20 \mathrm{U} \\
20 \mathrm{U}\end{array}$ & $\begin{array}{l}10 \mathrm{U} \\
10 \mathrm{U} \\
10 \mathrm{U} \\
10 \mathrm{U} \\
10 \mathrm{U} \\
10 \mathrm{U}\end{array}$ & $\begin{array}{l}10 U \\
10 U \\
10 U \\
10 U \\
10 U \\
10 U\end{array}$ & $\begin{array}{l}10 U \\
10 U \\
10 U \\
10 U \\
10 U \\
10 U\end{array}$ & $\begin{array}{l}10 \mathrm{U} \\
19 \\
10 \mathrm{U} \\
10 \mathrm{U} \\
63 \\
10 \mathrm{U}\end{array}$ & $\begin{array}{l}10 \mathrm{U} \\
12 \\
10 \mathrm{U} \\
10 \mathrm{U} \\
10 \\
10 \mathrm{U}\end{array}$ \\
\hline $\begin{array}{l}0-c 3 \\
0-c 3 \\
0-c 3 \\
0-c 3 \\
0-c 3\end{array}$ & $\begin{array}{l}1 \\
2 \\
3 \\
4 \\
5\end{array}$ & $\begin{array}{l}G \\
G \\
G \\
G \\
G\end{array}$ & $\begin{array}{rl}60 & U \\
100 U \\
50 U \\
100 U \\
60 U\end{array}$ & $\begin{array}{l}10 \mathrm{U} \\
10 \mathrm{U} \\
10 \mathrm{U} \\
10 \mathrm{U} \\
10 \mathrm{U}\end{array}$ & $\begin{array}{l}10 U \\
10 U \\
10 U \\
10 U \\
10 U\end{array}$ & $\begin{array}{l}10 \mathrm{U} \\
10 \mathrm{U} \\
10 \mathrm{U} \\
10 \mathrm{U} \\
10 \mathrm{U}\end{array}$ & $\begin{array}{l}10 U \\
10 U \\
10 U \\
10 U \\
10 U\end{array}$ & $\begin{array}{ll}10 & U \\
10 & U \\
10 & U \\
10 & U \\
10 & U\end{array}$ \\
\hline $\begin{array}{l}0-C 1 \\
0-C 1 \\
0-C 1 \\
0-C 1 \\
0-C 1 \\
0-C 1\end{array}$ & $\begin{array}{ll}1 & \\
2 & \operatorname{Rep} 1 \\
2 & \operatorname{Rep} 2 \\
3 & \\
4 & \\
5 & \end{array}$ & $\begin{array}{l}G \\
G \\
G \\
G \\
G \\
G\end{array}$ & $\begin{array}{rl}60 & U \\
60 & U \\
60 & U \\
100 & U \\
60 & U \\
60 U\end{array}$ & $\begin{array}{l}10 U \\
10 U \\
10 U \\
10 U \\
10 U \\
10 U\end{array}$ & $\begin{array}{l}10 U \\
10 U \\
10 U \\
10 U \\
10 U \\
10 U\end{array}$ & $\begin{array}{l}10 U \\
10 U \\
10 U \\
10 U \\
10 U \\
10 U\end{array}$ & $\begin{array}{l}10 U \\
10 U \\
10 U \\
10 U \\
10 U \\
10 U\end{array}$ & $\begin{array}{ll}10 & U \\
10 & U \\
10 & U \\
10 & U \\
10 & U \\
10 & U\end{array}$ \\
\hline $\begin{array}{l}1-C 2 \\
1-C 2 \\
1-C 2 \\
1-C 2 \\
1-C 2\end{array}$ & $\begin{array}{l}1 \\
2 \\
3 \\
4 \\
5\end{array}$ & $\begin{array}{l}F \\
F \\
F \\
F \\
F\end{array}$ & $\begin{array}{l}40 U \\
40 U \\
51 \\
40 U \\
40 U\end{array}$ & $\begin{array}{l}10 U \\
10 U \\
10 U \\
10 U \\
10 U\end{array}$ & $\begin{array}{l}10 \mathrm{U} \\
10 \mathrm{U} \\
10 \mathrm{U} \\
10 \mathrm{U} \\
10 \mathrm{U}\end{array}$ & $\begin{array}{ll}10 & U \\
10 & U \\
10 & U \\
10 & U \\
10 & U\end{array}$ & $\begin{array}{l}10 U \\
10 U \\
10 U \\
10 U \\
10 U\end{array}$ & $\begin{array}{ll}10 & U \\
10 & U \\
10 & U \\
10 & U \\
10 & U\end{array}$ \\
\hline $\begin{array}{l}0-C 2 \\
0-C 2 \\
0-C 2 \\
0-C 2 \\
0-C 2\end{array}$ & $\begin{array}{l}1 \\
2 \\
3 \\
4 \\
5\end{array}$ & $\begin{array}{l}F \\
F \\
F \\
F \\
F\end{array}$ & $\begin{array}{c}40 \mathrm{U} \\
65 \\
40 \mathrm{U} \\
120 \\
40 \mathrm{U}\end{array}$ & $\begin{array}{l}10 U \\
10 U \\
10 U \\
10 U \\
10 U\end{array}$ & $\begin{array}{l}10 U \\
10 U \\
10 U \\
10 U \\
10 U\end{array}$ & $\begin{array}{l}10 U \\
10 U \\
10 U \\
10 U \\
10 U\end{array}$ & $\begin{array}{l}10 U \\
10 U \\
10 U \\
10 U \\
10 U\end{array}$ & $\begin{array}{ll}10 & U \\
10 & U \\
10 & U \\
10 & U \\
10 & U\end{array}$ \\
\hline $\begin{array}{l}I-C .3 \\
1-C .3 \\
1-C 3 \\
1-C . C 3 \\
I=C .=3 \\
1-C 3\end{array}$ & $\begin{array}{ll}1 & \\
2 & \\
3 & \\
4 & R e p 1 \\
4 & R e p 2 \\
5 & \end{array}$ & $\begin{array}{l}F \\
F \\
F \\
F \\
F \\
F\end{array}$ & $\begin{array}{l}40 U \\
40 U \\
40 U \\
40 U \\
45 \\
40 U .\end{array}$ & $\begin{array}{l}10 U \\
10 U \\
10 U \\
10 U \\
10 U \\
10 U\end{array}$ & $\begin{array}{l}100 \\
100 \\
100 \\
100 \\
100 \\
100\end{array}$ & $\begin{array}{l}10 U \\
10 U \\
10 U \\
10 U \\
10 U \\
10 U\end{array}$ & $\begin{array}{l}10 U \\
10 U \\
10 U \\
10 U \\
10 U \\
10 U\end{array}$ & $\begin{array}{ll}10 & U \\
10 & U \\
10 & U \\
10 & U \\
10 & U \\
10 & U\end{array}$ \\
\hline $\begin{array}{l}I-C 4 \\
I-C 4 \\
I-C 4 \\
I-C 4 \\
I-C 4\end{array}$ & $\begin{array}{l}1 \\
2 \\
3 \\
4 \\
5\end{array}$ & $\begin{array}{l}c \\
c \\
c \\
c \\
c\end{array}$ & $\begin{array}{l}30 \mathrm{U} \\
48 \\
30 \mathrm{U} \\
31 \\
30 \mathrm{U}\end{array}$ & $\begin{array}{l}10 \mathrm{U} \\
10 \mathrm{U} \\
10 \mathrm{U} \\
10 \mathrm{U} \\
10 \mathrm{U}\end{array}$ & 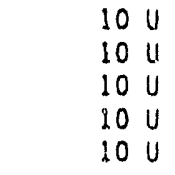 & $\begin{array}{l}10 \mathrm{U} \\
10 \mathrm{U} \\
10 \mathrm{U} \\
10 \mathrm{U} \\
10 \mathrm{U}\end{array}$ & $\begin{array}{l}14 \\
10 U \\
10 U \\
10 U \\
10 U\end{array}$ & $\begin{array}{ll}10 & U \\
10 & 11 \\
10 & U \\
10 & U \\
10 & U\end{array}$ \\
\hline $\begin{array}{l}1-C 8 \\
1-C B \\
1-C 8 \\
1-C 8 \\
1-C 8\end{array}$ & $\begin{array}{l}1 \\
2 \\
3 \\
4 \\
5\end{array}$ & $\begin{array}{l}F \\
F \\
F \\
F \\
F\end{array}$ & $\begin{array}{l}40 \mathrm{U} \\
40 \mathrm{U} \\
64 \\
40 \mathrm{U} \\
40 \mathrm{U}\end{array}$ & $\begin{array}{l}10 U \\
10 U \\
10 U \\
10 U \\
10 U\end{array}$ & $\begin{array}{l}10 U \\
10 U \\
10 U \\
10 U \\
10 U\end{array}$ & $\begin{array}{l}10 U \\
10 U \\
10 U \\
10 U \\
10 U\end{array}$ & $\begin{array}{l}10 \mathrm{U} \\
10 \mathrm{U} \\
10 \mathrm{U} \\
10 \mathrm{U} \\
10 \mathrm{U}\end{array}$ & $\begin{array}{ll}10 & U \\
10 & U \\
10 U \\
10 U \\
10 U\end{array}$ \\
\hline
\end{tabular}

PHASE III B 
IABLE L.5. (contd)

\begin{tabular}{|c|c|c|c|c|c|c|c|c|}
\hline \multirow[b]{2}{*}{$\begin{array}{l}\text { Sediment } \\
\text { Treatment }\end{array}$} & \multirow[b]{2}{*}{ Replicate } & \multirow[b]{2}{*}{ Batch } & \multicolumn{5}{|c|}{ N. caecoides PAHs (ka/ $\mathrm{kg}$ wet weight) } & \multirow[b]{2}{*}{$\begin{array}{l}\text { Anthr- } \\
\text { acene }\end{array}$} \\
\hline & & & $\begin{array}{l}\text { Naphtha- } \\
\text { lene }\end{array}$ & $\begin{array}{l}\text { Acenaph- } \\
\text { thylene }\end{array}$ & $\begin{array}{l}\text { Acenaph- } \\
\text { thene }\end{array}$ & Eluorene & $\begin{array}{l}\text { Phenan- } \\
\text { threne }\end{array}$ & \\
\hline $\begin{array}{l}I-T 5 \\
I-T 5 \\
I-T 5 \\
I-T 5 \\
I-T 5\end{array}$ & $\begin{array}{l}1 \\
2 \\
3 \\
4 \\
5\end{array}$ & $\begin{array}{l}c \\
c \\
c \\
c \\
c\end{array}$ & $\begin{array}{l}30 \mathrm{U} \\
30 \mathrm{U} \\
30 \mathrm{U} \\
30 \mathrm{U} \\
30 \mathrm{U}\end{array}$ & $\begin{array}{l}10 U \\
10 U \\
10 U \\
10 U \\
10 U\end{array}$ & $\begin{array}{l}10 u \\
10 u \\
37 \\
10 u \\
10 U\end{array}$ & $\begin{array}{l}10 U \\
10 U \\
64 \\
10 U \\
10 U\end{array}$ & $\begin{array}{c}29 \\
10 \mathrm{U} \\
120 \\
10 \mathrm{U} \\
18\end{array}$ & $\begin{array}{l}10 U \\
10 U \\
15 \\
10 U \\
10 U\end{array}$ \\
\hline $\begin{array}{l}R-A C \\
R-A C \\
R-A C \\
R-A C \\
R-A C\end{array}$ & $\begin{array}{l}1 \\
2 \\
3 \\
4 \\
5\end{array}$ & $\begin{array}{l}B \\
B \\
B \\
B \\
B\end{array}$ & $\begin{array}{l}30 \mathrm{U} \\
37 \\
30 \mathrm{U} \\
30 \mathrm{U} \\
30 \mathrm{U}\end{array}$ & $\begin{array}{l}10 U \\
10 U \\
10 U \\
10 U \\
10 U\end{array}$ & $\begin{array}{l}10 U \\
10 U \\
10 U \\
10 U \\
10 U\end{array}$ & $\begin{array}{l}10 U \\
10 U \\
10 U \\
10 U \\
10 U\end{array}$ & $\begin{array}{l}10 U \\
10 U \\
10 U \\
10 U \\
10 U\end{array}$ & $\begin{array}{l}10 U \\
10 U \\
10 U \\
10 U \\
10 U\end{array}$ \\
\hline $\begin{array}{l}R-A M \\
R-A M \\
R-A M \\
R-A M \\
R-A M\end{array}$ & $\begin{array}{l}1 \\
2 \\
3 \\
4 \\
5\end{array}$ & $\begin{array}{l}A \\
A \\
A \\
A \\
A\end{array}$ & $\begin{array}{l}50 U \\
50 U \\
50 U \\
50 U \\
50 U\end{array}$ & $\begin{array}{l}10 U \\
10 U \\
10 U \\
10 U \\
10 U\end{array}$ & $\begin{array}{ll}10 & U \\
10 & U \\
10 & U \\
10 & U \\
10 & U\end{array}$ & $\begin{array}{l}10 \mathrm{U} \\
10 \mathrm{U} \\
10 \mathrm{U} \\
10 \mathrm{U} \\
10 \mathrm{U}\end{array}$ & $\begin{array}{l}12 \\
10 u \\
10 U \\
14 \\
11\end{array}$ & $\begin{array}{l}10 \mathrm{U} \\
10 \mathrm{U} \\
10 \mathrm{U} \\
10 \mathrm{U} \\
10 \mathrm{U}\end{array}$ \\
\hline $\begin{array}{l}R-B F \\
R-B F \\
R-B F \\
R-B F \\
R-B F\end{array}$ & $\begin{array}{l}1 \\
2 \\
3 \\
4 \\
5\end{array}$ & $\begin{array}{l}A \\
A \\
A \\
A \\
A\end{array}$ & $\begin{array}{l}50 \mathrm{U} \\
50 \mathrm{U} \\
50 \mathrm{U} \\
50 \mathrm{U} \\
50 \mathrm{U}\end{array}$ & 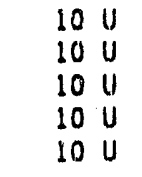 & $\begin{array}{ll}10 & U \\
10 & U \\
10 & U \\
10 & U \\
10 & U\end{array}$ & $\begin{array}{l}10 \mathrm{U} \\
10 \mathrm{U} \\
10 \mathrm{U} \\
10 \mathrm{U} \\
10 \mathrm{U}\end{array}$ & $\begin{array}{ll}10 & U \\
10 & U \\
10 U \\
10 U \\
10 U\end{array}$ & $\begin{array}{ll}10 & U \\
10 & U \\
10 & U \\
10 & U \\
10 & U\end{array}$ \\
\hline $\begin{array}{l}R=O S \\
R-O S \\
R=O S \\
R-O S \\
R-O S\end{array}$ & $\begin{array}{l}1 \\
2 \\
3 \\
4 \\
5\end{array}$ & $\begin{array}{l}B \\
B \\
B \\
B \\
B\end{array}$ & $\begin{array}{l}10 U \\
30 U \\
30 U \\
30 U \\
30 U\end{array}$ & $\begin{array}{ll}10 & U \\
10 & U \\
10 & U \\
10 & U \\
10 & U\end{array}$ & $\begin{array}{ll}10 & U \\
10 & U \\
10 & U \\
10 & U \\
10 U\end{array}$ & $\begin{array}{l}10 \mathrm{U} \\
10 \mathrm{U} \\
10 \mathrm{U} \\
10 \mathrm{U} \\
10 \mathrm{U}\end{array}$ & $\begin{array}{l}10 U \\
10 U \\
10 U \\
10 U \\
10 U\end{array}$ & $\begin{array}{l}10 u \\
10 U \\
10 U \\
10 U \\
10 U\end{array}$ \\
\hline $\begin{array}{l}R-P C \\
R-P C \\
R-P C \\
R-P C \\
R-P C \\
R-P C\end{array}$ & $\begin{array}{lll}1 & \operatorname{Rep} & 1 \\
1 & \operatorname{Rep} & 2 \\
2 & & \\
3 & & \\
4 & & \\
5 & & \end{array}$ & $\begin{array}{l}B \\
B \\
B \\
B \\
B \\
B\end{array}$ & $\begin{array}{l}30 U \\
30 U \\
30 U \\
30 U \\
30 U \\
30 U\end{array}$ & $\begin{array}{l}10 u \\
10 U \\
10 U \\
10 U \\
10 U \\
10 U\end{array}$ & $\begin{array}{ll}10 & U \\
10 & U \\
10 & U \\
10 & U \\
10 & U \\
10 & U\end{array}$ & $\begin{array}{l}10 U \\
10 U \\
10 U \\
10 U \\
10 U \\
10 U\end{array}$ & $\begin{array}{ll}10 U \\
10 U \\
10 U \\
10 U \\
10 U \\
10 U\end{array}$ & $\begin{array}{l}10 U \\
10 U \\
10 U \\
10 U \\
10 U \\
10 U\end{array}$ \\
\hline $\begin{array}{l}R-P F \\
R-P F \\
R=P F \\
R \cdot P F \\
R-P F \\
R=P F\end{array}$ & $\begin{array}{lll}1 & & \\
2 & & \\
3 & \operatorname{Rep} 1 \\
3 & \operatorname{Rep} 2 \\
4 & & \\
5 & & \end{array}$ & $\begin{array}{l}A \\
A \\
A \\
A \\
A \\
A\end{array}$ & $\begin{array}{l}50 U \\
50 U \\
50 U \\
50 U \\
50 U \\
50 U\end{array}$ & $\begin{array}{l}10 U \\
10 U \\
10 U \\
10 U \\
10 U \\
10 U\end{array}$ & $\begin{array}{ll}10 & U \\
10 & U \\
10 & U \\
10 & U \\
10 & U \\
10 & U\end{array}$ & $\begin{array}{l}10 U \\
10 U \\
10 U \\
10 U \\
10 U \\
10 U\end{array}$ & $\begin{array}{ll}10 U \\
10 U \\
10 U \\
10 U \\
10 U \\
10 U\end{array}$ & $\begin{array}{ll}10 & U \\
10 & 0 \\
10 & U \\
10 & U \\
10 & U \\
10 & 0\end{array}$ \\
\hline $\begin{array}{l}C-S B \\
C-S B \\
C-S B \\
C-S B \\
C-S B\end{array}$ & $\begin{array}{l}1 \\
2 \\
3 \\
4 \\
5\end{array}$ & $\begin{array}{l}B \\
B \\
B \\
B \\
E\end{array}$ & $\begin{array}{l}30 U \\
30 U \\
30 U \\
30 U \\
30 U\end{array}$ & $\begin{array}{l}10 U \\
10 U \\
10 U \\
10 U \\
10 U\end{array}$ & $\begin{array}{ll}10 & U \\
10 & U \\
10 & U \\
10 & U \\
10 & U\end{array}$ & $\begin{array}{l}10 U \\
10 U \\
10 U \\
10 U \\
10 U\end{array}$ & $\begin{array}{l}10 U \\
10 U \\
10 U \\
10 U \\
10 U\end{array}$ & $\begin{array}{ll}10 & 0 \\
10 & 0 \\
10 & U \\
10 & U \\
10 & U\end{array}$ \\
\hline $\begin{array}{l}C-N E \\
C-N E \\
C-H E \\
C-N E \\
C-N E\end{array}$ & $\begin{array}{l}1 \\
2 \\
3 \\
4 \\
5\end{array}$ & $\begin{array}{l}A \\
A \\
A \\
A \\
A\end{array}$ & $\begin{array}{l}50 \mathrm{U} \\
50 \mathrm{U} \\
50 \mathrm{U} \\
50 \mathrm{U} \\
50 \mathrm{U}\end{array}$ & $\begin{array}{l}10 U \\
10 U \\
10 U \\
10 U \\
10 U\end{array}$ & $\begin{array}{ll}10 & U \\
10 & U \\
10 & U \\
10 & u \\
10 & U\end{array}$ & $\begin{array}{l}10 U \\
10 U \\
10 U \\
10 U \\
10 U\end{array}$ & $\begin{array}{l}10 U \\
10 U \\
10 U \\
10 U \\
10 U\end{array}$ & $\begin{array}{l}10 u \\
10 \\
10 \\
10 \\
10\end{array}$ \\
\hline
\end{tabular}

(a) Undeterted above given concentration. 


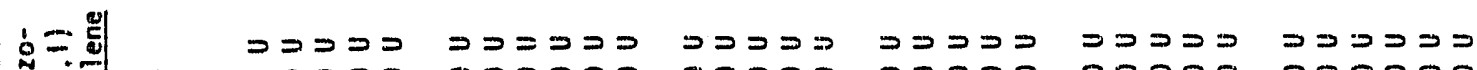

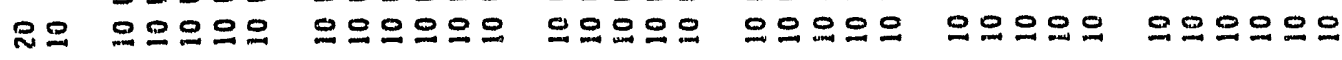

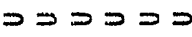

$$
=
$$

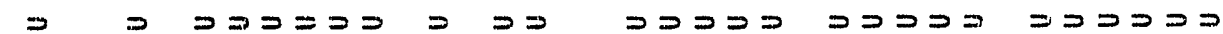

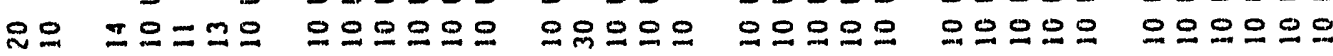

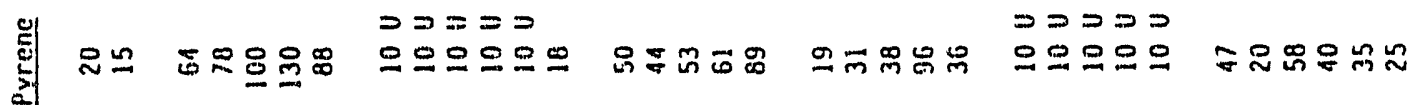




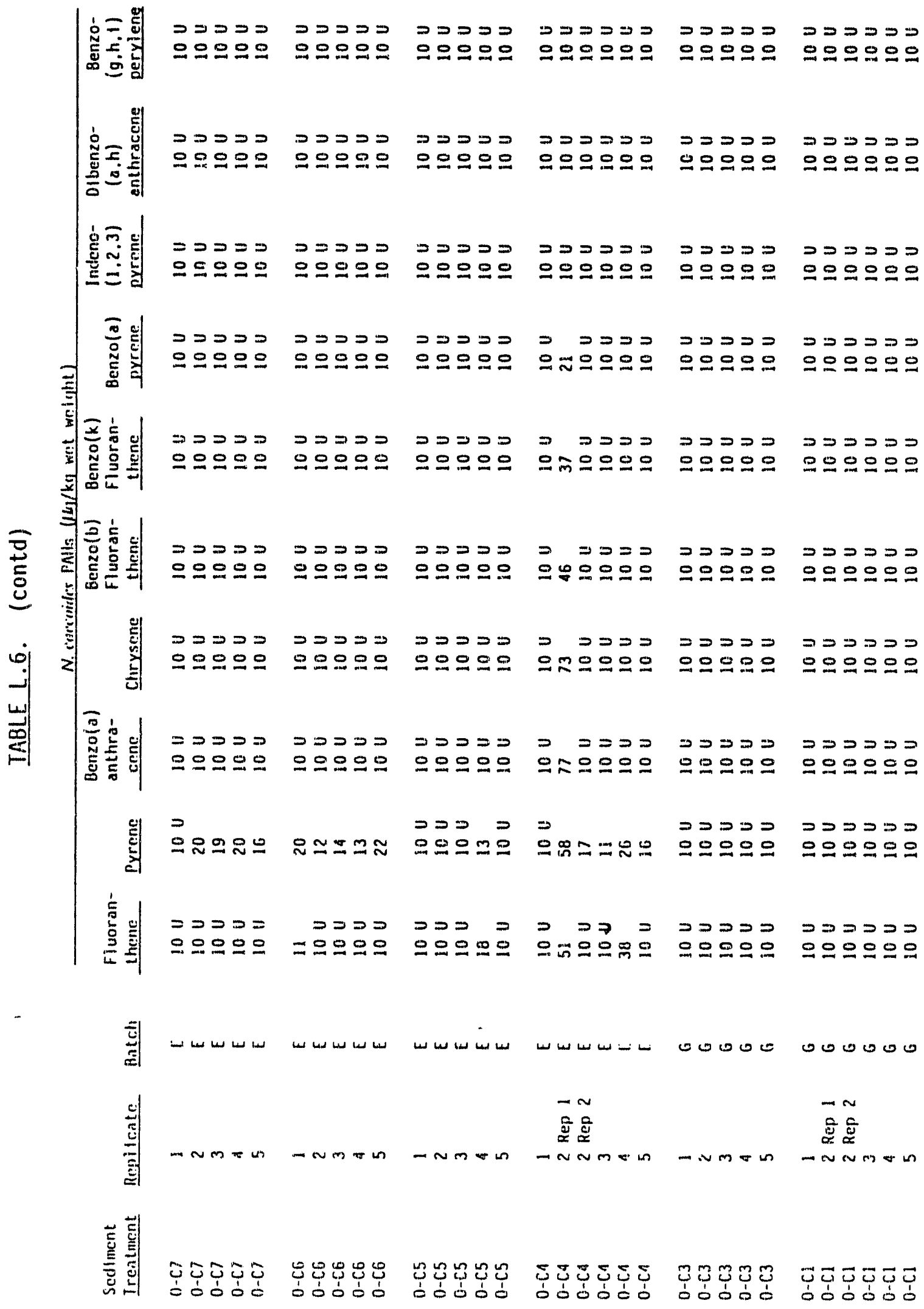




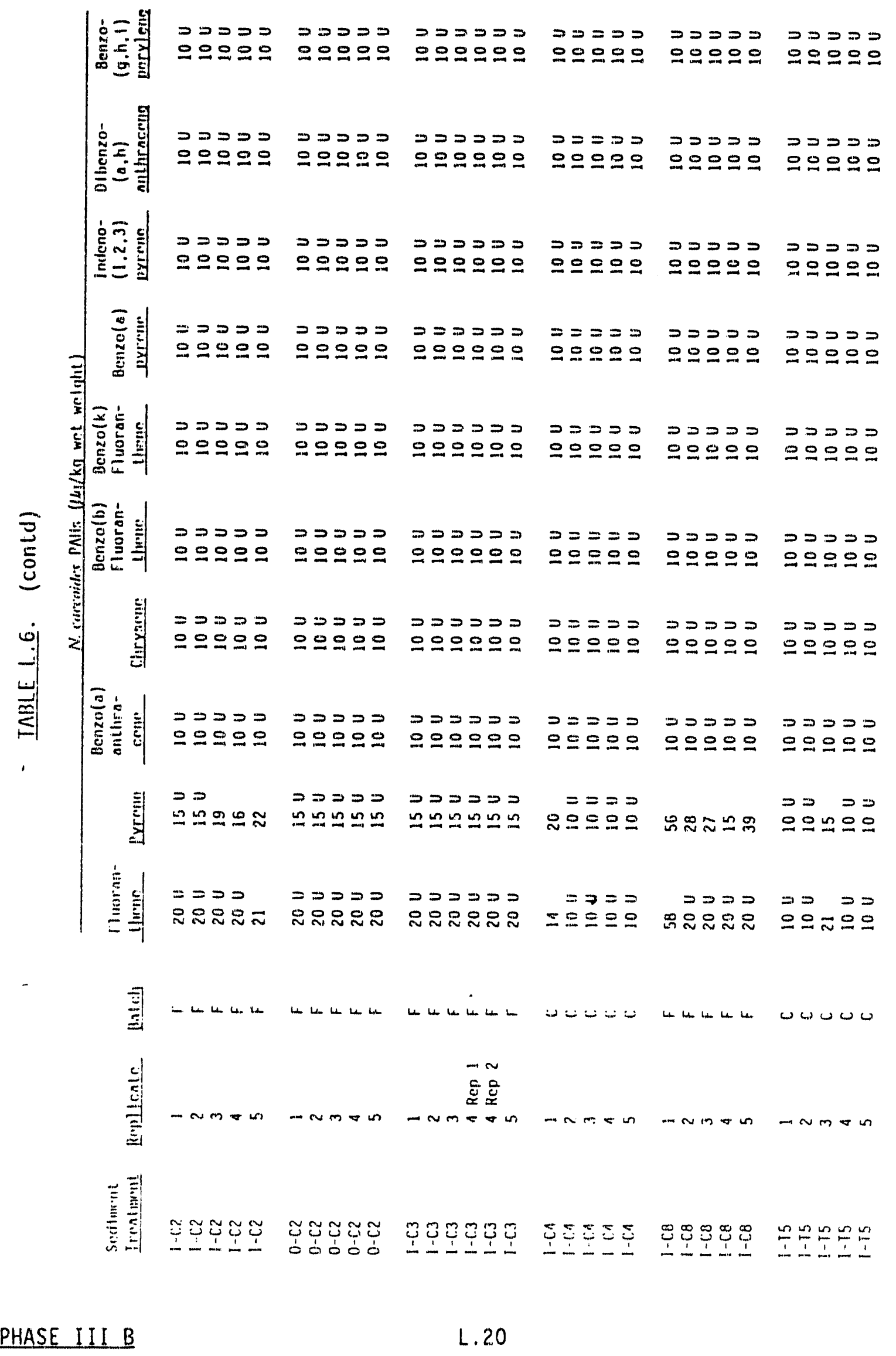




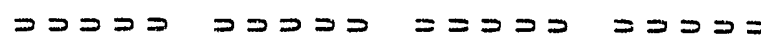

으으으으으 으으으으으 므으으으으 으으응으

$\rightarrow$ ว $\supset$ ว

$\supset \supset \supset \supset \supset \supset$

$\vec{P} \geq \vec{\partial}$

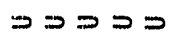

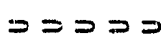

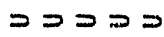

으으오으오

으으으으오

으으으으으

으으으으으

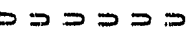

으으모으으으

$\supset \supset \supset \supset \supset=$

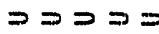

$==\geq>>$

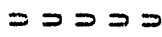

으으오으으

으으으으으

오으오으으

$\vec{D} \mathrm{x}>\mathrm{s}$

으으으으오으

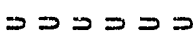

$\supset>>=$

$\Rightarrow \supset \supset \supset$

= $>>$

고 $\rightarrow \geq$

$\vec{D}=\vec{D}=$

으으어으으오

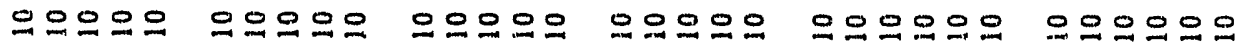

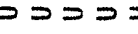

I $=2$

$==$ = $=$

$>==>$

오오으으으

으오으으으

으오오으으

으으오으오

ㄱ $\supset \geq \geq$

으으으으드으

$\supset \supset \supset \supset \supset \supset$

\section{$\Rightarrow$}

일.

옹우

フ ニ

フコン

$\geq \geq \geq \geq \geq \geq$

으으오으으으

$\supset \supset \supset \supset$

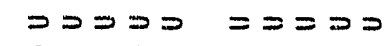

$=\supset \supset \supset$

フココ

コココ

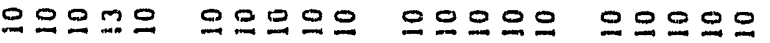

으으으으으으

응ㅇㅁㅇㅇㅇㅇㅇㅡ

تِّ

$\infty \infty_{\infty} \infty \quad<<<<<<<<$

$=\equiv \infty \infty$

$\infty \infty \infty \infty \infty$

$<<<<<<$

올

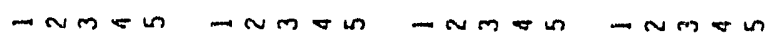

ì

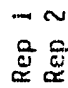

- ivm $<$ us

-Wmmon

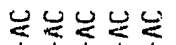

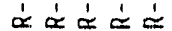

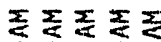

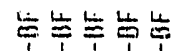

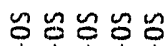

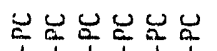

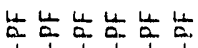

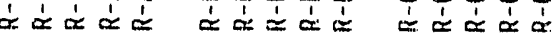




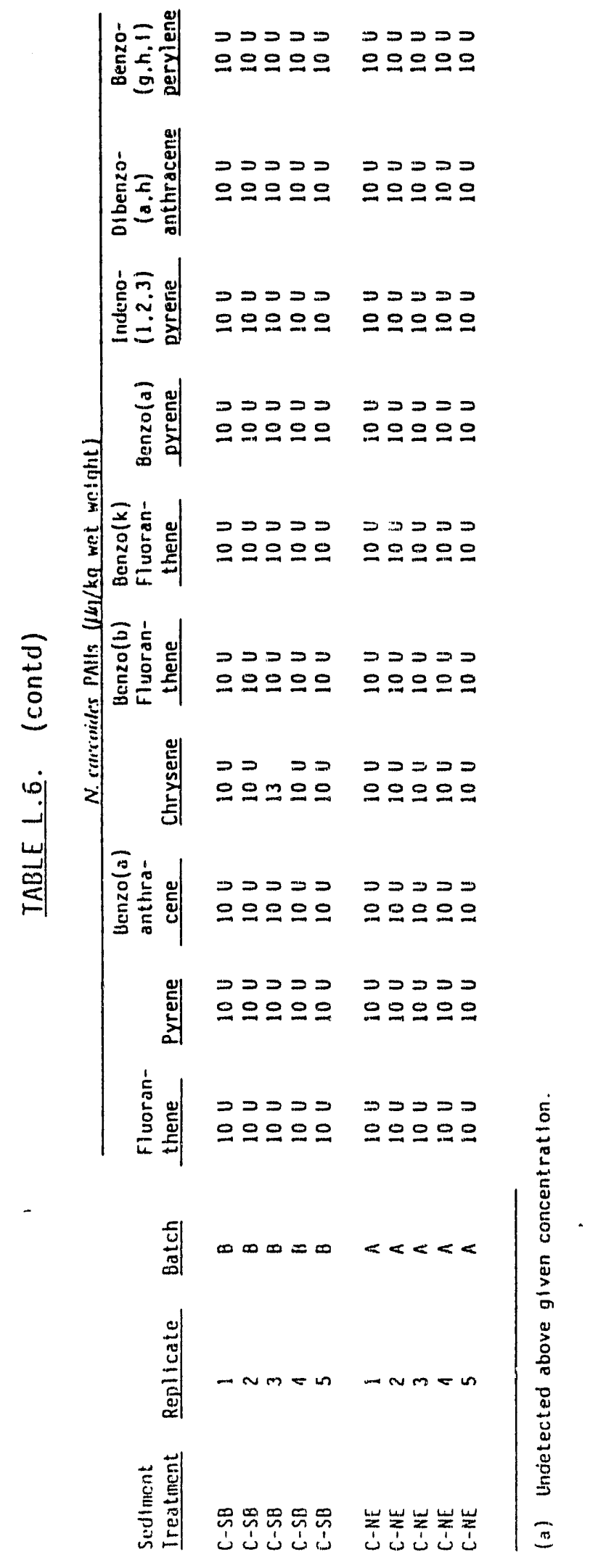


TABLE L.7. Quality Control Data for Low Molecular Weight Polynuclear Aromatic Hydrocarbons (PAHs), Wet Weight, in Tissue of N. caecoides, Oakland Harbor Phase III B Project

Sample
Method Blanks
Blank $A$
Blank B
Blank C

N. caecoides PAHs ( $\mu_{q} / \mathrm{kg}$ wet weight)

Acenaph- Acenaph- Phenan- Anthra-

Naphthalene thylene $\begin{aligned} & \text { Acenaph- Acenaph- } \\ & \text { thene }\end{aligned}$ Fluorene threne $\begin{aligned} & \text { Phenan- Anthra- } \\ & \text { cene }\end{aligned}$

Matrix Sptkes

Blank $A$

Blank A MS

Amount Recovered

Spike Amount

Percent Recovery

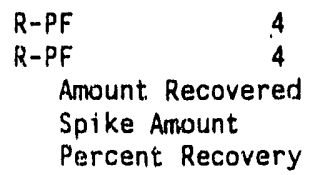

$\begin{array}{ll}50 U^{(a)} & 10 U \\ 30 U & 10 U \\ 30 U & 10 U \\ 30 U & 10 U \\ 20 U & 10 U \\ 40 U & 10 U \\ 60 U & 10 U\end{array}$

$\begin{array}{lll}10 U & 10 U \\ 10 U & 10 U \\ 10 U & 10 U \\ 10 U & 10 U \\ 10 U & 10 U \\ 10 U & 10 U \\ 10 U & 10 U\end{array}$

$10 U$
$10 U$
$10 U$
$10 U$
$10 U$
$10 U$
$10 U$

$10 \mathrm{U}$

$10 \mathrm{U}$

$\begin{array}{ll}A & 50 U^{(a)} \\ B & 30 U \\ C & 30 U \\ D & 30 U \\ E & 20 U \\ F & 40 U \\ G & 60 U\end{array}$

$10 \mathrm{U}$

$10 \mathrm{U}$

$10 \mathrm{U}$

$10 \mathrm{U} \quad 10 \mathrm{U}$

$10 \mathrm{U} \quad 10 \mathrm{U}$

$\begin{array}{lll}10 U & 10 U & 10 U \\ 10 U & 10 U & 10 U\end{array}$

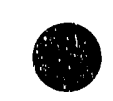

Blank $B$

Blank B MS

Amount Recovered Spike Amount

Percent Recovery

Blank B MSD

Amount Recovered

Spike Amount

Percent Recovery

MS/MSD RPD

MS/MSD I-Stat

R-OS
R-OS
- Amount Recovered
Spike Amount
Percent Recovery

Blank C

Blarik C MS

Amount Recovered

Spike Amount

Percent Recovery

Blank C MSD

Amount Recovered

Spike Amount

$\begin{array}{ll}A & 50 U \\ A & 120 \\ & 120 \\ & 100 \\ & 120 \%\end{array}$

$10 \mathrm{U}$

100

100

100

$120 \% \quad 100 \%$

A

$50 U$
110
110
100

$10 \mathrm{U}$

100

100

100
$100 \%$

$10 U$
120
120
100
$120 \%$

$\begin{array}{lll}10 U & 10 U & 10 U \\ 100 & 120 & 100 \\ 100 & 120 & 100 \\ 100 & 100 & 100 \\ 100 \% & 120 \% & 100 \%\end{array}$

$110 \%$

B

$30 \mathrm{U}$

40

40

100

$40 \%$

B

120

120

100

$120 \%$

$10 \mathrm{U}$

10
100
100

100

100

$100 \%$

$10 U$
120
120
100
$120 \%$

$\begin{array}{lll}10 U & 10 U & 10 U \\ 110 & 120 & 110 \\ 110 & 120 & 110 \\ 100 & 100 & 100 \\ 110 \% & 120 \% & 110 \%\end{array}$

Percent Recovery

$\begin{array}{ccc} & 100 \% & 0 \% \\ & 0.50 & 0.00 \\ \text { B } & & \\ B & 30 u & 10 U \\ & 59 & 85 \\ & 59 & 85 \\ & 100 & 100 \\ & 59 \% & 85 \% \\ C & & \\ C & 30 u & 10 U \\ & 140 & 97 \\ & 140 & 97 \\ & 100 \text { (b) } & 100 \\ & 140 \% \text { (b) } & 97 \% \\ C & & \\ & 160 & 100 \\ & 160 & 100 \\ & 100 & 100 \\ & 160 \% \text { (b) } & 100 \%\end{array}$

$\begin{array}{lllll}10 U & 10 U & 10 U & 10 U & 10 U \\ 100 & 120 & 130 & 120 & 110 \\ 100 & 120 & 130 & 120 & 110 \\ 100 & 100 & 100 & 100 & 100 \\ 100 \% & 120 \% & 130 \% & 120 \% & 110 \% \\ & & & & \\ 100 & 120 & 100 & 110 & 1410 \\ 100 & 120 & 100 & 110 & 1410 \\ 100 & 100 & 100 & 100 & 100 \\ 100 \% & 120 \% & 100 \% & 110 \% & 1410 \%(b)\end{array}$

$\begin{array}{ccccc}0 \% & 0 \% & 26 \% & 9 \% & 171 \% \\ 0.00 & 20.00 & 0.13 & 0.04 & 0.86\end{array}$

$\begin{array}{llcc}10 U & 10 U & 10 U & 10 U \\ 110 & 110 & 99 & 88 \\ 110 & 110 & 99 & 88 \\ 100 & 100 & 100 & 100 \\ 110 \% & 11.0 \% & 99 \% & 88 \%\end{array}$

$\begin{array}{llll}10 u & 10 U & 10 U & 10 U \\ 110 & 100 & 120 & 110 \\ 110 & 100 & 120 & 110 \\ 100 & 100 & 100 & 100 \\ 110 \% & 100 \% & 120 \% & 110 \%\end{array}$

$\begin{array}{llll}120^{\circ} & 100 & 120 & 110 \\ 120 & 100 & 120 & 110 \\ 100 & 100 & 100 & 100 \\ 120 \% & 100 \% & 120 \% & 110 \%\end{array}$


IABLE L.7. (contd)

\begin{tabular}{|c|c|c|c|c|c|c|c|}
\hline \multirow[b]{2}{*}{ Sample } & \multirow[b]{2}{*}{ Batch } & \multicolumn{6}{|c|}{ N. caecoides PAHs $(\mu \mathrm{g} / \mathrm{kg}$ wet weight) } \\
\hline & & Naphthalene & $\begin{array}{l}\text { Acenaph- } \\
\text { thylene }\end{array}$ & $\begin{array}{l}\text { Acenaph- } \\
\text { thene }\end{array}$ & Fluorene & $\begin{array}{l}\text { Phenan- } \\
\text { threne }\end{array}$ & $\begin{array}{l}\text { Anthra- } \\
\text { cene }\end{array}$ \\
\hline $\begin{array}{l}\text { MS/MSD RPD } \\
\text { MS/MSD I-Stat }\end{array}$ & & $\begin{array}{l}13 \% \\
0.07\end{array}$ & 0.02 & 0.04 & 0.00 & 0.00 & 0.00 \\
\hline $\begin{array}{l}0-C 13 \quad 1 \text { MS } \\
\text { O-C13 Anount Recovered } \\
\text { Spike Amuunt } \\
\text { Percent Recovery }\end{array}$ & $\begin{array}{l}\mathrm{C} \\
\mathrm{C}\end{array}$ & $\begin{array}{l}32 \\
142 \\
110 \\
100 \\
110 \%\end{array}$ & $\begin{array}{l}10 U \\
100 \\
100 \\
100 \\
100 \%\end{array}$ & $\begin{array}{l}10 U \\
110 \\
110 \\
100 \\
110 \%\end{array}$ & $\begin{array}{l}10 \mathrm{U} \\
110 \\
110 \\
100 \\
110 \%\end{array}$ & $\begin{array}{r}19 \\
118 \\
99 \\
100 \\
99 \%\end{array}$ & $\begin{array}{l}10 \mathrm{U} \\
110 \\
110 \\
100 \\
110 \%\end{array}$ \\
\hline $\begin{array}{l}\text { Bìank D } \\
\text { Blank D MS } \\
\text { Amount Recovered } \\
\text { Spike Arsount } \\
\text { Percent Recovery }\end{array}$ & $\begin{array}{l}0 \\
0\end{array}$ & $\begin{array}{l}30 \mathrm{U} \\
110 \\
110 \\
100 \\
110 \%\end{array}$ & $\begin{array}{l}10 U \\
100 \\
100 \\
100 \\
100 \%\end{array}$ & $\begin{array}{l}100 \\
110 \\
110 \\
100 \\
110 \%\end{array}$ & $\begin{array}{l}10 U \\
120 \\
120 \\
100 \\
120 \%\end{array}$ & $\begin{array}{l}10 \mathrm{U} \\
190 \\
190 \\
100 \\
190 \%(b)\end{array}$ & $\begin{array}{l}10 U \\
120 \\
120 \\
100 \\
120 \%\end{array}$ \\
\hline $\begin{array}{l}\text { Blank D MSO } \\
\text { Amount Recovered } \\
\text { Spike Amount } \\
\text { Percent Recovery }\end{array}$ & 0 & $\begin{array}{l}120 \\
120 \\
100 \\
120 \%\end{array}$ & $\begin{array}{l}100 \\
100 \\
100 \\
100 \%\end{array}$ & $\begin{array}{l}120 \\
120 \\
100 \\
120 \%\end{array}$ & $\begin{array}{l}110 \\
110 \\
100 \\
110 \%\end{array}$ & $\begin{array}{l}120 \\
120 \\
100 \\
120 \%\end{array}$ & $\begin{array}{l}110 \\
110 \\
100 \\
110 \%\end{array}$ \\
\hline $\begin{array}{l}\text { MS/MSD RPD } \\
\text { MS/MSD I-St at }\end{array}$ & & 0.04 & $0.00^{0 \%}$ & 0.04 & 0.04 & $\begin{array}{l}\quad 45 \% \\
0.23\end{array}$ & 0.04 \\
\hline $\begin{array}{l}0-C 8 \\
0-C 8 \\
\text { Amount Recovered } \\
\text { Spike Amount } \\
\text { Percent Recovery }\end{array}$ & $\begin{array}{l}D \\
D\end{array}$ & $\begin{array}{l}30 U \\
120 \\
120 \\
100 \\
120 \%\end{array}$ & $\begin{array}{l}10 U \\
110 \\
110 \\
100 \\
110 \%\end{array}$ & $\begin{array}{l}10 U \\
100 \\
100 \\
100 \\
100 \%\end{array}$ & $\begin{array}{l}10 U \\
100 \\
100 \\
100 \\
100 \%\end{array}$ & $\begin{array}{l}10 U \\
160 \\
160 \\
100 \\
160 \%(b)\end{array}$ & $\begin{array}{l}10 \mathrm{U} \\
110 \\
110 \\
100 \\
110 \%\end{array}$ \\
\hline $\begin{array}{l}\text { Blank E } \\
\text { Blank E MS } \\
\text { Aniount Recovered } \\
\text { Spike Amount } \\
\text { Percent Recovery }\end{array}$ & $\begin{array}{l}E \\
E\end{array}$ & $\begin{array}{l}20 \mathrm{U} \\
110 \\
110 \\
100 \\
110 \%\end{array}$ & $\begin{array}{c}10 \mathrm{U} \\
99 \\
99 \\
100 \\
99 \%\end{array}$ & $\begin{array}{l}10 U \\
100 \\
100 \\
100 \\
100 \%\end{array}$ & $\begin{array}{l}10 \mathrm{u} \\
410 \\
410 \\
100 \\
410 \%(\mathrm{~b})\end{array}$ & $\begin{array}{l}10 U \\
110 \\
110 \\
100 \\
110 \%\end{array}$ & $\begin{array}{c}10 \mathrm{U} \\
99 \\
99 \\
100 \\
99 \%\end{array}$ \\
\hline $\begin{array}{l}\text { 81ank E MSD } \\
\text { Amount Recovered } \\
\text { Spike Amount } \\
\text { Percent Recovery }\end{array}$ & $E$ & $\begin{array}{l}120 \\
120 \\
100 \\
120 \%\end{array}$ & $\begin{array}{c}77 \\
77 \\
100 \\
77 \%\end{array}$ & $\begin{array}{l}120 \\
120 \\
100 \\
120 \%\end{array}$ & $\begin{array}{l}100 \\
100 \\
100 \\
100 \%\end{array}$ & $\begin{array}{l}110 \\
110 \\
100 \\
110 \%\end{array}$ & $\begin{array}{c}90 \\
90 \\
100 \\
90 \%\end{array}$ \\
\hline $\begin{array}{l}\text { MS/MSD RPD } \\
\text { MS/MSD I-Stat }\end{array}$ & & 0.04 & 0.13 & $\begin{array}{l}18 \% \\
0.09\end{array}$ & $\begin{array}{l}122 \% \\
0.61\end{array}$ & $0.00 \%$ & $\begin{array}{l}10 \% \\
0.05\end{array}$ \\
\hline $\begin{array}{l}0-C G \quad 2 \\
0-C 6 \\
\text { Amount Recovered } \\
\text { Spike Amount } \\
\text { Percent Recovery }\end{array}$ & $\begin{array}{l}E \\
E\end{array}$ & $\begin{array}{l}20 U \\
100 \\
100 \\
100 \\
100 \%\end{array}$ & $\begin{array}{l}10 \mathrm{U} \\
97 \\
97 \\
100 \\
97 \%\end{array}$ & $\begin{array}{l}10 \mathrm{~V} \\
110 \\
110 \\
100 \\
110 \%\end{array}$ & $\begin{array}{l}100 \\
110 \\
110 \\
100 \\
110 \%\end{array}$ & $\begin{array}{l}10 U \\
110 \\
110 \\
100 \\
110 \%\end{array}$ & $\begin{array}{l}10 U \\
120 \\
120 \\
100 \\
120 \%\end{array}$ \\
\hline $\begin{array}{l}\text { Blank F } \\
\text { Blank F MS } \\
\text { Amount Recovered } \\
\text { Spike Amount } \\
\text { Percent Recovery }\end{array}$ & $\begin{array}{l}F \\
F\end{array}$ & $\begin{array}{c}40 \mathrm{U} \\
43 \\
43 \\
100 \\
43 \%\end{array}$ & $\begin{array}{c}10 U \\
90 \\
90 \\
100 \\
90 \%\end{array}$ & $\begin{array}{l}10 U \\
110 \\
110 \\
100 \\
110 \%\end{array}$ & $\begin{array}{l}10 U \\
120 \\
120 \\
100 \\
120 \%\end{array}$ & $\begin{array}{l}10 U \\
110 \\
110 \\
100 \\
110 \%\end{array}$ & $\begin{array}{l}10 \mathrm{U} \\
110 \\
110 \\
100 \\
110 \%\end{array}$ \\
\hline
\end{tabular}

PHASE III B 
TABLE L.7. (contd)

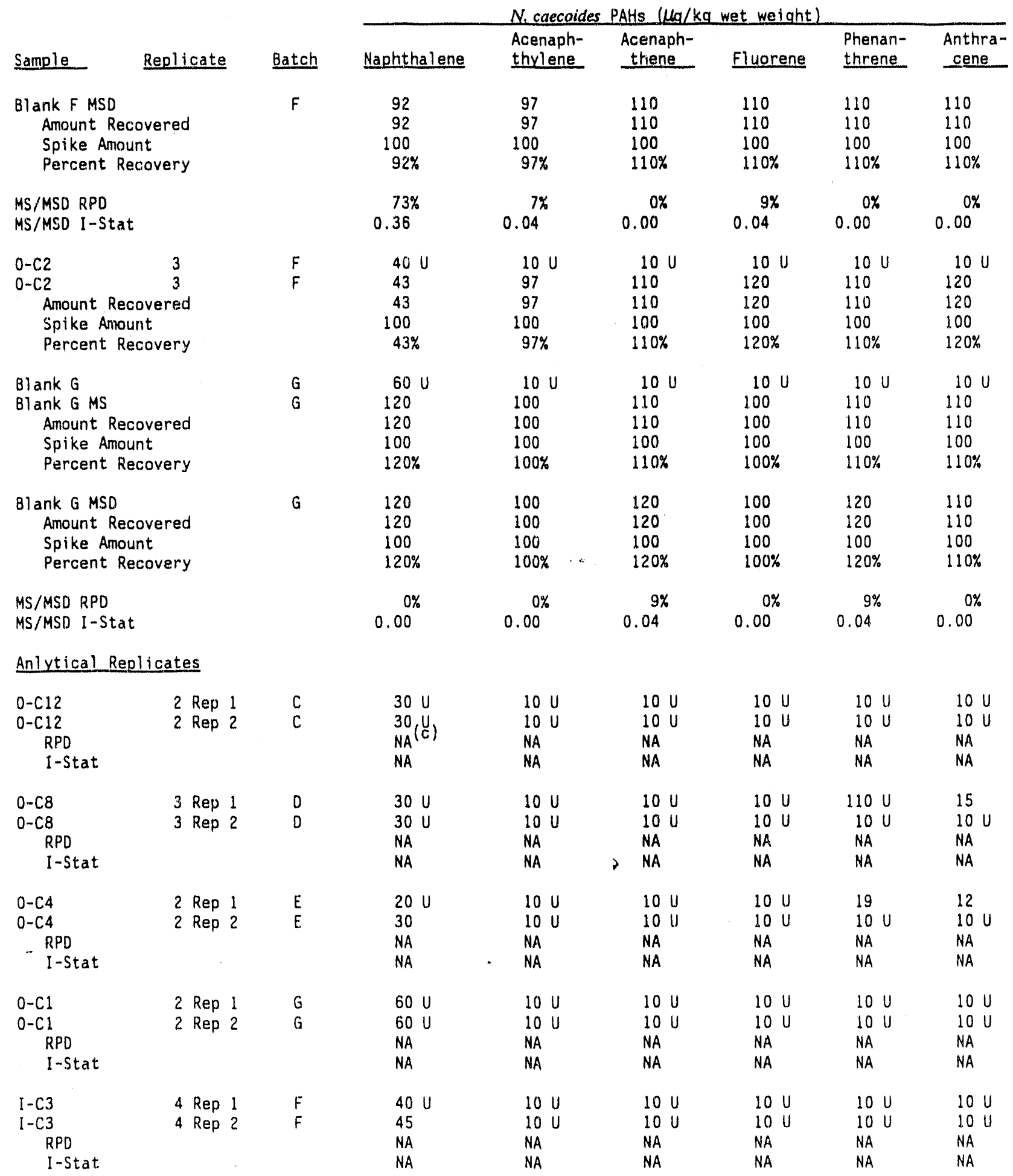


TABLE L.7. (contd)

\begin{tabular}{|c|c|c|c|c|c|c|c|c|}
\hline \multirow[b]{2}{*}{ Sample } & \multirow[b]{2}{*}{ Replicate } & \multirow[b]{2}{*}{ Batch } & \multicolumn{6}{|c|}{ N. caecoides PAHs ( $\mu \mathrm{g} / \mathrm{kg}$ wet weight) } \\
\hline & & & Naphthalene & $\begin{array}{l}\text { Acenaph- } \\
\text { thylene }\end{array}$ & $\begin{array}{l}\text { Acenaph- } \\
\text { thene }\end{array}$ & Eluorene & $\begin{array}{l}\text { Phenan- } \\
\text { threne }\end{array}$ & $\begin{array}{l}\text { Anthra- } \\
\text { cene }\end{array}$ \\
\hline $\begin{array}{l}R-P C \\
R-P C \\
\quad R P D \\
I \text {-Stat }\end{array}$ & $\begin{array}{ll}1 & \operatorname{Rep} 1 \\
1 & \operatorname{Rep} 2\end{array}$ & $\begin{array}{l}B \\
B\end{array}$ & $\begin{array}{l}30 U \\
30 U \\
N A \\
N A\end{array}$ & $\begin{array}{l}10 U \\
10 U \\
N A \\
N A\end{array}$ & $\begin{array}{l}10 U \\
10 U \\
N A \\
N A\end{array}$ & $\begin{array}{l}10 \mathrm{U} \\
10 \mathrm{U} \\
N A \\
N A\end{array}$ & $\begin{array}{l}10 U \\
10 U \\
N A \\
N A\end{array}$ & $\begin{array}{l}10 U \\
10 U \\
N A \\
N A\end{array}$ \\
\hline $\begin{array}{l}R-P F \\
R-P F \\
R P D \\
I-S \text { tat }\end{array}$ & $\begin{array}{l}3 \operatorname{Rep} 1 \\
3 \operatorname{Rep} 2\end{array}$ & $\begin{array}{l}A \\
A\end{array}$ & $\begin{array}{l}50 U \\
50 U \\
N A \\
N A\end{array}$ & $\begin{array}{l}10 U \\
10 U \\
N A \\
N A\end{array}$ & $\begin{array}{l}10 U \\
10 U \\
N A \\
N A\end{array}$ & $\begin{array}{l}10 U \\
10 U \\
N A \\
N A\end{array}$ & $\begin{array}{l}10 U \\
10 U \\
N A \\
N A\end{array}$ & $\begin{array}{l}10 U \\
10 U \\
N A \\
N A\end{array}$ \\
\hline
\end{tabular}

(a) Undetected above given concentration.

(b) Outside of target quality contral range.

(c) Not applicable. 


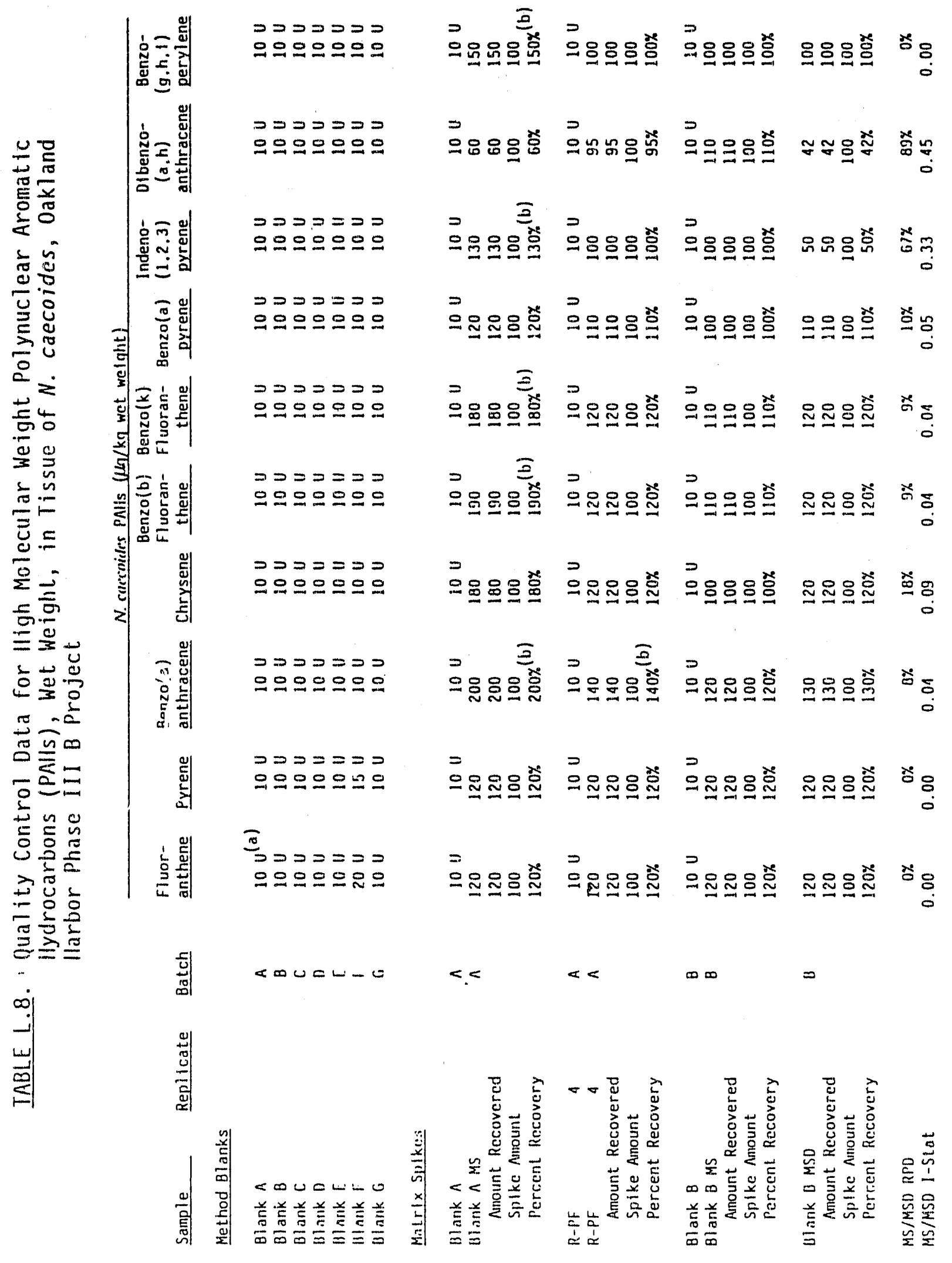




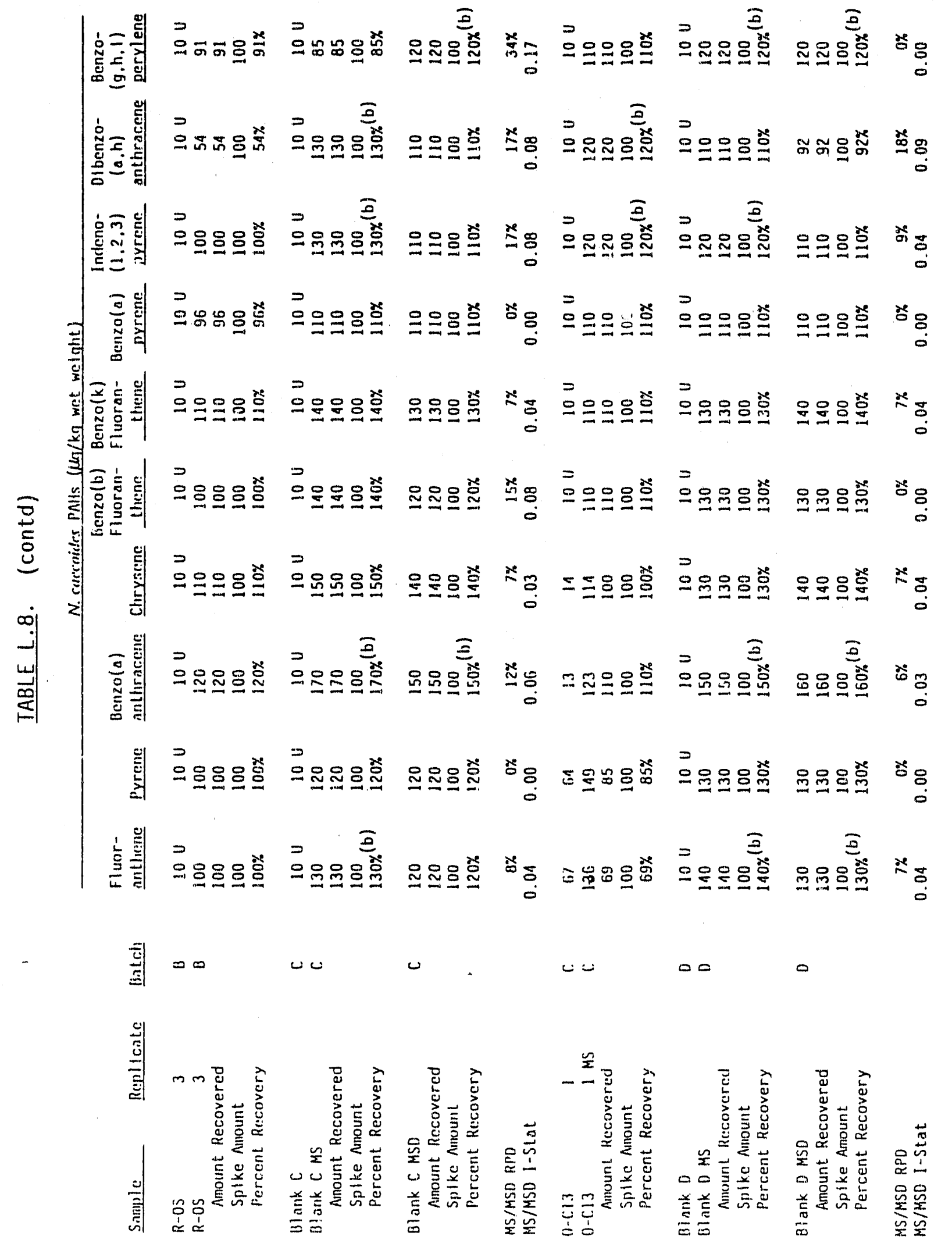

PHASE III B 
|

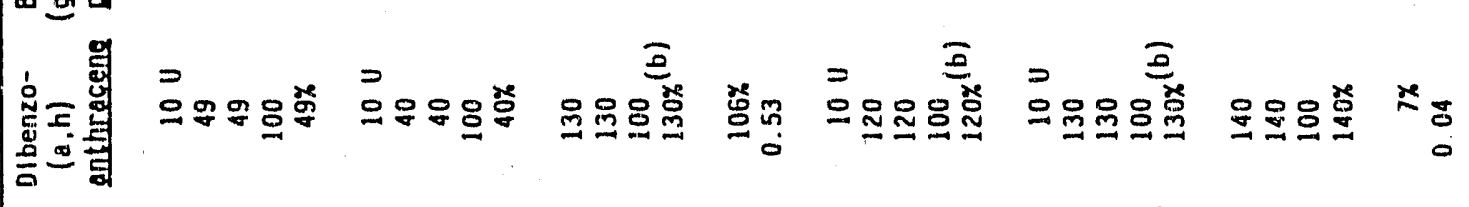

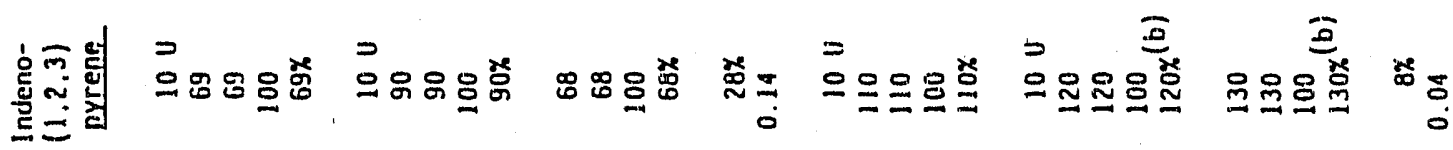

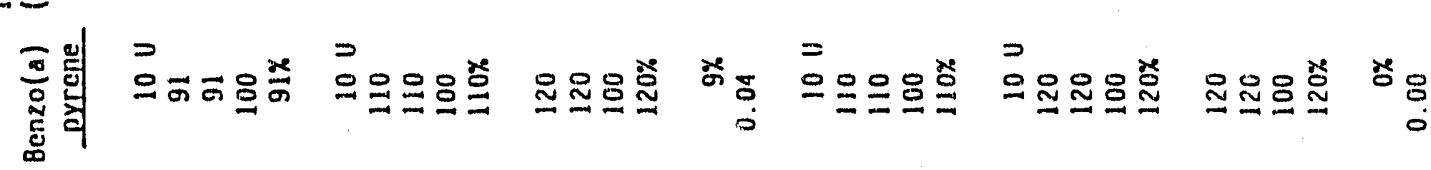

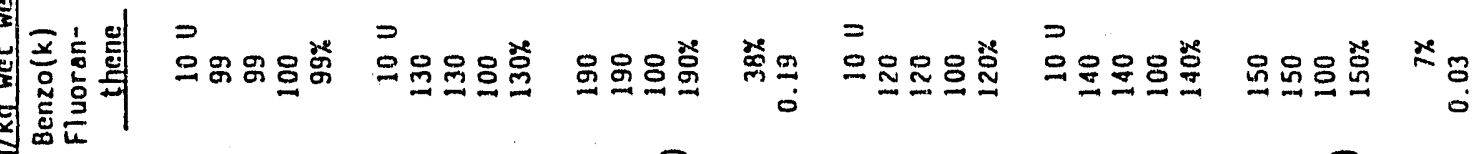

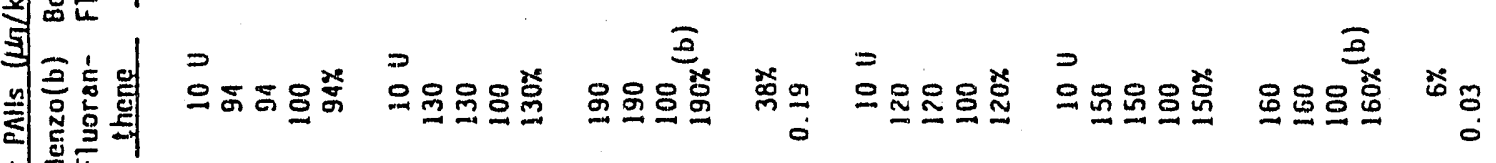

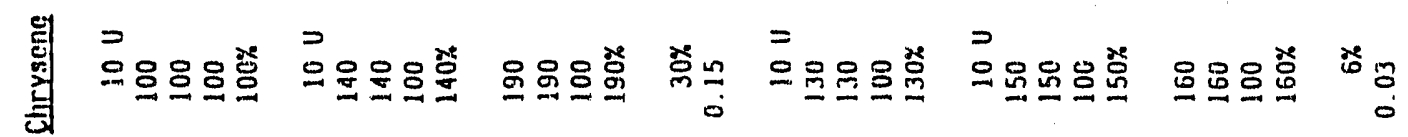

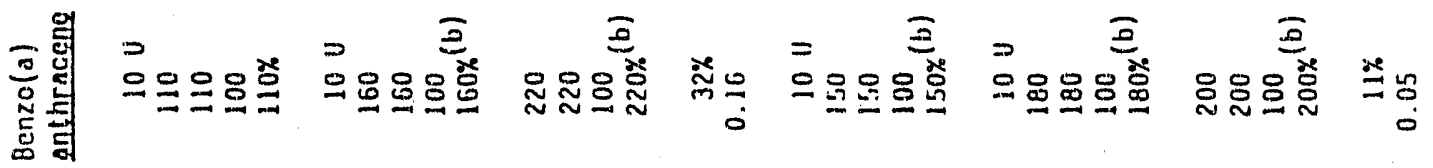

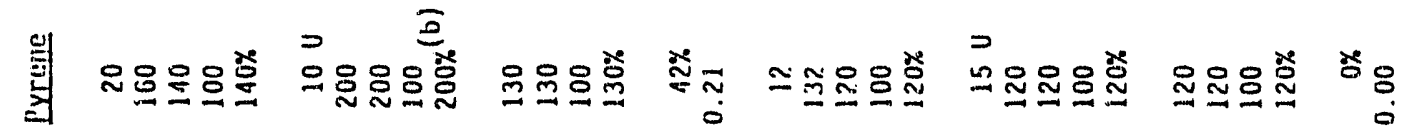

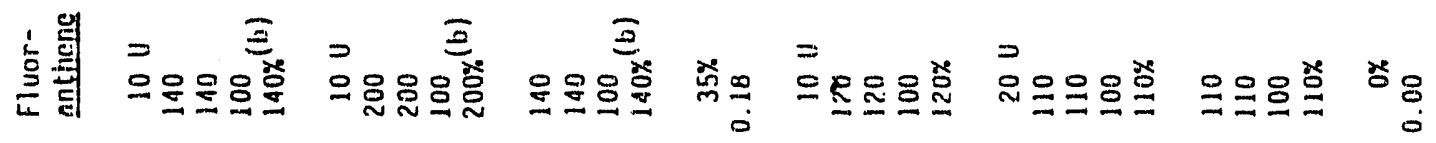

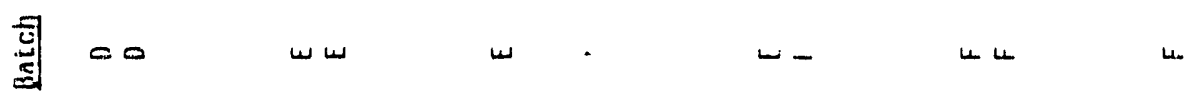

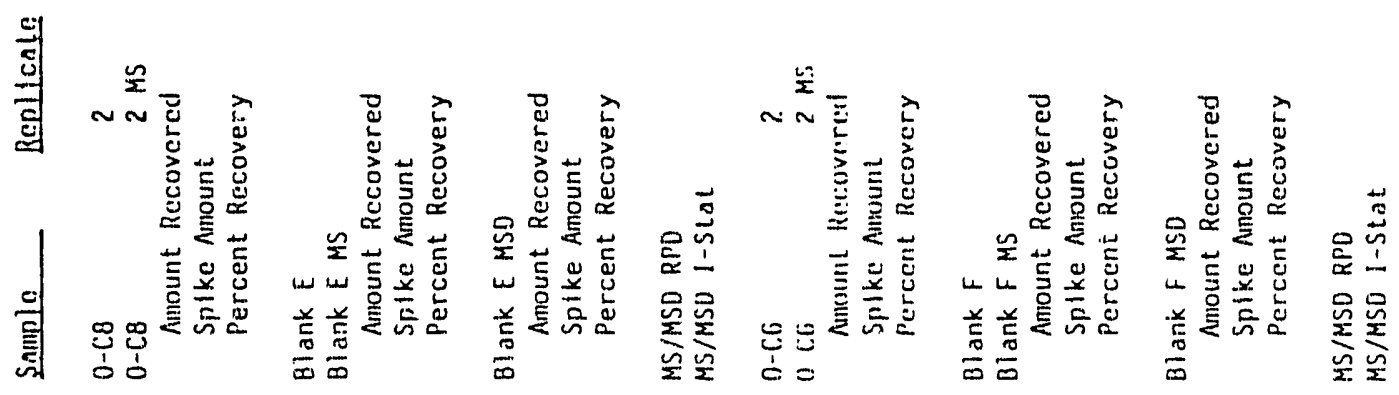




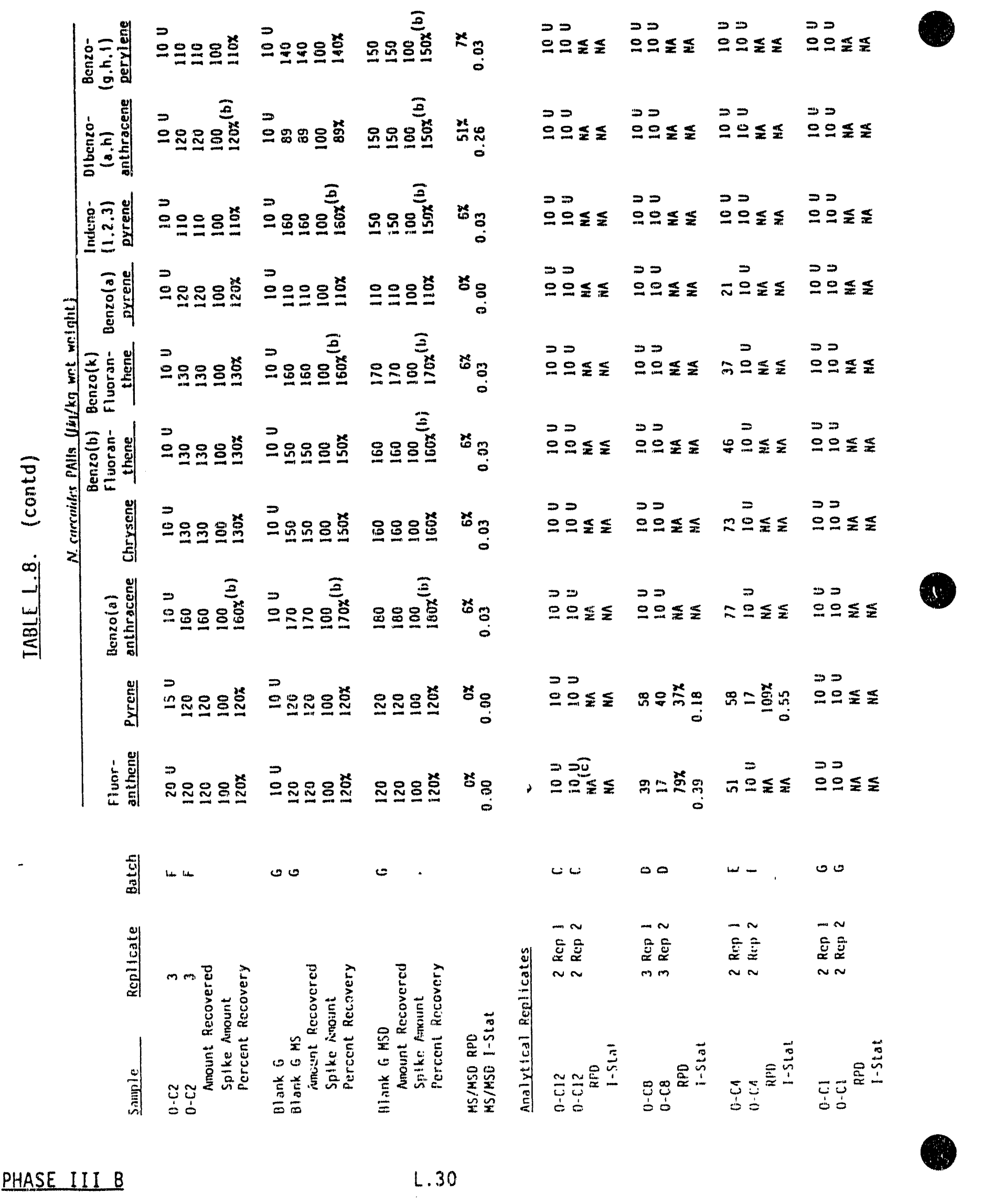




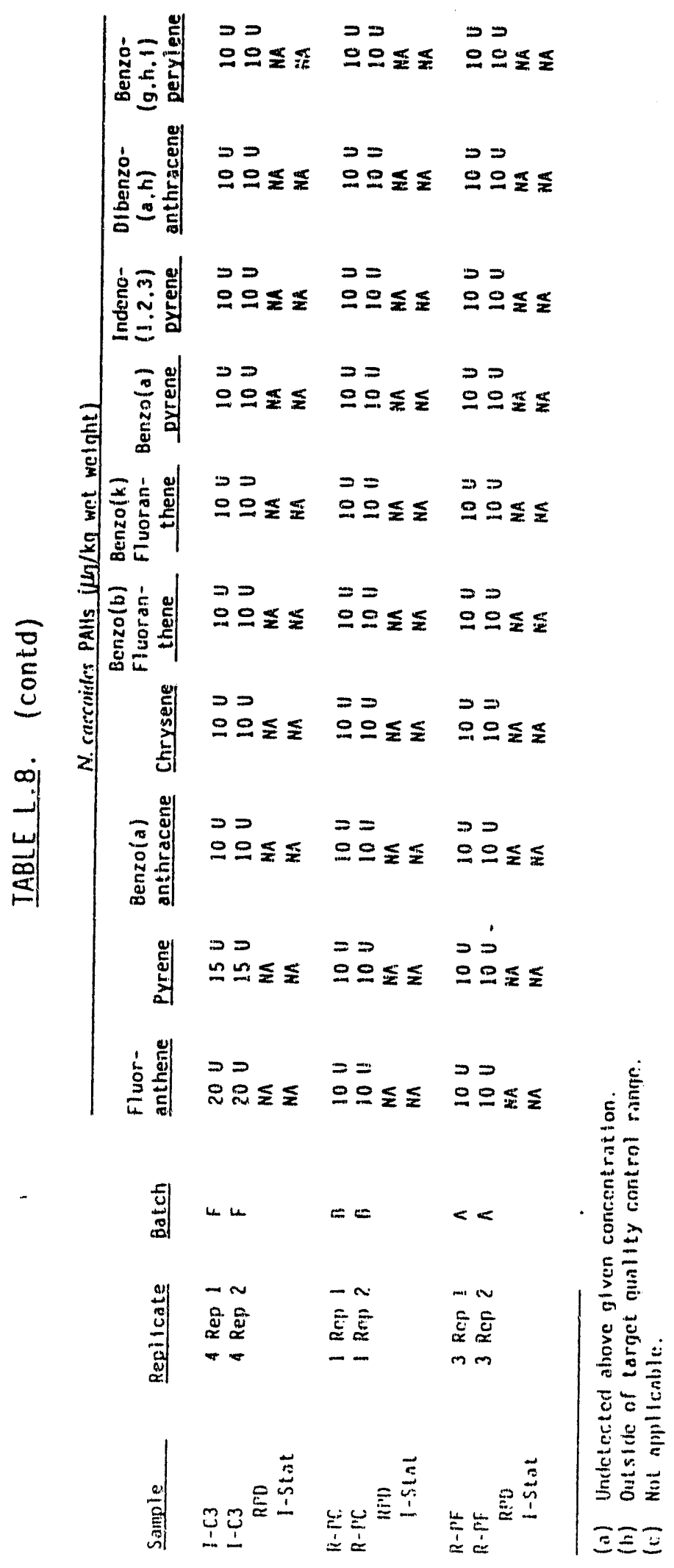


TABLE L.9. Surrogate Percent Recoveries for Polynuclear Aromatic Hydrocarbons (PAHs) in Tissue of $N$. caecoides, Oakland Harbor Phase III B Project

\begin{tabular}{|c|c|}
\hline $\begin{array}{l}\text { Sediment } \\
\text { Treatment }\end{array}$ & Replicate \\
\hline $\begin{array}{l}0-C 13 \\
0-C 13 \\
0-C 13 \\
0-C 13 \\
0-C 13\end{array}$ & $\begin{array}{l}1 \\
2 \\
3 \\
4 \\
5\end{array}$ \\
\hline $\begin{array}{l}0-C 12 \\
0-C 12 \\
0-C 12 \\
0-C 12 \\
0-C 12 \\
0-C 12\end{array}$ & $\begin{array}{lll}1 & & \\
2 & \text { Rep } & 1 \\
2 & \text { Rep } & 2 \\
3 & & \\
4 & & \\
5 & & \end{array}$ \\
\hline $\begin{array}{l}0-C 11 \\
0-C 11 \\
0-C 11 \\
0-C 11 \\
0-C 11\end{array}$ & $\begin{array}{l}1 \\
2 \\
3 \\
4 \\
5\end{array}$ \\
\hline $\begin{array}{l}0-C 10 \\
0-C 10 \\
0-C 10 \\
0-C 10 \\
0-C 10\end{array}$ & $\begin{array}{l}1 \\
2 \\
3 \\
4 \\
5\end{array}$ \\
\hline $\begin{array}{l}0-c 9 \\
0-c 9 \\
0-c 9 \\
0-c 9 \\
0 . c 9\end{array}$ & $\begin{array}{l}1 \\
2 \\
3 \\
4 \\
5\end{array}$ \\
\hline $\begin{array}{l}0-C 8 \\
0-C 8 \\
0-C 8 \\
0-C 8 \\
0-C 8 \\
0-C 8\end{array}$ & $\begin{array}{lll}1 & & \\
2 & & \\
3 & \operatorname{Rep} & 1 \\
3 & \operatorname{Rep} & 2 \\
4 & & \\
5 & & \end{array}$ \\
\hline $\begin{array}{l}0-c 7 \\
0-C 7 \\
0-c 7 \\
0-C 7 \\
0-C 7\end{array}$ & $\begin{array}{l}1 \\
2 \\
3 \\
4 \\
5\end{array}$ \\
\hline
\end{tabular}

Surrogate Percent Recoveries

Naph- Acenaph- Phenan- Benzo(a)
thalene thene threne pyrene

$c$
$C$
$C$

d8 $d 10$

d 10 d12

$\begin{array}{ll}\mathrm{C} & 76 \\ \mathrm{C} & 74 \\ \mathrm{C} & 65\end{array}$

76
74
72
65
62

80

84

80

87

83

81

79

75

77

70

74

69

65

$20^{(a)}$

76

82

69

75

68

43

80

58

74

73

85

60

62

71

76

75

69

75

70

77

81

70

90

97

100

75

81

79

77

88

110

89

93

90

86

86

89

79

93

95

82

$36 \quad 81$

52

81
59

96

88

74

83

66

88

77

87

93

60

38

56

87

96

$64 \quad 58$

$\begin{array}{llll}59 & 79 & 92 & 85\end{array}$

$71, \quad 85 \quad 97 \quad 88$

$74 \cdot 91$

100

49

80

95

97

99

90

76

$16^{(a)}$

100

96

79

91
75

92

91

59

82

89

90

83

85

97

97

85

87

87

64

96

100

$E$
$E$
$E$
$E$

70

95

100

110

68

100

110

64

98

93

94

98

61

87

90

97

65

91

96

97

PHASE $111 \mathrm{~B}$ 
TABLE L.9. (contd)

Sediment

\begin{tabular}{ccccc} 
& & \multicolumn{3}{c}{ Surrogate Percent Recoveries } \\
\cline { 3 - 4 } & Naph- & Acenaph- & Phenan- & Benzo(a) \\
thalene & thene & threne & pyrene \\
Replicate Batch & d8 & d10 & d10 & d12 \\
\hline
\end{tabular}

$0-c 6$

$0-C 6$

$0-C 6$

$0-C 6$

$0-\mathrm{C} 6$

$0-C 5$

$0-C 5$

$0-C 5$

$0-C 5$

$0-C 5$

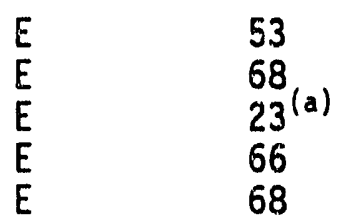

75

97

95

1
2
3
4
5

98

67

1

3
4
5

$E$
$E$
$E$
$E$

100

80

100

100

100

100

87

94

97
100

95

91

58

58

59

90

86

94

89

100

120

110

0-C4

$0-C 4$

$0-C 4$

$0-C 4$

$0-C 4$

O-C4

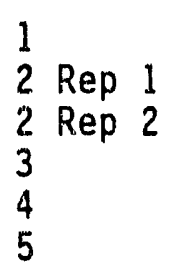

$E$
$E$
$E$
$E$
$E$

$39^{(a)}$

82

$34^{(a)}$

77

60

61 (a)

82

86

88

72

88

93

90

91

92

92

110

$0-c 3$

$0-C 3$

$0-C 3$

1

2

$0-C 3$

$0-C 3$

$G$
$G$
$G$
$G$
$G$

$110 \quad 100$

110

110

100

81

110

100

5

95

100

81

80

77

79

80

97

100

99

98

98
94

$0-C 1$

O-CI

O-Cl

O-C1

O-CI

$0-C 1$

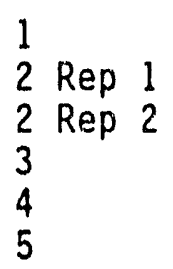

120

100

100

100

110

80

110

100

78

79

79

74

100

110

100

82

91

100

94

92

95

98

I $-\mathrm{C} 2$

$\mathrm{I}-\mathrm{C2}$

I-C2

1

$4^{(a)}$

61

84

50

78

63

I $-\mathrm{C} 2$

3

$\mathrm{I}-\mathrm{C} 2$

60

92

93

96

74

82.

69

88

$0-c 2$

$0-C 2$

$0-C 2$

$0-\mathrm{C2}$

$0-\mathrm{C} 2$

5

47
53
62
68
71

81

81

90

99

98

78

90

89

86

76

92

88

$87 \quad 87$

$99 \quad 90$

$81 \quad 84$

$94 \quad 92$

$98 \quad 97$

2
3
4
5

PHASE III B 
TABLE L.9. (contd)

Sediment

Ireatment

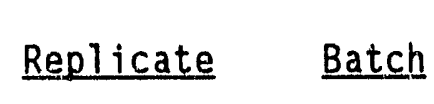

\section{I $-\mathrm{CH}$}

I $-\mathrm{C} 3$

$\mathrm{I}-\mathrm{C} 3$

$\mathrm{I}-\mathrm{C} 3$

I $-\mathrm{C} 3$

I-C3

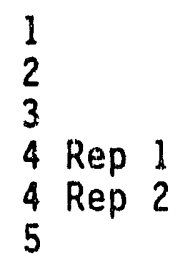

$F$
$F$
$F$
$F$
$F$
$F$

I $-\mathrm{C} 4$

I-C4

I-C4

1-C4

I-C4

I $-\mathrm{CB}$

I $-C 8$

I-C8

I $-C 8$

I $-\mathrm{CB}$

I - T5

I-T5

I $-T 5$

I $-T 5$

I-T5

$R-A C$

$R-A C$

$R=A C$

$R-A C$

$R-A C$

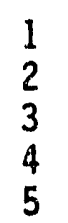

$c$
$c$
$c$
$c$
$c$

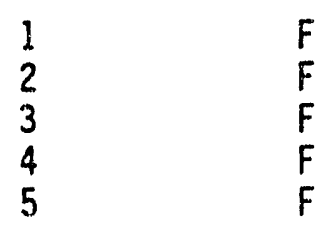

$F$
$F$
$F$
$F$
$F$

1

2

4

R-AM

$R^{-}-A M$

$R-A M$

$R-A M$

$R \cdots A M$

$R-B F$

$R \cdot B F$

$R-B F$

$R-B F$

$R-B F$

$c$
$c$
$c$
$c$
$c$

50

71

73

50

68

Surrogate Percent Recoveries

\begin{tabular}{|c|c|c|c|}
\hline $\begin{array}{l}\text { Naph- } \\
\text { thalene } \\
\text { d8 } \\
\end{array}$ & $\begin{array}{c}\text { Acenaph- } \\
\text { thene } \\
\text { d10 } \\
\end{array}$ & $\begin{array}{l}\text { Phenan- } \\
\text { threne } \\
\text { d10 } \\
\end{array}$ & $\begin{array}{c}\text { Benzo(a) } \\
\text { pyrene } \\
\mathrm{d} 12 \\
\end{array}$ \\
\hline $\begin{array}{l}57 \\
63 \\
52 \\
56 \\
52 \\
58\end{array}$ & $\begin{array}{l}91 \\
98 \\
81 \\
83 \\
82 \\
85\end{array}$ & $\begin{array}{r}97 \\
100 \\
84 \\
87 \\
88 \\
89\end{array}$ & $\begin{array}{r}94 \\
100 \\
87 \\
97 \\
90 \\
90\end{array}$ \\
\hline $\begin{array}{l}61 \\
77 \\
78 \\
72 \\
52\end{array}$ & $\begin{array}{l}66 \\
82 \\
85 \\
78 \\
78\end{array}$ & $\begin{array}{l}68 \\
86 \\
87 \\
80 \\
83\end{array}$ & $\begin{array}{l}64 \\
79 \\
82 \\
78 \\
80\end{array}$ \\
\hline $\begin{array}{l}21^{(\mathrm{a})} \\
45 \\
55 \\
34^{(\mathrm{a})} \\
38\end{array}$ & $\begin{array}{l}89 \\
84 \\
85 \\
87 \\
56\end{array}$ & $\begin{array}{r}100 \\
89 \\
95 \\
96 \\
60\end{array}$ & $\begin{array}{r}100 \\
89 \\
95 \\
81 \\
60\end{array}$ \\
\hline
\end{tabular}

5

90

99

100

88

63

79

84

57

79

68

83

88

63

82

65

78

82

58

$B$
$B$
$B$
$B$
$B$

94

97

77

77

78

85

93

70

100

$\begin{array}{ll}A & 100 \\ A & 110\end{array}$

A $\quad 100$

100

94

86

95

75

75

A $\quad 94$

110

A $\quad 98$

98

94

78

77

74

68

74

85

89

87

81

88

$\begin{array}{ll}A & 98 \\ A & 67\end{array}$

96

74

75

50

77

75

86

86

83

75

81

A $\quad 99$

A

99

99

97

78

86

57

92

83 
TABLE L.9. (contd)

Sediment

Ireatment Replicate Batch

$R-O S$

R-OS

$R-O S$

$\mathrm{R}-\mathrm{OS}$

$\mathrm{R}-\mathrm{OS}$

1
2
3
4
5

B
$B$
$B$
$B$
$B$

1 Rep 1

1 Rep 2

$R-P C$
$R-P C$

$R-P C$

$R-P C$

$R-P C$

$R-P C$

2

3

4
5

$R-P F$

$R-P F$

$R-P F$

$R-P F$

$R-P F$

$R-P F$

$C-S B$

$C-S B$

$C-S B$

$C-S B$

C-SB

C-NE

$\mathrm{C}-\mathrm{NE}$

$\mathrm{C}-\mathrm{NE}$

C.NE

C-NE

$\begin{array}{llll}1 & & & \text { A } \\ 2 & & & A \\ 3 & \operatorname{Rep} & 1 & A \\ 3 & \operatorname{Rep} 2 & \\ 4 & & A \\ 5 & & & A \\ 3 & & & A\end{array}$

$\begin{array}{rr}\text { B } & 110 \\ \text { B } & 110 \\ \text { B } & 94 \\ \text { B } & 100 \\ \text { B } & 110 \\ \text { B } & 99\end{array}$

100

99

100

100

98

95

97
89
120
89
92
110

95

86

110

96

98

98

93
56
110
84
99

100

65

100

97

88

$27^{(a)}$

93

79

87

99

96

98

96

A

96

94

cent Recoveries Phenan- Benzo(a) threne pyrene $\mathrm{d} 10 \quad \mathrm{~d} 12$

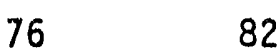

$67 \quad 69$

$79 \quad 86$

$72 \quad 79$

$72 \quad 85$

$84 \quad 87$

$83 \quad 92$

$84 \quad 100$

$86 \quad 92$

$80 \quad 93$

$\begin{array}{ll}76 & 81\end{array}$

$74 \quad 81$

$66 \quad 72$

$83 \quad 91$

$\begin{array}{ll}78 & 89\end{array}$

$\begin{array}{ll}76 & 87\end{array}$

$\begin{array}{ll}76 & 89\end{array}$

$81 \quad 89$

$54 \quad 56$

$83 \quad 98$

$79 \quad 91$

$72 \quad 74$

$70 \quad 81$

$\begin{array}{ll}69 & 79\end{array}$

$73 \quad 81$

$74 \quad 83$

$72 \quad 79$

(a) Surrogate recovery outside of target qual ity control range of $40-120 \%$. Two of three quantitation surrogates greater than $20 \%$ on all samples, QC requirements met; however, quantitation of lighter PAHs may have been affected on samples with recoveries $<20 \%$. Re-extraction not possible because entire tissue sample was used for analysis. 


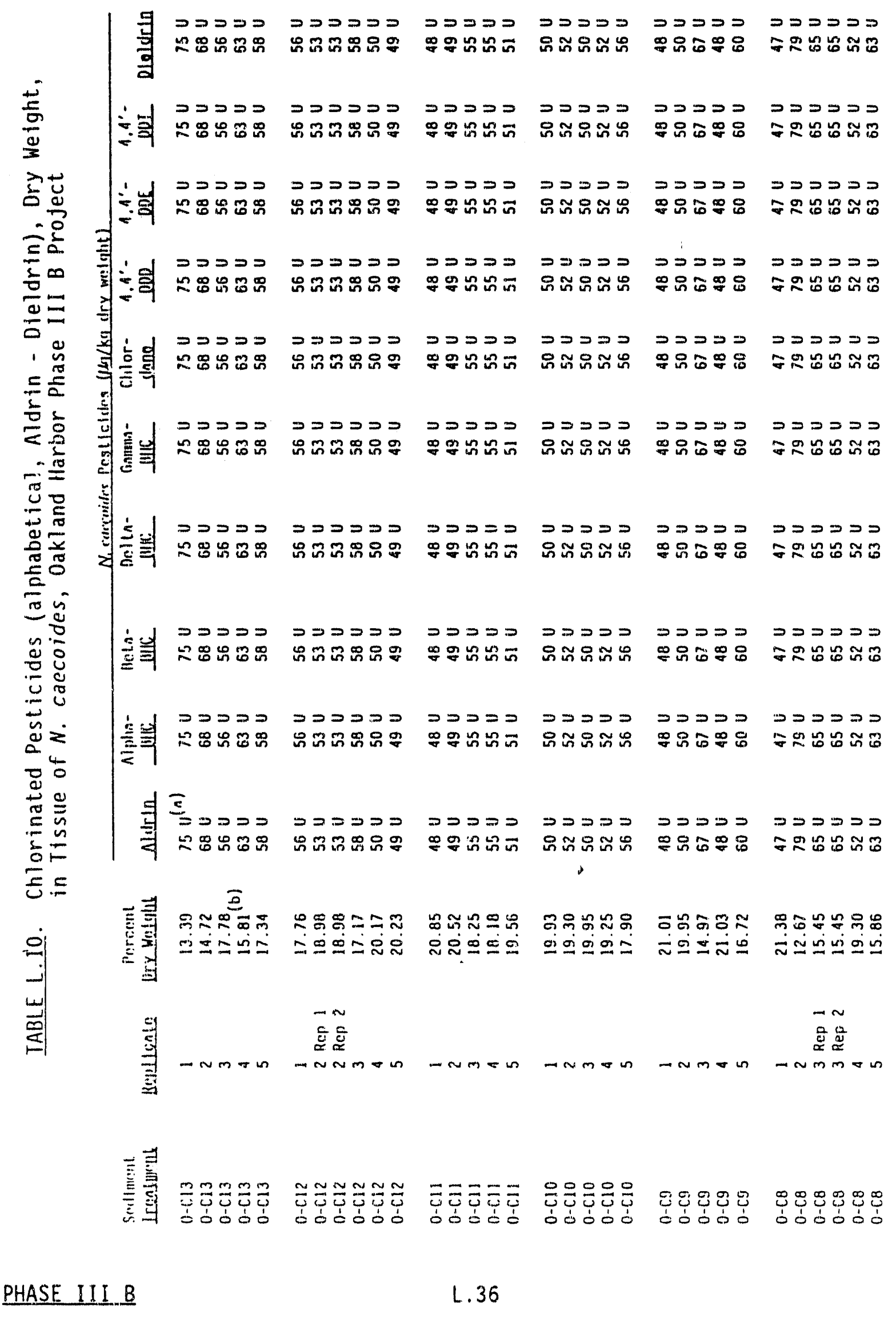




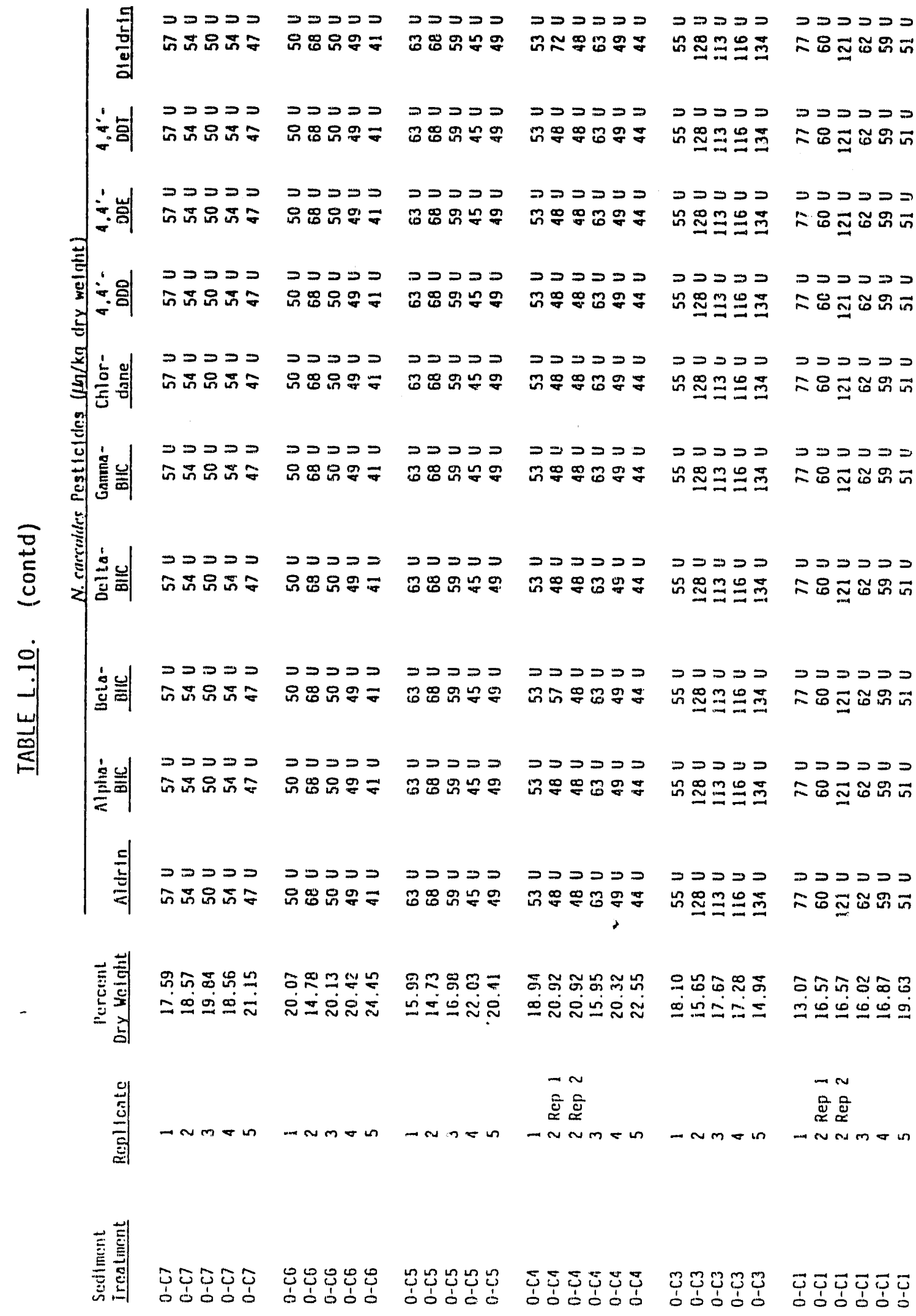




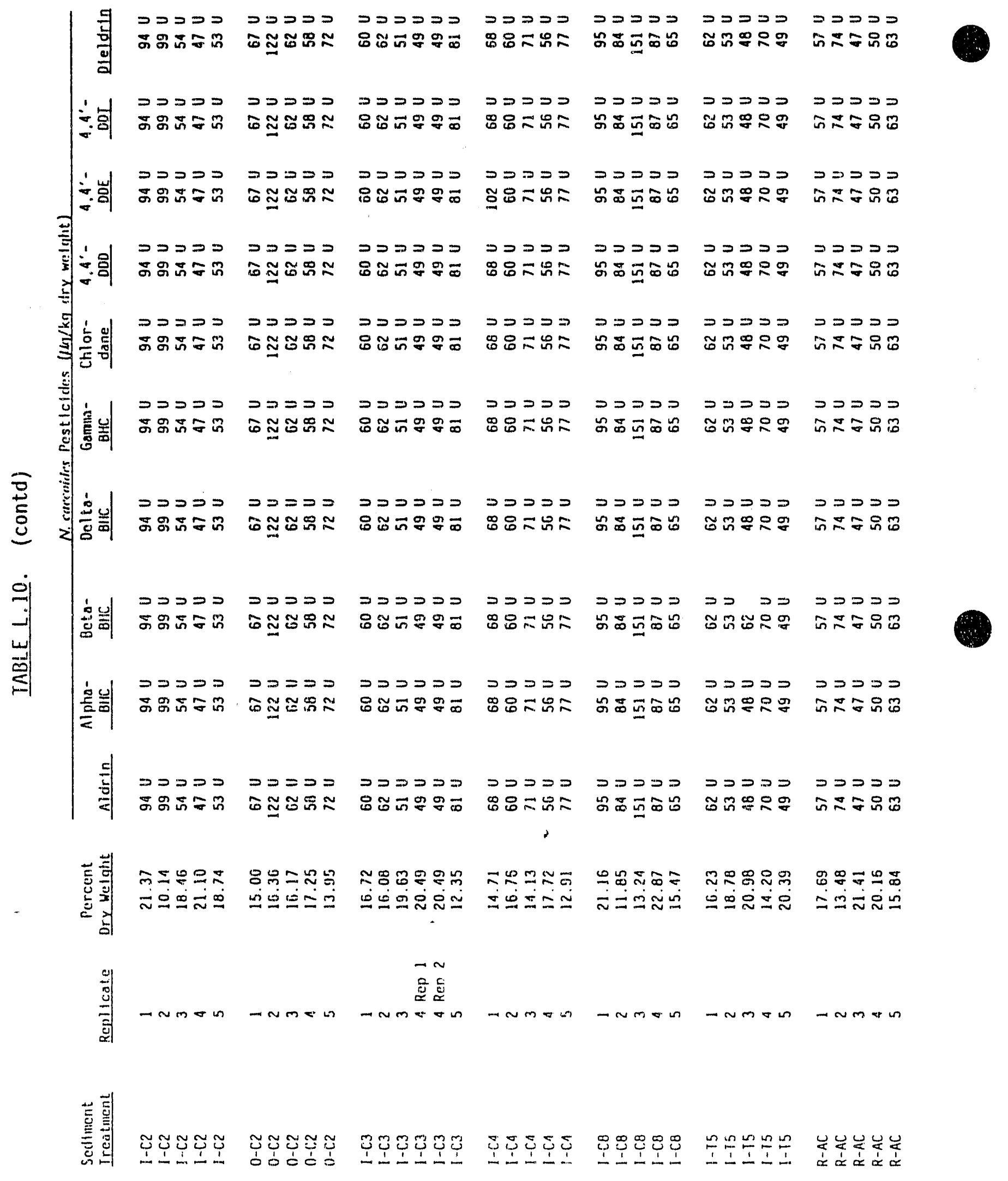




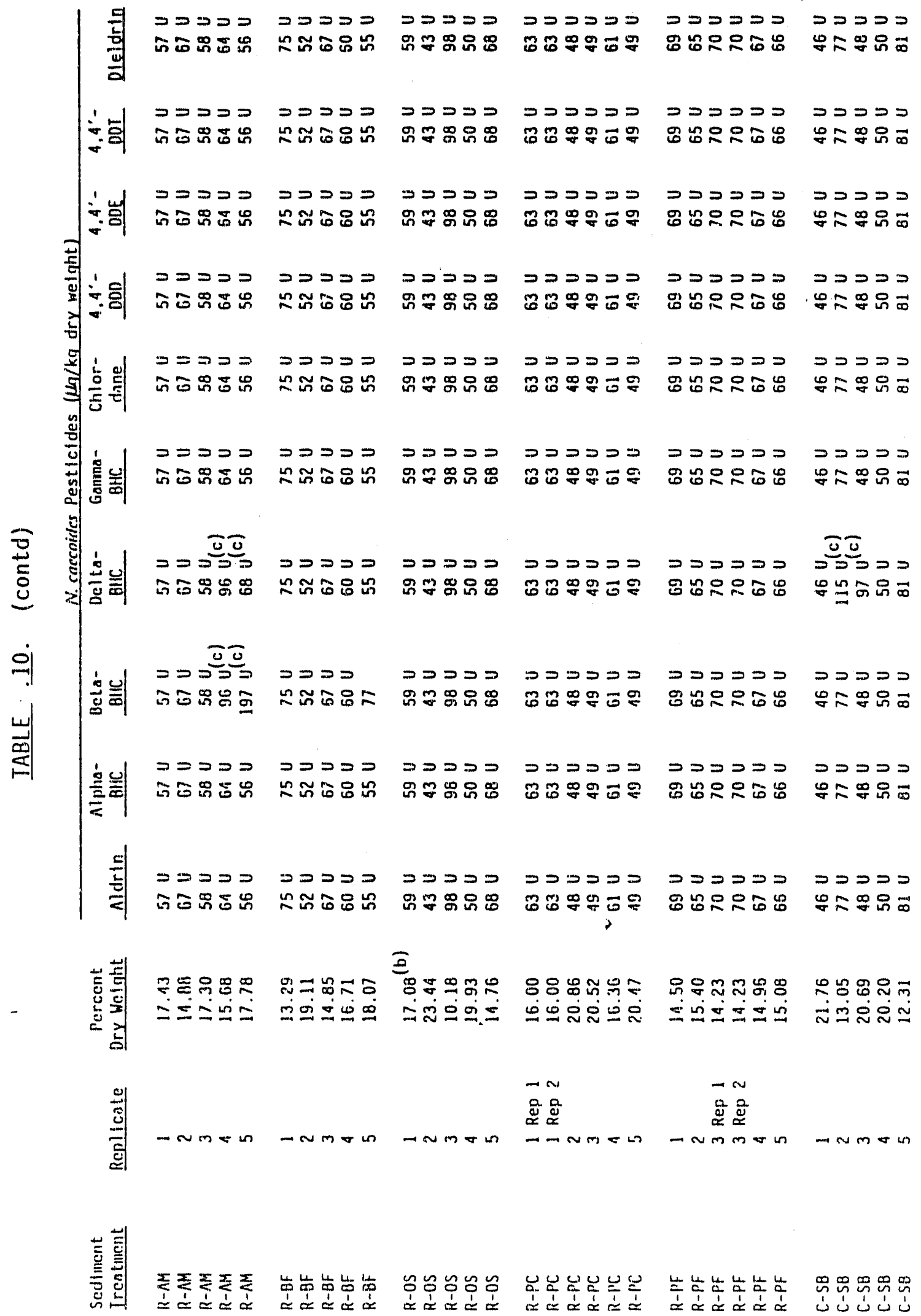




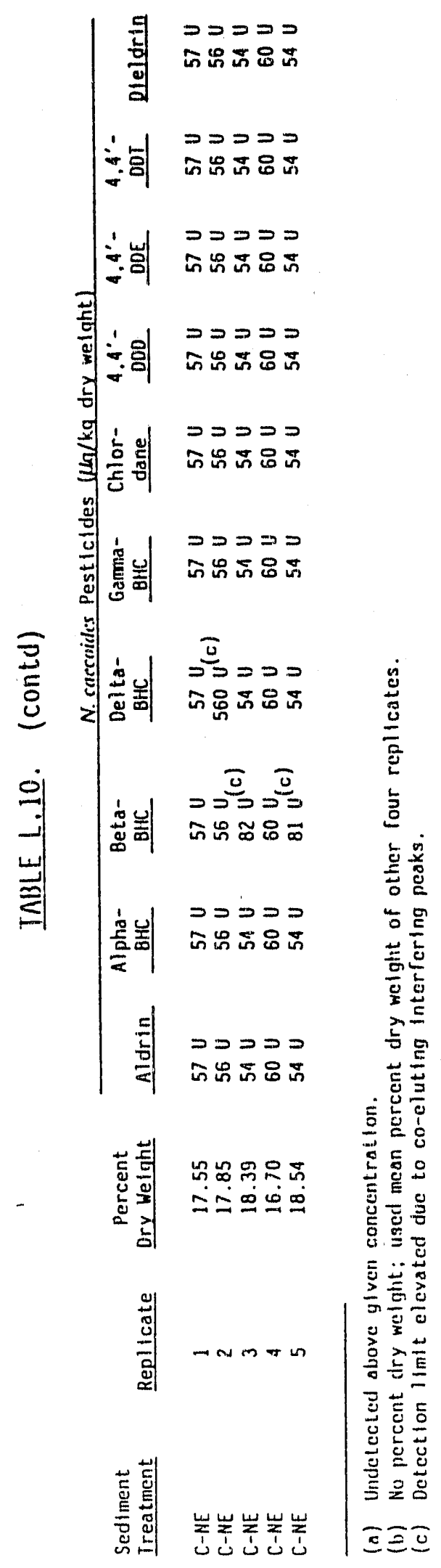




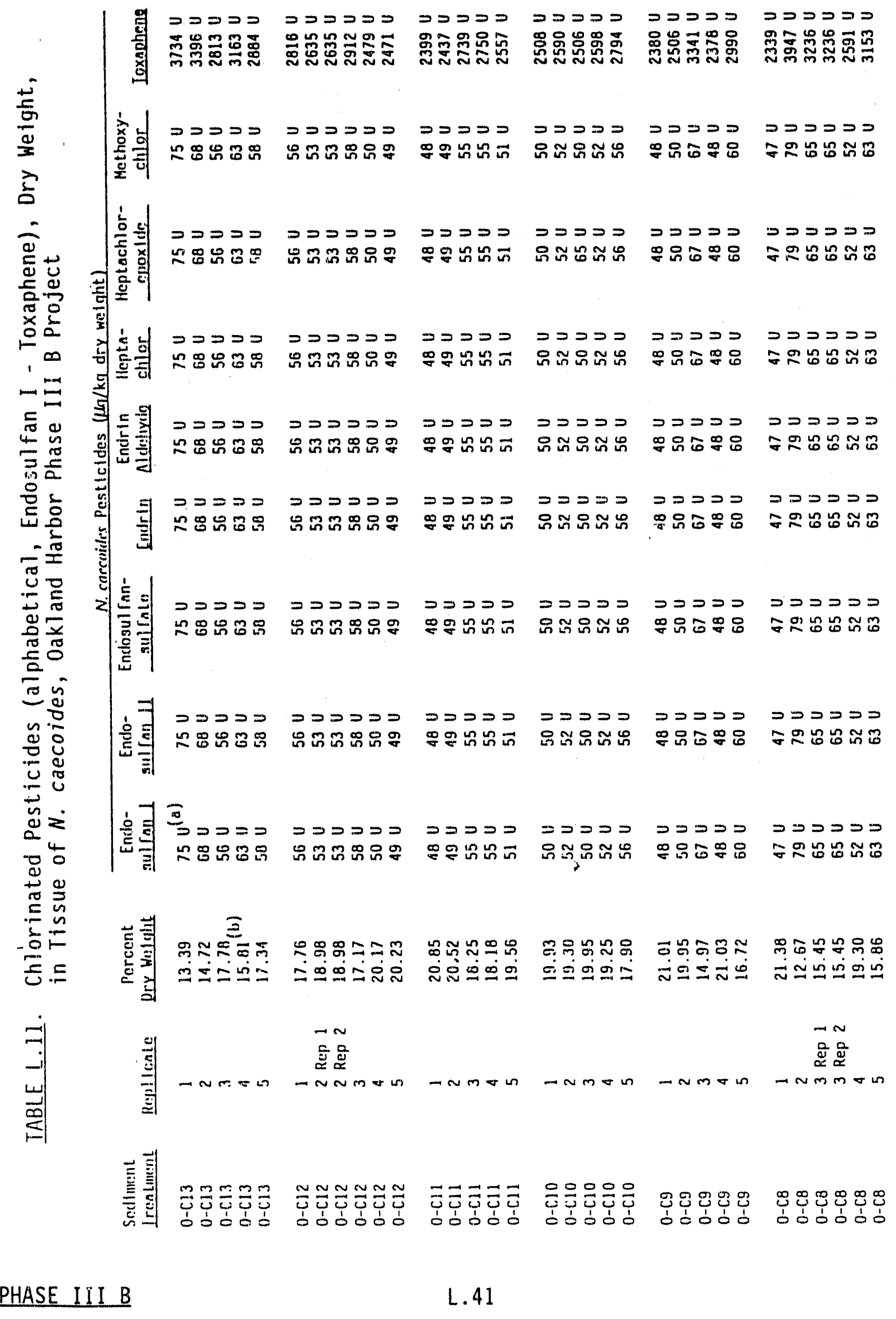




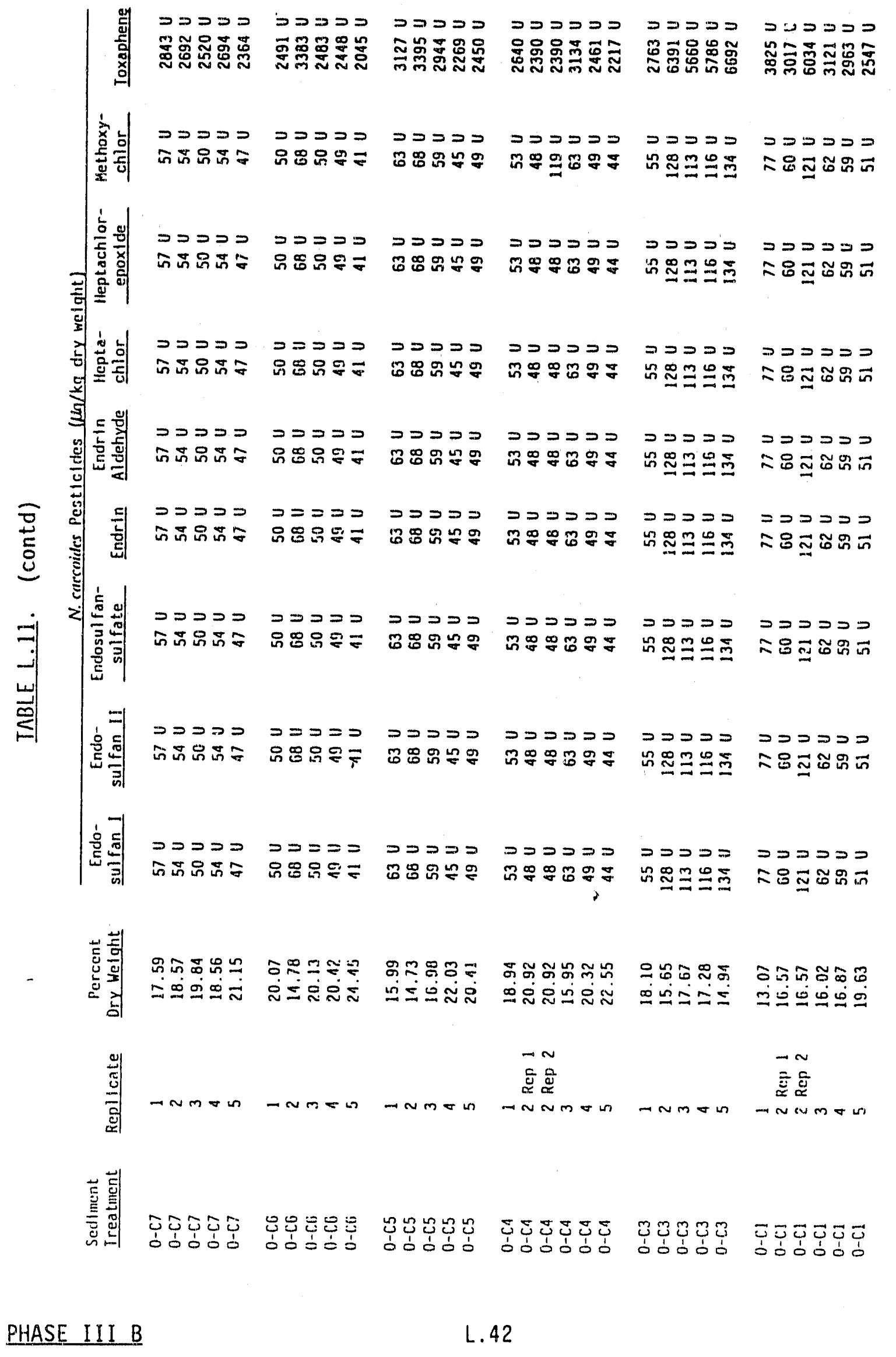




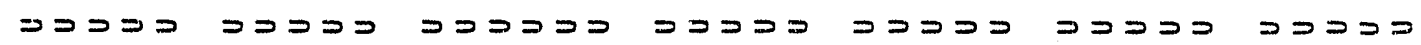

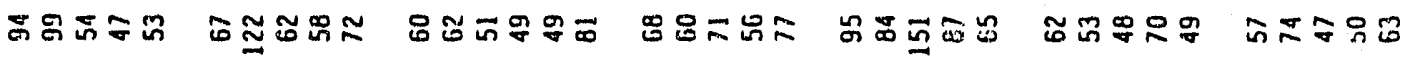

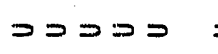

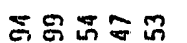

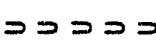

$\supset \supset \supset \supset \geq \supset$

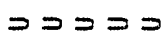

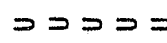

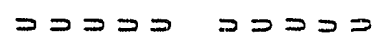

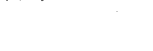

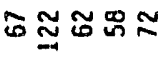

ำ

멍묘ㅇㅛㅛ

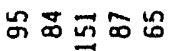

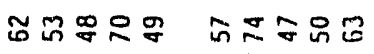

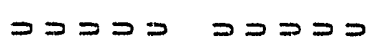

ว フコว

吕名品舟

ธ్ָ̃⿻

웅 ㄸำ

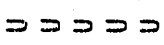

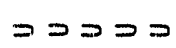

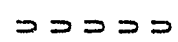

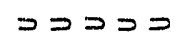

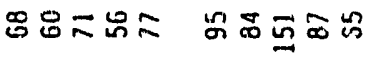

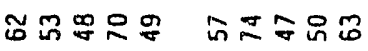

$=\geq=9$

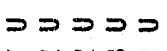

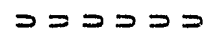

$==0=$

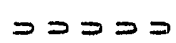

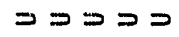

$\Rightarrow \supset \supset \supset$

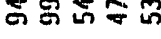

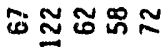

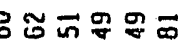

里咨品

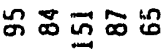

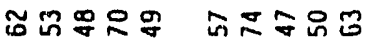

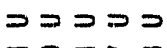

$\Rightarrow>D>0$

$>>>0 D 0$

$p=0=0$

$>\supset \supset \supset$

DDPDD $>>D P$ ธํํํำ

ธิกัธ

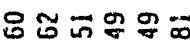

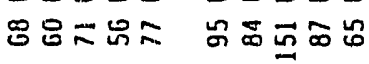

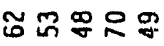

的东品星

올콜

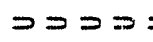

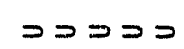

ว $>\geq \geq \geq$

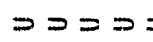

$>>=0$

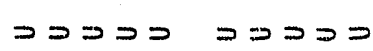

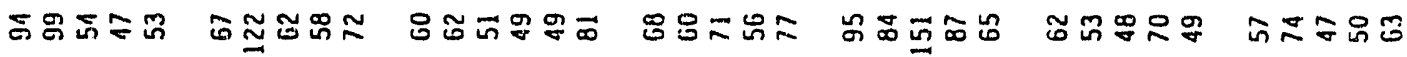

을흡

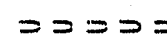

ธ马ํำ

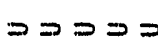

$\supset \supset \supset \supset つ=$

$==ン=$

$\supset \supset \supset \supset$

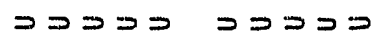
ธิ๊ ్ํำ

g요요

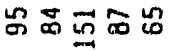

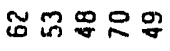

珨尔员是

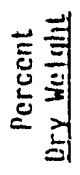

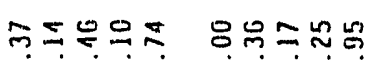

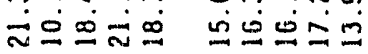

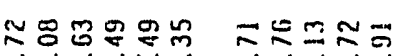

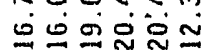

$\leq \dot{\Sigma} \pm \pm$

$0 \ln _{\infty} \approx$

ㄲํ용유 용 シ் $=\dot{\sim} \dot{\sim} \dot{0}$

콥

$\rightarrow \sim$

올 돔

-nmon mam-

$-\sim m=\nabla n$

$-n m-$

- Nmo

$m \sim m=m$

总氡

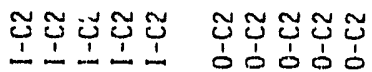

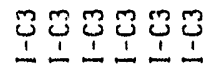

¿广SS

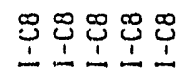

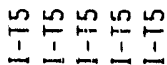

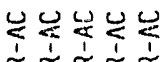




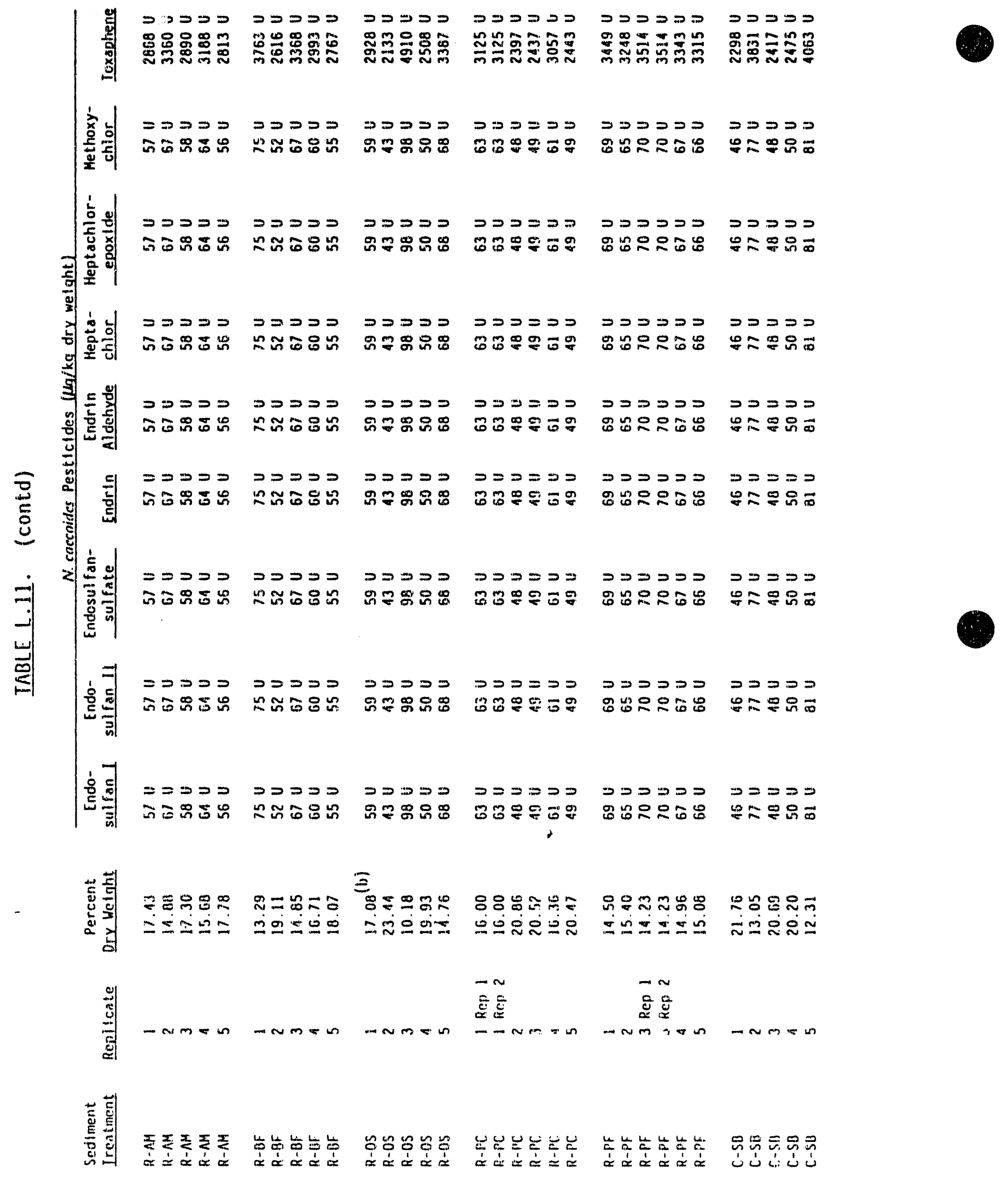




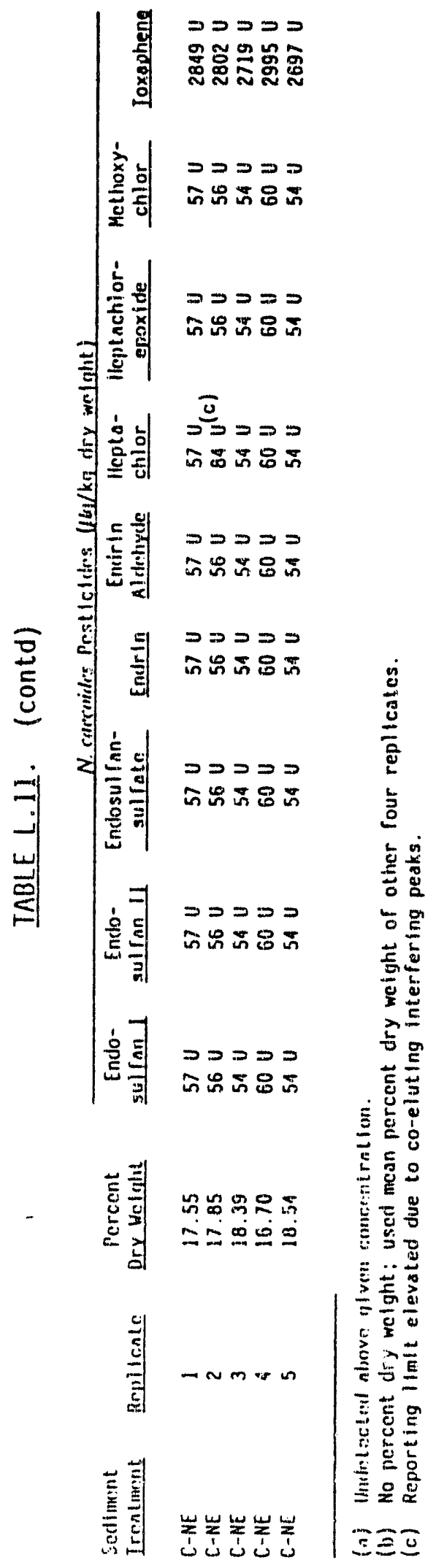




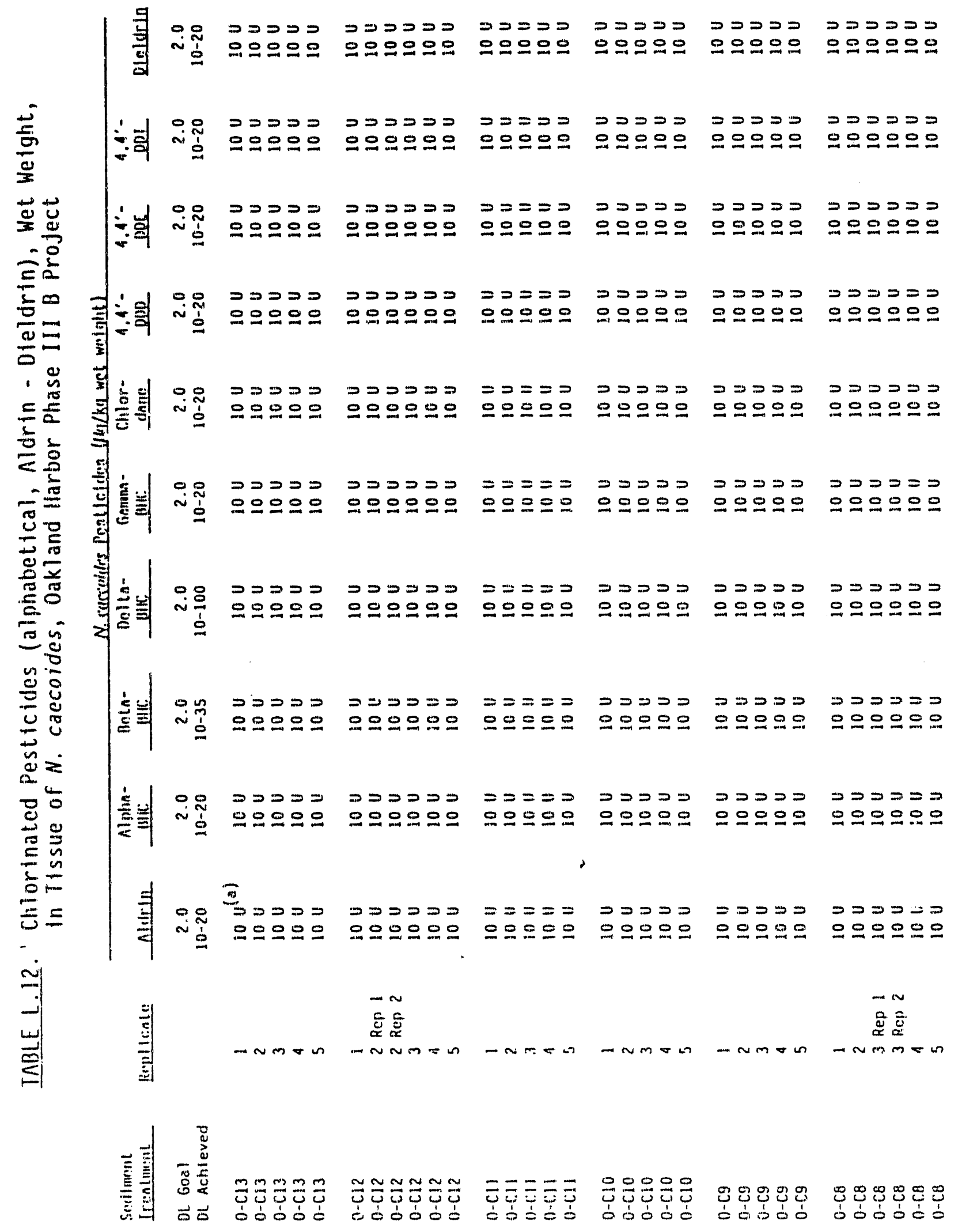




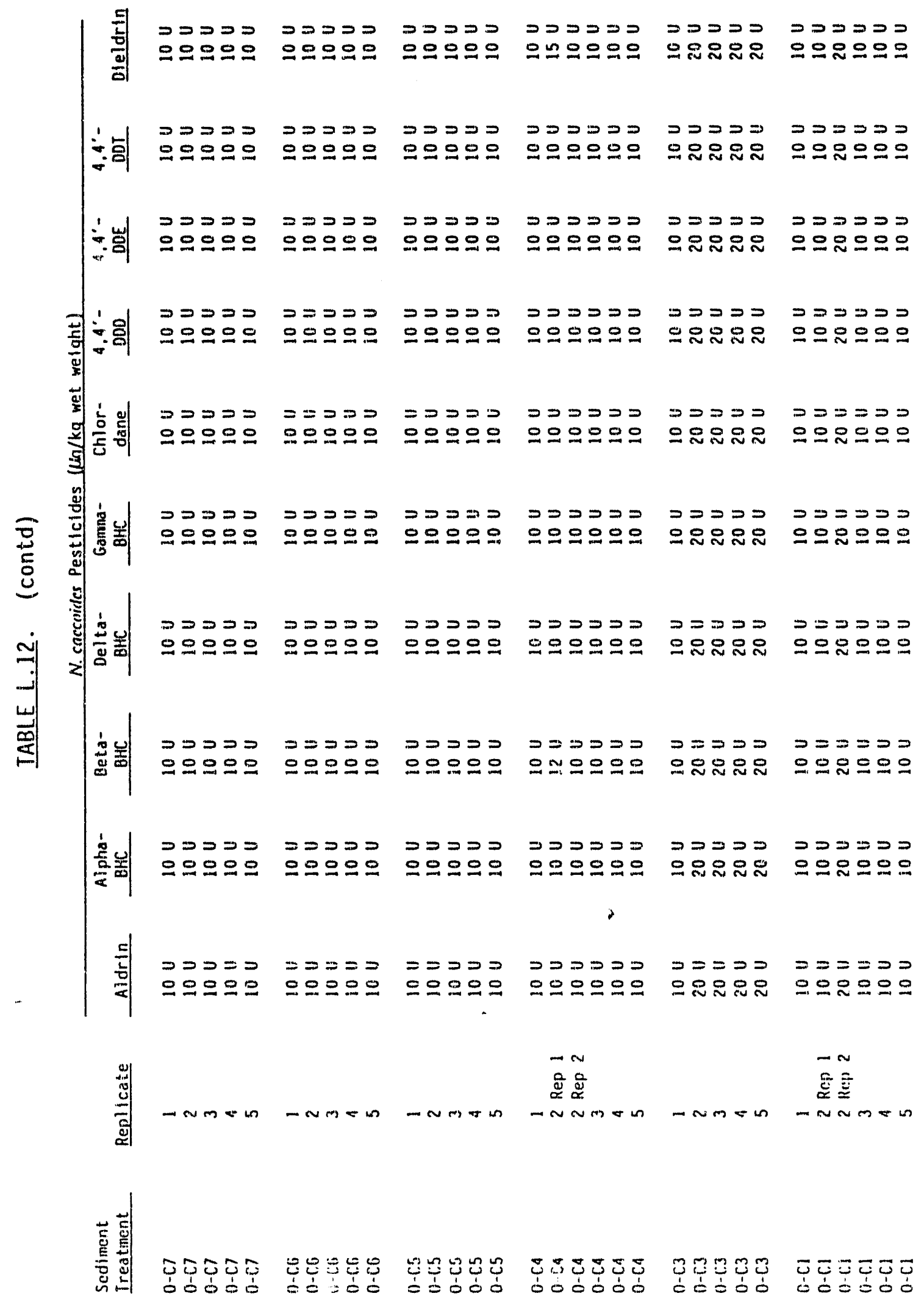




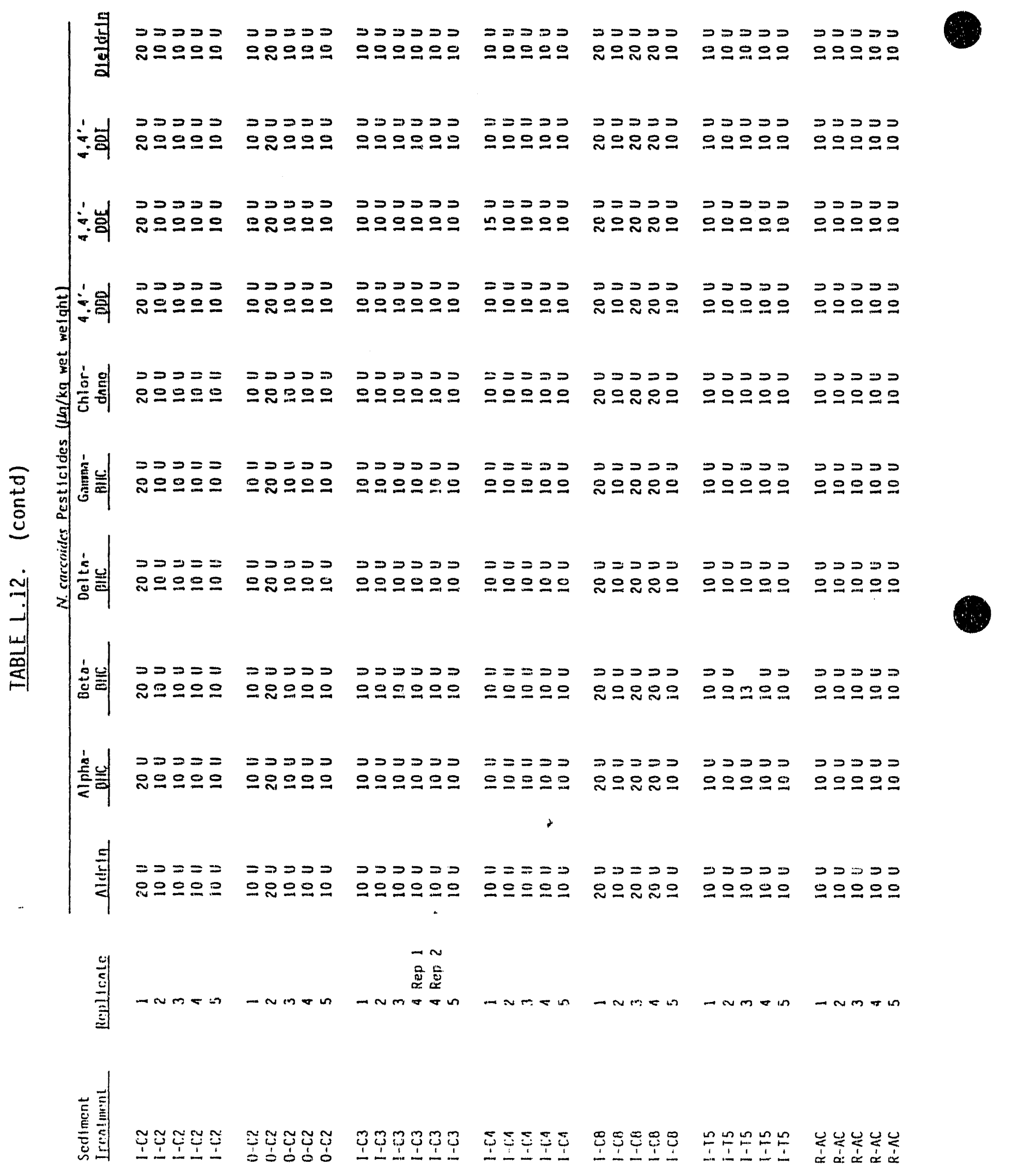




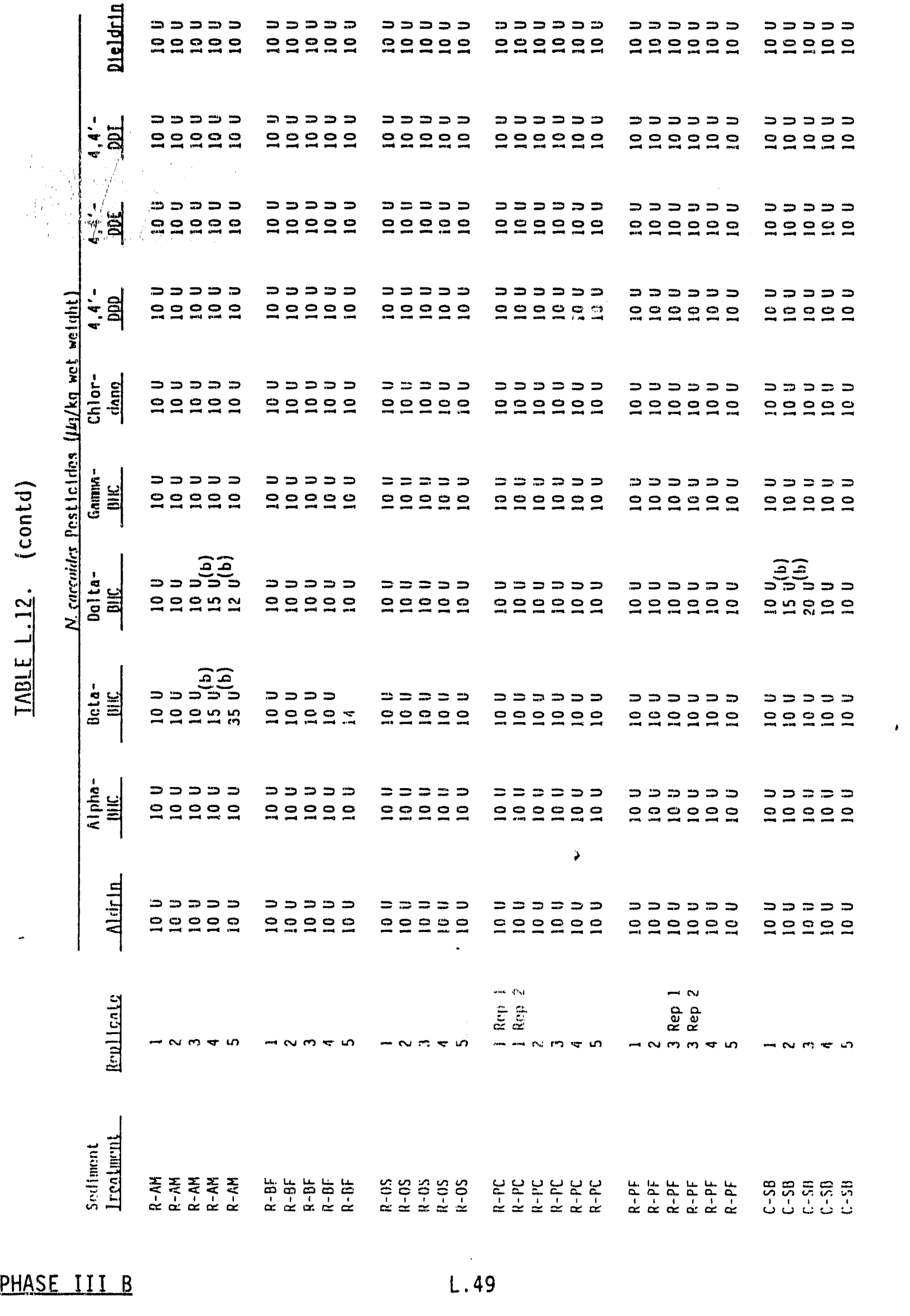




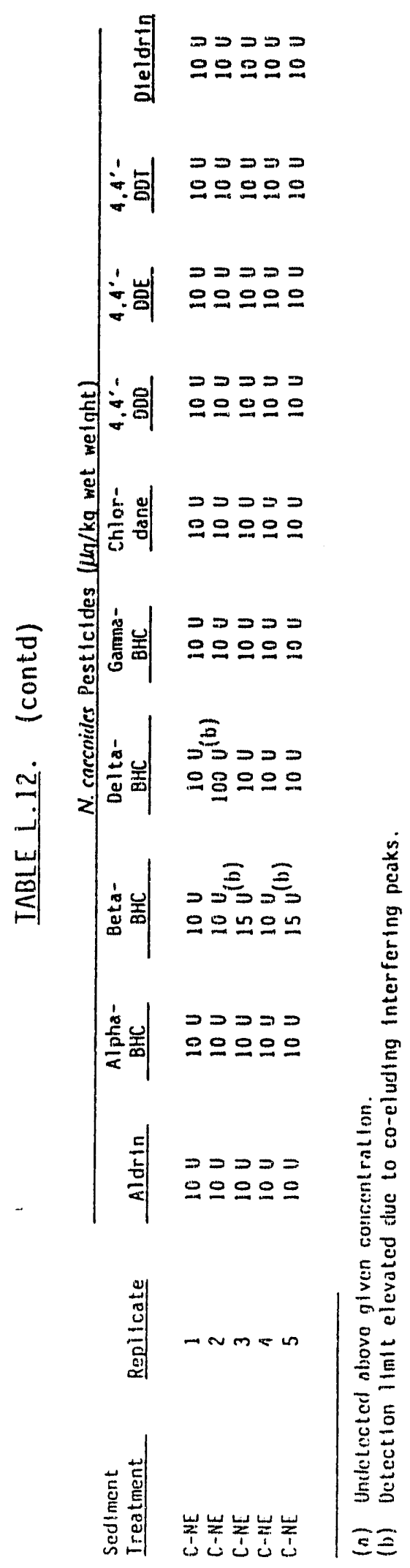

PHASE III B 


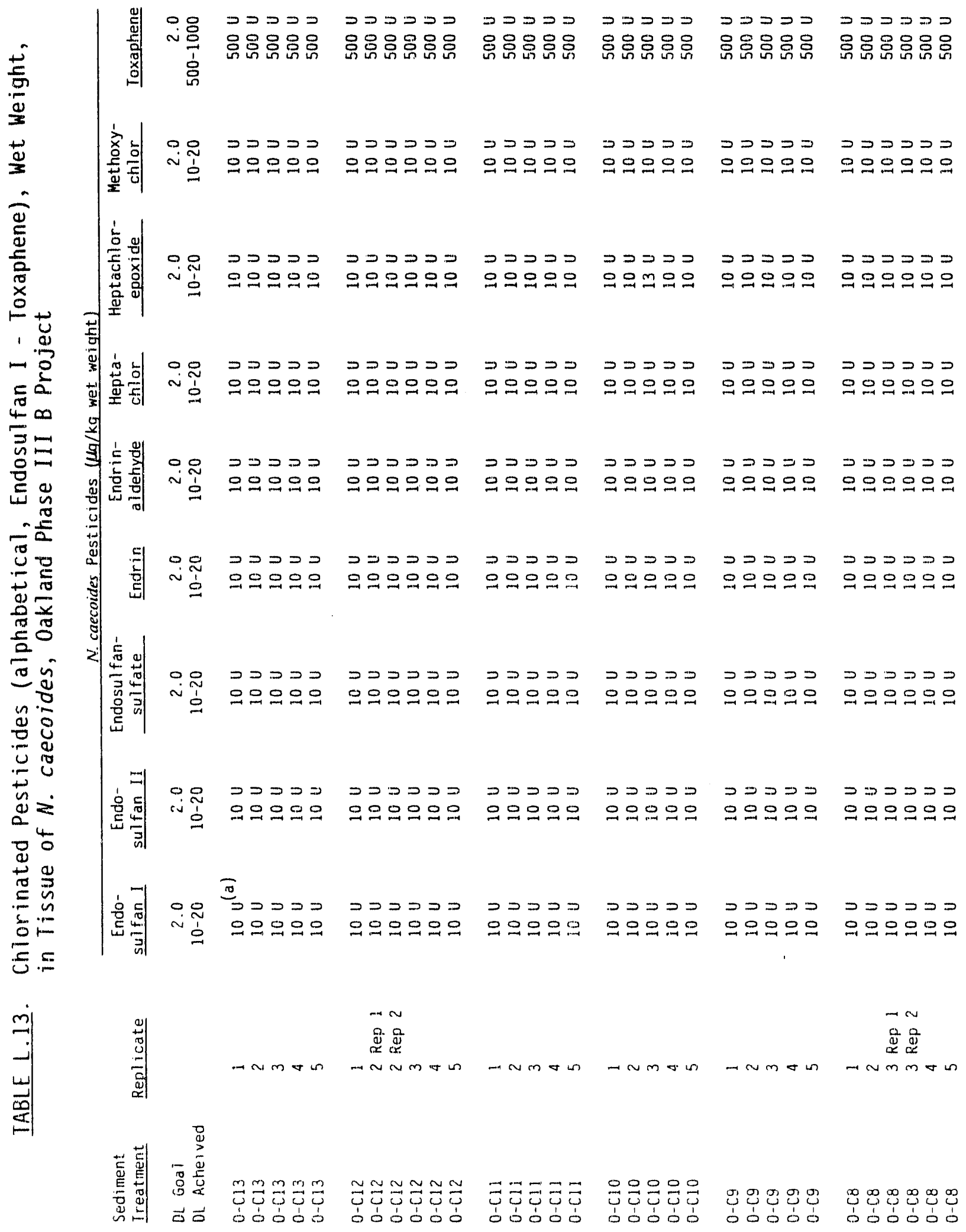




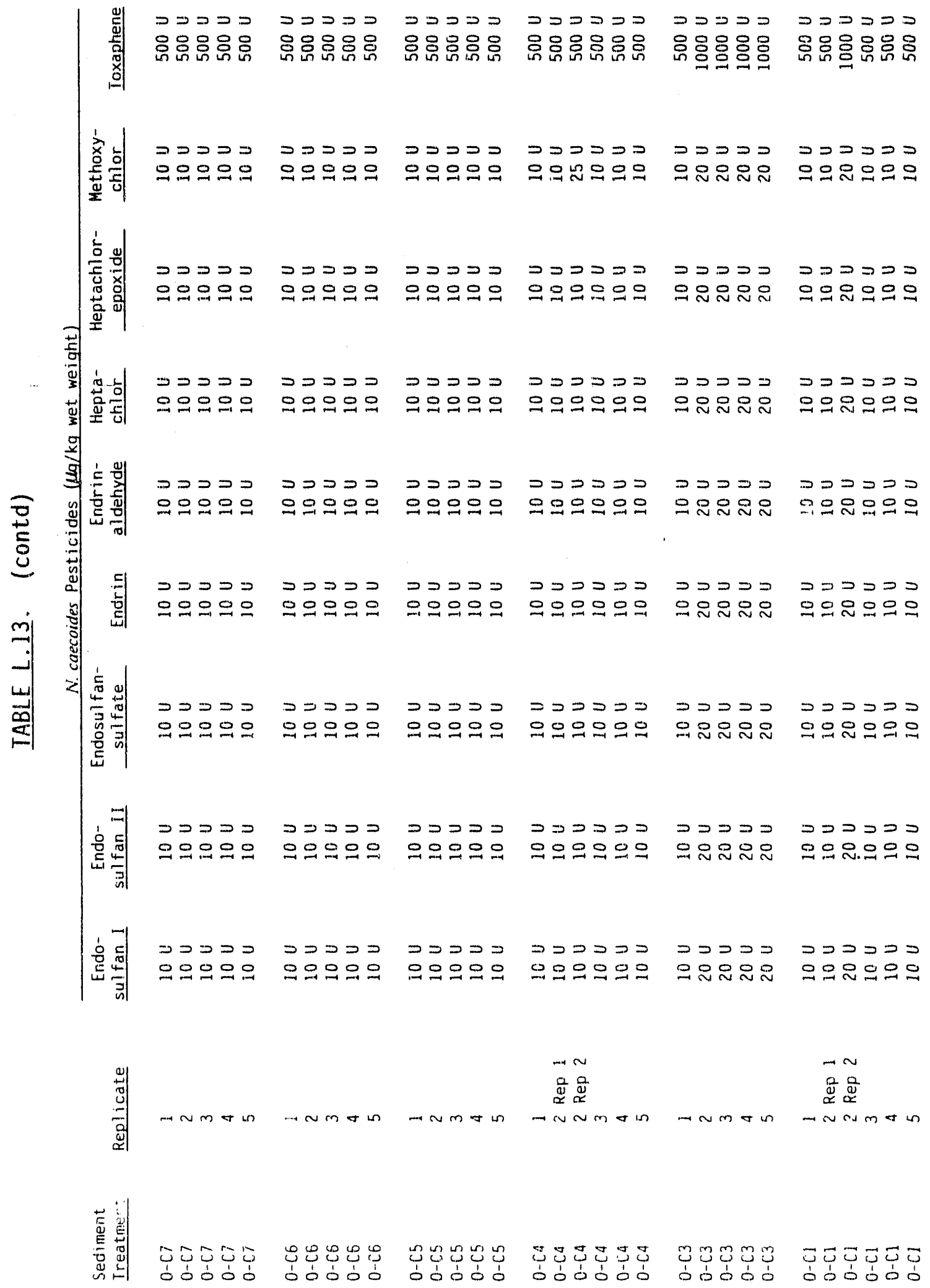




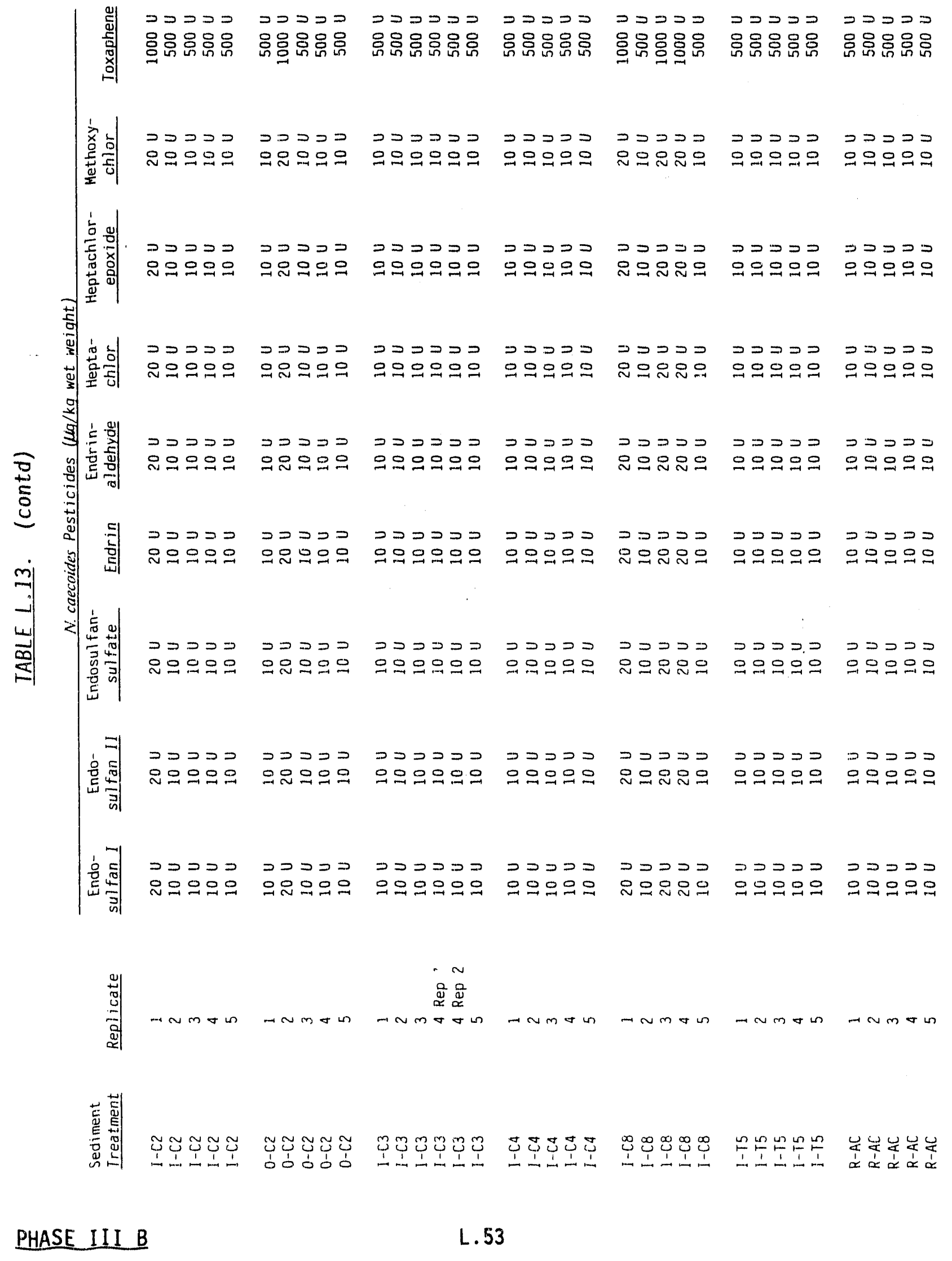




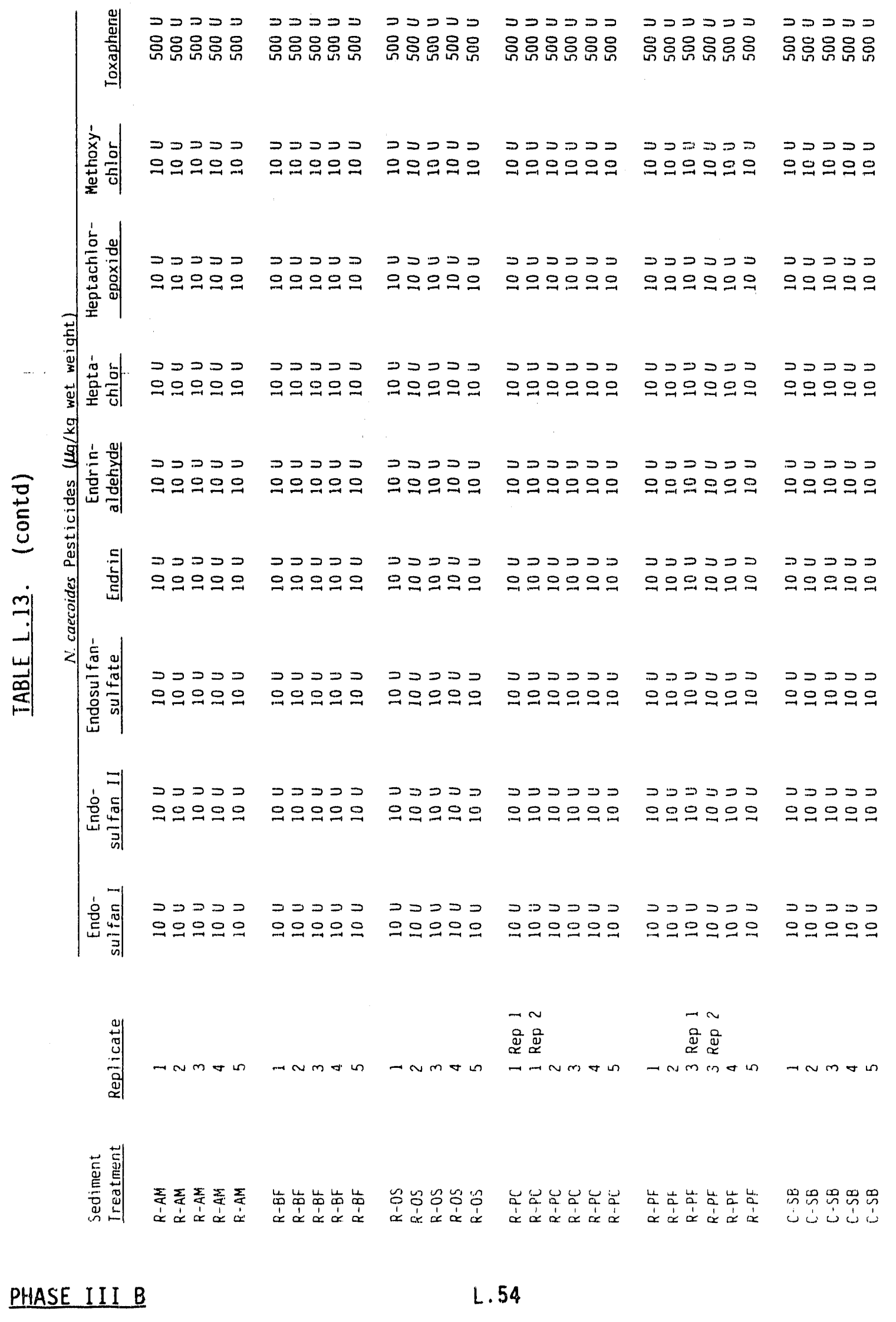




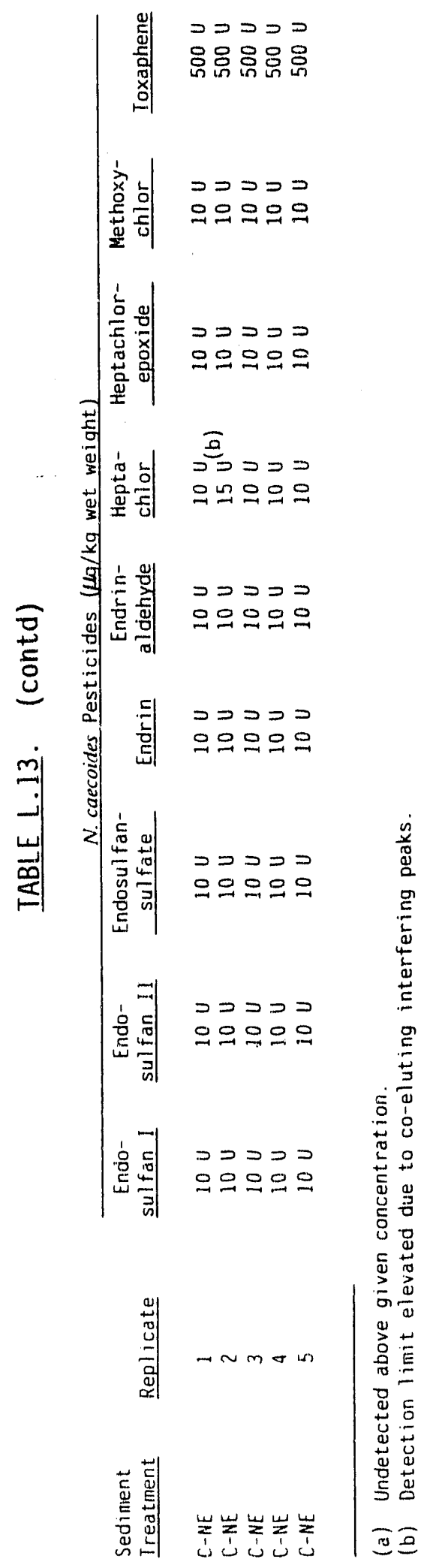

PHASE III B 


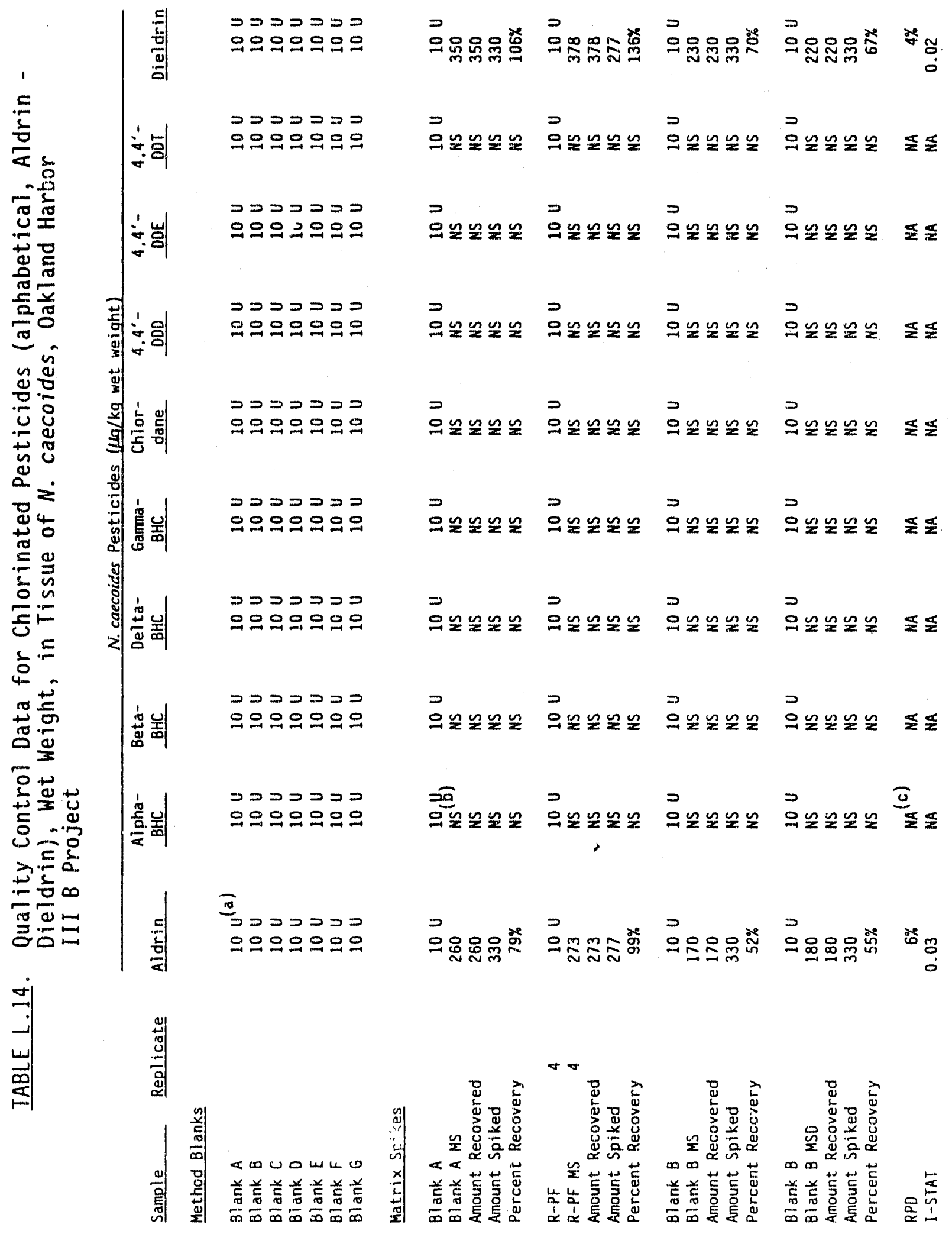

PHASE III B

L. 56 


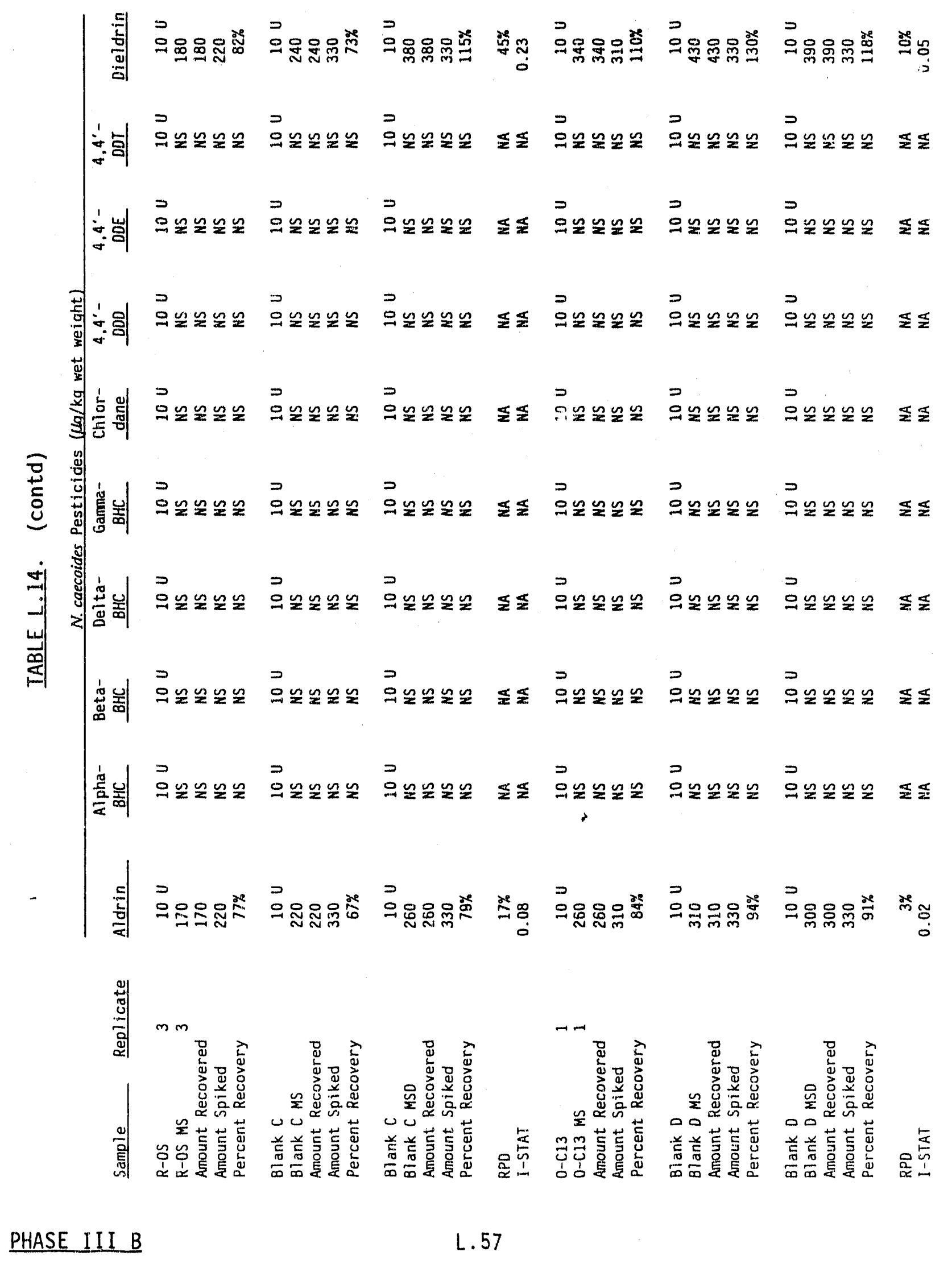




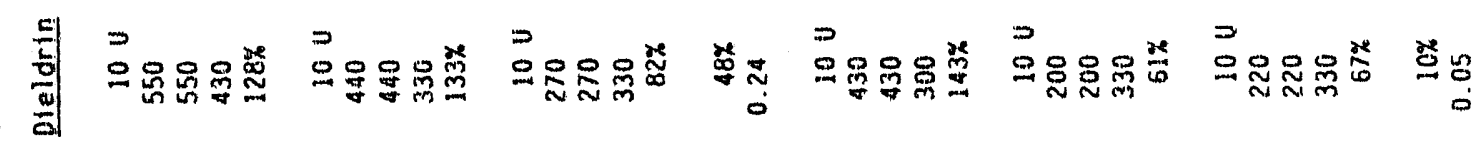

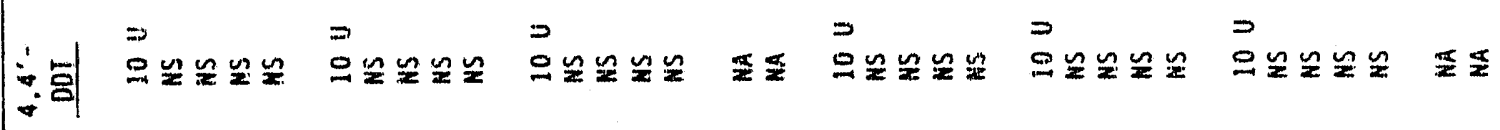

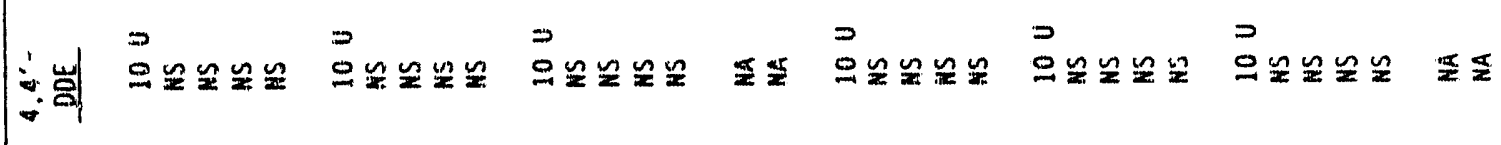

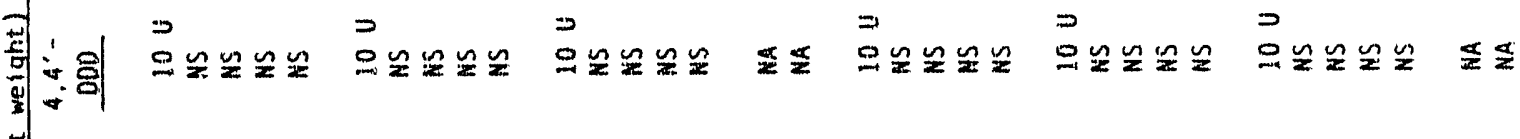

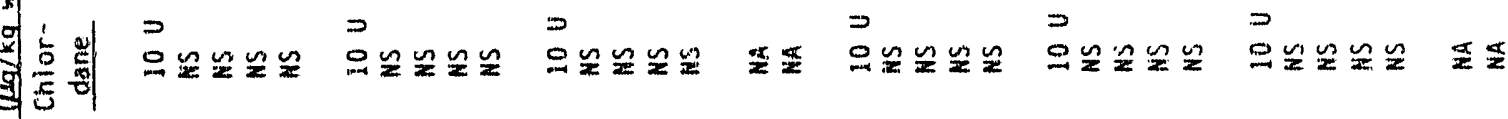

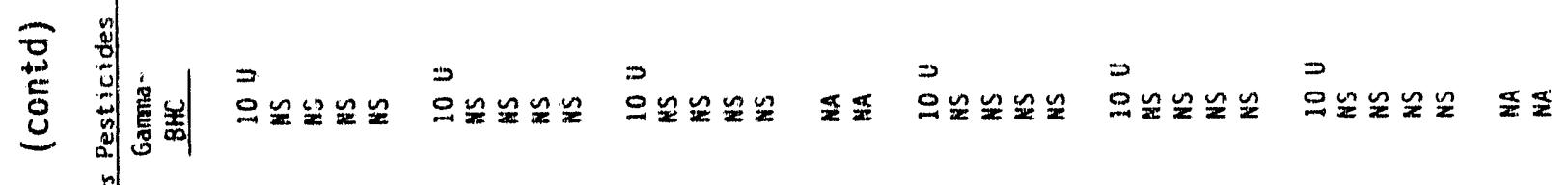

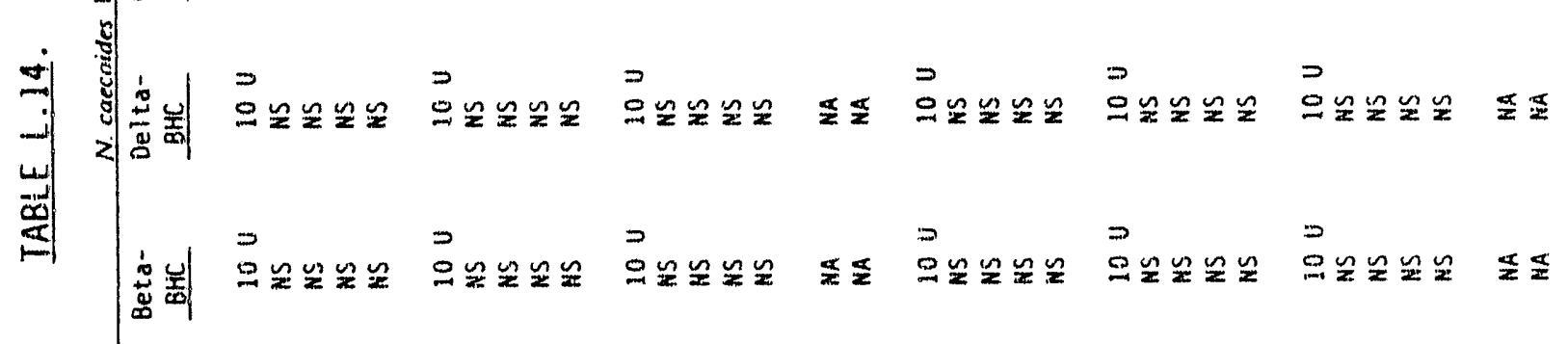

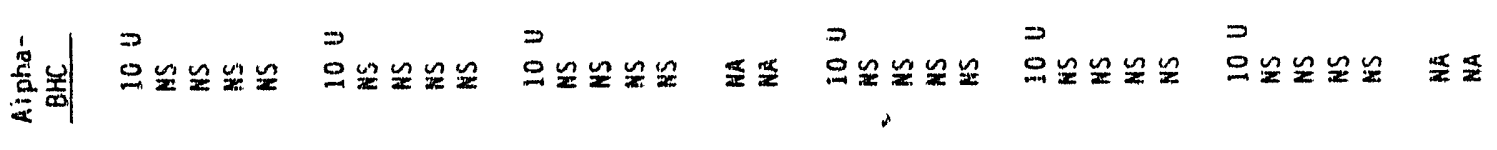

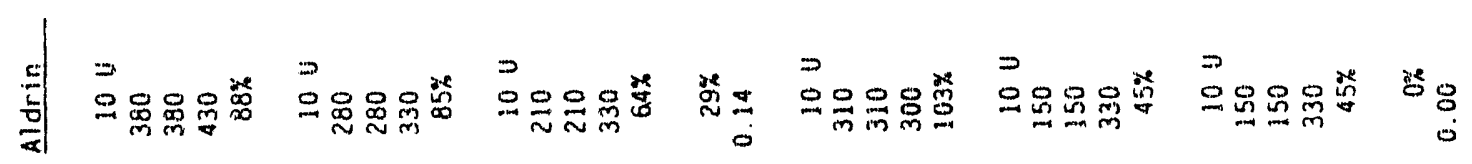

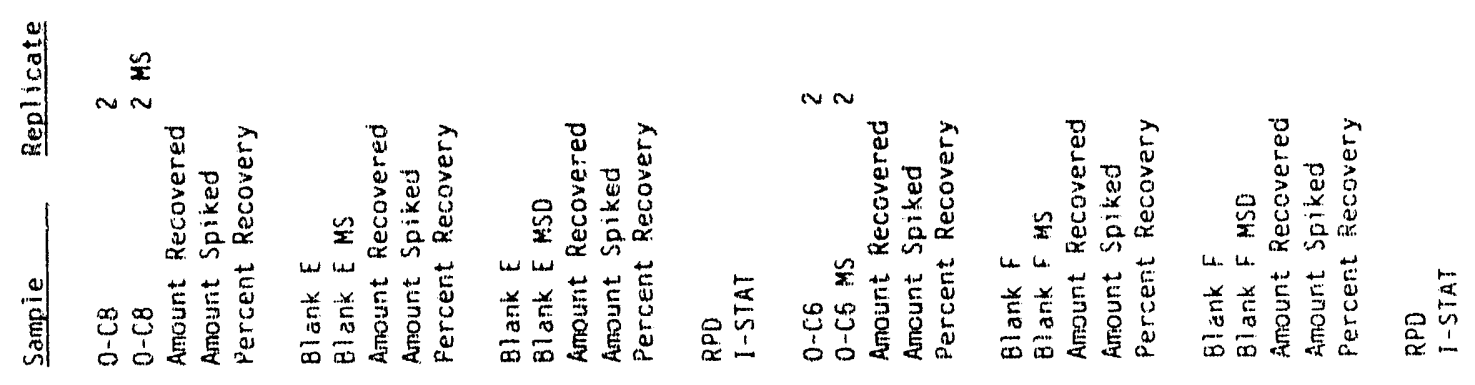




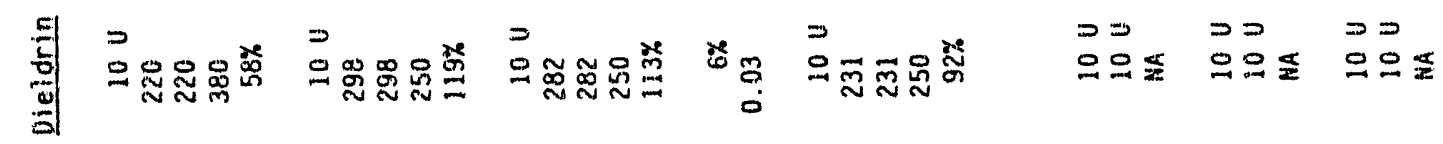

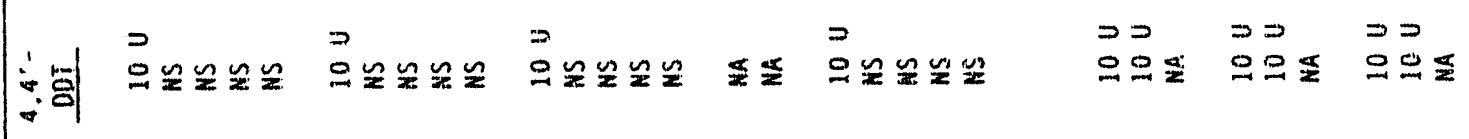

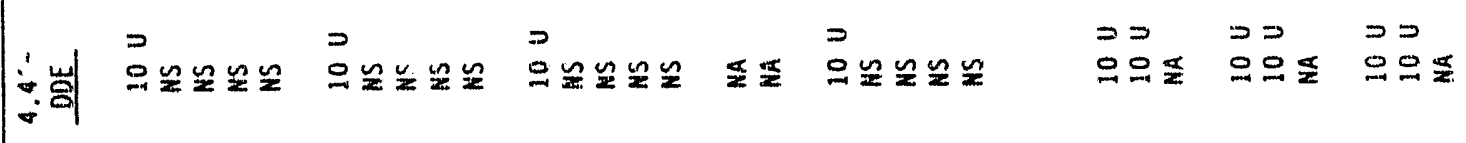
具:

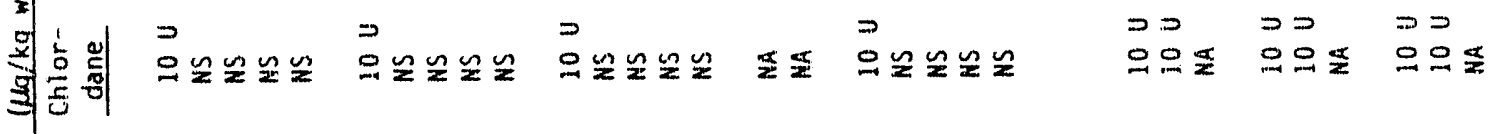

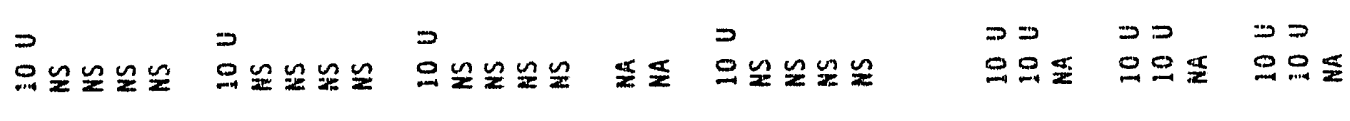

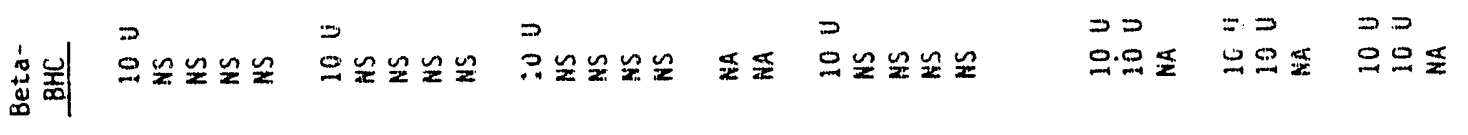

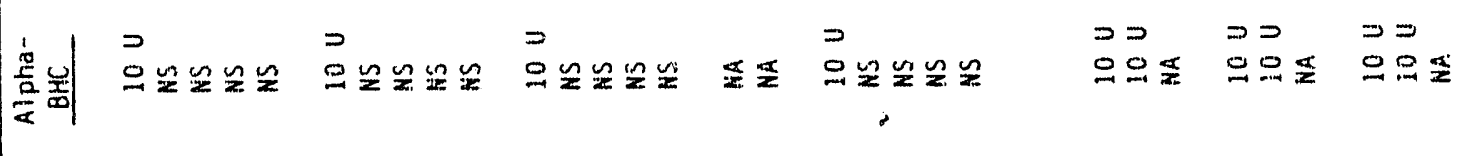
䒺

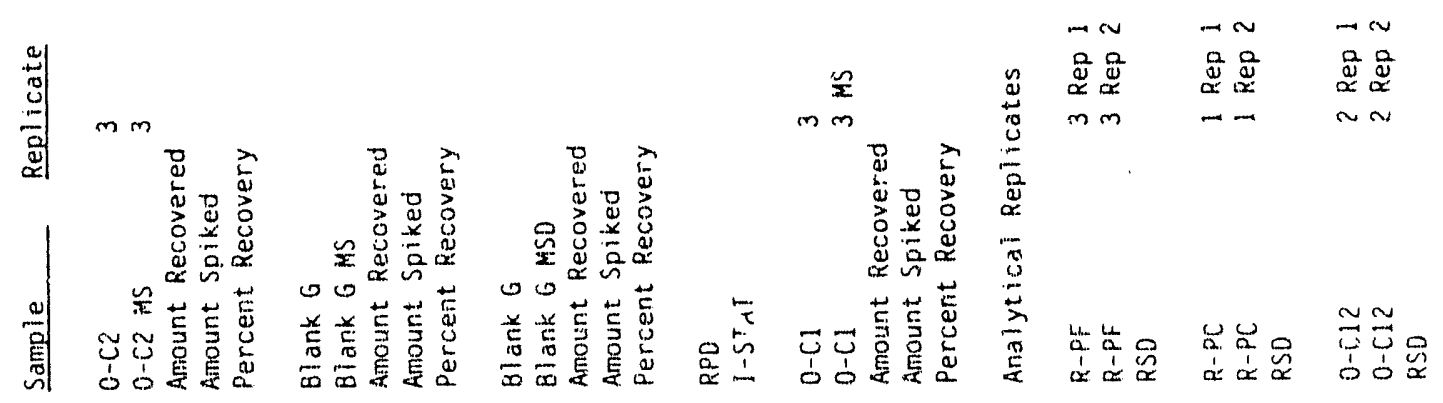




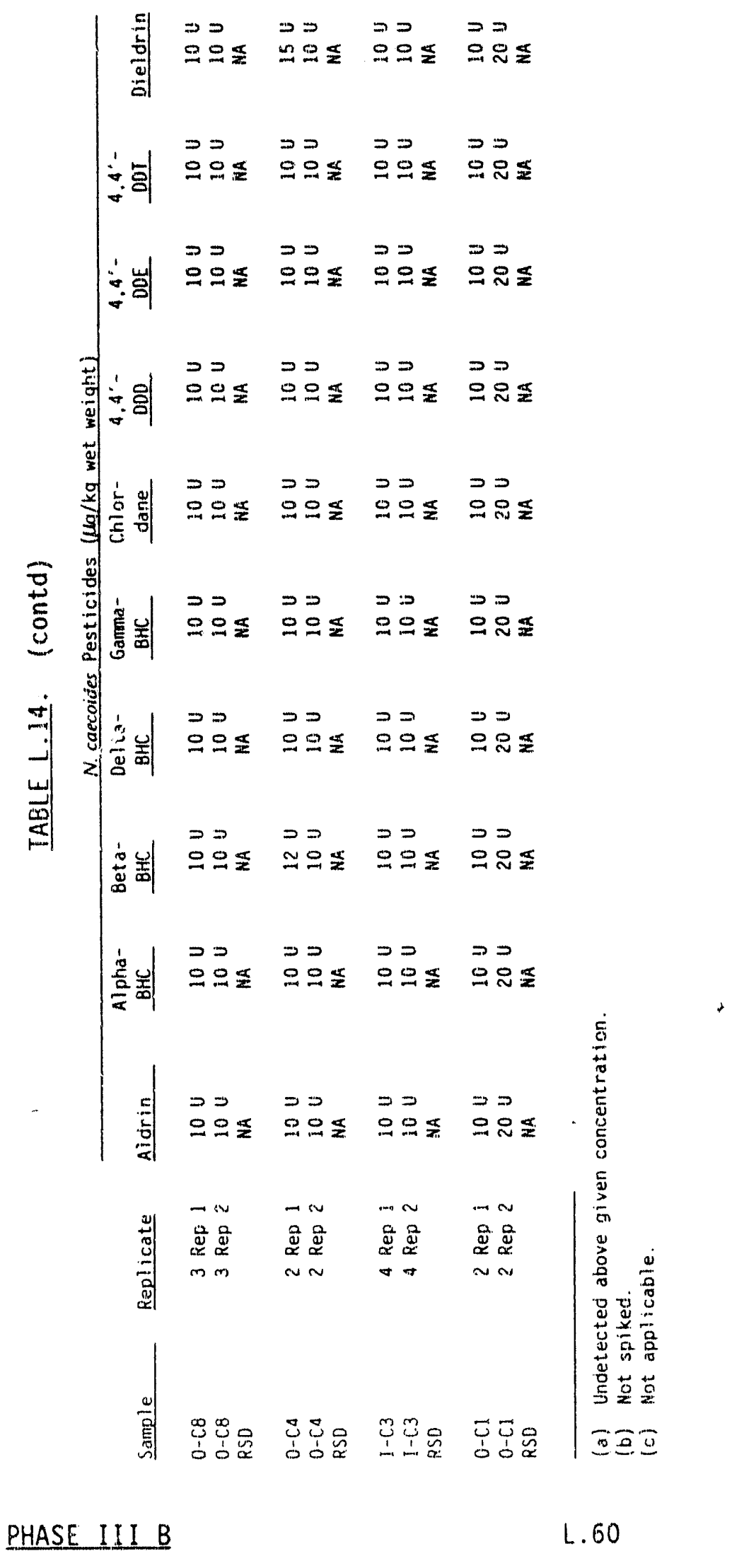




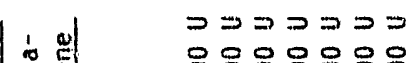

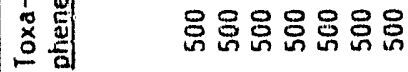

$\cdot$

焉

"

兽

$\stackrel{5}{+}$

可这

万世

至

ठํㅛ

$>0$

$\pm \frac{1}{2} 0$

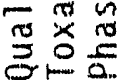

要

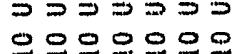

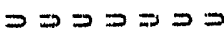

으으오으으유으

จ $\supset \supset \supset \supset \supset$

으응ㅇㅇ으으으

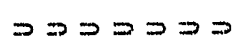

으으으음으응

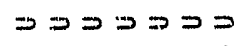

음으으이으으으

จ $\neg コ จ$

웅으응으으응

$\supset \supset \supset \supset \supset \supset$

우옹ㅇㅇㅇㅇㅇㅇㅇㅇㅜ

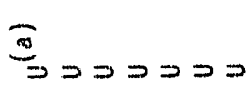
유으으으오으응

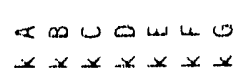
总总总总总总落

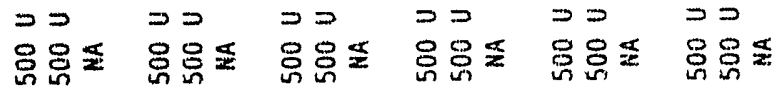

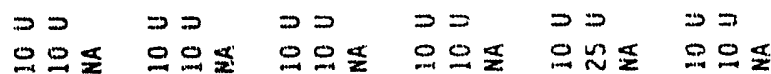

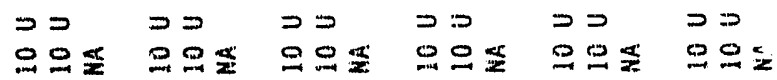

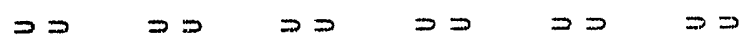

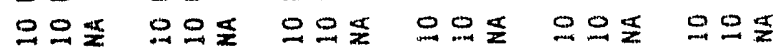

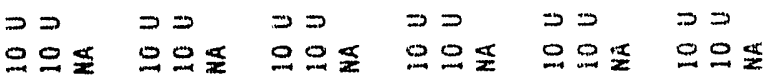

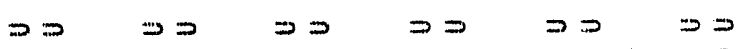

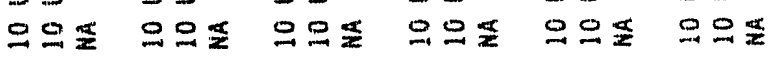

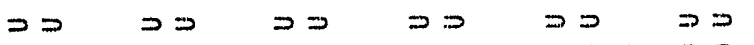

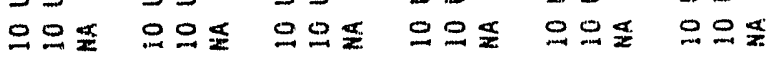
틈

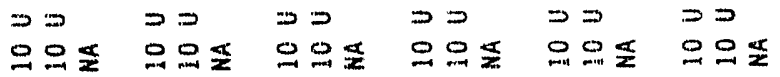

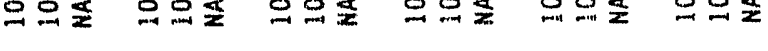

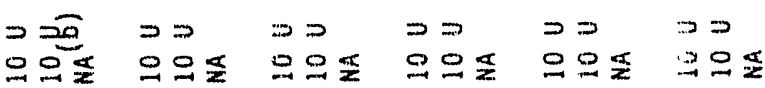

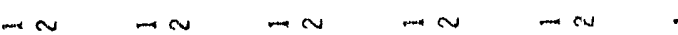

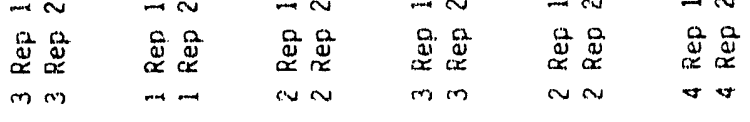

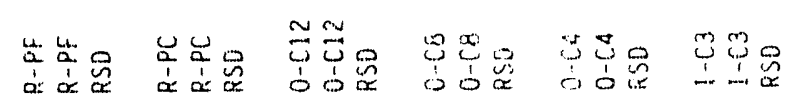




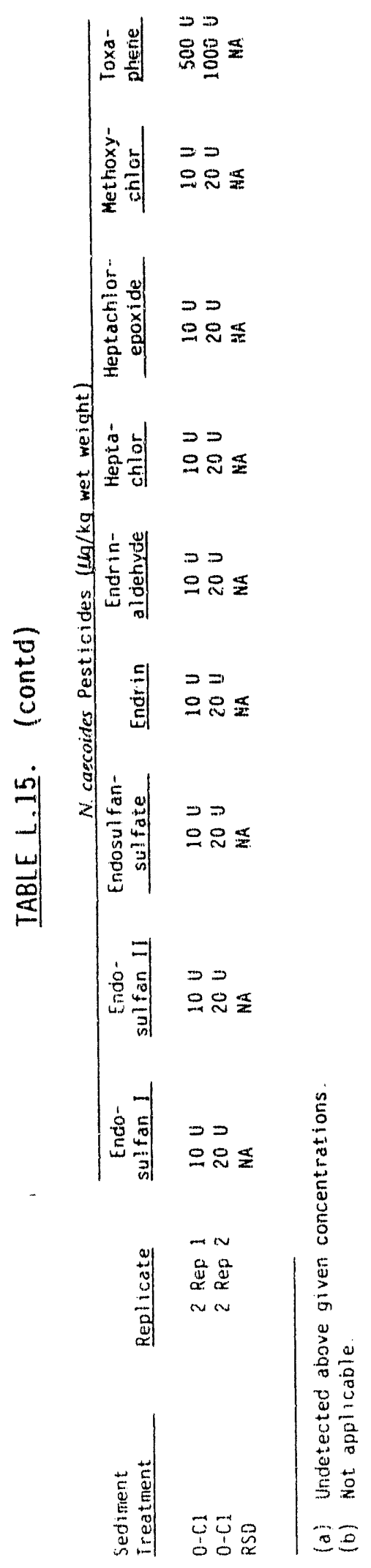

PHASE III B 


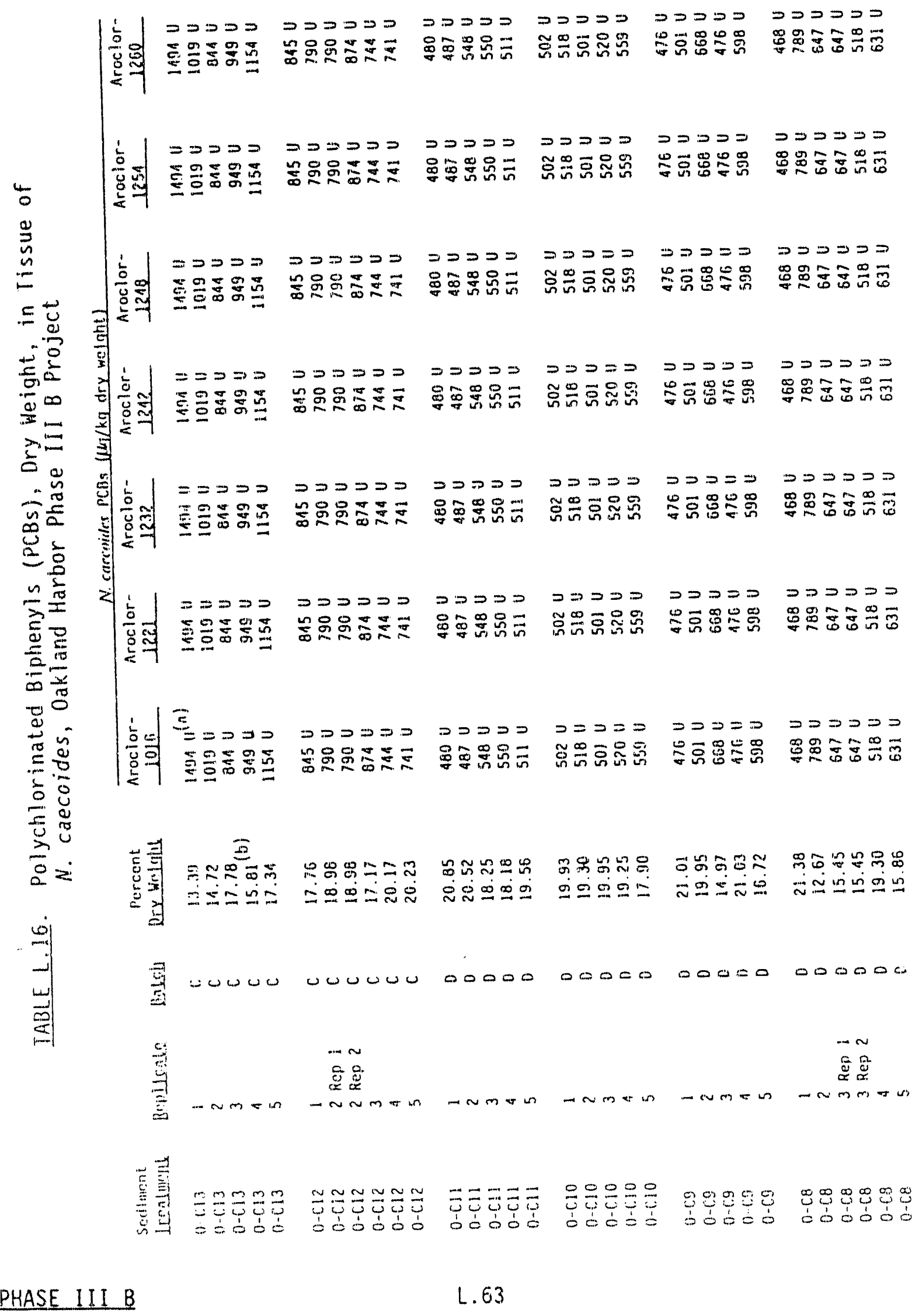




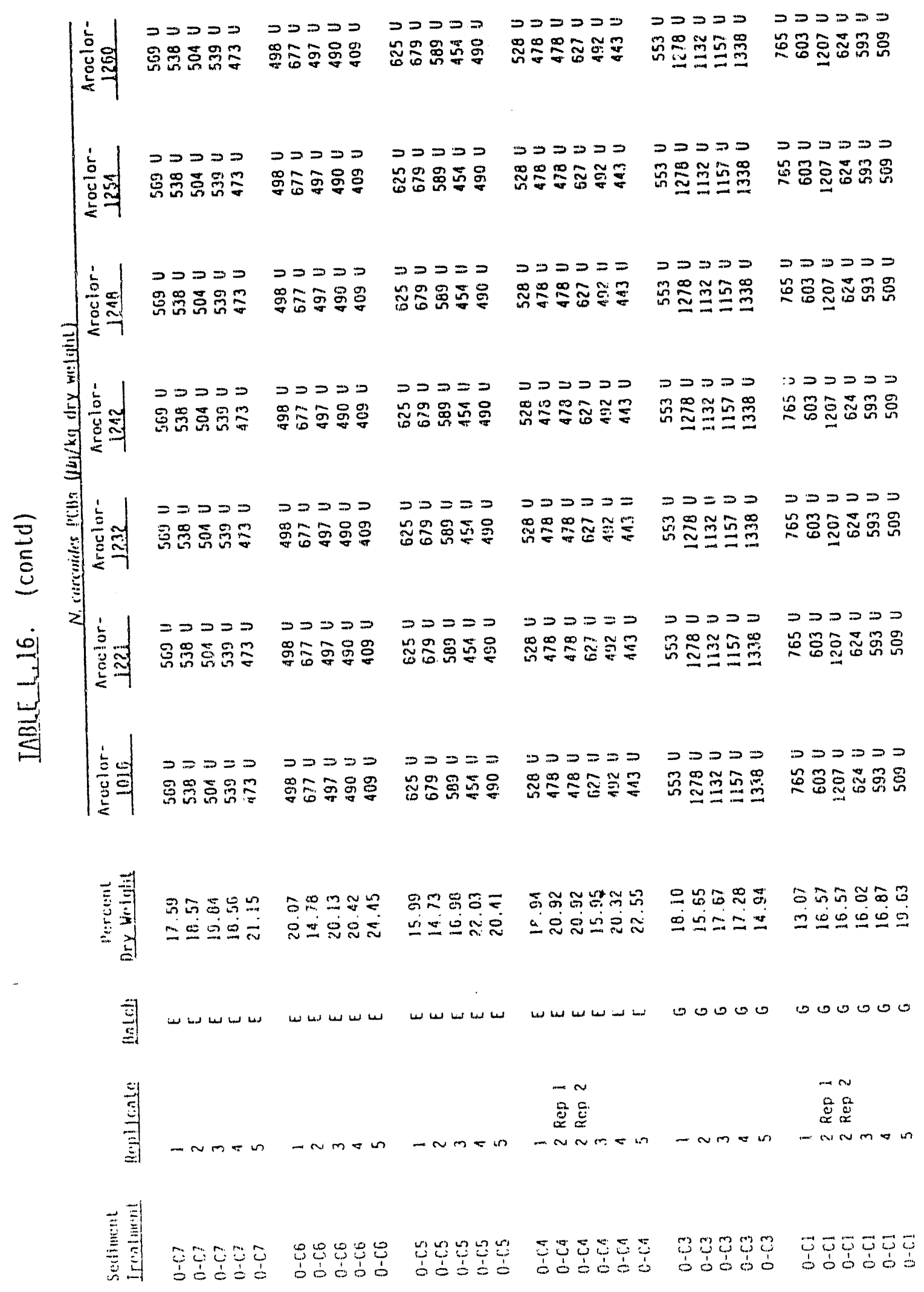




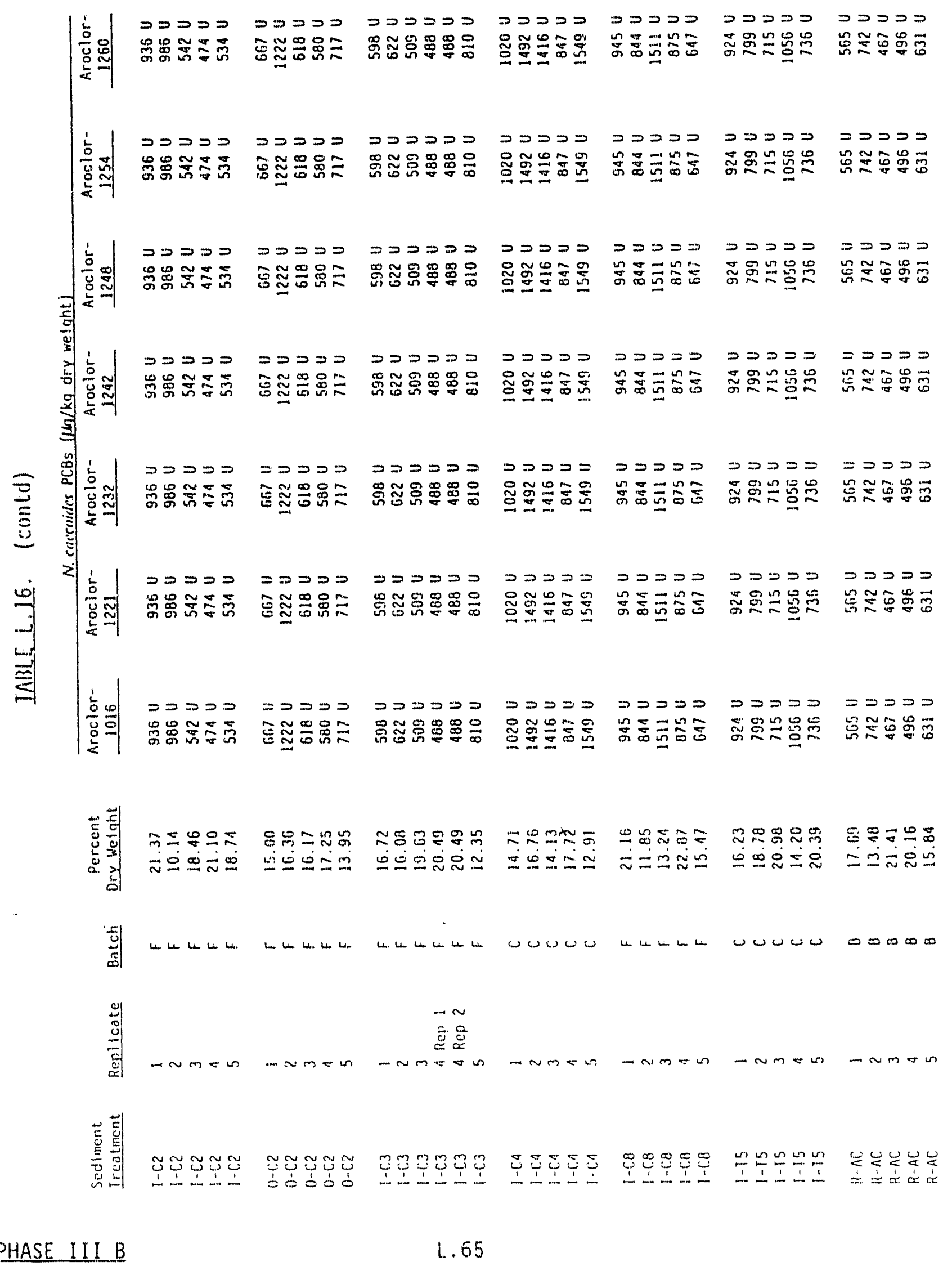




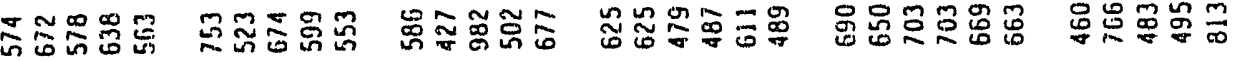

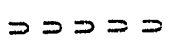

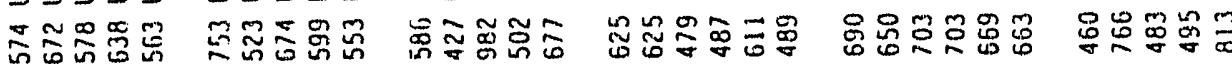

$: \supset \supset \supset \supset$

$\supset \supset \supset \supset \supset \supset$

$==\geq \supset \supset$

$\supset \supset \supset \supset$

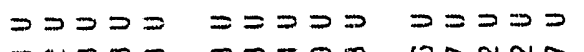

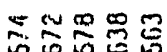

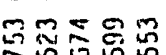

$\supset$ フコン

$==>\supset \supset$

$\supset \supset \supset \supset \supset$

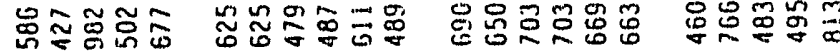

\section{$\supset \supset \supset \supset$}

$>\supset \supset \supset$
$\sim \sim \infty$

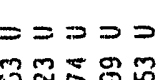

$\vec{P}>\supset \supset$

$>>>>P=$

$\supset=\supset \supset \supset \supset$

$\supset \supset \supset \supset$

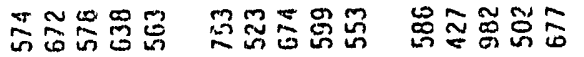

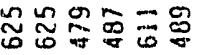

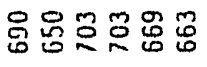

品怘带岸产

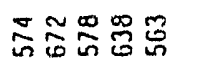

$s>3>0$

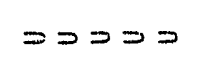

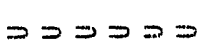

$\supset=\supset \supset \supset \supset$

$\supset \supset \supset \supset$

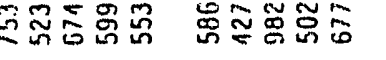

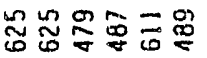

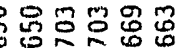

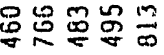

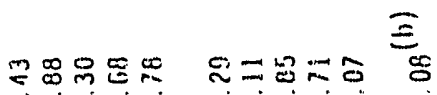

$\therefore=2$ in

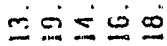

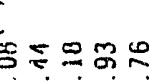

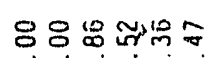

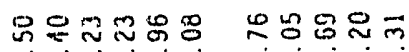

$-x-1$

$\therefore \dot{\sim} \dot{\theta} \dot{0}$

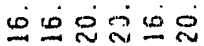

$\leq \dot{\Delta} \leq \dot{0}$

元要远只

苟

$<<<<$

$<<<<$

$\infty=\infty \infty$

$\infty \infty \infty \infty=\infty$

$<<<<<<$

$\infty \omega$

嵌

$\rightarrow n m+m \quad-n m \in m \quad-n m \in b$

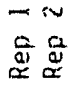

$=0$

- - vmen - oumbu

$\rightarrow \sim m \leqslant m$

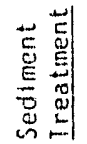

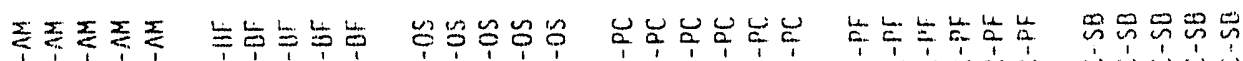

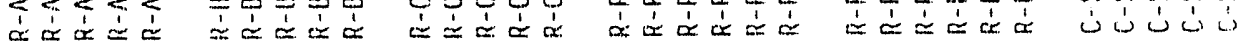




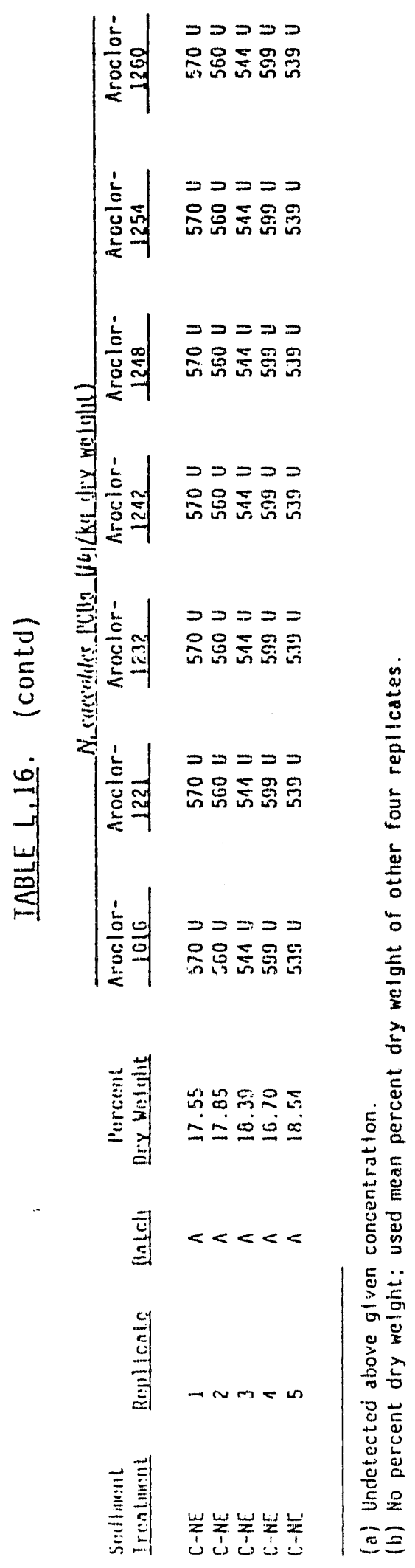

PHASE III B 


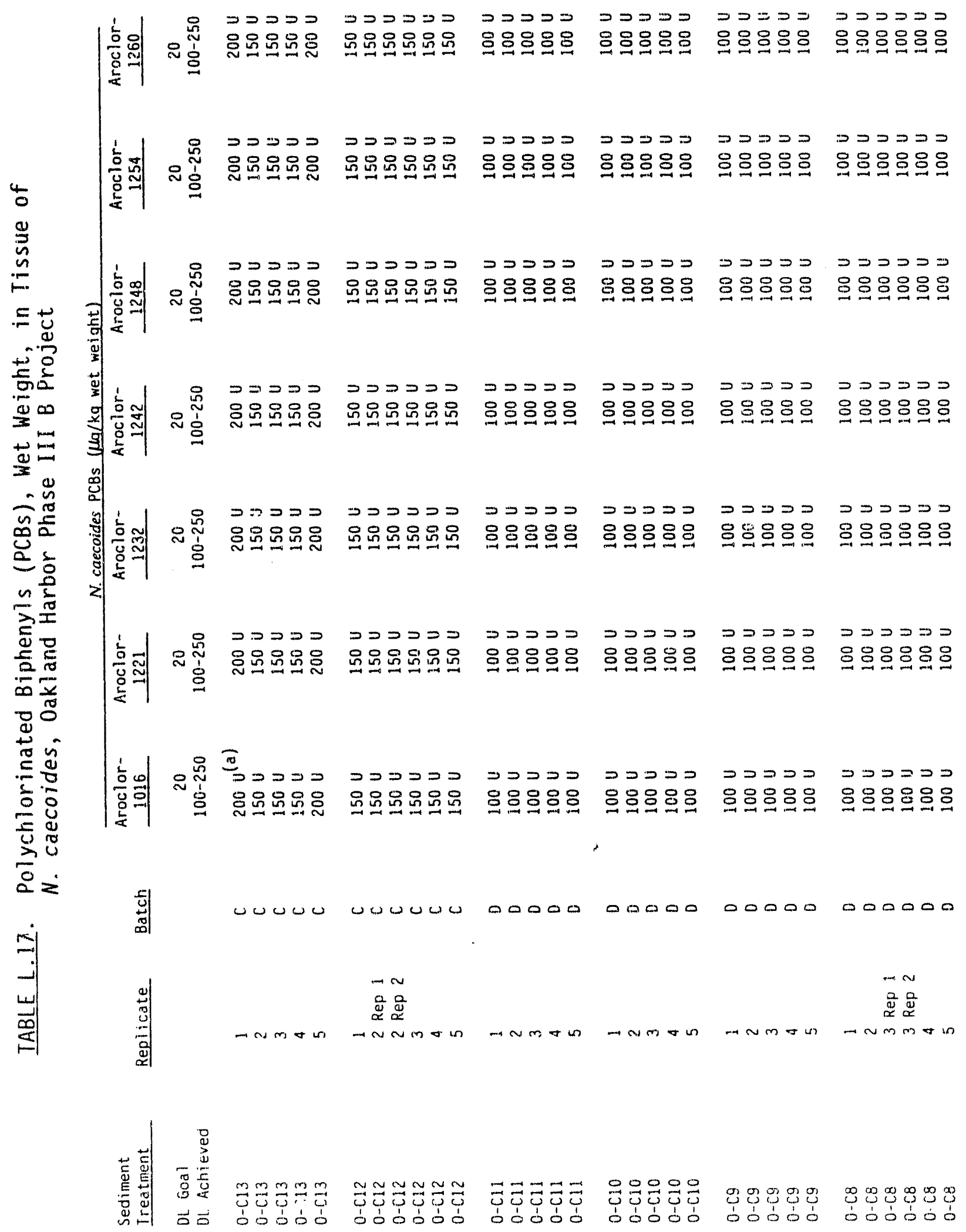




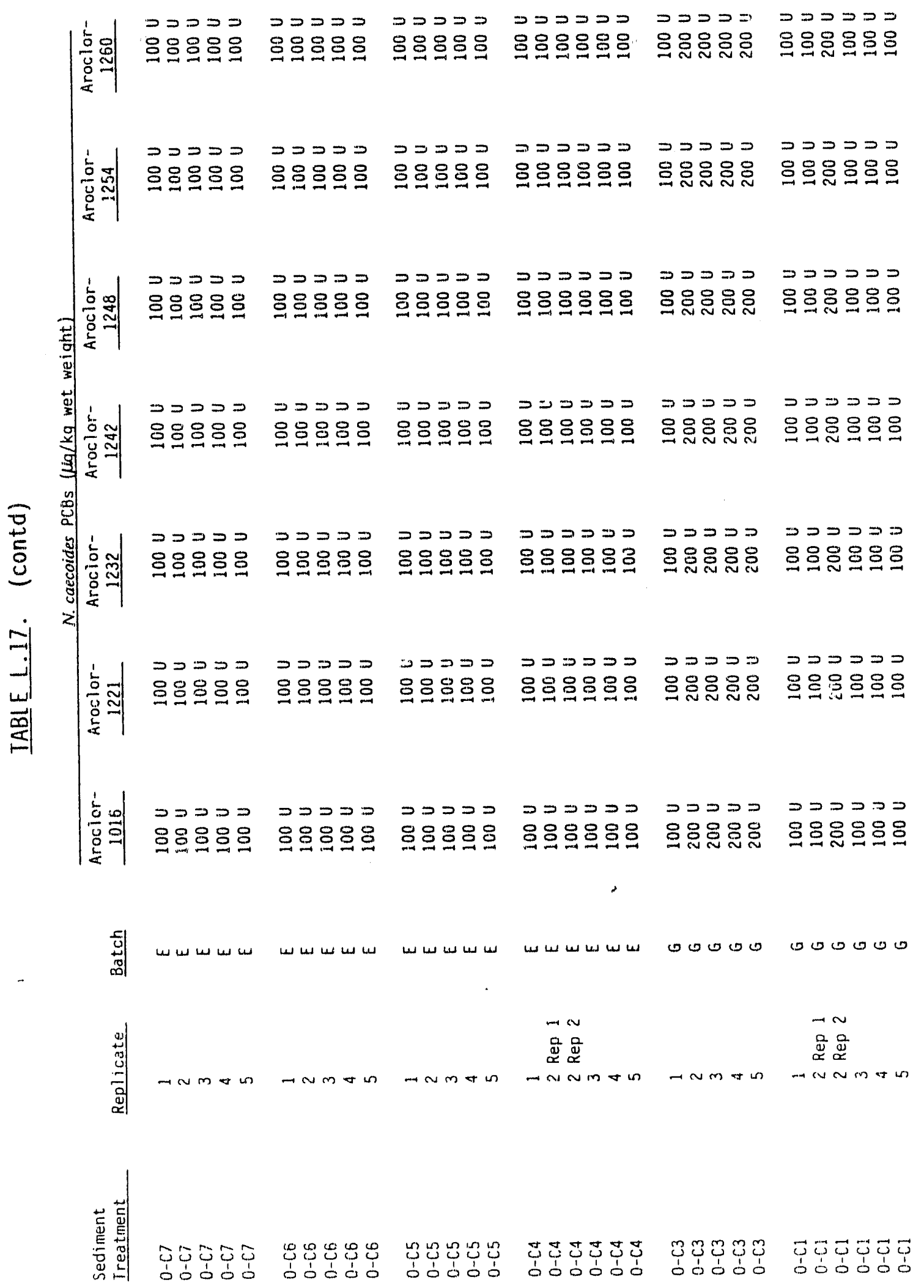




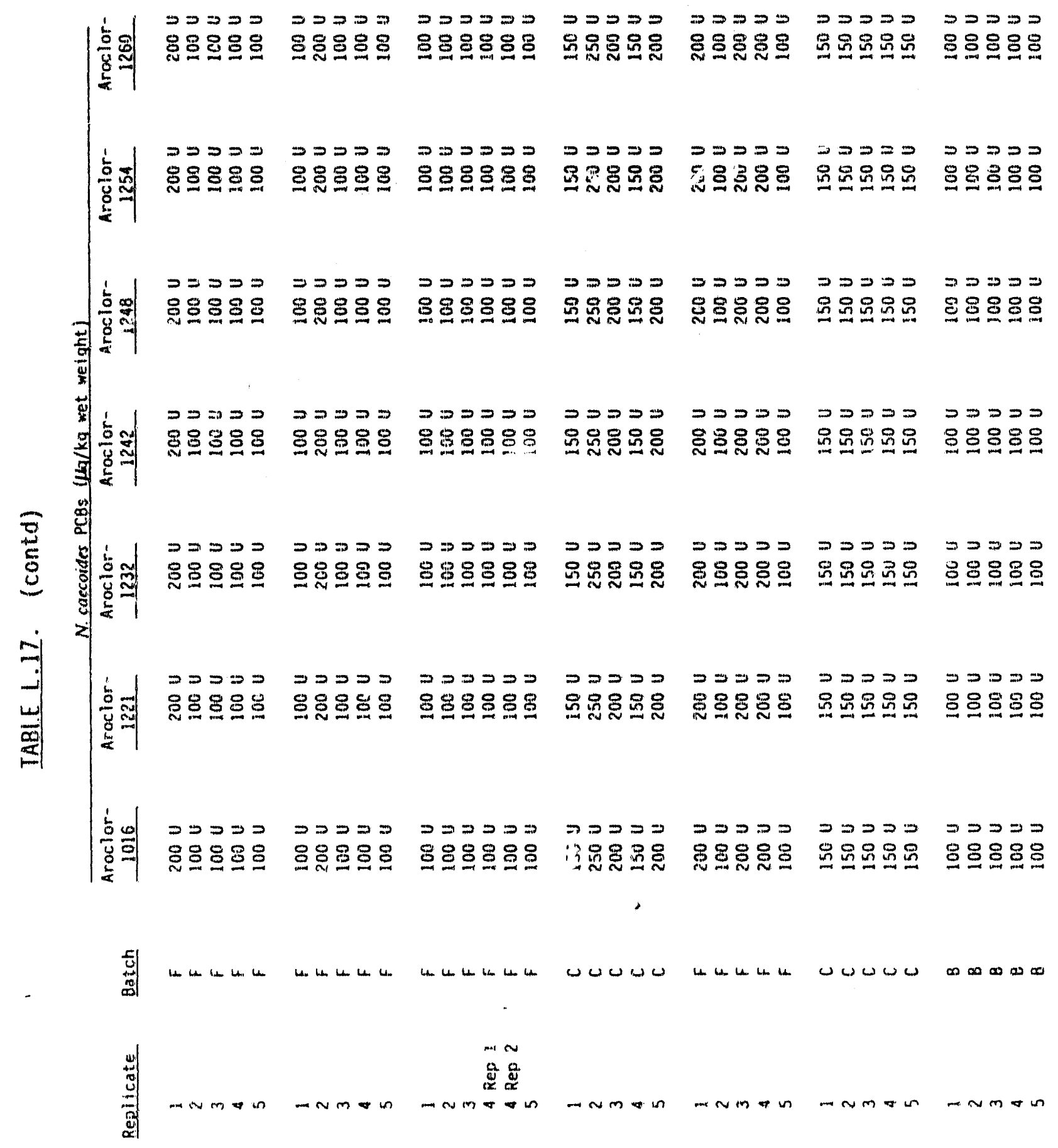

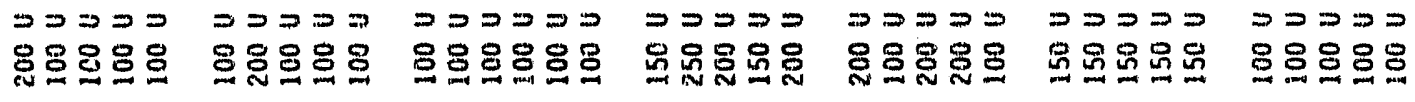

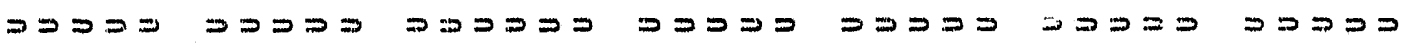

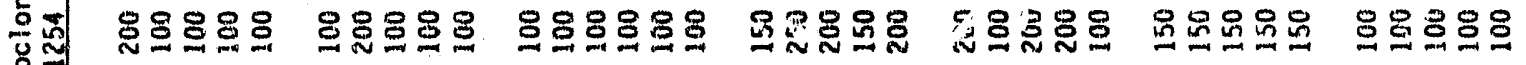

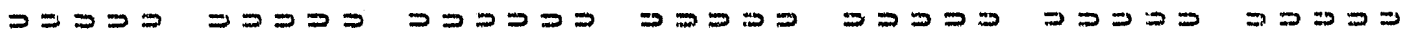

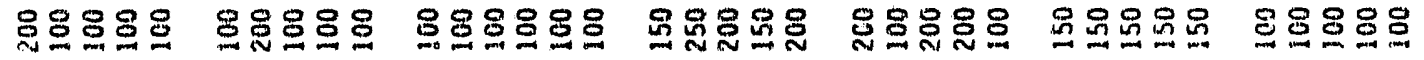

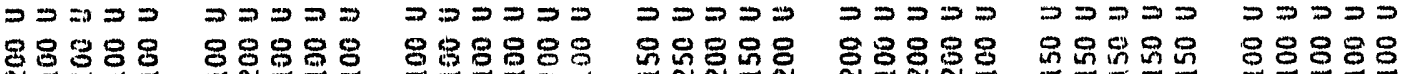

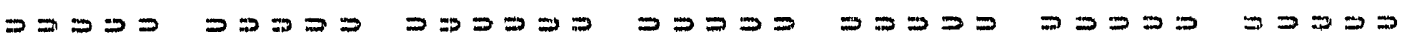

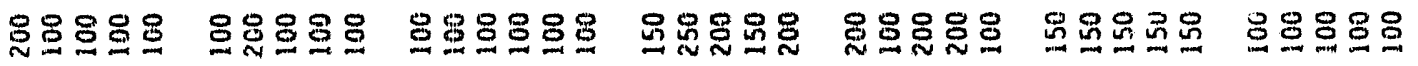

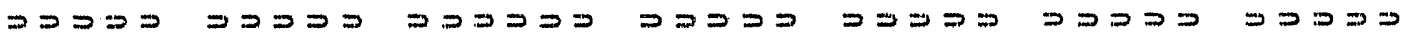

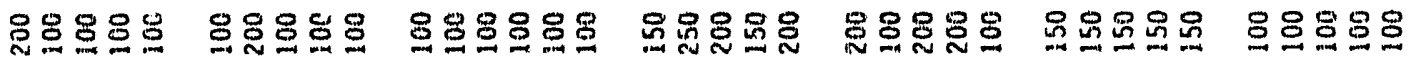

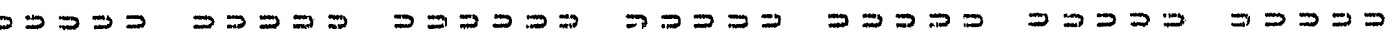

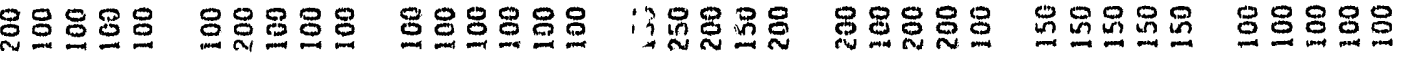




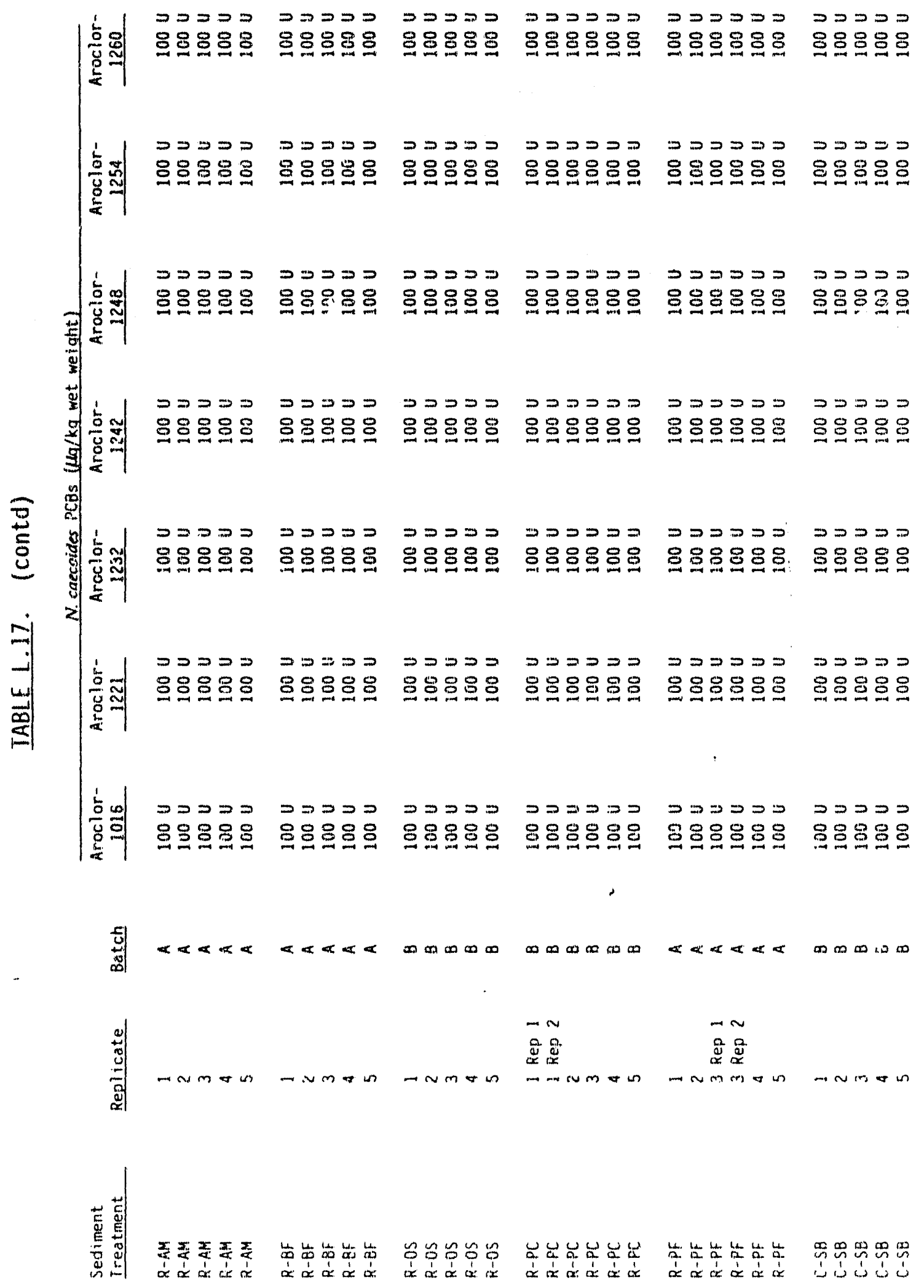




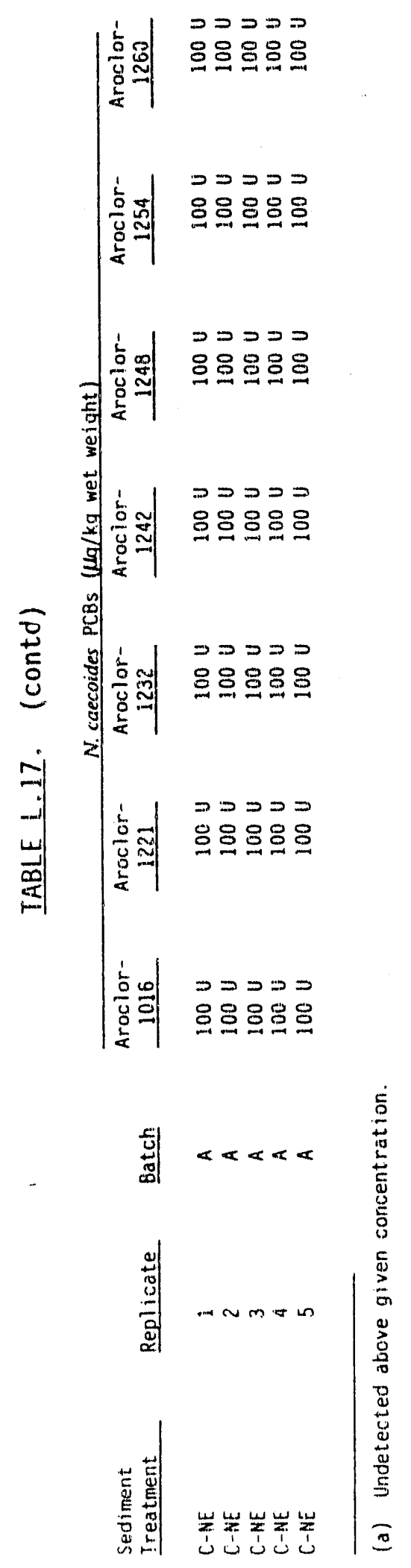

PHASE III $\mathrm{B}$ 


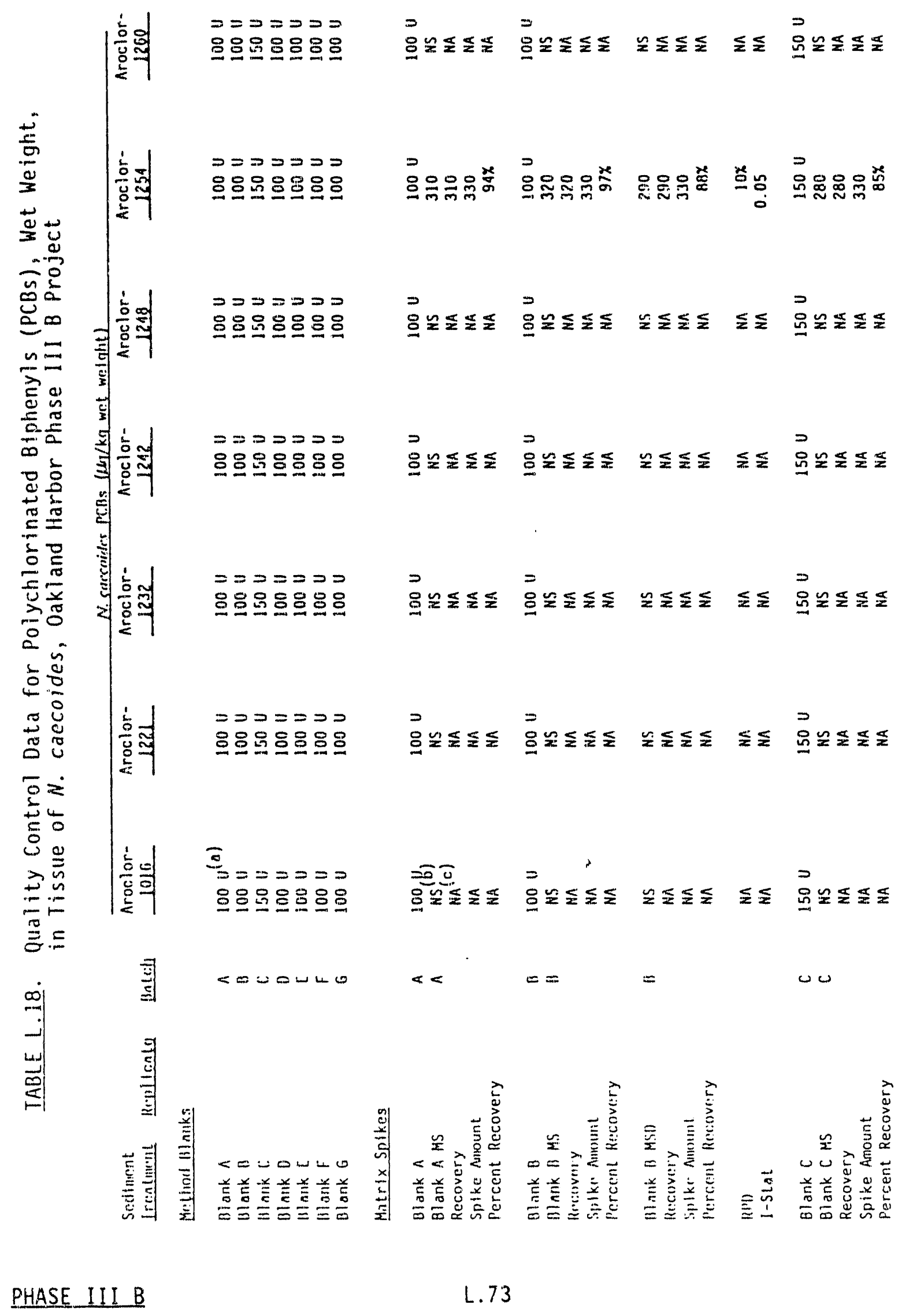


噒

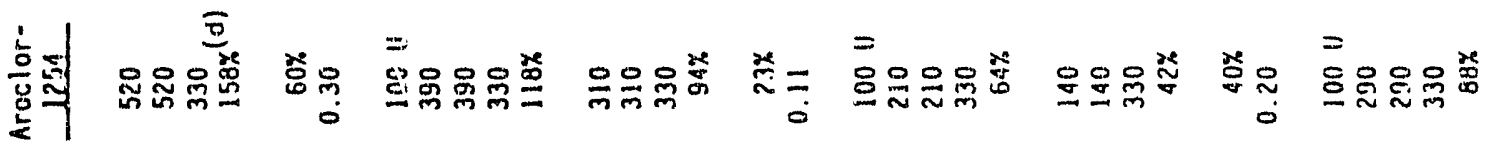

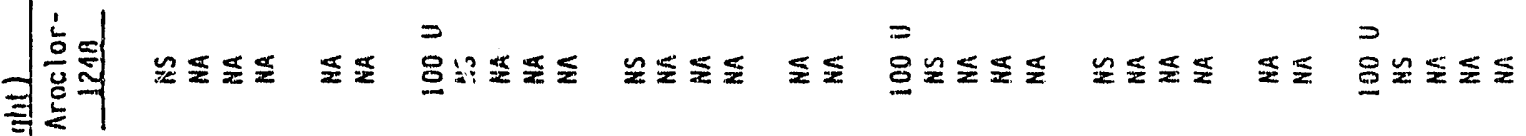
촐

至

朝期

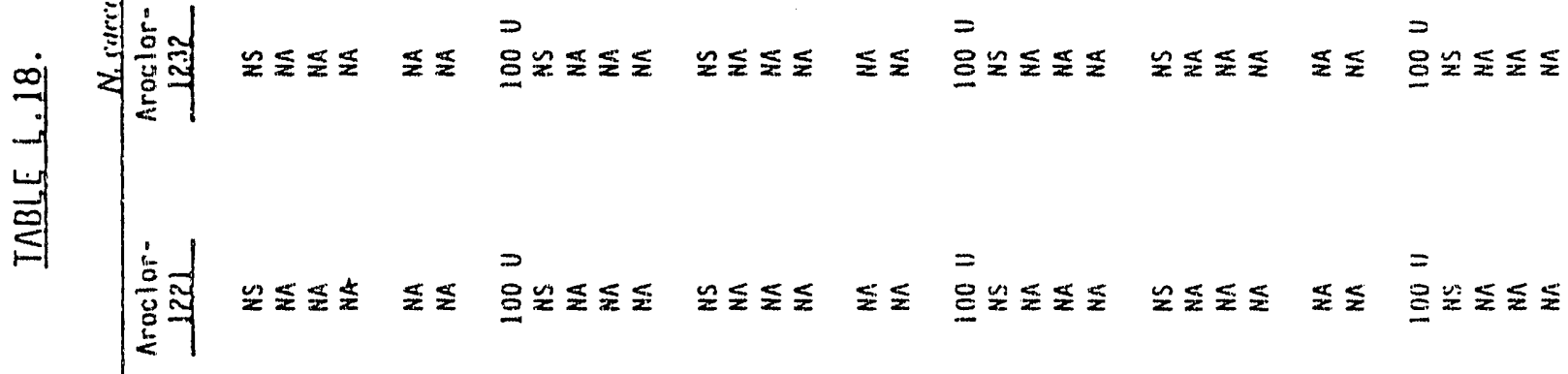

勘

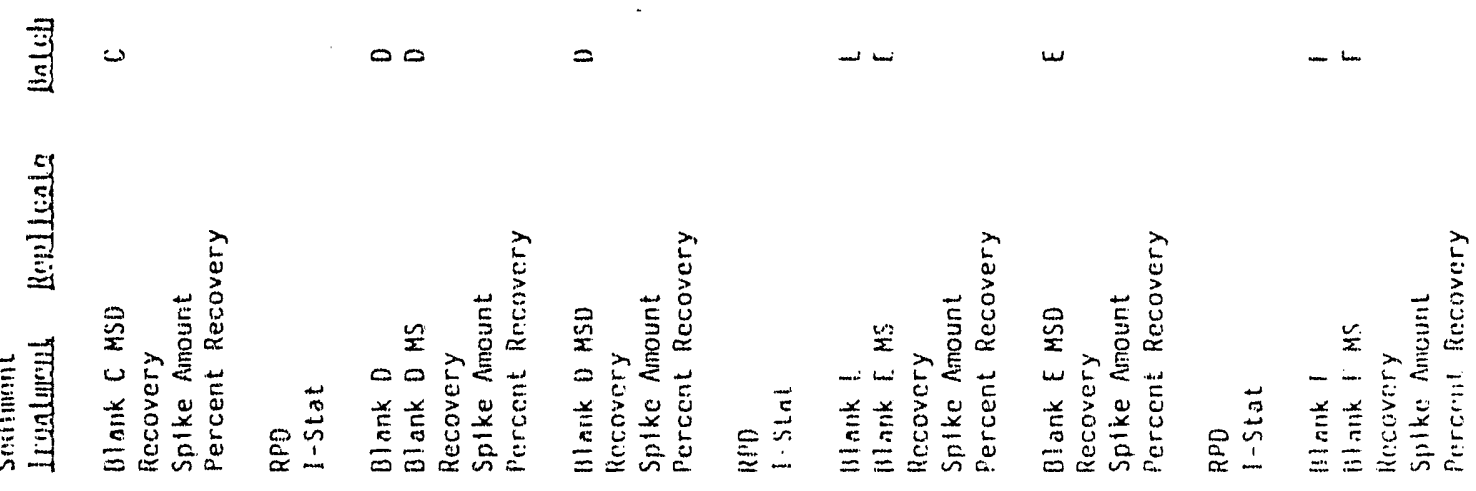

PHASE III B

L. 74 


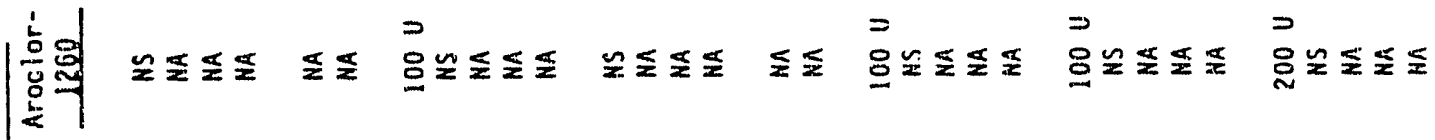

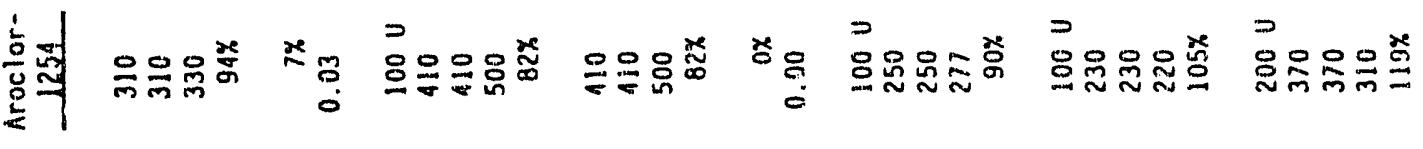

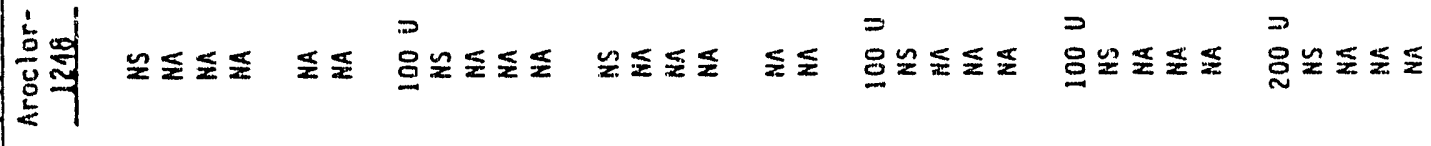

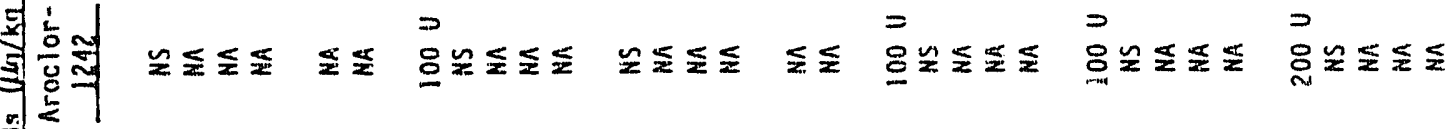

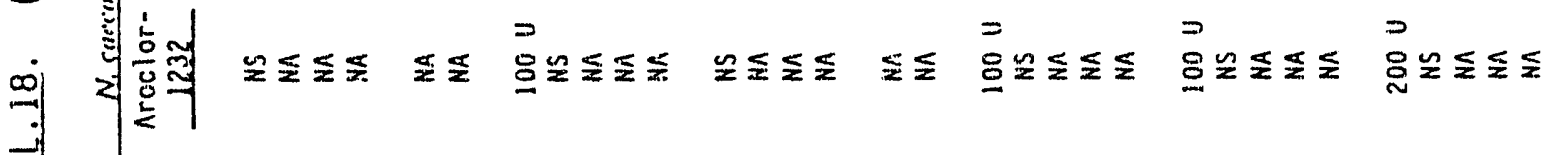

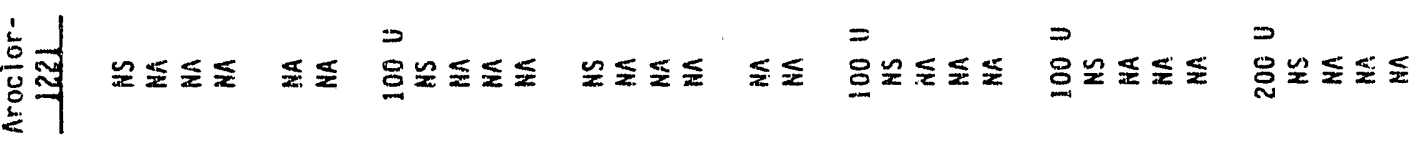

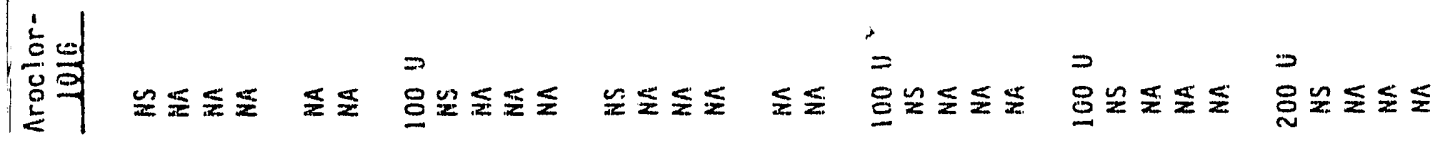

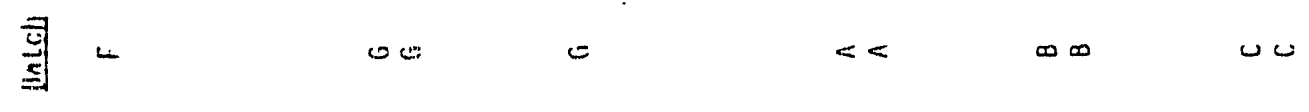

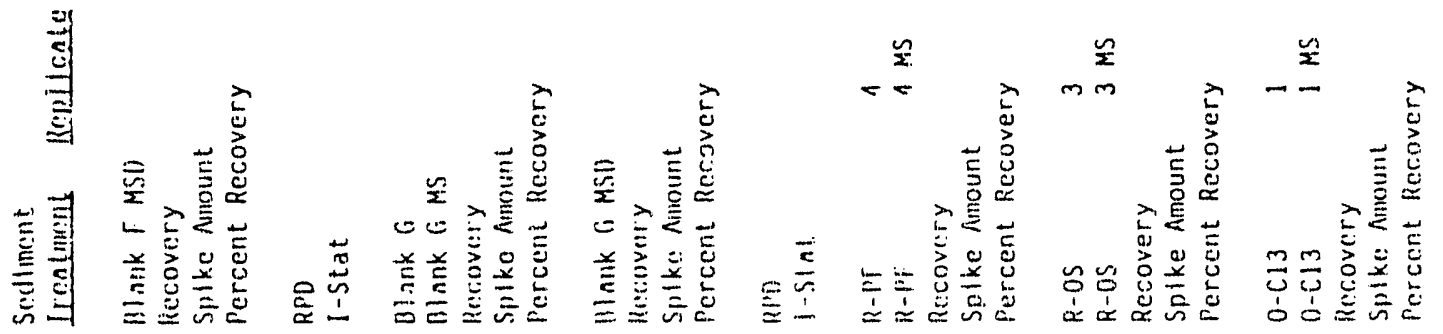




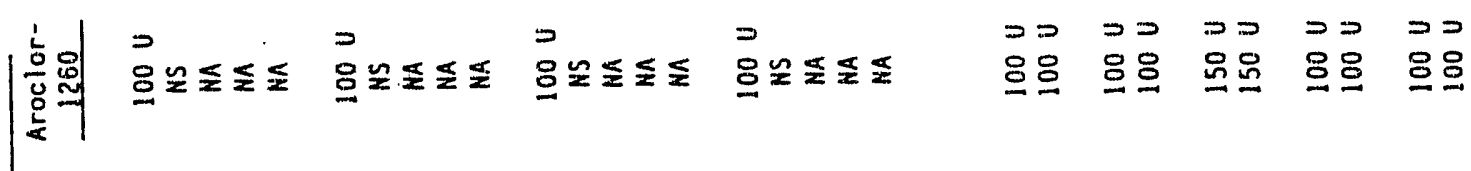

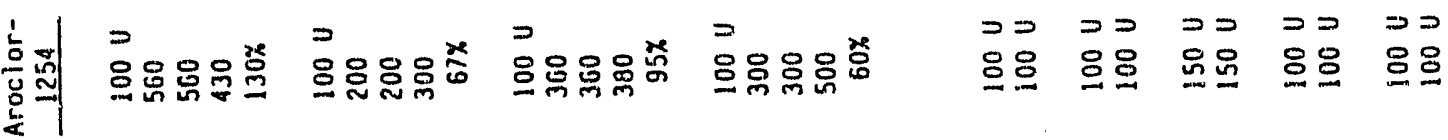

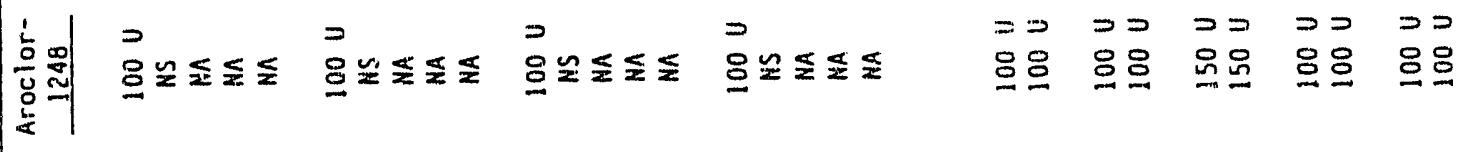

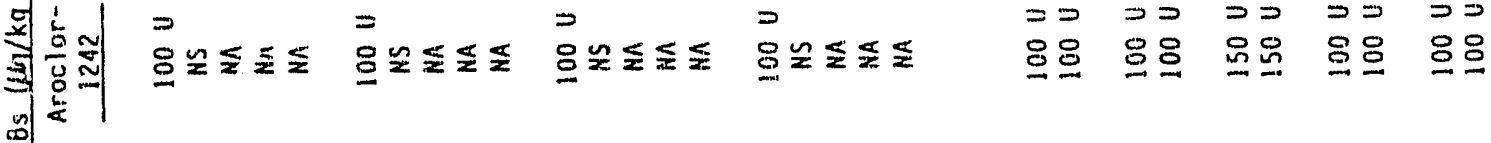

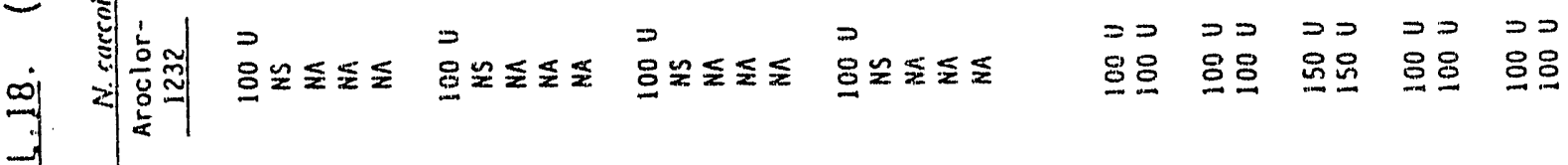

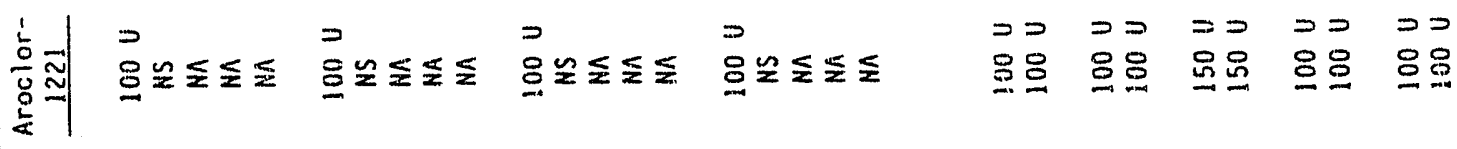

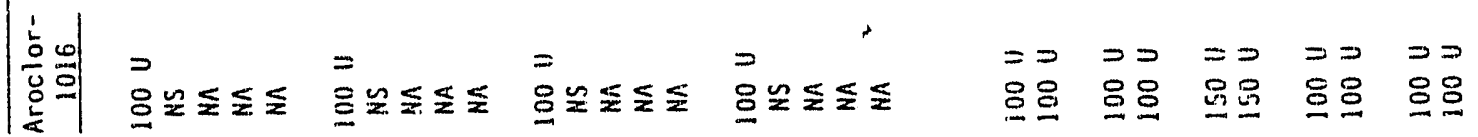
吉 00 W L

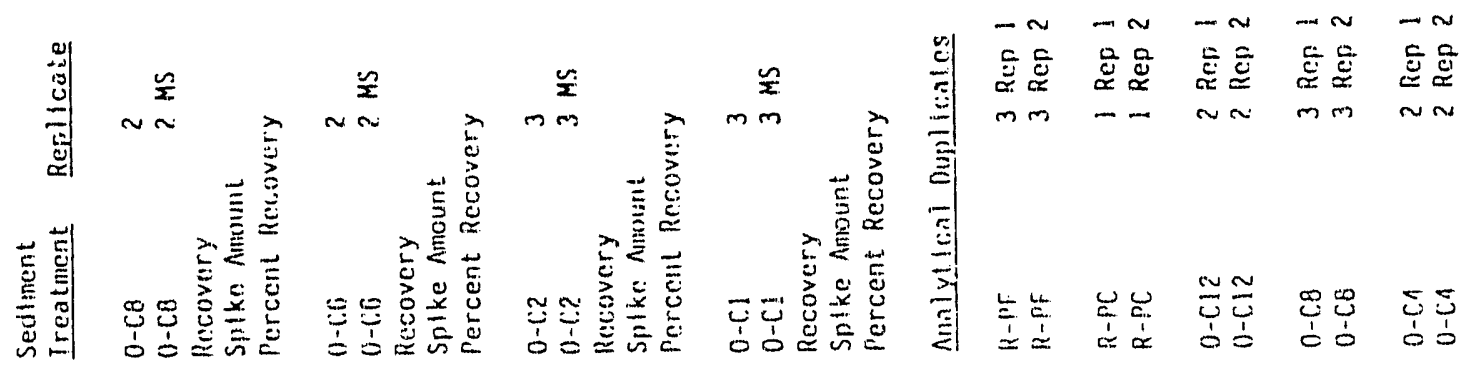




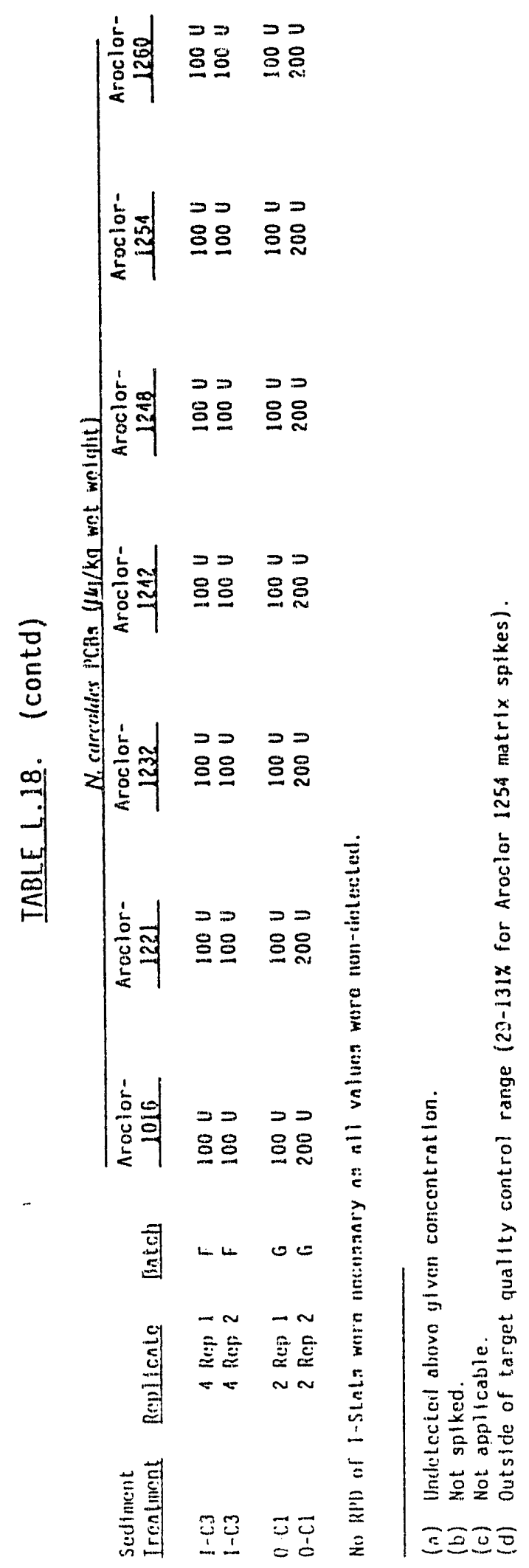

PHASE III $B$ 
TABLE L.19. Surrogate Percent Recoveries for Polychlorinated Biphenyls (PCBs) and Chlorinated Pesticides in Tissue of N. caecoides, Dakland Harbor Phase III B Project

\begin{tabular}{|c|c|c|c|}
\hline $\begin{array}{l}\text { Sediment } \\
\text { Treatment }\end{array}$ & Replicate & Batch & $\begin{array}{l}\text { Dibutyl- } \\
\text { chlorendate } \\
\text { Surrogate } \\
\text { Recovery } \% \\
\end{array}$ \\
\hline $\begin{array}{l}0-C 13 \\
0-C 13 \\
0-C 13 \\
0-C 13 \\
0-C 13\end{array}$ & $\begin{array}{l}1 \\
? \\
3 \\
4 \\
5\end{array}$ & $\begin{array}{l}c \\
c \\
c \\
c \\
c\end{array}$ & $\begin{array}{l}\text { NSA (a) } \\
\text { NSA } \\
\text { NSA } \\
\text { NSA } \\
\text { NSA }\end{array}$ \\
\hline $\begin{array}{l}0-C 12 \\
0-i 12 \\
0-C 12 \\
0-C 12 \\
0-C 12 \\
0-C 12\end{array}$ & $\begin{array}{lll}1 & & \\
2 & \text { Rep } & 1 \\
2 & \text { Rep } 2 \\
3 & & \\
4 & & \\
5 & & \end{array}$ & $\begin{array}{l}c \\
c \\
c \\
c \\
c \\
c\end{array}$ & $\begin{array}{l}\text { NSA } \\
\text { NSA } \\
\text { NSA } \\
\text { NSA } \\
\text { NSA } \\
\text { NSA }\end{array}$ \\
\hline $\begin{array}{l}0-C 11 \\
0-C 11 \\
0-C 11 \\
0-C 11 \\
0-C 11\end{array}$ & $\begin{array}{l}1 \\
2 \\
3 \\
4 \\
5\end{array}$ & $\begin{array}{l}D \\
D \\
D \\
D \\
D\end{array}$ & $\begin{array}{l}59 \\
50 \\
66 \\
59 \\
72\end{array}$ \\
\hline $\begin{array}{l}0-C 10 \\
0-C 10 \\
0-C 10 \\
0-C 10 \\
0-C 10\end{array}$ & $\begin{array}{l}1 \\
2 \\
3 \\
4 \\
5\end{array}$ & $\begin{array}{l}D \\
D \\
D \\
D \\
D\end{array}$ & $\begin{array}{l}77 \\
51 \\
52 \\
62 \\
44\end{array}$ \\
\hline $\begin{array}{l}0-C 9 \\
0-C 9 \\
0-C 9 \\
0-C 9 \\
0-C 9\end{array}$ & $\begin{array}{l}1 \\
2 \\
3 \\
4 \\
5\end{array}$ & $\begin{array}{l}D \\
D \\
D \\
D \\
D\end{array}$ & $\begin{array}{l}70 \\
61 \\
65 \\
75 \\
90\end{array}$ \\
\hline $\begin{array}{l}0-C 8 \\
0-C 8 \\
0-C 8 \\
0-C 8 \\
0-C 8 \\
0-C 8\end{array}$ & $\begin{array}{lll}1 & & \\
2 & & \\
3 & \operatorname{Rep} 1 \\
3 & \operatorname{Rep} 2 \\
4 & & \\
5 & & \end{array}$ & $\begin{array}{l}D \\
D \\
D \\
D \\
D \\
D\end{array}$ & $\begin{array}{l}70 \\
50 \\
46 \\
57 \\
78 \\
62\end{array}$ \\
\hline $\begin{array}{l}0-C 7 \\
0-C 7 \\
0-C 7 \\
0-C 7 \\
0-C 7\end{array}$ & $\begin{array}{l}1 \\
2 \\
3 \\
4 \\
5\end{array}$ & $\begin{array}{l}E \\
E \\
E \\
E \\
E\end{array}$ & $\begin{array}{r}98 \\
102 \\
94 \\
104 \\
108\end{array}$ \\
\hline & & L. 78 & \\
\hline
\end{tabular}


TABLE L.19. (contd)

\begin{tabular}{|c|c|c|c|}
\hline $\begin{array}{l}\text { Sediment } \\
\text { Ireatment }\end{array}$ & Replicate & Batch & $\begin{array}{c}\text { Dibutyl - } \\
\text { chlorendate } \\
\text { Surrogate } \\
\text { Recovery \% } \\
\end{array}$ \\
\hline $\begin{array}{l}0-c 6 \\
0-c 6 \\
0-c 6 \\
0-c 6 \\
0-c 6\end{array}$ & $\begin{array}{l}1 \\
2 \\
3 \\
4 \\
5\end{array}$ & $\begin{array}{l}E \\
E \\
E \\
E \\
E\end{array}$ & $\begin{array}{r}99 \\
107 \\
78 \\
89 \\
92\end{array}$ \\
\hline $\begin{array}{l}0-C 5 \\
0-C 5 \\
0-C 5 \\
0-C 5 \\
0-C 5\end{array}$ & $\begin{array}{l}1 \\
2 \\
3 \\
4 \\
5\end{array}$ & $\begin{array}{l}E \\
E \\
E \\
E \\
E\end{array}$ & $\begin{array}{r}116 \\
129 \\
89 \\
79 \\
109\end{array}$ \\
\hline $\begin{array}{l}0-C 4 \\
0-C 4 \\
0-C 4 \\
0-C 4 \\
0-C 4 \\
0-C 4\end{array}$ & $\begin{array}{lll}1 & & \\
2 & R e p & 1 \\
2 & R e p & 2 \\
3 & & \\
4 & & \\
5 & & \end{array}$ & $\begin{array}{l}E \\
E \\
E \\
E \\
E \\
E\end{array}$ & $\begin{array}{r}123 \\
113 \\
107 \\
74 \\
74 \\
61\end{array}$ \\
\hline $\begin{array}{l}0-C 3 \\
0-C 3 \\
0-C 3 \\
0-C 3 \\
0-C 3\end{array}$ & $\begin{array}{l}1 \\
2 \\
3 \\
4 \\
5\end{array}$ & $\begin{array}{l}G \\
G \\
G \\
G \\
G\end{array}$ & $\begin{array}{r}83 \\
78 \\
96 \\
74 \\
100\end{array}$ \\
\hline $\begin{array}{l}0-C 1 \\
0-C 1 \\
0-C 1 \\
0-C 1 \\
0-C 1 \\
0-C 1\end{array}$ & $\begin{array}{lll}1 & & \\
2 & \text { Rep } & 1 \\
2 & \operatorname{Rep} & 2 \\
3 & & \\
4 & & \\
5 & & \end{array}$ & $\begin{array}{l}G \\
G \\
G \\
G \\
G \\
G\end{array}$ & $\begin{array}{r}84 \\
89 \\
106 \\
95 \\
94 \\
68\end{array}$ \\
\hline $\begin{array}{l}I-C 2 \\
I-C 2 \\
I-C 2 \\
I-C 2 \\
I-C 2\end{array}$ & $\begin{array}{l}1 \\
2 \\
3 \\
4 \\
5\end{array}$ & $\begin{array}{l}F \\
F \\
F \\
F \\
F\end{array}$ & $\begin{array}{r}77 \\
34 \\
52 \\
65 \\
106\end{array}$ \\
\hline $\begin{array}{l}0-C 2 \\
0-C 2 \\
0-C 2 \\
0-C 2 \\
0-C 2\end{array}$ & $\begin{array}{l}1 \\
2 \\
3 \\
4 \\
5\end{array}$ & $\begin{array}{l}F \\
F \\
F \\
F \\
F\end{array}$ & $\begin{array}{l}75 \\
81 \\
78 \\
87 \\
86\end{array}$ \\
\hline
\end{tabular}


IABLE L.19. (contd)

\begin{tabular}{|c|c|c|c|}
\hline $\begin{array}{l}\text { Sediment } \\
\text { Ireatment }\end{array}$ & Replicate & Batch & $\begin{array}{l}\text { Dibutyl- } \\
\text { chlorendate } \\
\text { Surrogate } \\
\text { Recovery } \% \\
\end{array}$ \\
\hline $\begin{array}{l}I-C 3 \\
I-C 3 \\
I-C 3 \\
I-C 3 \\
I-C 3 \\
I-C 3\end{array}$ & 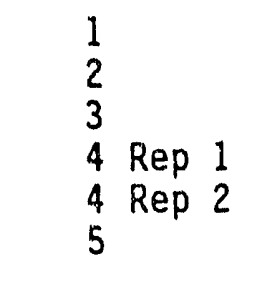 & $\begin{array}{l}F \\
F \\
F \\
F \\
F \\
F\end{array}$ & $\begin{array}{r}102 \\
90 \\
63 \\
80 \\
91 \\
81\end{array}$ \\
\hline $\begin{array}{l}I-C 4 \\
I-C 4 \\
I-C 4 \\
I-C 4 \\
I-C 4\end{array}$ & $\begin{array}{l}1 \\
2 \\
3 \\
4 \\
5\end{array}$ & $\begin{array}{l}c \\
c \\
c \\
c \\
c\end{array}$ & $\begin{array}{l}\text { NSA } \\
\text { NSA } \\
\text { NSA } \\
\text { NSA } \\
\text { NSA }\end{array}$ \\
\hline $\begin{array}{l}I-C 8 \\
I-C 8 \\
I-C 8 \\
I-C 8 \\
I-C 8\end{array}$ & $\begin{array}{l}1 \\
2 \\
3 \\
4 \\
5\end{array}$ & $\begin{array}{l}F \\
F \\
F \\
F \\
F\end{array}$ & $\begin{array}{l}98 \\
68 \\
76 \\
89 \\
78\end{array}$ \\
\hline $\begin{array}{l}I-T 5 \\
I-T 5 \\
I-T 5 \\
I-T 5 \\
I-T 5\end{array}$ & $\begin{array}{l}1 \\
2 \\
3 \\
4 \\
5\end{array}$ & $\begin{array}{l}c \\
c \\
c \\
c \\
c\end{array}$ & $\begin{array}{l}\text { NSA } \\
\text { NSA } \\
\text { NSA } \\
\text { NSA } \\
\text { NSA }\end{array}$ \\
\hline $\begin{array}{l}R-A C \\
R-A C \\
R-A C \\
R-A C \\
R-A C\end{array}$ & $\begin{array}{l}1 \\
2 \\
3 \\
4 \\
5\end{array}$ & $\begin{array}{l}B \\
B \\
B \\
B \\
B\end{array}$ & $\begin{array}{l}71 \\
68 \\
68 \\
56 \\
77\end{array}$ \\
\hline $\begin{array}{l}\text { R-AM } \\
\text { R-AM } \\
\text { R-AM } \\
\text { R-AM } \\
\text { R-AM }\end{array}$ & $\begin{array}{l}1 \\
2 \\
3 \\
4 \\
5\end{array}$ & $\begin{array}{l}A \\
A \\
A \\
A \\
A\end{array}$ & $\begin{array}{l}69 \\
72 \\
86 \\
84 \\
81\end{array}$ \\
\hline $\begin{array}{l}R-B F \\
R-B F \\
R-B F \\
R-B F \\
R-B F\end{array}$ & $\begin{array}{l}1 \\
2 \\
3 \\
4 \\
5\end{array}$ & $\begin{array}{l}A \\
A \\
A \\
A \\
A\end{array}$ & $\begin{array}{l}77 \\
67 \\
58 \\
66 \\
68\end{array}$ \\
\hline
\end{tabular}


TABLE L.19. (contd)

\begin{tabular}{|c|c|c|c|}
\hline $\begin{array}{l}\text { Sediment } \\
\text { Ireatment }\end{array}$ & Replicate & Batch & $\begin{array}{l}\text { Dibutyl- } \\
\text { chlorendate } \\
\text { Surrogate } \\
\text { Recovery } \% \\
\end{array}$ \\
\hline $\begin{array}{l}R-O S \\
R-O S \\
R-O S \\
R-O S \\
R-O S\end{array}$ & $\begin{array}{l}1 \\
2 \\
3 \\
4 \\
5\end{array}$ & $\begin{array}{l}B \\
B \\
B \\
B \\
B\end{array}$ & $\begin{array}{r}81 \\
58 \\
77 \\
90 \\
119\end{array}$ \\
\hline $\begin{array}{l}R-P C \\
R-P C \\
R-P C \\
R-P C \\
R-P C \\
R-P C\end{array}$ & $\begin{array}{lll}1 & \text { Rep } 1 \\
1 & \operatorname{Rep} 2 \\
2 & & \\
3 & & \\
4 & & \\
5 & & \end{array}$ & $\begin{array}{l}B \\
B \\
B \\
B \\
B \\
B\end{array}$ & $\begin{array}{r}121 \\
76 \\
154 \\
66 \\
82 \\
90\end{array}$ \\
\hline $\begin{array}{l}R-P F \\
R-P F \\
R-P F \\
R-P F \\
R-P F \\
R-P F\end{array}$ & $\begin{array}{lll}1 & & \\
2 & & \\
3 & \operatorname{Rep} & 1 \\
3 & \operatorname{Rep} & 2 \\
4 & & \\
5 & & \end{array}$ & $\begin{array}{l}A \\
A \\
A \\
A \\
A \\
A\end{array}$ & $\begin{array}{l}70 \\
64 \\
65 \\
56 \\
78 \\
79\end{array}$ \\
\hline $\begin{array}{l}C-S B \\
C-S B \\
C-S B \\
C-S B \\
C-S B\end{array}$ & $\begin{array}{l}1 \\
2 \\
3 \\
4 \\
5\end{array}$ & $\begin{array}{l}B \\
B \\
B \\
B \\
B\end{array}$ & $\begin{array}{l}61 \\
56 \\
84 \\
55 \\
82\end{array}$ \\
\hline $\begin{array}{l}C-N E \\
C-N E \\
C-N E \\
C-N E \\
C-N E\end{array}$ & $\begin{array}{l}1 \\
2 \\
3 \\
4 \\
5\end{array}$ & $\begin{array}{l}A \\
A \\
A \\
A \\
A\end{array}$ & $\begin{array}{l}51 \\
58 \\
64 \\
65 \\
63\end{array}$ \\
\hline
\end{tabular}

(a) No surrogate added: Aroclor 1254 spike solutions inadvertantly added instead of surrogate. 


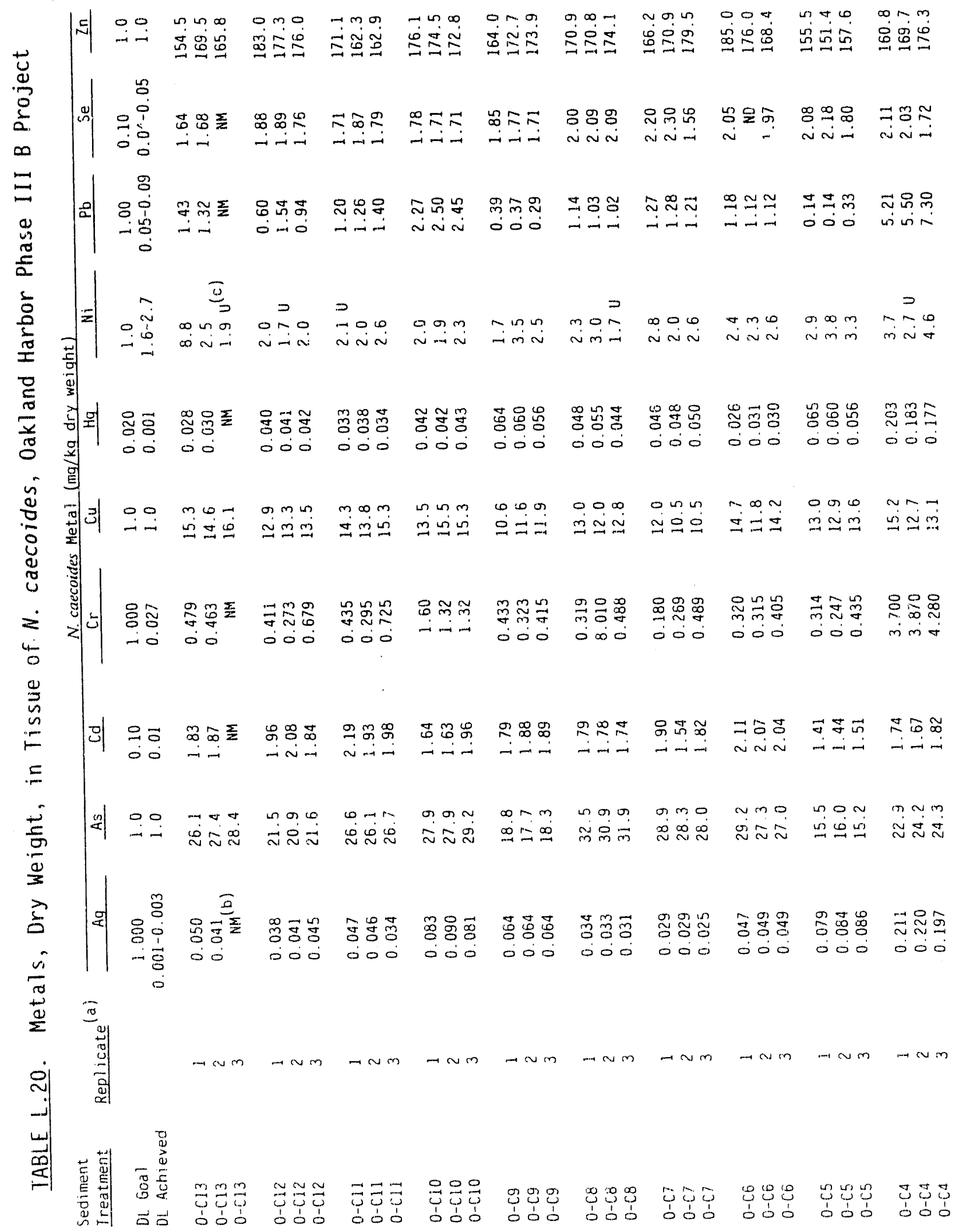

PHASE III B 


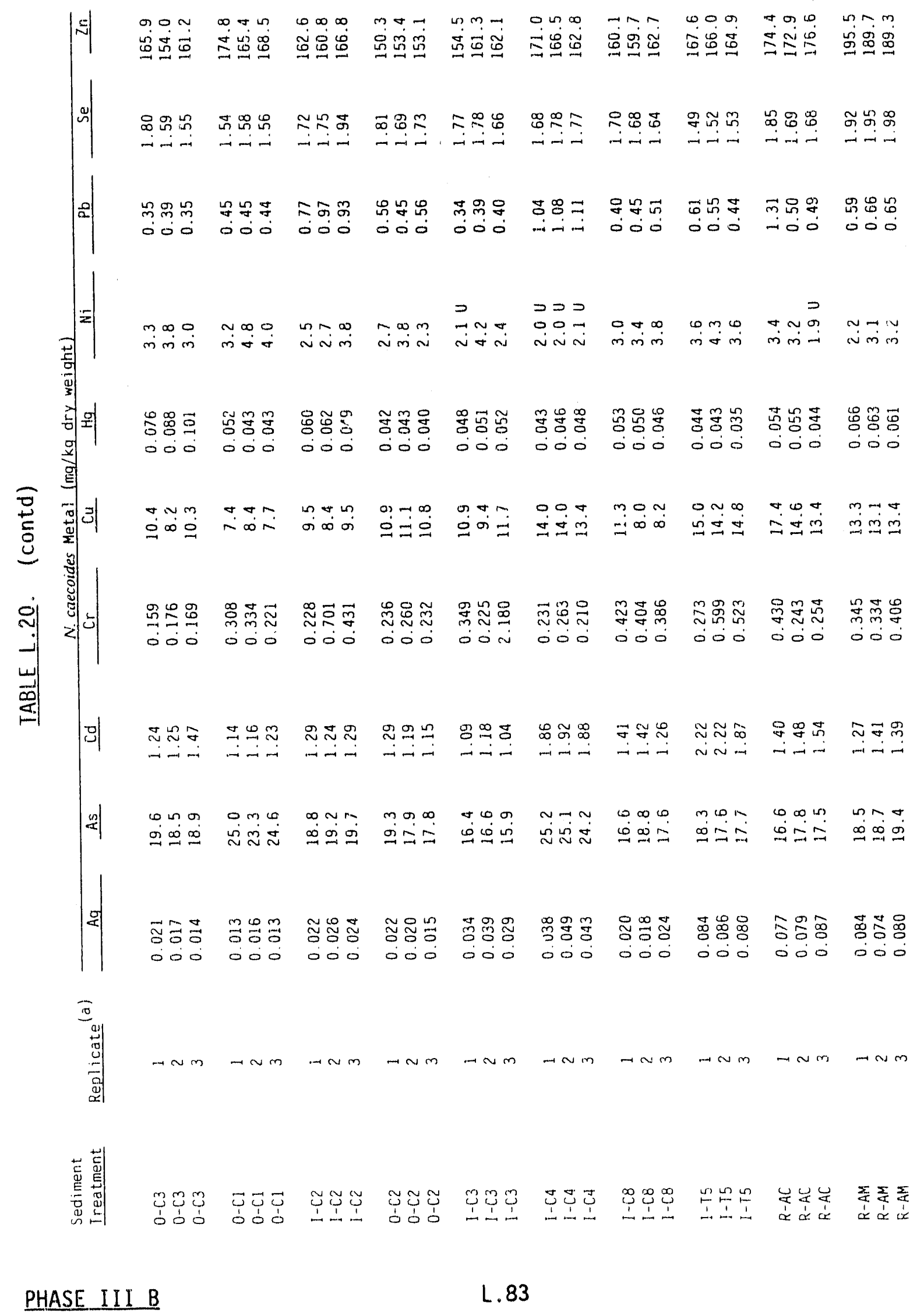




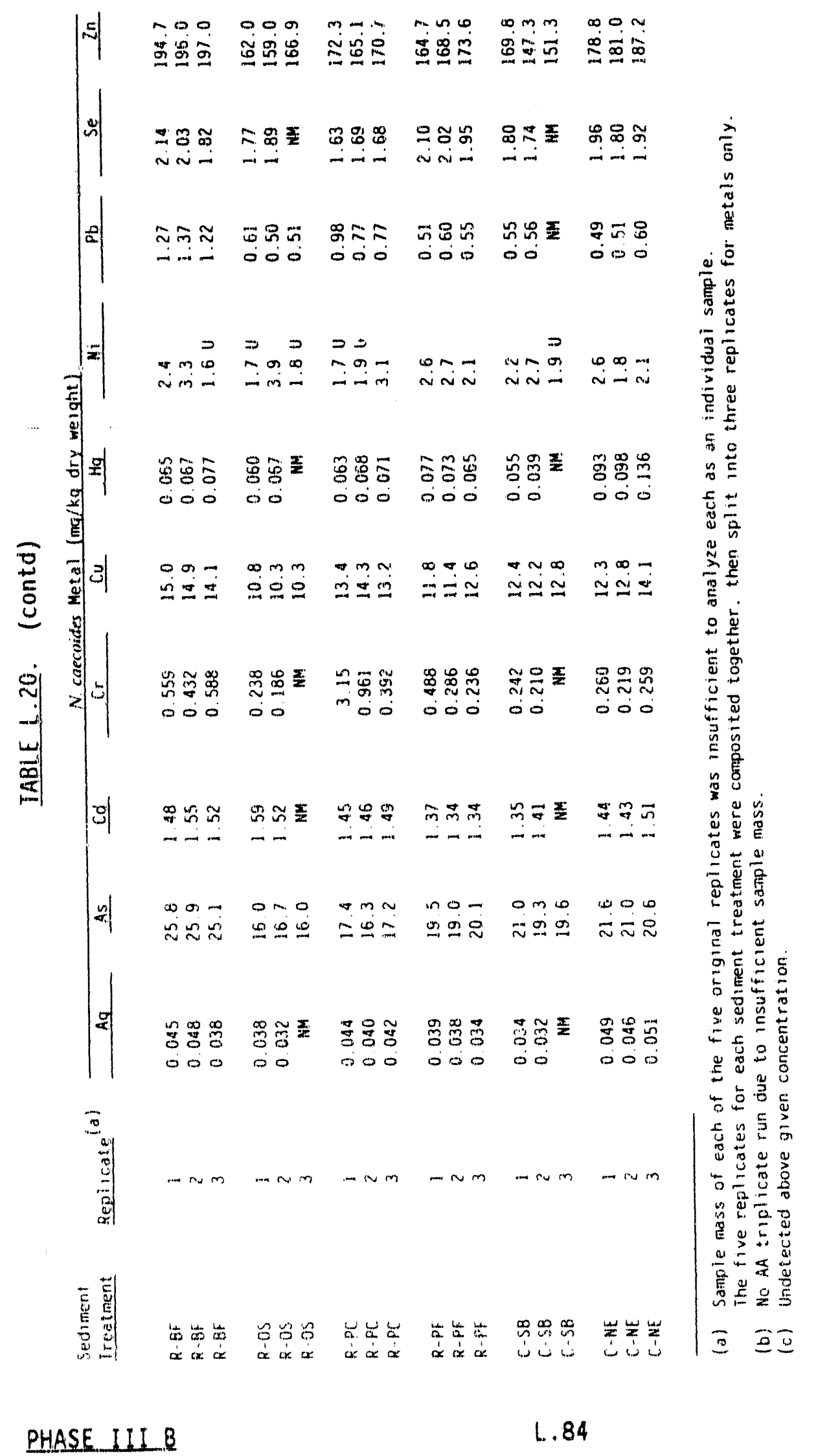




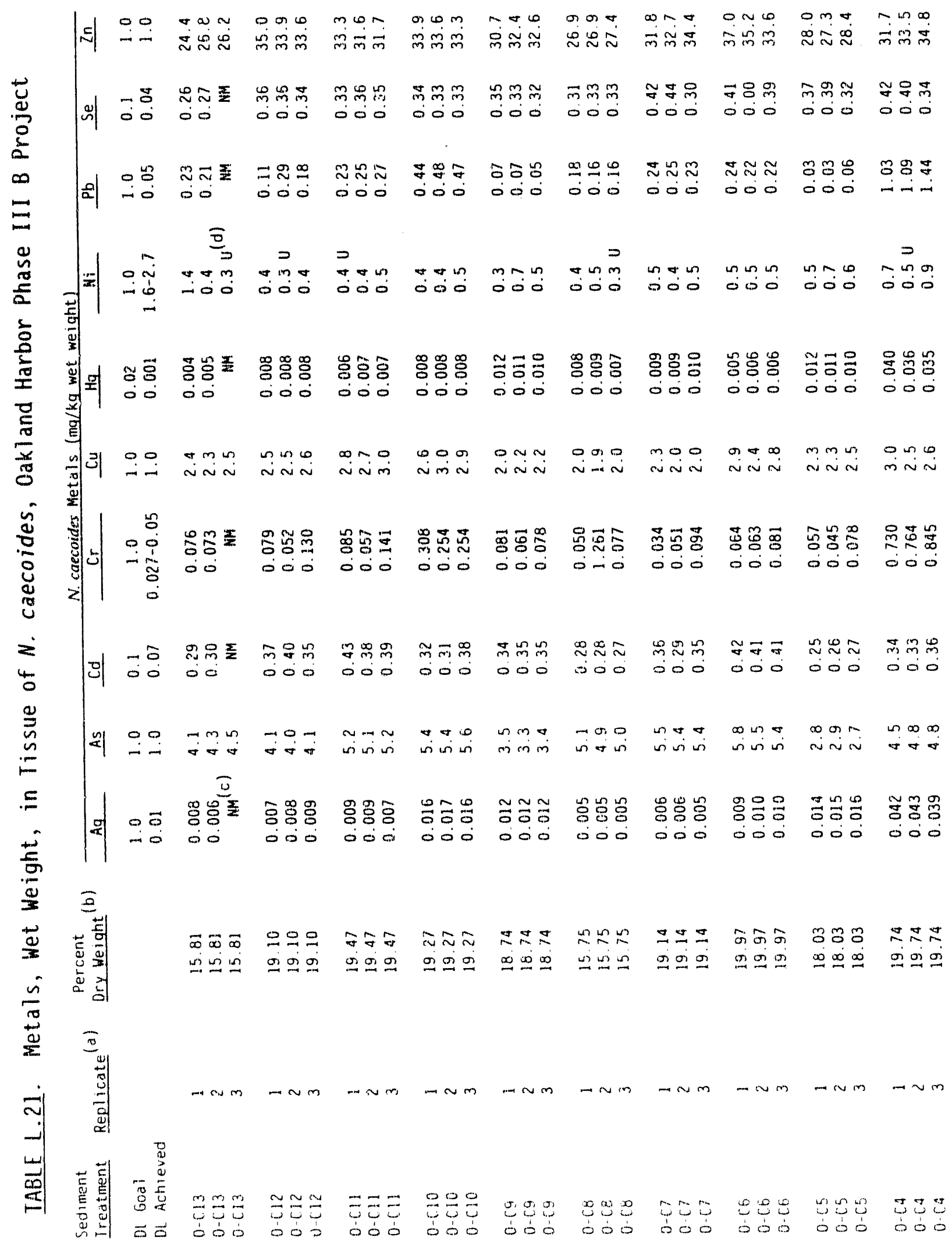

PHASE III $\mathrm{B}$ 


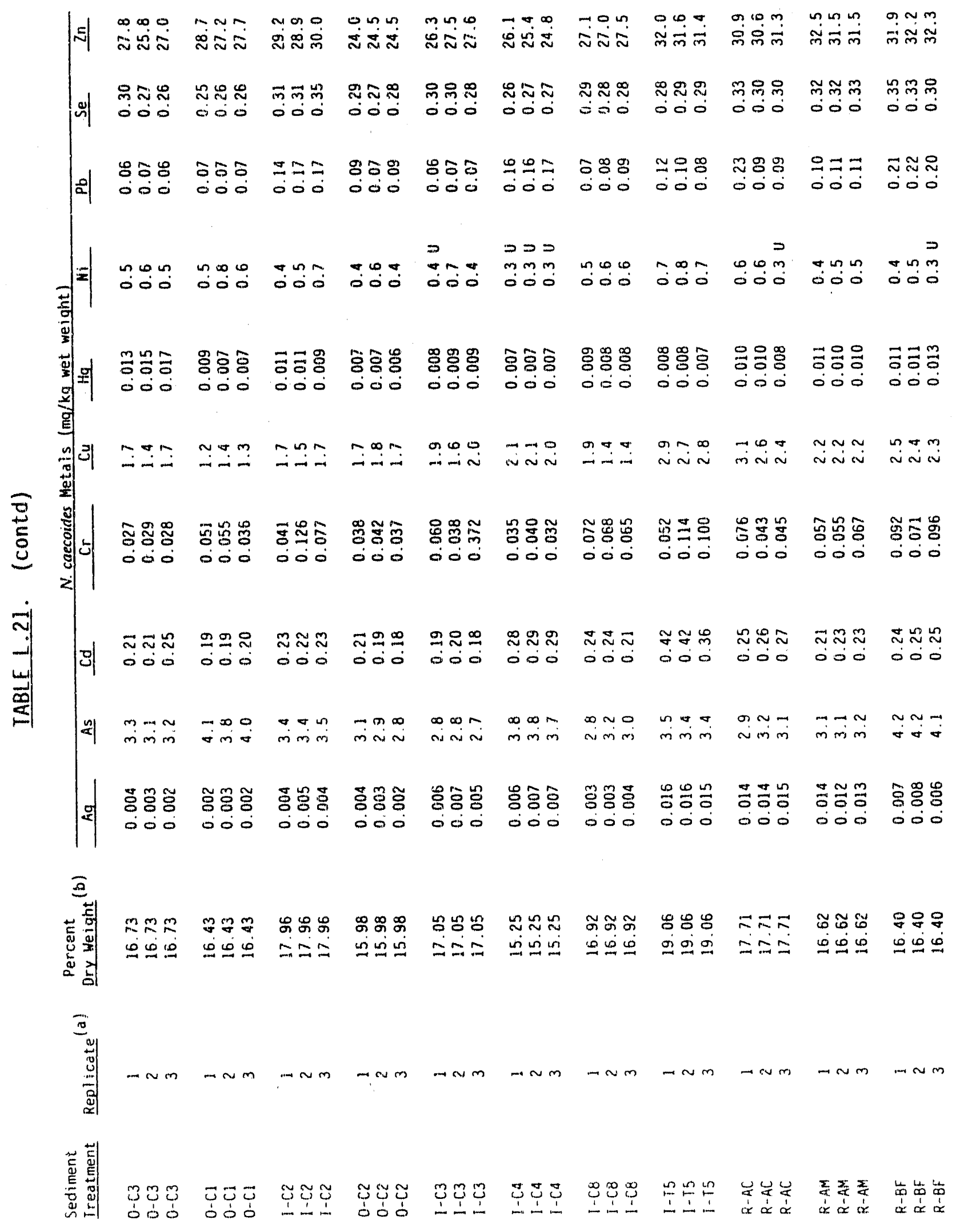

PHASE III B 


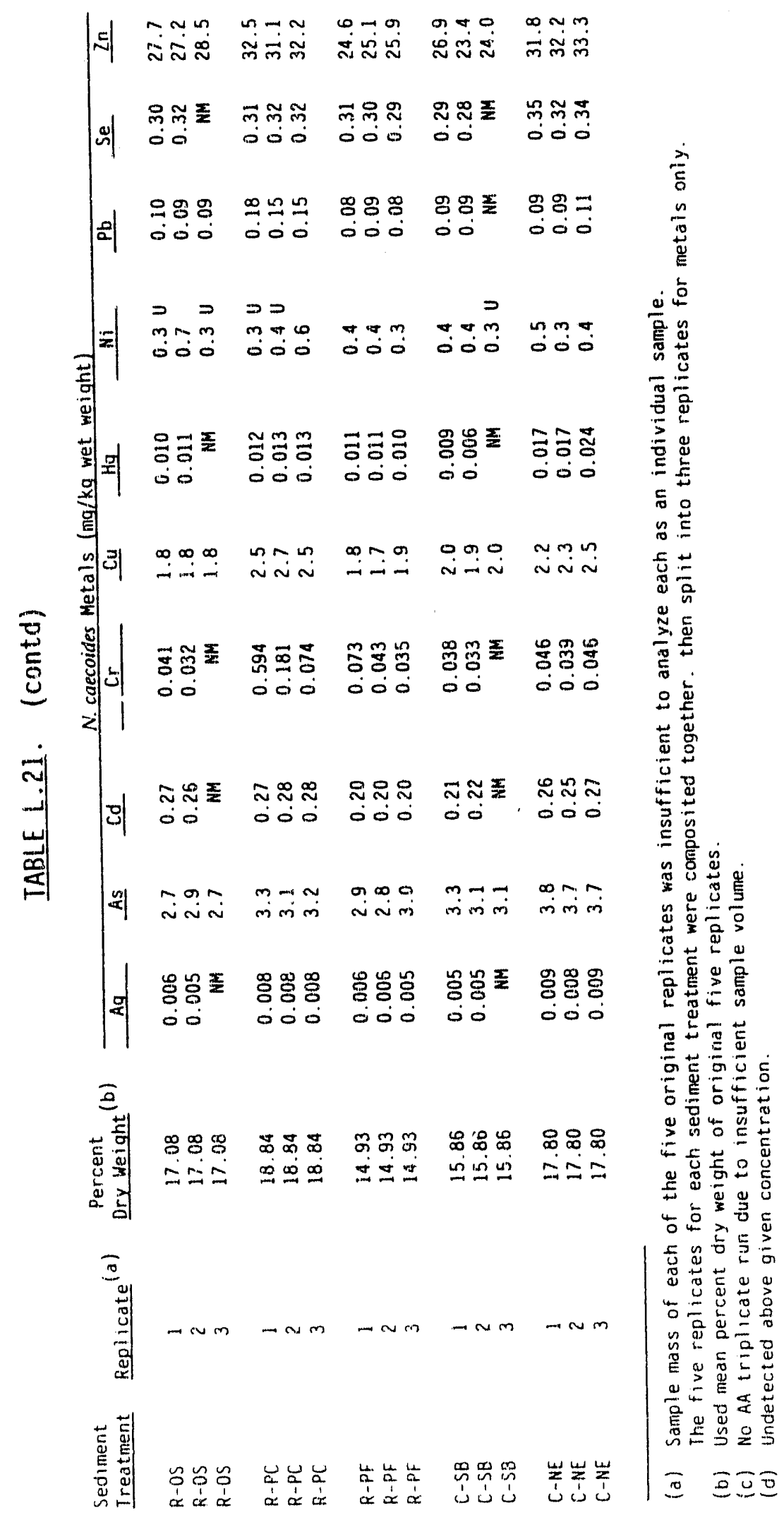




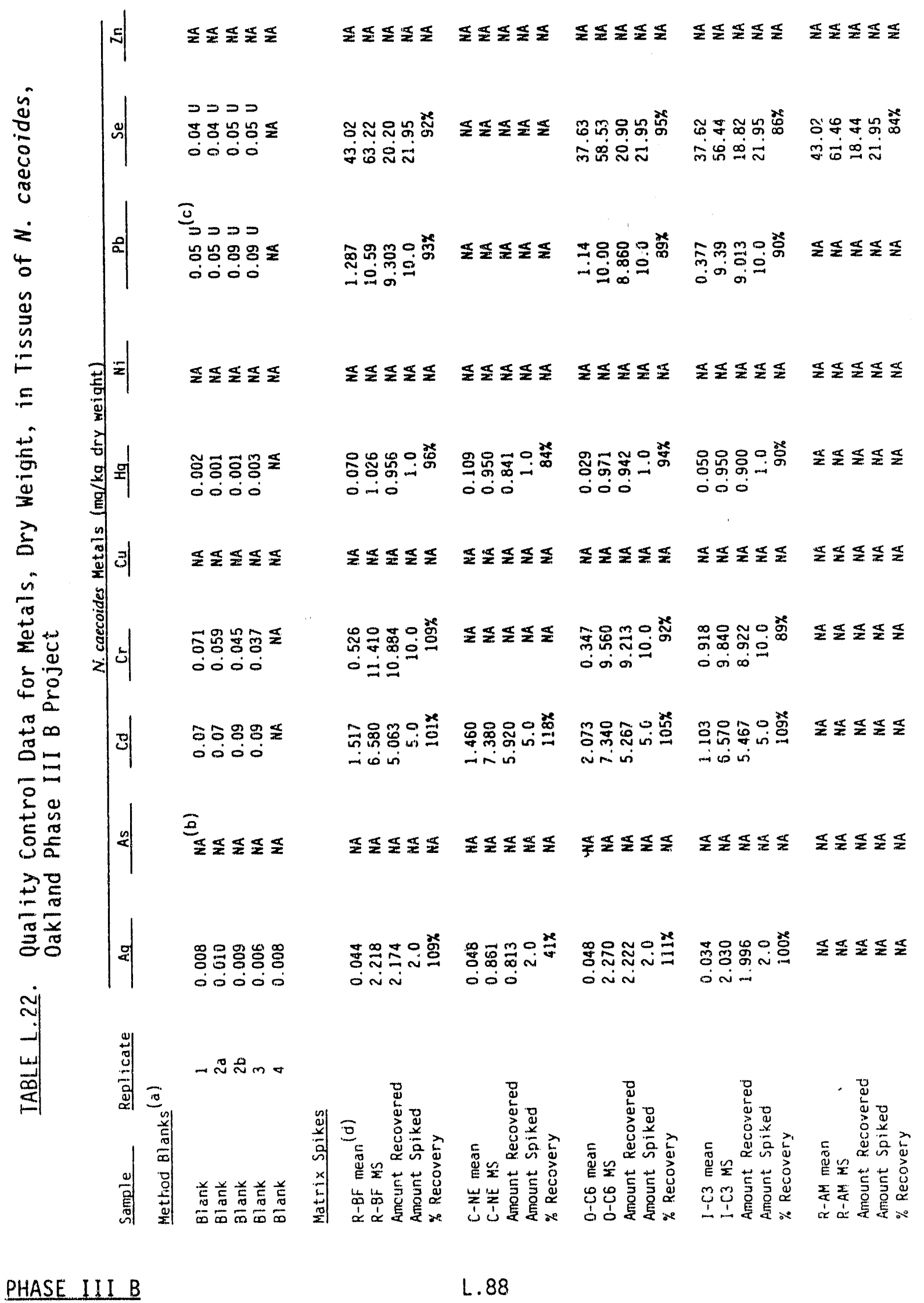




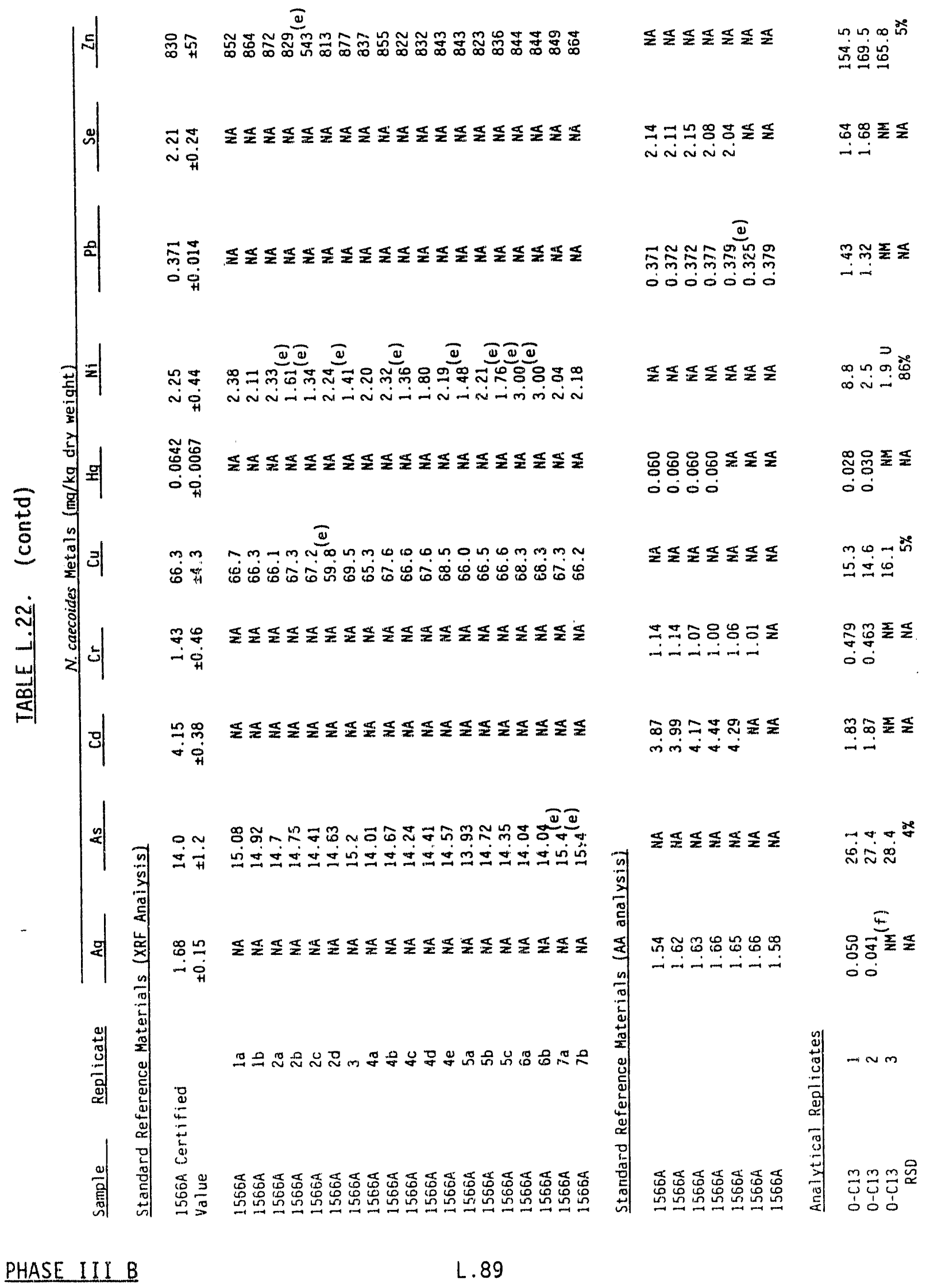




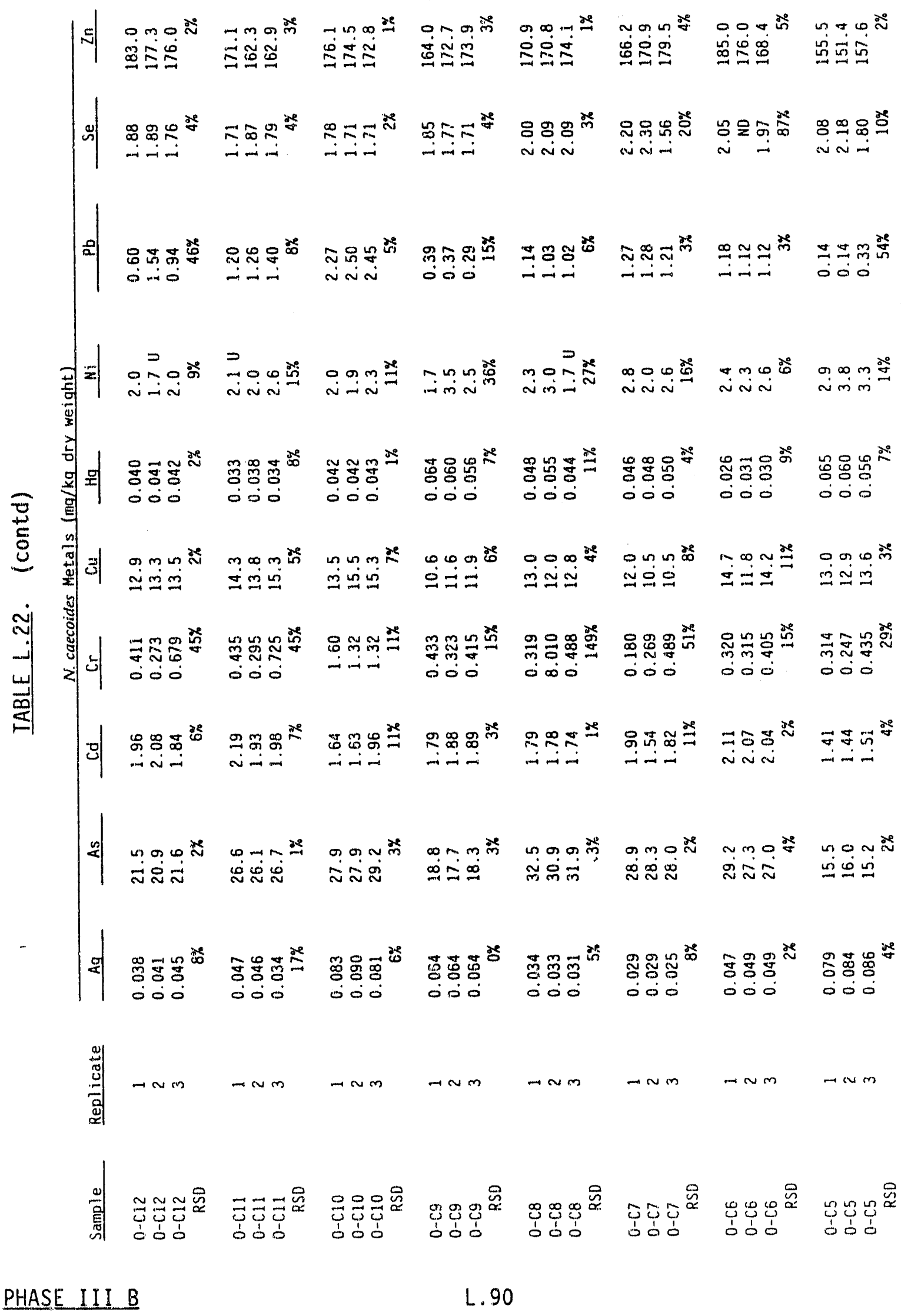




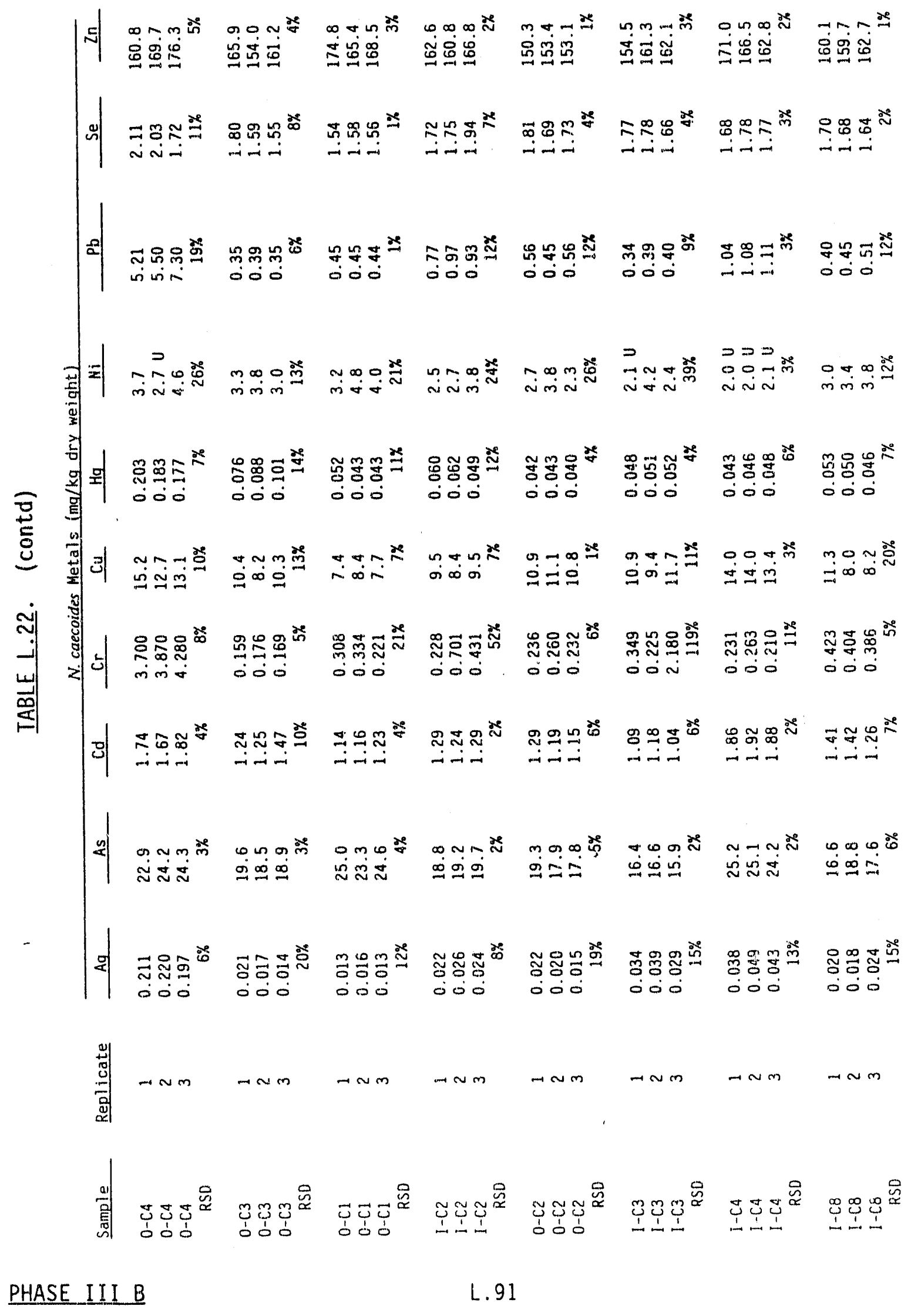




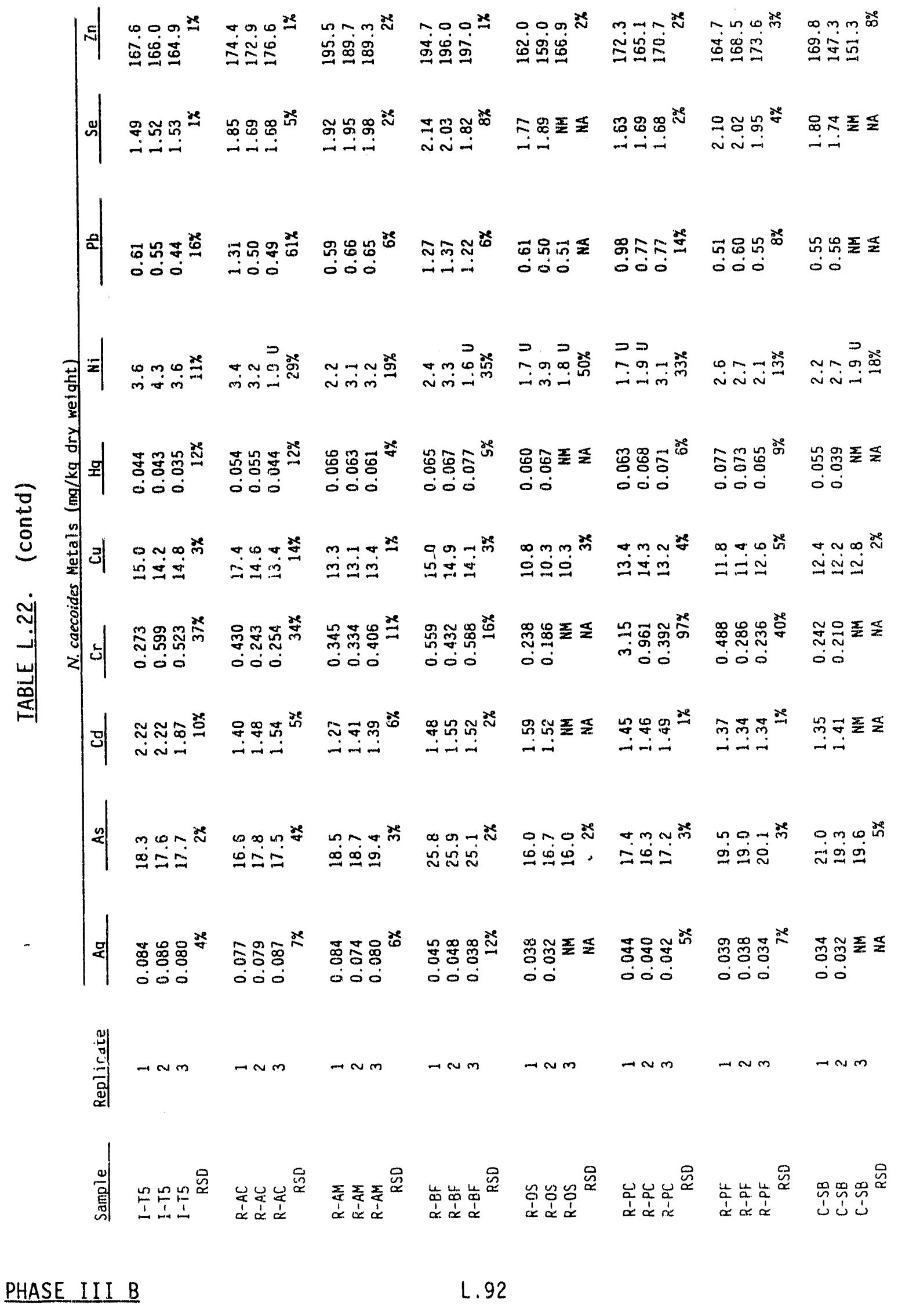




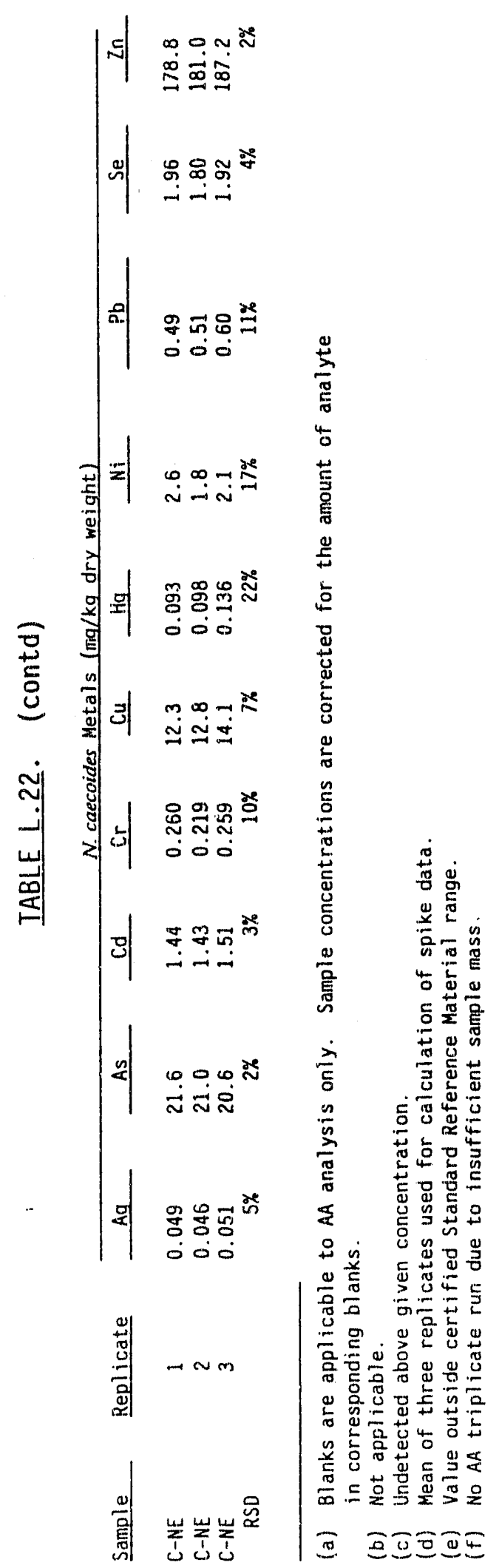

PHASE III $B$ 


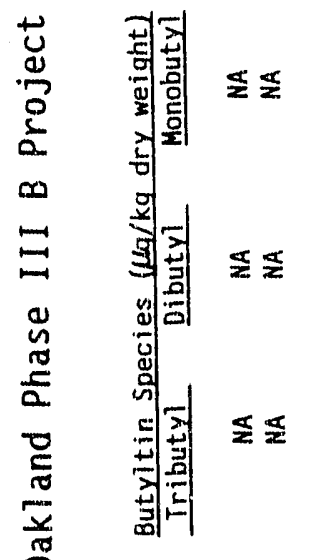

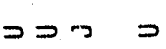

कणत

ப்่น்

$\supset \supset \supset \supset$

or on

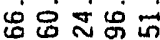

วつコつコー

n

$\neg \supset \supset コ コ$

a) $\rightarrow$ a $\infty$

守密递

ココココココ

a $\rightarrow$ N

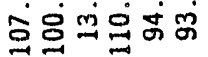

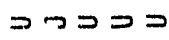

$\because \forall$ a

宓㲾守守

$\supset$ แココ

0 -

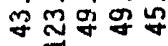

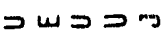

tin $\rightarrow$ in un

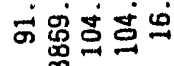

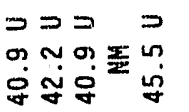

$\supset \supset \supset$

mot 0 皮

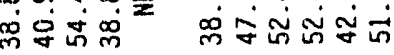

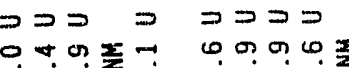

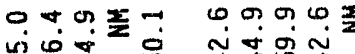

$\supset \supset コ コ コ コ$

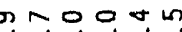

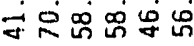

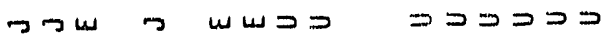
ம.?

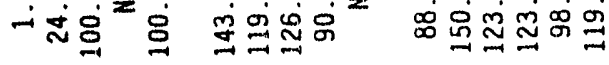

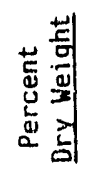

【屯

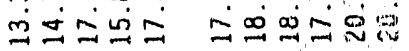

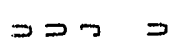

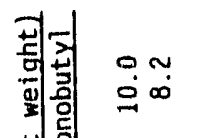

กบด

$\infty \dot{\infty} \sim \dot{\infty}$

コココั つつ

$\sim \sim \sim \operatorname{sun}$

$\infty \infty \infty \infty \infty \infty \infty$
冓踏赵 is

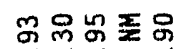

क्ष

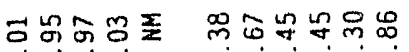

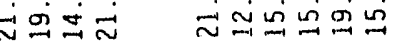

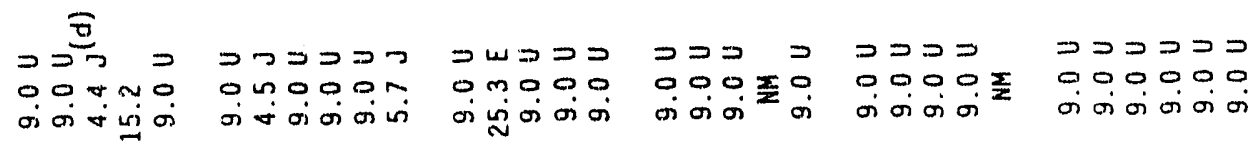

플

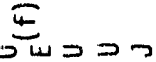
$0000 \%$ 00000 o 0 in 000

बiं

วコココ

ココつつ

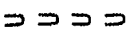

วつフコว ก

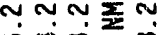

$\sim \sim \sim \sim ⿻$

דִ in $\infty \infty \infty$

कंषं

ว $ว$ מ

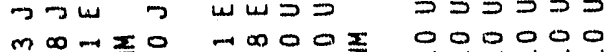
○+்

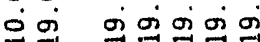

ธ่

茂

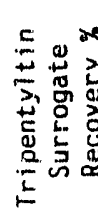

\section{드를}

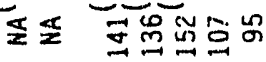

$\because \underset{0}{ }$

องอลำ

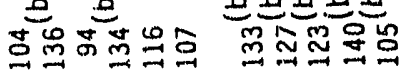

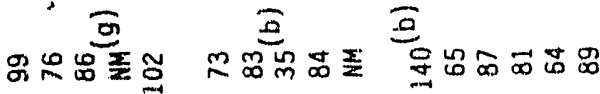

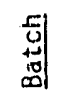

$$
\rightarrow-\rightarrow-\rightarrow
$$
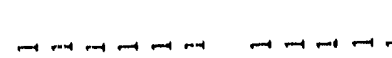

$\rightarrow \sim$

웜 윰

$-\pi \sim m \& \operatorname{son}$

$\rightarrow \sim N m+4$

in

苟

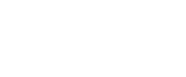

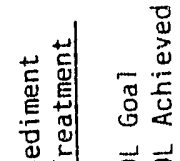

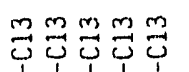

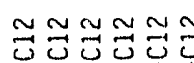
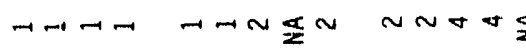

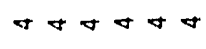

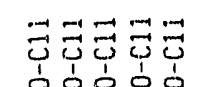

운운원운움

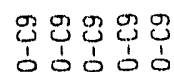

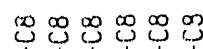

$\rightarrow \sim$

맘 वे

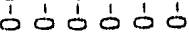




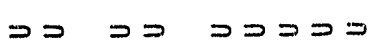

mo

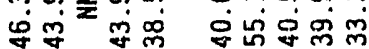

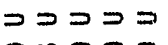

0 moon

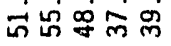

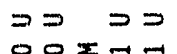

भig

د $\supset \supset \supset \supset \supset \supset$

の N 잔

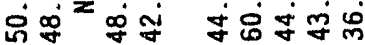

D $\geq \geq$

0

د

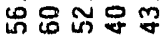

$\supset \Rightarrow \quad \supset \supset$

의 $x+\infty$

过 웜

$\supset \supset \supset \supset$

No 0 -

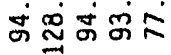

$\supset \supset \supset \supset$

$\infty 0$ \% $N-$

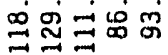

$\supset \supset \supset \supset$

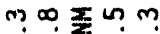

음

$\rightarrow \quad 20$

- 0 +0

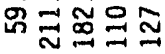

วココつ

$\infty m \infty \infty$ or

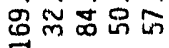
का का०

$\infty$ 垈兘家

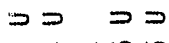

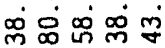

$\supset \supset \supset$ og 0 un un

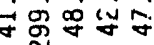

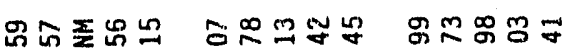
=
ตั $\infty \dot{0} \sim$
음 舟出品
ธิธกัญ

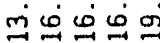


$P \geq \geq \geq \geq D$ $m \infty \forall \sim$ 额守品品和品

フココココ - $\forall$ ष

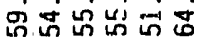

$\supset \supset \supset \supset \supset$ ר n

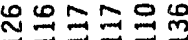

$\supset \supset \supset \supset$ ก - no

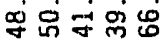

$\rightarrow \supset コ \supset \supset$ טก⿻上丨

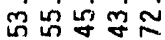

$\supset \supset \supset \supset د$ $\infty \sim \infty-\infty$

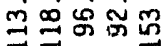

$\supset \supset \supset \supset$ +

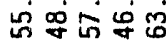

ב $\supset$ ב or un of at

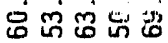

372 un onn

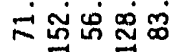

$\supset \supset \supset \supset \supset$ थ $\infty 001$

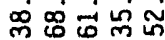

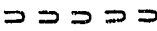
m

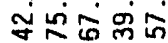

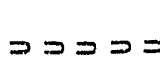
$\infty m w-\infty$ ตั

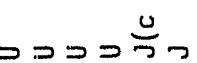
ฯ

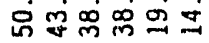

วาว Tn过官守的

コココつコ NNRNー

$\exists \supset \supset D$ - in $\infty$ o

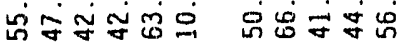

$\rightarrow \rightarrow$ 量 $\forall$ on 0 \%

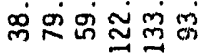

山

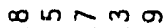

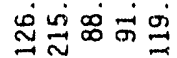

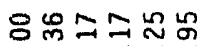
ஸ்
ำ نั

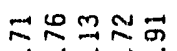

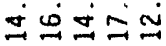

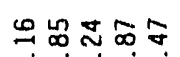
$\ddot{\sim}=\dot{\sim} \sim$

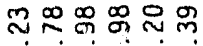
車只总过

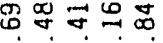
표료

$\supset=D \supset D$ บ $\sim \sim \sim \sim$

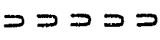
$\sim \sim \sim \sim \infty$

วココว $\sim \sim \sim \sim N$

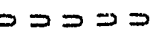
ก $\sim \sim \sim \sim ⿻ 上 丨$

כココつつ $\sim \sim \sim \sim \infty 0$

$\supset \supset \supset \supset$ $\infty \infty \infty \infty \infty \infty \infty$

$\infty \infty \infty \infty \infty$

$\infty \infty \infty \infty \infty$

$\infty \infty \infty \infty \infty$

$\infty \infty \infty \sim m$

$\sim \sim \sim N N$ $\infty \infty \infty \infty \infty$

$\supset \supset=D$ 00000

วコつコン フココン 00000 कं कांकांक

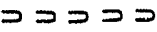
00000

$\neg \supset \supset \supset \supset \neg$ $00000 N$

कांण कां

$\supset \supset \supset \supset \supset$ 00000 का बांबां

का का

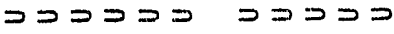
00000000000

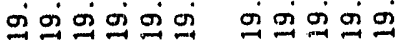

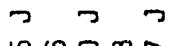
in $00 \infty n$ 잉ำ

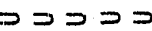
00000

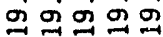

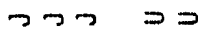
$\sim 0+600$

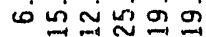

w $+00+0$

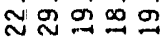

a

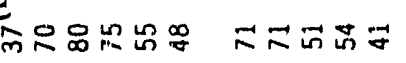

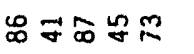

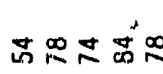

\section{를

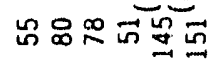

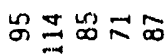

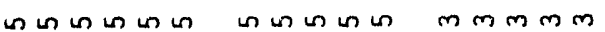

$\bullet \omega \infty \omega 0$

$m m m m \rightarrow-$

$\sim \sim \sim \sim \sim$

$\rightarrow n$

产

월 월

$\rightarrow \sim m m$ on

$-\infty m+\infty$

$\rightarrow$ nem in

$-\sim m \forall \ln$

$\vec{a}$

$\rightarrow \sim m$ m n

$\rightarrow \sim m \forall n$

岂苞

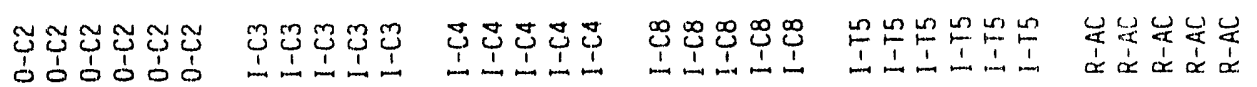




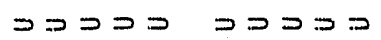
$\infty \infty-0 \infty$

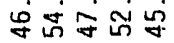

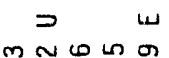
$\stackrel{9}{9} \stackrel{0}{\Xi}$

$\supset \supset \supset \supset$ Oก⿻

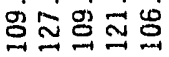
o) 0 Nㅇㅇㅇㅇㅇ

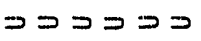
กNOOON กิ

$\supset \supset \supset \supset=$ oष 0 N

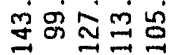

سا سا س כ כד N क 由 m m N

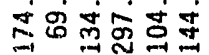
ב

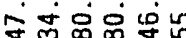

$\supset コ コ コ コ$ क $\rightarrow-\infty \infty$

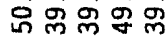

$\leadsto \supset \supset \supset \supset$ a 0 m n

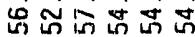
$\supset \supset \supset \supset \supset$ แ

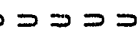
on $\infty$ 孚学出多

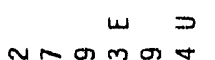

$\supset \supset \supset \supset \supset D$ N

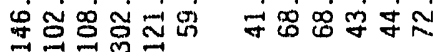

つつワ $\supset \supset コ コ コ コ$ o in ono

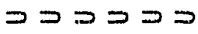
$m \pi 0$ \&

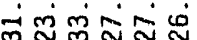

$m \infty \infty \infty m$

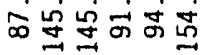

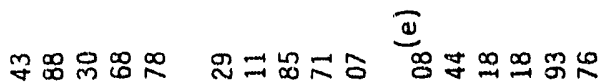

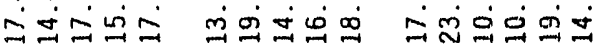

8 舟政嵌 மㅇํํํํ

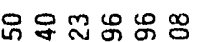

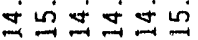

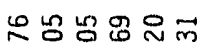

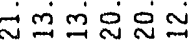

$\supset コ コ コ$ N $N$ N $\infty \infty \infty \infty$

$\neg コ コ コ \supset$ N $\sim \sim \sim$ $\infty \infty \infty \infty \infty$

$\neg \supset \supset \supset \supset$ $\sim \sim \sim \sim m \sim$ $\infty \infty \infty \infty \infty \infty$

フココンフ $\sim \sim \sim \sim \sim$ $\infty \infty \dot{\infty} \infty \infty$

$\supset コ コ コ コ コ$ $\sim \sim \sim \sim \sim \sim$ $\infty \infty \infty \infty \infty \infty$

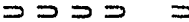
$\sim \sim \sim \sim \omega \sim$ $\infty \infty \infty \infty \infty$

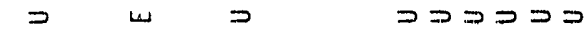
loon 000000

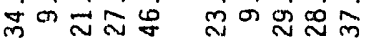

0.000
000000 जंकांबंकां

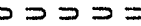
00000 कांकांक

$\omega=$ $\simeq \infty$ un $\sim$ บ त

$\supset コ コ コ コ$ 000000

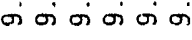

$\supset \supset \supset \supset \supset$ 00000 क्ष

$\supset コ \supset \supset \supset$ 00000 ஸें

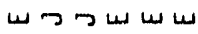

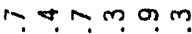

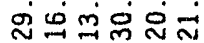

כコつつ 我窃

วコココวコ 0 이이웅

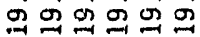

つココココ 000000 웍웜어요 a

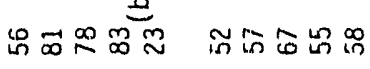

?

요욤ำ

2

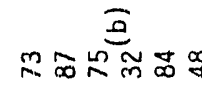

$\underline{2}$

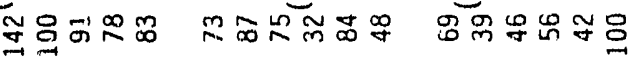

竞

ம

MnN

$\sim \sim \sim \sim \sim \sim$

$\sim \sim \sim m ! n$

6)

nann

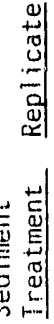

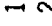

옹 윰

tNon

$\rightarrow \sim m+$ n

HNmm t

$r N m$ ar

웜 웜

월 웜

TNNm

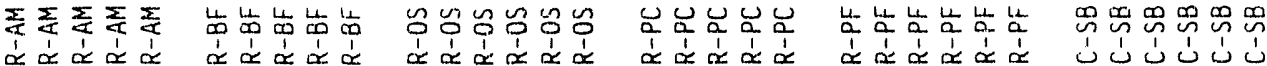



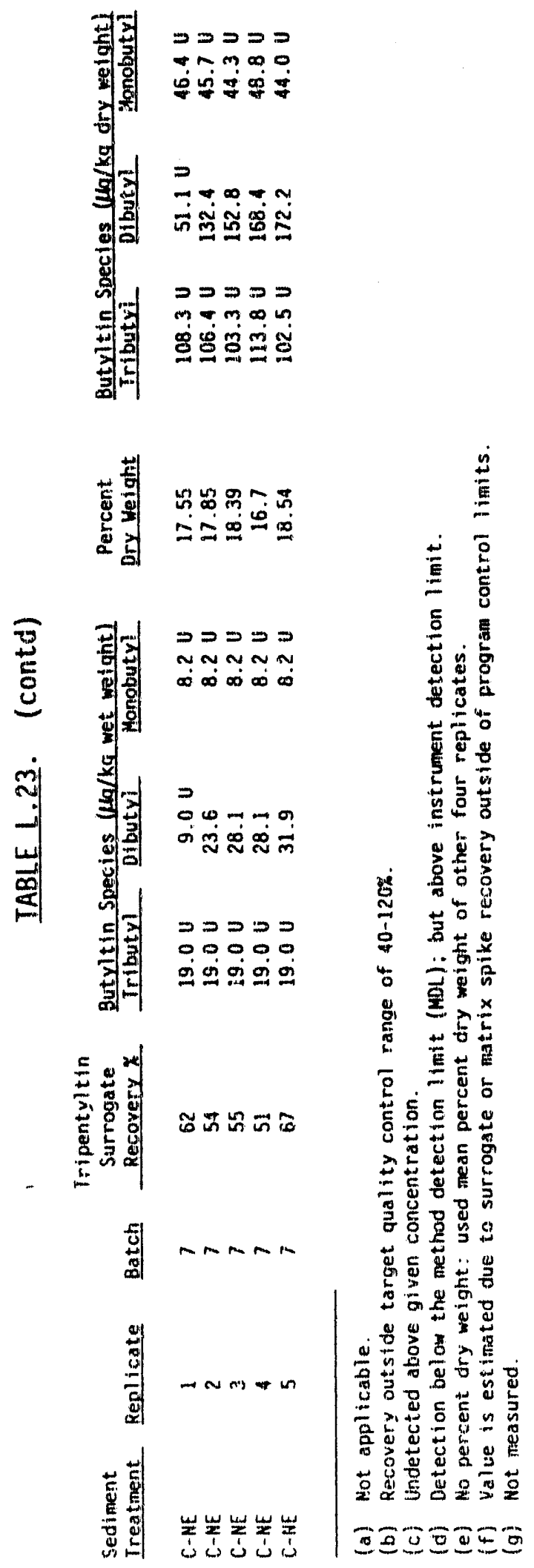

PHASE III 
TABLE L.24. Quality Control Data for Butyltins, Wet Weight, in Tissue of N. caecoides, Oakland Harbor Phase III B Project

\begin{tabular}{|c|c|c|c|c|c|c|}
\hline \multirow[b]{2}{*}{ Sample } & \multirow[b]{2}{*}{ Replicate } & \multirow[b]{2}{*}{ Batch } & \multirow{2}{*}{$\begin{array}{l}\text { Tripentyltin } \\
\text { Surrogate } \\
\text { Recovery } \% \\
\end{array}$} & \multicolumn{3}{|c|}{ Butyltin Species $(\mu \mathrm{g} / \mathrm{kg}$ wet weight) } \\
\hline & & & & Iributy] & Dibutyl & onobuty \\
\hline \multicolumn{7}{|c|}{ Method Blanks } \\
\hline $\begin{array}{l}\text { Blank } \\
\text { Blank } \\
\text { Blank } \\
\text { Blank } \\
\text { Blank } \\
\text { Blank } \\
\text { Blank }\end{array}$ & & $\begin{array}{l}1 \\
2 \\
3 \\
4 \\
5 \\
6 \\
7\end{array}$ & $\begin{array}{r}93 \\
78 \\
112 \\
81 \\
78 \\
42 \\
57\end{array}$ & $\begin{array}{l}26.2 \mathrm{~K}^{(\mathrm{a})} \\
19.0 \mathrm{U} \\
11.5 \mathrm{~K} \\
19.0 \mathrm{U} \\
19.0 \mathrm{U} \\
19.0 \mathrm{U} \\
19.0 \mathrm{U}\end{array}$ & $\begin{array}{l}9.0 U^{(b)} \\
9.0 U \\
9.0 U \\
9.0 U \\
9.0 U \\
9.0 U \\
9.0 U\end{array}$ & $\begin{array}{l}8.2 U \\
8.2 U \\
8.2 U \\
8.2 U \\
8.2 U \\
8.2 U \\
8.2 U\end{array}$ \\
\hline
\end{tabular}

\section{Matrix Spikes}

JE73 MS

Amount Recovered

Amount Spiked

Percent Recovery

JE74 MSD

Amount Recovered

Amount Spiked

Percent Recovery

MS/MSO RPD

MS/MSD I-Stat

JE75 MS

Amount Recovered

Amount Spiked

Percent Recovery

JE.78 MSD

Amount Recovered

Amount Spiked

Percent Recovery

MS/MSD RPD

MS/MSD I-Stat

JE 77 MS

Amount Recovered

Amount Spiked

Percent Recovery

JE76 MSD

Amount Recovered

Amount Spiked

Percent Recovery

MS/MSO RPD

MS/MSD I-Stat

JE79 MS

Amount Recovered

Amount Spiked

Percent. Recovery

\begin{tabular}{|c|c|c|c|c|}
\hline 1 & 90 & $\begin{array}{r}59.4 \\
59.4 \\
64.9 \\
91 \%\end{array}$ & $\begin{array}{l}50.2 \\
50.2 \\
53.4 \\
94 \%\end{array}$ & $\begin{array}{l}10.9 \\
10.9 \\
49.0 \\
22 \%\end{array}$ \\
\hline \multirow[t]{2}{*}{1} & 90 & $\begin{array}{l}40.2 \\
40.2 \\
65.6 \\
61 \%\end{array}$ & $\begin{array}{l}42.7 \\
42.7 \\
53.9 \\
79 \%\end{array}$ & $\begin{array}{l}8.2 U \\
8.2 \text { (U) } \\
N S \text { (c) } \\
N A\end{array}$ \\
\hline & & $\begin{array}{c}40 \% \\
0.20\end{array}$ & $\begin{array}{c}17 \% \\
0.09\end{array}$ & $\begin{array}{l}\text { NA } \\
\text { NA }\end{array}$ \\
\hline 2 & 78 & $\begin{array}{l}93.6 \\
93.6 \\
62.6 \\
150 \%(e)\end{array}$ & $\begin{array}{l}68.0 \\
68.0 \\
51.4(\mathrm{e}) \\
132 \%\end{array}$ & $\begin{array}{l}14.5 \\
14.5 \\
47.3 \\
31 \%\end{array}$ \\
\hline \multirow[t]{2}{*}{2} & 49 & $\begin{array}{l}110.0 \\
110.0 \\
57.8 \\
190 \%(\mathrm{e})\end{array}$ & $\begin{array}{l}87.5 \\
87.5 \\
47.5 \\
184 \%(e)\end{array}$ & $\begin{array}{l}12.7 \\
12.7 \\
43.6 \\
29 \%\end{array}$ \\
\hline & & $\begin{array}{l}24 \% \\
0.12\end{array}$ & $\begin{array}{l}33 \% \\
0.16\end{array}$ & 0.03 \\
\hline 3 & 80 & $\begin{array}{l}40.5 \\
40.5 \\
65.7 \\
62 \%\end{array}$ & $\begin{array}{l}34.3 \\
34.3 \\
54.0 \\
64 \%\end{array}$ & $\begin{array}{c}8.2 \mathrm{U} \\
8.2 \mathrm{U} \\
\mathrm{NS} \\
\mathrm{NA}\end{array}$ \\
\hline 3 & 89 & $\begin{array}{r}49.7 \\
49.7 \\
63.4 \\
78 \%\end{array}$ & $\begin{array}{l}41.1 \\
41.1 \\
52.1 \\
79 \%\end{array}$ & $\begin{array}{l}11.7 \\
11.7 \\
47.9 \\
24 \%\end{array}$ \\
\hline & & $\begin{array}{l}24 \% \\
0.12\end{array}$ & $\begin{array}{l}200 \% \\
1.00\end{array}$ & $\begin{array}{l}N A \\
N A\end{array}$ \\
\hline 4 & 53 & $\begin{array}{l}50.7 \\
50.7 \\
62.0 \\
82 \%\end{array}$ & $\begin{array}{l}48.9 \\
48.9 \\
51.0 \\
96 \%\end{array}$ & $\begin{array}{c}8.2 U \\
8.2 U \\
N S \\
N A\end{array}$ \\
\hline
\end{tabular}

PHASE III B 
TABLE L.24. (contd)

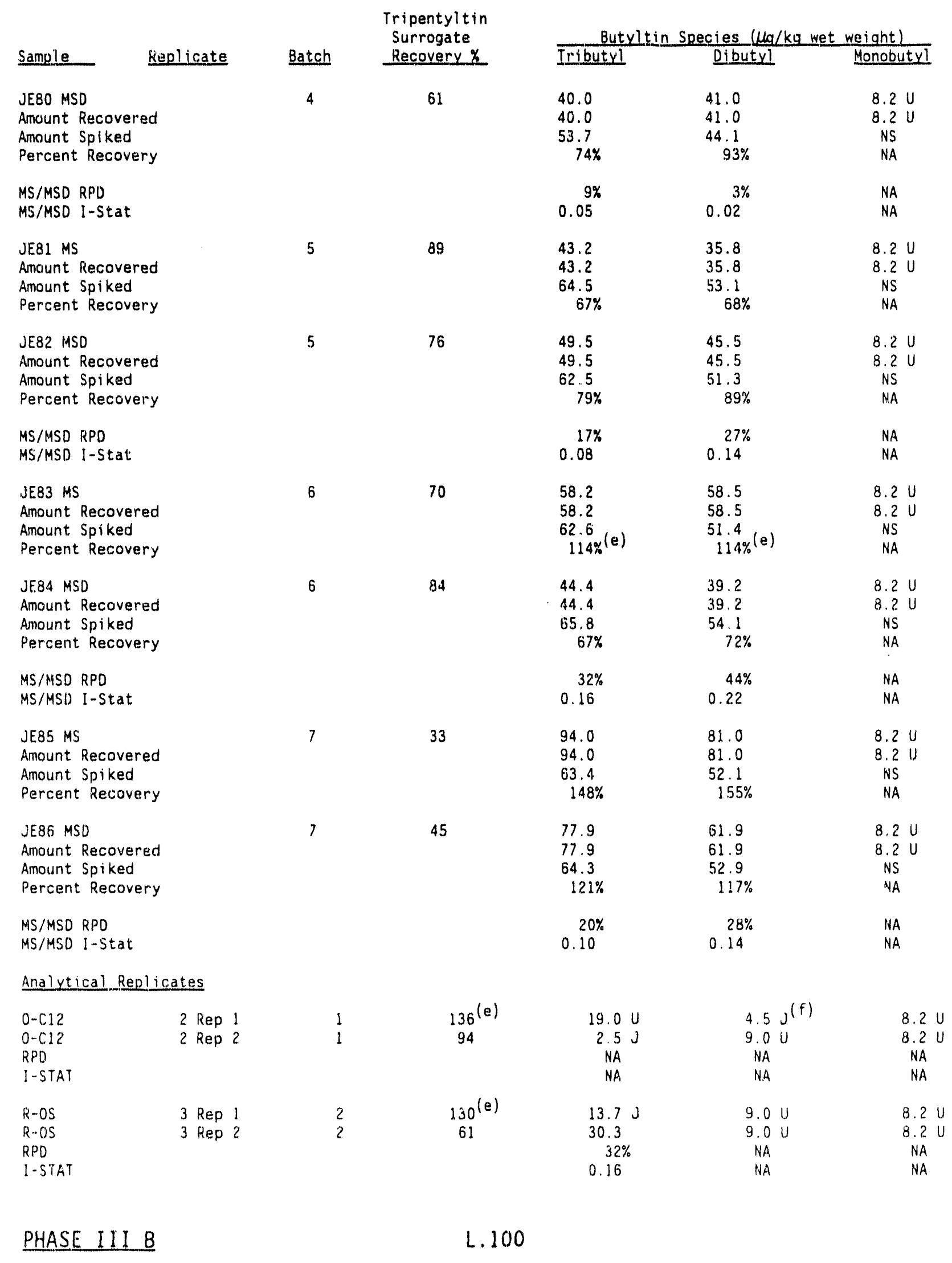


TABL.E L.24. (contd)

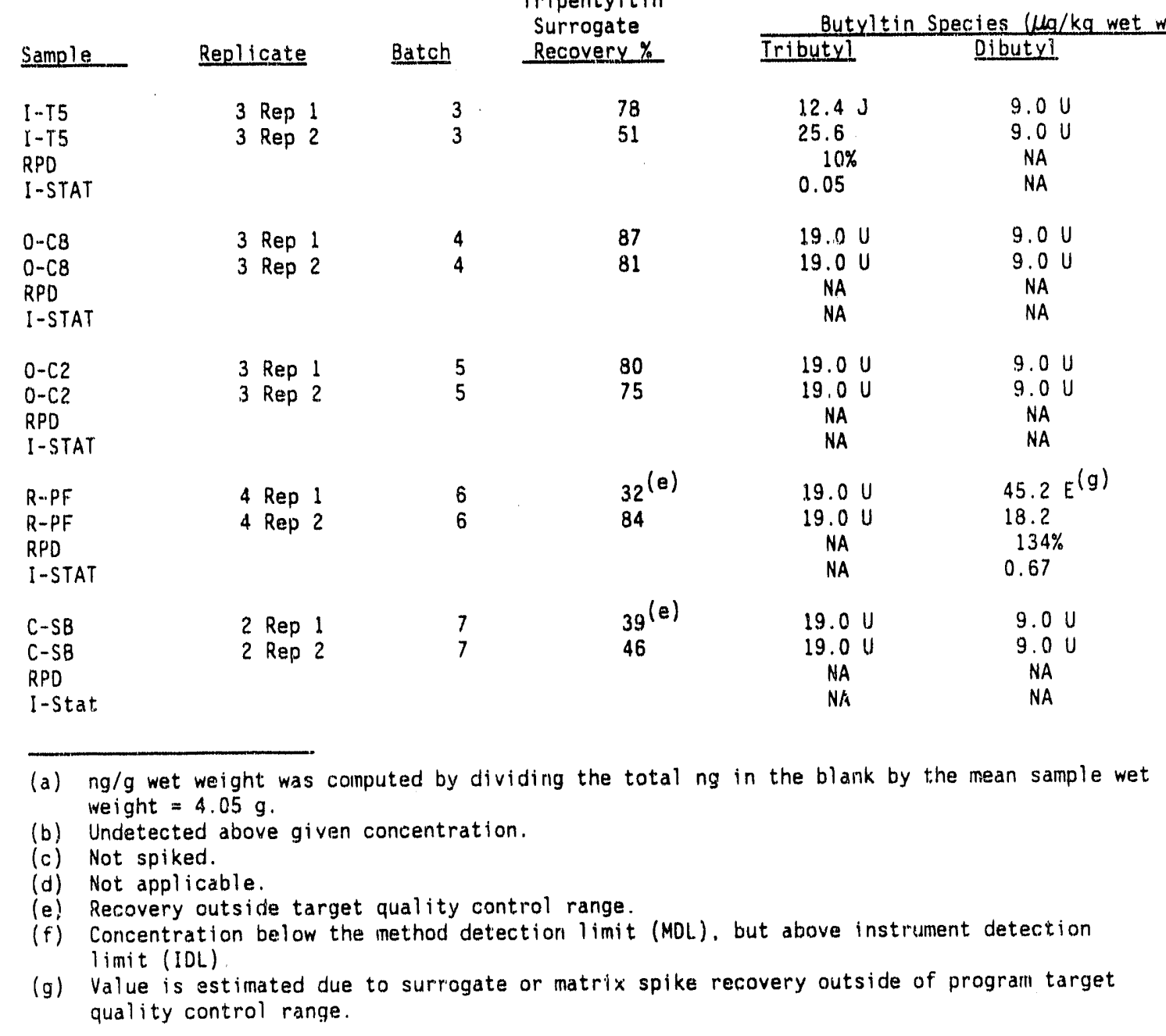


APPENDIX M

STATISTICALANALYSIS OEM. NASUTA BIOACCUMULATION 
TABLEM.1. Mean Concentration and Statistical Grouping for Naphthalene in $M$. nasuta Tissue

\begin{tabular}{|c|c|c|}
\hline $\begin{array}{l}\text { Sediment } \\
\text { Treatment }\end{array}$ & $\begin{array}{c}\text { Mean Tissue } \\
\text { Concentration } \\
\text { (ug/kg dry weight) }\end{array}$ & $\begin{array}{l}\text { Statistical } \\
\text { Grouping }\end{array}$ \\
\hline $\begin{array}{l}\text { R-AM } \\
\text { R-PC } \\
\text { R-OS } \\
\text { R-AC } \\
\text { R-PF } \\
\text { O-C11 } \\
\text { R-BF } \\
\text { O-C8 } \\
\text { O-C10 } \\
\text { O-C6 } \\
\text { O-C5 } \\
\text { O-C9 } \\
\text { O-C1 } \\
\text { O-C3 }\end{array}$ & $\begin{array}{c}65 \mathrm{U} \\
69 \mathrm{U} \\
69 \mathrm{U} \\
76 \mathrm{U} \\
77 \mathrm{U} \\
82 \\
97 \mathrm{U} \\
110 \\
157 \\
159 \\
166 \\
181 \\
198 \\
189\end{array}$ & $\begin{array}{l}\mathrm{A} \\
\mathrm{A} \\
\mathrm{A} \\
\mathrm{A} \\
\mathrm{A} \\
\mathrm{A} \\
\mathrm{AB} \\
\mathrm{ABC} \\
\mathrm{BC} \\
\mathrm{BC} \\
\mathrm{C} \\
\mathrm{C} \\
\mathrm{C} \\
\mathrm{C}\end{array}$ \\
\hline
\end{tabular}

TABLEM.2. ANOVA Results for Napthalene in $M$. nasuta Tissue

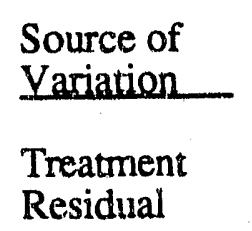

\begin{tabular}{rrrr}
$\begin{array}{l}\text { Sum of } \\
\text { Squares }\end{array}$ & d.f. & $\begin{array}{l}\text { Mean } \\
\text { Square }\end{array}$ & E-Ratio \\
\cline { 2 - 4 } 11.582 & 13 & 0.891 & 13.092 \\
4.219 & 62 & 0.068 &
\end{tabular}
Significance Level
0.0001


TABLEM.3. Mean Concentration and Statistical Grouping for Phenanthrene in M. nasuta Tissue

$\begin{array}{ccc}\begin{array}{c}\text { Sediment } \\ \text { Treatment }\end{array} & \begin{array}{c}\text { Mean Tissue } \\ \text { Concentration } \\ (\mu \mathrm{g} / \mathrm{kg} \text { dry weight })\end{array} & \begin{array}{c}\text { Statistical } \\ \text { Grouping }\end{array} \\ \text { R-PC } & 69 \mathrm{U} & \mathrm{A} \\ \text { R-OS } & 69 \mathrm{U} & \mathrm{A} \\ \text { R-AC } & 76 \mathrm{U} & \mathrm{A} \\ \text { R-PF } & 77 \mathrm{U} & \mathrm{A} \\ \text { O-C10 } & 80 & \mathrm{~A} \\ \text { R-AM } & 86 & \mathrm{~A} \\ \text { O-C6 } & 84 & \mathrm{~A} \\ \text { R-BF } & 97 \mathrm{U} & \mathrm{A} \\ \text { O-C5 } & 98 & \mathrm{~A}\end{array}$

TABLEM.4. ANOVA Results for Phenanthrene in $M$. nasuta Tissue

Source of

Variation

Treatment

Residual
Sum of

Squares

.546

2.322

8
42

Mean

Square

.068

.055
E-Ratio

1.234
Significance

Ievel

.3034 
TABLEM.5. Mean Concentration and Statistical Grouping for Fluoranthene in $M$. nasuta Tissue

\section{Sediment \\ Treatment}

R-PC
R-OS
I-C8
R-AC
R-PF
O-C5
O-C4
R-BF
O-C9
O-C7
O-C6
O-C10
O-C11
R-AM
O-C8
O-C13

Mean Tissue

Concentration

$(4 \mathrm{~g} / \mathrm{kg}$ dry weight)

\section{$69 \mathrm{U}$}

$69 \mathrm{U}$

75

$76 \mathrm{U}$

$77 \mathrm{U}$

89

95

$97 \mathrm{U}$

101

125

129

134

123

149

163

170
Statistical

Grouping.
A
A
$\mathrm{AB}$
$\mathrm{AB}$
$A B$
$A B C$
$A B C D$
$A B C D$
$A B C D$
$A B C D$
$A B C D$
$A B C D$
$\mathrm{ABCD}$
$\mathrm{BCD}$
$\mathrm{CD}$

TABLEM.6. ANOVA Results for Fluoranthene in $M$. nasuta tissue

\begin{tabular}{llllll}
$\begin{array}{l}\text { Source of } \\
\text { Variation }\end{array}$ & $\begin{array}{l}\text { Sum of } \\
\text { Squares }\end{array}$ & d.f. & $\begin{array}{c}\text { Mean } \\
\text { Square }\end{array}$ & E-Ratio & $\begin{array}{c}\text { Significance } \\
\text { Level }\end{array}$ \\
\cline { 3 - 5 } & & & & & \\
Treatment & 6.489 & 15 & 0.433 & 4.878 & 0.0001 \\
Residual & 6.208 & 70 & 0.089 & &
\end{tabular}


TABLE M.7. Mean Concentration and Statistical Grouping for Pyrene in M. nasuta Tissue

\begin{tabular}{|c|c|c|}
\hline $\begin{array}{l}\text { Sediment } \\
\text { Treatment }\end{array}$ & $\begin{array}{c}\text { Mean Tissue } \\
\text { Concentration } \\
(\mu \mathrm{g} / \mathrm{kg} \text { dry weight })\end{array}$ & $\begin{array}{l}\text { Statistical } \\
\text { Grouping }\end{array}$ \\
\hline $\begin{array}{l}\text { R-PC } \\
\text { R-OS } \\
\text { O-C3 } \\
\text { I-C3 } \\
\text { R-PF } \\
\text { O-C9 } \\
\text { R-BF } \\
\text { R-AC } \\
\text { O-C5 } \\
\text { I-C2 } \\
\text { O-C12 } \\
\text { O-C4 } \\
\text { O-C6 } \\
\text { O-C7 } \\
\text { R-AM } \\
\text { O-C10 } \\
\text { I-C8 } \\
\text { O-C8 } \\
\text { O-C11 } \\
\text { O-C13 }\end{array}$ & $\begin{array}{l}69 \mathrm{U} \\
69 \mathrm{U} \\
72 \\
76 \\
77 \mathrm{U} \\
95 \\
97 \mathrm{U} \\
139 \\
114 \\
179 \\
129 \\
132 \\
228 \\
226 \\
233 \\
284 \\
407 \\
336 \\
350 \\
591\end{array}$ & $\begin{array}{l}\text { A } \\
\text { A } \\
\text { A } \\
\text { A } \\
\text { A } \\
\text { A } \\
\text { AB } \\
\text { ABC } \\
\text { ABC } \\
\text { ABCD } \\
\text { ABCD } \\
\text { ABCD } \\
\text { BCDE } \\
\text { BCDE } \\
\text { CDE } \\
\text { DEF } \\
\text { EF } \\
\text { EF } \\
\text { EF } \\
\text { F }\end{array}$ \\
\hline
\end{tabular}

TABLE. M.8. ANOVA Results for Pyrene in M. nasuta Tissue

\begin{tabular}{lccrcc}
$\begin{array}{l}\text { Source of } \\
\text { Variation }\end{array}$ & $\begin{array}{l}\text { Sum of } \\
\text { Squares }\end{array}$ & d.f. & $\begin{array}{c}\text { Mean } \\
\text { Square }\end{array}$ & E-Ratio & $\begin{array}{c}\text { Significance } \\
\text { Level }\end{array}$ \\
\cline { 3 - 5 } & & & & & \\
Treatment & 42.633 & 19 & 2.244 & 14.245 & 0.0001 \\
Residual & 13.862 & 88 & 0.158 & &
\end{tabular}


TABLEM.9. Mean Concentration and Statistical Grouping for Benzo(a)anthracene in $M$. nasuta Tissue

\begin{tabular}{lcc} 
Sediment & $\begin{array}{c}\text { Mean Tissue } \\
\text { Concentration } \\
\text { Treatment }\end{array}$ & $\begin{array}{c}\text { Statistical } \\
\text { Gg/kg dry weight })\end{array}$ \\
Grouping \\
R-AM & $65 \mathrm{U}(\mathrm{a})$ & $\mathrm{A}$ \\
R-PC & $69 \mathrm{U}$ & $\mathrm{AB}$ \\
R-OS & $69 \mathrm{U}$ & $\mathrm{AB}$ \\
O-C11 & 74 & $\mathrm{AB}$ \\
R-AC & $76 \mathrm{U}$ & $\mathrm{AB}$ \\
R-PF & $77 \mathrm{U}$ & $\mathrm{AB}$ \\
O-C8 & 81 & $\mathrm{AB}$ \\
O-C10 & 83 & $\mathrm{AB}$ \\
R-BF & $97 \mathrm{U}$ & $\mathrm{B}$ \\
\hline
\end{tabular}

(a) Undetected in all replicates; value is mean of dry weight detection limits

TABLE M.10. ANOVA Results for Benzo(a)anthracene in M. nasuta Tissue

Source of

Variation

Treatment

Residual
Sum of

Squares

0.535

1.197

\begin{tabular}{|c|c|}
\hline & $\begin{array}{l}\text { Mean } \\
\text { Square }\end{array}$ \\
\hline $\begin{array}{r}8 \\
40\end{array}$ & $\begin{array}{l}0.067 \\
0.030\end{array}$ \\
\hline
\end{tabular}

F-Ratio

Significance

2.233

Level

0.0450

$\mathrm{AB}$

$A B$

$A B$

B 
TABLEM.11. Mean Concentration and Statistical Grouping for Chrysene in M. nasuta Tissue

\begin{tabular}{ccc} 
Sediment & $\begin{array}{c}\text { Mean Tissue } \\
\text { Concentration } \\
\text { Treatment }\end{array}$ & $\begin{array}{c}\text { Statistical } \\
\text { Grg/kgdry weight })\end{array}$ \\
Grouping \\
R-PC & $69 \mathrm{U}$ (a) & $\mathrm{A}$ \\
R-AM & 73 & $\mathrm{~A}$ \\
I-C8 & 74 & $\mathrm{~A}$ \\
O-C7 & 74 & $\mathrm{~A}$ \\
R-OS & 75 & $\mathrm{~A}$ \\
R-PF & $77 \mathrm{U}$ & $\mathrm{A}$ \\
O-C13 & 81 & $\mathrm{~A}$ \\
O-C9 & 87 & $\mathrm{~A}$ \\
O-C8 & 96 & $\mathrm{~A}$ \\
R-BF & $97 \mathrm{U}$ & $\mathrm{A}$ \\
R-AC & 98 & $\mathrm{~A}$ \\
O-C10 & 106 & $\mathrm{~A}$ \\
O-C11 & 108 & $\mathrm{~A}$ \\
\hline
\end{tabular}

(a) Undetected in all replicates, value is mean of dry weight detection limits

TABLE M.12. ANOVA Results for Chrysene in $M$. nasuta Tissue

\begin{tabular}{|c|c|c|c|c|c|}
\hline $\begin{array}{l}\text { Source of } \\
\text { Variation }\end{array}$ & $\begin{array}{l}\text { Sum of } \\
\text { Squares }\end{array}$ & d.f. & $\begin{array}{l}\text { Mean } \\
\text { Square }\end{array}$ & F-Ratio & $\begin{array}{c}\text { Significance } \\
\text { Level }\end{array}$ \\
\hline $\begin{array}{l}\text { Treatment } \\
\text { Residual }\end{array}$ & $\begin{array}{l}1.306 \\
3.051\end{array}$ & $\begin{array}{l}12 \\
56\end{array}$ & $\begin{array}{l}0.109 \\
0.054\end{array}$ & 1.997 & 0.0417 \\
\hline
\end{tabular}


TABLEM.13. Mean Concentration and Statistical Grouping for Benzo(b)fluoranthene in $M$. nasuta Tissue

\begin{tabular}{ccc} 
Sediment & $\begin{array}{c}\text { Mean Tissue } \\
\text { Concentration } \\
\text { Treatment }\end{array}$ & $\begin{array}{c}\text { Statistical } \\
\text { (ug/kg dry weight) }\end{array}$ \\
Grouping \\
R-AM & $65 \mathrm{U}$ (a) & $\mathrm{A}$ \\
R-PC & $69 \mathrm{U}$ & $\mathrm{A}$ \\
O-C11 & 69 & $\mathrm{~A}$ \\
R-OS & $69 \mathrm{U}$ & $\mathrm{A}$ \\
O-C7 & 74 & $\mathrm{~A}$ \\
R-AC & $76 \mathrm{U}$ & $\mathrm{A}$ \\
R-PF & $77 \mathrm{U}$ & $\mathrm{A}$ \\
O-C8 & 78 & $\mathrm{~A}$ \\
O-C10 & 80 & $\mathrm{~A}$ \\
O-C13 & 83 & $\mathrm{~A}$ \\
R-BF & $97 \mathrm{U}$ & $\mathrm{AB}$ \\
I-C8 & 125 & $\mathrm{~B}$ \\
\hline
\end{tabular}

(a) Undetected in all replicates; value is mean of dry weight detection limits

TABLE M.14. ANOVA Results for Benzo(b)fluoranthene in $M$. nasuta Tissue

\begin{tabular}{|c|c|c|c|c|c|}
\hline $\begin{array}{l}\text { Source of } \\
\text { Variation }\end{array}$ & $\begin{array}{l}\text { Sum of } \\
\text { Squares }\end{array}$ & d.f. & $\begin{array}{l}\text { Mean } \\
\text { Square }\end{array}$ & F-Ratio & $\begin{array}{c}\text { Significance } \\
\text { Level }\end{array}$ \\
\hline $\begin{array}{l}\text { Treatment } \\
\text { Residual }\end{array}$ & $\begin{array}{l}1.586 \\
1.561\end{array}$ & $\begin{array}{l}11 \\
52\end{array}$ & $\begin{array}{l}0.144 \\
0.030\end{array}$ & 4.805 & 0.0001 \\
\hline
\end{tabular}


TABLEM.15. Mean Concentration and Statistical Grouping for Benzo(a)pyrene in M. nasuta Tissue

$\begin{array}{cc}\text { Sediment } & \begin{array}{c}\text { Mean Tissue } \\ \text { Concentration } \\ \text { Treatment }\end{array} \\ \text { (ug/kg dry weight }\end{array}$

Statistical

Grouping

A

A

A

A

A

A

A

A

A

(a) Undetected in all replicates; value is mean of dry weight detection limits

TABLE M.16. ANOVA Results for Benzo(a)pyrene in $M$. nasuta Tissue

Source of

Yariation

Treatment

Residual
Sum of

Squares

$\frac{\text { d.f }}{8}$

.604

1.229
Mean

Square

0.075

0.03 ?
F-Ratio

2.333
Significance

Level

0.0381 
TABLEM.17. Mean Concentration and Statistical Grouping for Silver in $M$. nasuta Tissue

$\begin{array}{lcc}\begin{array}{c}\text { Sediment } \\ \text { Ireatment }\end{array} & \begin{array}{c}\text { Mean Tissue } \\ \text { Concentration } \\ \text { (mg/kg dry weight) }\end{array} & \begin{array}{c}\text { Statistical } \\ \text { Srouping }\end{array} \\ \text { O-C13 } & 0.289 & \mathrm{~A} \\ \text { O-C11 } & 0.274 & \mathrm{~A} \\ \text { O-C6 } & 0.304 & \mathrm{AB} \\ \text { O-C9 } & 0.411 & \mathrm{ABC} \\ \text { O-C10 } & 0.385 & \mathrm{ABC} \\ \text { O-C4 } & 0.352 & \mathrm{ABC} \\ \text { O-C7 } & 0.443 & \mathrm{ABC} \\ \text { R-PC } & 0.458 & \mathrm{ABC} \\ \text { R-PF } & 0.396 & \mathrm{ABC} \\ \text { O-C12 } & 0.483 & \mathrm{ABC} \\ \text { R-BF } & 0.407 & \mathrm{ABC} \\ \text { R-AM } & 0.438 & \mathrm{ABCD} \\ \text { O-C5 } & 0.793 & \mathrm{ABCDE} \\ \text { O-C8 } & 0.765 & \mathrm{ABCDE} \\ \text { R-AC } & 0.972 & \mathrm{BCDE} \\ \text { R-OS } & 0.980 & \mathrm{CDE} \\ \text { O-C2 } & 1.212 & \mathrm{DE} \\ \text { O-C3 } & 1.208 & \mathrm{DE} \\ \text { I-C2 } & 1.238 & \mathrm{DE} \\ \text { O-C1 } & 1.232 & \mathrm{DE} \\ \text { I-C8 } & 1.400 & \mathrm{E} \\ \text { I-C3 } & 1.446 & \mathrm{E}\end{array}$

TABLEM.18. ANOVA Results for Silver in M. nasuta Tissue

\begin{tabular}{|c|c|c|c|c|c|}
\hline $\begin{array}{l}\text { Source of } \\
\text { Variation }\end{array}$ & $\begin{array}{l}\text { Sum of } \\
\text { Squares }\end{array}$ & d.f. & $\begin{array}{l}\text { Mean } \\
\text { Square }\end{array}$ & E-Ratio & $\begin{array}{c}\text { Significance } \\
\text { Level }\end{array}$ \\
\hline $\begin{array}{l}\text { Treatment } \\
\text { Residual }\end{array}$ & $\begin{array}{l}42.173 \\
23.199\end{array}$ & $\begin{array}{r}21 \\
100\end{array}$ & $\begin{array}{l}2.008 \\
0.232\end{array}$ & 8.657 & 0.0001 \\
\hline
\end{tabular}


TABLEM.19. Mean Concentration and Statistical Grouping for Arsenic in $M$. nasuta Tissue

$\begin{array}{ccc}\begin{array}{c}\text { Sediment } \\ \text { Treament }\end{array} & \begin{array}{c}\text { Mean Tissue } \\ \text { Concentration } \\ \text { (mo/kgdry weight) }\end{array} & \begin{array}{c}\text { Statistical } \\ \text { Grouping }\end{array} \\ \text { O-C11 } & 16.8 & \mathrm{~A} \\ \text { R-AM } & 17.8 & \mathrm{~A} \\ \text { O-C10 } & 18.3 & \mathrm{~A} \\ \text { O-C7 } & 18.5 & \mathrm{~A} \\ \text { O-C9 } & 18.5 & \mathrm{~A} \\ \text { O-C6 } & 18.7 & \mathrm{~A} \\ \text { O-C13 } & 19.0 & \mathrm{~A} \\ \text { R-PC } & 19.6 & \mathrm{~A} \\ \text { R-PF } & 19.6 & \mathrm{~A} \\ \text { O-C4 } & 20.7 & \mathrm{~A} \\ \text { O-C8 } & 21.4 & \mathrm{~A} \\ \text { I-C2 } & 20.9 & \mathrm{~A} \\ \text { O-C12 } & 21.8 & \mathrm{~A} \\ \text { O-C5 } & 22.1 & \mathrm{~A} \\ \text { R-BF } & 21.9 & \mathrm{~A} \\ \text { O-C2 } & 21.9 & \mathrm{~A} \\ \text { R-OS } & 22.4 & \mathrm{~A} \\ \text { O-C3 } & 22.6 & \mathrm{~A} \\ \text { O-C1 } & 22.9 & \mathrm{~A} \\ \text { I-C3 } & 23.1 & \mathrm{~A} \\ \text { I-C8 } & 23.3 & \mathrm{~A} \\ \text { R-AC } & 23.7 & \mathrm{~A} \\ & & \end{array}$

IABLEM.20. ANOVA Results for Arsenic in M. nasuta Tissue

\begin{tabular}{|c|c|c|c|c|c|}
\hline $\begin{array}{l}\text { Source of } \\
\text { Variation }\end{array}$ & $\begin{array}{l}\text { Sum of } \\
\text { Squares }\end{array}$ & d.f. & $\begin{array}{l}\text { Mean } \\
\text { Square }\end{array}$ & E-Ratio & $\begin{array}{c}\text { Significance } \\
\text { Level }\end{array}$ \\
\hline $\begin{array}{l}\text { Treatment } \\
\text { Residual }\end{array}$ & $\begin{array}{l}1.311 \\
2.885\end{array}$ & $\begin{array}{r}21 \\
100\end{array}$ & $\begin{array}{l}0.062 \\
0.029\end{array}$ & 2.163 & 0.0059 \\
\hline
\end{tabular}


TABLEM.21. Mean Concentration and Statistical Grouping for Cadmium in $M$. nasuta Tissue

$\begin{array}{lcc}\begin{array}{c}\text { Sediment } \\ \text { Treatment }\end{array} & \begin{array}{c}\text { Mean Tissue } \\ \text { Concentration } \\ \text { (mg/kgdry weight) }\end{array} & \begin{array}{c}\text { Statistical } \\ \text { Grouping }\end{array} \\ \text { O-C11 } & 0.19 & \mathrm{~A} \\ \text { O-C7 } & 0.21 & \mathrm{~A} \\ \text { O-C10 } & 0.23 & \mathrm{AB} \\ \text { O-C6 } & 0.22 & \mathrm{AB} \\ \text { O-C13 } & 0.23 & \mathrm{AB} \\ \text { R-AM } & 0.23 & \mathrm{AB} \\ \text { O-C8 } & 0.25 & \mathrm{AB} \\ \text { R-PC } & 0.24 & \mathrm{AB} \\ \text { R-OS } & 0.25 & \mathrm{AB} \\ \text { R-BF } & 0.25 & \mathrm{AB} \\ \text { O-C4 } & 0.27 & \mathrm{AB} \\ \text { R-PF } & 0.26 & \mathrm{AB} \\ \text { O-C9 } & 0.26 & \mathrm{AB} \\ \text { O-C2 } & 0.29 & \mathrm{AB} \\ \text { O-C12 } & 0.31 & \mathrm{AB} \\ \text { R-AC } & 0.30 & \mathrm{AB} \\ \text { O-C1 } & 0.31 & \mathrm{AB} \\ \text { O-C5 } & 0.33 & \mathrm{AB} \\ \text { I-C8 } & 0.33 & \mathrm{AB} \\ \text { O-C3 } & 0.33 & \mathrm{AB} \\ \text { I-C2 } & 0.37 & \mathrm{~B} \\ \text { I-C3 } & 0.38 & \mathrm{~B} \\ \text { I-C3 } & & \end{array}$

TABLEM.22. ANOVA Results for Cadmium in $M$. nasuta Tissue

\begin{tabular}{|c|c|c|c|c|c|}
\hline $\begin{array}{l}\text { Source of } \\
\text { Variation }\end{array}$ & $\begin{array}{l}\text { Sum of } \\
\text { Squares }\end{array}$ & d.f. & $\begin{array}{l}\text { Mean } \\
\text { Square }\end{array}$ & E-Ratio & $\begin{array}{c}\text { Significance } \\
\text { Level }\end{array}$ \\
\hline $\begin{array}{l}\text { Treatment } \\
\text { Residual }\end{array}$ & $\begin{array}{l}4.594 \\
7.612\end{array}$ & $\begin{array}{r}21 \\
100\end{array}$ & $\begin{array}{l}0.219 \\
0.076\end{array}$ & 2.874 & 0.0002 \\
\hline
\end{tabular}


TABLEM.23. Mean Concentration and Statistical Grouping for Chromium in M. nasuta Tissue

\begin{tabular}{ccc}
$\begin{array}{c}\text { Sediment } \\
\text { Treatment }\end{array}$ & $\begin{array}{c}\text { Mean Tissue } \\
\text { Concentration } \\
\text { (mg/kg dry weight) }\end{array}$ & $\begin{array}{c}\text { Statistical } \\
\text { Grouping }\end{array}$ \\
\cline { 3 - 3 } R-PC & 1.09 & $\mathrm{~A}$ \\
R-AC & 1.13 & $\mathrm{AB}$ \\
R-AM & 1.12 & $\mathrm{AB}$ \\
I-C3 & 1.34 & $\mathrm{ABC}$ \\
O-C7 & 1.66 & $\mathrm{ABC}$ \\
O-C1 & 1.67 & $\mathrm{ABC}$ \\
I-C2 & 1.81 & $\mathrm{ABC}$ \\
R-BF & 1.79 & $\mathrm{ABCD}$ \\
O-C9 & 1.97 & $\mathrm{ABCD}$ \\
O-C6 & 1.86 & $\mathrm{BCD}$ \\
O-C3 & 1.85 & $\mathrm{BCD}$ \\
O-C13 & 1.93 & $\mathrm{BCD}$ \\
I-C8 & 1.92 & $\mathrm{BCD}$ \\
R-PF & 1.98 & $\mathrm{BCD}$ \\
O-C1 & 1.97 & $\mathrm{BCD}$ \\
O-C5 & 2.01 & $\mathrm{CD}$ \\
O-C8 & 2.02 & $\mathrm{CD}$ \\
O-C2 & 2.03 & $\mathrm{CD}$ \\
O-C4 & 2.17 & $\mathrm{CD}$ \\
R-OS & 2.24 & $\mathrm{CD}$ \\
O-C10 & 2.23 & $\mathrm{CD}$ \\
O-C12 & 3.13 & $\mathrm{D}$ \\
& &
\end{tabular}

TABLEM.24. ANOVA Results for Chromium in $M$. nastua Tissue

$\begin{array}{lcrccc}\begin{array}{l}\text { Source of } \\ \text { Variation }\end{array} & \begin{array}{l}\text { Sum of } \\ \text { Squares }\end{array} & \text { d.f. } & \begin{array}{c}\text { Mean } \\ \text { Square }\end{array} & \text { E-Ratio } & \begin{array}{c}\text { Significance } \\ \text { Level }\end{array} \\ \text { Treatment } & 8.105 & 21 & 0.386 & 5.794 & 0.0001 \\ \text { Residual } & 6.661 & 100 & 0.067 & & \end{array}$


TABLEM.25. Mean Concentration and Statistical Grouping for Copper in $M$. nasuta Tissue

$\begin{array}{ccc}\text { Sediment } & \begin{array}{c}\text { Mean Tissue } \\ \text { Concentration } \\ \text { Treatment }\end{array} & \begin{array}{c}\text { Statistical } \\ \text { Grokgdry weight) }\end{array} \\ \text { R-AM } & 14.0 & \mathrm{~A} \\ \text { O-C11 } & 14.5 & \mathrm{~A} \\ \text { R-BF } & 14.5 & \mathrm{~A} \\ \text { R-PF } & 15.3 & \mathrm{~A} \\ \text { O-C13 } & 17.3 & \mathrm{~A} \\ \text { O-C4 } & 16.6 & \mathrm{AB} \\ \text { O-C10 } & 18.2 & \mathrm{AB} \\ \text { O-C9 } & 17.9 & \mathrm{AB} \\ \text { O-C7 } & 20.2 & \mathrm{ABC} \\ \text { R-PC } & \mathrm{ABCD} \\ \text { O-C5 } & 21.8 & \mathrm{ABCDE} \\ \text { O-C6 } & 24.9 & \mathrm{ABCDE} \\ \text { O-C12 } & \mathrm{ABCDE} \\ \text { O-C8 } & 22.6 & \mathrm{ABCDE} \\ \text { R-AC } & 27.0 & \mathrm{ABCDE} \\ \text { R-OS } & 28.6 & \mathrm{ABCDE} \\ \text { O-C3 } & 31.1 & \mathrm{BCDE} \\ \text { O-C2 } & 32.1 & \mathrm{BCDE} \\ \text { O-C1 } & 36.4 & \mathrm{CDE} \\ \text { I-C2 } & 39.2 & \mathrm{DE} \\ \text { I-C3 } & 41.1 & \mathrm{E} \\ \text { I-C8 } & 42.1 & \mathrm{E}\end{array}$

TABLEM.26. ANOVA Results for Copper in $M$. nasuta Tissue

\begin{tabular}{lrrrrr}
$\begin{array}{l}\text { Source of } \\
\text { Variation }\end{array}$ & $\begin{array}{c}\text { Sum of } \\
\text { Squares }\end{array}$ & d.f. & $\begin{array}{l}\text { Mean } \\
\text { Square }\end{array}$ & E-Ratio & $\begin{array}{c}\text { Significance } \\
\text { Ievel }\end{array}$ \\
\hline Treatment & 20.928 & 21 & 0.997 & 6.605 & 0.0001 \\
Residual & 15.089 & 100 & 0.151 & &
\end{tabular}


TABLE M.27. Mean Concentration and Statistical Grouping for Mercury in M. nasuta Tissue

\begin{tabular}{lll} 
Sediment & $\begin{array}{c}\text { Mean Tissue } \\
\text { Concentration } \\
\text { Ireatment }\end{array}$ & $\begin{array}{c}\text { Statistical } \\
\text { Grouping }\end{array}$ \\
\cline { 2 - 3 } O-C11 & 0.045 & $\mathrm{~A}$ \\
O-C13 & 0.052 & $\mathrm{AB}$ \\
R-AM & 0.054 & $\mathrm{AB}$ \\
O-C9 & 0.056 & $\mathrm{AB}$ \\
O-C6 & 0.058 & $\mathrm{AB}$ \\
R-PC & 0.063 & $\mathrm{AB}$ \\
R-BF & 0.059 & $\mathrm{AB}$ \\
O-C10 & 0.061 & $\mathrm{AB}$ \\
O-C7 & 0.063 & $\mathrm{AB}$ \\
R-PF & 0.062 & $\mathrm{AB}$ \\
O-C12 & 0.066 & $\mathrm{AB}$ \\
O-C5 & 0.072 & $\mathrm{AB}$ \\
O-C4 & 0.069 & $\mathrm{AB}$ \\
O-C8 & 0.074 & $\mathrm{ABC}$ \\
R-AC & 0.083 & $\mathrm{ABCD}$ \\
R-OS & 0.085 & $\mathrm{ABCD}$ \\
O-C2 & 0.089 & $\mathrm{BCDE}$ \\
O-C3 & 0.142 & $\mathrm{CDEF}$ \\
I-C3 & 0.147 & $\mathrm{DEF}$ \\
I-C8 & 0.165 & $\mathrm{EF}$ \\
I-C2 & 0.231 & $\mathrm{FG}$ \\
O-C1 & 0.411 & $\mathrm{G}$
\end{tabular}

TABLEM.28. ANOVA Results for Mercury in $M$. nasuta Tissue

\begin{tabular}{|c|c|c|c|c|c|}
\hline $\begin{array}{l}\text { Source of } \\
\text { Variation }\end{array}$ & $\begin{array}{l}\text { Sum of } \\
\text { Squares }\end{array}$ & d.f. & $\begin{array}{l}\text { Mean } \\
\text { Square }\end{array}$ & E-Ratie & $\begin{array}{c}\text { Significance } \\
\text { Level }\end{array}$ \\
\hline $\begin{array}{l}\text { Treatment } \\
\text { Residual }\end{array}$ & $\begin{array}{r}30.890 \\
9.267\end{array}$ & $\begin{array}{r}21 \\
100\end{array}$ & $\begin{array}{l}1.471 \\
0.093\end{array}$ & 15.873 & 0.0001 \\
\hline
\end{tabular}


TABLEM.29. Mean Concentration and Statistical Grouping for Nickel in $M$. nasuta Tissue

$\begin{array}{ccc}\begin{array}{c}\text { Sediment } \\ \text { Treatment }\end{array} & \begin{array}{c}\text { Mean Tissue } \\ \text { Concentration } \\ \text { (mg/kgdry weight) }\end{array} & \begin{array}{c}\text { Statistical } \\ \text { Grouping }\end{array} \\ \text { R-AC } & 2.57 & \mathrm{~A} \\ \text { R-AM } & 2.47 & \mathrm{~A} \\ \text { R-PC } & 2.69 & \mathrm{~A} \\ \text { O-C11 } & 2.70 & \mathrm{~A} \\ \text { I-C2 } & 2.92 & \mathrm{AB} \\ \text { R-PF } & 2.98 & \mathrm{AB} \\ \text { O-C10 } & 3.50 & \mathrm{AB} \\ \text { I-C3 } & 3.70 & \mathrm{AB} \\ \text { O-C9 } & 3.61 & \mathrm{AB} \\ \text { I-C8 } & 3.71 & \mathrm{AB} \\ \text { O-C6 } & 3.78 & \mathrm{AB} \\ \text { O-C4 } & \mathrm{AB} \\ \text { O-C3 } & 3.95 & \mathrm{AB} \\ \text { O-C8 } & 3.91 & \mathrm{AB} \\ \text { O-C7 } & 3.92 & \mathrm{AB} \\ \text { R-BF } & 4.62 & \mathrm{AB} \\ \text { O-C2 } & 4.04 & \mathrm{AB} \\ \text { O-C1 } & 4.39 & \mathrm{AB} \\ \text { O-C5 } & 4.73 & \mathrm{AB} \\ \text { O-C12 } & 4.77 & \mathrm{AB} \\ \text { O-C13 } & 5.31 & \mathrm{~B} \\ \text { R-OS } & 9.54 & \mathrm{~B} \\ & 9.33 & \end{array}$

TABLEM.30. ANOVA Results for Nickel in M. nastua Tissue

\begin{tabular}{|c|c|c|c|c|c|}
\hline $\begin{array}{l}\text { Source of } \\
\text { Variation }\end{array}$ & $\begin{array}{l}\text { Sum of } \\
\text { Squares }\end{array}$ & d.f. & $\begin{array}{l}\text { Mean } \\
\text { Square }\end{array}$ & E-Ratio & $\begin{array}{c}\text { Significance } \\
\text { Level }\end{array}$ \\
\hline $\begin{array}{l}\text { Treaument } \\
\text { Residual }\end{array}$ & $\begin{array}{r}9.583 \\
16.096\end{array}$ & $\begin{array}{r}21 \\
100\end{array}$ & $\begin{array}{l}0.456 \\
0.161\end{array}$ & 2.835 & 0.0003 \\
\hline
\end{tabular}


TABLEM.31. Mean Concentration and Statistical Grouping for Lead in $M$. nasuta Tissue

$\begin{array}{lcc}\text { Sediment } & \begin{array}{c}\text { Mean Tissue } \\ \text { Concentration } \\ \text { Treatment }\end{array} & \begin{array}{c}\text { Statistical } \\ \text { (mg/kgdry weight) }\end{array} \\ \text { Orouping } \\ \text { O-C9 } & 1.46 & \mathrm{~A} \\ \text { R-PF } & 1.24 & \mathrm{AB} \\ \text { O-C11 } & 1.30 & \mathrm{AB} \\ \text { R-AM } & 1.44 & \mathrm{AB} \\ \text { R-BF } & 1.61 & \mathrm{ABC} \\ \text { O-C13 } & 1.82 & \mathrm{ABCD} \\ \text { O-C6 } & 1.90 & \mathrm{ABCD} \\ \text { R-PC } & 2.23 & \mathrm{ABCD} \\ \text { O-C10 } & 2.15 & \mathrm{ABCDE} \\ \text { O-C4 } & 2.06 & \mathrm{ABCDE} \\ \text { O-C12 } & 2.43 & \mathrm{ABCDE} \\ \text { O-C7 } & \mathrm{ABCDE} \\ \text { O-C5 } & 2.45 & \mathrm{ABCDE} \\ \text { R-OS } & 3.42 & \mathrm{ABCDE} \\ \text { R-AC } & 2.85 & \mathrm{BCDE} \\ \text { O-C8 } & 3.49 & \mathrm{CDE} \\ \text { O-C1 } & 4.47 & \mathrm{CDE} \\ \text { I-C3 } & 4.11 & \mathrm{CDE} \\ \text { I-C2 } & 4.12 & \mathrm{DE} \\ \text { O-C3 } & 4.25 & \mathrm{DE} \\ \text { I-C8 } & 4.27 & \mathrm{DE} \\ \text { O-C2 } & 4.26 & \mathrm{E} \\ \text { O-C } & 5.17 & \end{array}$

TABLEM.32. ANOVA Results for Lead in M. nasuta Tissue

Source of

Variation.

Treatment

Residual
Sum of

Squares

24.870

18.727

d.f
21
100

Mean

Square

1.184

0.187
Significance

Level

0.0001 
TABLEM.33. Mean Concentration and Statistical Grouping for Selenium in M. nasuta Tissue

$\begin{array}{lcc}\begin{array}{c}\text { Sediment } \\ \text { Treatment }\end{array} & \begin{array}{c}\text { Mean Tissue } \\ \text { Concentration } \\ \text { (mg/kg dry weight) }\end{array} & \begin{array}{c}\text { Statistical } \\ \text { Grouping }\end{array} \\ \text { R-AM } & 1.22 & \mathrm{~A} \\ \text { O-C4 } & 1.30 & \mathrm{~A} \\ \text { O-C10 } & 1.35 & \mathrm{AB} \\ \text { O-C5 } & 1.41 & \mathrm{AB} \\ \text { R-PF } & 1.38 & \mathrm{AB} \\ \text { O-C13 } & 1.40 & \mathrm{AB} \\ \text { R-BF } & 1.49 & \mathrm{AB} \\ \text { O-C11 } & 1.47 & \mathrm{AB} \\ \text { O-C9 } & 1.51 & \mathrm{AB} \\ \text { O-C7 } & 1.63 & \mathrm{AB} \\ \text { O-C6 } & 1.59 & \mathrm{AB} \\ \text { O-C3 } & 1.65 & \mathrm{AB} \\ \text { O-C8 } & 1.86 & \mathrm{AB} \\ \text { O-C1 } & 1.78 & \mathrm{AB} \\ \text { O-C12 } & 1.88 & \mathrm{AB} \\ \text { R-AC } & 1.92 & \mathrm{AB} \\ \text { O-C2 } & 1.90 & \mathrm{AB} \\ \text { R-PC } & 1.95 & \mathrm{AB} \\ \text { I-C2 } & 1.94 & \mathrm{AB} \\ \text { I-C3 } & 2.00 & \mathrm{AB} \\ \text { R-OS } & 2.08 & \mathrm{AB} \\ \text { I-C8 } & 2.30 & \mathrm{~B} \\ \text { In } & & \end{array}$

TABLEM.34. ANOVA Results for Selenium in $M$. nasuta Tissue

\begin{tabular}{|c|c|c|c|c|c|}
\hline $\begin{array}{l}\text { Source of } \\
\text { Yariation }\end{array}$ & $\begin{array}{l}\text { Sum of } \\
\text { Squares }\end{array}$ & d.f. & $\begin{array}{l}\text { Mean } \\
\text { Square }\end{array}$ & E-Ratio & $\begin{array}{c}\text { Significance } \\
\text { Level }\end{array}$ \\
\hline $\begin{array}{l}\text { Treatment } \\
\text { Residual }\end{array}$ & $\begin{array}{l}3.786 \\
6.383\end{array}$ & $\begin{array}{r}21 \\
100\end{array}$ & $\begin{array}{l}0.180 \\
0.064\end{array}$ & 2.825 & 0.0003 \\
\hline
\end{tabular}


TABLEM.35 . Mean Concentration and Statistical Grouping for Zinc in $M$. nasuta Tissue

\begin{tabular}{ccc}
$\begin{array}{c}\text { Sediment } \\
\text { Treatment }\end{array}$ & $\begin{array}{c}\text { Mean Tissue } \\
\text { Concentration } \\
\text { (mg/kg dry weight) }\end{array}$ & $\begin{array}{c}\text { Statistical } \\
\text { Grouping }\end{array}$ \\
\cline { 2 - 3 } O-C11 & 70.8 & $\mathrm{~A}$ \\
O-C3 & 82.6 & $\mathrm{AB}$ \\
R-AM & 83.7 & $\mathrm{AB}$ \\
O-C10 & 87.9 & $\mathrm{AB}$ \\
O-C6 & 85.9 & $\mathrm{AB}$ \\
O-C7 & 88.8 & $\mathrm{AB}$ \\
O-C13 & 90.4 & $\mathrm{AB}$ \\
R-BF & 90.3 & $\mathrm{AB}$ \\
O-C2 & 91.7 & $\mathrm{AB}$ \\
O-C9 & 92.6 & $\mathrm{AB}$ \\
R-PC & 91.6 & $\mathrm{AB}$ \\
R-PF & 93.0 & $\mathrm{AB}$ \\
O-C4 & 95.7 & $\mathrm{AB}$ \\
O-C8 & 96.5 & $\mathrm{AB}$ \\
O-C1 & 97.5 & $\mathrm{AB}$ \\
I-C3 & 102.3 & $\mathrm{AB}$ \\
O-C12 & $\mathrm{AB}$ \\
I-C8 & 104.1 & $\mathrm{AB}$ \\
R-OS & 104.7 & $\mathrm{AB}$ \\
I-C2 & 104.2 & $\mathrm{AB}$ \\
O-C5 & 112.5 & $\mathrm{~B}$ \\
R-AC & 113.0 & $\mathrm{~B}$
\end{tabular}

TABLEM.36. ANOVA Results for Zinc in M. nasuta Tissue

\begin{tabular}{lcrrrr}
$\begin{array}{l}\text { Source of } \\
\text { Variation }\end{array}$ & $\begin{array}{l}\text { Sum of } \\
\text { Squares }\end{array}$ & d.f. & $\begin{array}{l}\text { Mean } \\
\text { Square }\end{array}$ & E-Ratio & $\begin{array}{c}\text { Significance } \\
\text { Level }\end{array}$ \\
\hline $\begin{array}{l}\text { Treatment } \\
\text { Residual }\end{array}$ & 1.657 & 21 & 0.079 & 2.026 & 0.0108 \\
& 3.896 & 100 & 0.039 & &
\end{tabular}


IABLEM.37. Mean Concentration and Statistical Grouping for Tributyltin in M. nasuta Tissue

\begin{tabular}{lll} 
Sediment & $\begin{array}{c}\text { Mean Tissue } \\
\text { Concentration } \\
\text { Treatment }\end{array}$ & $\begin{array}{c}\text { Statistical } \\
\text { Grouping }\end{array}$ \\
\cline { 2 - 3 } R-PC & 10.4 & $\mathrm{~A}$ \\
O-C3 & 11.0 & AE \\
I-C3 & 11.1 & AB \\
O-C13 & 11.8 & ABC \\
R-OS & 11.9 & ABC \\
O-C12 & 12.4 & ABCD \\
O-C2 & 13.3 & ABCDE \\
O-C6 & 13.8 & ABCDEF \\
I-C2 & 14.9 & ABCDEF \\
R-AC & 14.7 & ABCDEFG \\
R-PF & ABCDEFG \\
O-C1 & 15.3 & BCDEFGH \\
O-C7 & 15.9 & BCDEFGH \\
O-C.5 & 16.7 & BCDEFGH \\
O-C4 & 16.7 & CDEFGH \\
O-C8 & 17.5 & DEFGH \\
R-AM & 18.0 & DEFGH \\
I-C8 & 18.4 & EFGH \\
R-BF & 18.4 & FGH \\
O-C11 & 19.9 & FGH \\
O-C10 & 20.3 & GH \\
O-C9 & 22.3 & H \\
& 23.4 &
\end{tabular}

TABLEM.38. ANOVA Results for Tributyltin in $M$. nasuta Tissue

\begin{tabular}{|c|c|c|c|c|c|}
\hline $\begin{array}{l}\text { Source of } \\
\text { Variation }\end{array}$ & $\begin{array}{l}\text { Sum of } \\
\text { Squares }\end{array}$ & d.f. & $\begin{array}{l}\text { Mean } \\
\text { Square }\end{array}$ & E-Ratio & $\begin{array}{c}\text { Significance } \\
\text { Level }\end{array}$ \\
\hline $\begin{array}{l}\text { Treatment } \\
\text { Residual }\end{array}$ & $\begin{array}{l}6.495 \\
3.373\end{array}$ & $\begin{array}{l}21 \\
98\end{array}$ & $\begin{array}{l}0.309 \\
0.034\end{array}$ & 8.985 & 0.0001 \\
\hline
\end{tabular}


TABLEM.39. Mean Concentration and Statistical Grouping for Dibutyltin in $M$. nasuta Tissue

$\begin{array}{ccc}\begin{array}{c}\text { Sediment } \\ \text { Treatment }\end{array} & \begin{array}{c}\text { Mean Tissue } \\ \text { Concentration } \\ \text { (ug/kg dry weight) }\end{array} & \begin{array}{c}\text { Statistical } \\ \text { Grouping }\end{array} \\ \text { O-C12 } & 6 & \mathrm{~A} \\ \text { O-C1 } & 6.8 & \mathrm{AB} \\ \text { O-C13 } & 6.9 & \mathrm{AB} \\ \text { I-C3 } & 7.1 & \mathrm{AB} \\ \text { R-AC } & 7.2 & \mathrm{AB} \\ \text { R-PC } & 8.3 & \mathrm{AB} \\ \text { O-C6 } & 7.7 & \mathrm{AB} \\ \text { I-C2 } & 7.8 & \mathrm{AB} \\ \text { O-C3 } & 8.4 & \mathrm{AB} \\ \text { I-C8 } & 8.5 & \mathrm{AB} \\ \text { O-C5 } & 9.1 & \mathrm{AB} \\ \text { O-C8 } & 9 & \mathrm{AB} \\ \text { R-PF } & 9.3 & \mathrm{AB} \\ \text { O-C7 } & 9.5 & \mathrm{AB} \\ \text { O-C9 } & 9.8 & \mathrm{AB} \\ \text { O-C10 } & 10.5 & \mathrm{AB} \\ \text { R-AM } & 10.3 & \mathrm{AB} \\ \text { O-C4 } & 10.9 & \mathrm{AB} \\ \text { O-C2 } & 12.6 & \mathrm{AB} \\ \text { O-C11 } & 11.4 & \mathrm{AB} \\ \text { R-OS } & 16 & \mathrm{AB} \\ \text { R-BF } & 12.9 & \mathrm{~B} \\ \text { - } & & \end{array}$

TABLEM.40. ANOVA Results for Dibutyltin in $M$. nasura 'Tissue

\begin{tabular}{|c|c|c|c|c|c|}
\hline $\begin{array}{l}\text { Source of } \\
\text { Variation }\end{array}$ & $\begin{array}{l}\text { Sum of } \\
\text { Squares }\end{array}$ & d.f. & $\begin{array}{l}\text { Mean } \\
\text { Square }\end{array}$ & E-Ratio & $\begin{array}{c}\text { Significance } \\
\text { Level }\end{array}$ \\
\hline $\begin{array}{l}\text { Treatment } \\
\text { Residual }\end{array}$ & $\begin{array}{r}4.621 \\
10.151\end{array}$ & $\begin{array}{l}21 \\
98\end{array}$ & $\begin{array}{l}0.220 \\
0.104\end{array}$ & 2.125 & 0.0071 \\
\hline
\end{tabular}


TABLEM.41. Mean Concentration and Statistical Grouping for Monobutyltin in M. nasuta Tissue

$\begin{array}{ccc}\begin{array}{c}\text { Sediment } \\ \text { Treatment }\end{array} & \begin{array}{c}\text { Mean Tissue } \\ \text { Concentration } \\ \text { (4g/kg dry weight })\end{array} & \begin{array}{c}\text { Statistical } \\ \text { Grouping }\end{array} \\ \text { O-C12 } & 2.2 & \mathrm{~A} \\ \text { O-C13 } & 2.5 & \mathrm{~A} \\ \text { R-PC } & 2.7 & \mathrm{~A} \\ \text { O-C6 } & 2.8 & \mathrm{~A} \\ \text { I-C3 } & 3.1 & \mathrm{~A} \\ \text { O-C. } & 3.3 & \mathrm{~A} \\ \text { O-C10 } & 3.2 & \mathrm{~A} \\ \text { O-C11 } & 3.3 & \mathrm{~A} \\ \text { R-OS } & 3.5 & \mathrm{~A} \\ \text { I-C2 } & 3.9 & \mathrm{~A} \\ \text { R-AC } & 4.1 & \mathrm{~A} \\ \text { I-C8 } & 4.7 & \mathrm{~A} \\ \text { R-AM } & 5.2 & \mathrm{~A} \\ \text { O-C1 } & 4.2 & \mathrm{~A} \\ \text { O-C2 } & 4.5 & \mathrm{~A} \\ \text { O-C4 } & 6.1 & \mathrm{~A} \\ \text { R-BF } & 5.2 & \mathrm{~A} \\ \text { O-C9 } & 5.9 & \mathrm{~A} \\ \text { O-C3 } & 5.4 & \mathrm{~A} \\ \text { O-C7 } & 9.5 & \mathrm{~A} \\ \text { R-PF } & 19 & \mathrm{~A} \\ \text { O-C8 } & 7.2 & \mathrm{~A}\end{array}$

TABLEM.42. ANOVA Results for Monobutyltin in M. nasuta Tissue

\begin{tabular}{|c|c|c|c|c|c|}
\hline $\begin{array}{l}\text { Source of } \\
\text { Variation }\end{array}$ & $\begin{array}{l}\text { Sum of } \\
\text { Squares }\end{array}$ & d.f. & $\begin{array}{l}\text { Mean } \\
\text { Square }\end{array}$ & E-Ratio & $\begin{array}{c}\text { Significance } \\
\text { Level }\end{array}$ \\
\hline $\begin{array}{l}\text { Treatment } \\
\text { Residual }\end{array}$ & $\begin{array}{l}11.261 \\
31.559\end{array}$ & $\begin{array}{l}21 \\
98\end{array}$ & $\begin{array}{l}0.536 \\
0.322\end{array}$ & 1.665 & 0.0499 \\
\hline
\end{tabular}




\section{APPENDIX N}

\section{STATISTICAL ANALYSIS OF N. CAECOIDES BIOACCUMULATION}


TABLEN.1. Mean Concentration and Statistical Grouping for Naphthalene in $N$. caecoides Tissue

\begin{tabular}{|c|c|c|}
\hline $\begin{array}{l}\text { Sediment } \\
\text { Treatment }\end{array}$ & $\begin{array}{c}\text { Mean } \\
\text { Concentration } \\
(\mu g / \mathrm{kg} \text { dry weight) }\end{array}$ & $\begin{array}{l}\text { Statistical } \\
\text { Grouping }\end{array}$ \\
\hline $\begin{array}{l}\text { O-C7 } \\
\text { O-C4 } \\
\text { O-C5 } \\
\text { O-C6 } \\
\text { R-OS } \\
\text { O-C11 } \\
\text { R-PC } \\
\text { O-C12 } \\
\text { R-AC } \\
\text { O-C8 } \\
\text { O-C13 } \\
\text { I-C4 } \\
\text { I-C3 } \\
\text { I-C2 } \\
\text { I-C8 } \\
\text { R-AM } \\
\text { R-BF } \\
\text { R-PF } \\
\text { O-C2 }\end{array}$ & $\begin{array}{l}107 \\
113 \\
116 \\
125 \\
167 \mathrm{U}(\mathrm{a}) \\
159 \\
166 \mathrm{U} \\
166 \\
185 \\
195 \\
201 \\
222 \\
238 \\
252 \\
289 \\
302 \mathrm{U} \\
310 \mathrm{U} \\
340 \mathrm{U} \\
379\end{array}$ & $\begin{array}{l}\text { A } \\
\text { AB } \\
\text { ABC } \\
\text { ABC } \\
\text { ABCD } \\
\text { ABCDE } \\
\text { ABCDE } \\
\text { ABCDE } \\
\text { ABCDEF } \\
\text { BCDEF } \\
\text { CDEFG } \\
\text { DEFG } \\
\text { DEFG } \\
\text { DEFG } \\
\text { EFG } \\
\text { FG } \\
\text { FG } \\
\text { G } \\
\text { G }\end{array}$ \\
\hline
\end{tabular}

(a) Undetected in ail replicates; value is mean of dry weight detection limits.

TABLEN.2. ANOVA Results for Naphthalene in N. caecoides Tissue

$\begin{array}{lrrrrr}\begin{array}{l}\text { Source of } \\ \text { Variation }\end{array} & \begin{array}{l}\text { Sum of } \\ \text { Squares }\end{array} & \text { d.f. } & \begin{array}{l}\text { Mean } \\ \text { Square }\end{array} & \text { E-Ratio } & \begin{array}{c}\text { Significance } \\ \text { Level }\end{array} \\ \text { Treatment } & 13.950 & 18 & 0.775 & 11.895 & 0.0001 \\ \text { Residual } & 5.343 & 82 & 0.065 & & \end{array}$


TABLEN.3. Mean Concentration and Statistical Grouping for Acenaphthene in $N$. caecoides Tissue

\begin{tabular}{l} 
Sediment \\
Treatment \\
R-PC \\
R-AC \\
R-AM \\
R-OS \\
R-BF \\
O-C13 \\
R-PF \\
I-T5 \\
\hline
\end{tabular}

Mean

Concentration

(ug/kg dry weight)

$55 \mathrm{U}(\mathrm{a})$
$58 \mathrm{U}$
$60 \mathrm{U}$
$63 \mathrm{U}$
$62 \mathrm{U}$
68
$68 \mathrm{U}$
82

Statistical

Grouping
A
A
A
A
A
A
A

(a) Undetected in all replicates; value is mean of dry weight detection limits.

TABLEN44. ANOVA Results for Acenaphthene in N. caecoides Tissue

Source of
Variation

Treatment

Residual

\section{Sum of} Squares

0.319

1.968
Mean

d.f. Square

0.046

0.058
Significance

E-Ratio

0.786

0.6035 
TABLEN.5. Mean Concentration and Statistical Grouping for Fluorene in N. caecoides Tissue

\begin{tabular}{l} 
Sediment \\
Treatment \\
R-PC \\
R-AC \\
R-AM \\
R-OS \\
R-BF \\
R-PF \\
I-T5 \\
O-C13 \\
\hline
\end{tabular}

Mean

Concentration

(ug/kg dry weight)

$55 \mathrm{U}(\mathrm{a})$

$58 \mathrm{U}$

$60 \mathrm{U}$

$63 \mathrm{U}$

$62 \mathrm{U}$

$68 \mathrm{U}$

108

102
Statistical

Grouping
A
A
A
A
A
A
A

(a) Undetected in all replicates; value is mean of dry weight detection limits.

TABLEN.6. ANOVA Results for Fluorene in N. caecoides Tissue

\begin{tabular}{|c|c|c|c|c|c|}
\hline $\begin{array}{l}\text { Source of } \\
\text { Yariation }\end{array}$ & $\begin{array}{l}\text { Sum of } \\
\text { Squanes }\end{array}$ & d.f. & $\begin{array}{l}\text { Mean } \\
\text { Square }\end{array}$ & E-Ratio & $\begin{array}{l}\text { Significance } \\
\text { Level }\end{array}$ \\
\hline $\begin{array}{l}\text { Treatment } \\
\text { Residual }\end{array}$ & $\begin{array}{l}0.857 \\
4.694\end{array}$ & $\begin{array}{r}7 \\
34\end{array}$ & $\begin{array}{l}0.122 \\
0.138\end{array}$ & 0.887 & 0.5271 \\
\hline
\end{tabular}


TABLEN. Mean Concentration and Statistical Grouping for Phenanthrene in N. caecoides Tissue

\begin{tabular}{l} 
Sediment \\
Treament \\
\hline R-PC \\
R-AC \\
O-C11 \\
R-BF \\
R-OS \\
O-C5 \\
R-PF \\
R-AM \\
I-C4 \\
O-C4 \\
O-C8 \\
O-C13 \\
I-T5 \\
\hline
\end{tabular}

Mean

Concentration

He/kg dry weight)

$55 \mathrm{U}(\mathrm{a})$

$58 \mathrm{U}$

58

$62 \mathrm{U}$

$63 \mathrm{U}$

70

$68 \mathrm{U}$

69

72

101

170

204

193

\section{Statistical}

Grouping

A

A

A

A

A

A

A

A

A

A

A

(a) Undetected in all replicates; value is mean of dry weight detection limits.

TABLEN.8. ANOVA Results for Phenanthrene in $N$. caecoides Tissue

\begin{tabular}{|c|c|c|c|c|c|}
\hline $\begin{array}{l}\text { Source of } \\
\text { Yariation }\end{array}$ & $\begin{array}{l}\text { Sum of } \\
\text { Squares }\end{array}$ & d.f & $\begin{array}{l}\text { Mean } \\
\text { Square }\end{array}$ & E-Ratio & $\begin{array}{c}\text { Significance } \\
\text { Level }\end{array}$ \\
\hline $\begin{array}{l}\text { Treatment } \\
\text { Residual }\end{array}$ & $\begin{array}{r}4.695 \\
17.345\end{array}$ & $\begin{array}{l}12 \\
56\end{array}$ & $\begin{array}{l}0.391 \\
0.310\end{array}$ & 1.263 & 0.2660 \\
\hline
\end{tabular}


TABLEN.2. Mean Concentration and Statistical Grouping for Anthracene in N. caecoides Tissue

\begin{tabular}{l} 
Sediment \\
Treatment \\
O-C4 \\
R-PC \\
R-AC \\
R-AM \\
R-OS \\
I-T5 \\
R-BF \\
O-C8 \\
R-PF \\
O-C13 \\
\hline
\end{tabular}

$\begin{gathered}\text { Mean } \\ \text { Concentration } \\ (\mu \mathrm{g} / \mathrm{kg} \text { dry weight) }\end{gathered}$
52
$55 \mathrm{U}(\mathrm{a})$
$58 \mathrm{U}$
$60 \mathrm{U}$
$63 \mathrm{U}$
61
$62 \mathrm{U}$
67
$68 \mathrm{U}$
72

Statistical

Grouping

(a) Undetected in all replicates; value is mean of dry weight detection limits.

TABLEN.10. ANOVA Results for Anthracene in N. caecoides Tissue

Source of

Variation

Treatment

Residual

\section{Surn of}

Squares

0.417

1.610
Mean

d.f. Square

0.046

0.037
A

A

A

A

A

A

A

A

A

$A$
$A$
$A$
$A$
$A$
$A$
$A$
$A$


TABLEN.11. Mean Concentration and Statistical Grouping for Fluoranthene in N. caecoides Tissue

\begin{tabular}{l} 
Sediment \\
Treatment \\
O-C6 \\
R-PC \\
R-AC \\
R-AM \\
R-OS \\
R-BF \\
O-C5 \\
I-T5 \\
R-PF \\
I-C4 \\
O-C11 \\
O-C10 \\
O-C4 \\
O-C8 \\
I-C2 \\
I-C8 \\
O-C13 \\
\hline
\end{tabular}

Mean

Concentration

(1/.g/kg dry weight)

52

$55 \mathrm{U}(\mathrm{a})$

61

$60 \mathrm{U}$

$63 \mathrm{U}$

$62 \mathrm{U}$

64

67

$68 \mathrm{U}$

72

87

146

106

104

121

162

208

\section{Statistical}

Grouping

A

A

$\mathrm{AB}$

$A B$

$A B$

$A B$

$A B$

$A B C$

$A B C$

$A B C$

$A B C$

$A B C$

$A B C$

$A B C$

$A B C$

BC

(a) Undetected in all replicates; value is mean of dry weight detection limits.

TABLEN.12. ANOVA Results for Fluoranthene in N. caecoides Tissue

\begin{tabular}{|c|c|c|c|c|c|}
\hline $\begin{array}{l}\text { Source of } \\
\text { Yariation }\end{array}$ & $\begin{array}{l}\text { Sum of } \\
\text { Squares }\end{array}$ & d.f & $\begin{array}{l}\text { Mean } \\
\text { Square }\end{array}$ & E-Ratio & $\begin{array}{c}\text { Significance } \\
\text { Level }\end{array}$ \\
\hline $\begin{array}{l}\text { Treatment } \\
\text { Residual }\end{array}$ & $\begin{array}{r}9.500 \\
14.665\end{array}$ & $\begin{array}{l}16 \\
72\end{array}$ & $\begin{array}{l}0.594 \\
0.204\end{array}$ & 2.915 & 0.0010 \\
\hline
\end{tabular}


TABLEN.13. Mean Concentration and Statistical Grouping for Pyrene in N. caecoides Tissue

\begin{tabular}{ccc}
$\begin{array}{c}\text { Sediment } \\
\text { Treatment }\end{array}$ & $\begin{array}{c}\text { Mean } \\
\text { Concentration } \\
\text { (ug/kg dry weight) }\end{array}$ & $\begin{array}{c}\text { Statistical } \\
\text { Grouping }\end{array}$ \\
\cline { 2 - 3 } R-PC & $55 \mathrm{U}(\mathrm{a})$ & $\mathrm{A}$ \\
O-C12 & 60 & $\mathrm{~A}$ \\
O-C5 & 59 & $\mathrm{~A}$ \\
I-T5 & 61 & $\mathrm{~A}$ \\
R-OS & $63 \mathrm{U}$ & $\mathrm{A}$ \\
R-BF & $62 \mathrm{U}$ & $\mathrm{A}$ \\
R-PF & $68 \mathrm{U}$ & $\mathrm{AB}$ \\
I-C4 & 80 & $\mathrm{AB}$ \\
O-C6 & 81 & $\mathrm{AB}$ \\
O-C7 & 89 & $\mathrm{ABC}$ \\
O-C4 & 113 & $\mathrm{ABCD}$ \\
I-C2 & 103 & $\mathrm{ABCD}$ \\
R-AM & 109 & $\mathrm{ABCDE}$ \\
R-AC & 159 & $\mathrm{BCDEF}$ \\
I-C8 & 205 & $\mathrm{CDEF}$ \\
O-C10 & 229 & $\mathrm{DEF}$ \\
O-C8 & 225 & $\mathrm{EF}$ \\
O-C11 & 307 & $\mathrm{FG}$ \\
O-C13 & 580 & $\mathrm{G}$ \\
& &
\end{tabular}

(a) Undetected in all replicates; value is mean of dry weight detection limits.

TABLEN.14. ANOVA Results for Pyrene in N. caecoides Tissue

\begin{tabular}{|c|c|c|c|c|c|}
\hline $\begin{array}{l}\text { Source of } \\
\text { Yariation }\end{array}$ & $\begin{array}{l}\text { Sum of } \\
\text { Squares }\end{array}$ & d.f. & $\begin{array}{l}\text { Mean } \\
\text { Square }\end{array}$ & E.Ratio & $\begin{array}{c}\text { Significance } \\
\text { Level }\end{array}$ \\
\hline $\begin{array}{l}\text { Treatment } \\
\text { Residual }\end{array}$ & $\begin{array}{r}39.658 \\
9.635\end{array}$ & $\begin{array}{l}18 \\
81\end{array}$ & $\begin{array}{l}2.203 \\
0.119\end{array}$ & 18.522 & 0.0001 \\
\hline
\end{tabular}


TABLEN.15. Mean Concentration and Statistical Grouping for Benzo(a)anthracene in $N$. caecoides Tissue

\begin{tabular}{l} 
Sediment \\
Treatment \\
R-PC \\
R-AC \\
R-AM \\
R-OS \\
R-BF \\
O-C13 \\
R-PF \\
O-C4 \\
\hline
\end{tabular}

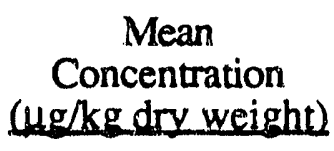

$55 \mathrm{U}(\mathrm{a})$

$58 \mathrm{U}$

$60 \mathrm{U}$

$63 \mathrm{U}$

$62 \mathrm{U}$

68

$68 \mathrm{U}$

104
Statistical

Grouping

A
A
A
A
A
A
A
A

(a) Undetected in ail replicates; value is mean of dry weight detection limits.

TABLEN.16. ANOVA Results for Benzo(a)anthracene in N. caecoides Tissue

Source of

Variation

Treatment

Residual
Sum of

Squares

0.311

4.285
Mean

Square

0.044

0.122
Significance

E-Ratio

0.363

0.9177 
TABLEN.17. Mean Concentration and Statistical Grouping for Chrysene in N. caecoides Tissue

\begin{tabular}{l} 
Sediment \\
Treatment \\
R-PC \\
R-AC \\
R-AM \\
R-OS \\
R-BF \\
R-PF \\
O-C11 \\
O-C4 \\
O-C13 \\
\hline
\end{tabular}

Mean
Concentration
(hg/kgdry wei

$55 \mathrm{U}(\mathrm{a})$
$58 \mathrm{U}$
$60 \mathrm{U}$
$63 \mathrm{U}$
$62 \mathrm{U}$
$68 \mathrm{U}$
71
101
75

Statistical

Grouping

(a) Undetected in all replicates; value is mean of dry weight detection limits.

TABLEN.18. ANOVA Results for Chrysene in N. caecoides Tissue

Source of

Variation

Treatment

Residual
Sum of

Squares

0.392

4.998
Mean

d.f. Square

8

39

0.049

0.128
A
A
A
A
A
A
A
A 
TABLEN.19. Mean Concentration and Statistical Grouping for Benzo(b)fluoranthene in $N$. caecoides Tissue

\begin{tabular}{l} 
Sediment \\
Treament \\
R-PC \\
R-AC \\
R-AM \\
R-OS \\
R-BF \\
R-PF \\
O-C11 \\
O-C4 \\
\hline
\end{tabular}

Mean

Concentration

(ug/kg dry weight)

$55 \mathrm{U}(\mathrm{a})$

$58 \mathrm{U}$

$60 \mathrm{U}$

$63 \mathrm{U}$

$62 \mathrm{U}$

$68 \mathrm{U}$

69

79
Statistical

Grouping
A
A
A
A
A
A
A
A

(a) Undetected in all replicates; value is mean of dry weight detection limits.

TABLEN.20. ANOVA Results for Benzo(b)fluoranthene in N. caecoides Tissue

Source of

Variation

Treatment

Residual
Sum of

Squares

0.183

3.361

$\begin{array}{cl}\text { d.f. } & \begin{array}{l}\text { Mean } \\ \text { Square }\end{array} \\ 7 & 0.026 \\ 35 & 0.096\end{array}$

0.096
Significance

E-Ratio

0.272

0.9608
Level 
TABLEN.21. Mean Concentrations and Statistical Grouping for Benzo(k)fluoranthene in $N$. caecoiales Tissue

\begin{tabular}{ccc}
$\begin{array}{c}\text { Sediment } \\
\text { Treatment }\end{array}$ & $\begin{array}{c}\text { Mean } \\
\text { Concentration } \\
\text { (4g/kgdry weight) }\end{array}$ & $\begin{array}{c}\text { Statistical } \\
\text { Grouping }\end{array}$ \\
\cline { 2 - 3 } R-PC & $55 \mathrm{U}(\mathrm{a})$ & $\mathrm{A}$ \\
R-AC & $58 \mathrm{U}$ & $\mathrm{A}$ \\
R-AM & $60 \mathrm{U}$ & $\mathrm{A}$ \\
R-OS & $63 \mathrm{U}$ & $\mathrm{A}$ \\
R-BF & $62 \mathrm{U}$ & $\mathrm{A}$ \\
R-PF & $68 \mathrm{U}$ & $\mathrm{A}$ \\
O-C11 & 70 & $\mathrm{~A}$ \\
O-C4 & 72 & $\mathrm{~A}$ \\
\hline
\end{tabular}

(a) Undetected in all replicates; value is mean of dry weight detection limits.

TABLEN.22. ANOVA Results for Benzo(k)fluoranthene in N. caecoides Tissue

Source of

Viriation

freatment

Residual
Sum of

Squares

0.166

2.936
Mean

Square

0.024

$35 \quad 0.084$

Significance

E-Ratio

0.283

0.9565 
TABLEN.23. Mean Concentrations and Statistical Grouping for Benzo(a)pyrene in $N$. caecoides Tissue

\begin{tabular}{ccc} 
Sediment & $\begin{array}{c}\text { Mean } \\
\text { Concentration } \\
\text { Treatment }\end{array}$ & $\begin{array}{c}\text { Statistical } \\
\text { Grouping }\end{array}$ \\
R-PC & $55 \mathrm{~kg}$ dry weight $)$ & $\mathrm{A}$ \\
R-AC & $58 \mathrm{U}$ & $\mathrm{A}$ \\
O-C4 & 60 & $\mathrm{~A}$ \\
R-AM & $60 \mathrm{U}$ & $\mathrm{A}$ \\
R-OS & $63 \mathrm{U}$ & $\mathrm{A}$ \\
R-BF & $62 \mathrm{U}$ & $\mathrm{A}$ \\
O-C11 & 67 & $\mathrm{~A}$ \\
R-PF & $68 \mathrm{U}$ & $\mathrm{A}$ \\
\hline
\end{tabular}

(a) Undetected in all replicates; value is mean of dry weight detection limits.

TABLEN24. ANOVA Results for Benzo(a)pyrene in N. caecoides Tissue

\begin{tabular}{|c|c|c|c|c|c|}
\hline $\begin{array}{l}\text { Source of } \\
\text { Yariation }\end{array}$ & $\begin{array}{l}\text { Sum of } \\
\text { Squares }\end{array}$ & d.f. & $\begin{array}{l}\text { Mean } \\
\text { Square }\end{array}$ & E-Ratio & $\begin{array}{c}\text { Significance } \\
\text { Level }\end{array}$ \\
\hline $\begin{array}{l}\text { Treatment } \\
\text { Residual }\end{array}$ & $\begin{array}{l}0.169 \\
1.857\end{array}$ & $\begin{array}{r}7 \\
35\end{array}$ & $\begin{array}{l}0.024 \\
0.053\end{array}$ & 0.456 & 0.8593 \\
\hline
\end{tabular}


TABLEN.25 . Mean Concentrations and Statistical Grouping for Silver in N. caecoides Tissue

\begin{tabular}{lcc}
$\begin{array}{c}\text { Sediment } \\
\text { Treatment }\end{array}$ & $\begin{array}{c}\text { Mean } \\
\text { Concentration } \\
\text { (mg/kg dry weight) }\end{array}$ & $\begin{array}{c}\text { Statistical } \\
\text { Grouping }\end{array}$ \\
\hline O-C1 & 0.014 & A \\
O-C3 & 0.017 & AB \\
O-C2 & 0.019 & AB \\
I-C8 & 0.021 & BC \\
I-C2 & 0.024 & BCD \\
O-C7 & 0.028 & CDE \\
O-C8 & 0.033 & DEF \\
I-C3 & 0.034 & DEF \\
R-OS & 0.035 & EFG \\
R-PF & 0.037 & EFG \\
O-C12 & 0.041 & FG \\
O-C11 & FG \\
R-PC & 0.042 & FG \\
I-C4 & 0.042 & FG \\
R-BF & 0.043 & FG \\
O-C13 & 0.044 & FGH \\
O-C6 & 0.046 & GH \\
O-C9 & 0.048 & HI \\
R-AM & 0.064 & I \\
R-AC & 0.079 & I \\
O-C5 & 0.081 & I \\
I-T5 & 0.083 & I \\
O-C10 & 0.083 & J \\
0-C4 & 0.085 & \\
& 0.209 &
\end{tabular}

TABLEN.26. Anova Results for Silver in N. caecoides Tissue

\begin{tabular}{|c|c|c|c|c|c|}
\hline $\begin{array}{l}\text { Source of } \\
\text { Variation }\end{array}$ & $\begin{array}{l}\text { Sum of } \\
\text { Squares }\end{array}$ & d.f. & $\begin{array}{l}\text { Mean } \\
\text { Square }\end{array}$ & E-Ratio & $\begin{array}{c}\text { Significance } \\
\text { Level }\end{array}$ \\
\hline $\begin{array}{l}\text { Treatment } \\
\text { Residual }\end{array}$ & $\begin{array}{r}26.577 \\
0.537\end{array}$ & $\begin{array}{l}23 \\
48\end{array}$ & $\begin{array}{l}0.156 \\
0.012\end{array}$ & 98.915 & 0.0001 \\
\hline
\end{tabular}


TABLEN.27. Mean Concentrations and Statistical Grouping for Arsenic in N. caecoides Tissue

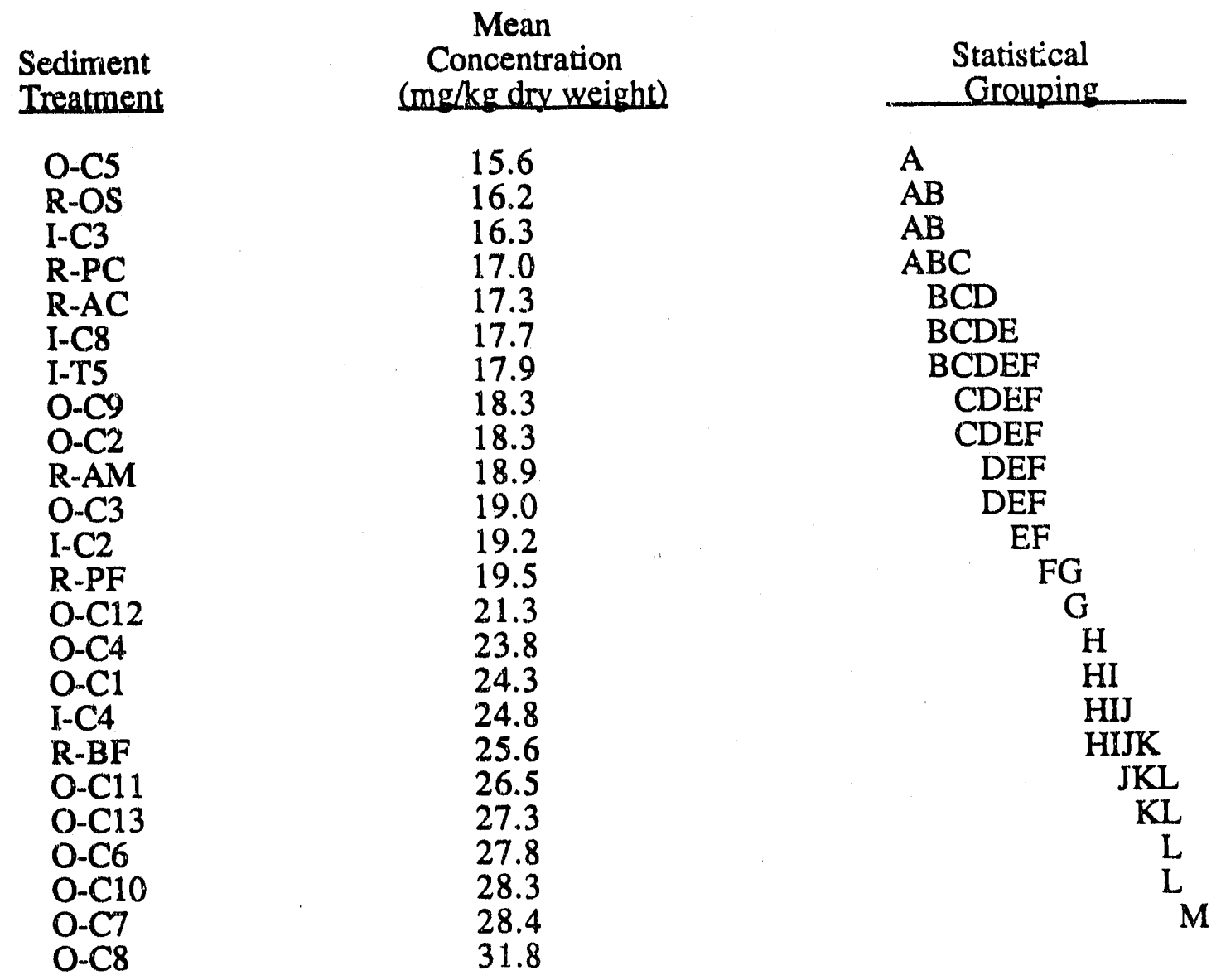

TABLEN.28. Anova Results for Arsenic in N. caecoides Tissue

\begin{tabular}{|c|c|c|c|c|c|}
\hline $\begin{array}{l}\text { Source of } \\
\text { Yariation }\end{array}$ & $\begin{array}{l}\text { Sum of } \\
\text { Squares }\end{array}$ & d.f. & $\begin{array}{l}\text { Mean } \\
\text { Square }\end{array}$ & E-Ratio & $\begin{array}{c}\text { Significance } \\
\text { Level }\end{array}$ \\
\hline $\begin{array}{l}\text { Treatment } \\
\text { Residual }\end{array}$ & $\begin{array}{l}3.263 \\
0.047\end{array}$ & $\begin{array}{l}23 \\
48\end{array}$ & $\begin{array}{l}0.142 \\
0.001\end{array}$ & 146.039 & 0.0001 \\
\hline
\end{tabular}


TABLEN.29. Mean Concentrations and Statistical Grouping for Cadmium in N. caecoides Tissue

\begin{tabular}{lcc}
$\begin{array}{c}\text { Sediment } \\
\text { Treatment }\end{array}$ & $\begin{array}{c}\text { Mean } \\
\text { Concentration } \\
\text { (mg/kg dry weight) }\end{array}$ & $\begin{array}{c}\text { Statistical } \\
\text { Grouping }\end{array}$ \\
\hline I-C3 & 1.10 & A \\
O-C1 & 1.18 & AB \\
O-C2 & 1.21 & ACB \\
I-C2 & 1.27 & ABCD \\
O-C3 & 1.32 & ABCDE \\
R-PF & 1.35 & BCDE \\
R-AM & 1.36 & BCDE \\
I-C8 & 1.36 & BCDE \\
O-C5 & 1.45 & CDEF \\
R-PC & 1.47 & DEF \\
R-AC & 1.47 & DEFG \\
R-BF & 1.52 & DEFG \\
R-OS & 1.56 & EFGH \\
O-C10 & 1.74 & FGHI \\
O-C4 & 1.74 & FGHI \\
O-C7 & 1.75 & FGHIJ \\
O-C8 & 1.77 & GHIJ \\
O-C13 & HIJ \\
O-C9 & 1.85 & HIJ \\
I-C4 & 1.85 & IJ \\
O-C12 & 1.89 & IJ \\
O-C11 & 1.96 & IJ \\
O-C6 & 2.03 & J \\
I-T5 & 2.07 & J \\
& 2.10 &
\end{tabular}

TABLEN.30. ANOVA Results for Cadmium in N. caecoides Tissue

\begin{tabular}{|c|c|c|c|c|c|}
\hline $\begin{array}{l}\text { Source of } \\
\text { Variation }\end{array}$ & $\begin{array}{l}\text { Sum of } \\
\text { Squares }\end{array}$ & d.f. & $\begin{array}{l}\text { Mean } \\
\text { Square }\end{array}$ & E-Ratio & $\begin{array}{c}\text { Significance } \\
\text { Level }\end{array}$ \\
\hline $\begin{array}{l}\text { Treatment } \\
\text { Residual }\end{array}$ & $\begin{array}{l}2.504 \\
0.153\end{array}$ & $\begin{array}{l}23 \\
46\end{array}$ & $\begin{array}{l}0.109 \\
0.003\end{array}$ & 32.744 & 0.0001 \\
\hline
\end{tabular}


TABLE N.31. Mean Concentrations and Statistical Grouping for Chromium in N. caecoides Tissue

$\begin{array}{lll}\begin{array}{l}\text { Sediment } \\ \text { Treatment }\end{array} & \begin{array}{c}\text { Mean } \\ \text { Concentration } \\ \text { (mg/kgdryweight) }\end{array} & \begin{array}{c}\text { Statistical } \\ \text { Grouping }\end{array} \\ \text { O-C3 } & 0.168 & \mathrm{~A} \\ \text { R-OS } & 0.212 & \mathrm{AB} \\ \text { I-C4 } & 0.235 & \mathrm{ABC} \\ \text { O-C2 } & 0.243 & \mathrm{ABC} \\ \text { O-C1 } & 0.288 & \mathrm{ABC} \\ \text { O-CC7 } & 0.313 & \mathrm{ABC} \\ \text { R-AC } & 0.309 & \mathrm{ABC} \\ \text { R-PF } & 0.337 & \mathrm{ABC} \\ \text { O-C5 } & 0.332 & \mathrm{ABC} \\ \text { O-C6 } & 0.347 & \mathrm{ABC} \\ \text { R-AM } & 0.362 & \mathrm{ABC} \\ \text { O-C9 } & 0.390 & \mathrm{ABC} \\ \text { I-C8 } & 0.404 & \mathrm{ABC} \\ \text { I-C2 } & 0.453 & \mathrm{ABC} \\ \text { O-C12 } & 0.454 & \mathrm{ABC} \\ \text { I-OT5 } & 0.465 & \mathrm{ABC} \\ \text { O-C11 } & 0.485 & \mathrm{ABC} \\ \text { O-C13 } & 0.471 & \mathrm{ABC} \\ \text { R-BF } & 0.526 & \mathrm{ABC} \\ \text { I-C3 } & 0.918 & \mathrm{ABC} \\ \text { R-PC } & 1.501 & \mathrm{BCD} \\ \text { O-C8 } & 2.939 & \mathrm{BCD} \\ \text { O-C10 } & 1.413 & \mathrm{CD} \\ \text { O-C4 } & 3.950 & \mathrm{D} \\ & & \end{array}$

TABLEN.32. ANOVA Results for Chromium in $N$. caecoides Tissue

\begin{tabular}{|c|c|c|c|c|c|}
\hline $\begin{array}{l}\text { Source of } \\
\text { Variation }\end{array}$ & $\begin{array}{l}\text { Sum of } \\
\text { Squares }\end{array}$ & d.f. & $\begin{array}{l}\text { Mean } \\
\text { Square }\end{array}$ & E-Ratio & $\begin{array}{c}\text { Significance } \\
\text { Level }\end{array}$ \\
\hline $\begin{array}{l}\text { Treatment } \\
\text { Residual }\end{array}$ & $\begin{array}{l}32.148 \\
14.555\end{array}$ & $\begin{array}{l}23 \\
46\end{array}$ & $\begin{array}{l}1.398 \\
0.316\end{array}$ & 4.417 & 0.0001 \\
\hline
\end{tabular}


TABLEN.33. Mean Concentrations and Statistical Grouping for Copper in N. caecoides Tissue

\begin{tabular}{lcc} 
Sediment & $\begin{array}{c}\text { Mean } \\
\text { Treatment }\end{array}$ & $\begin{array}{c}\text { Statistical } \\
\text { Grouping } \\
\text { (m) } / \text { kg dry weight) }\end{array}$ \\
\hline O-C1 & 7.8 & A \\
I-C8 & 9.2 & AB \\
I-C2 & 9.1 & AB \\
O-C3 & 9.7 & ABC \\
R-OS & 10.5 & BCD \\
I-C3 & 10.7 & BCDE \\
O-C2 & 10.9 & BCDEF \\
O-C7 & 11.0 & BCDEF \\
O-C9 & 11.4 & BCDEFG \\
R-PF & 11.9 & CDEFGH \\
O-C8 & 12.6 & DEFGHI \\
O-C5 & 13.2 & DEFGHI \\
O-C12 & 13.2 & DEFGHI \\
R-AM & 13.3 & DEFGHI \\
O-C6 & 13.6 & EFGHI \\
O-C4 & 13.7 & FGHI \\
R-PC & 13.6 & FGHI \\
I-C4 & 13.8 & FGHI \\
O-C11 & 14.5 & GHI \\
R-BF & 14.7 & HI \\
I-T5 & 14.7 & HI \\
O-C10 & 14.8 & HI \\
R-AC & 15.1 & HI \\
O-C13 & 15.3 & I \\
& &
\end{tabular}

TABLEN.34. ANOVA Results for Copper in N. caecoides Tissue

\begin{tabular}{|c|c|c|c|c|c|}
\hline $\begin{array}{l}\text { Source of } \\
\text { Yariation }\end{array}$ & $\begin{array}{l}\text { Sum of } \\
\text { Squares }\end{array}$ & d.f. & $\begin{array}{l}\text { Mean } \\
\text { Square }\end{array}$ & E-Ratio & $\begin{array}{c}\text { Significande } \\
\text { Level }\end{array}$ \\
\hline $\begin{array}{l}\text { Treatment } \\
\text { Residual }\end{array}$ & $\begin{array}{l}2.399 \\
0.299\end{array}$ & $\begin{array}{l}23 \\
48\end{array}$ & $\begin{array}{l}0.104 \\
0.006\end{array}$ & 16.725 & 0.0001 \\
\hline
\end{tabular}


TABLEN.35. Mean Concentrations and Statistical Grouping for Mercury in N. caecoides Tissue

\begin{tabular}{lcc} 
Sediment & $\begin{array}{c}\text { Mean } \\
\text { Concentration } \\
\text { Treatment }\end{array}$ & $\begin{array}{c}\text { Statistical } \\
\text { Grouping }\end{array}$ \\
\hline O-C6 & 0.029 & A \\
O-C13 & 0.029 & A \\
O-C11 & 0.035 & AB \\
I-T5 & 0.041 & BC \\
O-C12 & 0.041 & BC \\
O-C2 & 0.042 & BC \\
O-C10 & 0.042 & BC \\
I-C4 & 0.046 & BCD \\
O-C1 & 0.046 & CD \\
O-C7 & 0.048 & CDE \\
O-C8 & 0.049 & CDEF \\
I-C8 & 0.050 & CDEF \\
I-C3 & 0.050 & CDEF \\
R-AC & 0.051 & CDEF \\
I-C2 & 0.057 & DEFG \\
O-C9 & 0.060 & EFG \\
R-OAM & 0.060 & FG \\
R-OS & 0.063 & FG \\
R-PC & 0.064 & GH \\
R-BF & 0.067 & GH \\
R-PF & 0.070 & GH \\
O-C3 & 0.072 & H \\
O-C4 & 0.088 & I \\
& &
\end{tabular}

TABLEN.36. ANOVA Results for Mercury in N. caecoides Tissue

\begin{tabular}{|c|c|c|c|c|c|}
\hline $\begin{array}{l}\text { Source of } \\
\text { Yariation }\end{array}$ & $\begin{array}{l}\text { Sum of } \\
\text { Squares }\end{array}$ & d.f. & $\begin{array}{l}\text { Mean } \\
\text { Square }\end{array}$ & E-Ratio & $\begin{array}{c}\text { Significance } \\
\text { Level }\end{array}$ \\
\hline $\begin{array}{l}\text { Treatment } \\
\text { Residual }\end{array}$ & $\begin{array}{l}9.771 \\
0.321\end{array}$ & $\begin{array}{l}23 \\
46\end{array}$ & $\begin{array}{l}0.425 \\
0.007\end{array}$ & 60.804 & 0.0001 \\
\hline
\end{tabular}


TABLEN.39. Mean Concentrations and Statistical Grouping for Nickel in N. caecoides Tissue

\begin{tabular}{|c|c|}
\hline $\begin{array}{l}\text { Sediment } \\
\text { Treatment }\end{array}$ & $\begin{array}{c}\text { Mean } \\
\text { Concentration } \\
\text { (mg/kg dry weight) }\end{array}$ \\
\hline $\begin{array}{l}\text { O-C12 } \\
\text { I-C4 } \\
\text { O-C10 } \\
\text { R-PC } \\
\text { O-C11 } \\
\text { O-C8 } \\
\text { R-OS } \\
\text { R-BF } \\
\text { O-C6 } \\
\text { O-C7 } \\
\text { R-PF } \\
\text { O-C9 } \\
\text { R-AC } \\
\text { I-C3 } \\
\text { R-AM } \\
\text { O-C2 } \\
\text { I-C2 } \\
\text { O-C5 } \\
\text { O-C3 } \\
\text { I-C8 } \\
\text { O-C13 } \\
\text { O-C4 } \\
\text { I-T5 } \\
\text { O-C1 }\end{array}$ & $\begin{array}{l}1.9 \\
2.0 \mathrm{U}(\mathrm{a}) \\
2.1 \\
2.2 \\
2.3 \\
2.3 \\
2.5 \\
2.4 \\
2.4 \\
2.5 \\
2.5 \\
2.6 \\
2.8 \\
2.9 \\
2.8 \\
2.9 \\
3.0 \\
3.3 \\
3.4 \\
3.4 \\
4.4 \\
3.7 \\
3.8 \\
4.0\end{array}$ \\
\hline
\end{tabular}

\section{Statistical} Grouping

A

A

A

A

A

A

A

A

A

A

A

A

A

A

A

A

A

A

A

A

A

(a) Undetected in all replicates; value is mean of dry weight detection limits.

TABLEN.38. ANOVA Results for Nickel in N. caecoides Tissue

Source of

Variation

Treatment

Residual
Sum of

Squares

3.141

3.974
Mean

d.f. Square

23

48

0.137

0.083
Significant

E-Ratio

1.649

0.0718 
TABLEN.39. Mean Concentrations and Statistical Grouping for Lead in N. caecoides Tissue

$\begin{array}{ccc}\begin{array}{c}\text { Sediment } \\ \text { Treatment }\end{array} & \begin{array}{c}\text { Mean } \\ \text { Concentration } \\ \text { (mg/kg dry weight }\end{array} & \begin{array}{c}\text { Statistical } \\ \text { Grouping }\end{array} \\ \text { O-C5 } & 0.20 & \mathrm{~A} \\ \text { O-C9 } & 0.35 & \mathrm{AB} \\ \text { O-C3 } & 0.36 & \mathrm{BC} \\ \text { I-C3 } & 0.38 & \mathrm{BC} \\ \text { O-C1 } & 0.45 & \mathrm{BCD} \\ \text { I-C8 } & 0.45 & \mathrm{BCD} \\ \text { O-C2 } & 0.52 & \mathrm{BCDE} \\ \text { I-T5 } & 0.53 & \mathrm{BCDE} \\ \text { R-OS } & 0.54 & \mathrm{BCDE} \\ \text { R-PF } & 0.55 & \text { BCDE } \\ \text { R-AM } & 0.63 & \text { BCDEF } \\ \text { R-AC } & 0.77 & \text { CDEFG } \\ \text { R-PC } & 0.84 & \text { DEFGH } \\ \text { I-C2 } & 0.89 & \text { EFGH } \\ \text { O-C12 } & 1.03 & \text { EFGH } \\ \text { O-C8 } & 1.06 & \text { FGH } \\ \text { I-C4 } & 1.08 & \text { FGH } \\ \text { O-C6 } & 1.14 & \text { FGH } \\ \text { O-C7 } & 1.25 & \text { GH } \\ \text { O-C11 } & 1.29 & \text { GHI } \\ \text { R-BF } & 1.29 & \text { GHI } \\ \text { O-C13 } & 1.38 & \text { HI } \\ \text { O-C10 } & 6.41 & \text { J } \\ \text { O-C4 } & & \end{array}$

TABLEN.40. ANOVA Results for Lead in N. caecoides Tissue

\begin{tabular}{|c|c|c|c|c|c|}
\hline $\begin{array}{l}\text { Source of } \\
\text { Variation }\end{array}$ & $\begin{array}{l}\text { Sum of } \\
\text { Squares }\end{array}$ & d.f. & $\begin{array}{l}\text { Mean } \\
\text { Square }\end{array}$ & E-Ratio & $\begin{array}{l}\text { Significant } \\
\text { Level }\end{array}$ \\
\hline $\begin{array}{l}\text { Treatment } \\
\text { Residual }\end{array}$ & $\begin{array}{r}35.376 \\
1.977\end{array}$ & $\begin{array}{l}23 \\
47\end{array}$ & $\begin{array}{l}1.538 \\
0.042\end{array}$ & 36.558 & 0.0001 \\
\hline
\end{tabular}


TABLEN.41. Mean Concentrations and Statistical Grouping for Selenium in N. caecoides Tissue

$\begin{array}{lcc}\begin{array}{c}\text { Sediment } \\ \text { Treatment }\end{array} & \begin{array}{c}\text { Mean } \\ \text { Concentration } \\ \text { (mg/kgdry weight) }\end{array} & \begin{array}{c}\text { Statistical } \\ \text { Grouping }\end{array} \\ \text { T-81 } & 1.51 & \mathrm{~A} \\ \text { O-C3 } & 1.56 & \mathrm{~A} \\ \text { O-C13 } & 1.65 & \mathrm{AB} \\ \text { R-PC } & 1.66 & \mathrm{ABC} \\ \text { I-C8 } & 1.67 & \mathrm{ABC} \\ \text { O-C10 } & 1.67 & \mathrm{ABC} \\ \text { I-C3 } & 1.73 & \mathrm{ABC} \\ \text { R-AC } & 1.74 & \mathrm{ABC} \\ \text { O-C2 } & 1.74 & \mathrm{ABC} \\ \text { I-C4 } & 1.74 & \mathrm{ABC} \\ \text { R-OS } & 1.74 & \mathrm{ABC} \\ \text { O-C12 } & 1.83 & \mathrm{ABC} \\ \text { O-C4 } & 1.84 & \mathrm{ABC} \\ \text { R-AM } & 1.95 & \mathrm{BC} \\ \text { O-C7 } & 1.95 & \mathrm{BC} \\ \text { R-BF } & 2.02 & \mathrm{BC} \\ \text { O-C6 } & 2.00 & \mathrm{BC} \\ \text { O-C5 } & 2.01 & \mathrm{BC} \\ \text { R-PF } & 2.02 & \mathrm{BC} \\ \text { O-C8 } & 2.02 & \mathrm{BC} \\ \text { G-C8 } & 2.06 & \mathrm{C}\end{array}$

TABLEN.42. ANOVA Results for Selenium in N. caecoides Tissue

Source of

Yariation

Treatment

Residual
Sum of

Squares

0.501

0.203

$d . f$
23
45

23
45
Mean Square

0.022

0.005
E-Ratio

4.819

Significant Level

0.0001 
TABLEN.43. Mean Concentrations and Statistical Grouping for Zinc in N. caecoides Tissue

\begin{tabular}{ccc} 
Sediment & $\begin{array}{c}\text { Mean } \\
\text { Concentration } \\
\text { Treatment }\end{array}$ & \multicolumn{1}{c}{$\begin{array}{c}\text { Statistical } \\
\text { Grouping }\end{array}$} \\
\cline { 3 - 3 } O-C2 & 152 dry weight) & A \\
O-C5 & 154.8 & AB \\
I-C3 & 159.3 & ABC \\
O-C3 & 160.4 & ABCD \\
I-C8 & 160.8 & ABCDE \\
R-OS & 162.6 & ABCDEF \\
O-C13 & 163.3 & ABCDEF \\
I-C2 & 163.4 & ABCDEF \\
O-C11 & 165.4 & ABCDEFG \\
I-T5 & 166.2 & BCDEFG \\
I-C4 & 166.8 & BCDEFG \\
O-C4 & 168.9 & CDEFG \\
R-PF & 168.9 & CDEFG \\
R-PC & 169.4 & CDEFG \\
O-C1 & CDEFG \\
O-C9 & 169.6 & CDEFG \\
O-C8 & 170.2 & CDEFG \\
O-C7 & 171.9 & CDEFG \\
O-C10 & 172.2 & DEFG \\
R-AC & 174.5 & EFG \\
O-C6 & 174.6 & FGH \\
O-C12 & 176.5 & GH \\
R-AM & 178.8 & HI \\
R-BF & 191.5 &
\end{tabular}

TABLEN.44. ANOVA Results for Zinc in N. caecoides Tissue

\begin{tabular}{|c|c|c|c|c|c|}
\hline $\begin{array}{l}\text { Source of } \\
\text { Variation }\end{array}$ & $\begin{array}{l}\text { Sum of } \\
\text { Squares }\end{array}$ & d.f. & $\begin{array}{l}\text { Mean } \\
\text { Square }\end{array}$ & E-Ratio & $\begin{array}{c}\text { Significance } \\
\text { Level }\end{array}$ \\
\hline $\begin{array}{l}\text { Treatment } \\
\text { Residual }\end{array}$ & $\begin{array}{l}0.232 \\
0.035\end{array}$ & $\begin{array}{l}23 \\
48\end{array}$ & $\begin{array}{l}0.010 \\
0.001\end{array}$ & 13.752 & 0.0001 \\
\hline
\end{tabular}


TABLEN.45. Mean Concentrations and Statistical Grouping for Tributyltin in N. caecoides Tissue

\begin{tabular}{cc}
$\begin{array}{c}\text { Sediment } \\
\text { Treatment }\end{array}$ & $\begin{array}{c}\text { Mean } \\
\text { Concentration } \\
\text { (ug/kg dry weight })\end{array}$ \\
O-C10 & 56.9 \\
R-PC & $65.7 \mathrm{UJ}(\mathrm{a})$ \\
O-C1 & 79.1 \\
I-T5 & 87.8 \\
I-C4 & 98.5 \\
R-AM & $114.9 \mathrm{U}(\mathrm{b})$ \\
O-C9 & 119.9 \\
R-BF & $117.8 \mathrm{U}$ \\
R-AC & 128.4 \\
R-PF & $128.0 \mathrm{U}$ \\
O-C3 & 138.2 \\
R-OS & 154.2 \\
O-C11 & 837.1 \\
& \\
\hline
\end{tabular}

Statistical
Grouping

A

A

A

A

A

A

A

A

A

A

A

A

(a) Undetected or detected below method detection limit in all replicates; concentration is mean of dry weight detection limits and detected values.

(b) Undetected in all replicates; concentration is mean of dry weight detection limits.

TABLEN.46. ANOVA Results for Tributyltin in N. caecoides Tissue

\begin{tabular}{l} 
Source of \\
Variation \\
\hline
\end{tabular}

Treatment

Residual
Sum of

Squares

13.450

37.012

d.f.
12
53

Mean

Square

1.121

0.698
Significance

F-Ratio

1.605

0.1185 
TABLEN.47. Mean Concentrations and Statistical Grouping for Dibutyltin in N. caecoides Tissue

\begin{tabular}{lc}
$\begin{array}{c}\text { Sediment } \\
\text { Treatment }\end{array}$ & $\begin{array}{c}\text { Mean } \\
\text { Concentration } \\
\text { (ug/kg dry weigh }\end{array}$ \\
R-PC & $48.3 \mathrm{U}(\mathrm{a})$ \\
R-AC & $52.0 \mathrm{U}$ \\
O-C13 & 60.0 \\
O-C11 & 62.1 \\
R-OS & $62.1 \mathrm{U}$ \\
I-C2 & 96.1 \\
O-C4 & 89.9 \\
O-C3 & 93.4 \\
R-PF & 140.2 \\
R-BF & 159.5 \\
R-AM & 164.3 \\
\hline
\end{tabular}

\section{Statistical \\ Grouping}

A
A
A
A
A
A
A
A
A
A
A

(a) Undetected in all replicates; concentration is mean of dry weight detection limits.

TABLEN.48. ANOVA Results for Dibutyltin in $N$. caecoides Tissue

\begin{tabular}{|c|c|c|c|c|c|}
\hline $\begin{array}{l}\text { Source of } \\
\text { Variation }\end{array}$ & $\begin{array}{l}\text { Sum of } \\
\text { Squares }\end{array}$ & d.f. & $\begin{array}{l}\text { Mean } \\
\text { Square }\end{array}$ & E-Ratio & $\begin{array}{c}\text { Significance } \\
\text { Level }\end{array}$ \\
\hline $\begin{array}{l}\text { Treatment } \\
\text { Residual }\end{array}$ & $\begin{array}{r}8.889 \\
13.857\end{array}$ & $\begin{array}{l}10 \\
45\end{array}$ & $\begin{array}{l}0.889 \\
0.308\end{array}$ & 2.887 & 0.0072 \\
\hline
\end{tabular}


TABLEN 49. Mean Concentrations and Statistical Grouping for Monobutyltin in N. caecoides Tissue

\begin{tabular}{ccc}
$\begin{array}{c}\text { Sediment } \\
\text { Ireatment }\end{array}$ & $\begin{array}{c}\text { Mean } \\
\text { Concentration } \\
(\mu / \text { /kg dry weight) }\end{array}$ & $\begin{array}{r}\text { Statist } \\
\text { Group }\end{array}$ \\
R-PC & $43.9 \mathrm{U}(\mathrm{a})$ & $\mathrm{A}$ \\
R-AC & $47.3 \mathrm{U}$ & $\mathrm{A}$ \\
O-C13 & 53.1 & $\mathrm{~A}$ \\
R-AM & $49.3 \mathrm{U}$ & $\mathrm{A}$ \\
R-BF & $50.5 \mathrm{U}$ & $\mathrm{A}$ \\
I-C2 & 51.8 & $\mathrm{~A}$ \\
R-OS & 57.5 & $\mathrm{~A}$ \\
R-PF & $54.9 \mathrm{U}$ & $\mathrm{A}$ \\
\hline
\end{tabular}

(a) Undetected in all replicates; concentration is mean of dry weight detection limits.

TABLEN.50. ANOVA Results for Monobutyltin in N. caecoides Tissue

Source of

Variation

Treatment

Residual

\section{Sum of}

Squares

0.224

2.292

$\begin{array}{cl}\text { d.f. } & \begin{array}{l}\text { Mean } \\ \text { Square }\end{array} \\ 7 & 0.032 \\ 34 & 0.067\end{array}$

$\frac{\text { E-Ratio }}{0.475}$

Significance

Level

0.8457 


\section{DISTRIBUTION}

No. of

Conies

\section{OFESITE}

2 DOE/Office of Scientific and Technical Information

D. Roberts

U.S. Army Corps of Engineers San Francisco District

211 Main Street

San Francisco, CA 94105

$5 \quad$ R. Chisholm

U.S. Army Corps of Engineers

San Francisco District

211 Main Street

San Francisco, CA 94105

J. Harari

U.S. Army Corps of Engineers

San Francisco District

211 Main Street

San Francisco, CA 94105

$2 \quad$ K. Guy

U.S. Army Corps of Engineers

San Francisco District

211 Main Street

San Francisco, CA 94105
No. of

Copies

QNSLTE

DOE Richland Eield Office

P. W. Kruger

36 Pacific Northwest Laboratory

E. S. Barrows

R. Cuello

R. M. Ecker

M. R. Finney

W. R. Gorst

N. P. Kohn (5)

L. F. Lefkovitz

H. L. Mayhew

J. A. Trelstad (2)

J. A. Ward (5)

J. Q. Word (10)

Publishing Coordination

Technical Report Files (5)

\section{Routing}

J. W. Falco

M. J. Graham

R. L. Skaggs

P.C. Hays (last) 

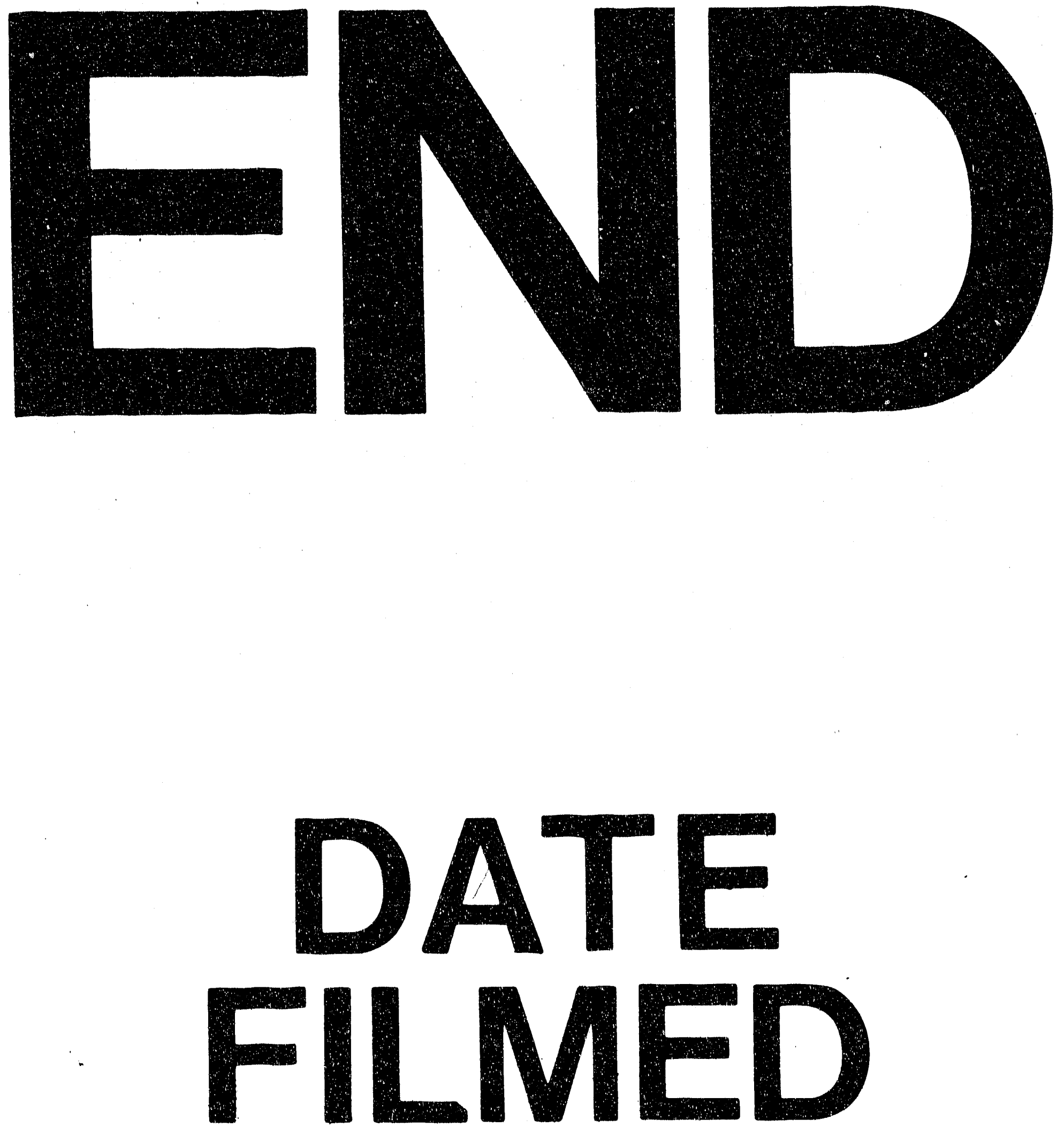

1

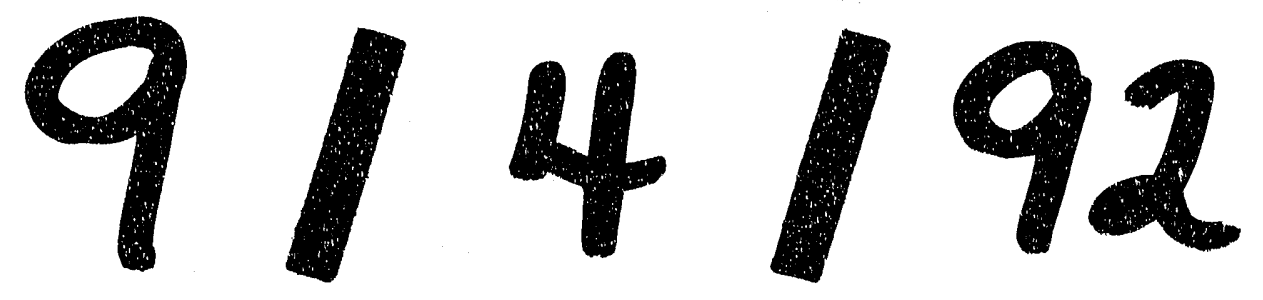


